\title{
Mechanical Properties of Thin Films
}

\author{
William D. Nix \\ Department of Materials Science and Engineering \\ Stanford University
}

An incomplete set of class notes for a graduate class at Stanford University: Materials Science and Engineering 353, Mechanical

Properties of Thin Films

January 2005

(some figures kindly provided by various Stanford students have not yet been fully acknowledged)

\author{
Prof. W.D. Nix \\ Department of Materials Science and Engineering \\ Stanford University \\ Stanford CA 94305-2205 \\ Phone: 650-725-2605 \\ Fax: $650-725-4034$ \\ E-mail: nix@stanford.edu
}


Materials Science and Engineering 353

Mechanical Properties of Thin Films
Winter Quarter 2005

Prof. W.D. Nix

\section{Introduction}

Thin Film = Layer of material with one dimension much smaller than the other two. Here we focus mainly on thin films attached to substrates, usually much thicker than the film in question.

Thin film materials have become technologically important in recent years. Some examples are:

- $\quad$ Microelectronic Integrated Circuits

- Magnetic Information Storage Systems

- Optical Coatings

- Wear Resistant Coatings

- Corrosion Resistant Coatings

Motivation for the use of materials in thin film form:

- $\quad$ Need For Small-Scale Devices (I.C.'s, magnetic storage)

- $\quad$ Physical Properties That Are Scale-Dependent (optical filters)

- $\quad$ Cost Benefits (use small amounts of expensive materials for coatings)

Although we typically think of thin film based devices in terms of their electronic, magnetic or optical properties, many such devices are limited by their mechanical properties -- important to understand mechanical properties of thin films 


\section{Thin Film Definitions}

\section{What is Thin?}

\section{Geometry - Mechanics}

Some of our attention will be focused on the thin substrates on which films are commonly deposited. Because the thicknesses of these substrates are usually much smaller than their lateral dimensions, simple beam bending mechanics can be used to describe their elastic response.

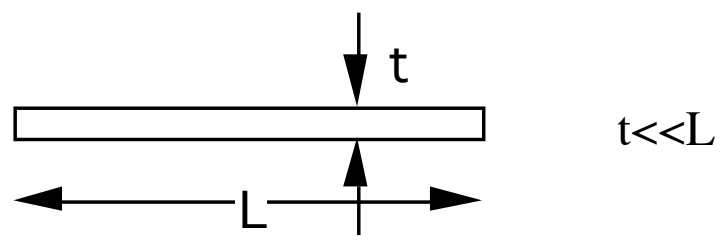

Thin plate - simple axial and bending stresses

Example: Si substrates:

$$
\begin{aligned}
& t \approx 0.5 \mathrm{~mm} \\
& L \approx 100 \mathrm{~mm}
\end{aligned} \frac{t}{L} \approx \frac{0.5}{100}=5 \times 10^{-3}
$$

Thin "Film"

Also, the films deposited on such substrates are usually much thinner than the thickness of the substrate and, of course, the film thickness is much, much smaller than the lateral dimensions of the substrate

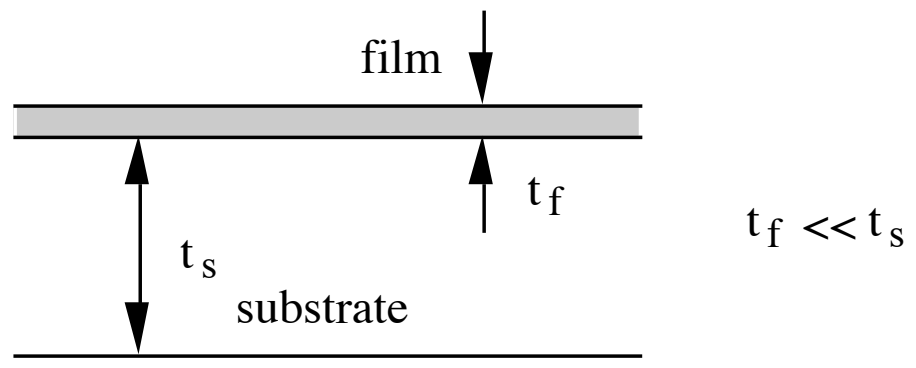

Example: $\quad$ Al film on Si substrate

$$
\begin{array}{ll}
t_{f} & \approx 1 \mu \mathrm{m} \\
t_{s} & \approx 0.5 \mathrm{~mm}=500 \mu \mathrm{m}
\end{array} \frac{t_{f}}{t_{s}}=\frac{1}{500}=2 \times 10^{-3}
$$




\section{Microstructure}

Film thickness comparable to microstructural dimension

$$
\begin{aligned}
& t_{f} \approx \text { grain size }=\mathrm{d} \\
& \mathrm{t}_{\mathrm{f}} \approx \text { dislocation spacing }=1 / \sqrt{\rho}
\end{aligned}
$$

Microstructural dimensions often controlled by film thickness -

$t_{f} \approx 1 \mu \mathrm{m}$. This results in finer microstructures than in bulk. This causes mechanical properties of thin films to be different from those of bulk materials.

Artificial Microstructures - quantum well structures

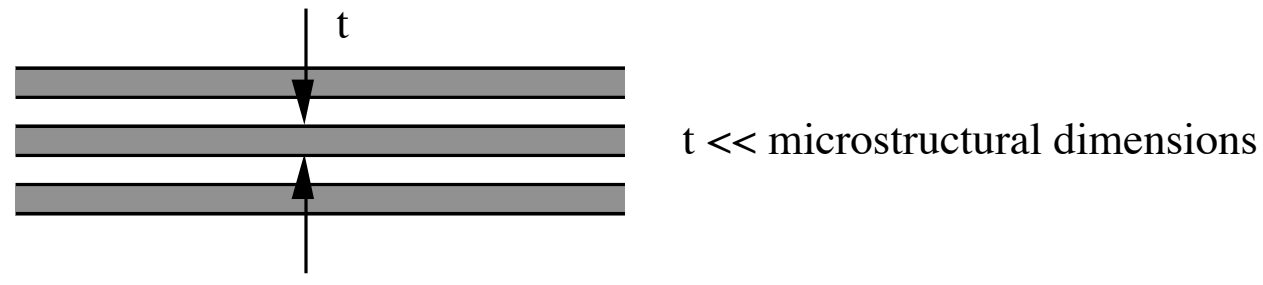

Atomistic Structure

Film thickness approaches mono-molecular dimensions

$t_{f} \approx a$ atomic dimension - not treated in this course

Note

Most thin films of interest are attached to substrates

\section{Thin Films in Technology}

* $\quad$ Microelectronic IC's

* Magnetic Storage

* Optical Filters

* Wear Resistant Coatings

* Corrosion Resistant Coatings 


\section{Complimentary Metal-Oxide-Semiconductor Transistor}

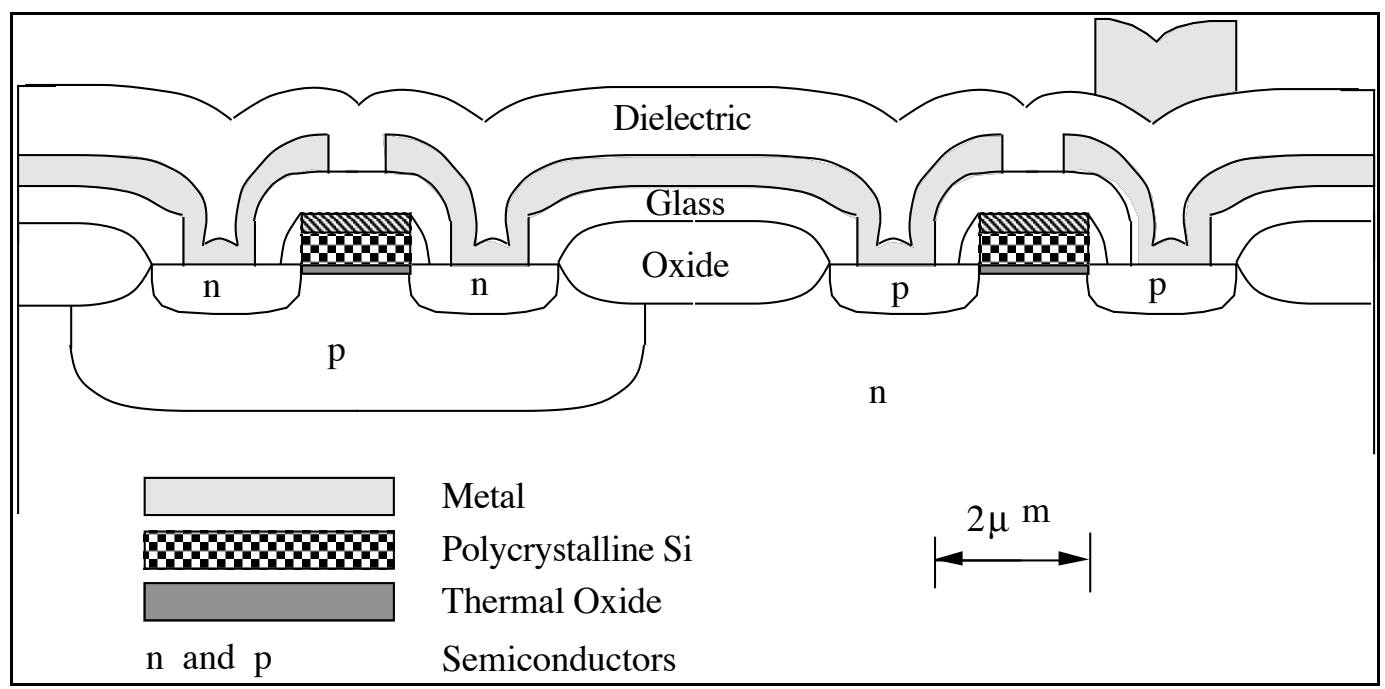

\section{Magnetic Head-Disk Interactions}

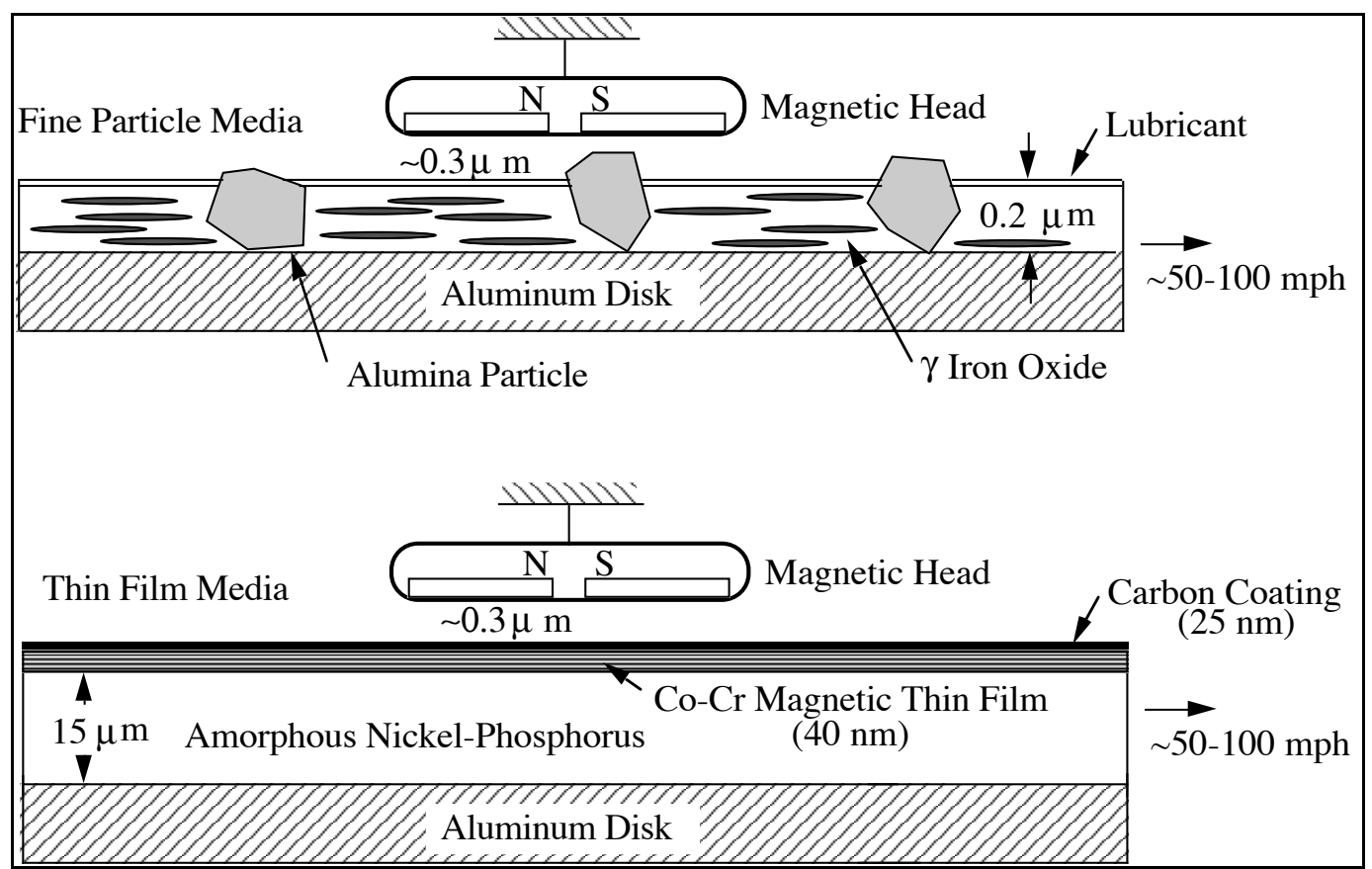


Thin Films in a Modern Microprocessor

AMD K6 Microprocessor (bottom section)

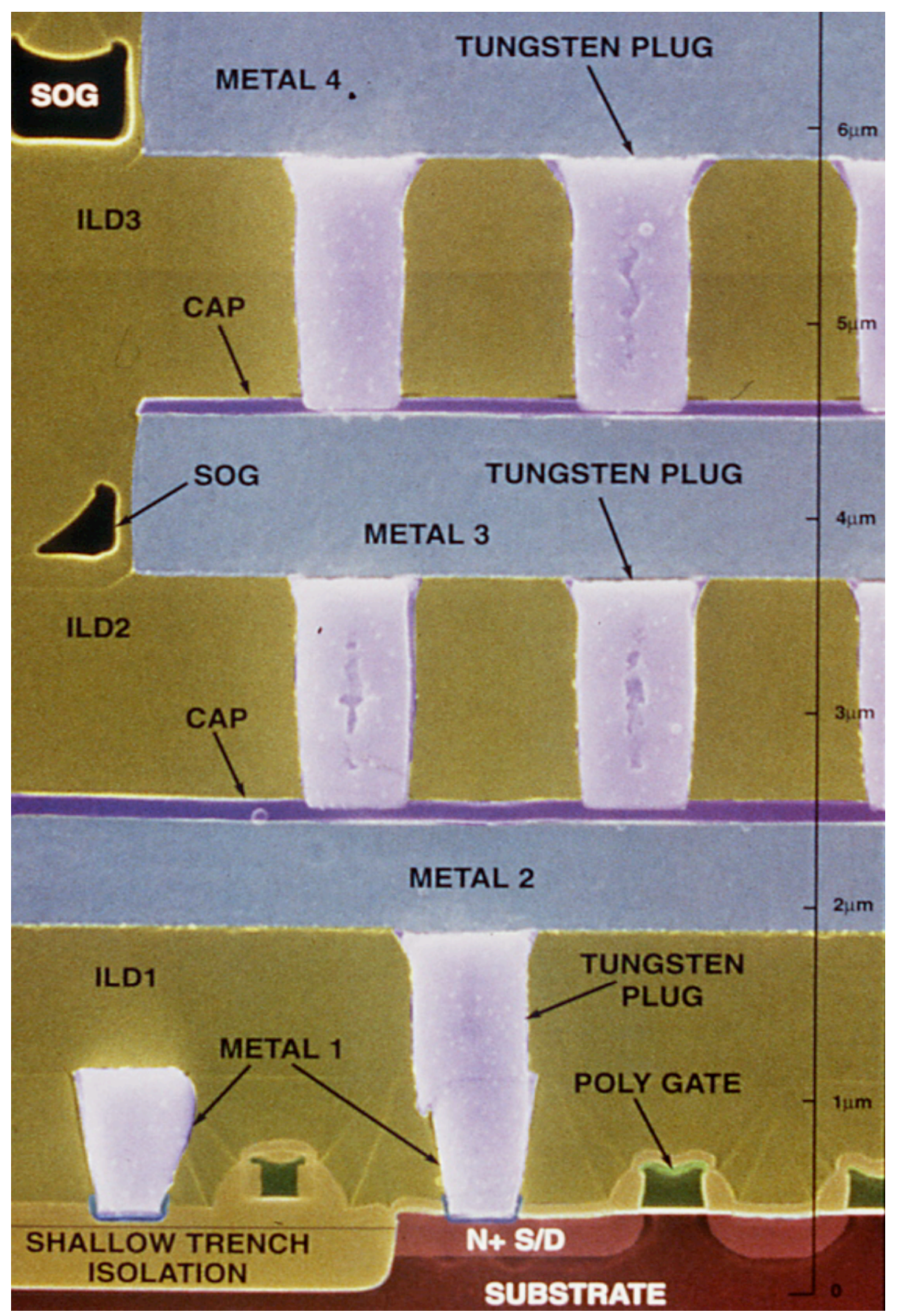


Thin Films in a Modern Microprocessor

AMD K6 Microprocessor (top section)

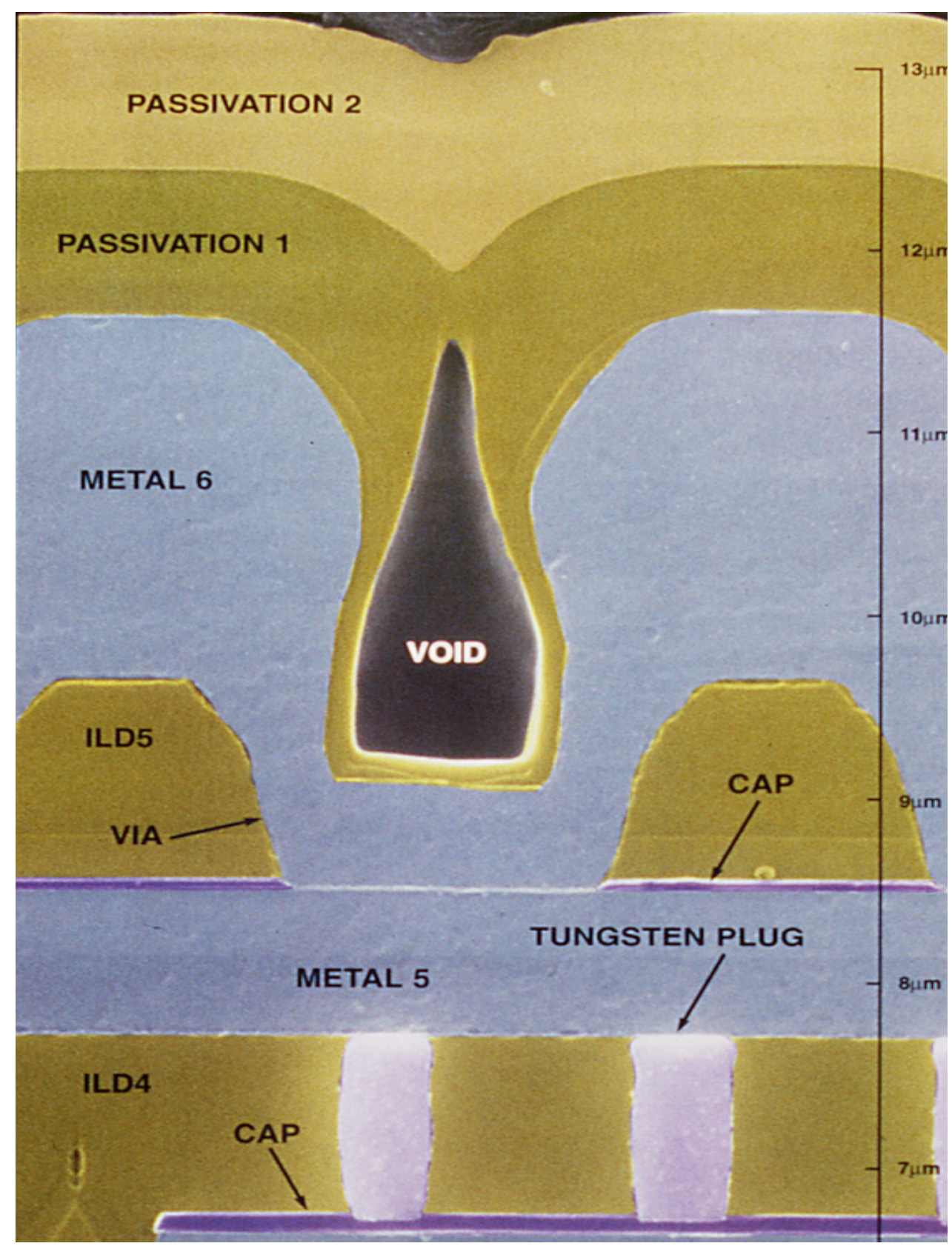




\section{Schematic Of Optical Thin Film Device}

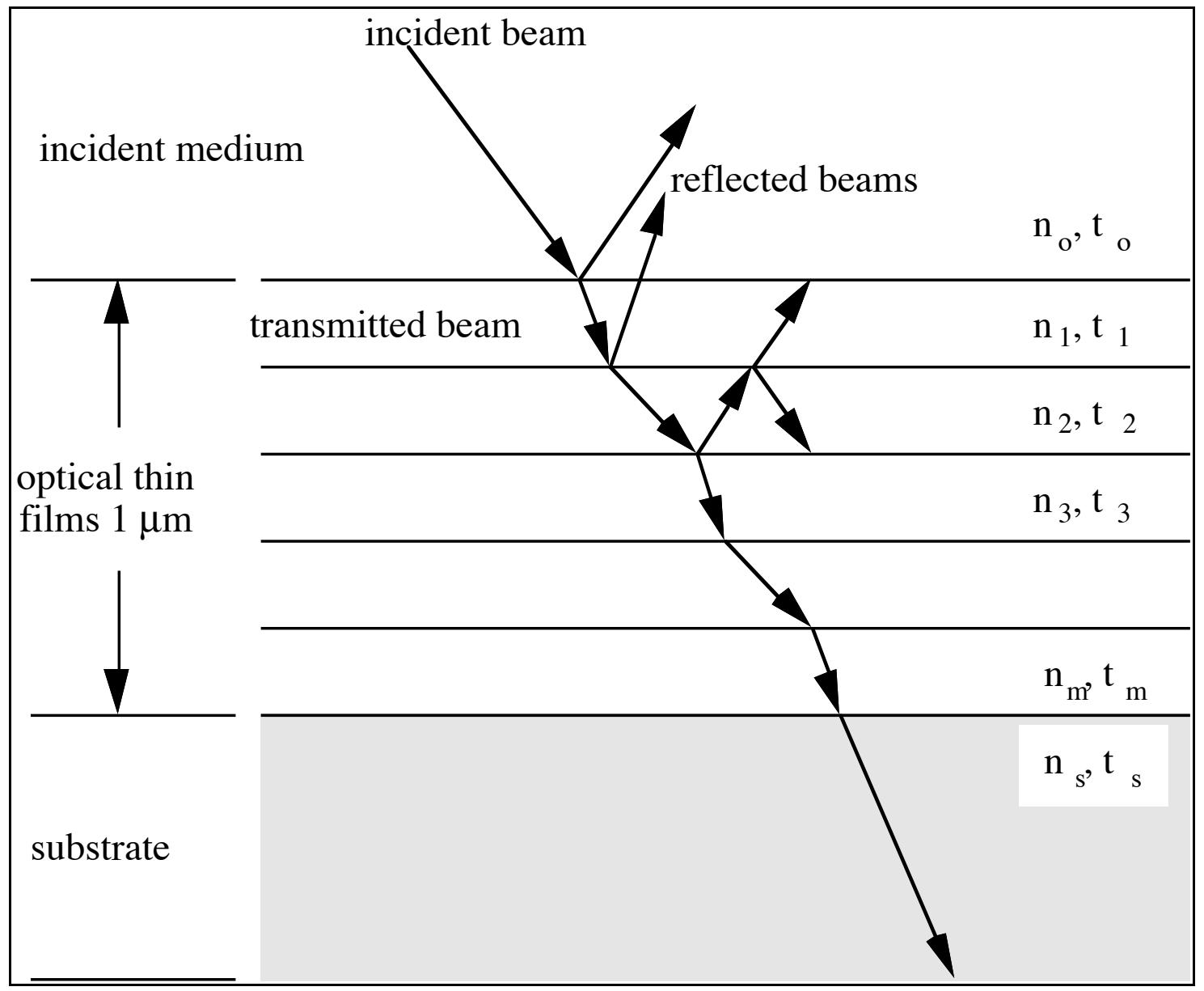

Schematic of an optical thin film device consisting of a substrate and several thin films. Each film has an index of refraction, $\mathrm{n}_{1}$, and thickness, $\mathrm{t}_{1}$. The incident beam is reflected and transmitted at each interface, although for clarity, only a few reflections are shown. One or more of the optically active materials may be absorbing. Each film has an optical thickness that is typically an integer multiple of a quarter of the wavelength of the radiation of interest. Coatings can be designed to either pass or block certain parts of the optical spectrum. The thicknesses of the films in these optical devices are so critical that thermal expansion and elastic strains (Poisson effects) have to be taken into account. For more information students may visit the website for Optical Coatings Laboratory, Inc. (OCLI) in Santa Rosa, CA, to see the amazing properties of these thin films:

\section{http:/ / www.ocli.com/}




\section{Mechanical Behavior Problems}

* Tolerance Problems

* Failure

* Wafer Cracking

* Thin Film Peeling

* Metal Crack Problem

* Electromigration Problem

\section{Elastic Deformation -- Tolerance Problems}

Tolerance Problems Associated with Thin Films

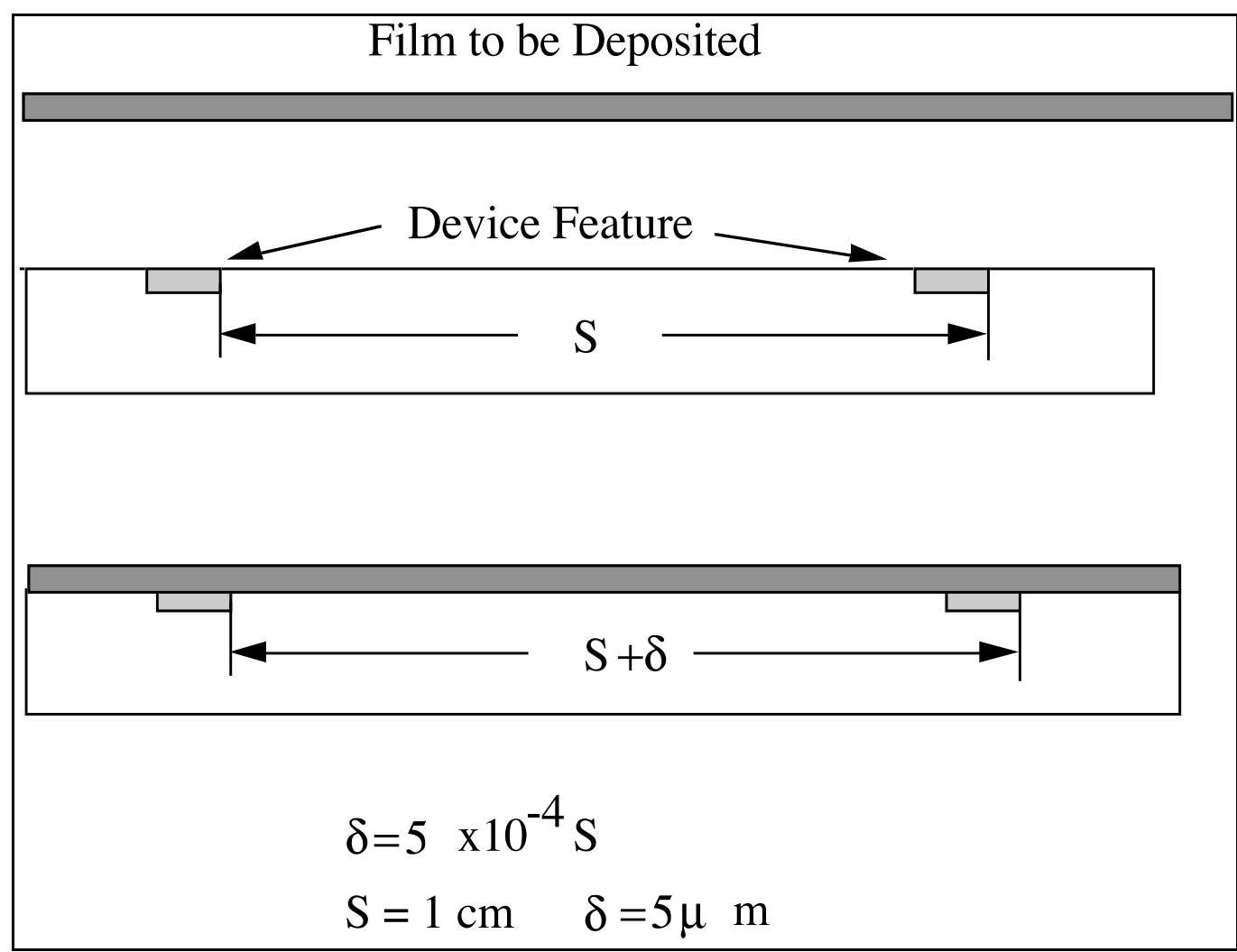


Example of possible tolerance problems associated with thin films

SCALPEL ${ }^{\mathrm{TM}}$ Bell Labs - SCattering with Angular Limitation Projection Electron-Beam Lithography

\section{http://www.bell-labs.com/projects/SCALPEL/description.html}

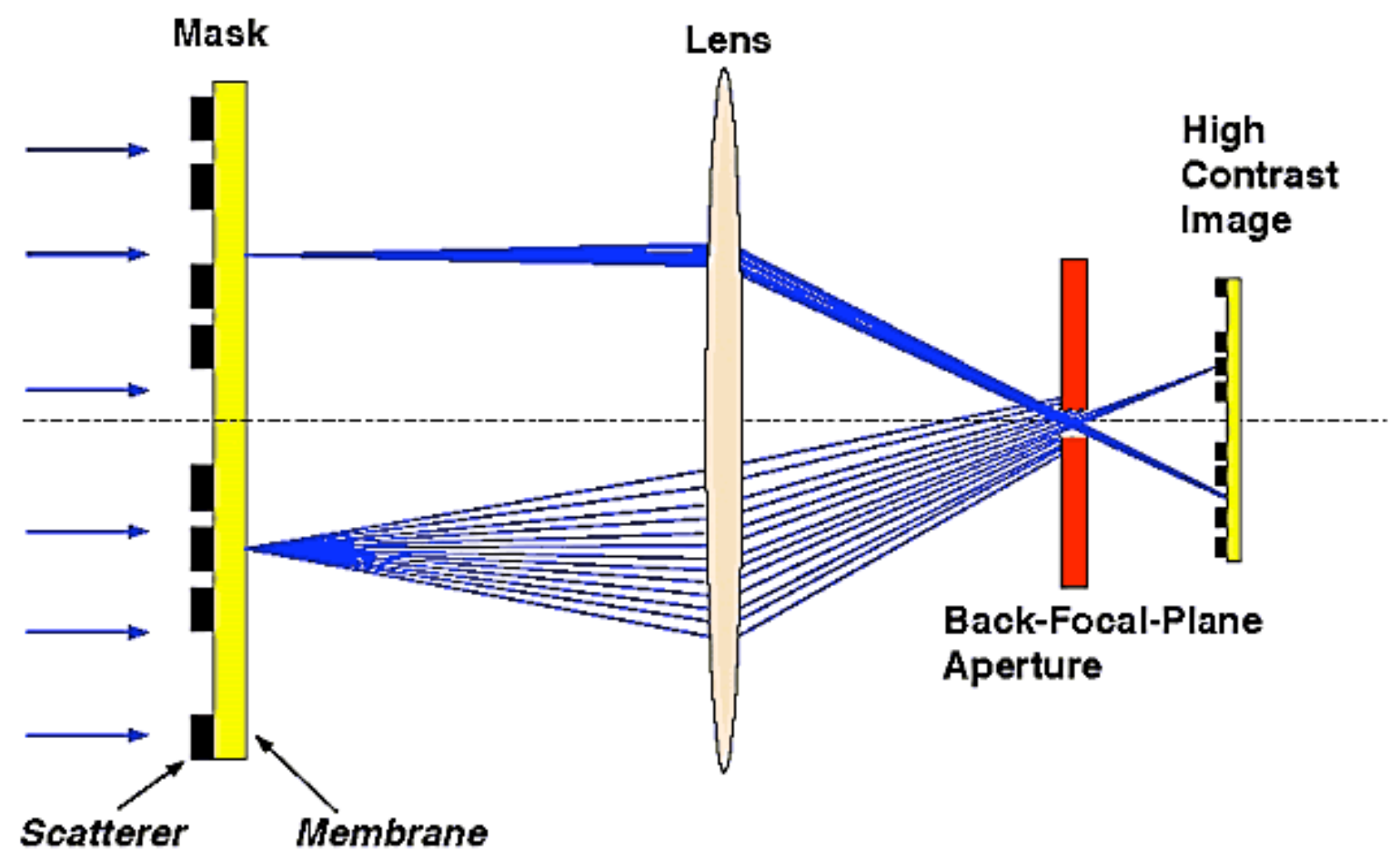

Figure 1. SCALPEL principle of operation.

SCALPEL technology is a form of e-beam lithography that depends critically on the distribution of strains in a thin film. As we shall see, islands of film attached to a thin substrate can cause distortions of the substrate, which in turn would lead to distortions in the projected image. So understanding the stresses and strains in these structures is a problem of technological significance.

The aspect of SCALPEL which differentiates it from previous attempts at projection electron-beam lithography is the mask. This consists of a low atomic number membrane covered with a layer of a high atomic number material: the pattern is delineated in the latter. While the mask is almost completely electrontransparent at the energies used $(100 \mathrm{keV})$, contrast is generated by utilizing the difference in electron scattering characteristics between the membrane and patterned materials. The membrane scatters electrons weakly and to small 
angles, while the pattern layer scatters them strongly and to high angles. An aperture in the back-focal plane of the projection optics blocks the strongly scattered electrons, forming a high contrast aerial image at the wafer plane (Figure 1). The functions of contrast generation and energy absorption are thus separated between the mask and the aperture. This means that very little of the incident energy is actually absorbed by the mask, minimizing thermal instabilities in the mask.

\section{Plastic Deformation}

Failure Associated with Inhomogeneous Plastic Flow

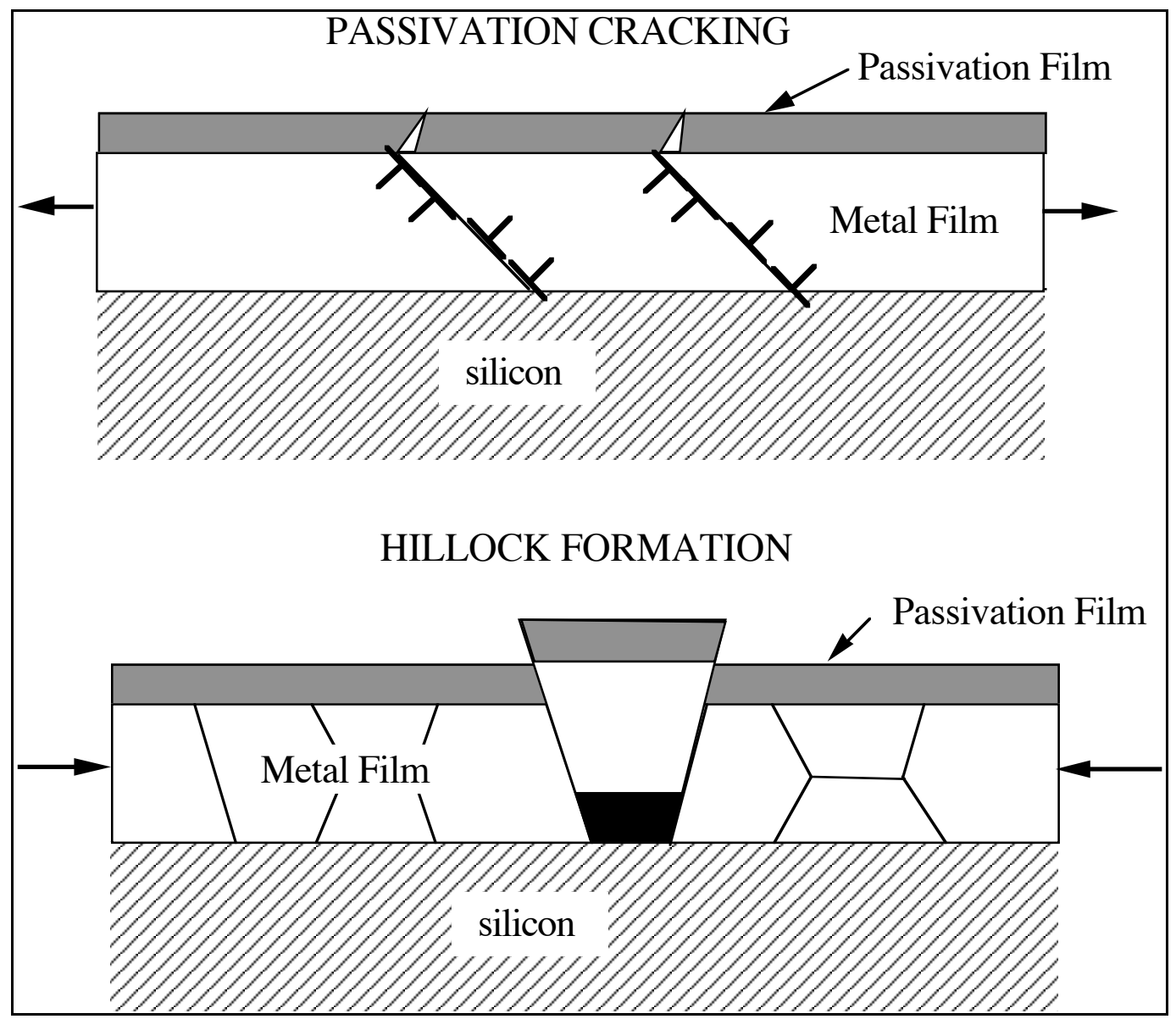




\section{"Bird's Beak" and Dislocations}

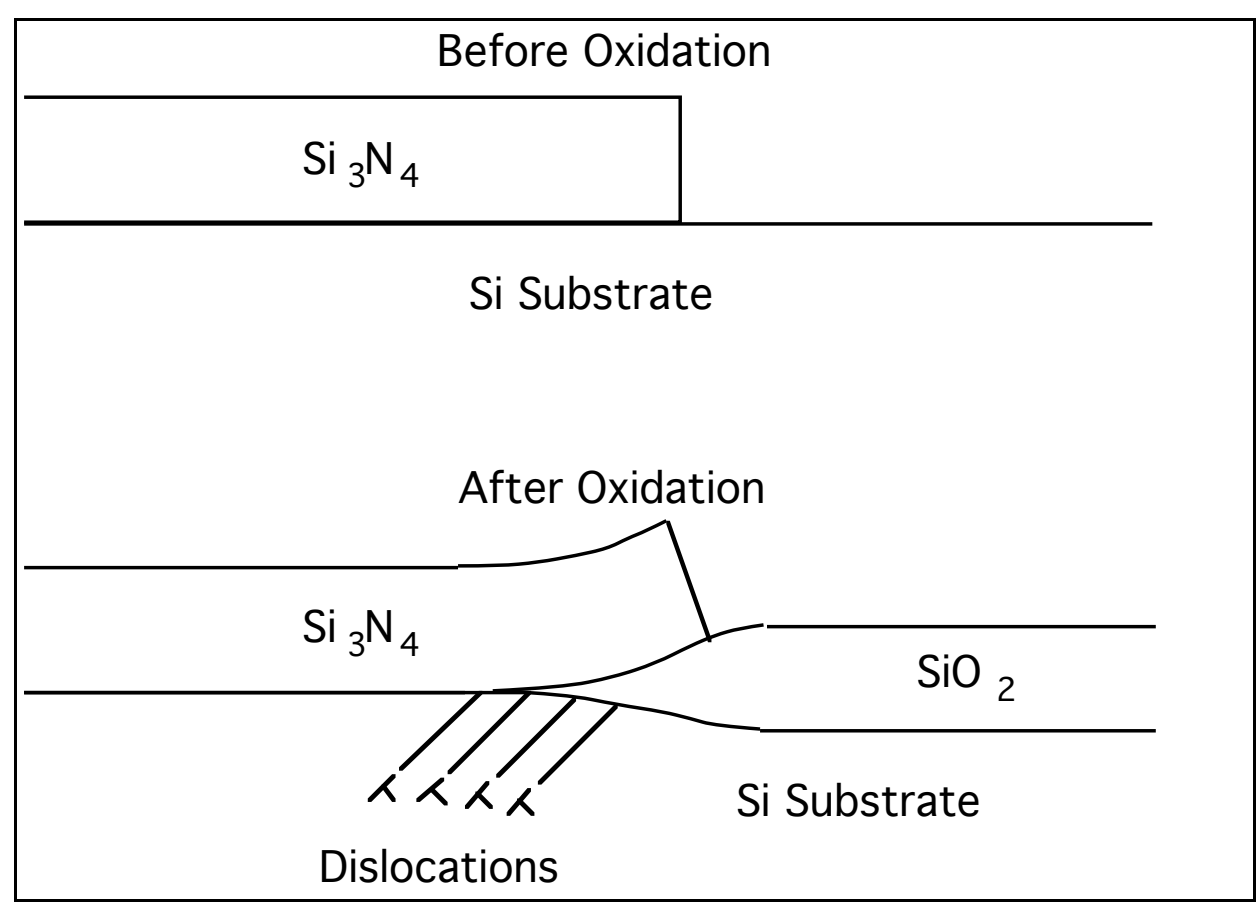

Formation of "Bird's Beak" and dislocations during LOCOS process with Silicon Nitride mask.

\section{Fracture}

Wafer Cracking

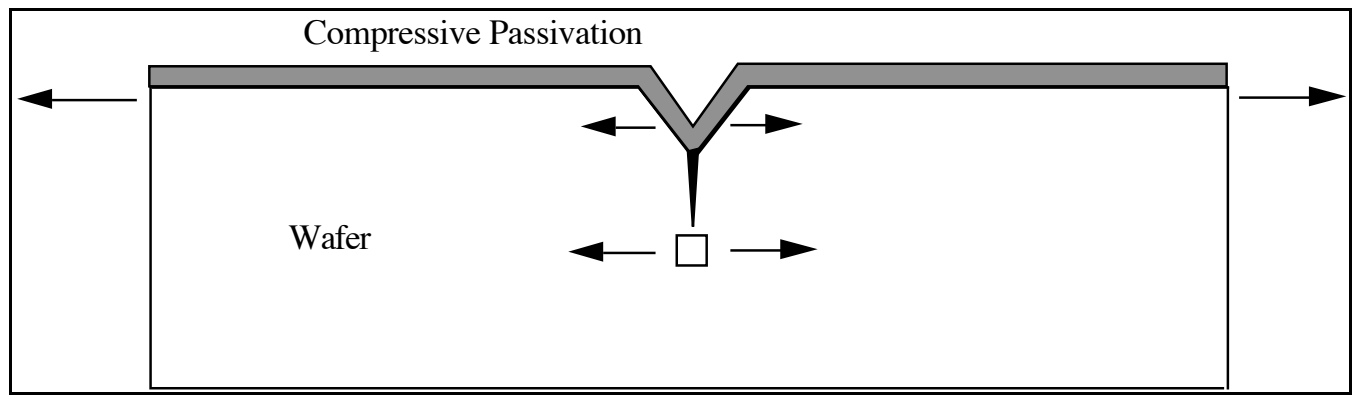




\section{Thin Film Debonding}

Thin Film Debonding
Thin Film Peeling
* Caused by Tensile Stresses
* Interfacial Strength is Important
Tensile Stress
Thin Film Buckling
* Caused by Compressive Stresses
* Interfacial Strength is Important




\section{CVD W Films/Lines}

Here we see CVD W lines that have debonded and curled up from the substrate . The curvature of the debonded lines shows that a stress gradient had existed in the film before it debonded from the substrate.

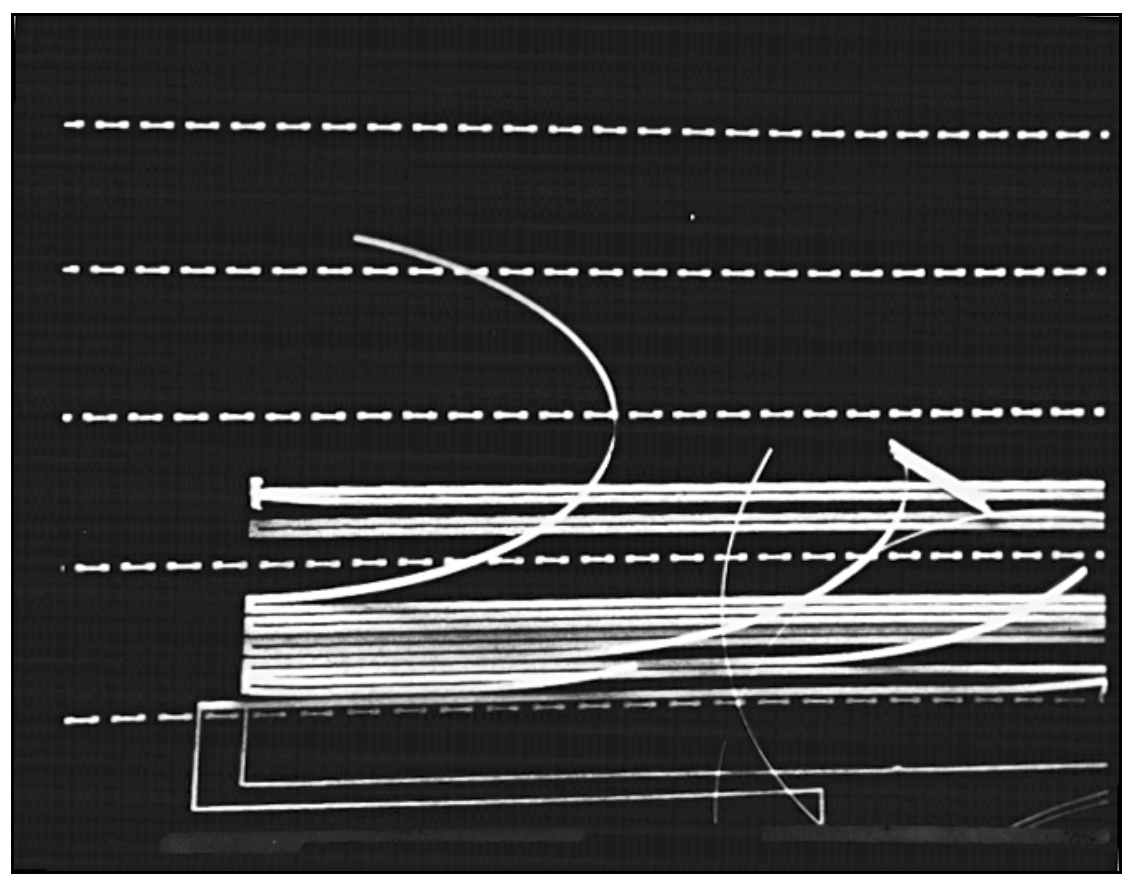


Telephone cord delaminations of a Fe/Pt multilayered film from a (001) MgO substrate
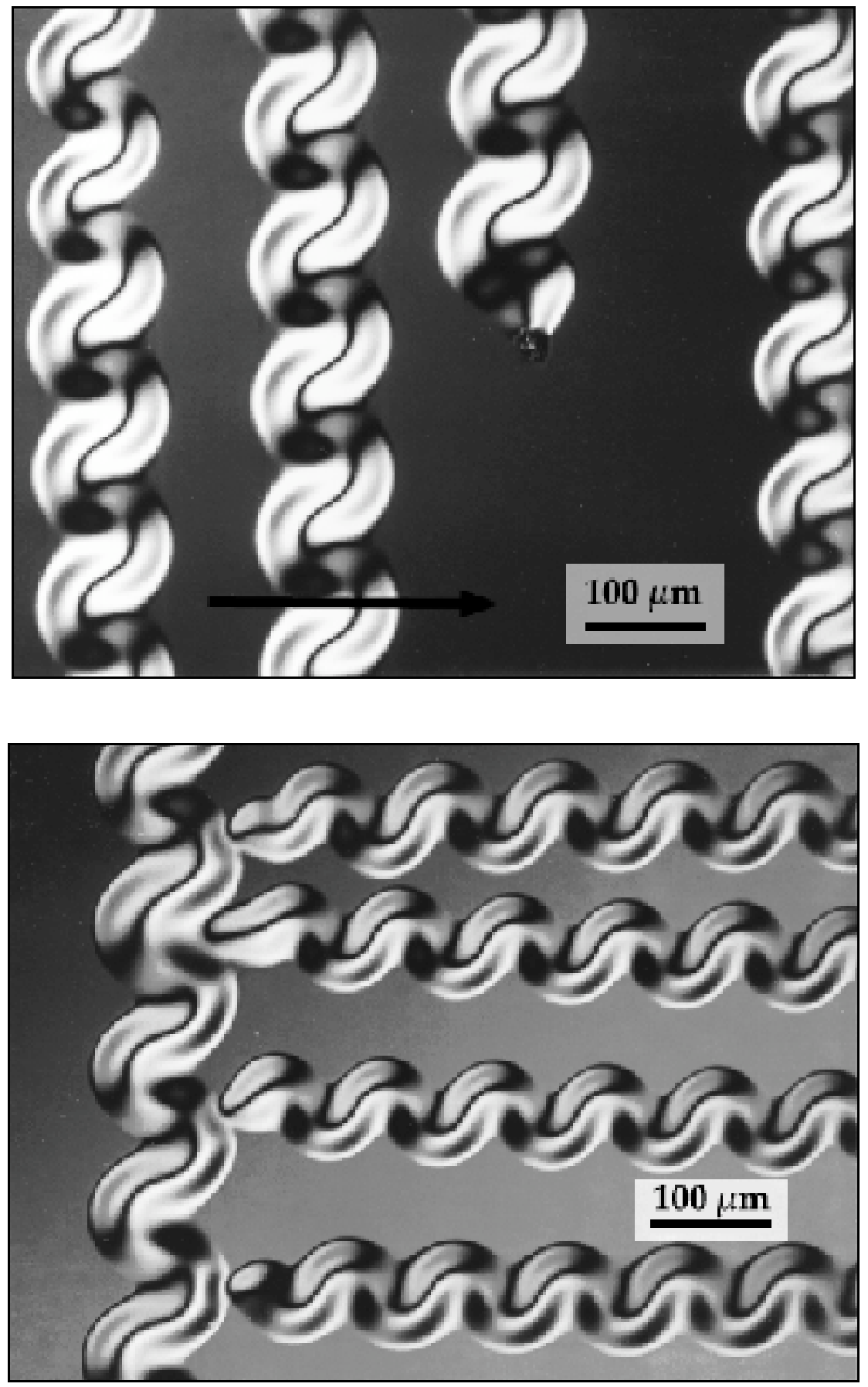


\section{Metal Crack Problem for Interconnect Metals}

\begin{tabular}{|ll|}
\hline & Metal Deposition \\
& Patterning \\
&
\end{tabular}




\section{Electromigration Problem for Interconnect Metals}

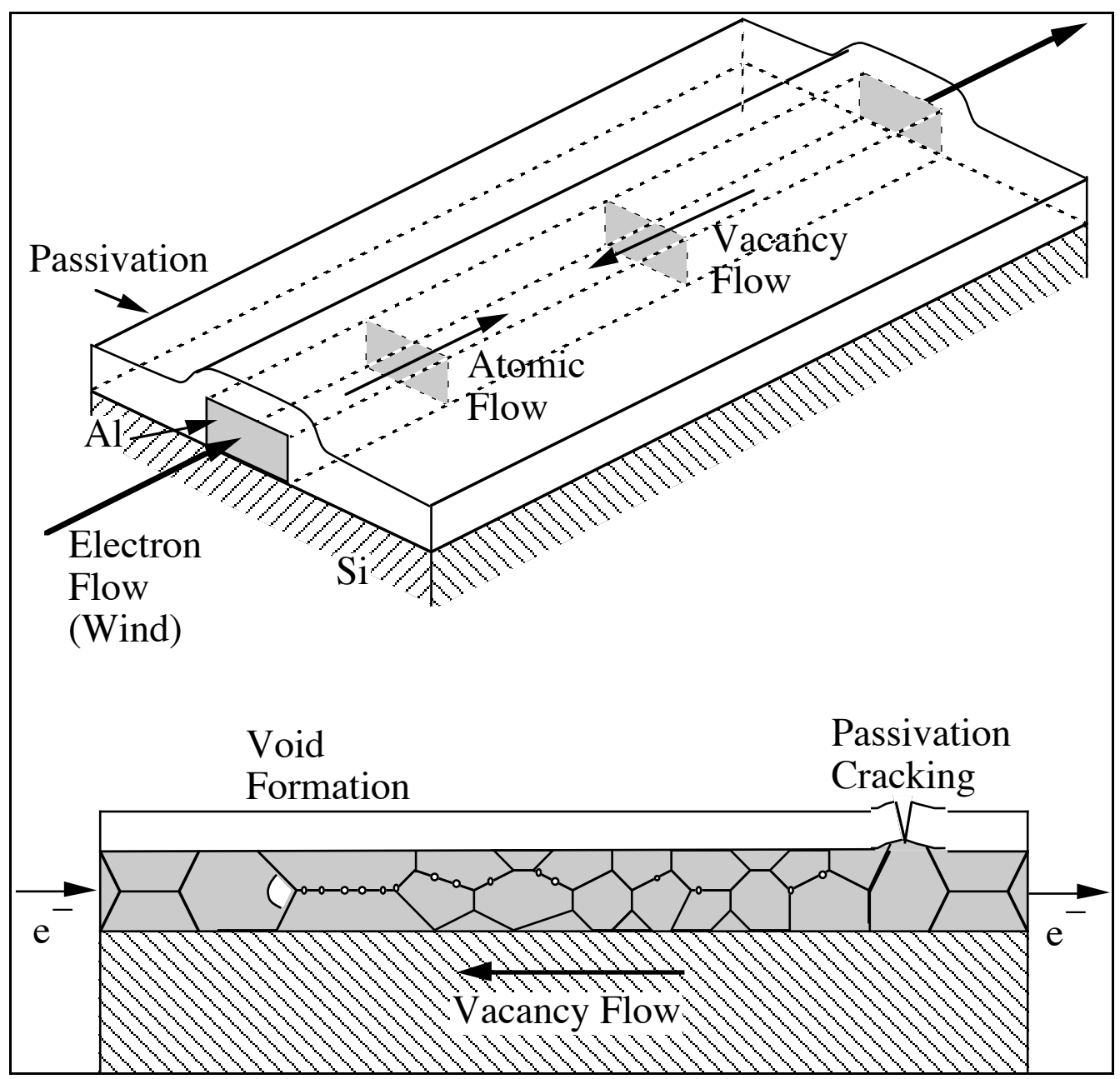




\section{Introduction to Sources of Stresses in Thin Films}

Accommodation of Misfit Between Film and Substrate

Thin Film $=t_{f}<<t_{S}$ ie. massive substrate

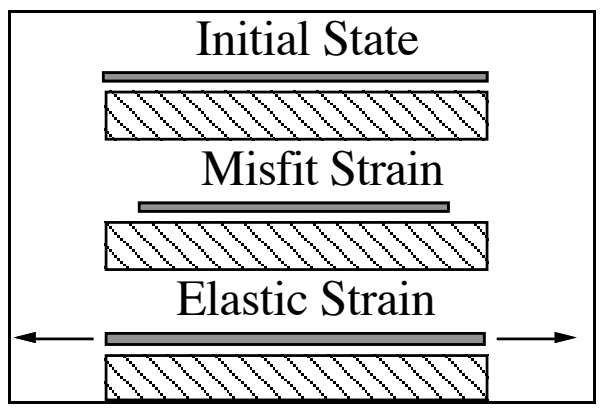

\section{Sources of Stress in Thin Films}

Thermal: $\quad \varepsilon_{\text {misfit }}=\left(\alpha_{\text {film }}-\alpha_{\text {substrate }}\right)\left(T-T_{o}\right)$

"Intrinsic": $\quad \varepsilon_{\text {misfit }}=\frac{\Delta \varepsilon_{\text {transformation }}}{3}$

Epitaxial: $\quad \varepsilon_{\text {misfit }}=\frac{a_{\text {film }}-a_{\text {substrate }}}{a_{\text {substrate }}}$

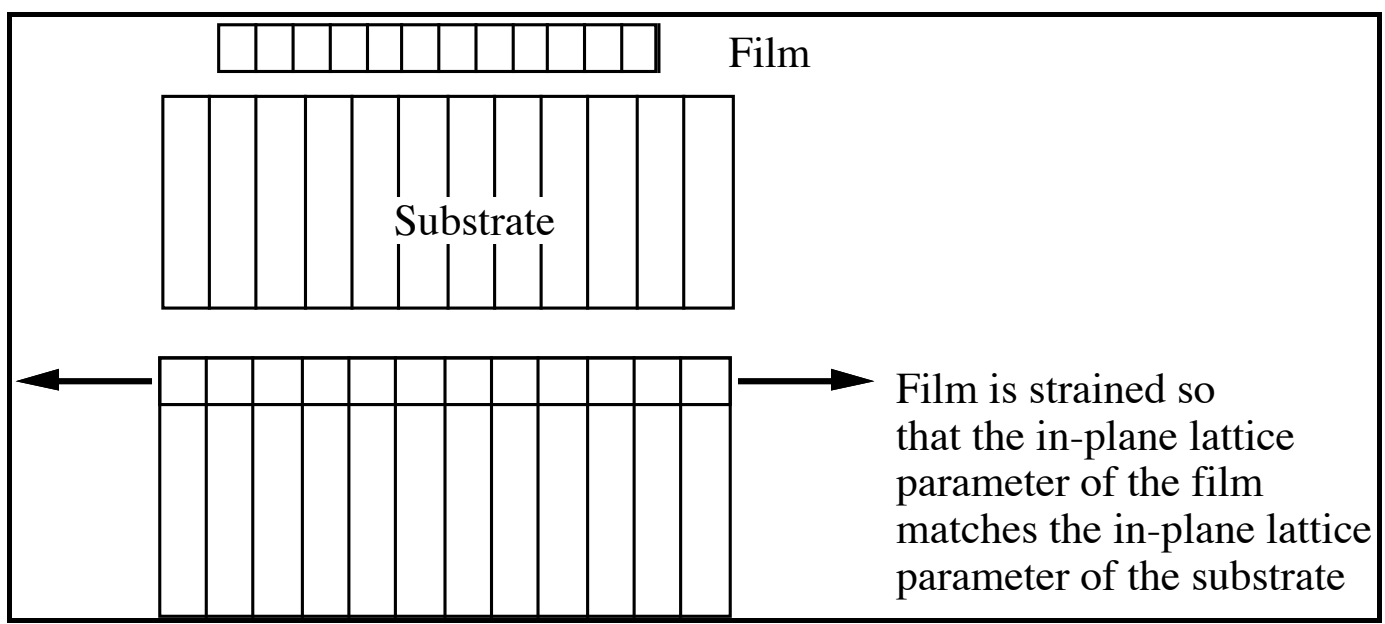




\section{Stresses in Film}

Exert stresses in substrate!

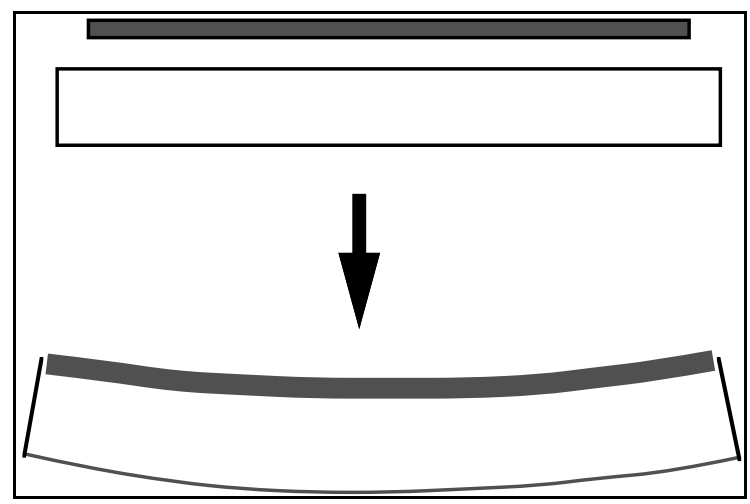

So we start with a discussion of basic mechanics of thin plates. 


\section{Mechanics of Thin Films and Substrates}

Basic results from Elements of Strength of Materials (Timoshenko \& Young)

Many results used in the study of mechanical properties of thin films are based on simple "mechanics of materials" solutions to mechanics problems. A central problem involves the biaxial bending of a thin plate.

\section{Biaxial Bending of Isotropic Plate}

Biaxial bending at isotropic plate (note : cubic crystals are biaxially isotropic in the $\{111\}$ and $\{100\}$ planes).

$M=$ moment per unit length applied along edges of plate.

Biaxial Bending of a Thin Plate

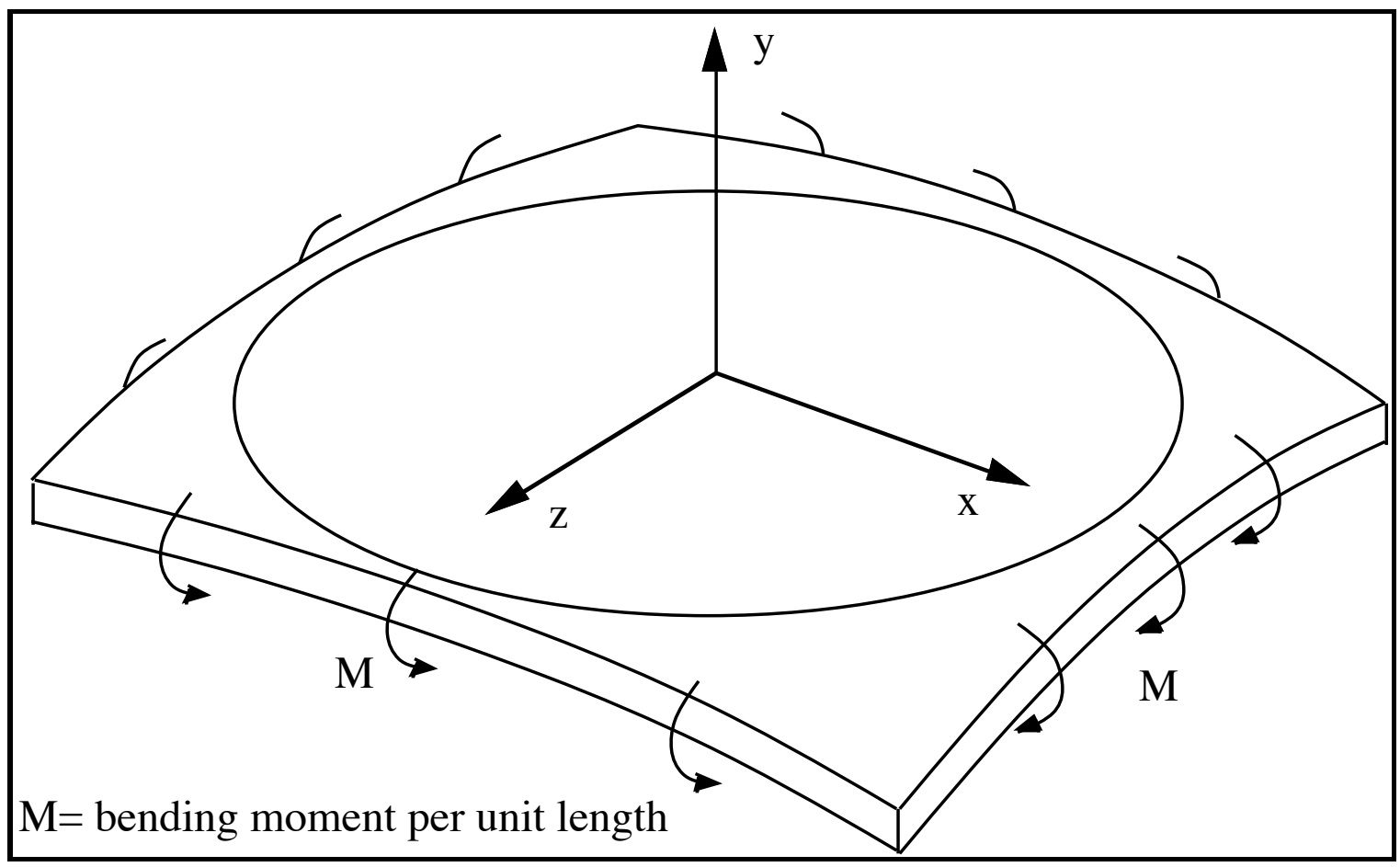




\section{Bending Stresses}

Find stress state in plate using basic solution for bending stresses:

Bending Moments and Stress Distribution

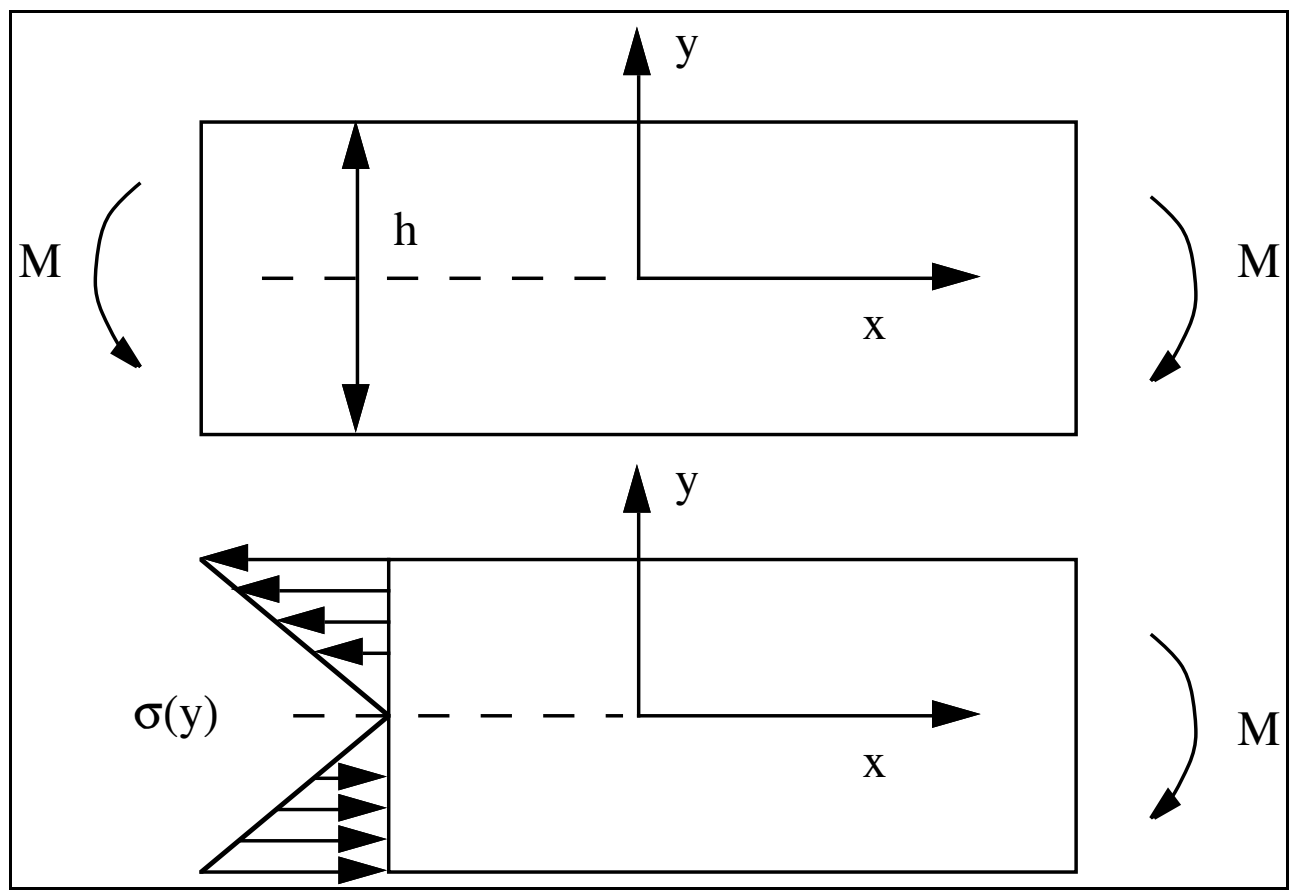

The biaxial stress distribution is (for pure bending):

$$
\sigma_{x x}=\alpha y\left(=\sigma_{z z}\right)
$$

as shown in the figure.

The stresses in the beam are related to the bending moment through the following relations. A simple free body analysis requires these results. First, the moment (per unit length along the edge) is related to the stresses in the plate by

$$
M=\int_{-h / 2}^{h / 2} \sigma_{x x} y d y=\int_{-h / 2}^{h / 2} \alpha y^{2} d y=\frac{\alpha h^{3}}{12}
$$

so we may find $\alpha$ : 


$$
\alpha=\frac{12 M}{h^{3}} .
$$

Thus the stresses are:

$$
\sigma_{x x}=\sigma_{z z}=\frac{12 M}{h^{3}} y
$$

with all other stress components being zero.

Note: we define a moment of the sign shown as positive because it produces positive stress in the positive y domain.

\section{Curvature}

\section{Curvature Relations}

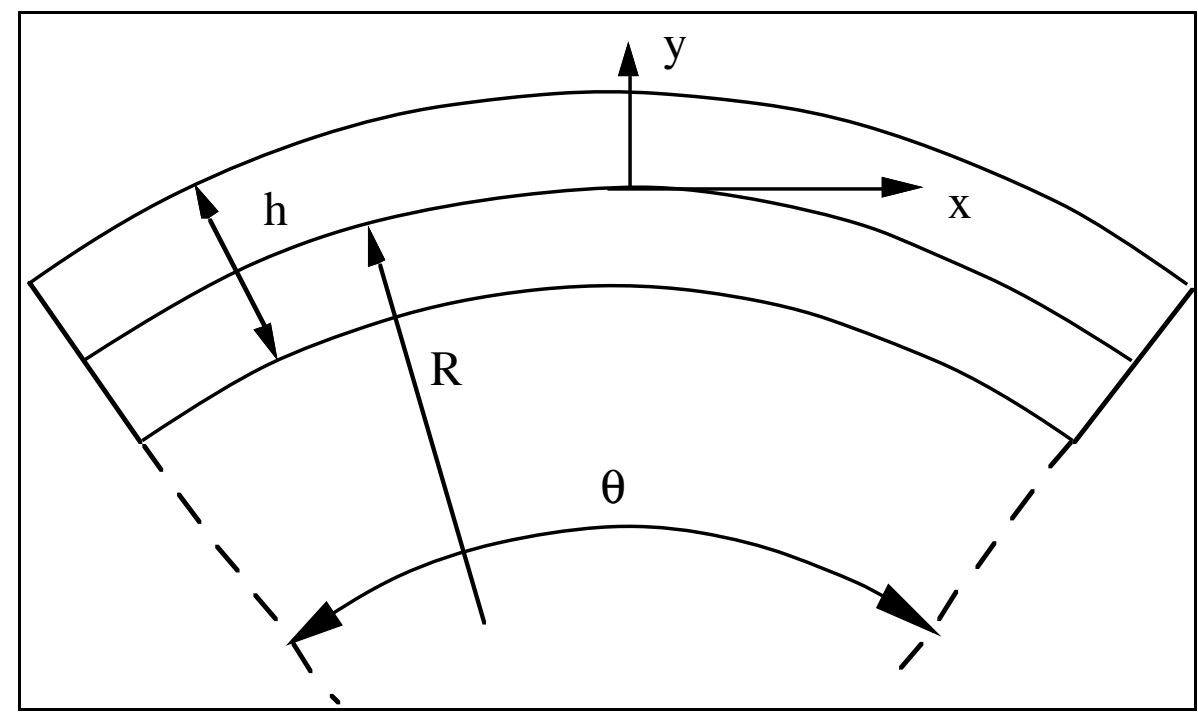

We start by relating the bending strain to the curvature. First, the tensile strain in the beam (plate) is, for pure bending,:

$$
\varepsilon_{x x}(y)=\frac{(R+y) \theta-R \theta}{R \theta}=\frac{y}{R}=-\kappa y
$$


This is obvious from the geometry of the bending. Using this expression we find the relation between the curvature and the strain in the plate is:

$$
\kappa \equiv-\frac{1}{R}=\frac{-\varepsilon_{x x}(y)}{y}
$$

Also, the diagram shows negative curvature by the following definition of curvature:

$$
\kappa=\frac{\frac{d^{2} u_{y}}{d x^{2}}}{\left(1+\left(\frac{d u_{y}}{d x}\right)^{2}\right)^{3 / 2}} \approx \frac{d^{2} u_{y}}{d x^{2}}
$$

\section{Curvature Associated with a Biaxial Bending Moment}

Above we showed that the curvature is related to the bending strain in the plate:

$$
\kappa=\frac{-\varepsilon_{x x}(y)}{y} .
$$

We must calculate $\varepsilon_{x x}(y)$ to find the curvature. We use Hooke's Law (isotropic). The strain is

$$
\varepsilon_{x x}=\frac{1}{E}\left(\sigma_{x x}-v\left(\sigma_{y y}+\sigma_{z z}\right)\right)
$$

where

$$
\sigma_{y y}=0 \text { and } \sigma_{x x}=\sigma_{z z} .
$$

Then the biaxial stress-strain relation is:

$$
\varepsilon_{x x}=\frac{(1-v)}{E} \sigma_{x x} .
$$


Specifically, the strain and stress are:

$$
\varepsilon_{x x}(y)=\frac{(1-v)}{E} \sigma_{x x}(y) .
$$

From the moment analysis above, the stress is related to the moment by

$$
\sigma_{x x}(y)=\frac{12 M}{h^{3}} y
$$

so that

$$
\varepsilon_{x x}(y)=\frac{(1-v)}{E} \frac{12 M}{h^{3}} y .
$$

Finally, the relation between the applied moment and the resulting curvature is

$$
\begin{aligned}
& \kappa=\frac{-\varepsilon_{x x}(y)}{y}=-\frac{(1-v)}{E} \frac{12 M}{h^{3}} \\
& \kappa=-\frac{(1-v)}{E} \frac{12 M}{h^{3}}
\end{aligned}
$$




\section{Deflection}

We now turn to the corresponding plate deflections or displacements.

\section{Deflection Associated With Biaxial Bending}

Consider displacement along a radius (either $\mathrm{x}$ or $\mathrm{r}$ ) from center

\section{Plate Deflection}

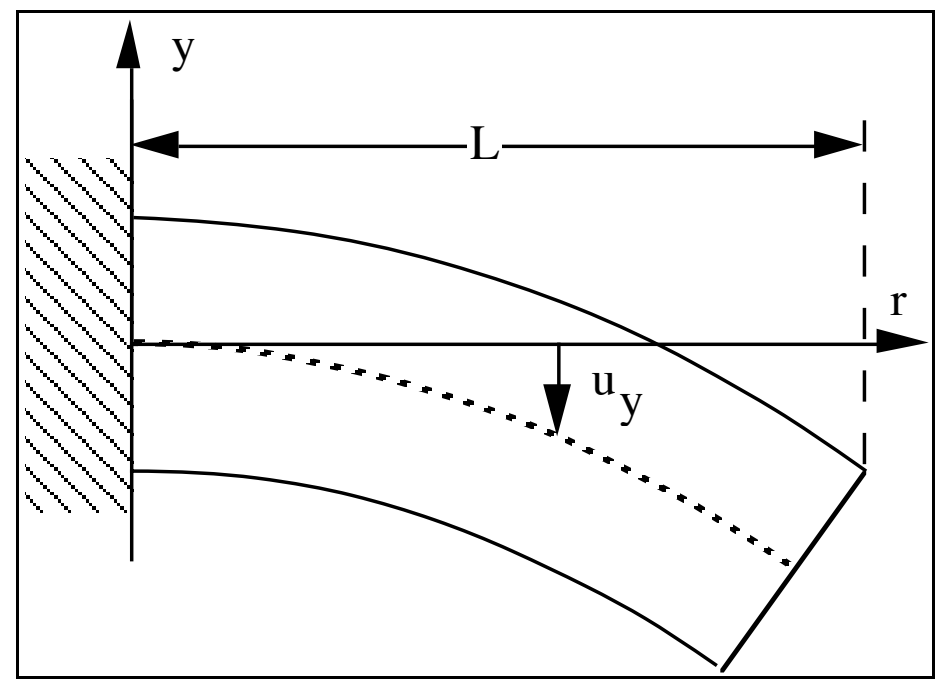

Negative curvature $\sim$ negative displacements.

$$
\frac{d^{2} u_{y}}{d r^{2}}=\kappa=-\frac{(1-v)}{E} \frac{12 M}{h^{3}}=\text { cons } \tan t
$$

The curvature is constant for pure bending. Integrate the equation to obtain:

$$
\frac{d u_{y}}{d r}=\kappa r+c_{1} .
$$

Apply the boundary condition: 


$$
\frac{d u_{y}}{d r}=0 \quad \text { at } \quad r=0 \text { to obtain } \quad c_{1}=0 .
$$

Integrate again to find:

$$
u_{y}=\frac{\kappa r^{2}}{2}+c_{2}
$$

with boundary condition:

$$
u_{y}=0 \quad \text { at } \quad r=0 \quad \text { to obtain } \quad c_{2}=0 \text {. }
$$

The result is:

$$
u_{y}=\kappa \frac{r^{2}}{2}=-\frac{(1-v)}{E} \frac{12 M}{h^{3}} \frac{r^{2}}{2}
$$

or, in terms of the cartesian coordinates in the plate

$$
u_{y}=-\frac{(1-v)}{E} \frac{12 M}{h^{3}} \frac{\left(x^{2}+z^{2}\right)}{2}
$$

Finally, at the edge of the plate $(r=L)$,

$$
u_{y}(r=L)=-\frac{(1-v)}{E} \frac{6 M L^{2}}{h^{3}}
$$

Note that a positive moment produces a negative curvature and also negative displacements

\section{Thin Film Stresses}

Single thin film on a flat substrate 
Consider a stress free thin film of thickness $t_{f}$ bonded to stress-free substrate of thickness $t_{s}>>t_{f}$

\section{Thin Film on a Substrate}

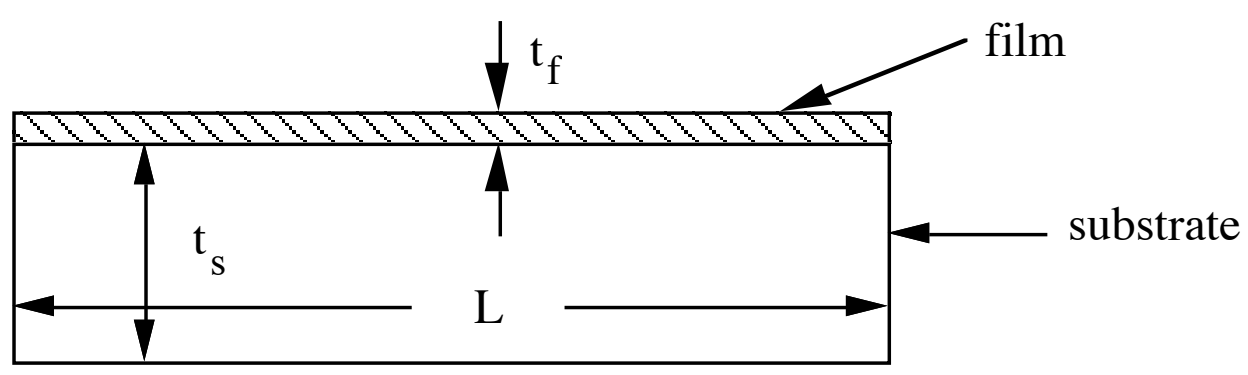

We also consider the typical case in which $L>>t_{S}$, so the substrate behaves as a plate.

Throughout this section the thought experiment on the following page is envisioned. A sequence of steps is made to arrive at a stressed film on an elastically deformed substrate. We start by imagining a stress-free film attached to a substrate, which is also stress-free. All thin film stresses are caused by the elastic accommodation of an incompatibility between the film and the substrate. It is best to imagine that the film is first removed from the substrate and allowed to change its dimensions relative to the substrate, that is, become incompatible with the substrate. Such unconstrained, transformational, deformation leads to no stresses in the film. Free thermal expansion or contraction and volume changes associated with phase transformations are examples. Once the transformation has occurred, the film is then elastically strained to again match the substrate. Of course external forces, shown in the diagram, are needed to cause this elastic deformation. The film is then bonded to the substrate and the external forces are released. The film will tend to return to its undeformed state but that deformation is resisted by the substrate, to which the film is attached. The forces being exerted onto the substrate cause it to deform as shown. In the case shown, the forces on the substrate cause it to compress and bend biaxially. Only the bending deformation is obvious in the diagram. 
Film Stress - Substrate Curvature Relations

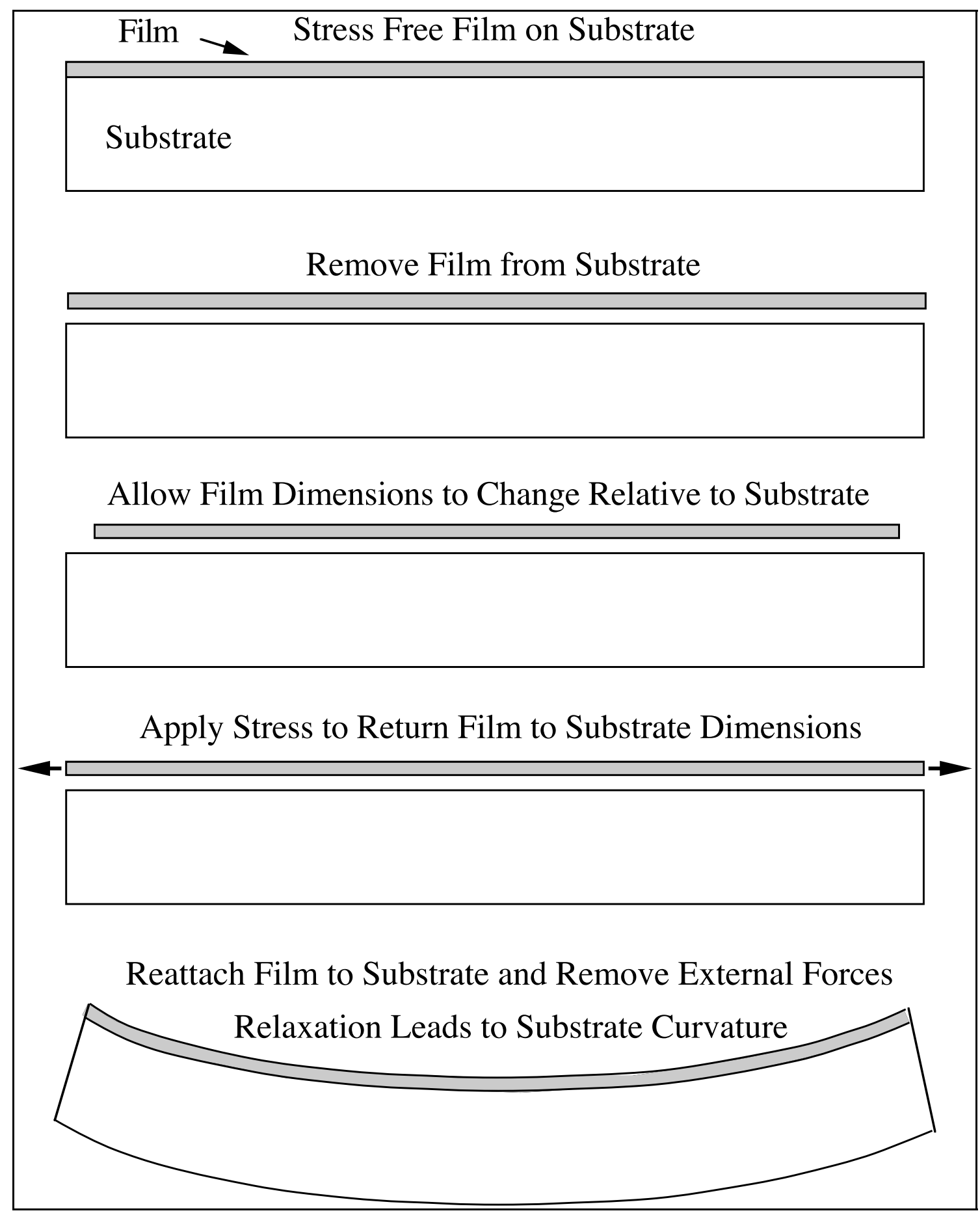


We wish to consider the stress in the film. Between the film and the substrate, stress can arise from incompatibilities, or misfit, caused by:

* Differences in thermal expansion,

* Phase transformations with volume changes,

* Densification of the film,

* $\quad$ Epitaxial effects

To understand the stresses and strains associated with the film and the related bending, we first imagine the film is free of the substrate. Then allow the film to shrink or expand relative to the substrate. The final state is then achieved by deforming both the film and substrate so that they again fit together perfectly.

For illustration, assume that the film shrinks relative to substrate. We say that the film has undergone a stress-free transformation, or misfit, strain relative to the substrate.

The Film and Substrate in a Stress-Free State (after the transformation or misfit)

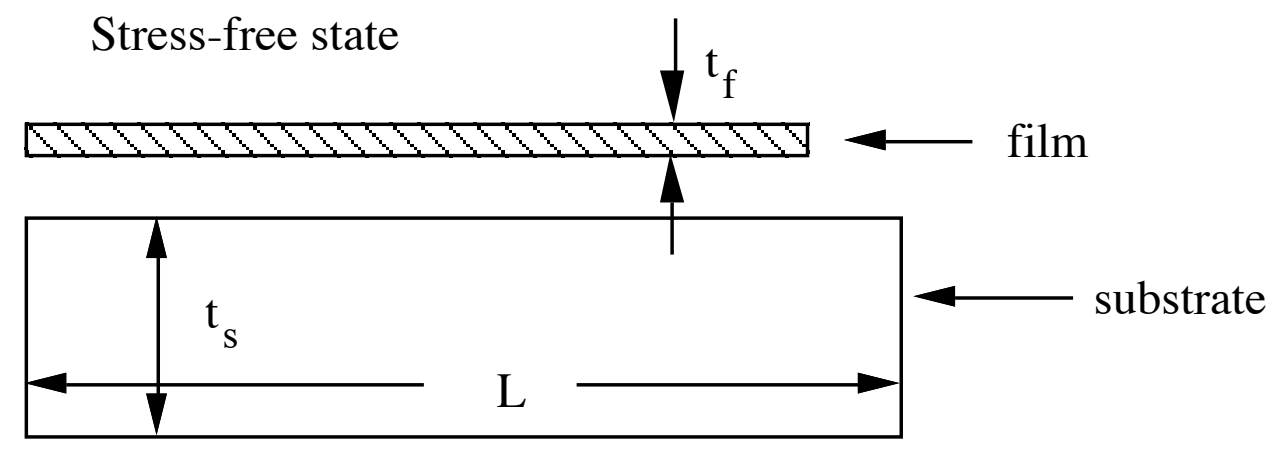

To fit the film and substrate back together, a biaxial tension force (per unit length) must be applied along the edge of the film.

\section{Stretching the Film}

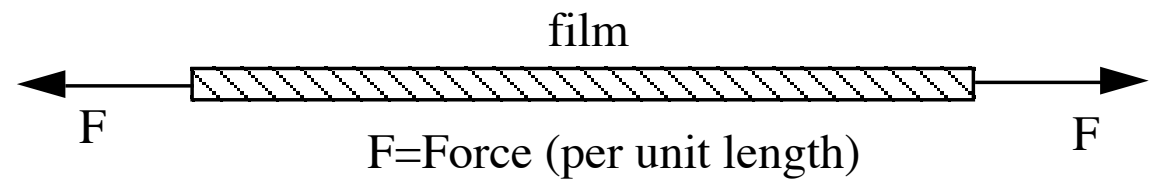

An equal and opposite force must be applied along the edge of the substrate. 


\section{Substrate Forces}

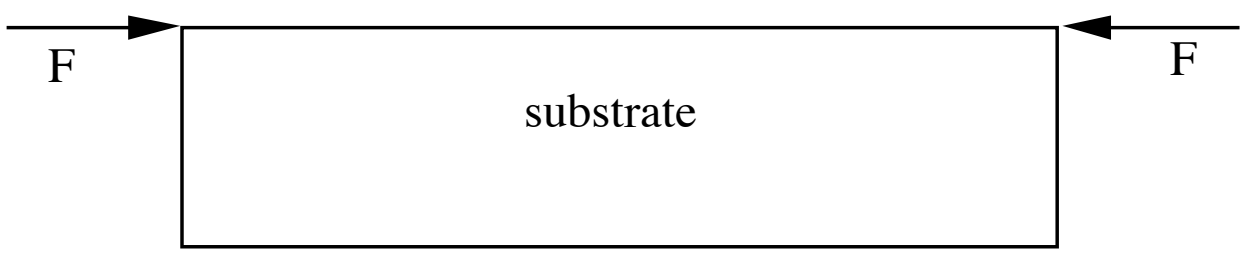

The tension forces in the film produce biaxial tension stresses, and the compressive force on the substrate produces biaxial compression and biaxial bending in the substrate.

\section{Film Stress - Curvature Relation}

Assume that a biaxial tension stress

$$
\sigma_{x x}=\sigma_{z z}=\sigma_{f}
$$

exists in the film as a result of the above processes. Then we have

\section{Film Stress}

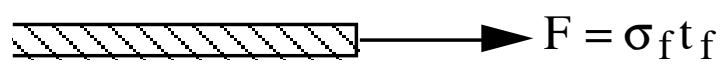

and in the substrate:

Substrate Forces

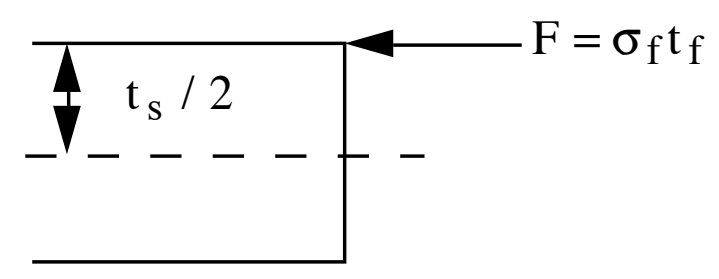

which is equivalent to 


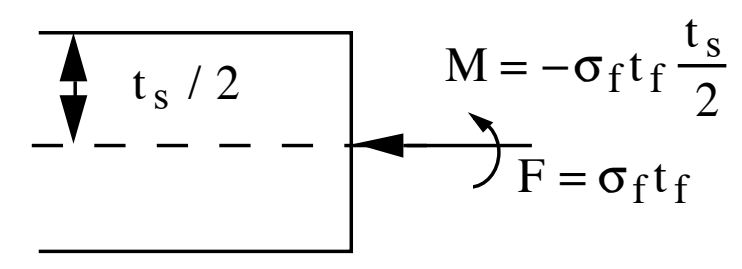

The bending of the substrate due to this moment is

$$
\begin{aligned}
& \kappa=-\frac{\left(1-v_{s}\right)}{E_{s}} \frac{12 M}{t_{s}^{3}}=-\frac{\left(1-v_{s}\right)}{E_{s}} \frac{12}{t_{s}^{3}}\left(-\sigma_{f} t_{f} \frac{t_{s}}{2}\right) \\
& \kappa=\frac{\left(1-v_{s}\right)}{E_{s}} \frac{6 \sigma_{f} t_{f}}{t_{s}^{2}}
\end{aligned}
$$

or the stress in film is

$$
\sigma_{f}=\left(\frac{E_{s}}{1-v_{s}}\right) \frac{t_{s}^{2}}{6 t_{f}} \kappa=\left(\frac{E_{s}}{1-v_{s}}\right) \frac{t_{s}^{2}}{6 t_{f} R}
$$

This is one of the most important relations in this course. It is the Stoney

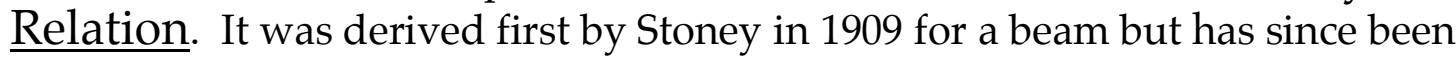
generalized to a plate.

The film stress-curvature relation shows that:

1. Film stress can be found by measuring the curvature of the substrate ( or change of curvature, $\Delta \kappa$, if not initially flat).

2. Results depend only on the dimensions of film and substrate and the elastic properties of substrate, not the properties of the film. With this feature, the film may be plastically deforming or undergoing phase transformations and still the measurement of the substrate curvature gives the current stress in the film. 


\section{Stresses in Film and Substrate (far from the edges of the film)}

(The edge effects will be discussed later.)

The stresses in the film are biaxial:

$$
\sigma_{x x}=\sigma_{z z}=\sigma_{f}(\text { all other components are zero })
$$

The stresses in the substrate are also biaxial but it is convenient to distinguish between the stresses associated with biaxial compression and those associated with biaxial bending:

$$
\sigma_{x x}=\sigma_{z z}=\sigma_{\text {compression }}+\sigma_{\text {bending }}
$$

These components can be calculated from the forces acting on the substrate as follows:

$$
\begin{aligned}
& \sigma_{\text {compression }}=-\frac{F}{t_{s}}=-\frac{t_{f}}{t_{s}} \sigma_{f}-\frac{\mathrm{t}_{\mathrm{s}}-\longleftarrow}{\mathrm{t}}=\sigma_{\mathrm{f}} \mathrm{t}_{\mathrm{f}} \\
& \sigma_{\text {bending }}=\frac{12 M}{t_{s}^{3} y}-\frac{\overline{\mathrm{t}_{\mathrm{s}}}}{2} \underline{\mathbf{V}}--\mathrm{M} \quad M=-\sigma_{f} t_{f} \frac{t_{s}}{2} \\
& \sigma_{\text {bending }}=-\frac{6 \sigma_{f} t_{f}}{t_{s}^{2}} y
\end{aligned}
$$

These stresses are illustrated in the diagrams below. 


\section{Stress Diagram:}

Stresses in Film and Substrate

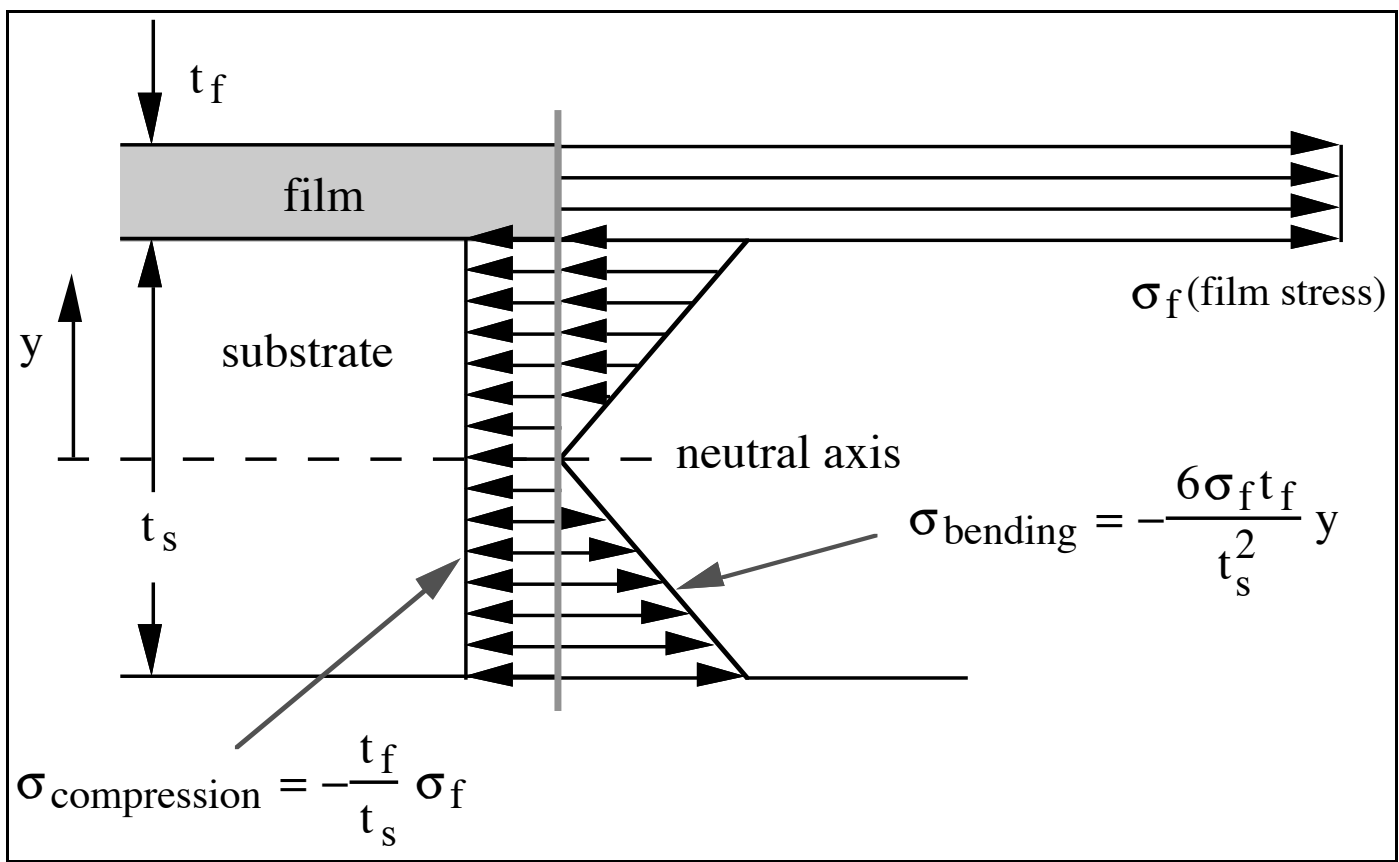

Stresses at the interface between film and substrate, where $y=t_{S} / 2$, are:

In the film: $\sigma_{x x}=\sigma_{f}$

In the substrate:

$$
\begin{aligned}
& \sigma_{x x}=\sigma_{\text {compression }}+\sigma_{\text {bending }} \\
& \sigma_{x x}\left(y=\frac{t_{s}}{2}\right)=-\frac{t_{f}}{t_{s}} \sigma_{f}-\frac{3 t_{f}}{t_{s}} \sigma_{f}=-\frac{4 t_{f}}{t_{s}} \sigma_{f}
\end{aligned}
$$


For typical geometries:

$$
\begin{aligned}
& t_{f}=1 \mu \mathrm{m} \\
& t_{s}=500 \mu \mathrm{m} \\
& \sigma_{x x}\left(\text { substrate, } y=\frac{t_{s}}{2}\right)=-\frac{4}{500} \sigma_{f}
\end{aligned}
$$

The substrate stresses are about 100 times smaller than the film stresses! It should be noted here that there are no significant tractions acting between the film and the substrate over most of the film/substrate interface. The forces transmitted between the film and the substrate act through the interface only near the edges of the film, usually just a few film thicknesses from the edge. We discuss this later.

\section{Calculation of Film Stresses from Misfit Strain}

The analysis above assumed the existence of a film stress $\sigma_{f}$. We now compute this stress from film/substrate mismatch.

Assume that a misfit exists between the film and substrate in the stress free state,

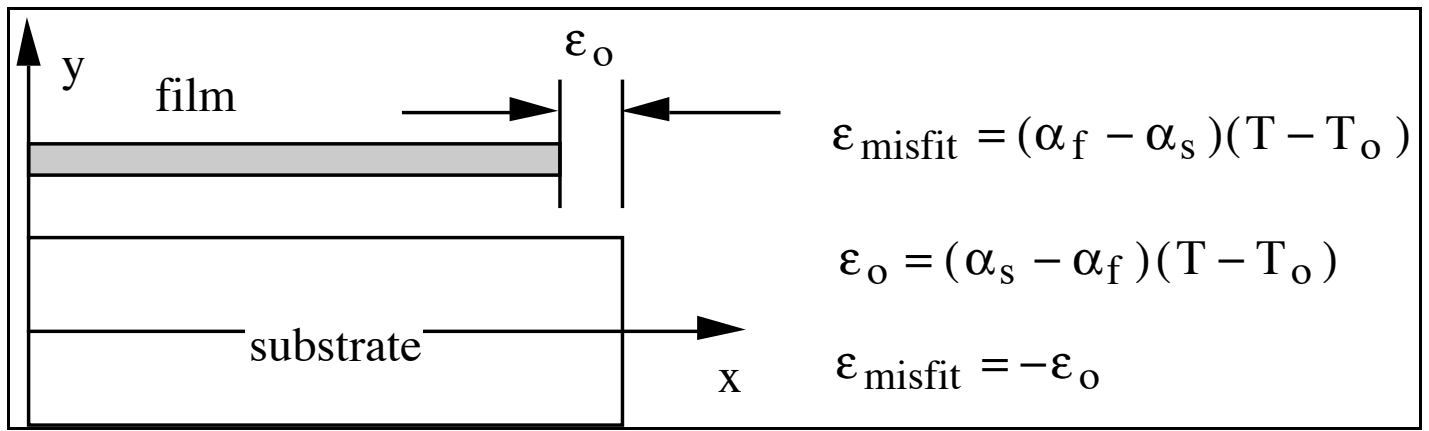

If the misfit were due to a difference in thermal expansion, then

$$
\varepsilon_{\text {misfit }}=\left(\alpha_{f}-\alpha_{s}\right)\left(T-T_{o}\right)
$$

It will be convenient to define

$$
\varepsilon_{o}=-\varepsilon_{\text {misfit }}=\left(\alpha_{s}-\alpha_{f}\right)\left(T-T_{o}\right)
$$


This is the strain that would have to be imposed to accommodate the misfit.

For the film and substrate to fit together perfectly, we must have

$$
\varepsilon_{f}^{\text {elastic }}+\varepsilon_{\text {misfit }}=\varepsilon_{s}^{\text {elastic }}\left(y=\frac{t_{s}}{2}\right)
$$

or

$$
\varepsilon_{f}^{\text {elastic }}-\varepsilon_{s}^{\text {elastic }}\left(y=\frac{t_{s}}{2}\right)+\varepsilon_{\text {misfit }}=0
$$

where

$$
\varepsilon_{f}^{\text {elastic }}=\left(\frac{1-v_{f}}{E_{f}}\right) \sigma_{f}
$$

and

$$
\varepsilon_{s}^{\text {elastic }}\left(y=\frac{t_{s}}{2}\right)=\left(\frac{1-v_{s}}{E_{s}}\right)\left(-4 \frac{t_{f}}{t_{s}} \sigma_{f}\right)
$$

so

$$
\left(\frac{1-v_{f}}{E_{f}}\right) \sigma_{f}-\left(\frac{1-v_{s}}{E_{s}}\right)\left(-4 \frac{t_{f}}{t_{s}} \sigma_{f}\right)=-\varepsilon_{\text {misfit }}=\varepsilon_{o}
$$

Solving for $\sigma_{f}$, the resulting expression for the stress in the film is:

$$
\sigma_{f}=\frac{\varepsilon_{o}}{\left[\left(\frac{1-v_{f}}{E_{f}}\right)+\left(\frac{1-v_{s}}{E_{s}}\right)\left(4 \frac{t_{f}}{t_{s}}\right)\right]}
$$

In the limiting cases: 
$\underline{\text { Rigid substrate case: }}\left(\frac{1-v_{s}}{E_{s}}\right) \rightarrow 0 \quad$ we have

$$
\sigma_{f}=\frac{E_{f}}{1-v_{f}} \varepsilon_{o}
$$

In this limit all the misfit strain is accommodated by strain in the film.

Elastically homogeneous case:

$$
\begin{aligned}
& \left(\frac{1-v_{f}}{E_{f}}\right)=\left(\frac{1-v_{s}}{E_{s}}\right) \\
& \sigma_{f}=\frac{E}{1-v} \frac{\varepsilon_{o}}{\left.1+4 \frac{t_{f}}{t_{s}}\right]} \approx \frac{E}{1-v} \varepsilon_{o}
\end{aligned}
$$

The previous relation is not valid for

$$
E_{s} \rightarrow 0 \quad \text { or } \quad \frac{t_{s}}{t_{f}} \rightarrow 0 .
$$

Thin film approximations break down in such cases. Next we consider the more general problem of mutiple films and substrates with arbitrary thicknesses and biaxial elastic moduli.

\section{General Treatment of Multiple Films of Arbitrary Thickness and Arbitrary Biaxial Elastic Moduli}

\section{The Problem}

Suppose that films of arbitrary thickness $t_{1}, t_{2}, t_{3}, \ldots t_{n}$ with arbitrary biaxial elastic moduli $B_{1}, B_{2}, B_{3} \ldots B_{n}$ are deposited onto a substrate of thickness $t_{0}$ with 
biaxial modulus $B_{0}$. As shown in the diagram below, the films are characterized by misfit strains, relative to the substrate, $\varepsilon_{1}^{\text {misfit }}, \varepsilon_{2}^{\text {misfit }}, \varepsilon_{3}^{\text {misfit }}, \ldots \ldots . \varepsilon_{n}^{\text {misfit }}$. The positions of the interfaces that mark the beginning of each film are denoted as $y_{1}$, $y_{2}, y_{3}, \ldots y_{n}$. Elastic accommodation of the misfit strains causes the entire multilayered structure to bend to a curvature $\kappa$. We wish to develop a general procedure for calculating the curvature caused by elastic accommodation of the misfit strains.

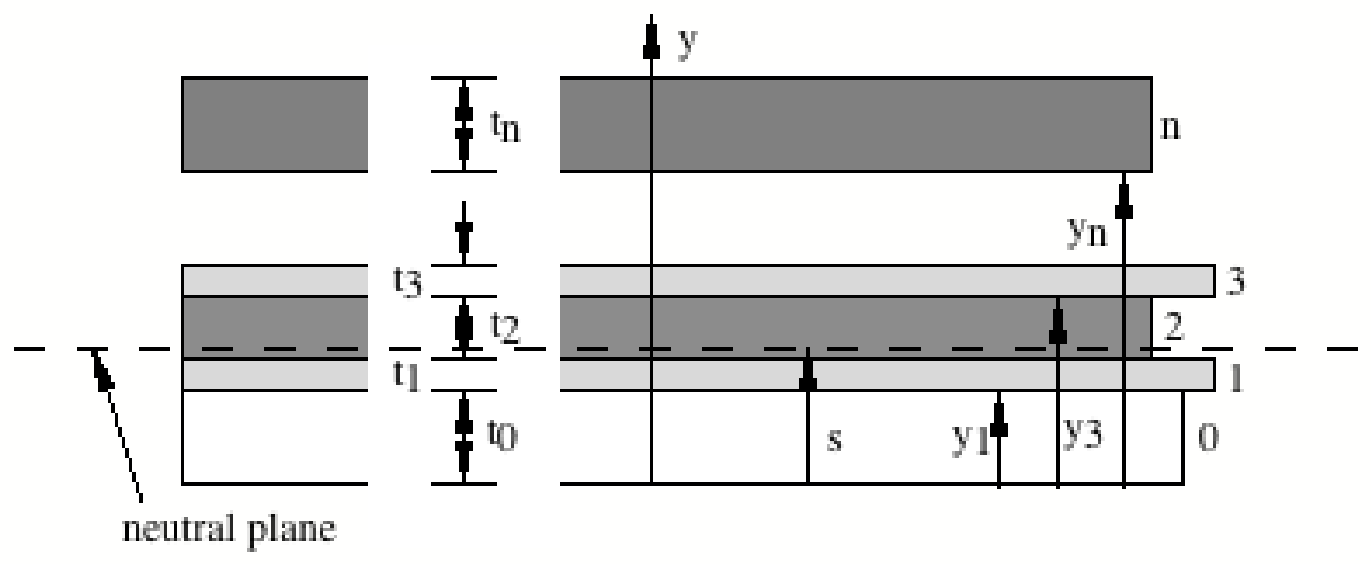

We need to know two quantities to determine the curvature produced when the misfit strains are accommodated elastically. One is the position of the neutral plane for bending, $s$ and the other is the bending stiffness of the multilayered plate, which we call, $B I$, where $B$ is the biaxial modulus and $I$ is the moment of enertia (per unit width) of the plate.

\section{Position of the Neutral Plane for Bending}

We first determine the position of the neutral plane for bending. This can be found by subjecting the plate to an arbitrary, uniform, in-plane elastic strain $\varepsilon$ (with no bending) and finding the position of plane about which there are no bending moments. This is, by definition, the position of the neutral plane. This condition may be expressed as

$$
M=0=\sum_{i=0}^{n} \int_{y_{i}}^{y_{i+1}} \varepsilon B_{i}(y-s) d y,
$$

or 


$$
0=\sum_{i=0}^{n} \int_{y_{i}}^{y_{i+1}} B_{i}(y-s) d y \text {. }
$$

For a multilayered plate this leads to

$$
s=\frac{1}{2} \frac{\sum_{i=0}^{n} B_{i}\left(y_{i+1}^{2}-y_{i}^{2}\right)}{\sum_{i=0}^{n} B_{i}\left(y_{i+1}-y_{i}\right)} \text {. }
$$

For a bilayer plate this leads to

$$
s=\frac{B_{o} t_{o}^{2}+B_{1}\left(t_{1}^{2}+2 t_{o} t_{1}\right)}{2\left(B_{o} t_{o}+B_{1} t_{1}\right)} \text {. }
$$

\section{Bending Stiffness}

We now turn to the bending stiffness of the multilayered plate. For reference it is useful to remember that the relationship between an applied bending moment (per unit width), $M$, and the resulting curvature, $\kappa$, for an elastically homogeneous plate is simply

$$
M=-\left(\frac{E}{1-v}\right) \frac{h^{3}}{12} \kappa
$$

It is useful to write this relation as

$$
M=-(B I) \kappa
$$

where $B=E /(1-v)$ is the biaxial modulus of the plate and $I=h^{3} / 12$ is the moment of enertia (per unit width) for bending. This product can be called the bending stiffness. For a multilayered plate with films of arbitrary thickness and arbitrary biaxial elastic moduli the curvature can be found from the known bending moment if the bending stiffness, $B I$, is known. 
We can find the bending stiffness for any multilayered plate by simply imposing a bending curvature onto the plate and calculating the necessary bending moment. Suppose a plate is deformed in pure bending with a curvature $\kappa$. Then the biaxial elastic strains everywhere in the plate may be expressed as

$$
\varepsilon=-\kappa(y-s) .
$$

This linear strain distribution is shown in the diagram below for a positive imposed curvature.

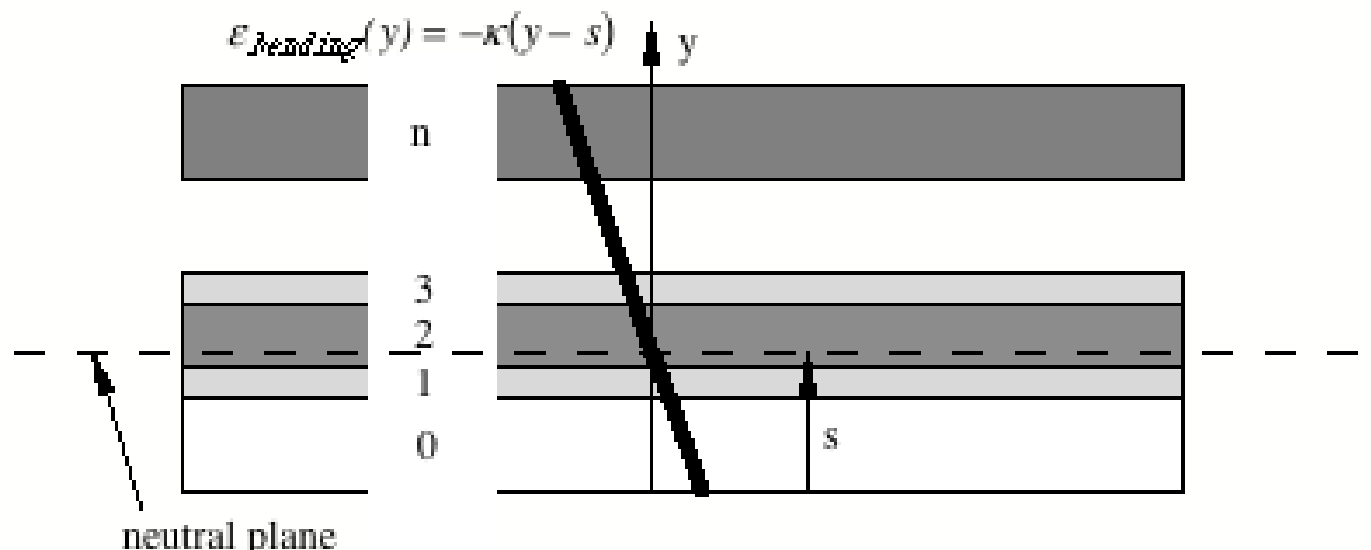

Now the biaxial stresses can be found at every point in the plate, using $\sigma=B \varepsilon$ and from that the bending moment needed to produce the imposed curvature can be calculated as

$$
M=\sum_{i=0}^{n} \int_{y_{i}}^{y_{i+1}}-\kappa B_{i}(y-s)^{2} d y
$$

Thus the bending stiffness, $B I$, can be calculated as

$$
B I=-\frac{M}{\kappa}=\sum_{i=0}^{n} \int_{y_{i}}^{y_{i+1}} B_{i}(y-s)^{2} d y \text {, }
$$

which leads to 


$$
B I=\sum_{i=0}^{n} \frac{B_{i}}{3}\left(\left(y_{i+1}-s\right)^{3}-\left(y_{i}-s\right)^{3}\right)
$$

For a bilayer plate the bending stiffness is

$$
B I=\frac{B_{0}^{2} t_{0}^{4}+B_{1}^{2} t_{1}^{4}+2 B_{0} B_{1} t_{0} t_{1}\left(2 t_{0}^{2}+3 t_{0} t_{1}+2 t_{1}^{2}\right)}{12\left(B_{0} t_{0}+B_{1} t_{1}\right)}
$$

\section{Curvature of a Multilayered Plate caused by Elastic Accommodation of Misfit Strains}

With the relationships developed above, we may now compute the curvature of a multilayered plate caused by the elastic accommodation of the misfit strains. If the layers shown in the first figure are elastically stretched or compressed to exactly match the dimensions of the substrate, then the elastic strains in the layers will be

$$
\varepsilon_{1}^{\text {elastic }}=-\varepsilon_{1}^{\text {misfit }}, \varepsilon_{2}^{\text {elastic }}=-\varepsilon_{2}^{\text {misfit }}, \ldots \ldots \varepsilon_{n}^{\text {elastic }}=-\varepsilon_{n}^{\text {misfit }}
$$

These elastic strains will require stresses in the layers, which can be maintained only if a bending moment, $M_{\text {restrain }}$, is applied to the plate. We call this $M_{\text {restrain }}$ because it is the bending moment that would have to be applied to keep the plate flat once the misfit strains have been accommodated elastically. It may be calculated as

$$
M_{\text {restrain }}=\sum_{i=1}^{n} \int_{y_{i}}^{y_{i+1}} \varepsilon_{i}^{\text {elastic }} B_{i}(y-s) d y
$$

Note that stresses are not developed in the substrate by these accommodations processes since the films are being deformed to match the substrate. Removal of this restraining moment is equivalent to superimposing a bending moment

$$
M=-M_{\text {restrain }}
$$

onto the multilayered plate. In terms of the misfit strains this bending moment is 


$$
M=\sum_{i=1}^{n} \int_{y_{i}}^{y_{i+1}} \varepsilon_{i}^{m i s f i t} B_{i}(y-s) d y
$$

Now the curvature of the plate can be directly calculated using

$$
\kappa=-\frac{M}{(B I)}
$$

where the bending stiffness, $B I$, is given by (9) and the bending moment, $M$, is given by (15). The result is

$$
\kappa=-\frac{\sum_{i=1}^{n} B_{i} \varepsilon_{i}^{m i s f i t}\left\{\frac{1}{2}\left(y_{i+1}^{2}-y_{i}^{2}\right)-s\left(y_{i+1}-y_{i}\right)\right\}}{\sum_{i=0}^{n} \frac{B_{i}}{3}\left(\left(y_{i+1}-s\right)^{3}-\left(y_{i}-s\right)^{3}\right)}
$$

For a bilayer plate in which the misfit strain of the one film is $\varepsilon_{1}^{m i s f i t}$, the resulting curvature is

$$
\kappa=-\varepsilon_{1}^{\text {misfit }} \frac{6 B_{0} B_{1} t_{0} t_{1}\left(t_{0}+t_{1}\right)}{\left(B_{0}^{2} t_{0}^{4}+2 B_{0} B_{1} t_{0} t_{1}\left(2 t_{0}^{2}+3 t_{0} t_{1}+2 t_{1}^{2}\right)+B_{1}^{2} t_{1}^{4}\right)}
$$

\section{Elastic Assumptions}

It should be noted that the procedure described here requires all of the layers to deform elastically only. No plastic deformation is allowed to occur in any of the layers. The reason for this requirement is that all of the layers contribute to the bending stiffness. For the case of a thin film on a substrate, the film is allowed to deform plastically because the film is so thin that it does not contribute to the bending stiffness For the multilayered structure yielding may occur when the films are deposited. That would only change the misfit that must be accommodated. But in the final state all of the layers must be elastic. 


\section{Multiple Films - Additive Effects}

First consider what happens when a moment per unit length $M$ is applied to a thin film/substrate composite along the edge of the composite.

Mechanics of a substrate with a thin film

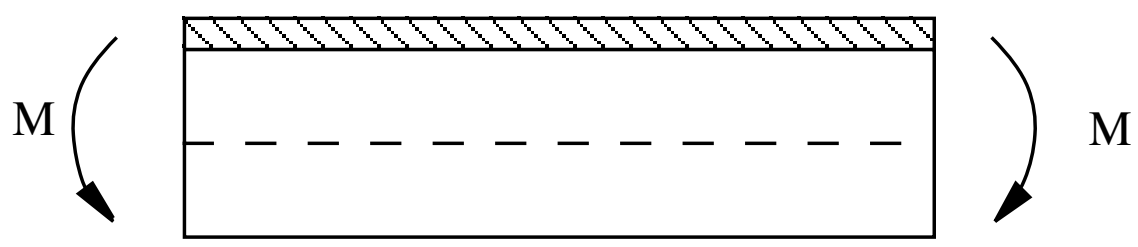

Because the film is extremely thin compared to the substrate, $t_{f}<<t_{s}$ the flexural rigidity of the film/substrate composite is the same as that for the substrate alone. So the resulting curvature is

$$
\kappa=-\left(\frac{1-v_{s}}{E_{s}}\right) \frac{12 M}{t_{s}^{3}} \quad \text {, as if the film were not there. }
$$

The bending of the substrate imposes an additional (small) strain on the film. (The film goes along for the ride.) The strain at the film/substrate interface is given by

$$
\varepsilon_{x x}\left(y=\frac{t_{s}}{2}\right)=\left(\frac{1-v_{s}}{E_{s}}\right) \frac{6 M}{t_{s}^{2}}
$$

Thus the increment in strain imposed on the film is also

$$
\Delta \varepsilon_{x x}^{f i l m}=\left(\frac{1-v_{s}}{E_{s}}\right) \frac{6 M}{t_{s}^{2}}
$$

Now we may consider the additive effects of multiple films. When a second film is deposited on top of the first, it may also bend the substrate if a misfit exists between it and the substrate. Any moment and bending caused by the second film would cause the strain in the first film to change (very slightly) due to the bending. 
Example: Effect of stress in a passivation film on the stress in an underlying metal film

A typical problem is to study compressive passivation; we wish to determine the stresses induced in the underlying film (metal film). Consider a metal film on a silicon substrate. Suppose a stress ( biaxial ) exists in the metal film and that the substrate is bent.

$\sigma_{f_{1}}=\sigma_{m}$

We will assume that the stress in the film is tensile.

Metal film on a silicon substrate (bending not shown)

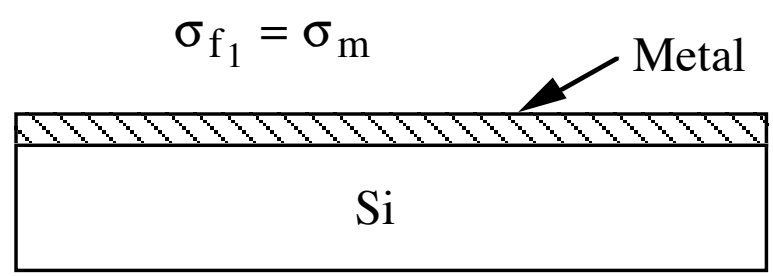

Now consider the effect of depositing a second film, on top of the metal film. This might be a passivation film which we will assume to be in compression. Assume the deposition of the second film does not change the chemical composition or defect structure of the first film. We look only at the induced stresses.

Passivation film on top of a metal film on a silicon substrate

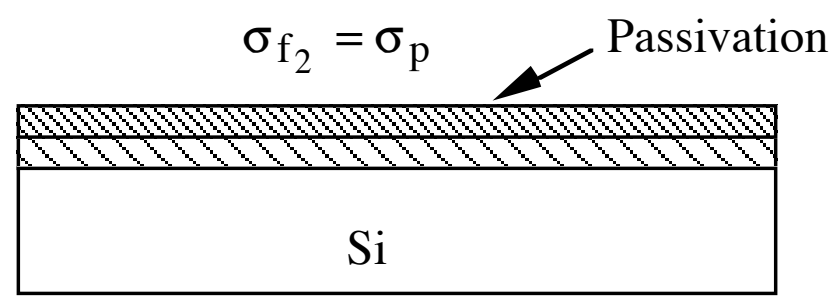

Let the stress in the passivation (due to mismatch with substrate) be $\sigma_{f_{2}}=\sigma_{p}$. The stress in the second film (the passivation) imposes a moment on the substrate and causes bending. 


\section{Passivation - substrate mechanics}

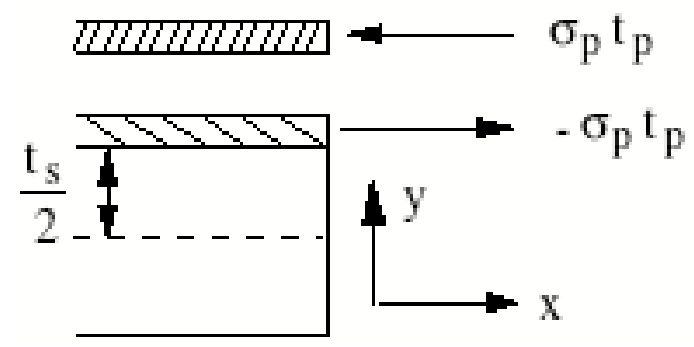

In the substrate:

$$
\begin{aligned}
& F_{s}=-\sigma_{p} t_{p} \\
& M_{s}=-\sigma_{p} t_{p} \frac{t_{s}}{2}
\end{aligned}
$$

The increment in strain on the metal film is then

$$
\begin{aligned}
& \Delta \varepsilon_{x x}^{\text {metal }}=\Delta \varepsilon_{x x}(\text { compression })+\Delta \varepsilon_{x x}(\text { bending }) \\
& \Delta \varepsilon_{x x}^{\text {metal }}=\left(\frac{1-v_{s}}{E_{s}}\right)\left(\frac{-\sigma_{p} t_{p}}{t_{s}}\right)+\left(\frac{1-v_{s}}{E_{s}}\right) \frac{6}{t_{s}^{2}}\left(-\sigma_{p} t_{p} \frac{t_{s}}{2}\right) \\
& \Delta \varepsilon_{x x}^{\text {metal }}=-\left(\frac{1-v_{s}}{E_{s}}\right) \frac{4 t_{p}}{t_{s}} \sigma_{p}
\end{aligned}
$$

and the corresponding increment in stress in the metal film is

$$
\Delta \sigma_{x x}^{\text {metal }}=\left(\frac{E_{m}}{1-v_{m}}\right) \Delta \varepsilon_{x x}^{\text {metal }}=-\left(\frac{E_{m}}{1-v_{m}}\right)\left(\frac{1-v_{s}}{E_{s}}\right) \frac{4 t_{p}}{t_{s}} \sigma_{p}
$$

If the metal film and substrate were elastically identical, i.e.,

$$
\left(\frac{E_{m}}{1-v_{m}}\right)=\left(\frac{E_{s}}{1-v_{s}}\right) \text { then }
$$




$$
\Delta \sigma_{x x}^{\text {metal }}=-\frac{4 t_{p}}{t_{s}} \sigma_{p}
$$

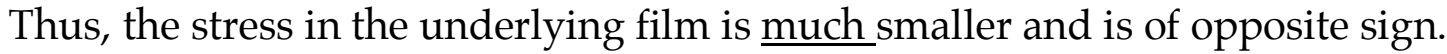

Since in the thin film approximation, $t_{p} / t_{s}<<1$, it follows that

$$
\Delta \sigma_{x x}^{\text {metal }}<<\sigma_{p}
$$

So, a compressive passivation (with $\sigma_{\mathrm{p}}$ negative) does produce a tension stress in a film beneath it, but the added stress in the film is extremely small, much smaller than the initial stress in the film.

To a good approximation, the stress in a given film depends only on its misfit with the substrate. The other films present have essentially no effect on the stress in a particular film.

\section{Additivity of Curvatures Produced by Thin Films}

Because the films of interest are all very thin compared to the thickness of the substrate, each film deposited imposes a separate bending moment which produces a curvature. Since the moments are additive, so are the resulting curvatures.

Consider $\mathrm{n}$ films of thickness $t_{1}, t_{2}, t_{3} \ldots t_{n^{\prime}}$ and stresses $\sigma_{1}, \sigma_{2}, \sigma_{3} \ldots \sigma_{n}$

\section{Substrate with multiple films}

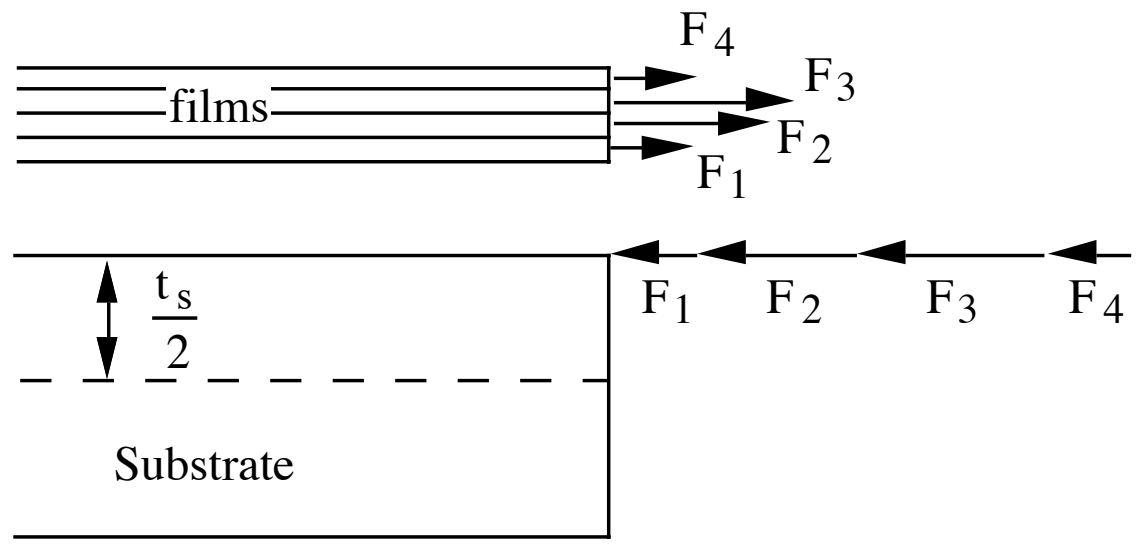


The bending moments are (per unit length) $M_{1}, M_{2}, M_{3} \ldots$

$$
M_{1}=-\sigma_{1} t_{1} \frac{t_{s}}{2} \text { etc. }
$$

Thus each film produces a curvature (change) $\Delta \kappa_{1}, \Delta \kappa_{2}, \Delta \kappa_{3} \ldots$

$$
\Delta \kappa_{1}=-\left(\frac{1-v_{s}}{E_{s}}\right) \frac{12 M_{1}}{t_{s}^{3}}=\left(\frac{1-v_{s}}{E_{s}}\right) \frac{6 \sigma_{1} t_{1}}{t_{s}^{2}}
$$

So for $\mathrm{n}$ films present

$$
\begin{aligned}
& \Delta \kappa_{\text {total }}=\Delta \kappa_{1}+\Delta \kappa_{2}+\Delta \kappa_{3}+\ldots \Delta \kappa_{n} \\
& \Delta \kappa_{\text {total }}=\left(\frac{1-v_{s}}{E_{s}}\right) \frac{6}{t_{s}^{2}}\left[\sigma_{1} t_{1}+\sigma_{2} t_{2}+\sigma_{3} t_{3} \ldots \sigma_{n} t_{n}\right]
\end{aligned}
$$

Here $\Delta \kappa_{\text {total }}$ is the change in curvature associated with the presence of $\mathrm{n}$ thin films on a substrate. The substrate need not be perfectly flat to use this formula. Thin films with uniform stresses cause the shape of the substrate to change by a constant curvature. We will see later that the stress in a film is found by measuring the shape of a substrate both before and after the film is deposited. The difference in shape is simply a uniform or constant curvature.

\section{Some Notes On Measuring Procedures:}

To find the stress in each film by the curvature method, the curvature change produced by that film alone must be found. If two films are present, it is necessary to make at least two curvature measurements (If the substrate is perfectly flat), or three (if the substrate is initially curved). A common procedure would involve :

1. Measure initial curvature (or shape) of substrate $\left(\kappa_{O}\right)$

2. Measure change in curvature after film 1 is deposited:calculate $\sigma_{1}$

3. Measure change in curvature after film 2 is deposited: calculate $\sigma_{2}$ 


\section{$\underline{\text { Natural order of films }}$}
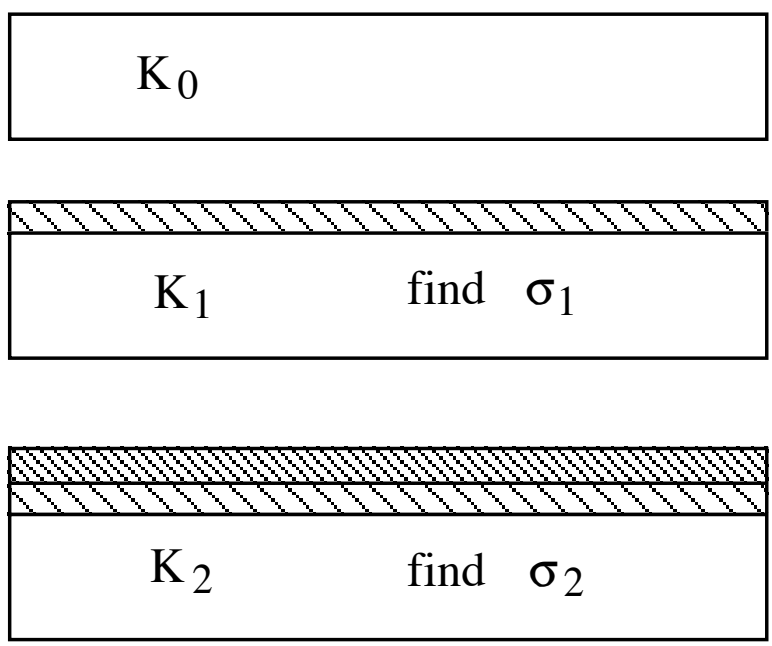

Even if $\kappa_{2}=\kappa_{0}$, it does not mean the films are stress free, even if the substrate is not elastically strained.

Another procedure would be :

1. Measure initial curvature, $\kappa_{O}$

2. Deposit both films 1 and 2 and, measure curvature change, then calculate $\sigma_{1} t_{1}+\sigma_{2} t_{2}$

3. Remove second film and measure $\kappa_{1}$ and calculate $\sigma_{1}$

Removal of one film

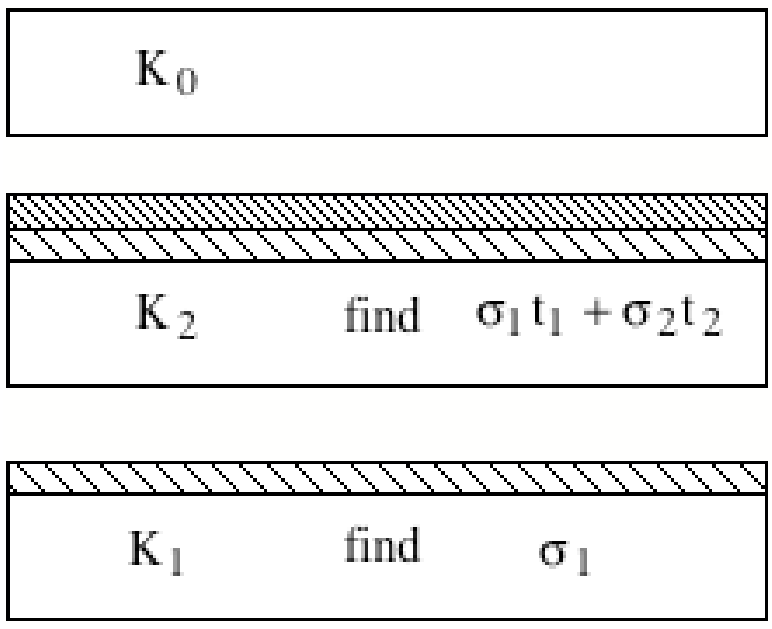


If a film is present and is not removable we can find $\sigma_{1}$ without the before and after steps, by backthinning of the substrate

\section{Back thinning of substrate.}
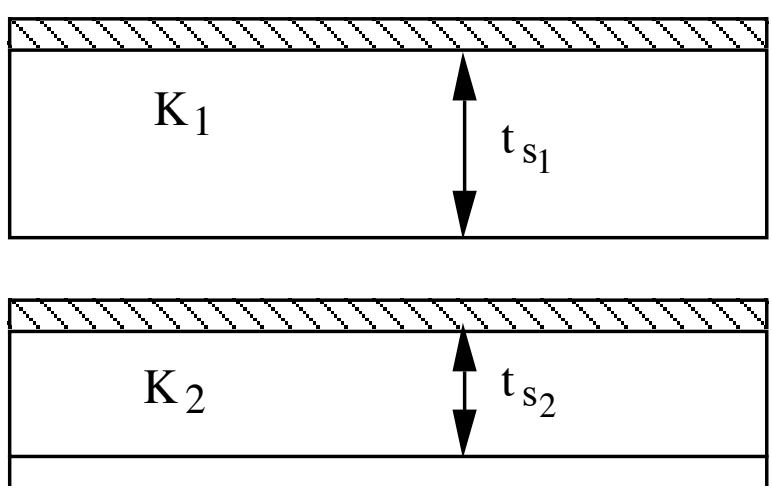

The curvatures are related to the stresses through the following relations:

$$
\begin{aligned}
& \kappa_{1}=\kappa_{0}+\frac{6 \sigma_{f} t_{f}}{t_{s_{1}}^{2}}\left(\frac{1-v_{s}}{E_{s}}\right) \\
& \kappa_{2}=\kappa_{0}+\frac{6 \sigma_{f} t_{f}}{t_{s_{2}}^{2}}\left(\frac{1-v_{s}}{E_{s}}\right)
\end{aligned}
$$

By subtracting the two relations we find

$$
\sigma_{f}=\left(\frac{E_{s}}{1-v_{s}}\right) \frac{\left(\kappa_{2}-\kappa_{1}\right)}{6 t_{f}\left(\frac{1}{t_{s_{2}}^{2}}-\frac{1}{t_{s_{1}}^{2}}\right)}
$$

\section{Interfacial Stresses}

Stresses in the film lead to forces on the substrate which, in turn, cause stresses to develop in the substrate. Thus, some forces must be transmitted across the film/substrate interface. But where? Only at the edges of the film!

Consider part of the film far from the edges of the film: 


\section{Stresses in film and substrate}

no significant tractions on the interface

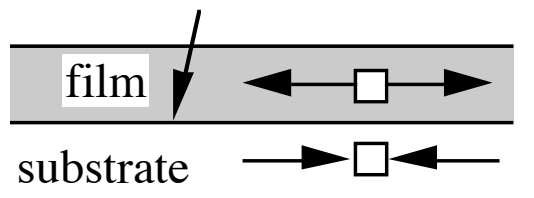

biaxial tension

biaxial compression

and bending

A simple force balance shows that very small normal tractions act on the filmsubstrate interface:

A force balance

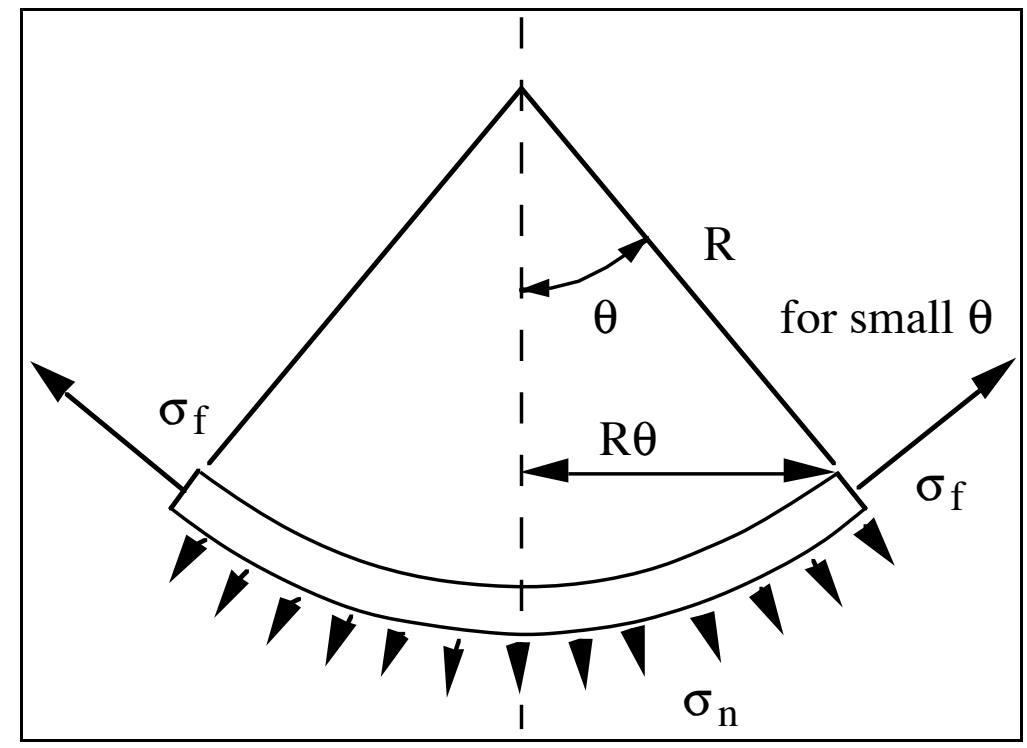

A free body analysis:

$$
\begin{aligned}
& F_{u p}=2 \pi(R \theta) \sigma_{f} t_{f} \sin \theta \\
& F_{\text {down }}=\sigma_{n} \pi(R \theta)^{2}
\end{aligned}
$$

Equating the forces: 


$$
\sigma_{n} \pi(R \theta)^{2}=2 \pi(R \theta) \sigma_{f} t_{f} \sin \theta
$$

The normal stress on the interface is then

$$
\sigma_{n}=\frac{2 t_{f}}{R} \sigma_{f}
$$

For

$$
\begin{aligned}
& t_{f} \approx 10^{-6} m \\
& R=100 m
\end{aligned}
$$

we get

$$
\sigma_{n}=2 \times 10^{-8} \sigma_{f}
$$

a very small stress indeed! 


\section{Edge Effects - Interfacial Shear Stresses}

Since thin film stresses cause the substrate to bend, there must be forces acting between the film and the substrate. As argued below, these interfacial forces are significant only near the edges of the film.

Consider a film with a misfit relative to the substrate:

Misfit strain

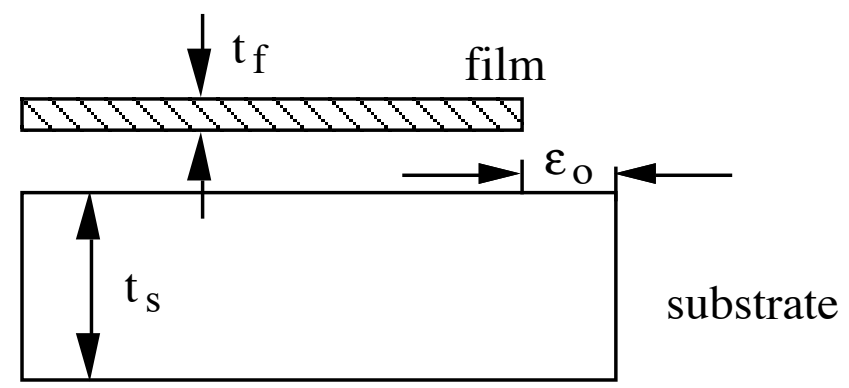

To join the film to the substrate we apply a biaxial tension stress $\sigma$ that is sufficient to stretch the film to match the substrate.

Film with external loading

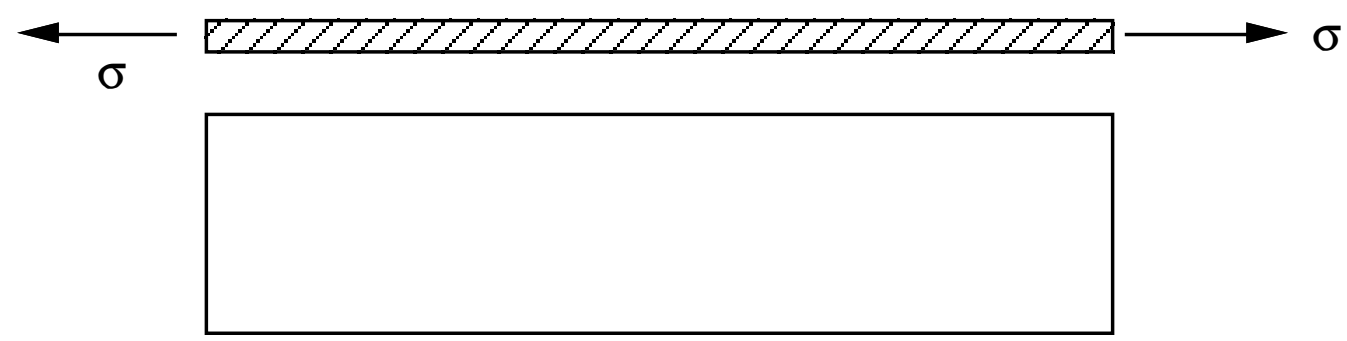

Now, we bond the stretched film to the substrate while maintaining the externally imposed biaxial stress. A magnified view of one edge is like: 


\section{External edge loading}

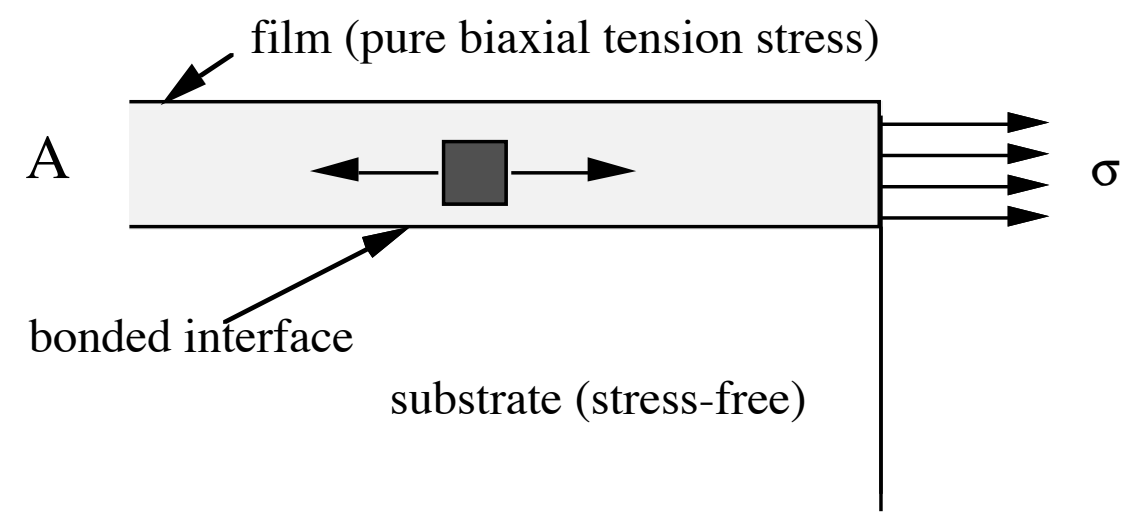

To find the final stress state in the film and substrate, we must superimpose edge forces to remove the external stress.

Removal of edge forces

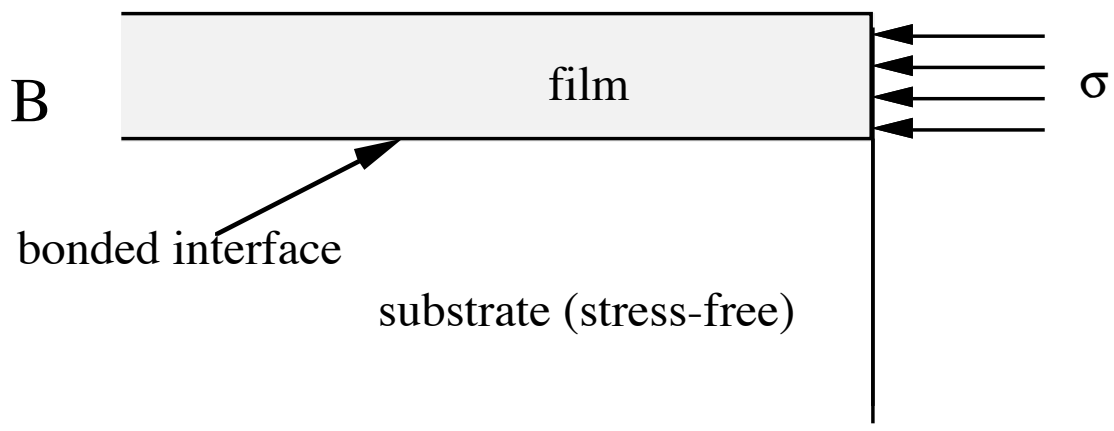

The stresses in the film near the edge are found by summing the stresses of states $A$ and B. Stress state A is pure biaxial tension in the film and stress state B is more complex. 


\section{Qualitative Remarks about Stress State B}

The interfacial shear stresses are contained entirely in stress state B.

Qualitatively, the shear stress $\sigma_{x y}$ on the interface must be

Interfacial shear stresses

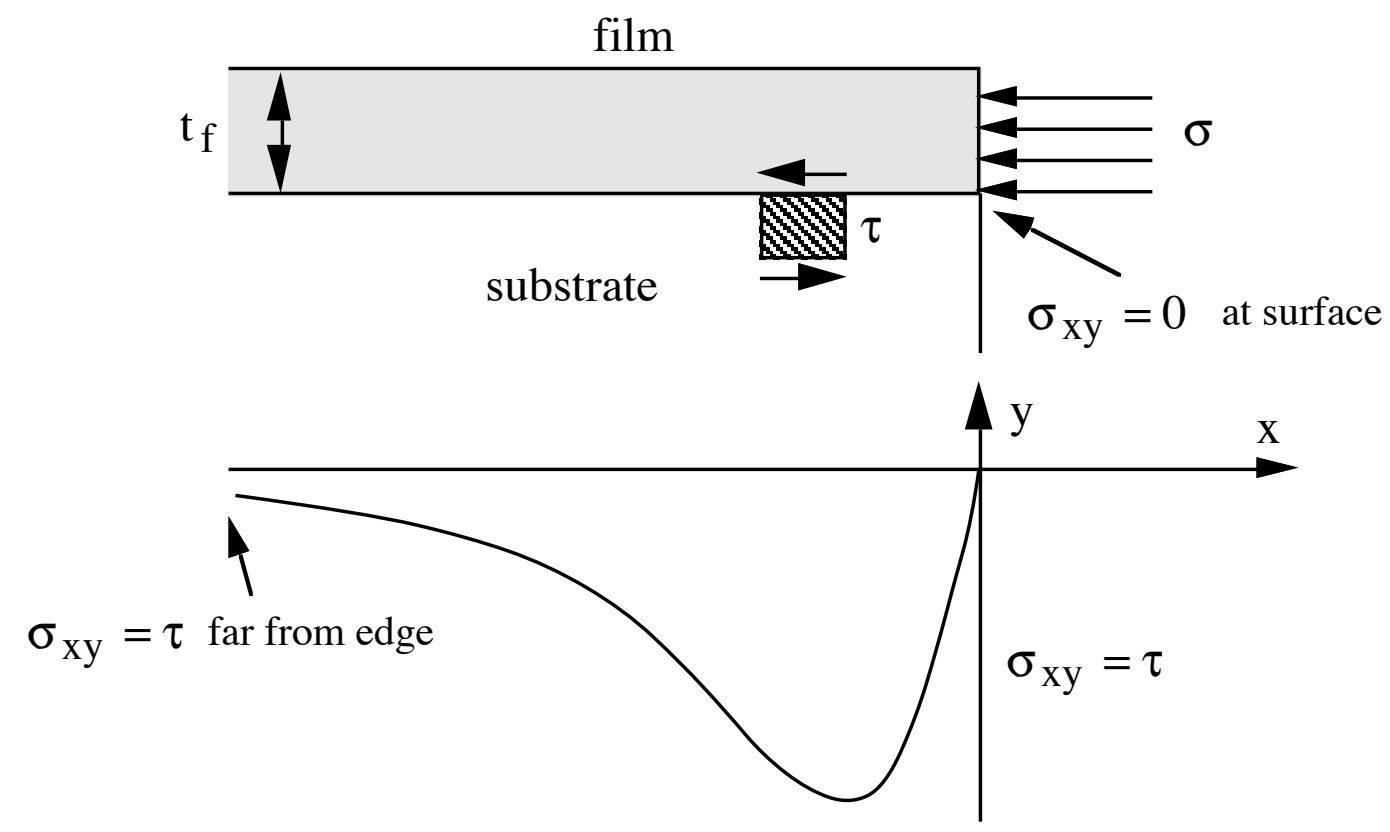

Force balance (the force per unit length of edge) requires

$$
\sigma t_{f}=\int_{-\infty}^{0} \sigma_{x y} d x=\int_{-\infty}^{0} \tau d x
$$

\section{Reviews of the topic of Near-edge Film Stresses have been made by}

M. Murakami, T.-S. Kuan and I.A. Blech, " Mechanical Properties of Thin Films on Substrates", Treatise on Mater. Sci. Tech., Vol. 24, (1982) 194.

Basic solutions given by Aleck (1949), and Tranter and Craggs (1947). Also, discussed by Doerner and Nix (1988). 


\section{A Simple Analysis Of Interfacial Shear Stresses}

Interfacial stress distribution
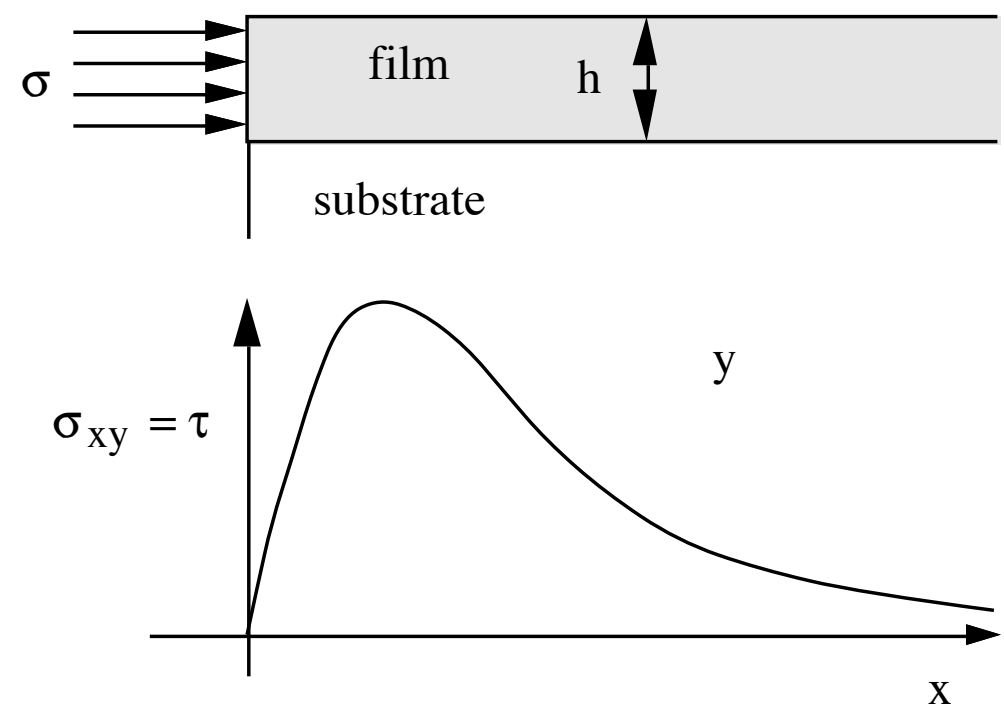

Assume:

$$
\tau=A \frac{x}{h} \exp \left(-B \frac{x}{h}\right)=A \alpha \exp (-B \alpha)
$$

Force equilibrium:

$$
\int_{0}^{\infty} \tau d x=\sigma h \quad \text { or } \quad \int_{0}^{\infty} \tau d \alpha=\sigma
$$

Define $\alpha_{m \text { ax }} \quad$ by

$$
\begin{aligned}
& \frac{d \tau}{d \alpha}=0 \\
& \frac{d \tau}{d \alpha}=A \alpha \exp (-B \alpha)(-B)+A \exp (-B \alpha)=0
\end{aligned}
$$




$$
[1-\alpha B] A \exp (-B \alpha)=0
$$

So we have

$$
\alpha_{\max }=\frac{1}{B}
$$

Apply force equilibrium:

$$
\int_{0}^{\infty} A \alpha \exp (-B \alpha) d \alpha=\sigma
$$

or

$$
\int_{0}^{\infty} \alpha \exp (-B \alpha) d \alpha=\frac{\sigma}{A}
$$

Integration by parts leads to:

$$
\int_{0}^{\infty} \alpha \exp (-B \alpha) d \alpha=\frac{1}{B^{2}}=\frac{\sigma}{A}
$$

So

$$
A=\sigma B^{2}=\frac{\sigma}{\alpha_{\max }^{2}}
$$

or

$$
\tau=\frac{\sigma \alpha}{\alpha_{\max }^{2}} \exp \left(-\frac{\alpha}{\alpha_{\max }}\right)
$$

The maximum interfacial shear stress is:

$$
\tau_{\max }=\tau\left(\alpha=\alpha_{\max }\right)=\frac{\sigma}{e \alpha_{\max }}
$$


For a rigid substrate the shear stress reaches maximum very near the edge of the film

Suppose $\alpha_{\max }=\frac{1}{4}$, then

$$
\tau_{\max }=\frac{4 \sigma}{e}=\frac{4}{2.7} \sigma
$$

Shear stress distribution for a rigid substrate

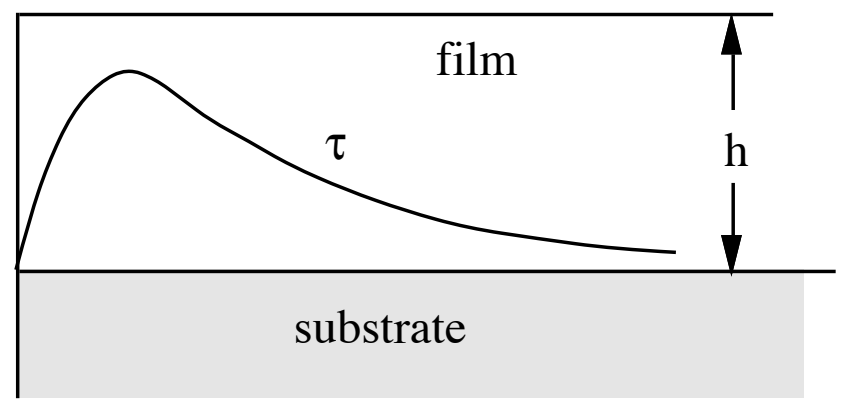

For compliant substrate, shear stress reaches maximum farther from edge of film

Suppose $\alpha_{\text {max }}=1$

$$
\tau_{\max }=\frac{\sigma}{e}=\frac{\sigma}{2.7}
$$

\section{Shear stress distribution for a compliant substrate}

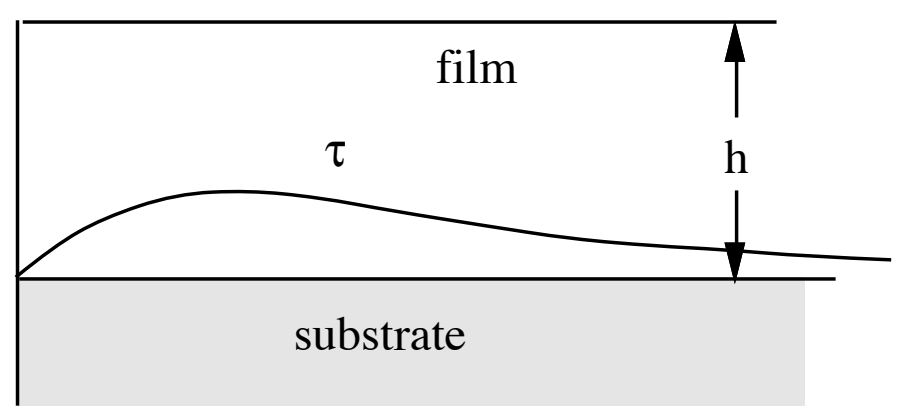

These estimates and calculations (see below) assume that the interface between the film and substrate is able to support the shear stresses required. If the 
interface is weak, the shear stress distribution will be controlled by the interfacial shear strength.

\section{Thermal Stresses in Al Lines on Si Substrates}

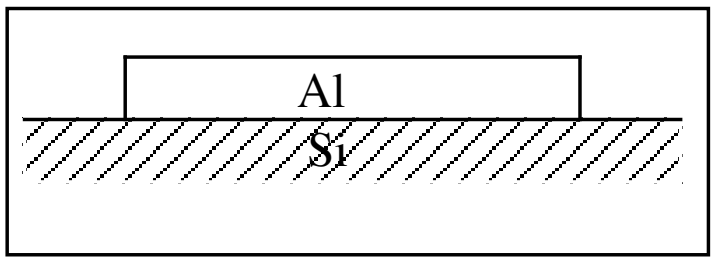

Stress free at $T=T_{0}$

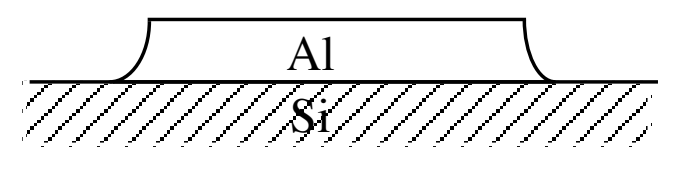

Thermal stresses at $\mathrm{T}=\mathrm{T}_{0}-\Delta \mathrm{T}$

\section{Lines}

* Typical patterned structure

* $\quad$ Permits study of stresses near edges

* $\quad$ Subject of MSE 352 --Stress Analysis

\section{References}

B.J. Aleck, J. Appl. Mech., $\underline{16} 118$ (1949)

I.A. Blech and A.A. Levi, J. Appl, Mech., $\underline{48} 442$ (1981)

A.I. Sauter, Ph.D. Stanford, 1991

M. Murakami, T.-S. Kuan, I.A. Blech, Treat. Mater. Sci. Tech. Vol. 24 (1982) 194.) 


\section{Plane Stress Problem (Aleck and Blech - Levi)}

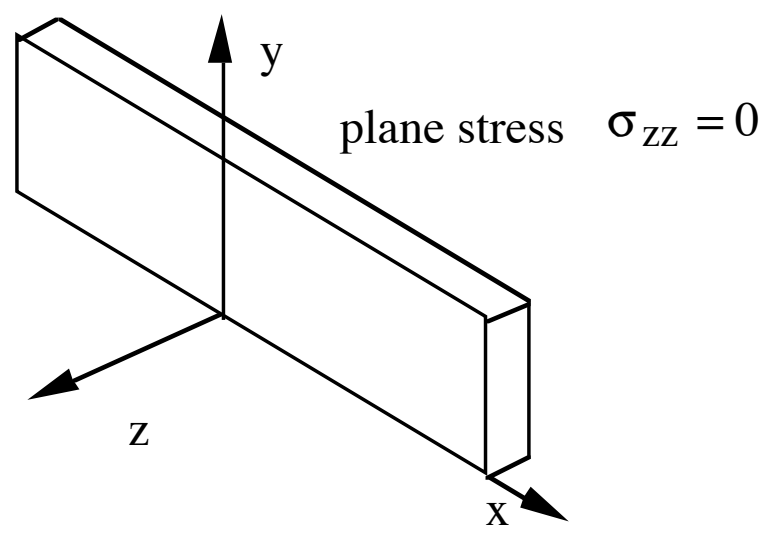

All thermal stresses normalized by: $\mathrm{E} \Delta \alpha \Delta \mathrm{T}$

\section{Plane Strain Problem (Sauter)}

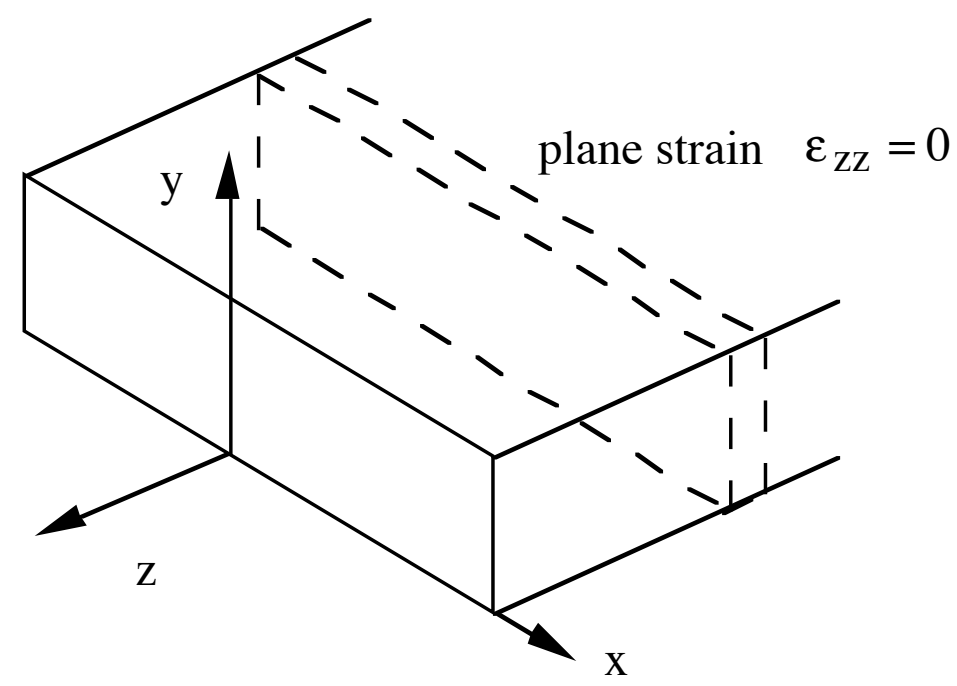

All thermal stresses normalized by: $\sigma=\frac{E}{1-v} \Delta \alpha \Delta T$ 


\section{Plane stress solution by Blech and Levi}

film elastically stretched, then bonded to substrate
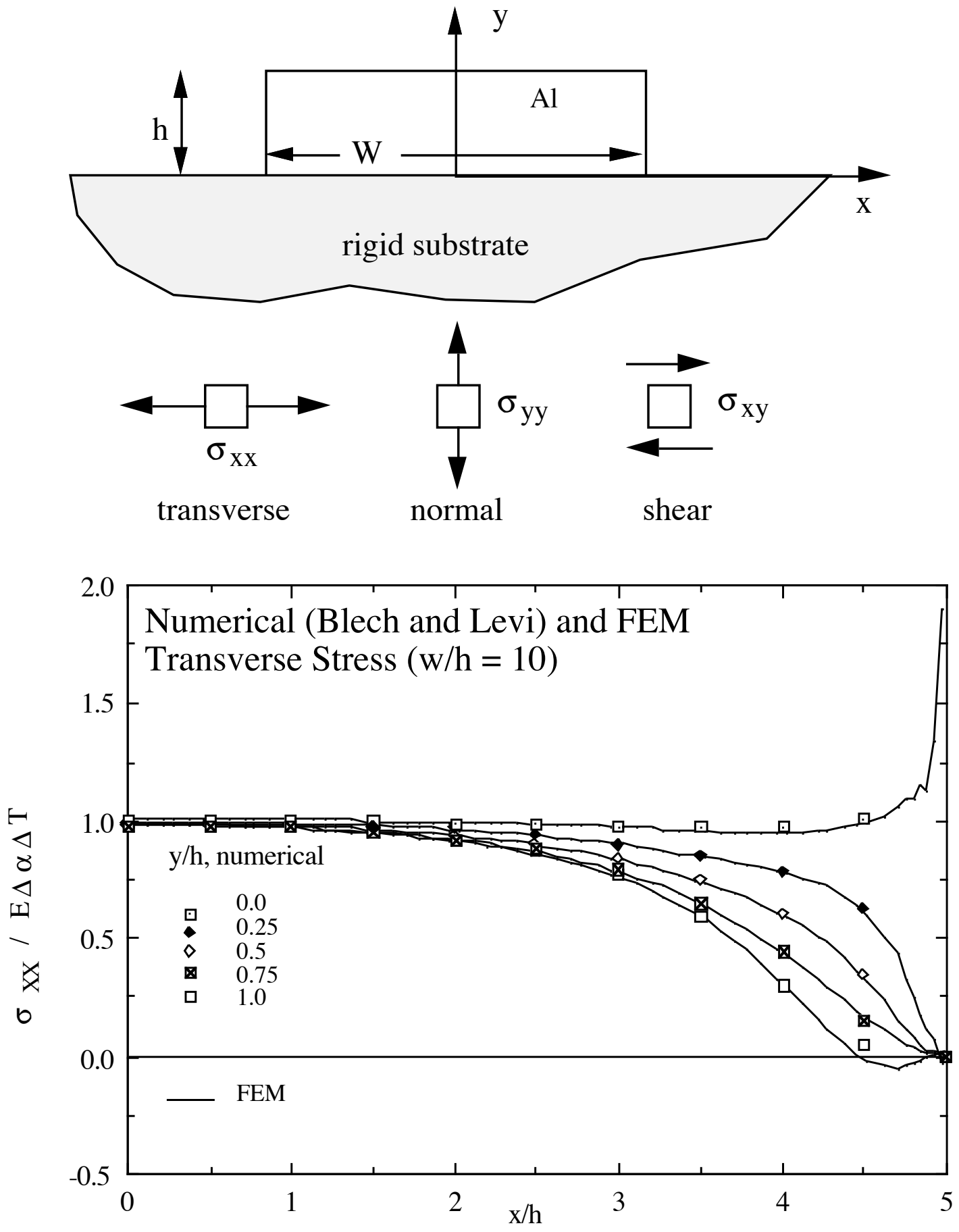


\section{FEM method.}

\section{Finite element geometry and problem}

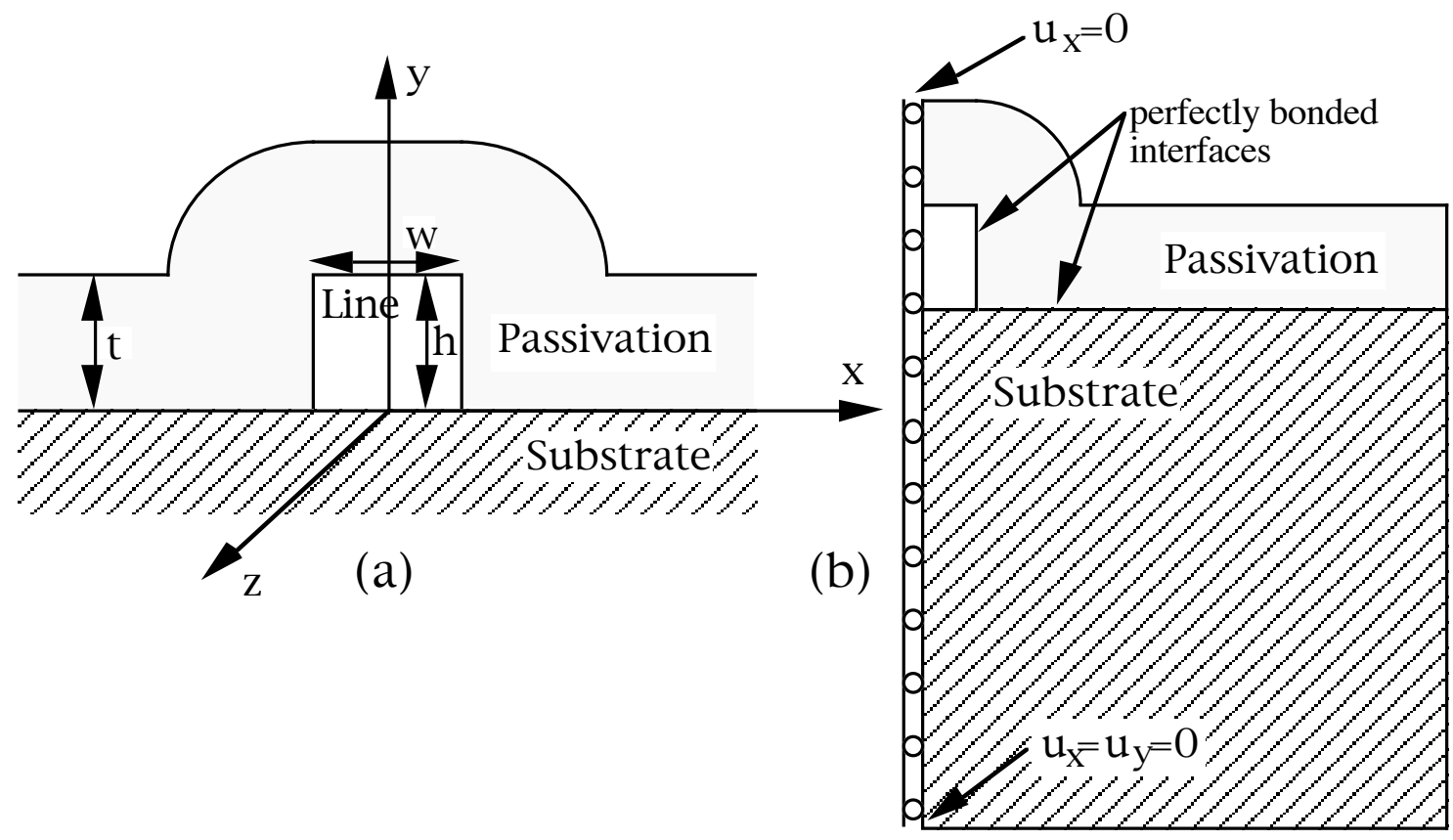

The stresses in all of the FEM plots shown on the following pages, taken from Anne Sauter-Mack's Ph.D. dissertation, are normalized by the biaxial thermal stress that would develop in an elastic film on a perfectly rigid substrate:

$$
\sigma=\frac{E}{1-v} \Delta \alpha \Delta T
$$


Transverse stresses in an unpassivated line
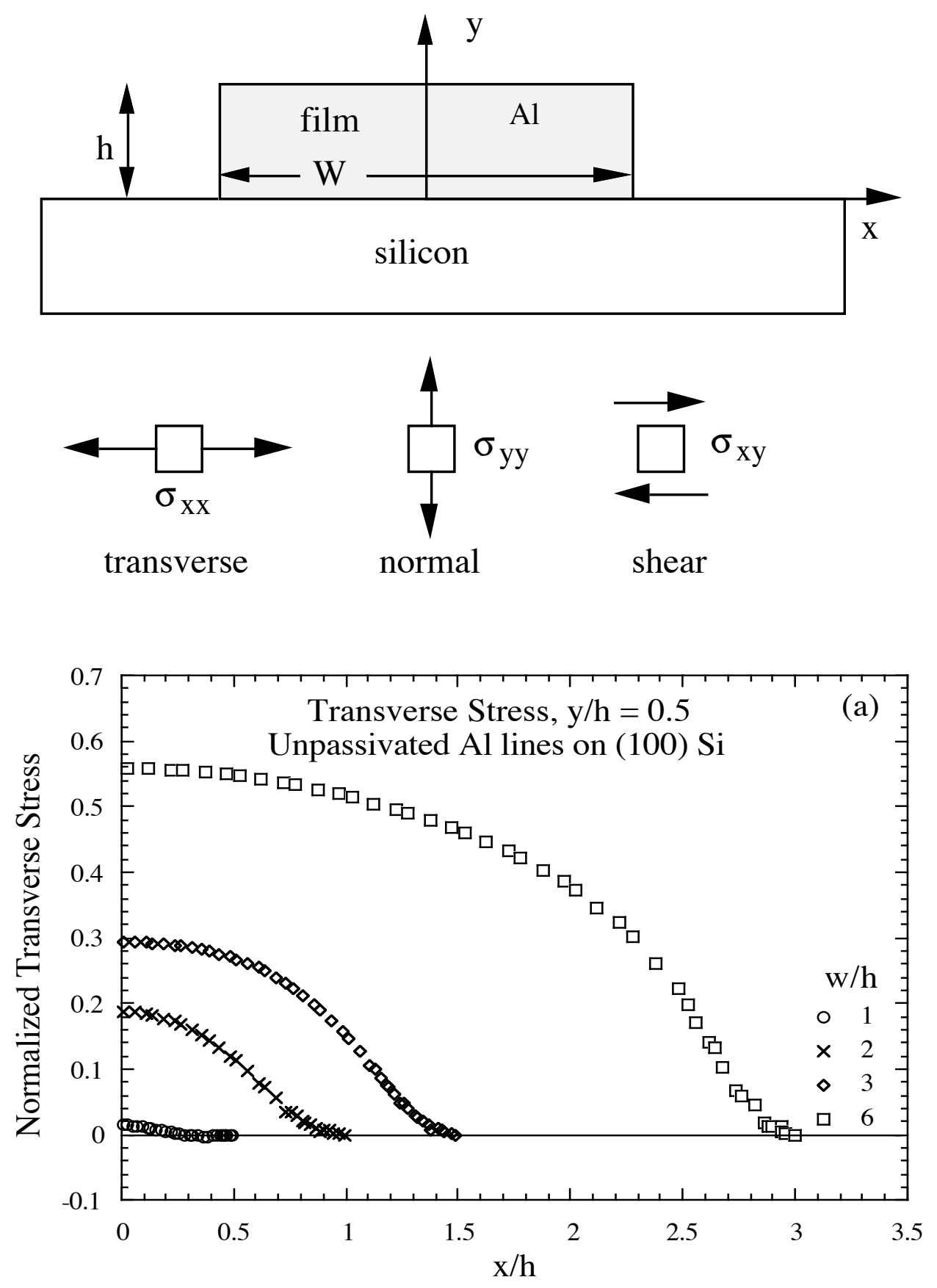
Transverse stresses in an unpassivated line
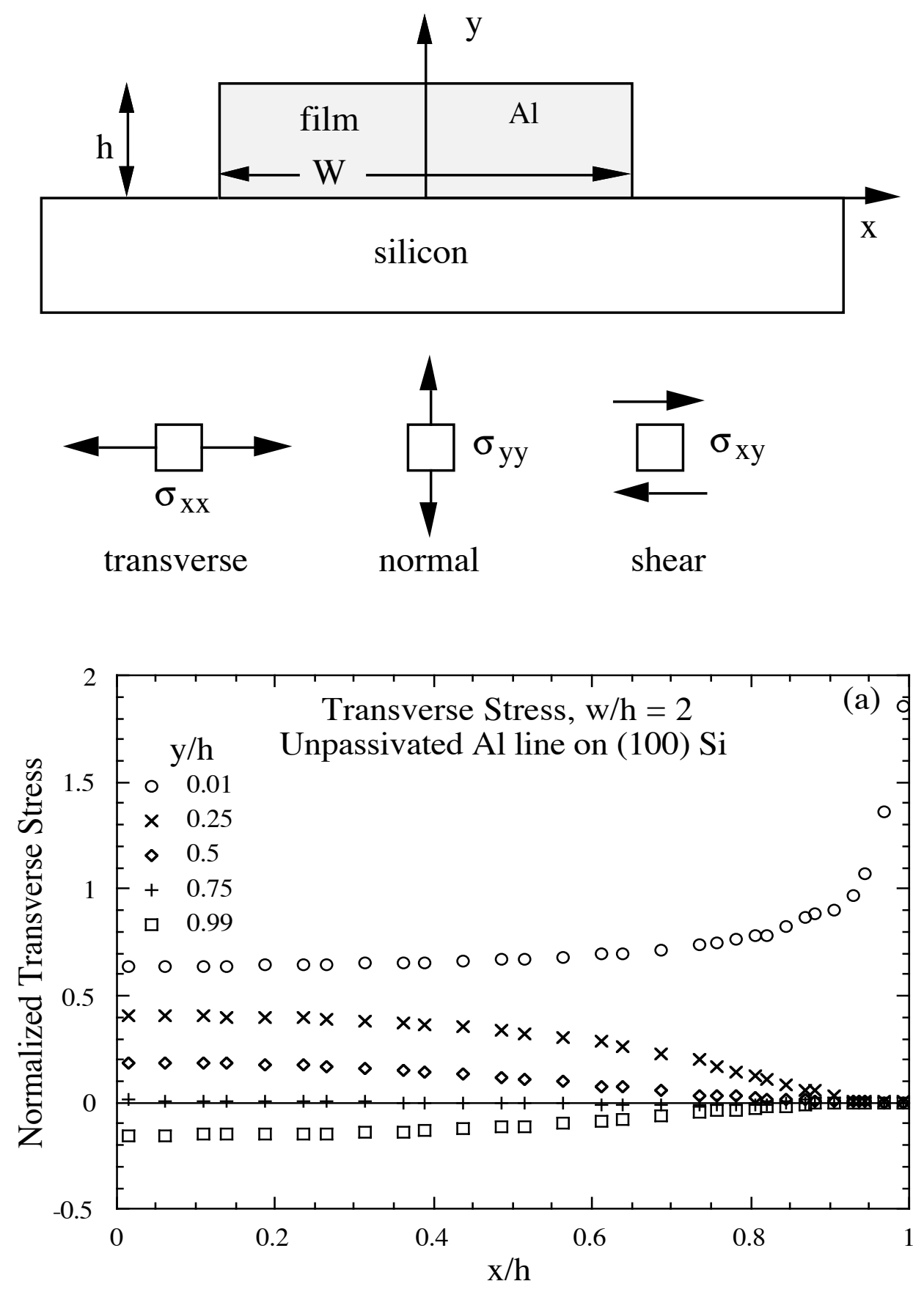
Shear stresses in an unpassivated line
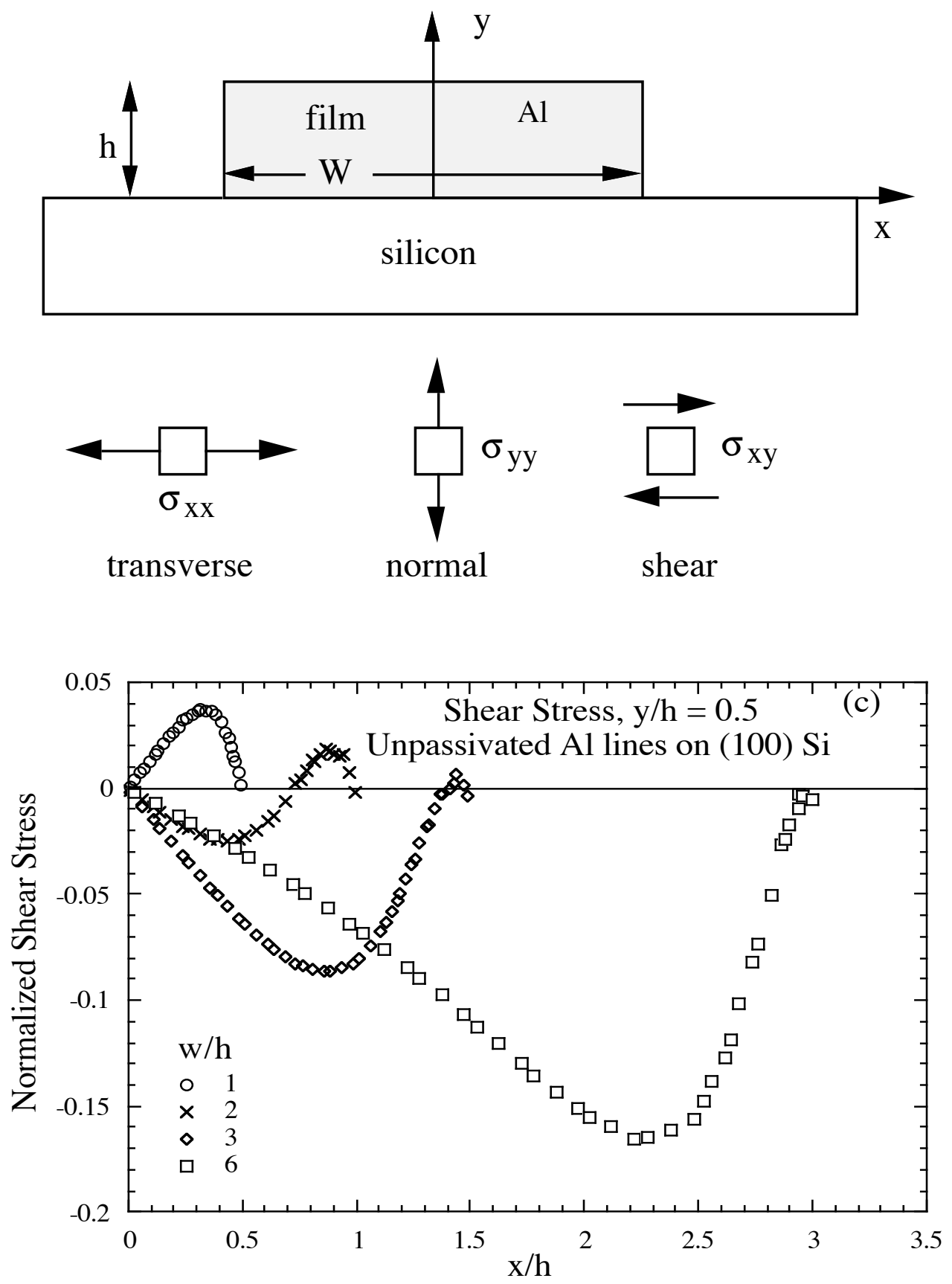

Stresses are "relaxed" in unpassivated narrow lines. 
Shear stresses in an unpassivated line
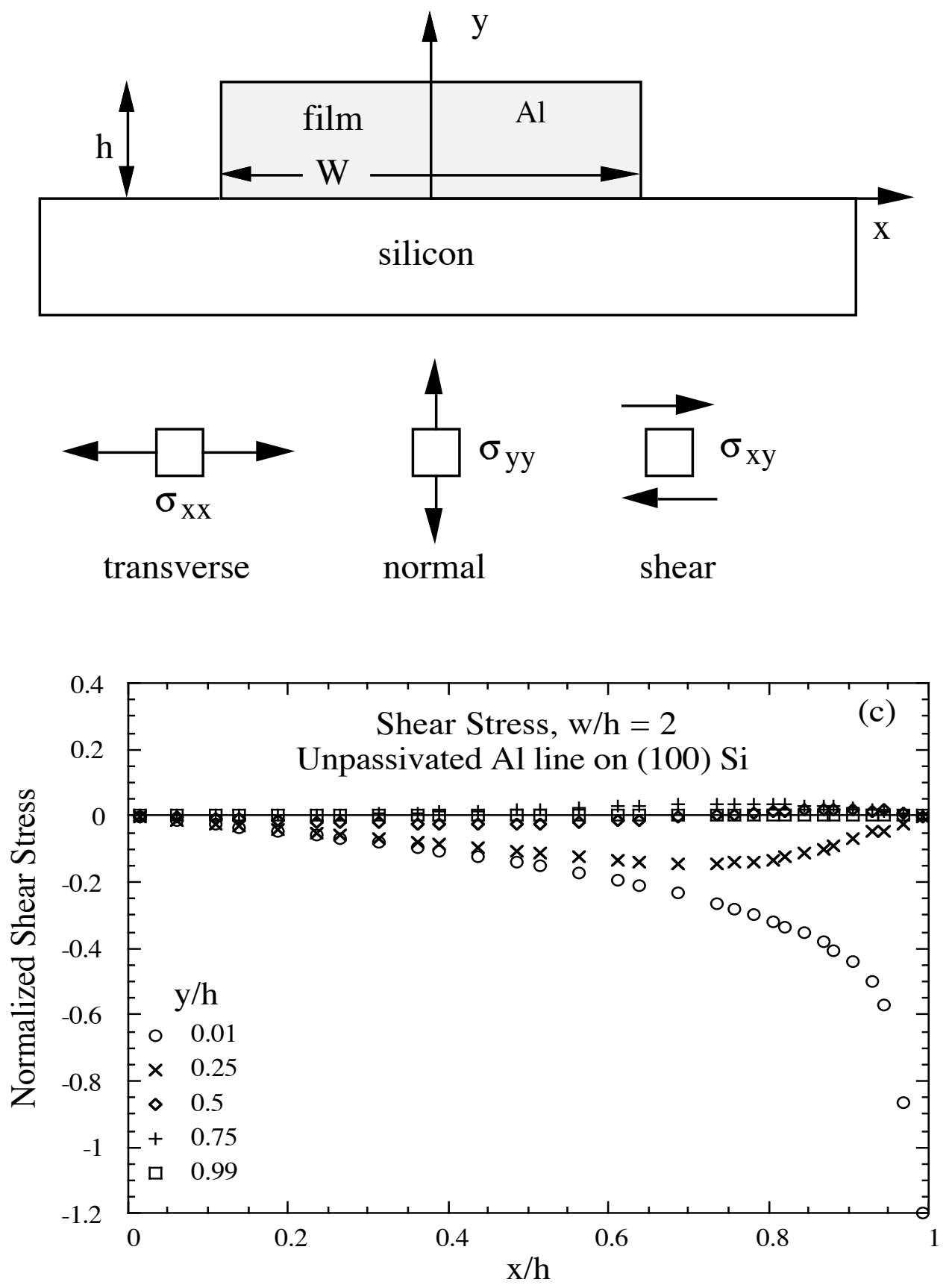
Longitudinal stresses in an unpassivated line
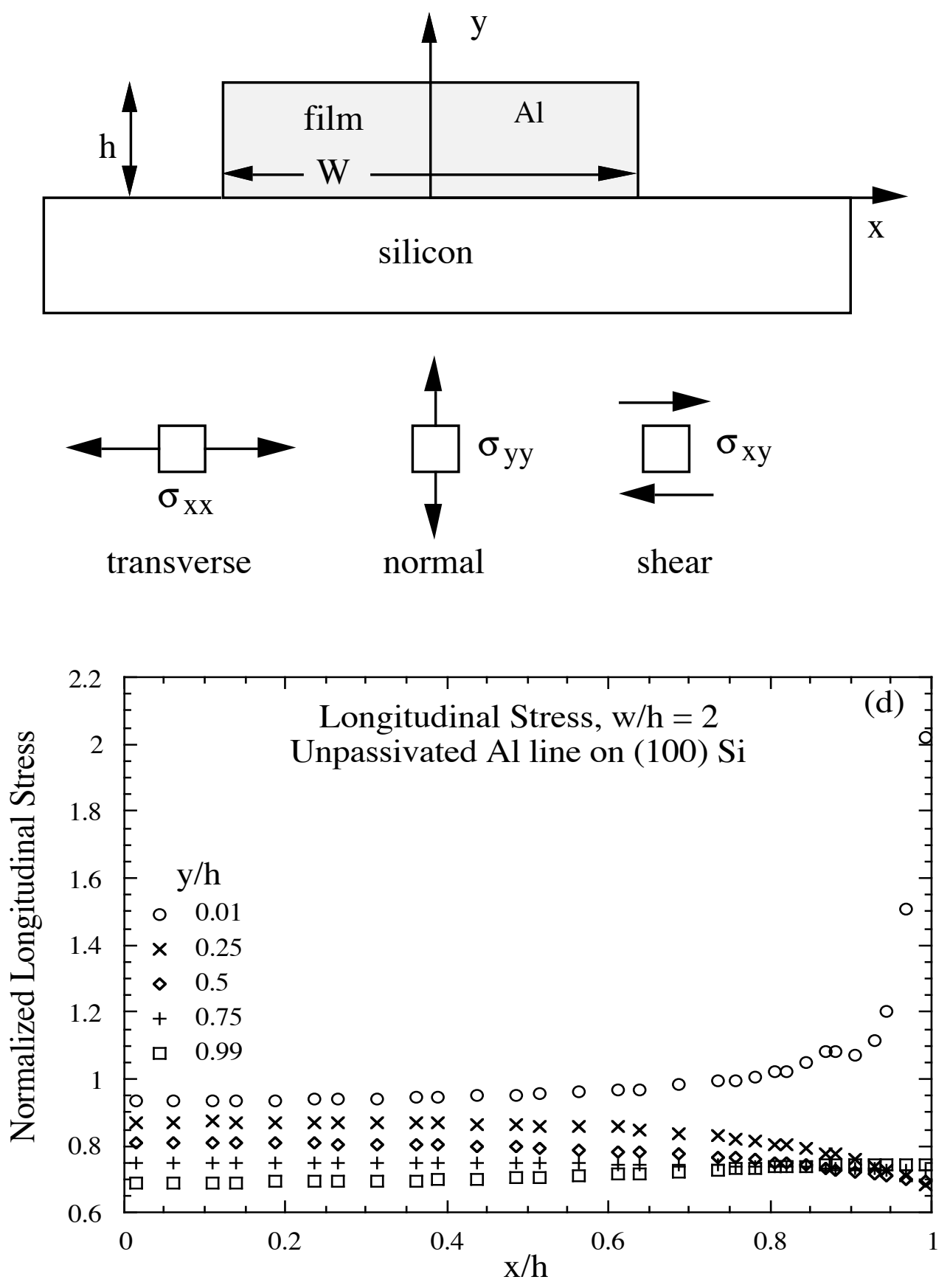
Normal Stresses in an unpassivated Line
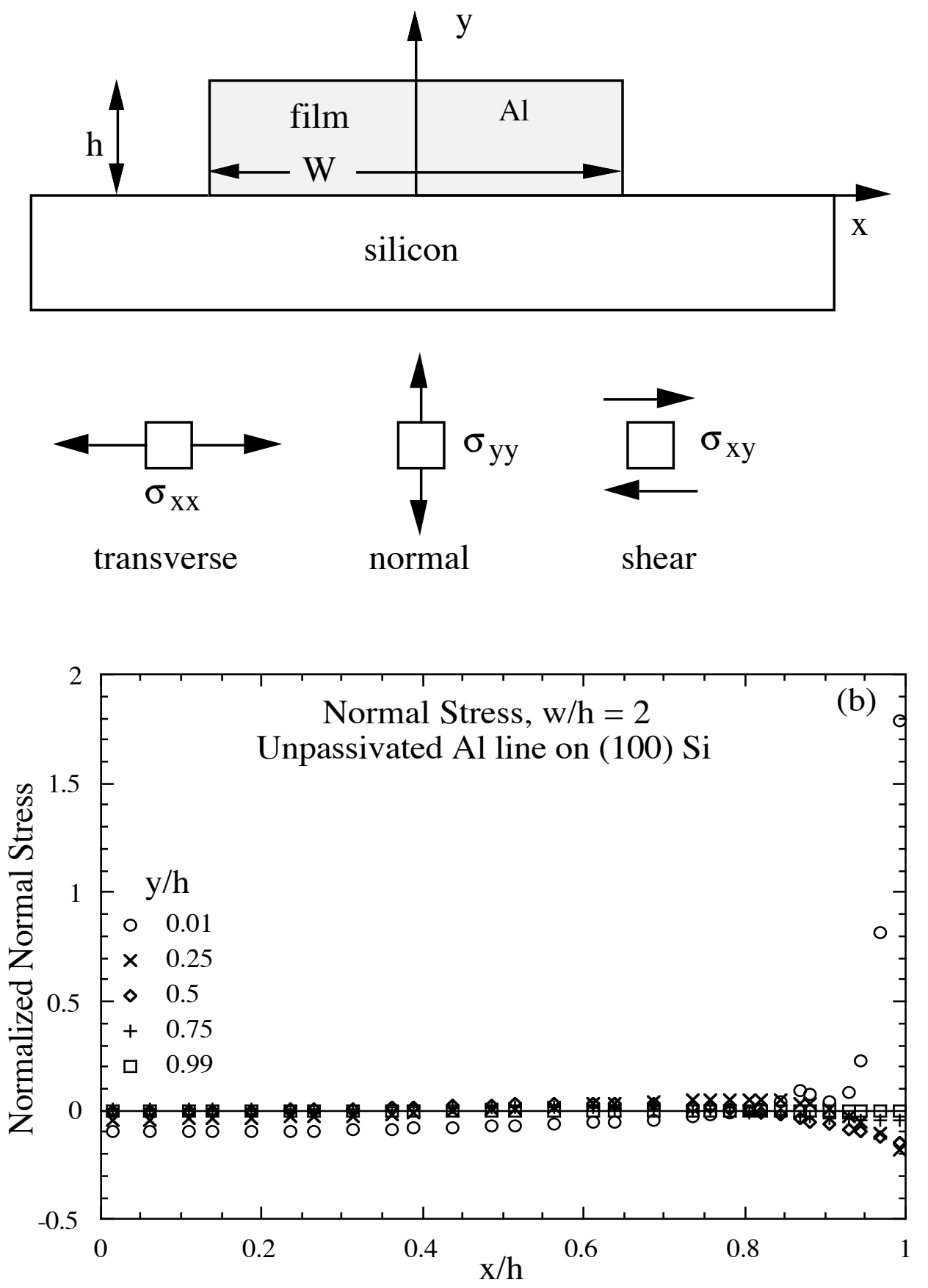
Hydrostatic Stresses in a Passivated Line
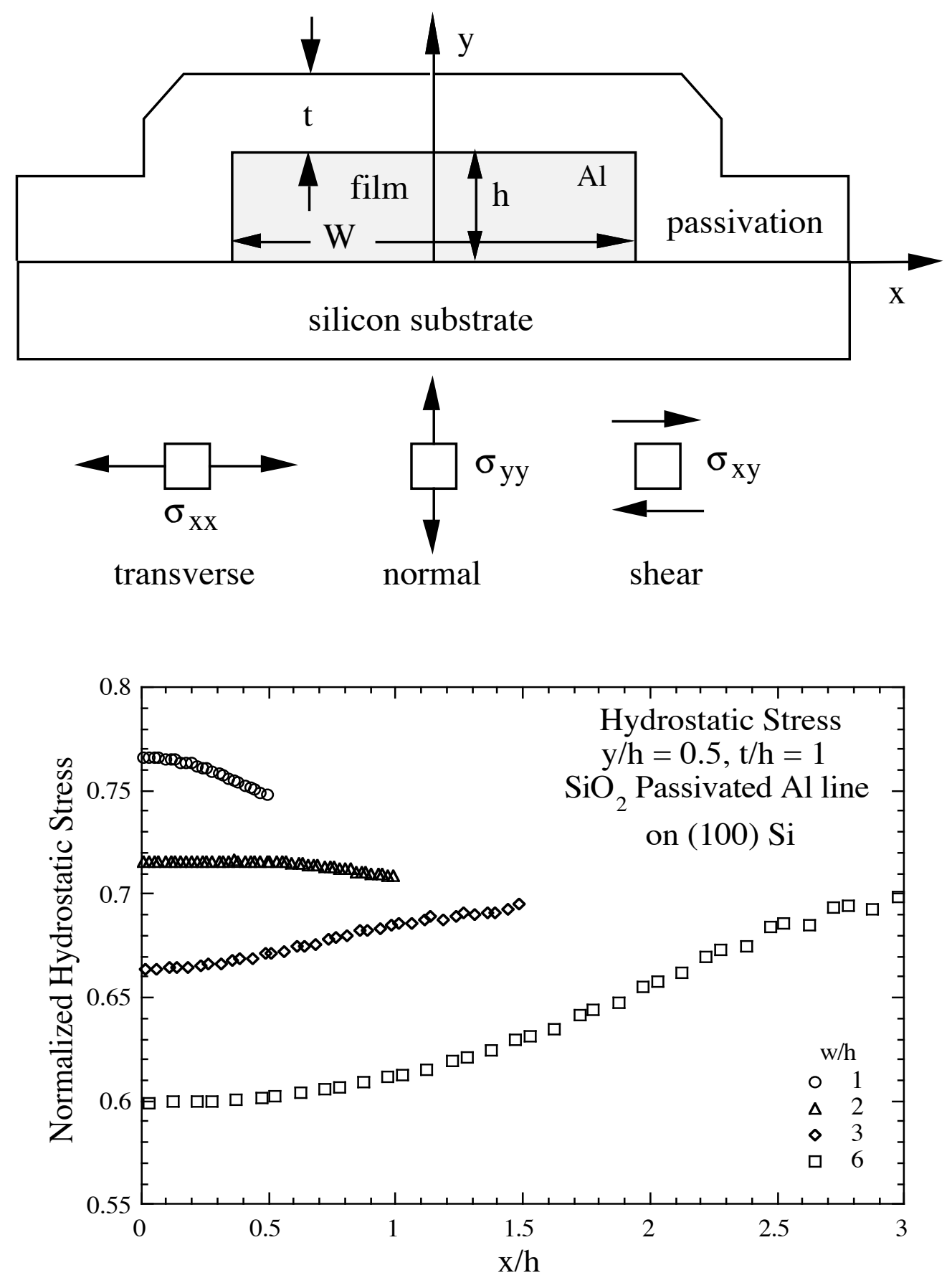
Normal Stresses in a passivated line
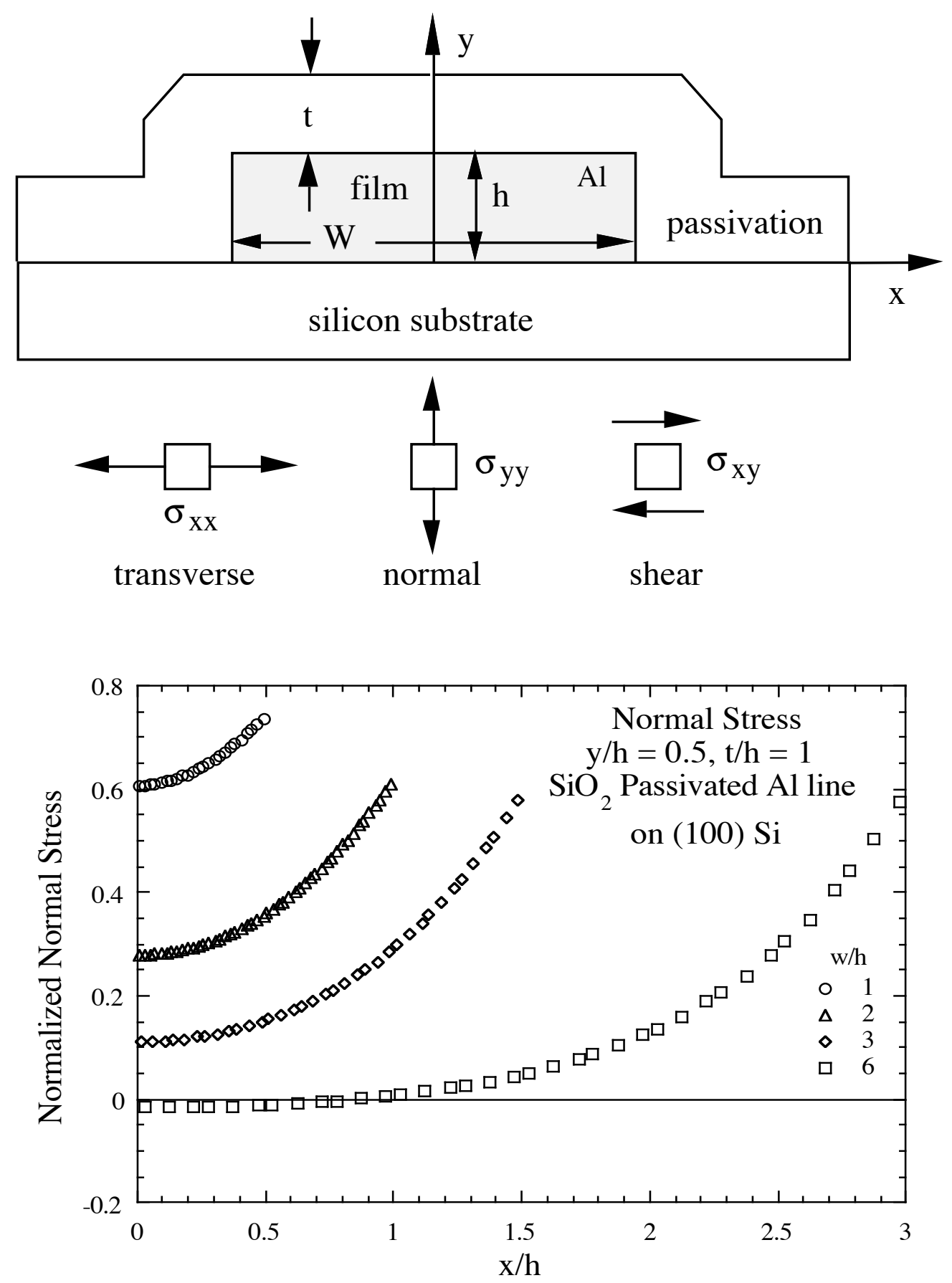

The figures above show clearly that the passivation inhibits the relaxations that occur in unpassivated line structures. Indeed, the stresses in passivated lines are highest in narrow lines with a square cross-section. As seen in the diagrams above, the stresses in the wider lines are reduced by the deflection of the thin 
passivation "plate" on top of the line. This top passivation is much stiffer when the line is narrow and this leads to much higher normal and hydrostatic stresses.

\section{Complex Stresses In Continuous Films}

Consider grain structure such that grain size $\approx \mathrm{t}_{\mathrm{f}}$ and allow relaxation by grain boundary and surface diffusion. We assume that no diffusion or sliding can occur along the film/substrate interface. This might be typical of a metal film on an oxidized Si substrate with an adhesion layer of $\mathrm{Ti}$ or $\mathrm{W}$ at the interface.

\section{Relaxation Of Stresses By Grain Boundary And Surface Diffusion.}

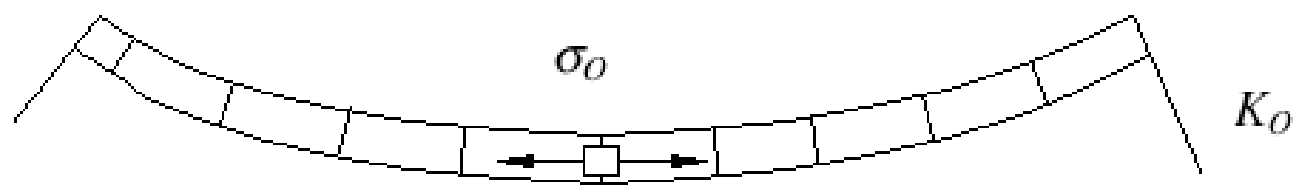

before diffusional relaxation

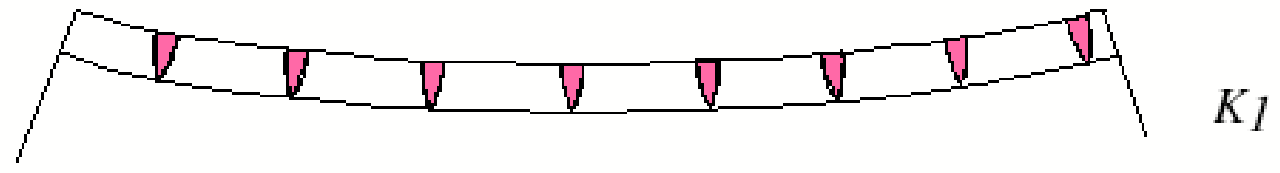

after complete grain boundary diffusional relaxation

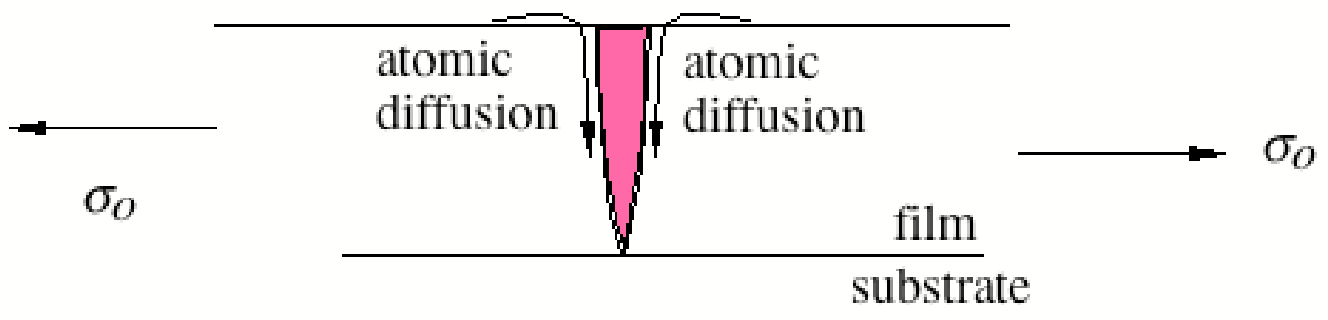

mechanism of grain boundary relaxation and curvature change 


\section{Relaxation Of Stresses By Grain Boundary And Surface Diffusion.}

In the fully relaxed state each grain is an "island" film. Large stresses are still present near the film substrate interface but the stresses are largely relaxed near the top of the film and the normal tractions are zero along the grain boundaries.

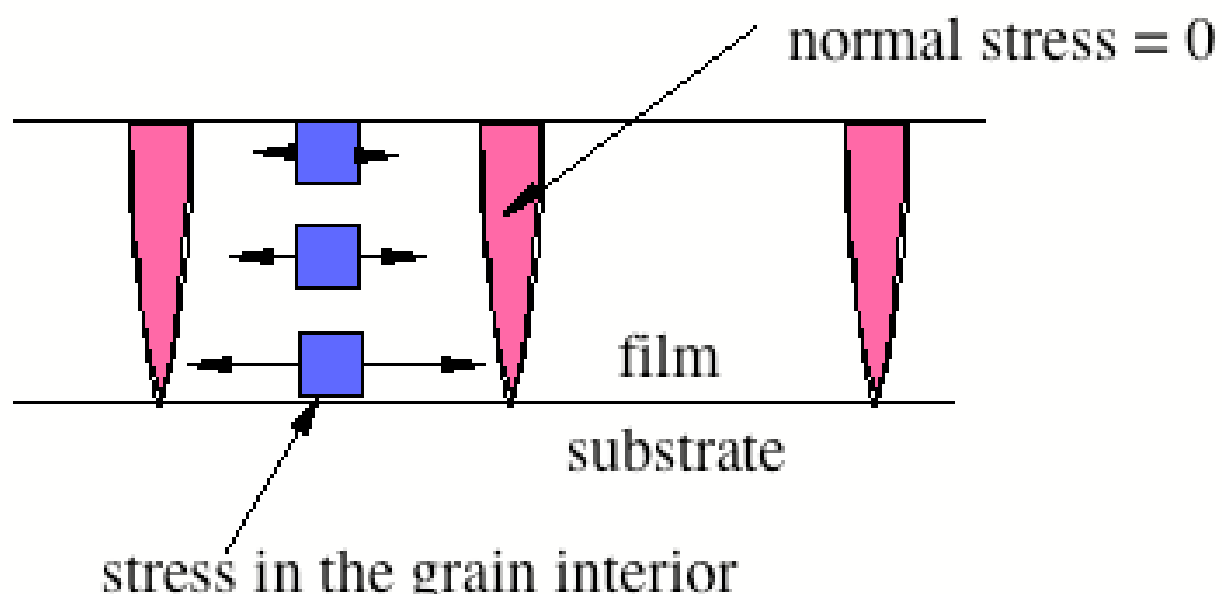

Two relations are needed to describe the development of such an inhomogeneous state of stress: one relates to the kinetics of stress relaxation at the grain boundary (more precisely, the relaxation of the normal tractions on the boundary), and the other relates to the corresponding change in curvature.

The first problem has been treated recently by H. Gao et al. (H. Gao, L. Zhang, W.D. Nix, C.V. Thompson and E. Arzt, "Crack-Like Grain Boundary Diffusion Wedges in Thin Metal Films," Acta Materialia, 47, 2865-2878 (1999).). One version of their treatment leads to the following kinetic law for stress relaxation in the grain boundary:

$$
\left(\frac{d \bar{\sigma}_{g b}}{d t}\right)=-\frac{\bar{\sigma}_{g b}}{\tau_{g b}}
$$

where $\bar{\sigma}_{g b}$ is the average normal stress in the grain boundary and

$$
\tau_{g b}=\frac{0.599\left(1-v_{f}^{2}\right) \pi t_{f}^{3} k T}{2 E_{f} \delta D_{g b} \Omega}
$$


is the time-constant for the relaxation process. Here, $t_{f}$ is the film thickness, $E_{f}$ and $v_{f}$ are the elastic properties of the film, $\delta D_{g b}$ is the grain boundary diffusivity and $\Omega$ is the atomic volume.

Because the stresses are relaxed only at the grain boundaries and not in the grain interiors, forces still act between the grains and the substrate, causing the latter to bend. But the induced curvature will be less than that for a continuous film. The curvature change induced by such a discontinuous film was treated by J.L. Beuth, (Int. J. Solids and Structures, 29, 1657-1675 (1992)) and later by John Hutchinson (private communication). They treated each diffusionally relaxed grain boundary as if it were a mode I crack in the film. With this treatment, the final curvature after complete diffusional relaxation at the grain boundaries can be expressed approximately as

$$
\kappa_{\text {relaxed }}=\frac{1-v_{s}}{E_{s}} \frac{6 t_{f} \sigma_{o}}{t_{s}^{2}}\left[1-H\left(d / t_{f}\right)\right]
$$

where $H\left(d / t_{f}\right)$ is the Hutchinson factor given by

$$
H\left(d / t_{f}\right)=\left(\frac{\pi t_{f}}{d}\right) \tanh \left(\frac{d}{\pi t_{f}}\right)
$$

where $d$ is the grain size (the spacing between grain boundaries) and $t_{f}$ is the film thickness and where $\sigma_{o}$ is the stress that would exist in the film if there were no grain boundary relaxation. Note that if $d>>t_{f}$, then $H\left(d / t_{f}\right)=0$ and the Stoney equation is recovered. On the other hand, if $d<<t_{f}$, then $H\left(d / t_{f}\right)=1$ and the induced curvature observed is small in spite of the stresses in the grains. These relations will be used to understand diffusion controlled plasticity in polycrystalline metal films on substrates. 


\section{Measurement Of Stresses In Thin Films}

\section{X-Ray Measurements}

X-ray diffraction technique - References:

M. Murakami, CRC Critical Reviews in Solid State and Materials Sciences Vol. 11, (1984) 317-354

James A. Bain, "Structural Characterization of Thin Metallic Films and Multilayers Using X-Ray Diffraction," Ph.D. Dissertation, Stanford University, 1993)

B. M. Clemens and J.A. Bain, "Stress Determination in Textured Thin Films using X-ray Diffraction," MRS Bulletin, vol.17, no.7, p. 46-51, July 1992

X-ray diffraction can be used to measure the spacing between crystallographic planes in a crystal. Measuring the plane spacing is equivalent to having an internal strain gage.

X-ray Diffraction

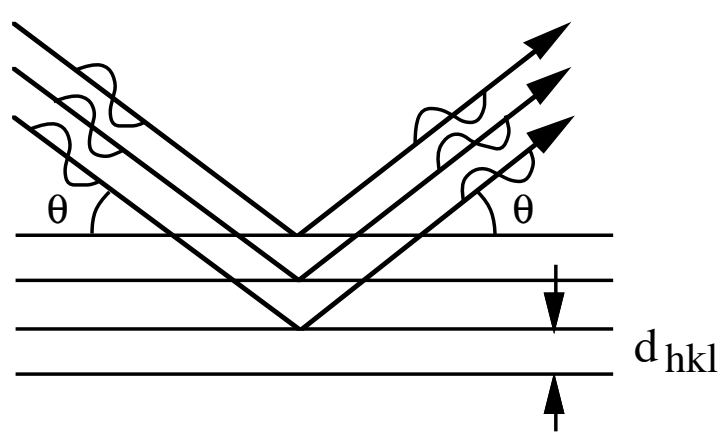

Bragg's Law for constructive interference in diffraction is:

$$
\lambda=2 d_{h k l} \sin \theta
$$

so for X-rays of a given wavelength, the crystallographic plane spacing can be found by measuring the Bragg angle: 


$$
d_{h k l}=\frac{\lambda}{2 \sin \theta}
$$

In a typical experiment, the intensity of the scattered $x$-ray beam is measured as a function of $\theta$.

\section{$\underline{\mathrm{X} \text {-ray diffraction peak }}$}

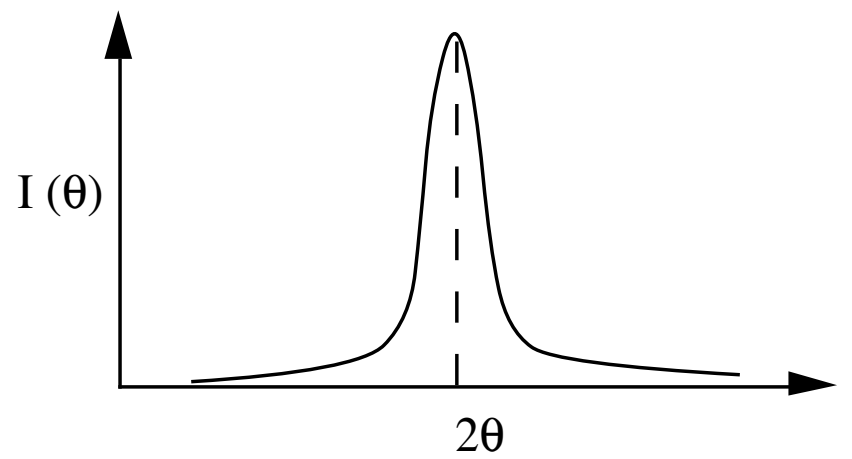

The "position" of the diffraction peak determines $d_{h k l}$.

When a crystal is subjected to stresses, the resulting elastic strains cause the " $d$ spacings" to change.

Example - film subjected to biaxial tension.

\section{Effect of stress on plane spacing}

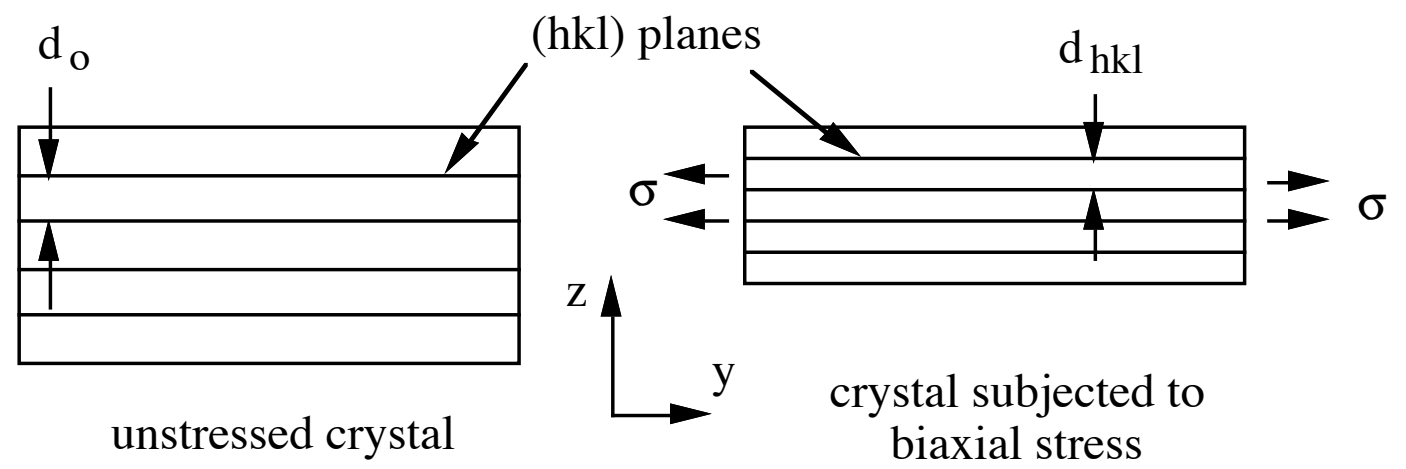


The elastic strain perpendicular to the plane of the film is then

$$
\varepsilon_{z z}=\frac{d_{h k l}-d_{o}}{d_{o}}
$$

The measurement of $d_{h k l}$ and $d_{o}$ must be done at the same temperature. Otherwise, the strain calculated in this way would include thermal strain which does not cause stresses to develop in the crystal. Also, it is generally not possible to use the "book value" of $d_{o}$ with a measured $d_{h k l}$ to determine the elastic strain in a thin film. As we will see, the $d_{o}$ must be measured for the particular film in question because slight changes in composition can cause changes in $d_{o}$. We note that for biaxial tension, $d_{h k l}<d_{o}$, if the $h k l$ planes lie parallel to the film plane, and $\varepsilon_{z z}$ is negative.

Suppose X-ray diffraction is used to measure $d_{h k l}$ and to compute $\varepsilon_{z z}$. How do we calculate $\varepsilon_{x x}=\varepsilon_{y y}$, or the stresses? - We use elasticity to calculate the other strains and the stresses.

\section{Determination of Stresses in Thin Films by Symmetric X-Ray Diffraction}

\section{$\underline{\text { Isotropic Elasticity }}$}

Biaxial stress acting on a thin film

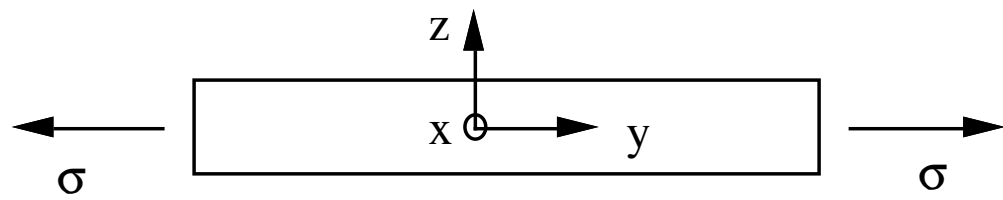

We first consider symmetric $x$-ray diffraction in which the scattering vector is perpendicular to the plane of the film. This permits a measurement of the spacing of those atomic planes that lie parallel to the plane of the film. This is called the symmetric reflecting geometry. 


\section{Symmetric Reflecting Geometry}

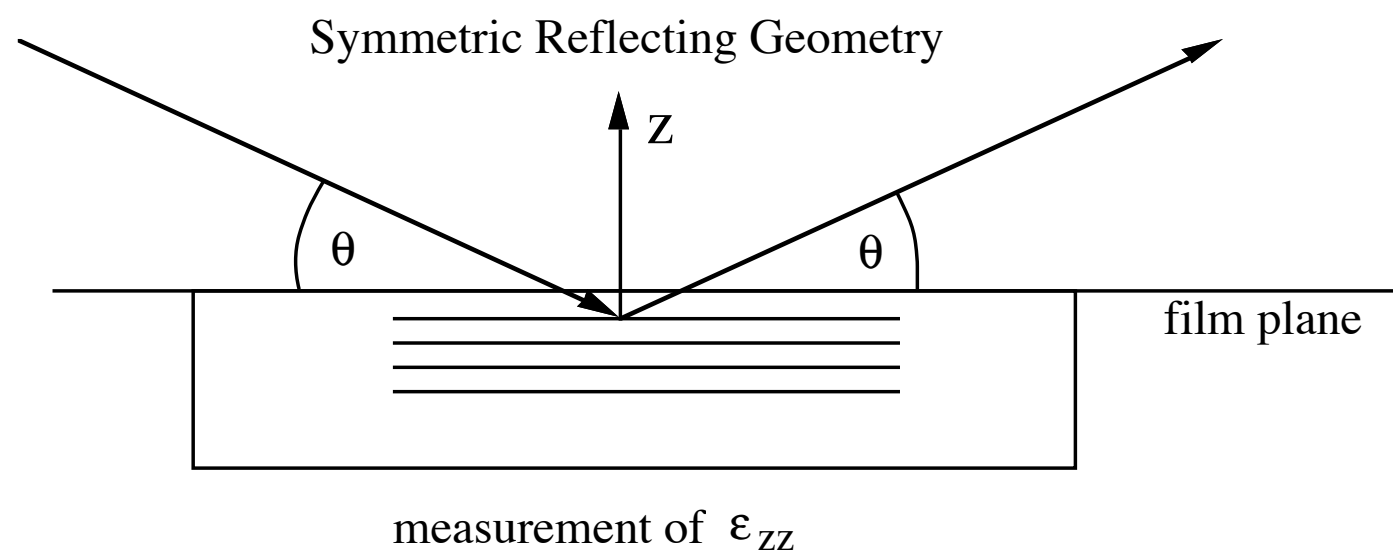

We may find the relation between the measured strain $\varepsilon_{z z}$ and the stresses in the film using the isotropic form of Hooke's Law:

$$
\varepsilon_{x x}=\varepsilon_{y y}=\frac{1}{E}\left[\sigma_{x x}-v\left(\sigma_{y y}+\sigma_{z z}\right)\right]
$$

but, $\sigma_{z z}=0$ for a thin film and $\sigma_{x x}=\sigma_{y y}=\sigma$ for the usual case of an equal biaxial stress, so

$$
\varepsilon_{x x}=\frac{1-v}{E} \sigma
$$

The corresponding normal strain is

$$
\begin{aligned}
& \varepsilon_{z z}=\frac{1}{E}\left[\sigma_{z z}-v\left(\sigma_{y y}+\sigma_{x x}\right)\right]=-\frac{2 v}{E} \sigma \\
& \varepsilon_{z z}=-\frac{2 v}{E} \sigma .
\end{aligned}
$$

So for isotropic elasticity the biaxial stress and the other strains can be found using 


$$
\begin{aligned}
& \sigma=-\frac{E}{2 v} \varepsilon_{z z} \\
& \varepsilon_{x x}=\varepsilon_{y y}=-\frac{1-v}{2 v} \varepsilon_{z z}
\end{aligned}
$$

Thus, measurement of $\varepsilon_{z z}$ by X-ray diffraction gives the biaxial stress, $\sigma$, and biaxial strains $\varepsilon_{x x}=\varepsilon_{y y}$ if the film is elastically isotropic.

But, most crystalline solids are not elastically isotropic. So an anisotropic elastic analysis is required. Next we give some results for cubic crystals.

\section{Summary of results for single crystal and fiber textured film}

\section{Cubic Films Subjected to Equal In-plane Strains}

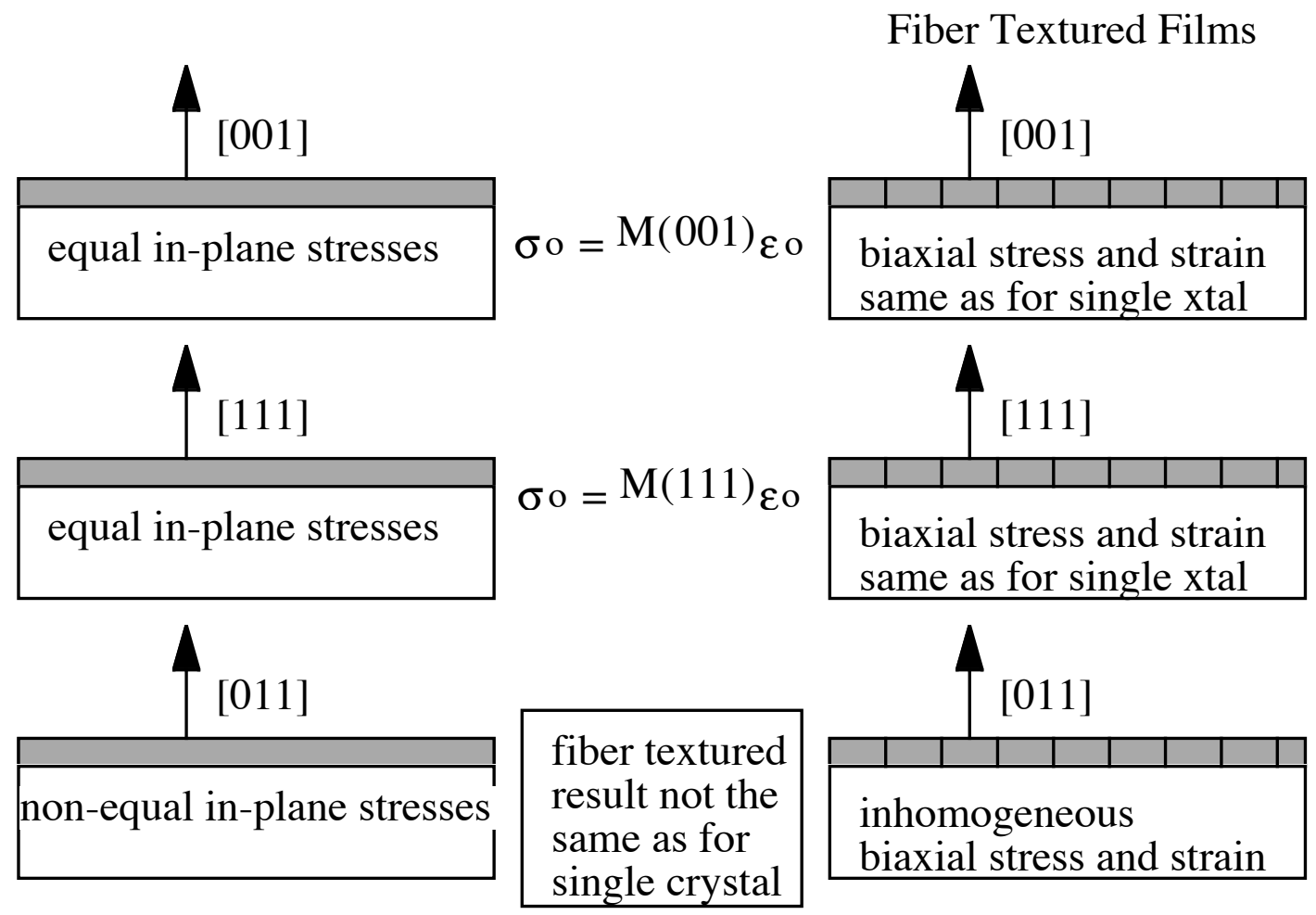




\section{Cubic Crystal Elasticity}

Most of the thin film materials under consideration are cubic materials for which Hooke's law can be described using either the stiffnesses, $c_{i j}$, or the compliances, $s_{i j}$. These matrix quantities are related to the stresses and strains in the coordinates of the cubic crystal as follows:

$$
\left[\begin{array}{c}
\sigma_{1} \\
\sigma_{2} \\
\sigma_{3} \\
\sigma_{4} \\
\sigma_{5} \\
\sigma_{6}
\end{array}\right]=\left[\begin{array}{cccccc}
c_{11} & c_{12} & c_{12} & 0 & 0 & 0 \\
c_{12} & c_{11} & c_{12} & 0 & 0 & 0 \\
c_{12} & c_{12} & c_{11} & 0 & 0 & 0 \\
0 & 0 & 0 & c_{44} & 0 & 0 \\
0 & 0 & 0 & 0 & c_{44} & 0 \\
0 & 0 & 0 & 0 & 0 & c_{44}
\end{array}\right]\left[\begin{array}{c}
\varepsilon_{1} \\
\varepsilon_{2} \\
\varepsilon_{3} \\
\varepsilon_{4} \\
\varepsilon_{5} \\
\varepsilon_{6}
\end{array}\right]
$$

and

$$
\left[\begin{array}{l}
\varepsilon_{1} \\
\varepsilon_{2} \\
\varepsilon_{3} \\
\varepsilon_{4} \\
\varepsilon_{5} \\
\varepsilon_{6}
\end{array}\right]=\left[\begin{array}{cccccc}
s_{11} & s_{12} & s_{12} & 0 & 0 & 0 \\
s_{12} & s_{11} & s_{12} & 0 & 0 & 0 \\
s_{12} & s_{12} & s_{11} & 0 & 0 & 0 \\
0 & 0 & 0 & s_{44} & 0 & 0 \\
0 & 0 & 0 & 0 & s_{44} & 0 \\
0 & 0 & 0 & 0 & 0 & s_{44}
\end{array}\right]\left[\begin{array}{c}
\sigma_{1} \\
\sigma_{2} \\
\sigma_{3} \\
\sigma_{4} \\
\sigma_{5} \\
\sigma_{6}
\end{array}\right] .
$$

Here the matrix subscripts can be related to the subscripts for the tensor quantities as $1=x x, 2=y y, 3=z z, 4=y z, 5=x z, 6=x y$. A more compact representation of these matrix operations is

$$
\sigma_{i}=c_{i j} \varepsilon_{j}
$$

and

$$
\varepsilon_{i}=s_{i j} \sigma_{j}
$$

These relations are used in all of the single crystal calculations given below. 


\section{(100) Single Crystal Films}

Assume that the cube plane is parallel to the film. Many epitaxial semiconducting films have this orientation; $\mathrm{Si} / \mathrm{SiGe}$ and $\mathrm{GaAs} / \mathrm{Si}$ are examples.

Film plane parallel to the cube plane (001)
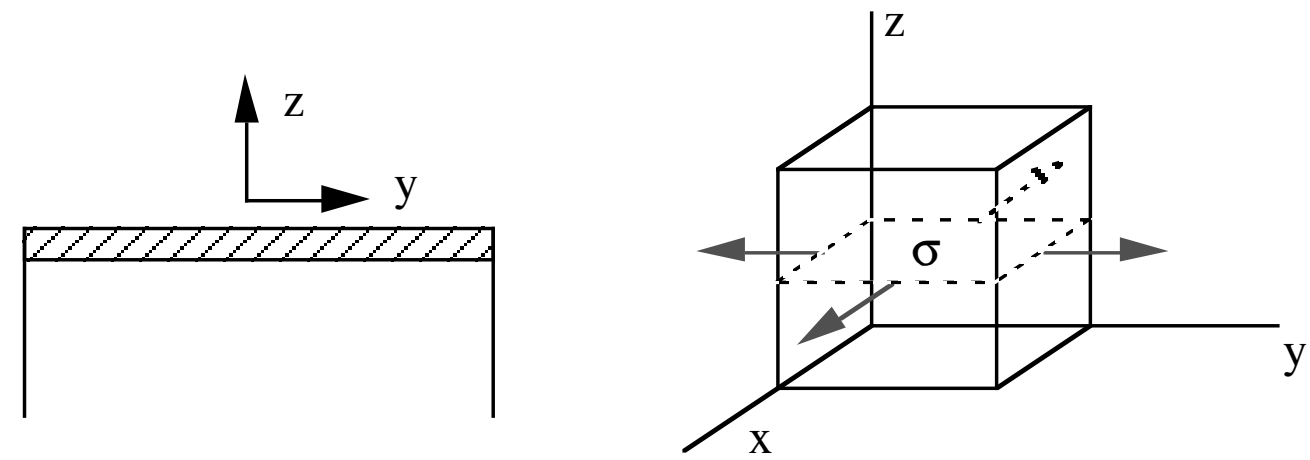

The stresses are

$$
\sigma_{1}=\sigma_{x x}=\sigma \quad \sigma_{2}=\sigma_{y y}=\sigma \quad \sigma_{3}=\sigma_{z z}=0
$$

Using compliances, the corresponding strains can be expressed as:

$$
\begin{aligned}
& \varepsilon_{x x}=\varepsilon_{y y}=\varepsilon_{1}=s_{11} \sigma_{1}+s_{12} \sigma_{2}+s_{12} \sigma_{3} \\
& \varepsilon_{x x}=\varepsilon_{y y}=\left(s_{11}+s_{12}\right) \sigma \\
& \varepsilon_{z z}=\varepsilon_{3}=s_{12} \sigma_{1}+s_{12} \sigma_{2}+s_{11} \sigma_{3}=2 s_{12} \sigma
\end{aligned}
$$

so,

$$
\begin{aligned}
& \sigma=\frac{1}{2 s_{12}} \varepsilon_{z z} \\
& \varepsilon_{x x}=\varepsilon_{y y}=\left(\frac{s_{11}+s_{12}}{2 s_{12}}\right) \varepsilon_{z z}
\end{aligned}
$$


For elastically anisotropic single crystal (001) films, these relations can be used to find the stress and the other strains from the measured strain.

\section{Using Stiffnesses:}

If the misfit strain is known (as in thermal mismatch problems) and one wishes to compute stresses and all strain components in the film when the film is attached to the substrate, then the stiffnesses are more useful. Suppose $-\varepsilon_{o}$ is biaxial misfit strain (the film has to be stretched to fit the substrate).

\section{Misfit strain}

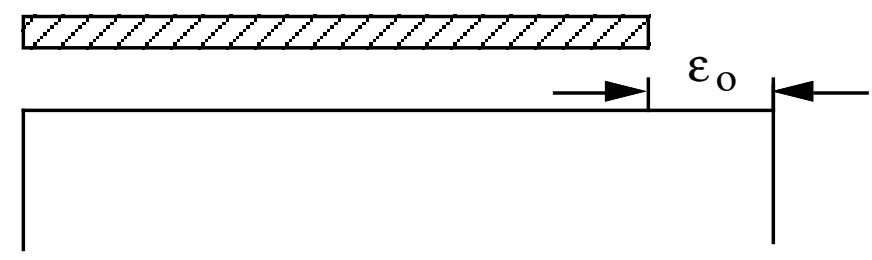

In this figure, $\varepsilon_{1}=\varepsilon_{2}=\varepsilon_{o} \quad$ and $\quad \sigma_{3}=0$.

Here the quantity $\varepsilon_{o}$ stands for the in-plane elastic strain in the film. It is exactly equal in magnitude (and opposite in sign) to the misfit strain only if the substrate is either infinitely thick or perfectly rigid. Of course, for usual substrate thicknesses the in-plane strain is very nearly the same in magnitude as the misfit strain, because most of the accommodation strain occurs in the film. For this strain state the stresses are

$$
\begin{aligned}
& \sigma=\sigma_{x x}=\sigma_{y y}=\sigma_{1}=c_{11} \varepsilon_{1}+c_{12} \varepsilon_{2}+c_{12} \varepsilon_{3} \\
& \sigma=\sigma_{x x}=\sigma_{y y}=\left(c_{11}+c_{12}\right) \varepsilon_{0}+c_{12} \varepsilon_{3}
\end{aligned}
$$

and

$$
\sigma_{3}=\sigma_{z z}=0=c_{12} \varepsilon_{1}+c_{12} \varepsilon_{2}+c_{11} \varepsilon_{3}
$$

Using this relation gives

$$
\varepsilon_{3}=-\frac{2 c_{12}}{c_{11}} \varepsilon_{o}
$$


The biaxial stress then becomes

$$
\sigma=\left(c_{11}+c_{12}\right) \varepsilon_{o}-\frac{2 c_{12}^{2}}{c_{11}} \varepsilon_{o}
$$

and the corresponding biaxial strain is

$$
\varepsilon_{o}=-\frac{c_{11}}{2 c_{12}} \varepsilon_{z z}
$$

Finally, in terms of the measured strain, the biaxial stress is

$$
\sigma=\left[\left(c_{11}+c_{12}\right)-\frac{2 c_{12}^{2}}{c_{11}}\right]\left(-\frac{c_{11}}{2 c_{12}} \varepsilon_{z z}\right)
$$

Thus, we can find the other strains and stress by measuring $\varepsilon_{z z}$.

This analysis also gives the biaxial elastic modulus for (100) cubic films

$$
B(001) \equiv \frac{\sigma}{\varepsilon_{o}}=c_{11}+c_{12}-\frac{2 c_{12}^{2}}{c_{11}}
$$

This will be useful in multilayer problems. For cubic crystals an equal biaxial strain in the (001) plane leads to an equal biaxial stress. As a consequence, the results developed here for single crystal (001) films apply to polycrystalline films with a perfect (001) fiber texture. We also note that this biaxial modulus should be used in place of the isotropic form of the biaxial modulus:

$$
B(\text { isotropic })=\frac{E}{1-v}
$$

for bending of (001) single crystal substrates. 
(001) fiber texture - biaxial elastic properties same as (001) single crystal film

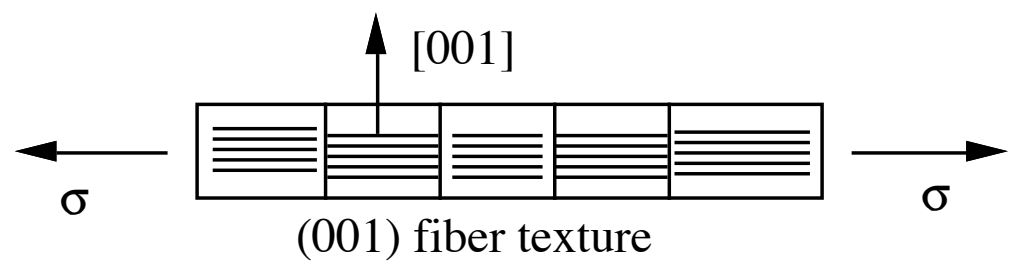

(111) single crystal films

Consider the (111) plane parallel to the plane of the film.

(111) single crystal films

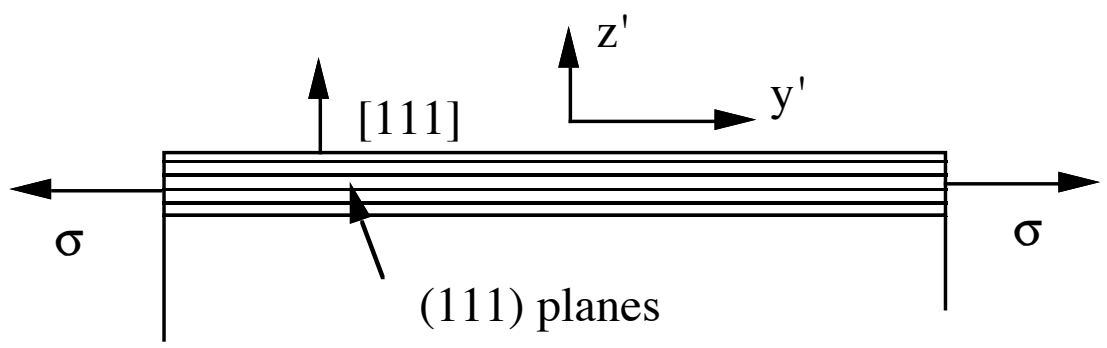

To find relations between stresses and strains in the $x^{\prime}, y^{\prime}, z^{\prime}$ coordinates, one must use coordinate transformations and Hooke's Law for cubic solids. Specifically, we take the film coordinates to be

$$
x^{\prime}=\frac{1}{\sqrt{6}}[2-\overline{1} 1] \quad y^{\prime}=\frac{1}{\sqrt{2}}[01 \overline{1}] \quad z^{\prime}=\frac{1}{\sqrt{3}}[111]
$$

The strain state in the film is given in the film coordinates as

$$
\varepsilon_{x^{\prime} x^{\prime}}=\varepsilon_{y^{\prime} y^{\prime}}=\varepsilon_{o} \quad \varepsilon_{z^{\prime} z^{\prime}}=\varepsilon^{*}
$$

and the stresses are

$$
\sigma_{x^{\prime} x^{\prime}}=\sigma_{y^{\prime} y^{\prime}}=\sigma_{o} \quad \sigma_{z^{\prime} z^{\prime}}=0
$$


Note that a cubic crystal is fully isotropic in the (111) plane. The solution to the elasticity problem involves either transforming the elastic constants to the $x^{\prime}$, $y^{\prime}, z^{\prime}$ coordinate system or transforming the given stresses and strains to the cubic coordinate system and using the $c_{i j}{ }^{\prime} s$ or $s_{i j}{ }^{\prime} s$ as appropriate. This involves the transformation of second rank tensors from one coordinate system to another.

Here we outline the steps that could be taken to determine the stresses in a film where the elastic strains are known or given. We may assume that the strains in the film are of the form:

$$
\varepsilon_{x^{\prime} x^{\prime}}=\varepsilon_{y^{\prime} y^{\prime}}=\varepsilon_{o} \quad \varepsilon_{z^{\prime} z^{\prime}}=\varepsilon^{*}
$$

where $\varepsilon_{o}$ is specified and $\varepsilon^{*}$ is known to be non-zero, but its value is not known at first. The task is to compute the corresponding stresses in the film.

We start by transforming the strains form the film coordinate system to the cube coordinate system, where Hooke's law can be used. For this we use the transformation law for second rank tensors,

$$
\varepsilon_{i j}=a_{i k^{\prime}} a_{j l^{\prime}} \varepsilon_{k^{\prime} l^{\prime}}
$$

where $\varepsilon_{k^{\prime} l^{\prime}}$ are the strains in the film coordinate system and $\varepsilon_{i j}$ are the corresponding strains in the cubic coordinate system, and $a_{i k^{\prime}}$ and $a_{j l}$ are the corresponding direction cosines relating the two coordinate systems. For example, using the coordinate system above,

$$
\begin{aligned}
& a_{x x^{\prime}}=\left[\begin{array}{lll}
1 & 0 & 0
\end{array}\right] \cdot \frac{1}{\sqrt{6}}[211]=\frac{--}{\sqrt{6}} \\
& a_{x y^{\prime}}=\left[\begin{array}{lll}
1 & 0 & 0
\end{array}\right] \cdot \frac{1}{\sqrt{2}}\left[\begin{array}{ll}
0 & 1
\end{array}\right]=0 \\
& \text { etc. }
\end{aligned}
$$

Once the tensor strains are found in the cubic coordinate system, we need to find the matrix strain components using

$$
\varepsilon_{1}=\varepsilon_{x x}, \varepsilon_{2}=\varepsilon_{y y}, \varepsilon_{3}=\varepsilon_{z z}, \varepsilon_{4}=2 \varepsilon_{y z}, \varepsilon_{5}=2 \varepsilon_{x z}, \varepsilon_{6}=2 \varepsilon_{x y}
$$


Now we may use Hooke's law with the stiffnesses to find all of the stresses in the cube coordinate system,

$$
\sigma_{i}=c_{i j} \varepsilon_{j}
$$

We then identify the matrix stresses using

$$
\sigma_{x x}=\sigma_{1}, \sigma_{y y}=\sigma_{2}, \sigma_{z z}=\sigma_{3}, \sigma_{y z}=\sigma_{4}, \sigma_{x z}=\sigma_{5}, \sigma_{x y}=\sigma_{6}
$$

Finally we again use the transformation law for second rank tensors to find the stresses in the film coordinate system,

$$
\sigma_{k^{\prime} l^{\prime}}=a_{k^{\prime} i} a_{l^{\prime} j} \sigma_{i j}
$$

At this point in the analysis an equation for the out-of-plane stress $\sigma_{z^{\prime} z^{\prime}}$ will be available. By setting $\sigma_{z^{\prime} z^{\prime}}=0$ we can find the unknown out-of-plane strain, $\varepsilon^{*}$. A process of this kind leads to the stresses in the film for the given biaxial strain. The solution to this (111) crystal problem gives a relation between the in-plane and out of plane strain,

$$
\varepsilon_{o}=-\varepsilon *\left[\frac{c_{11}+2 c_{12}+4 c_{44}}{2 c_{11}+4 c_{12}-4 c_{44}}\right]
$$

This comes from condition that $\sigma_{z^{\prime} z^{\prime}}=0$.

The stress is given by

$$
\sigma=\frac{6 c_{44}\left(c_{11}+2 c_{12}\right)}{c_{11}+2 c_{12}+4 c_{44}} \varepsilon_{o}
$$

where the coefficient defines the biaxial modulus in the (111) plane

$$
B(111) \equiv \frac{\sigma}{\varepsilon_{o}}=\frac{6 c_{44}\left(c_{11}+2 c_{12}\right)}{c_{11}+2 c_{12}+4 c_{44}}
$$

So, if the $\varepsilon_{z^{\prime} z^{\prime}}$ strain is measured with x-rays 


$$
\varepsilon_{z^{\prime} z^{\prime}}=\frac{d_{111}-d_{o}}{d_{o}}=\varepsilon^{*}
$$

then, the in-plane strain, $\varepsilon_{o}$ and the biaxial stress, $\sigma$, can be directly computed. Because the (111) plane in cubic crystals is fully isotropic, the results for (111) single crystal films can be used to describe polycrystalline films with a perfect (111) fiber texture.

Again we note that this biaxial modulus, B(111), should be used in place of the isotropic form of the biaxial modulus:

$$
B(\text { isotropic })=\frac{E}{1-v}
$$

for bending of (111) single crystal substrates.

(111) fiber texture

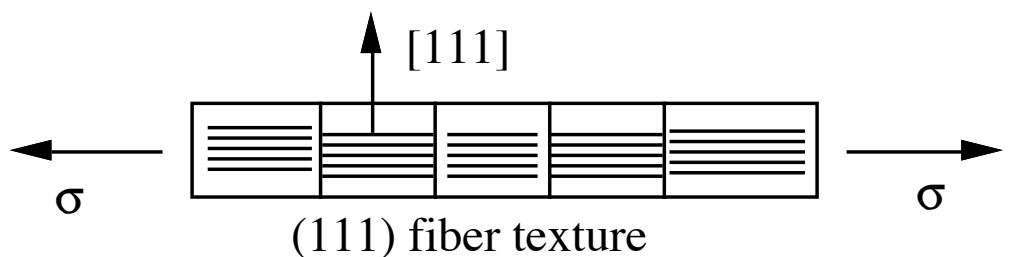

(110) single crystal films

For completeness we now consider the more uncommon case of single crystal films with the (011) planes lying parallel to the plane of the film. We can derive exact results for single crystal films with this orientation. But because equal biaxial strains in the (011) plane do not lead to equal biaxial stresses, the single crystal results cannot be used for polycrystalline films with the (011) fiber texture. No simple solutions exist for these polycrystalline films. 


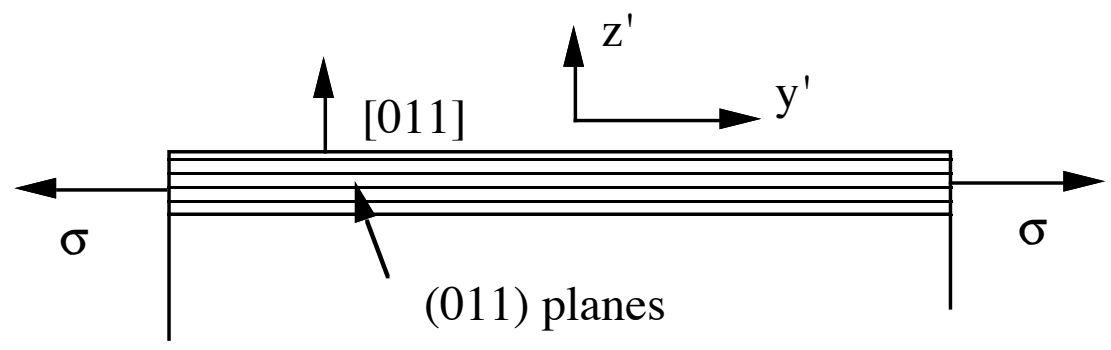

Here we take

$$
x^{\prime}=[100] \quad y^{\prime}=\frac{1}{\sqrt{2}}[011] \quad z^{\prime}=\frac{1}{\sqrt{2}}[011]
$$

Because the misfit strain is usually "equal biaxial", we assume that the strain state in the film after attachment to the substrate is:

$$
\varepsilon_{x^{\prime} x^{\prime}}=\varepsilon_{y^{\prime} y^{\prime}}=\varepsilon_{o} \quad \varepsilon_{z^{\prime} z^{\prime}}=\varepsilon^{*}
$$

as before.

However, cubic crystals are not isotropic in the (011) plane, so

$$
\sigma_{x^{\prime} x^{\prime}} \neq \sigma_{y^{\prime} y^{\prime}} \quad \sigma_{z^{\prime} z^{\prime}}=0
$$

The solution to the (011) problem is

$$
\varepsilon_{o}=-\varepsilon *\left[\frac{c_{11}+c_{12}+2 c_{44}}{c_{11}+3 c_{12}-2 c_{44}}\right]
$$

and the stresses are:

$$
\sigma_{x^{\prime} x^{\prime}}=-\frac{\left(c_{11}+c_{12}\right)\left(c_{11}+c_{12}+2 c_{44}\right)}{2\left(c_{11}+3 c_{12}-2 c_{44}\right)} \varepsilon^{*}+c_{12} \varepsilon^{*}
$$




$$
\begin{aligned}
& \sigma_{y^{\prime} y^{\prime}}=-\frac{\left(2 c_{11}+6 c_{12}+4 c_{44}\right)\left(c_{11}+c_{12}+2 c_{44}\right)}{4\left(c_{11}+3 c_{12}-2 c_{44}\right)} \varepsilon^{*} \\
& +\frac{\left(2 c_{11}+2 c_{12}-4 c_{44}\right)}{4} \varepsilon^{*}
\end{aligned}
$$

These relations also permit one to find the biaxial modulus in the different directions.

These would be defined as :

$$
\begin{gathered}
\sigma_{x^{\prime} x^{\prime}}=B(100) \varepsilon_{o} \\
\sigma_{y^{\prime} y^{\prime}}=B(011) \varepsilon_{o}
\end{gathered}
$$




\section{Stresses In Polycrystalline Films}

Often thin films have a strong crystallographic texture, so that one particular plane is parallel to the film. For $\mathrm{Pb}$ on $\mathrm{Si}$ the texture is $\mathrm{a}<111>$ fiber texture.

Polycrystalline film with columnar grain structure and (111) fiber texture

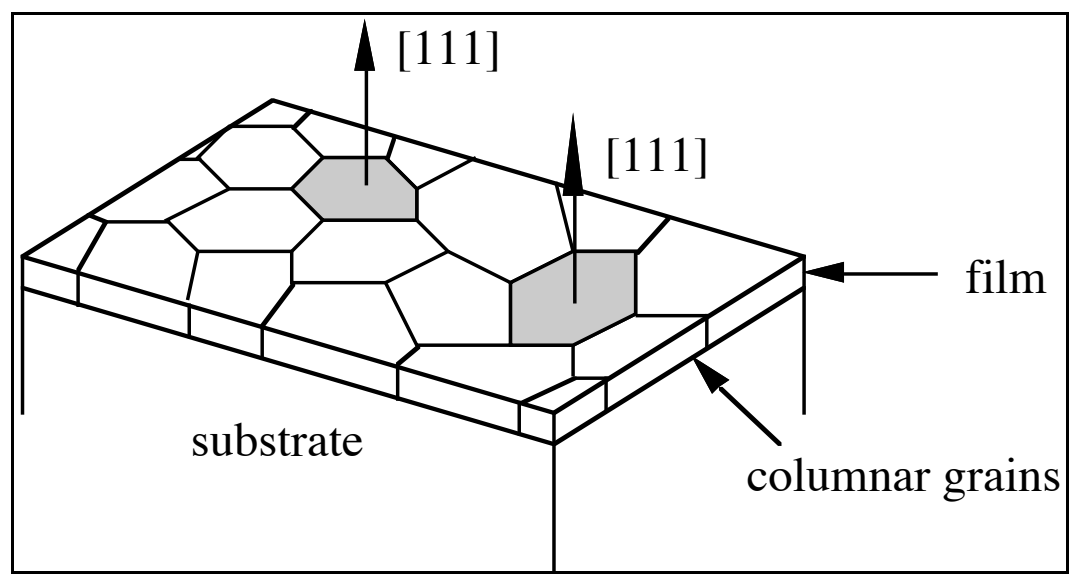

Therefore, stresses in the film are found by measuring $d_{111}$. Conversion to stress is the same as if the film were a single crystal. (Even if the film did not have a strong texture, the x-ray technique applies to polycrystalline films. Fewer grains would diffract and intensities would be lower for randomly oriented grains.)

Murakami has measured $d_{h k l}$ in $\mathrm{Pb}$ (111) films as a function of temperature and film thickness. (See M. Murakami, Thin Solid Films 55 (1978) 101-111.) The results are instructive as they permit a study of elastic and plastic deformation of the film.. 
Measurements of (111) d spacings for Pb films on Si substrates (schematic)

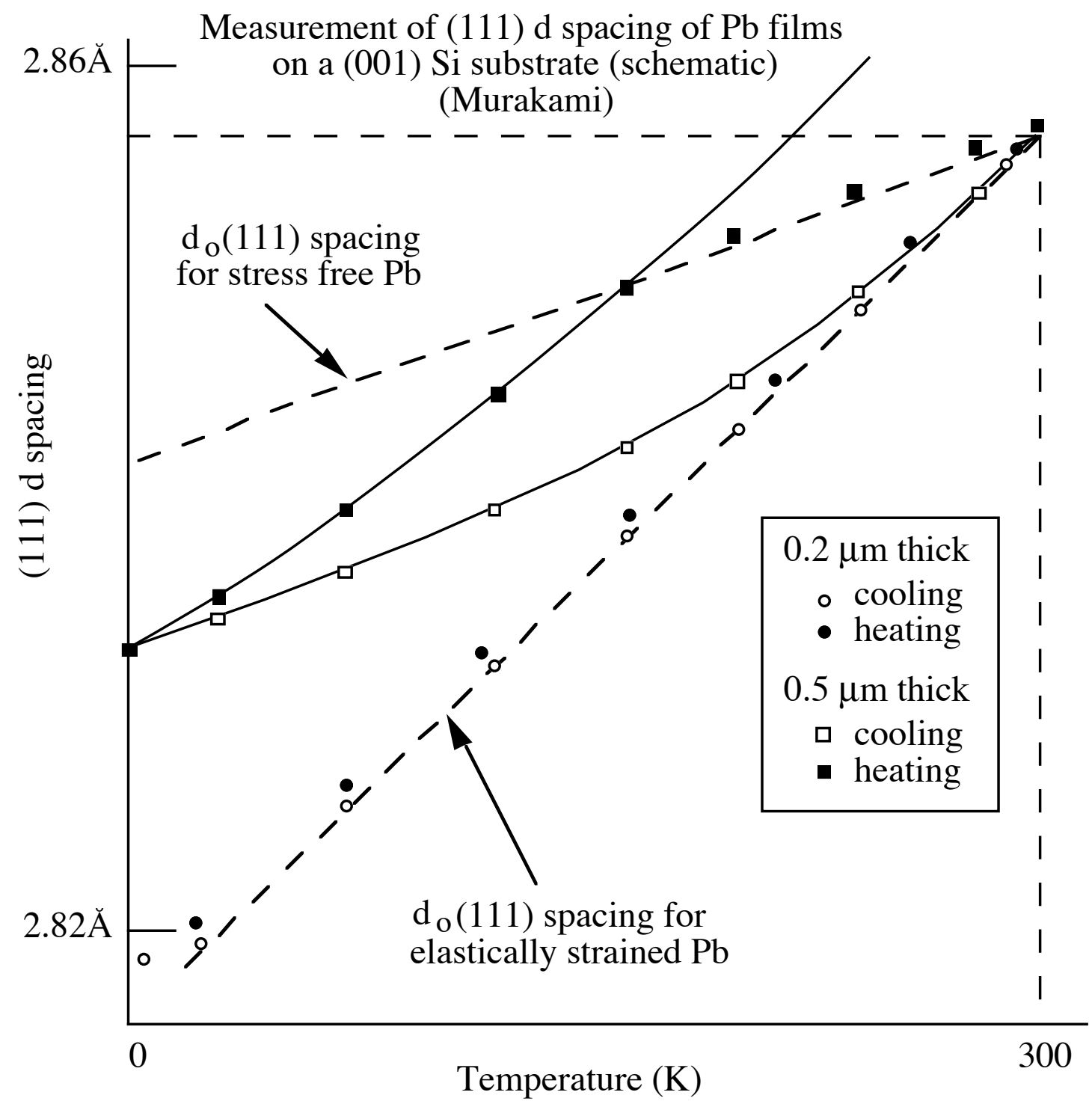

These results may be interpreted as follows. The $0.2 \mu \mathrm{m}$ thick $\mathrm{Pb}$ film is so strong that it deforms elastically on cooling and heating. The $0.5 \mu \mathrm{m} \mathrm{Pb}$ film initially deforms elastically but at about $200 \mathrm{~K}$ it begins to deform plastically and the elastic strain remains about constant on cooling. On heating the thicker film deforms elastically as it unloads and then begins to yield plastically at about 170 $\mathrm{K}$. All of the results can be displayed by calculating the biaxial stress in the film as a function of temperature. The results for the thicker film are shown in the figure below. Here we see the elastic- plastic transitions discussed here. 
Stress - Temperature Plot for a $0.5 \mu \mathrm{m} \mathrm{Pb}$ film on a Si substrate

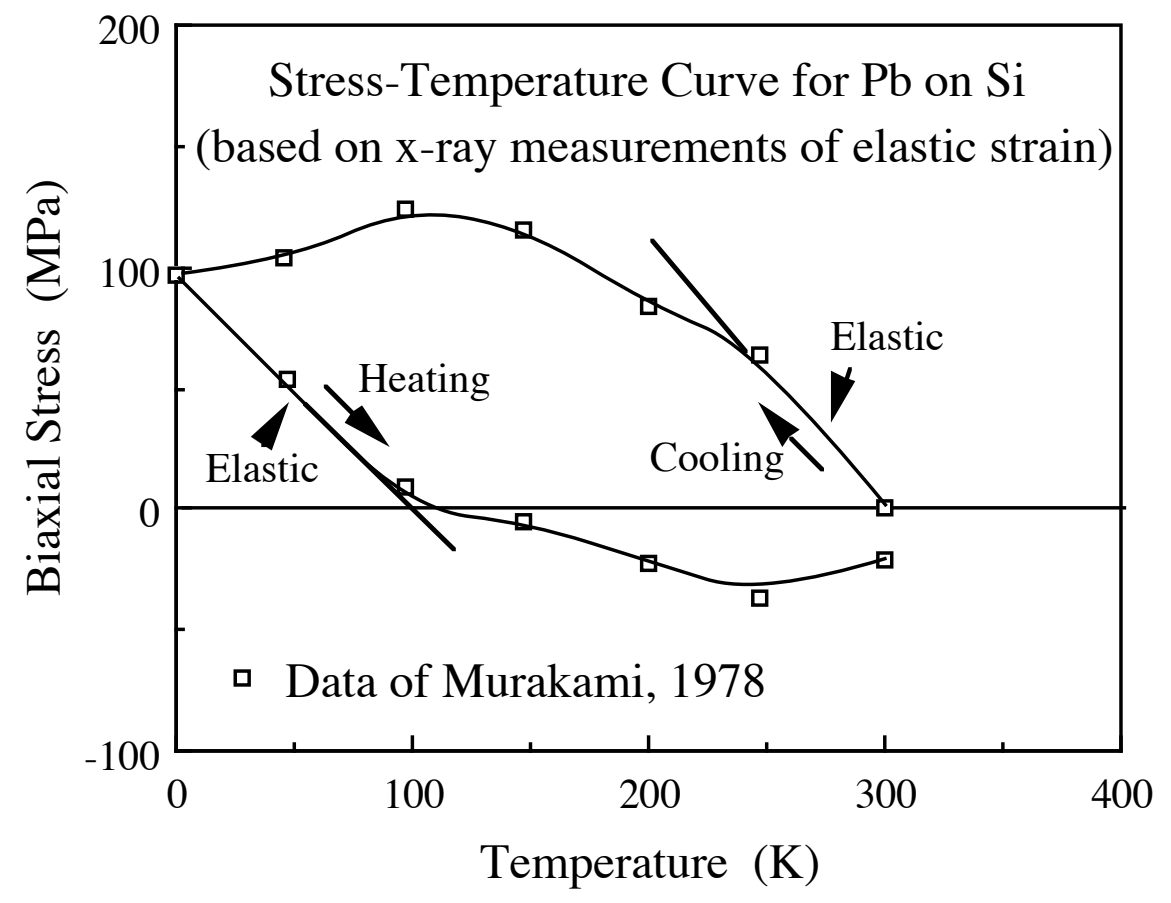

\section{Determination of Stresses in Thin Films by Non-symmetric X-Ray Diffraction}

The stress measurements discussed above are all based on a single measurement of the strain - namely the strain in the film perpendicular to the plane of the film, $\varepsilon_{z z}$. The other strains and the stresses have been calculated using Hooke's law. Here we discuss the measurement of in-plane components of the strain using non-symmetrical $x$-ray diffraction geometry. The scattering vector must have a component parallel to the plane of the film in order to measure in-plane strain components. Diffraction from planes that lie at an angle to the plane of the film are shown in the figure below. 
Non-symmetric reflecting geometry

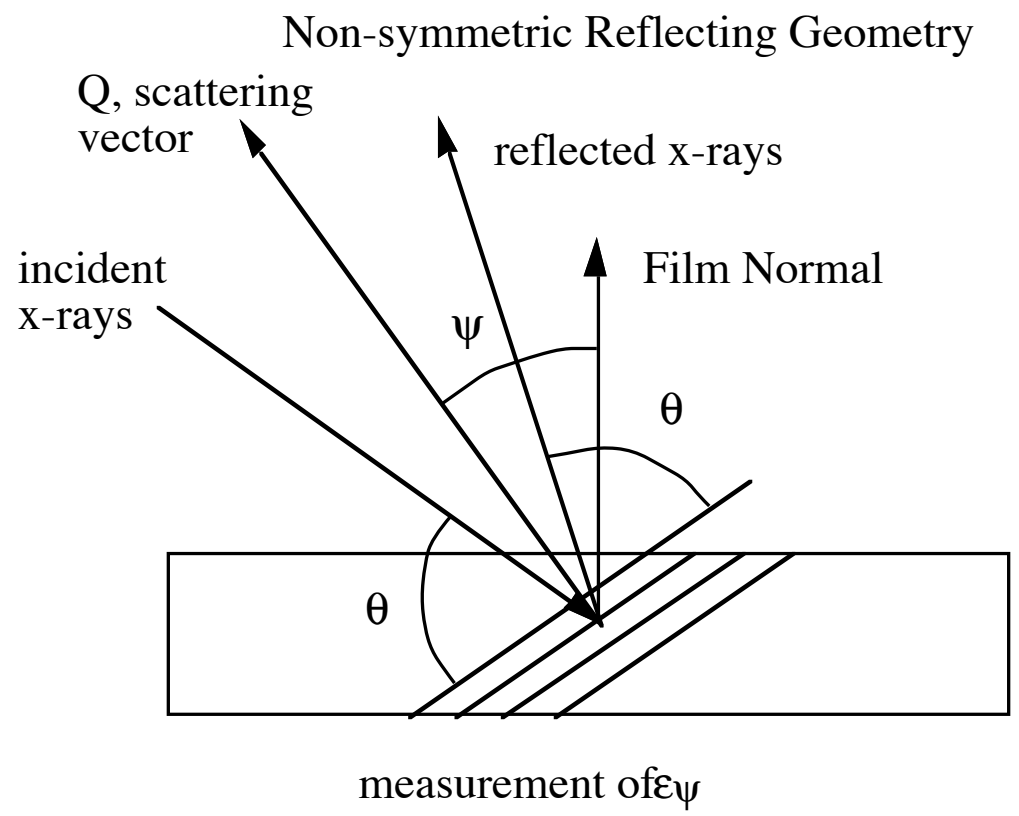

Sample geometry for non-symmetric x-ray diffraction

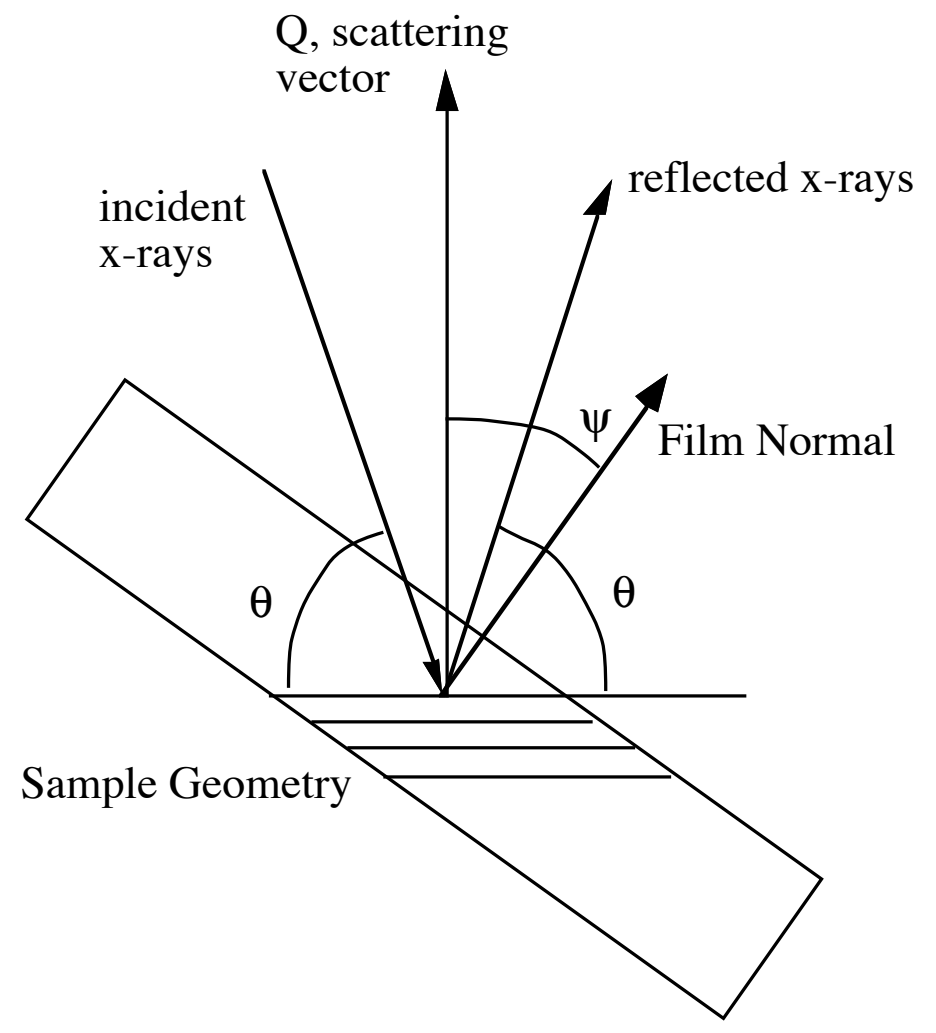


Any state of strain can be described in terms of the principal strains, the axial strains in the principal coordinate system. In this coordinate system all of the shear stress components are zero. For the case of thin films subjected to homogeneous states of strain, the thin film geometry requires the principal coordinates to lie parallel and perpendicular to the plane of the film. In the coordinate system shown in the figure below the shear strains $\varepsilon_{13}$ and $\varepsilon_{23}$ must be zero (for all ordinary crystalline materials) because the corresponding shear stresses $\sigma_{13}$ and $\sigma_{23}$ are zero (because the surface of the film is traction free).

\section{Principal coordinates for strains in a thin film}

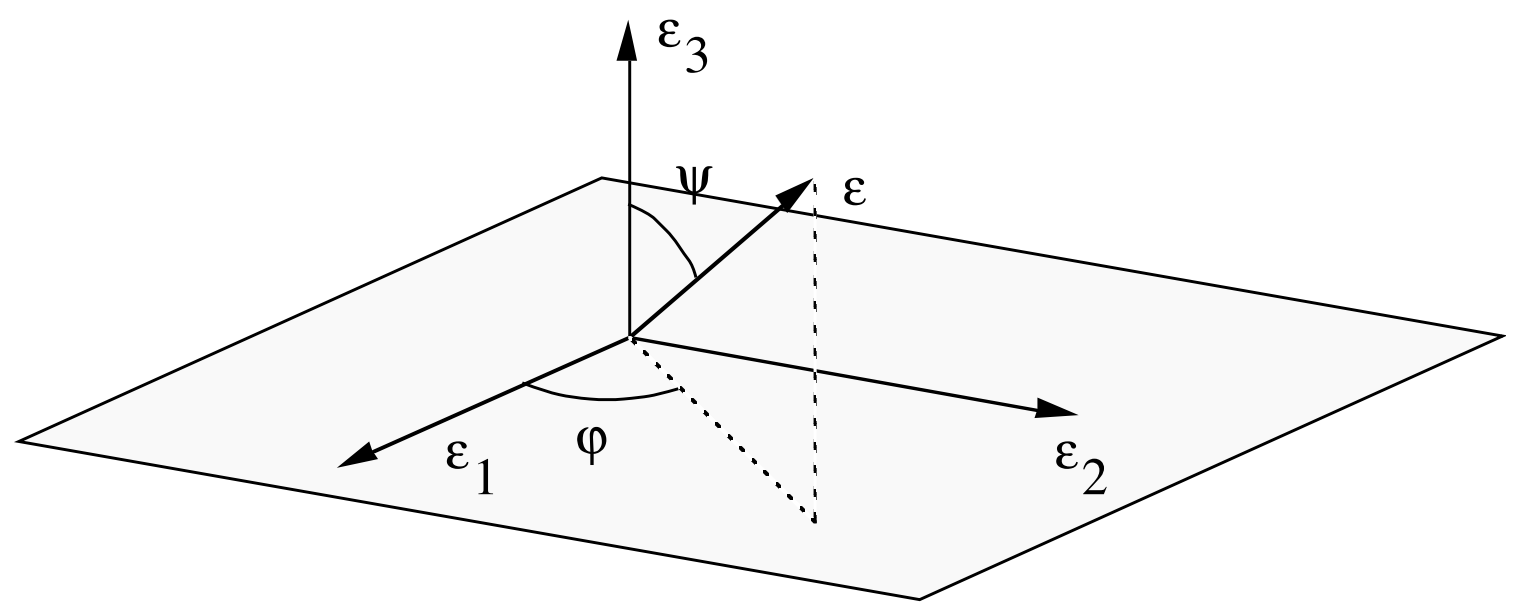

The axial strain in any arbitrary direction can be described using what is sometimes called the strain ellipse. Equivalently the axial strain in any arbitrary direction (call it the $\alpha$ direction) can be found by transforming the components of the strain tensor to the direction in question. For this we use the same transformation law used earlier for stresses, namely

$$
\begin{aligned}
& \varepsilon_{\alpha \alpha}=a_{\alpha k} a_{\alpha l} \varepsilon_{k l} \\
& \varepsilon_{\alpha \alpha}=a_{\alpha 1} a_{\alpha 1} \varepsilon_{11}+a_{\alpha 2} a_{\alpha 2} \varepsilon_{22}+a_{\alpha 3} a_{\alpha 3} \varepsilon_{33} \\
& \varepsilon_{\alpha \alpha}=a_{\alpha 1} a_{\alpha 1} \varepsilon_{1}+a_{\alpha 2} a_{\alpha 2} \varepsilon_{2}+a_{\alpha 3} a_{\alpha 3} \varepsilon_{3} \\
& \varepsilon_{\alpha \alpha}=a_{\alpha 1}^{2} \varepsilon_{1}+a_{\alpha 2}^{2} \varepsilon_{2}+a_{\alpha 3}^{2} \varepsilon_{3}
\end{aligned}
$$

Using the direction cosines in this transformation we have

$$
\varepsilon=a_{1}^{2} \varepsilon_{1}+a_{2}^{2} \varepsilon_{2}+a_{3}^{2} \varepsilon_{3}
$$


where the direction cosines are:

$$
\begin{aligned}
& a_{1}=\sin \psi \cos \varphi \\
& a_{2}=\sin \psi \sin \varphi \\
& a_{3}=\cos \psi
\end{aligned}
$$

yielding

$$
\varepsilon=\varepsilon_{1} \sin ^{2} \psi \cos ^{2} \varphi+\varepsilon_{2} \sin ^{2} \psi \sin ^{2} \varphi+\varepsilon_{3} \cos ^{2} \psi
$$

This relation gives the axial strain in any arbitrary direction given the principal strains in the film.

We now use Hooke's Law to find the corresponding stresses in the film. Using the isotropic form of Hooke's law we have

$$
\begin{aligned}
& \varepsilon_{1}=\frac{1}{E}\left(\sigma_{1}-v\left(\sigma_{2}+\sigma_{3}\right)\right) \\
& \varepsilon_{2}=\frac{1}{E}\left(\sigma_{2}-v\left(\sigma_{3}+\sigma_{1}\right)\right) \\
& \varepsilon_{3}=\frac{1}{E}\left(\sigma_{3}-v\left(\sigma_{1}+\sigma_{2}\right)\right)
\end{aligned}
$$

Inserting these expressions into the expression above we find

$$
\varepsilon_{\psi \varphi}=\frac{1+v}{E}\left[\sigma_{1} \cos ^{2} \varphi+\sigma_{2} \sin ^{2} \varphi\right] \sin ^{2} \psi+\frac{1}{E}\left[\sigma_{3}-v\left(\sigma_{1}+\sigma_{2}\right)\right]
$$

for the axial strain in any direction in the film.

If we limit our attention to a thin film with an equal-biaxial stress state

$$
\begin{aligned}
& \sigma_{1}=\sigma_{2}=\sigma \\
& \sigma_{3}=0
\end{aligned}
$$

the result is 


$$
\begin{aligned}
& \varepsilon_{\psi \varphi}=\frac{1+v}{E} \sigma\left[\cos ^{2} \varphi+\sin ^{2} \varphi\right] \sin ^{2} \psi-\frac{1}{E}[2 v \sigma] \\
& \varepsilon_{\psi}=\frac{1+v}{E} \sigma \sin ^{2} \psi-\frac{1}{E}[2 v \sigma] \\
& \varepsilon_{\psi}=\frac{1+v}{E} \sigma \sin ^{2} \psi-\frac{2 v}{E} \sigma
\end{aligned}
$$

This relation is important because it shows that the strain in the film should vary linearly with $\sin ^{2} \psi$ where $\psi$ is the angle between the scattering vector and the film plane normal.

We note that the strain is zero when

$$
\sin ^{2} \psi=\frac{2 v}{1+v}
$$

This relation permits a determination of the strain-free reflecting plane spacing. In particular, using the usual strain definition,

$$
\varepsilon_{\psi}=\frac{d_{\psi}-d_{o}}{d_{o}}
$$

we get

$$
\begin{aligned}
& d_{\psi}=d_{o}\left(\varepsilon_{\psi}+1\right)=d_{o}+d_{o} \varepsilon_{\psi} \\
& d_{\psi}=\left[d_{o}-\frac{2 v}{E} \sigma d_{o}\right]+\left[\frac{1+v}{E} \sigma d_{o}\right] \sin ^{2} \psi
\end{aligned}
$$

So the spacing $d_{\psi}$ is expected to depend linearly on $\sin ^{2} \psi$ and to be equal to the strain-free spacing, $d_{o}$, when 


$$
\begin{aligned}
& d_{o}=\left[d_{o}-\frac{2 v}{E} \sigma d_{o}\right]+\left[\frac{1+v}{E} \sigma d_{o}\right] \sin ^{2} \psi \\
& 1=\left[1-\frac{2 v}{E} \sigma\right]+\left[\frac{1+v}{E} \sigma\right] \sin ^{2} \psi \\
& 0=-\frac{2 v}{E} \sigma+\left[\frac{1+v}{E} \sigma\right] \sin ^{2} \psi \\
& \sin ^{2} \psi=\frac{2 v}{1+v}
\end{aligned}
$$

Equivalently, the strain free lattice parameter can be measured as a part of the experiment using this relation, assuming the elastic properties (Poisson's ratio) are reasonably well known. This is necessary because the exact composition and equilibrium lattice parameter of the film may not be known with sufficient precision to determine the strain very accurately. Indeed, the principal advantage of the non-symmetric technique for measuring stresses in films over the simple symmetric technique is that the stress-free plane spacing can be determined as part of the measurement and need not be known separately. This is an important advantage because the strain depends on the difference in plane spacings and large error can result if both spacings are not known with precision.

\section{Use of (422) planes for (111) Texture}

Many FCC metal thin films exhibit strong (111) fiber textures. For such films the (422) reflecting planes are particularly useful for thin film stress measurements because different $\{422\}$ planes can be found at different angles to the film plane normal. The crystal structure diagram shows the angles made by two $<422>$ directions with the [111] film plane normal. 


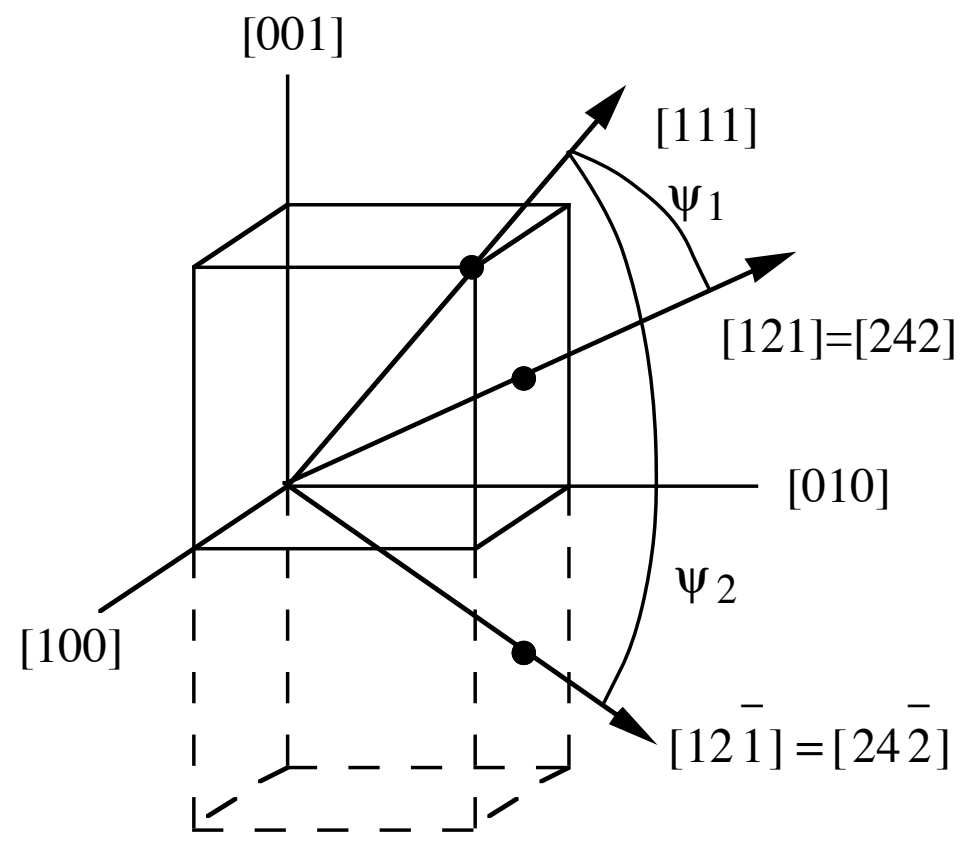

For the cubic films with a strong (111) fiber texture the (422) plane normals will be found at the following angles:

$$
\begin{aligned}
\cos \psi_{1} & =\frac{1}{\sqrt{3}}[111] \cdot \frac{1}{\sqrt{6}}[121]=\frac{4}{3 \sqrt{2}} \\
\psi_{1} & =19.44^{\circ} \sin ^{2} \psi_{1}=0.110 \\
\cos \psi_{2} & =\frac{1}{\sqrt{3}}[111] \cdot \frac{1}{\sqrt{6}}[12 \overline{1}]=\frac{2}{3 \sqrt{2}} \\
\psi_{2} & =61.86^{\circ} \sin ^{2} \psi_{2}=0.777
\end{aligned}
$$

Diffraction from these two (422) planes leads to two scattering vector cones for cubic metals with a strong (111) fiber texture as shown in the following figure: 
Scattering vector cones from a polycrystalline film with a $<111>$ fiber texture
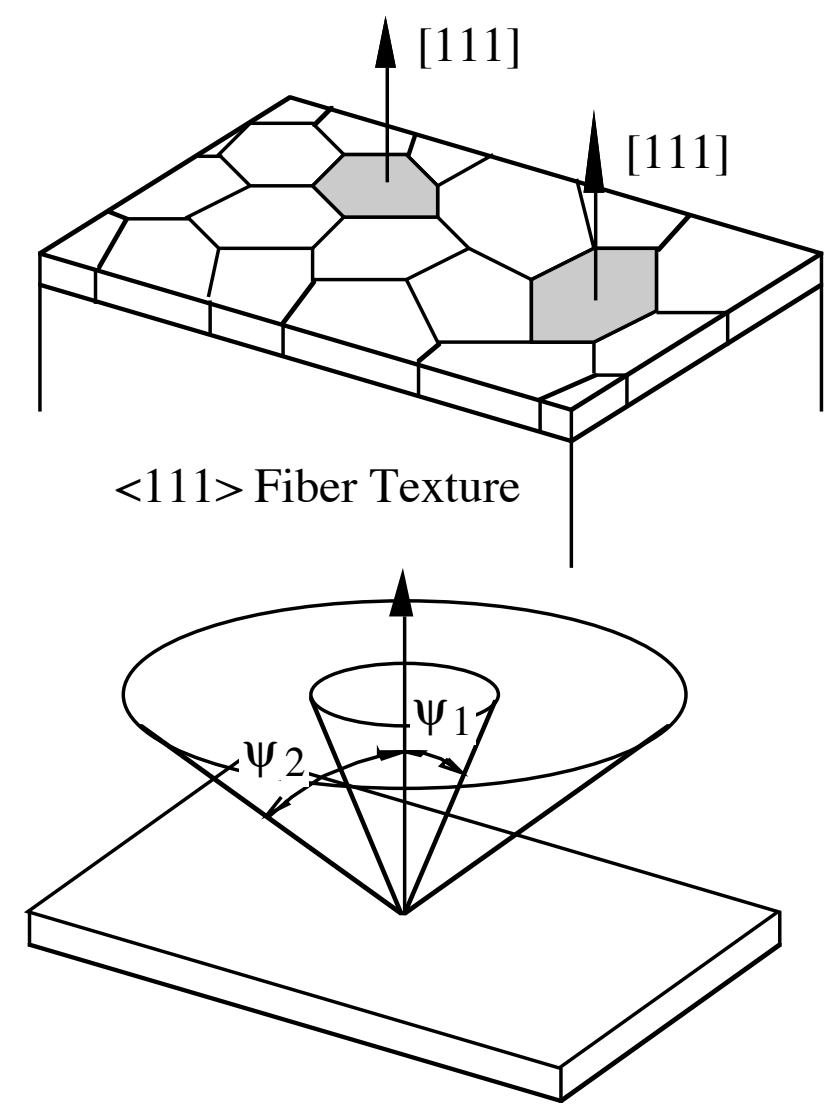

(422) scattering vector cones

Measuring the (422) plane spacing, $d_{\psi}$, for the two scattering vectors leads to two points on the $d_{\psi}$ vs $\sin ^{2} \psi$ plot. This is shown in the following figure. 
Typical non-symmetric diffraction results for (111) textured Al thin film

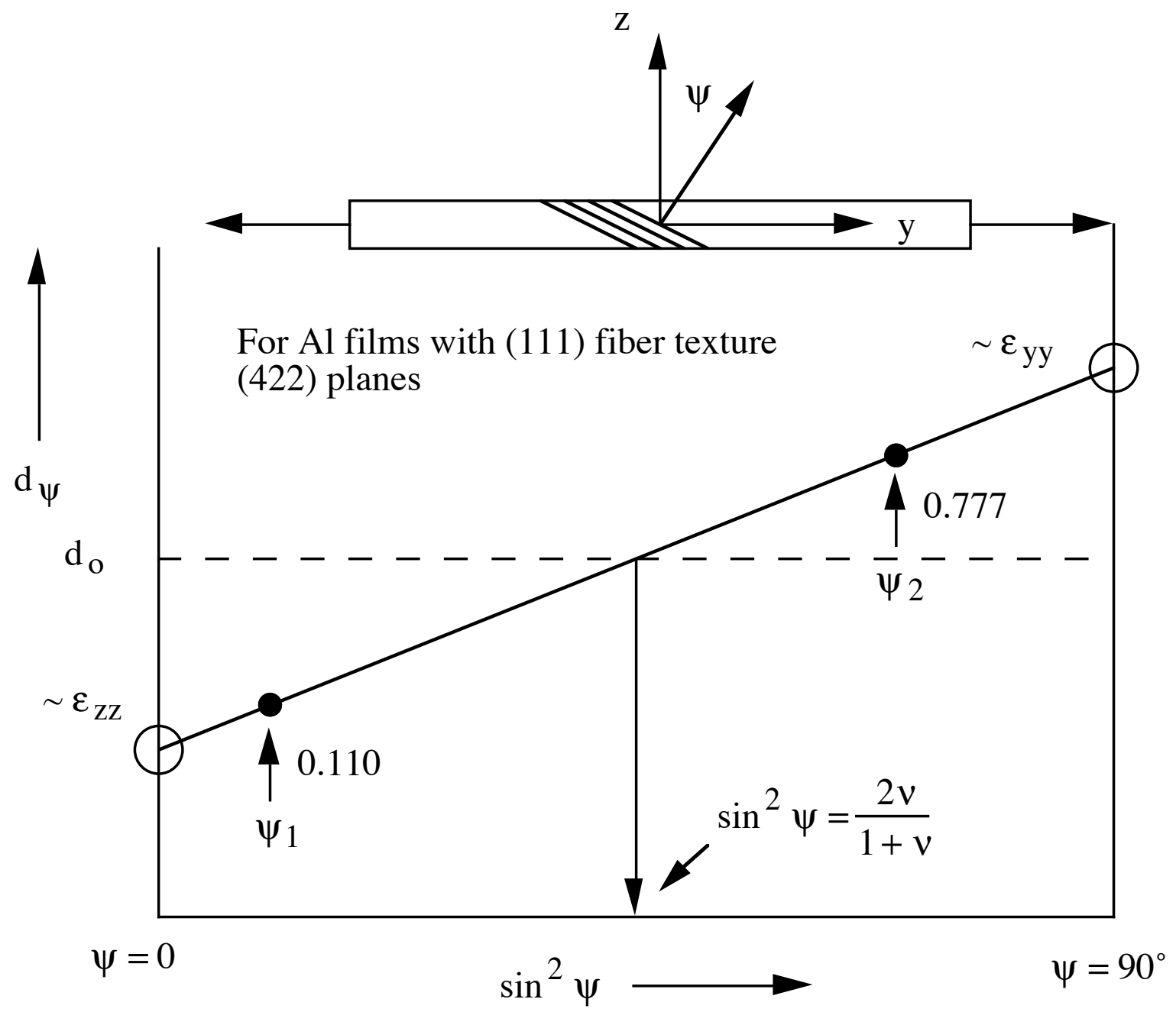

The straight line in this figure permits a calculation of the strain as a function of the angle $\psi$. For the point $\psi=0$ the strain calculated is $\varepsilon_{z z}$, the same quantity found by symmetric $x$-ray diffraction. For the limit $\psi=90^{\circ}$ the measured plane spacing leads to the in-plane strain, $\varepsilon_{y y}$. 


\section{Experimental results for aluminum thin films}

Aluminum is sufficiently isotropic elastically for the following relation to be used

$$
\sin ^{2} \psi=\frac{2 v}{1+v}
$$

Poisson's ratio for aluminum is about 0.34 , so the stress-free plane spacing will be found at

$$
\sin ^{2} \psi=0.5 .
$$

This result is used to establish the stress-free plane spacing for aluminum thin films.

\section{X-ray measurements of stresses in aluminum films}

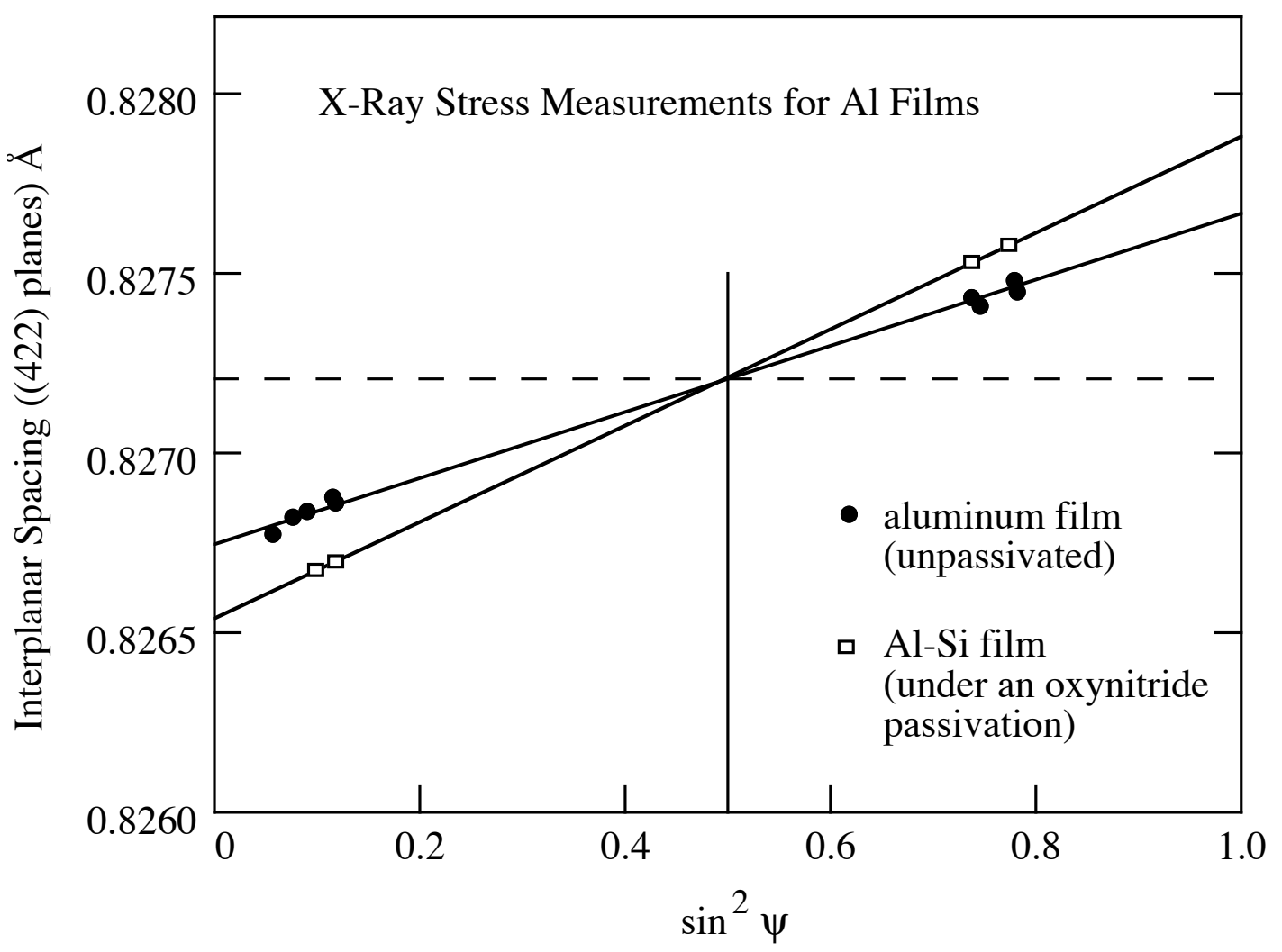


Textured films with elastic anisotropy

As shown above, the strain in any arbitrary direction can be expressed in terms of the principal strains as follows:

$$
\varepsilon_{\psi}=\varepsilon_{1} \sin ^{2} \psi \cos ^{2} \varphi+\varepsilon_{2} \sin ^{2} \psi \sin ^{2} \varphi+\varepsilon_{3} \cos ^{2} \psi
$$

For the case of a thin film subjected to an equal-biaxial strain state,

$$
\varepsilon_{1}=\varepsilon_{2}
$$

The strain can be then expressed as

$$
\begin{aligned}
& \varepsilon_{\psi}=\varepsilon_{2} \sin ^{2} \psi\left(\sin ^{2} \varphi+\cos ^{2} \varphi\right)+\varepsilon_{3} \cos ^{2} \psi \\
& \varepsilon_{\psi}=\varepsilon_{2} \sin ^{2} \psi+\varepsilon_{3} \cos ^{2} \psi
\end{aligned}
$$

\section{Textured films with (001) Growth Texture}

For films with the (001) fiber texture subjected to an equal-biaxial strain state, the in-plane strains can be expressed as

$$
\varepsilon_{1}=\varepsilon_{2}=\left(\frac{s_{11}+s_{12}}{2 s_{12}}\right) \varepsilon_{3}
$$

and the corresponding biaxial stress is

$$
\sigma=\frac{1}{2 s_{12}} \varepsilon_{3}
$$

These relations are the same as those for single crystal (001) films. For equalbiaxial strain states, (001) fiber textured films behave elastically like (001) single crystal films. Solving for the three principal strains we have 


$$
\begin{aligned}
& \varepsilon_{3}=2 s_{12} \sigma \\
& \varepsilon_{1}=\varepsilon_{2}=\left(\frac{s_{11}+s_{12}}{2 s_{12}}\right) 2 s_{12} \sigma=\left(s_{11}+s_{12}\right) \sigma
\end{aligned}
$$

Inserting these relations into the expression for $\varepsilon_{\psi}$, we have

$$
\begin{aligned}
& \varepsilon_{\psi}=\left(s_{11}+s_{12}\right) \sigma \sin ^{2} \psi+2 s_{12} \sigma \cos ^{2} \psi \\
& \varepsilon_{\psi}=\left(s_{11}+s_{12}\right) \sigma \sin ^{2} \psi+2 s_{12} \sigma\left(1-\sin ^{2} \psi\right) \\
& \varepsilon_{\psi}=\frac{d_{\psi}-d_{o}}{d_{o}}=\left(s_{11}-s_{12}\right) \sigma \sin ^{2} \psi+2 s_{12} \sigma
\end{aligned}
$$

We note that $\varepsilon_{\psi}=0$ when

$$
0=\left(s_{11}-s_{12}\right) \sigma \sin ^{2} \psi+2 s_{12} \sigma
$$

This leads to

$$
\sin ^{2} \psi=\frac{2 s_{12}}{s_{11}-s_{12}}
$$

If the compliances of the (001) fiber textured film are known, the angle at which the stress free pane spacing would be found is given by this expression. A similar analysis using the stiffnesses leads to

$$
\sin ^{2} \psi=\frac{2 c_{12}}{c_{11}+2 c_{12}}
$$




\section{Textured films with (111) Growth Texture}

Thin films having the (111) fiber texture are fully isotropic in the plane of the film. In this case it can be shown that the elastic strain in a film subjected to a biaxial state of stress can be described by

$$
\varepsilon_{\psi}=\frac{d_{\psi}-d_{o}}{d_{o}}=\sigma\left(\frac{2 s_{11}+4 s_{12}-s_{44}}{3}+\frac{s_{44}}{2} \sin ^{2} \psi\right)
$$

The unstrained lattice plane spacing, $d_{o}$, is found by setting $\varepsilon_{\psi}=0$, which leads to

$$
\sin ^{2} \psi=\frac{2 s_{44}-4 s_{11}-8 s_{12}}{3 s_{44}}
$$

\section{Unstrained Lattice Parameter Measurements and Thermal Expansion Coefficients}

In the treatments given above it is necessary to determine the unstrained dspacing, $d_{o}$, using the elastic constants of the film and the known crystallographic texture of the film. This is usually a reliable process, as the elastic properties for most crystalline materials are quite well-known. For a completely new thin film material, for which the elastic constants are unknown, it still may be possible to determine $d_{o}$ from the $d_{\psi} v s \cdot \sin ^{2} \psi$ measurements. If the sign of the biaxial stress in the film is changed, say by thermal cycling, then the $d_{\psi} v s \cdot \sin ^{2} \psi$ plots will have very different different slopes and will cross at one point, indicating the $d_{o}$ for that material. Thus $d_{o}$ can be determined without any knowledge of the elastic properties. This technique has been used in a study of stresses in Al and Au films by Cornella et al. Appl. Phys. Lett., Vol. 71, No. 20, (1997). Typically, heating the film to a high temperature and then cooling to the measurement temperature leads to a tension stress in the film, while first cooling the film to a low temperature and then heating to the measurement temperature produces a compressive stress. The signs of the slopes of the $d_{\psi} v s \cdot \sin ^{2} \psi$ plots are different and the point of intersection of the two lines can be accurately determined. The following figure indicates the principle of the technique. 


\section{Experimental Technique for Determining $d_{o}$}

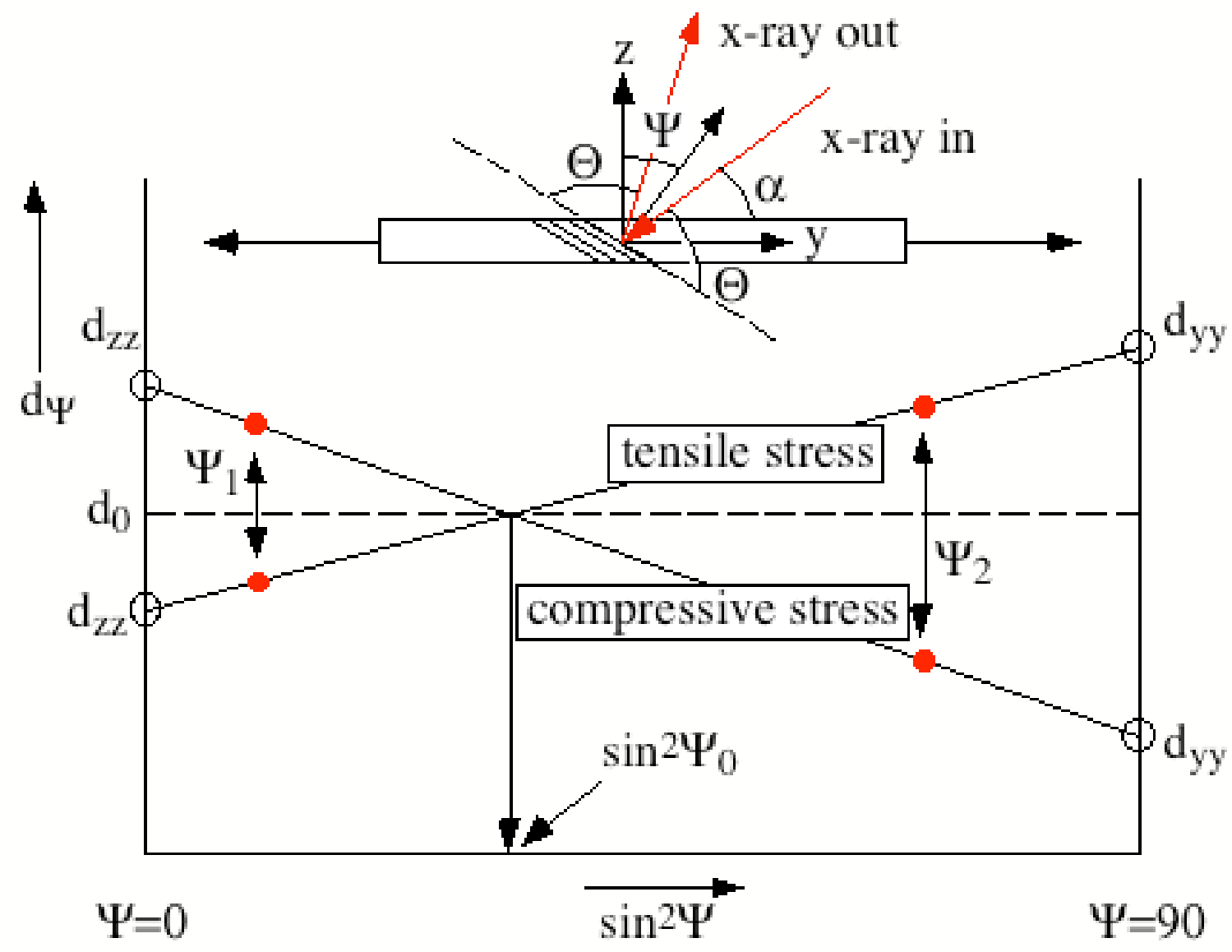

Kraft and Nix (Journal of Applied Physics, $\underline{83}$, 3035-3038 (1998)) used this technique to measure the thermal expansion coefficient of an $\mathrm{Al}$ film on a Si substrate. Some of their results are shown as $d_{\psi} v s \cdot \sin ^{2} \psi$ obtained at different temperatures during both heating (compression) and cooling (tension). The results directly give the thermal expansion of the film, even though it is still attached to and constrained by the substrate! 
$d_{\psi} v s \cdot \sin ^{2} \psi$ Plots for an Al film on a Si Substrate in both Compression and Tension

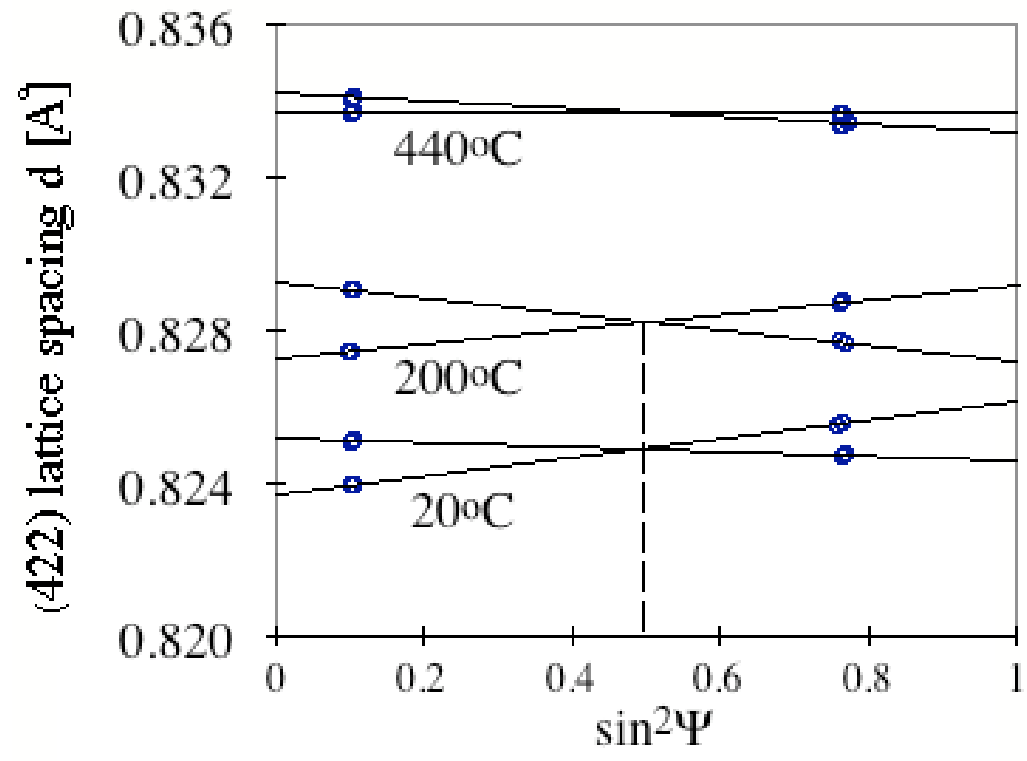

Thermal Expansion Coefficient of Al Film Determined by X-Ray Technique

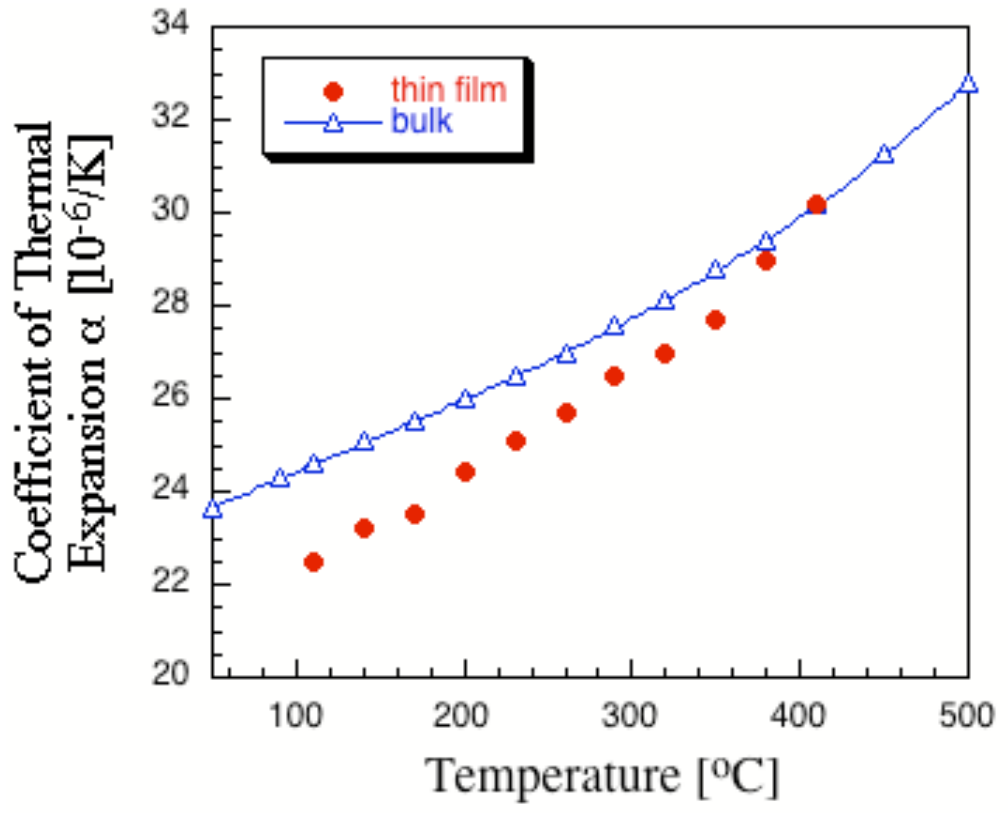


Film Stress Determined using Multiple Reflecting Planes

In the data shown above one particular set of diffracting planes, $\{422\}$, was used to measure the elastic strain in different directions in the film. This is possible because different $\{422\}$ planes can be found at different angles to the film plane normal. But this restricts the strain measurements to certain directions in the film. For strongly textured films the reflecting plane normals are found only at discrete angles. If strain measurements in other directions are needed, then other reflecting planes would have to be used. In this case one cannot make a plot of $d_{\psi} v s \cdot \sin ^{2} \psi$ because the $d_{o}$ 's are different for the different reflecting planes. The following analysis in needed when different reflecting planes are used.

First, the elastic strain in any direction can be expressed as

$$
\varepsilon_{\psi}=\frac{d_{h k l}-d_{h k l}^{o}}{d_{h k l}^{o}}
$$

where for cubic solids

$$
d_{h k l}^{o}=\frac{a_{o}}{\sqrt{h^{2}+k^{2}+l^{2}}}
$$

These relations lead to

$$
\begin{gathered}
\varepsilon_{\psi}=\frac{d_{h k l} \sqrt{h^{2}+k^{2}+l^{2}}}{a_{o}}-1 \\
d_{h k l} \sqrt{h^{2}+k^{2}+l^{2}}=a_{o}\left(1+\varepsilon_{\psi}\right)
\end{gathered}
$$

We see that $d_{h k l} \sqrt{h^{2}+k^{2}+l^{2}}$ in linearly proportional to the strain in the film. Thus, when different reflecting planes are used, the plot should be $d_{h k l} \sqrt{h^{2}+k^{2}+l^{2}} v s \cdot \sin ^{2} \psi$. All of the data should fall on a common straight line in such a plot. 
$d_{\psi} v s \cdot \sin ^{2} \psi$ Plot Using Multiple Reflections

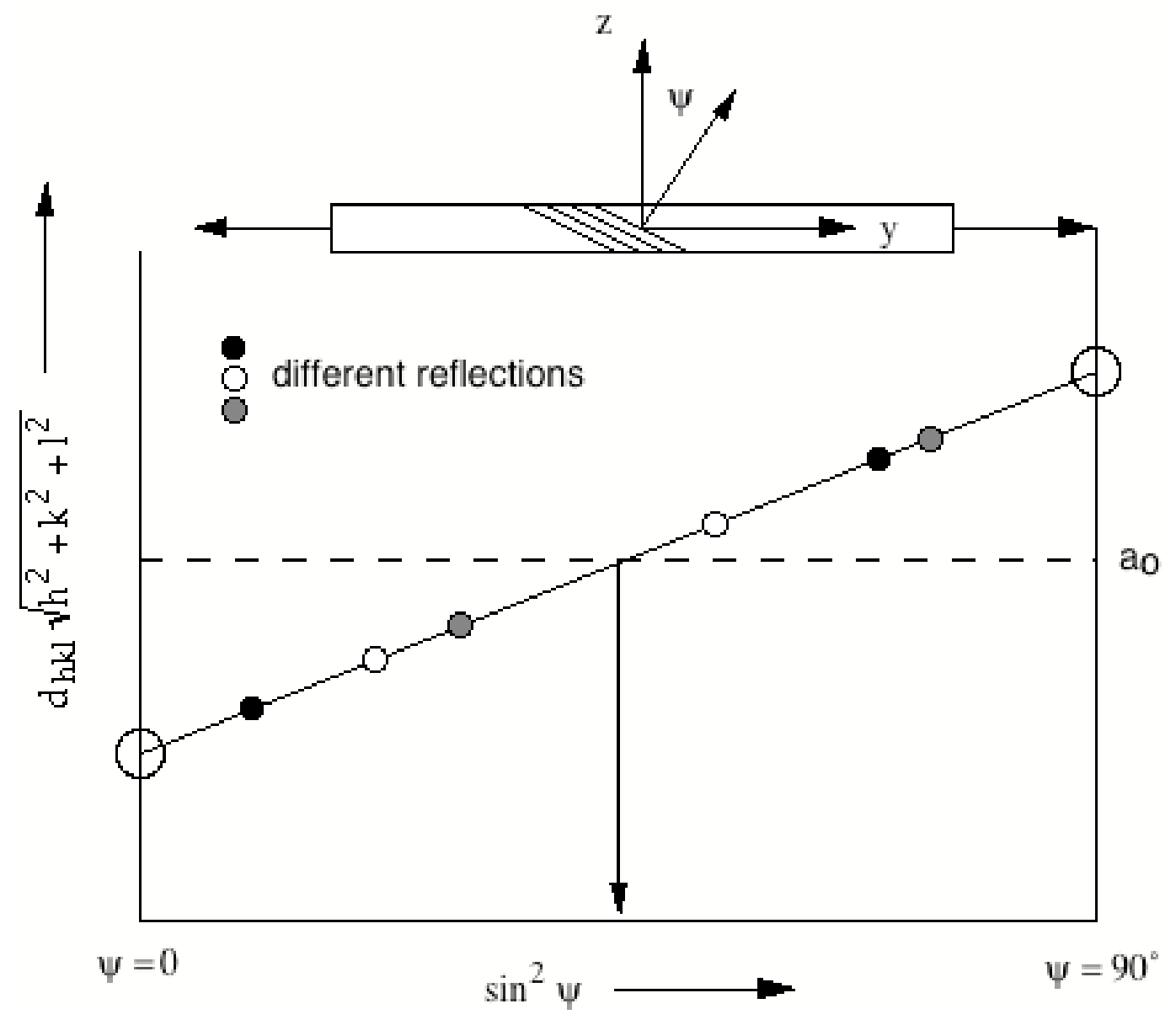


$d_{\psi} v s \cdot \sin ^{2} \psi$ Plot Using Multiple Reflections for a $0.8 \mu \mathrm{m}$ Thick Gold

$\underline{\text { Film }}$

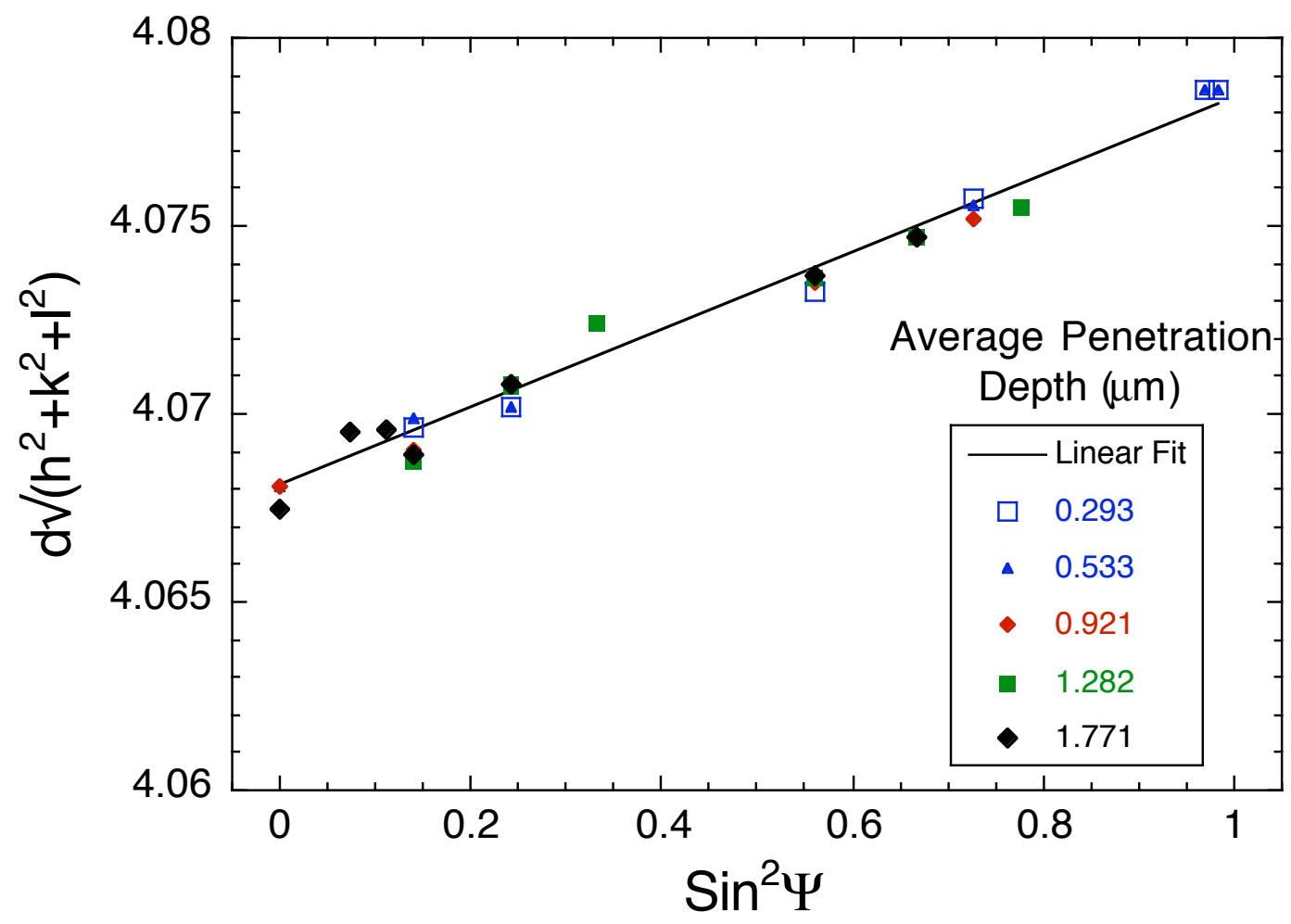

\section{Applications to Plastic Deformation of Aluminum Films}

The x-ray methods we have discussed for thin films have been used to study the evolution of stress in metal films as a function of thermal cycling. The data below is taken from the Ph.D. dissertation of R. Venkatraman. We note that the stresses found by $x$-ray diffraction are close to those found by the wafer curvature methods to be discussed later. X-ray diffraction measurements offer more information about the strain state in the film. For example, the broadening of the diffraction peak can be measured in addition to its position. This provides information about the defect state of the film. The line broadening associated with cooling the aluminum film from high temperature is shown in the figure below. The broadening at low temperatures is a sure sign of high dislocation densities in the film. This clearly shows that plastic deformation by dislocation motion and multiplication occurs on cooling. 
Comparison with wafer curvature data (R. Venkatraman)

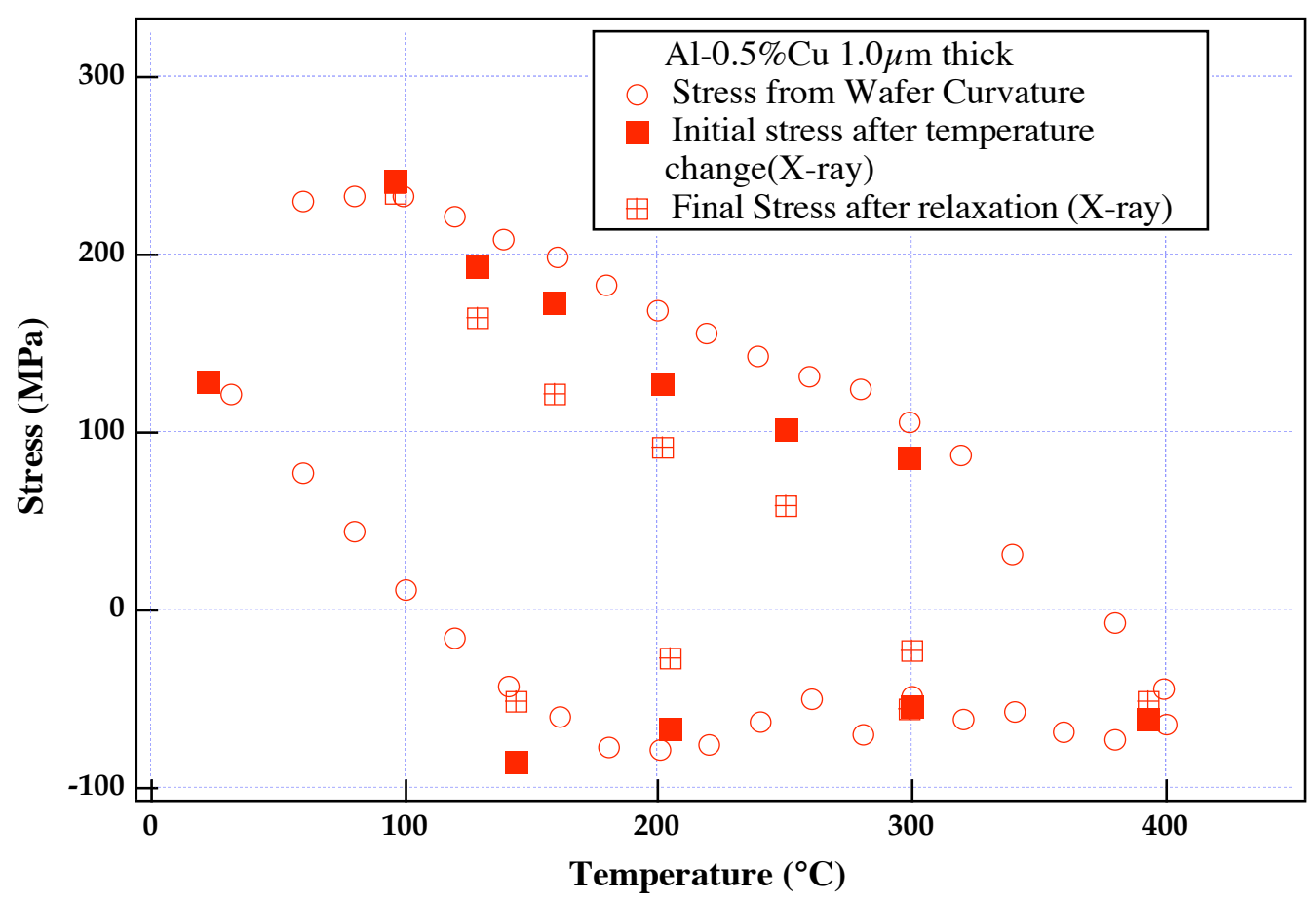

\section{Line Broadening on cooling in a Al-0.5\%Cu thin film (R. Venkatraman)}

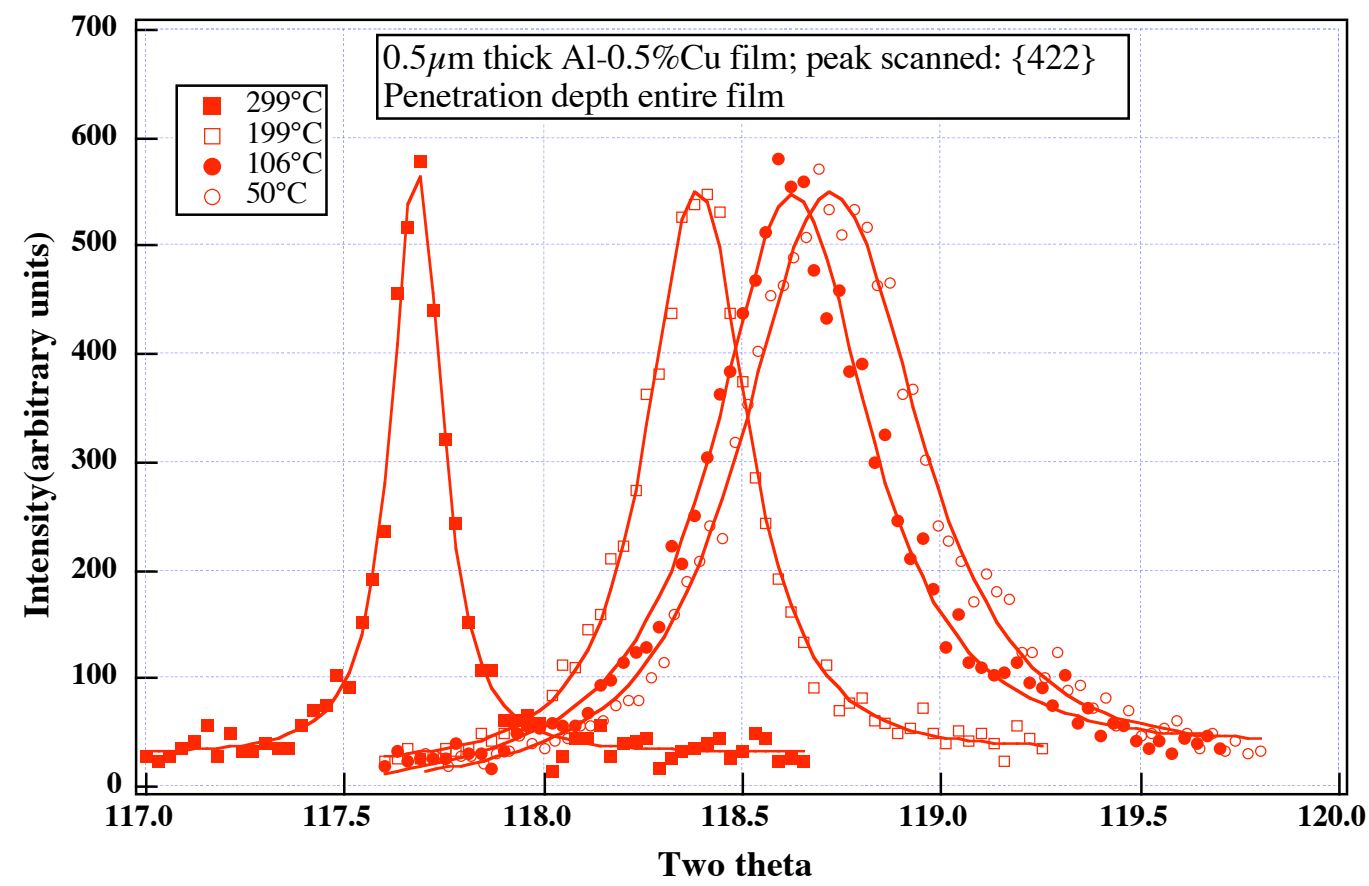




\section{$\underline{\text { Stress measurements in line structures }}$}

Stresses in passivated aluminum lines have been measured by x-ray diffraction (Flinn and Chiang). They extended the non-symmetric x-ray diffraction method discussed above to find the average principal strains and stresses in long continuous lines of aluminum under a passivation.

Consider the passivated metal lines shown in the following diagram. The passivation was deposited at an elevated temperature and the entire structure was subsequently cooled to room temperature. Such a thermal treatment causes the following principal stresses (and strains) to develop in the lines:

$$
\sigma_{x x}, \sigma_{y y}, \sigma_{z z} \quad \text { and } \quad \varepsilon_{x x}, \varepsilon_{y y}, \varepsilon_{z z}
$$

By measuring the (422) plane spacings for scattering vectors at different angles from the $z$ axis, it is possible to determine the principal strains: $\varepsilon_{x x}, \varepsilon_{y y}, \varepsilon_{z z}$. Using an appropriate elasticity analysis leads to the principal stresses: $\sigma_{x x}, \sigma_{y y}, \sigma_{z z}$. For metal films having the (111) fiber texture, the (422) planes produce scattering vector cones at two different angles as discussed above. Thus diffraction can be found at the angles shown in the figure. By investigating scattering vectors along both "parallel" and "perpendicular" paths it is possible to determine all of the principal strains in the lines. 
Parallel and perpendicular paths for non-symmetric x-ray scattering from lines

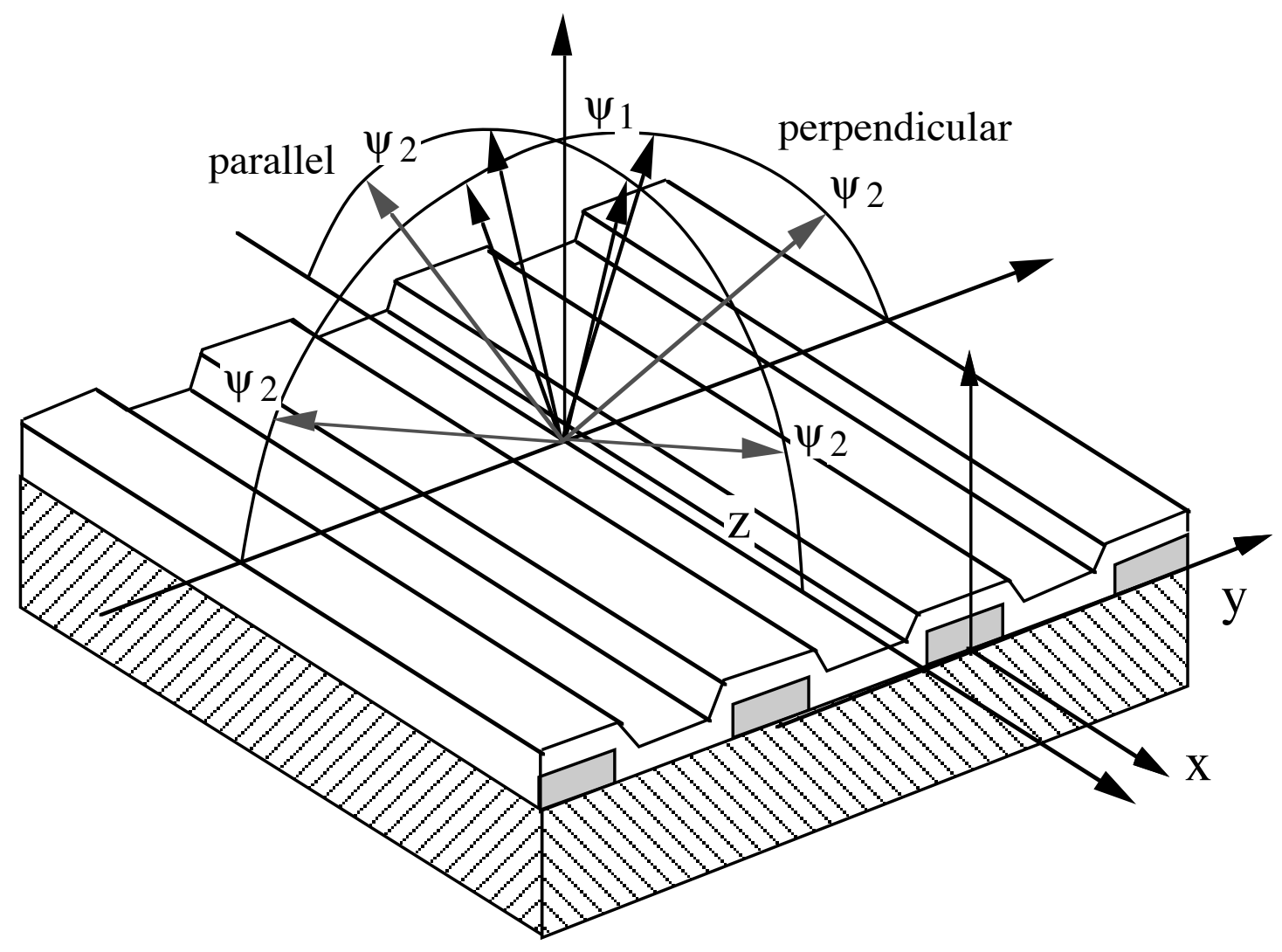

As discussed above, the strain in any direction can be expressed as

$$
\varepsilon_{\psi}=\varepsilon_{x} \sin ^{2} \psi \cos ^{2} \varphi+\varepsilon_{y} \sin ^{2} \psi \sin ^{2} \varphi+\varepsilon_{z} \cos ^{2} \psi
$$

where we have replaced $1,2,3$ with $x, y, z$ in this relation.

Along the "perpendicular" path we have

$$
\varphi=\frac{\pi}{2} \quad \text { so that } \quad \cos ^{2} \varphi=0 \quad \text { and } \quad \sin ^{2} \varphi=1
$$

For this "path" the strain $\varepsilon_{\psi}$ is 


$$
\begin{aligned}
& \varepsilon_{\psi}(\text { perpendicular })=\varepsilon_{y} \sin ^{2} \psi+\varepsilon_{z} \cos ^{2} \psi=\varepsilon_{y} \sin ^{2} \psi+\varepsilon_{z}\left(1-\sin ^{2} \psi\right) \\
& \varepsilon_{\psi}(\text { perpendicular })=\left(\varepsilon_{y}-\varepsilon_{z}\right) \sin ^{2} \psi+\varepsilon_{z}
\end{aligned}
$$

Again using

$$
\varepsilon_{\psi}=\frac{d_{\psi}-d_{o}}{d_{o}}
$$

the measured plane spacing along the "perpendicular" path will be

$$
d_{\psi}(\text { perpendicular })=d_{o}\left[\left(1+\varepsilon_{z}\right)+\left(\varepsilon_{y}-\varepsilon_{z}\right) \sin ^{2} \psi\right] .
$$

We see from this result that the strains transverse to the lines, $\varepsilon_{y}$, and normal to the lines $\varepsilon_{z}$ can be measured using the "perpendicular" path.

Along the "parallel" path we have

$$
\varphi=0 \quad \text { so that } \cos ^{2} \varphi=1 \text { and } \sin ^{2} \varphi=0 .
$$

For this path the strain $\varepsilon_{\psi}$ is

$$
\begin{aligned}
& \varepsilon_{\psi}(\text { parallel })=\varepsilon_{x} \sin ^{2} \psi+\varepsilon_{z} \cos ^{2} \psi=\varepsilon_{x} \sin ^{2} \psi+\varepsilon_{z}\left(1-\sin ^{2} \psi\right) \\
& \varepsilon_{\psi}(\text { parallel })=\left(\varepsilon_{x}-\varepsilon_{z}\right) \sin ^{2} \psi+\varepsilon_{z}
\end{aligned}
$$

Again using

$$
\varepsilon_{\psi}=\frac{d_{\psi}-d_{o}}{d_{o}}
$$

the measured plane spacing along the "parallel" path will be

$$
d_{\psi}(\text { parallel })=d_{o}\left[\left(1+\varepsilon_{z}\right)+\left(\varepsilon_{x}-\varepsilon_{z}\right) \sin ^{2} \psi\right]
$$


We see from this result that the strains along the lines, $\varepsilon_{x}$, and normal to the lines $\varepsilon_{z}$ can be measured using the "parallel" path. Thus, by using nonsymmetric x-ray scattering both "parallel" and "perpendicular" to the lines we can determine the three principal strains in the lines. The corresponding stresses could be calculated using Hooke's law.

$\underline{\text { X-ray measurements of strains in passivated lines }}$

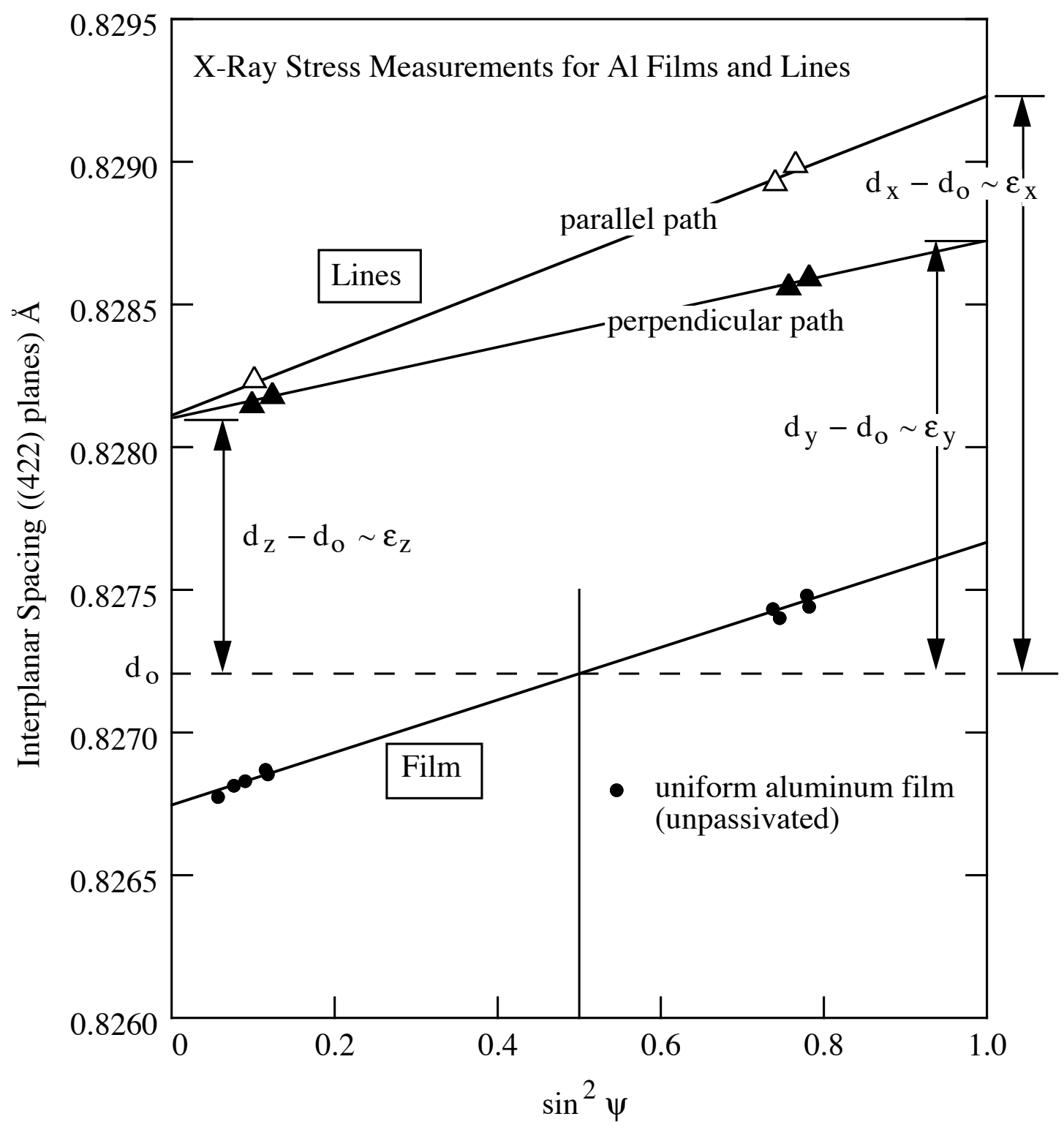

The results of these measurements have been compared with the results of finite element calculations. The comparison is shown is the table below. 


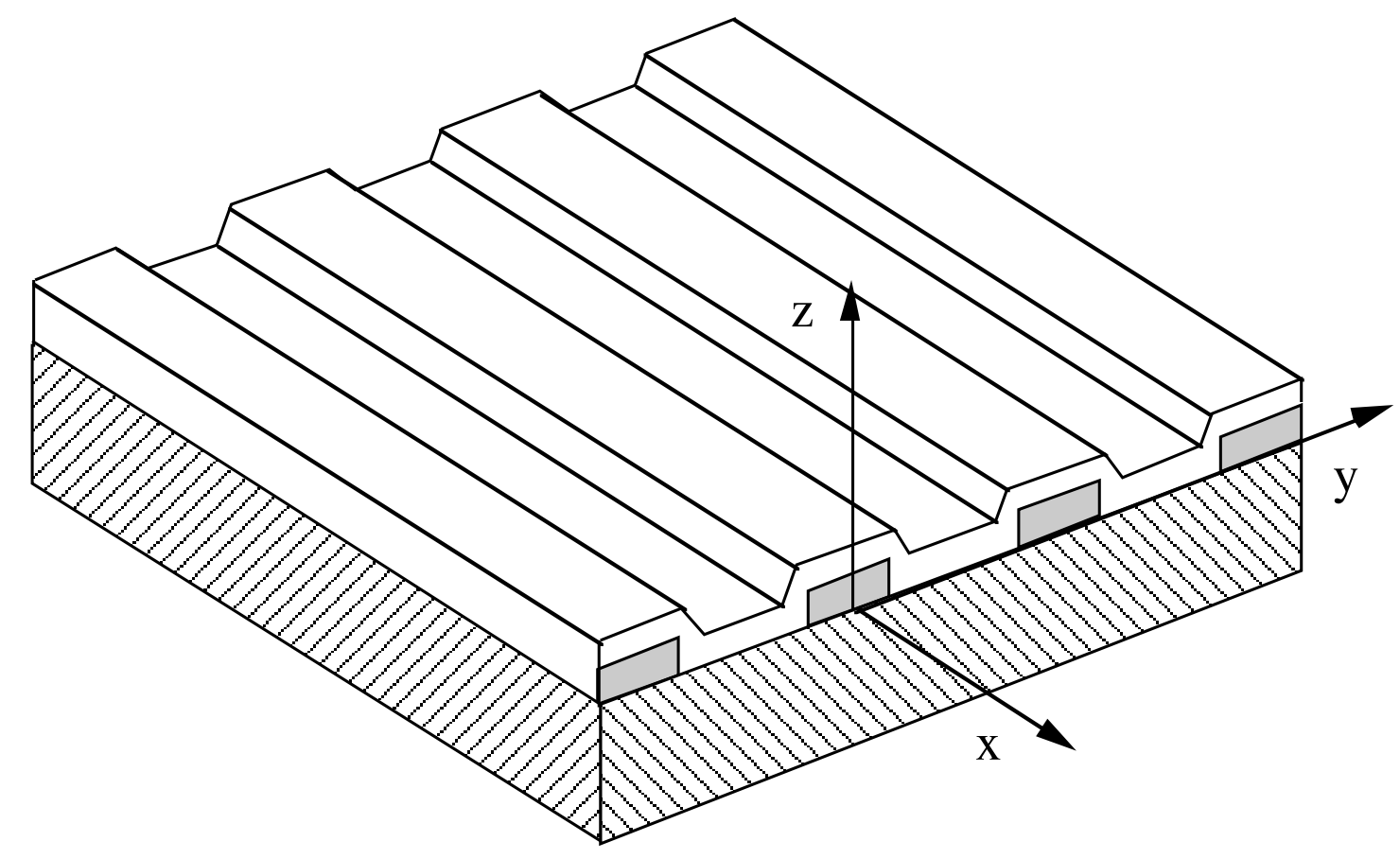

Average Stresses in Units of MPa

\begin{tabular}{|l|l|l|l|}
\hline Sample & $\sigma_{x}$ & $\sigma_{y}$ & $\sigma_{z}$ \\
\hline Uniform Film (x-ray) & 160 & 160 & 0 \\
\hline $\begin{array}{l}\text { Passivated Lines (x-ray) } \\
\text { (Flinn and Chiang) }\end{array}$ & 538 & 453 & 362 \\
\hline $\begin{array}{l}\text { Finite Element Calculation } \\
\text { (Sauter (now Mack)) }\end{array}$ & 559 & 489 & 376 \\
\hline
\end{tabular}




\section{Strain Distribution in Thin Films}

In much of the previous discussion we have assumed that the stresses and strains in films are uniform except at the edges. This need not be true. There is no reason why bending stresses cannot also exist in the film. Suppose we have a biaxial stress state as follows.

Stress distribution in a film

$$
\sigma_{\mathrm{Xx}}=\sigma_{\mathrm{XX}}=\sigma
$$

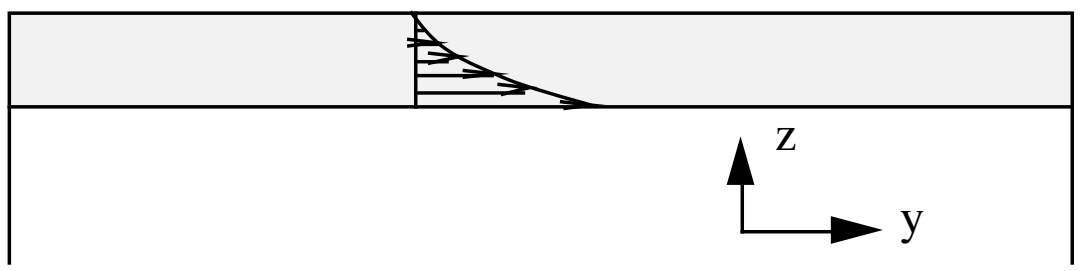

We have something like this when diffusional relaxation occurs in polycrystalline films.

\section{Diffusional relaxation leading to a biaxial stress gradient in a film}

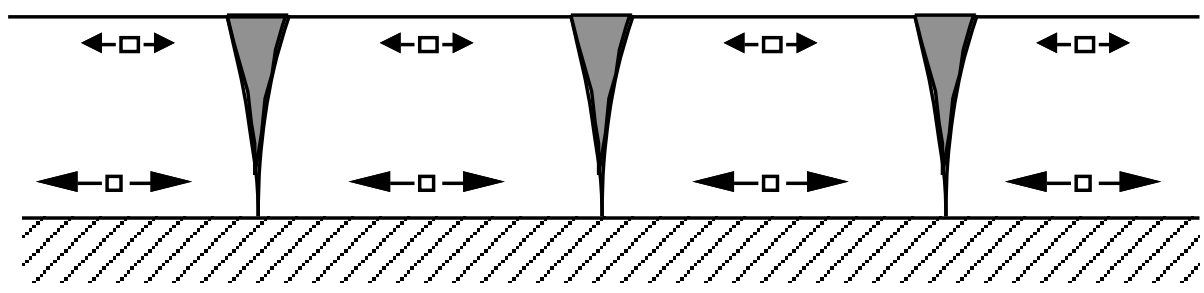

How can this stress (or strain) distribution be measured? The most direct

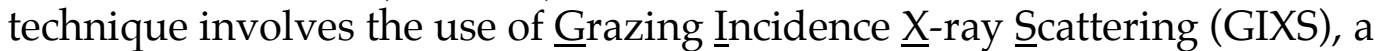
technique for measuring atomic plane spacings (for planes lying perpendicular to plane of the film) as a function of depth from the surface.

\section{Grazing Incidence X-Ray Scattering (GIXS)}

GIXS was developed by people at SSRL, and used by Doerner and Brennan (JAP 1988) to measure the strain (stress) distributions in Al films on Si. It has since been used by R. Venkatraman, P. Besser and R. Vinci and others in the Bravman group to study the strain distributions in thin metal films. 


\section{$\underline{\text { Negative Index of Refraction }}$}

The GIXS technique is based on Snell's law. When x-rays are incident on a medium with a higher index of refraction, the path changes as shown in the following diagram.

\section{$\underline{X}$-rays incident on a solid with a lower index of refraction}

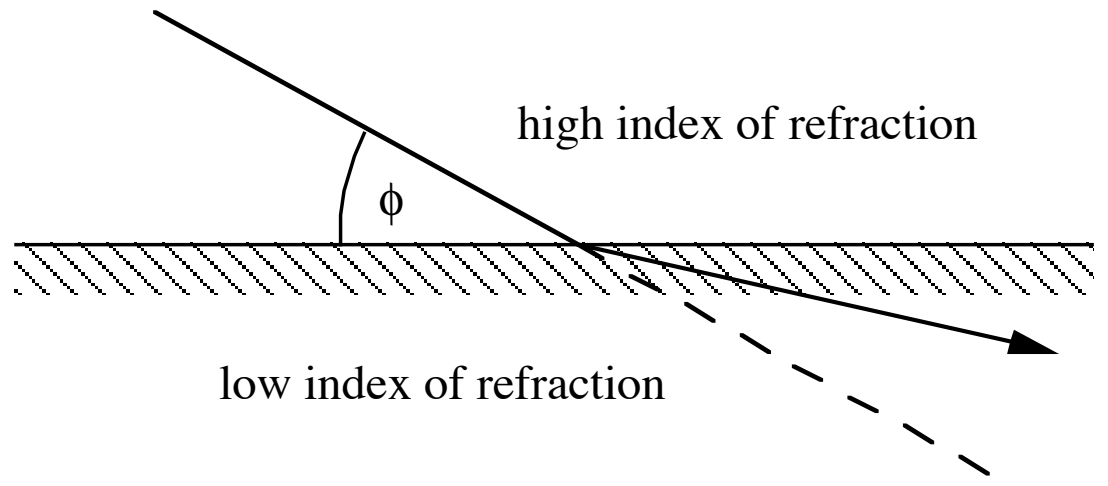

Below a critical angle $\phi_{c}$, the x-rays are reflected out of rather than into sample. The penetration of $\mathrm{x}$-rays into the sample therefore depends very sensitively on the incident angle $\phi$.

\section{$\underline{\text { X-rays incident on a solid below a critical angle }}$}

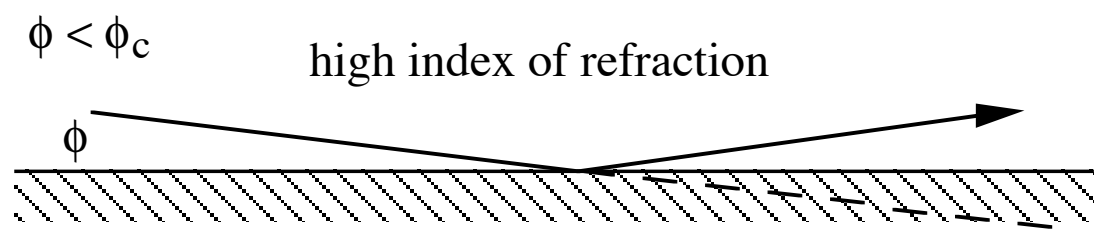

low index of refraction

The surface sensitivity can be shown by showing the x-ray penetration depth as a function of the angle of incidence of the x-rays. Here we see that the critical angle for aluminum is about 0.2 degrees. 


\section{Penetration depth as a function of incident angle (Pure Al, $8700 \mathrm{eV}$ )}

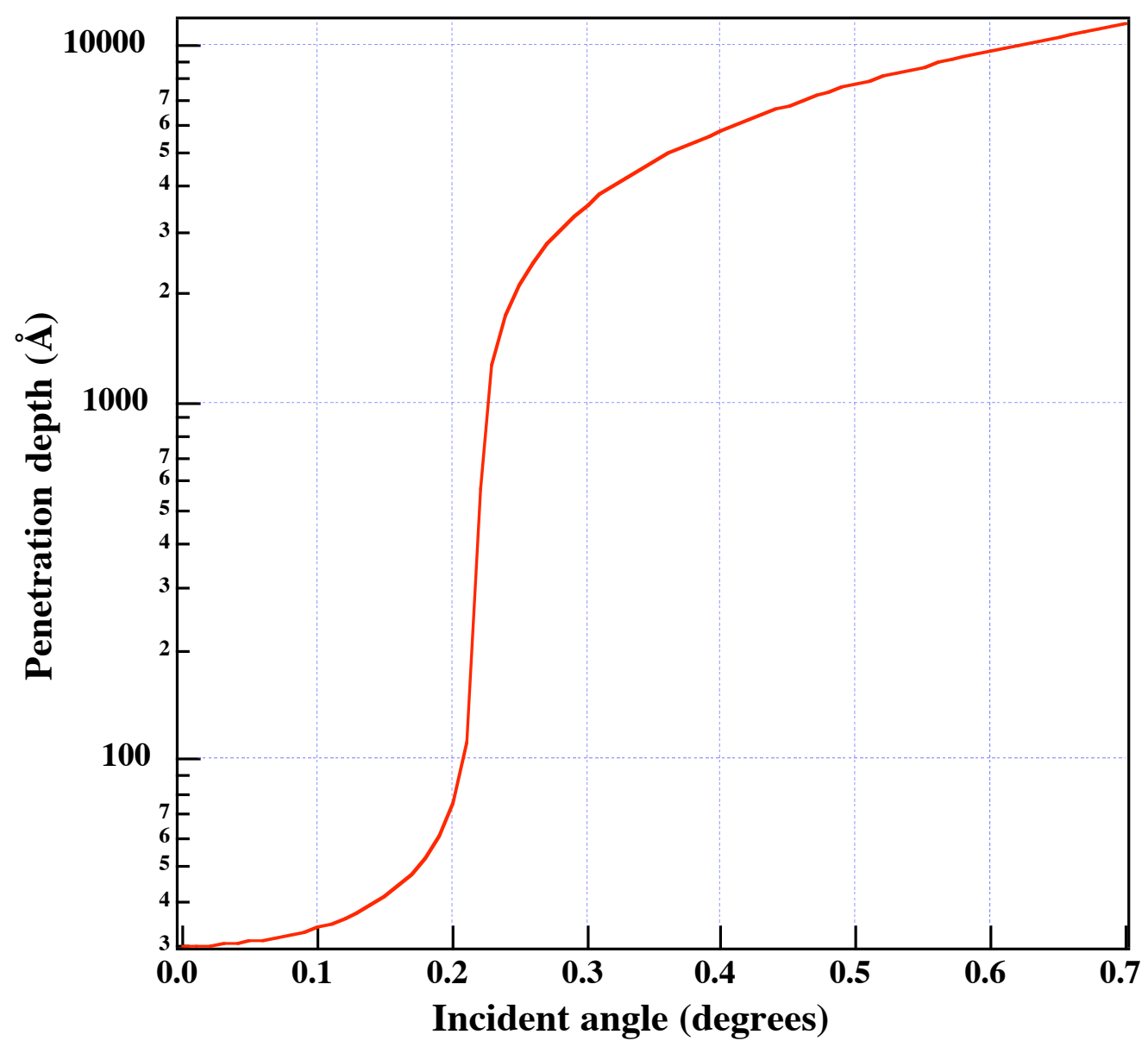

By varying the incident angle, $\phi$, it is possible to sample different depths into the film. Thus we can measure the strains at various depths with this technique.

By measuring the scattered intensity as a function of the Bragg angle $2 \theta$ (see GIXS diagram) it is possible to measure spacing of planes that are perpendicular to the plane of the film. So, the in-plane strain, $\varepsilon_{o}$, is measured directly. 


\section{GIXS Geometry}

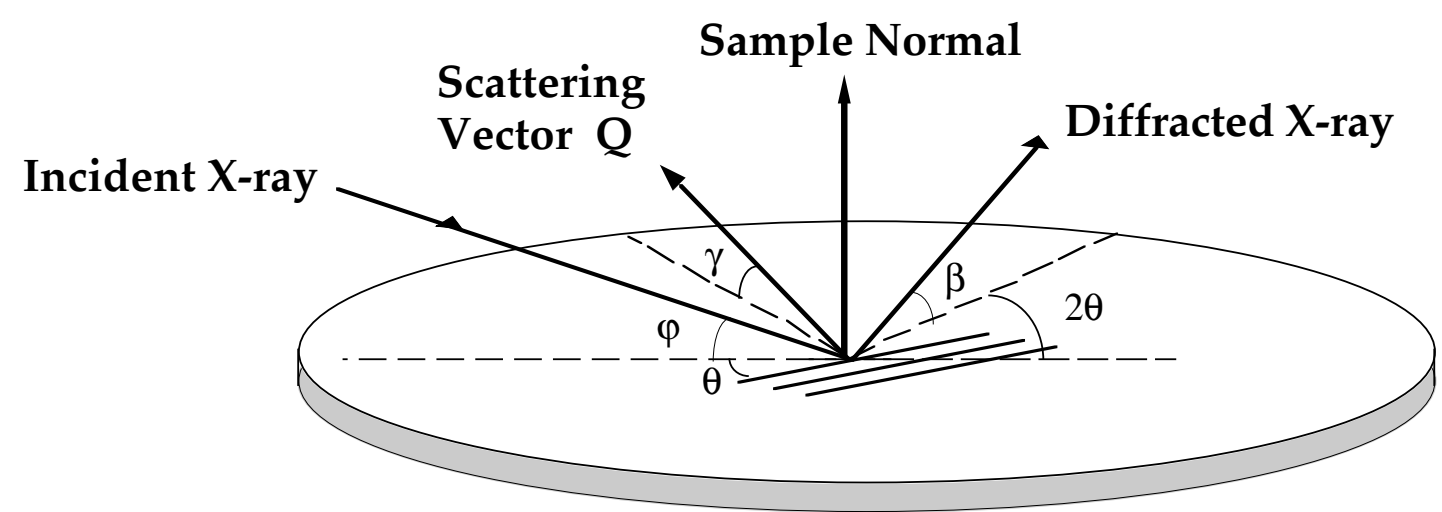

Some results from the work of $\mathrm{R}$. Venkatraman $\left(\mathrm{Al}-0.5 \% \mathrm{Cu}, 144^{\circ} \mathrm{C}\right.$, heating)

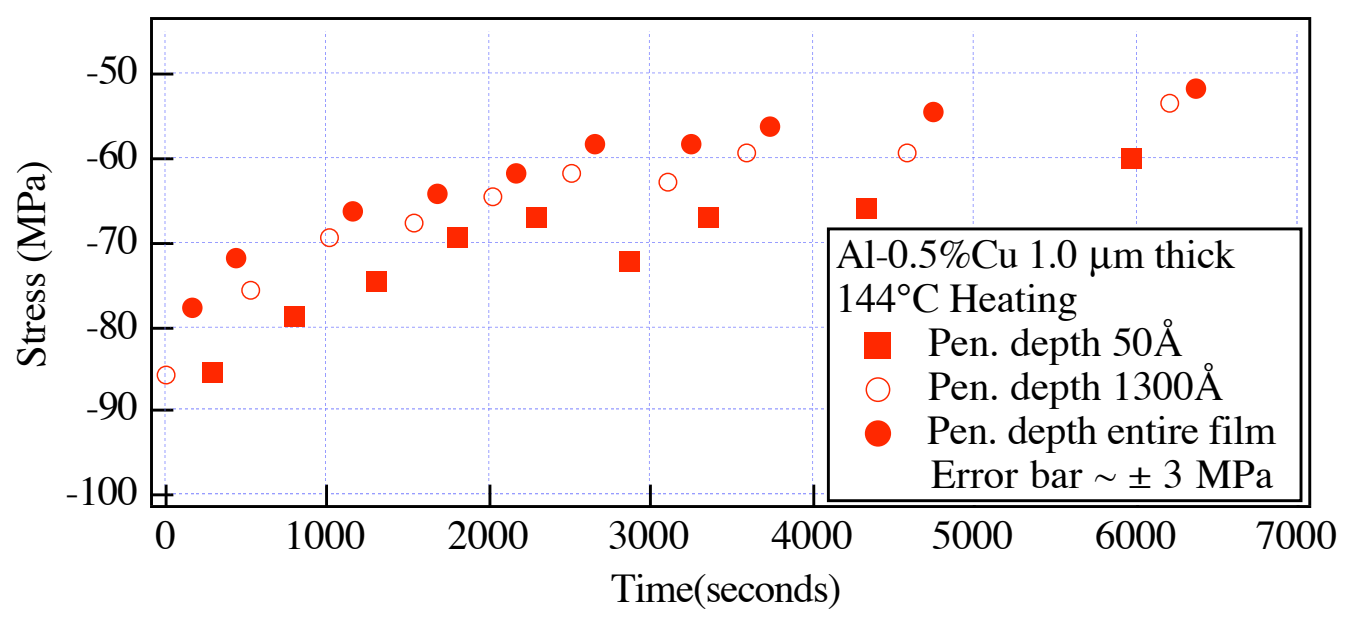

Here we note that the stresses near the surface are larger than those in the bulk of the film, perhaps indicating a near-surface resistance to deformation during heating. Perhaps the oxide present on the surface of the film is responsible for the higher stresses near the surface. 
Some results from the work of $\mathrm{R}$. Venkatraman $\left(\mathrm{Al}-0.5 \% \mathrm{Cu}, 205^{\circ} \mathrm{C}\right.$, heating)

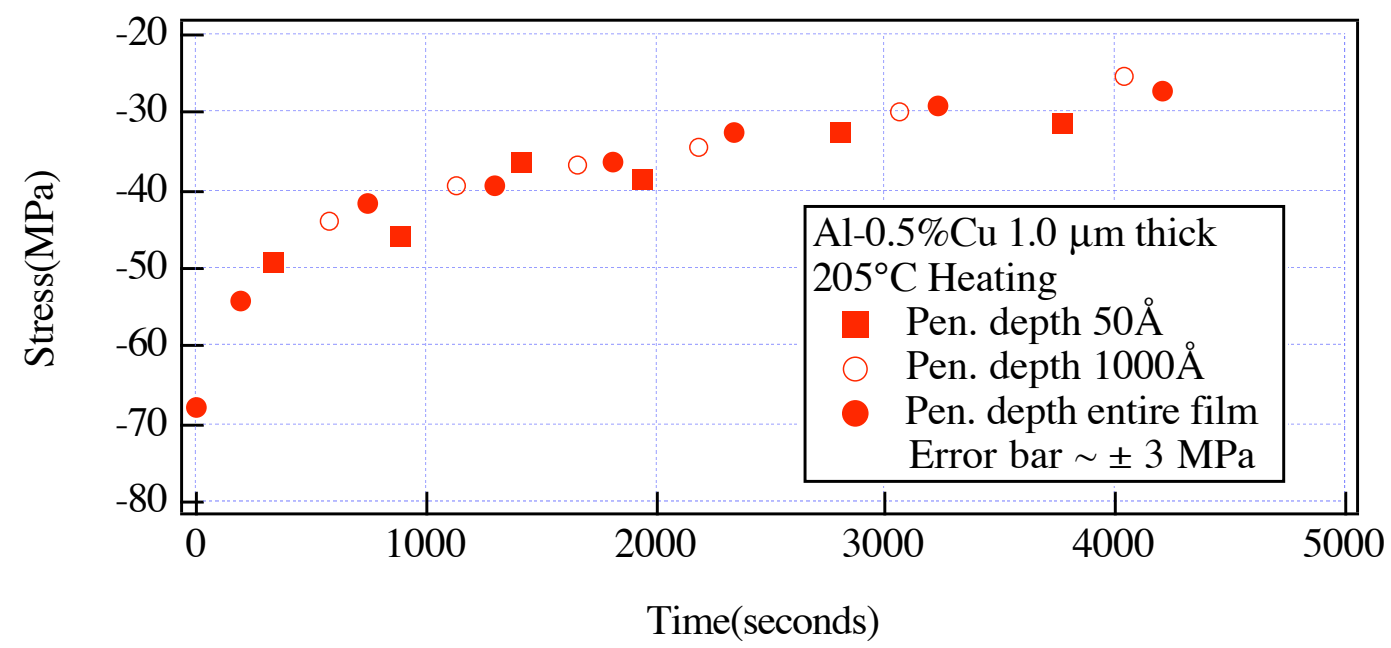

By the time the temperature has reached $200^{\circ} \mathrm{C}$ the differences between the surface and the bulk of the film have disappeared. This is not unexpected as the bulk of the grains can be relaxed by dislocation as well as diffusional processes at higher temperatures.

Some results from the work of $\mathrm{R}$. Venkatraman $\left(\mathrm{Al}-0.5 \% \mathrm{Cu}, 202^{\circ} \mathrm{C}\right.$, cooling)

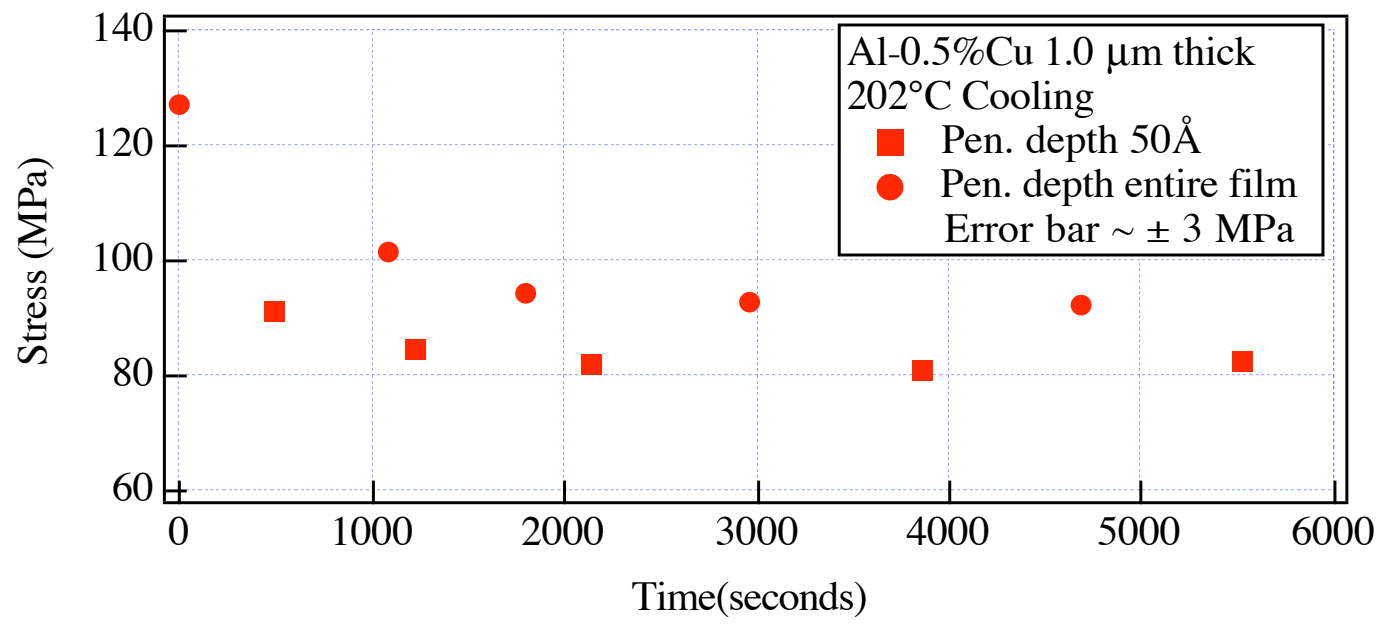

On cooling we again see some differences in stress at different depths, perhaps indicating the role of near surface relaxations on cooling. The "final" strain distribution in the film at $96^{\circ} \mathrm{C}$ is shown in the following figure. Although the stresses do decrease near the surface, the gradients are found only very near the 
surface. Over most of the thickness of the film the stresses are essentially uniform.

\section{Final Strain distribution at $96^{\circ} \mathrm{C}$ at "long" times}

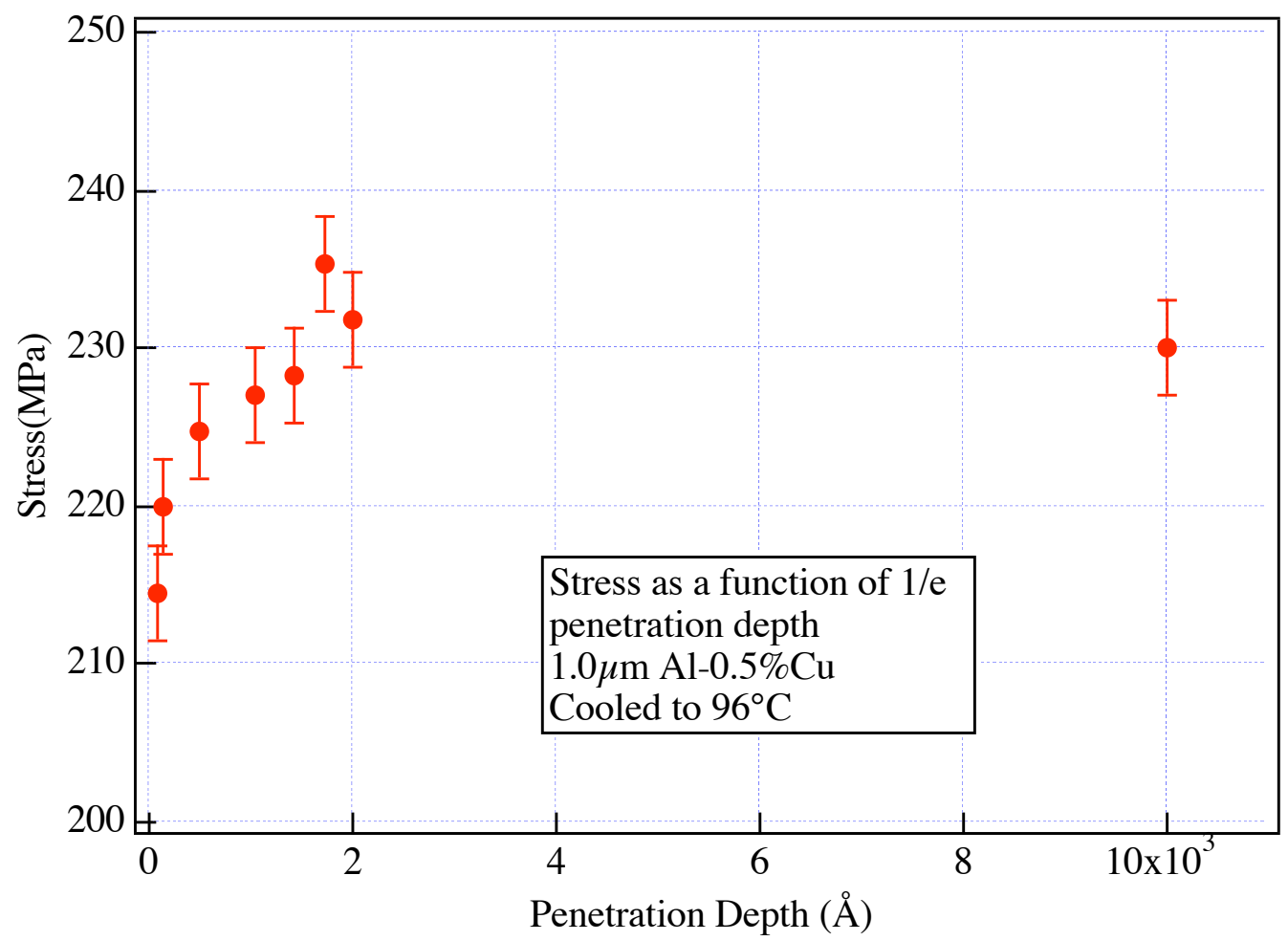

The surface relaxation with respect to the bulk is similar to results obtained by Doerner and Brennan (J.App.Phys. 63, 126 (1988) ). 
Strain distribution in aluminum films determined by the GIXS technique (M.F. Doerner and S. Brennan, 1988)

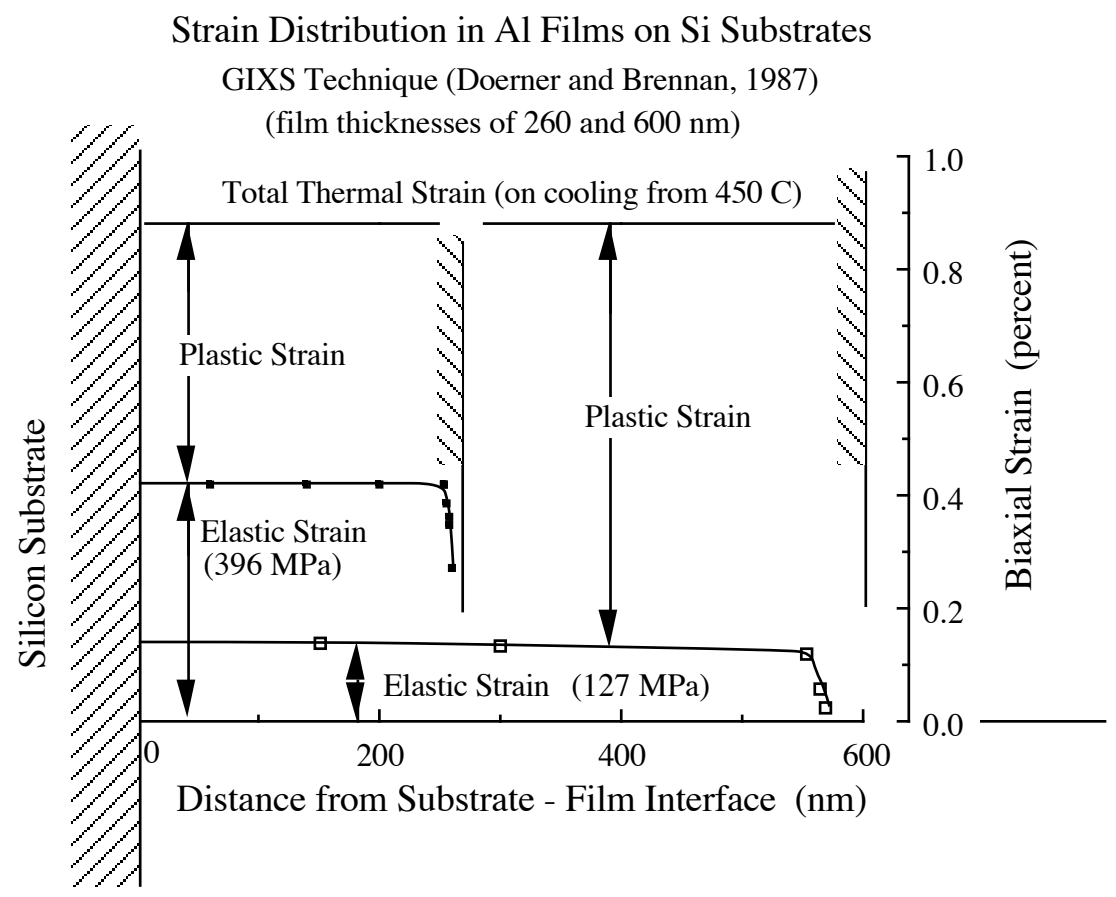

\section{Non-Symmetric, Depth Dependent X-ray Diffraction}

The problem of strain gradients in thin films has been addressed more recently by Leung et al. (O.S. Leung, A. Munkholm, S. Brennan and W.D. Nix, "A Search for Plastically-Induced Strain Gradients in Gold Thin Films on Silicon Substrates using Depth-sensitive X-Ray Diffraction," Journal of Appl. Phys., 88, 1389-1396 (2000).), who used a non-symmetric x-ray diffraction technique to determine whether significant gradients of strain are responsible for the high strengths of thin films. The beauty of the non-symmetric diffraction technique developed by Brennan and Munkholm is that a particular set of diffracting planes in a particular set of grains can be studied as a function of the depth of penetration of the x-rays. The figure below shows the basic diffracting geometry and the following figures show that the measured elastic strains are essentially independent of depth of penetration. The conclusion is that the elastic strains are nearly uniform through the thickness of the film. 
Non-symmetric x-ray diffraction technique for studying depth dependence of strain in thin films

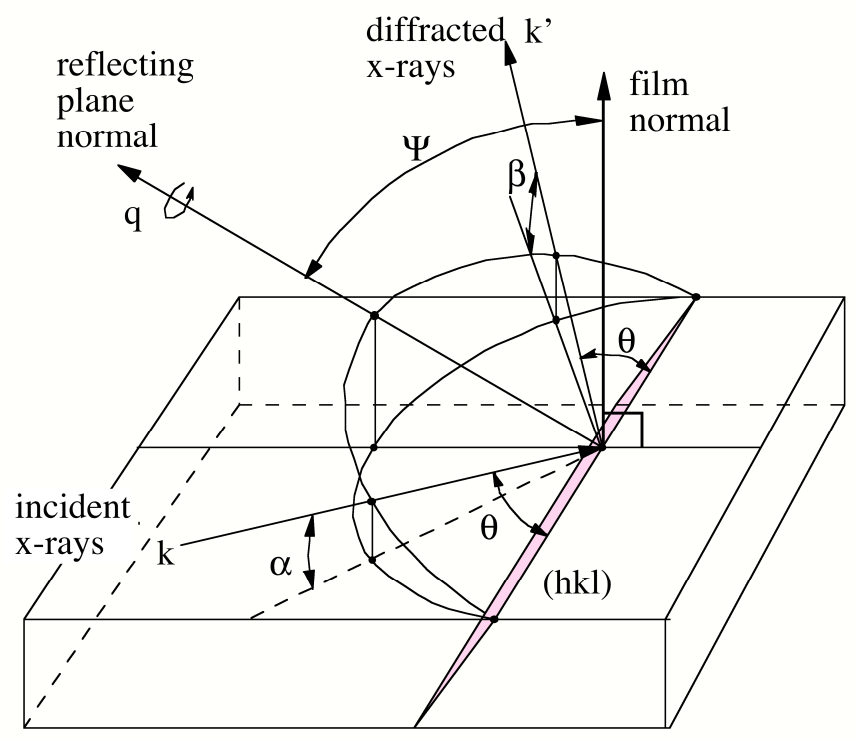

$d_{\psi} v s \cdot \sin ^{2} \psi$ as a function of $\mathrm{x}$-ray penetration depth (using multiple reflections) for a $0.8 \mu \mathrm{m}$ thick gold film

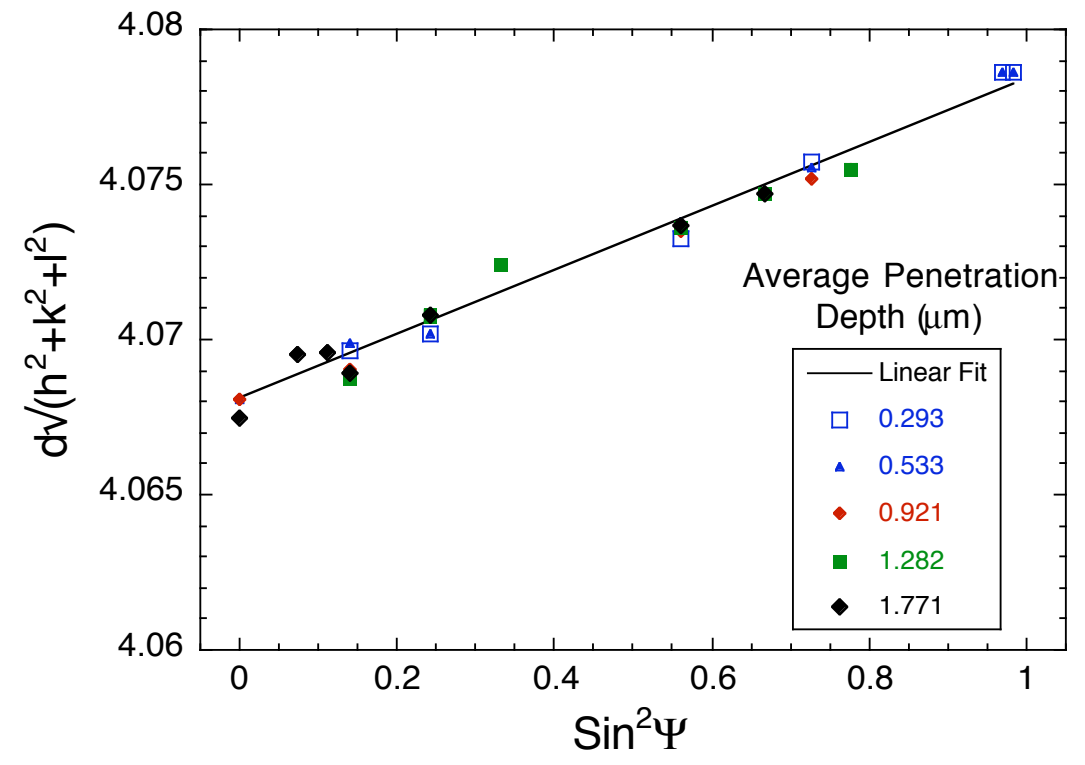


Stress as a function of $x$-ray penetration depth for gold films of different thichness using the non-symmetric, depth dependent $x$-ray diffraction technique.

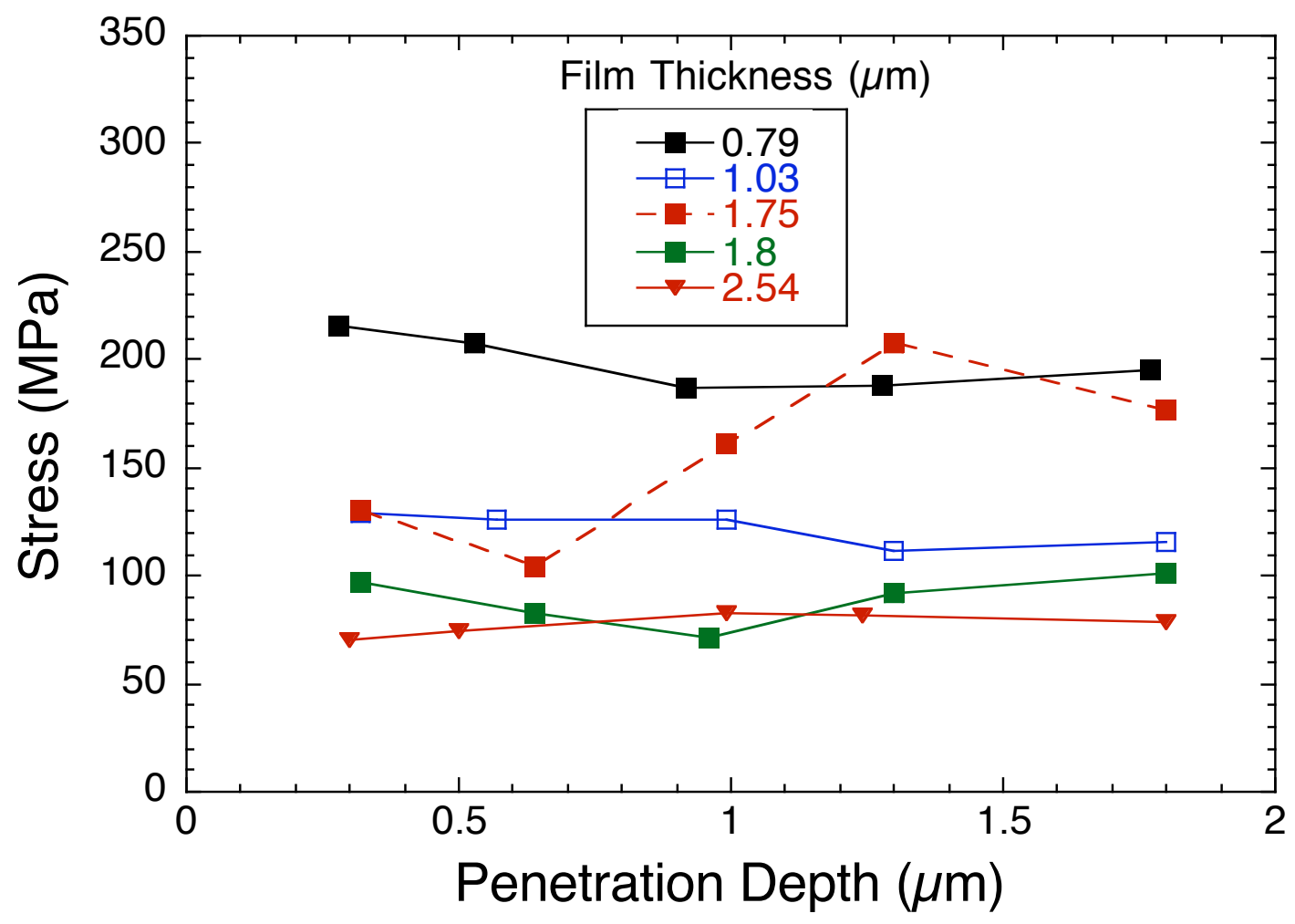




\section{Beam Deflection Techniques}

The x-ray techniques described above give direct information about stresses and strains in films and they permit the determination of stresses and strains in localized regions or patterned structures. One can determine local stresses and strains. But, the technique is relatively slow and expensive instrumentation is required. Also, it is difficult to use $\mathrm{x}$-ray techniques to determine stresses in growing films. For this purpose, beam deflection techniques can be used.

Consider a substrate in the form of cantilever beam with dimensions $L \gg W \gg t_{s}$.

\section{Substrate with film fixed to support}

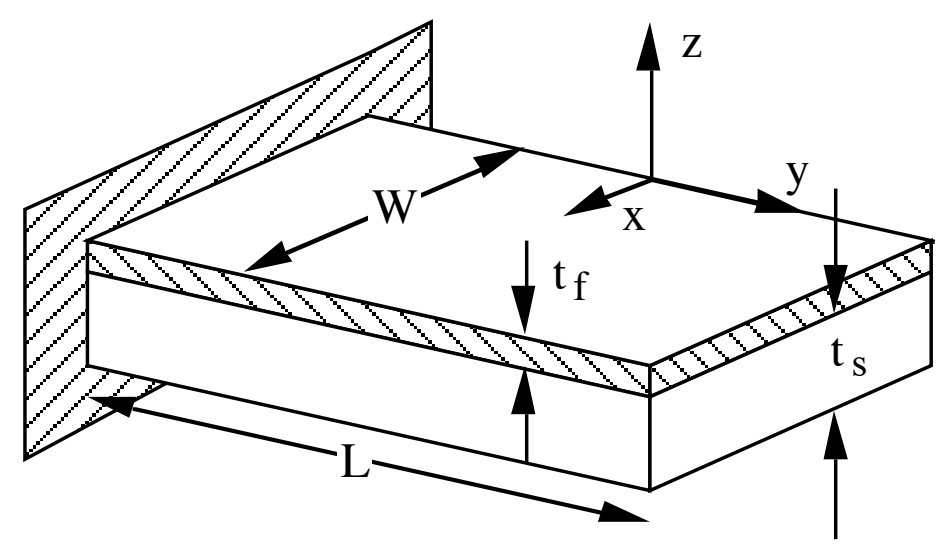

Under these conditions, the beam bends as a wafer does and we may use the relations discussed earlier to compute the defection at the end of the beam.

$$
u_{z}(y=L)=-\left(\frac{1-v}{E}\right)_{s} \frac{6 M L^{2}}{t_{s}^{3}}
$$

where the moment (per unit length) along the edge is

$$
M=-\sigma_{f} t_{f} \frac{t_{s}}{2}
$$

SO 


$$
u_{z}(y=L)=\left(\frac{1-v}{E}\right)_{s} \frac{3 \sigma_{f} t_{f} L^{2}}{t_{s}^{2}}
$$

This relation assumes that the substrate bends into shape of spherical shell -biaxial bending may not be valid if the substrate is short and wide--due to constraint of support! Ideally, the substrate should be clamped at a point rather than along an edge. Such point clamping would allow the substrate to bend to a spherical shape.

Substrate bending during thin film deposition

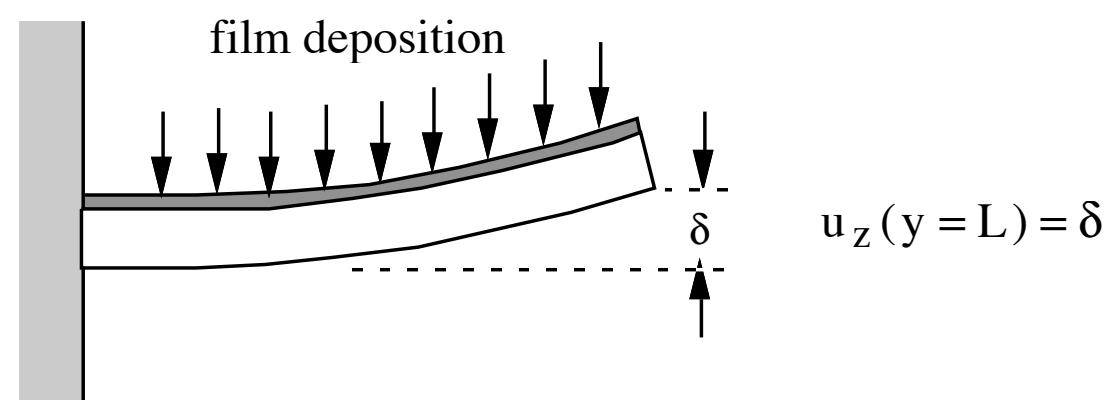

Solving for the film stress

$$
\sigma_{f}=\left(\frac{E}{1-v}\right)_{s} \frac{t_{s}^{2}}{3 L^{2} t_{f}} \delta
$$

Such simply supported beams can be mounted in deposition chambers. Measuring $\delta$ during film deposition gives $\sigma_{f} t_{f}$ from which $\sigma_{f}$ can be found if $t_{f}$ is known.

\section{Wafer Curvature Methods}

The most popular technique for measuring thin film stress is based on measurements of the curvature of the substrate on which the film is deposited. The method is relatively fast (faster than x-rays, typically) and quite accurate (higher resolution than simple beam deflection).

As shown earlier the stress in the film is given by 


$$
\sigma_{f}=\left(\frac{E}{1-v}\right)_{s} \frac{t_{s}^{2}}{6 t_{f} R}=\left(\frac{E}{1-v}\right)_{s} \frac{t_{s}^{2}}{6 t_{f}} \Delta \kappa
$$

where $\Delta \kappa$ is the curvature change induced by the stress in the film. In most systems, the curvature (or radius of curvature) is measured by scanning a laser beam across the wafer (or substrate) and deflecting the position of the reflected beam. The geometry shown below is easy to analyze.

\section{Measurement of Substrate Curvature by the Optical Level Technique}

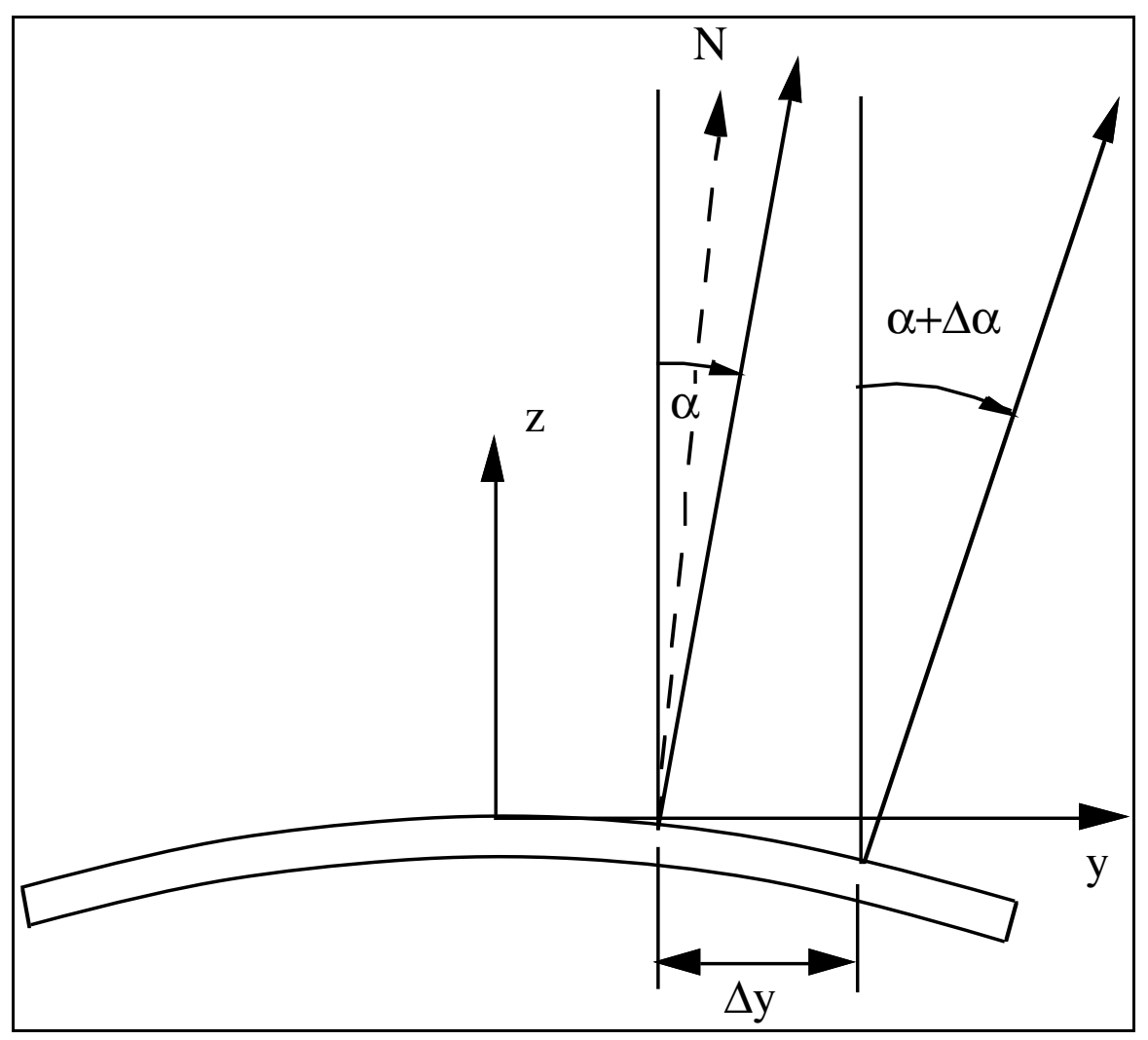

The curvature of the substrate can be found by reflecting a laser beam from the surface of the substrate and measuring the angle of reflection as a function of position on the substrate. At any point the slope of the surface, $s$, is, for the usual case of nearly flat substrates,

$$
\text { slope }=s=\left[\frac{d u_{z}}{d y}\right]=-\frac{\alpha}{2}
$$

Here the angle $\alpha$ is angle between the incident laser beam and the reflected beam. From the geometry it is clear that slope is given by the inclination of the 
substrate normal, or $\alpha / 2$. We note that the slope is negative for positive values of $\alpha$. The curvature is, by definition, the derivative of the slope with respect to position:

$$
\frac{1}{R}=\kappa=\frac{d^{2} u_{z}}{d y^{2}}=\frac{d s}{d y}=-\frac{1}{2} \frac{d \alpha}{d y}
$$

Thus, the curvature can be found by measuring the angle of reflection as a function of position.

\section{Measurement of Substrate Curvature by the Optical Level Technique}

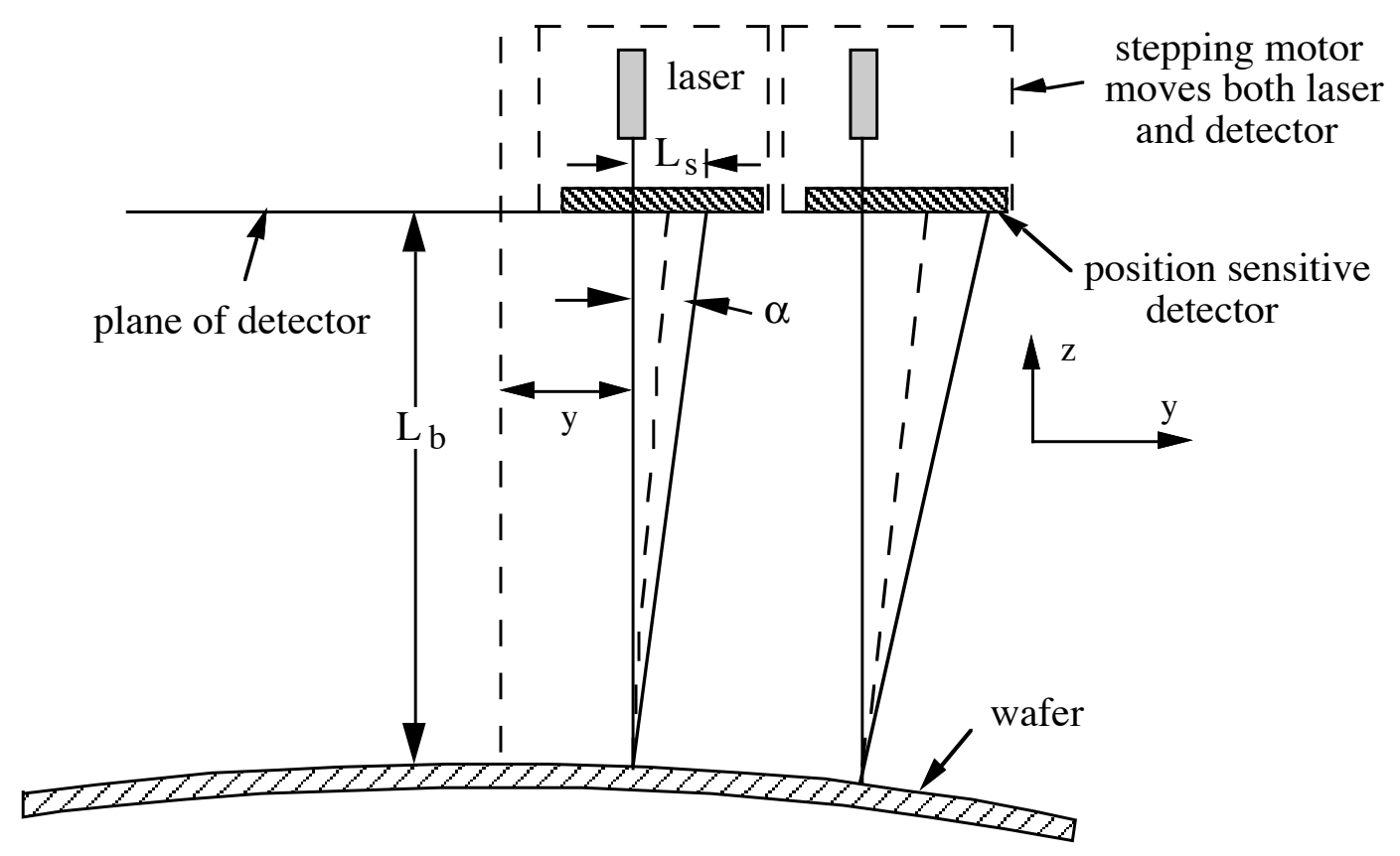

Because the angle of incidence is equal to the angle of reflection,

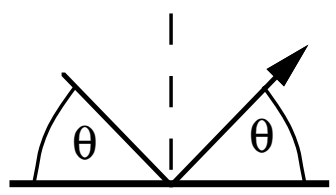

and since

$$
\frac{d u_{z}}{d y} \approx 0
$$


it follows that the angle $\alpha$ at any point is, to a very good approximation

$$
\alpha \approx \frac{L_{s}}{L_{b}}
$$

so the curvature is

$$
\begin{aligned}
& \kappa=\frac{d^{2} u_{z}}{d y^{2}}=\frac{d s}{d y}=-\frac{1}{2} \frac{d \alpha}{d y} \\
& \kappa=-\frac{1}{2} \frac{d}{d y}\left(\frac{L_{s}}{L_{b}}\right) .
\end{aligned}
$$

So the curvature is found by plotting $\frac{L_{s}}{L_{b}}$ vs. $y$, and then taking the slope.

Plot of optical lever as a function of diametral distance for a spherical mirror. (Radius of curvature, $R=38.2 \mathrm{~m}$.)

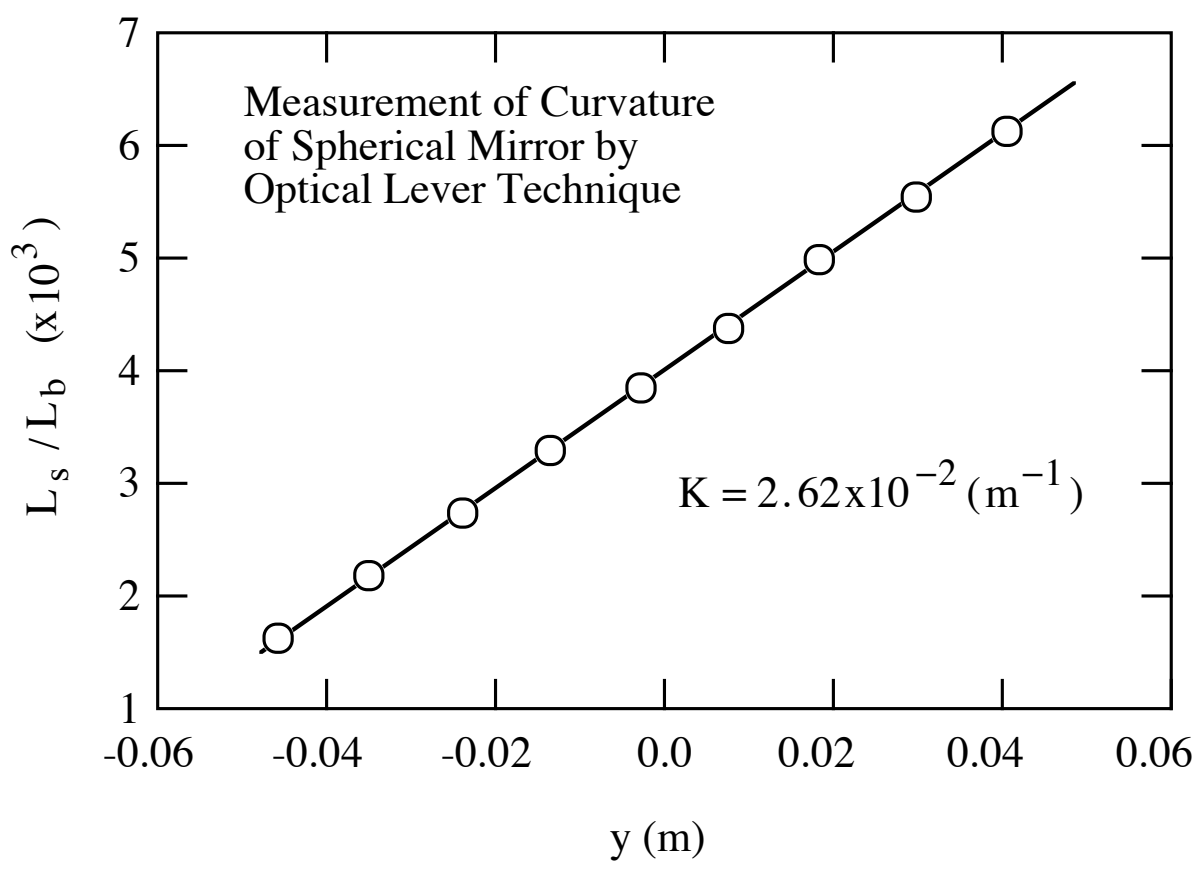


Laser Scanning Technique for Measuring Substrate Curvature (schematic)

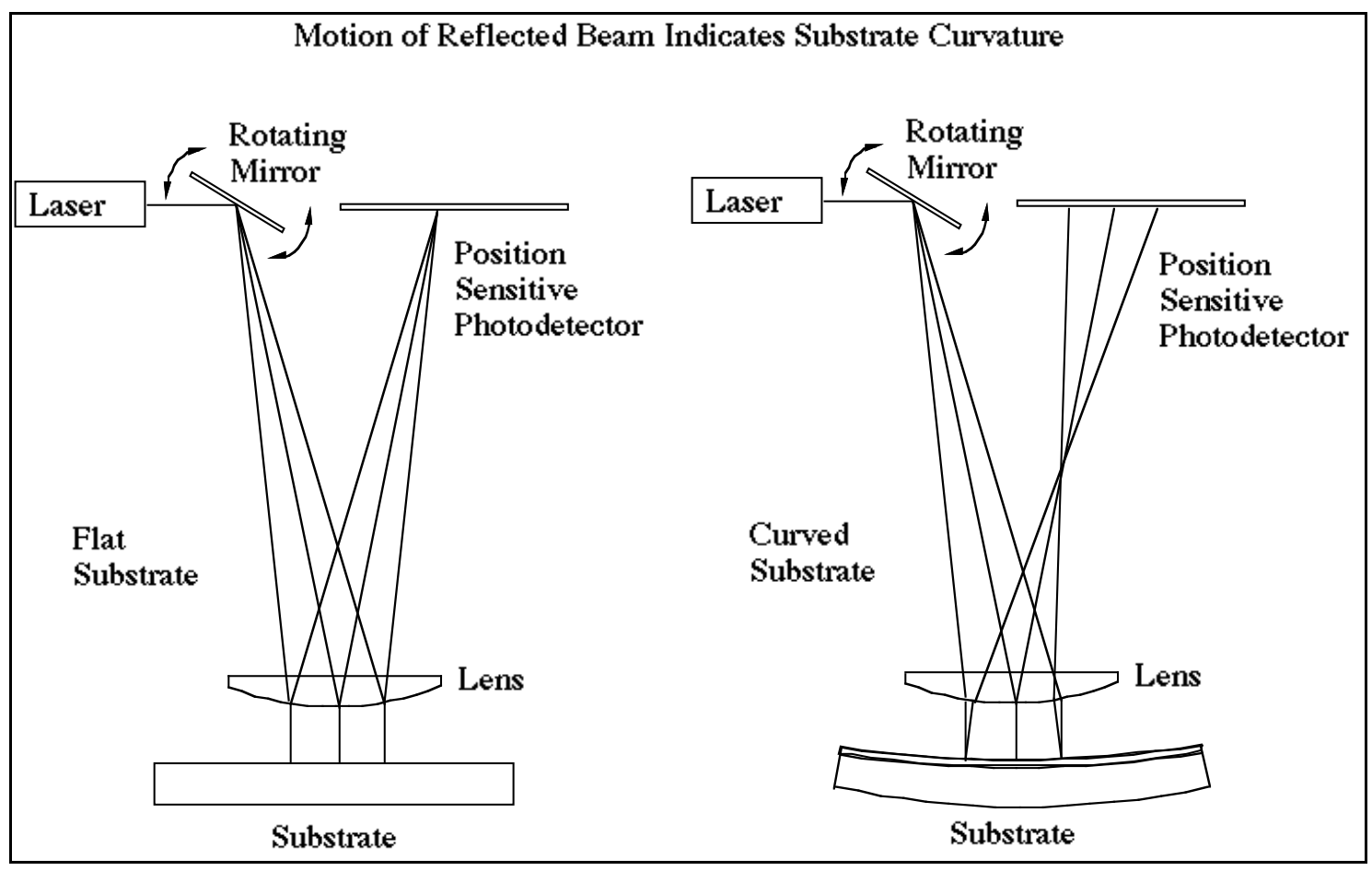

\section{Other Substrate Curvature Techniques}

The figure above shows how the laser beam can be scanned quickly using a mirror mounted on a rotational stepper motor. Such instruments are much faster than those requiring both the laser source and the detector to be moved relative to the substrate. Moving the laser and detector, as shown in the diagram earlier, also has the disadvantage that "bumps in the roadway" for the trolley will lead to noise in the data.

More recently laser beam splitting techniques have replaced laser scanning methods for measuring substrate curvature, especially if rapid measurements are required, as in the case of stress measurements during film deposition. As shown in the figure below, an incident laser beam is split into multiple beams by an etalon and the multiple parallel beams then strike the sample and reflect to a CCD camera. The spacing between the spots at the CCD camera indicates the curvature of the surface. Because the positions of all of the spots are recorded simultaneously, very rapid curvature changes can be detected and measured. The etalon-based beam splitting system was developed J.A. Floro, E. Chason and S.R. Lee at Sandia National Laboratories in Albuquerque (MSR Symposium, Vol. 381 (1995)) and is now commercially available. 
Etalon-Based Substrate Curvature Measuring System (diagram courtesy Vidya Ramaswami, Stanford)

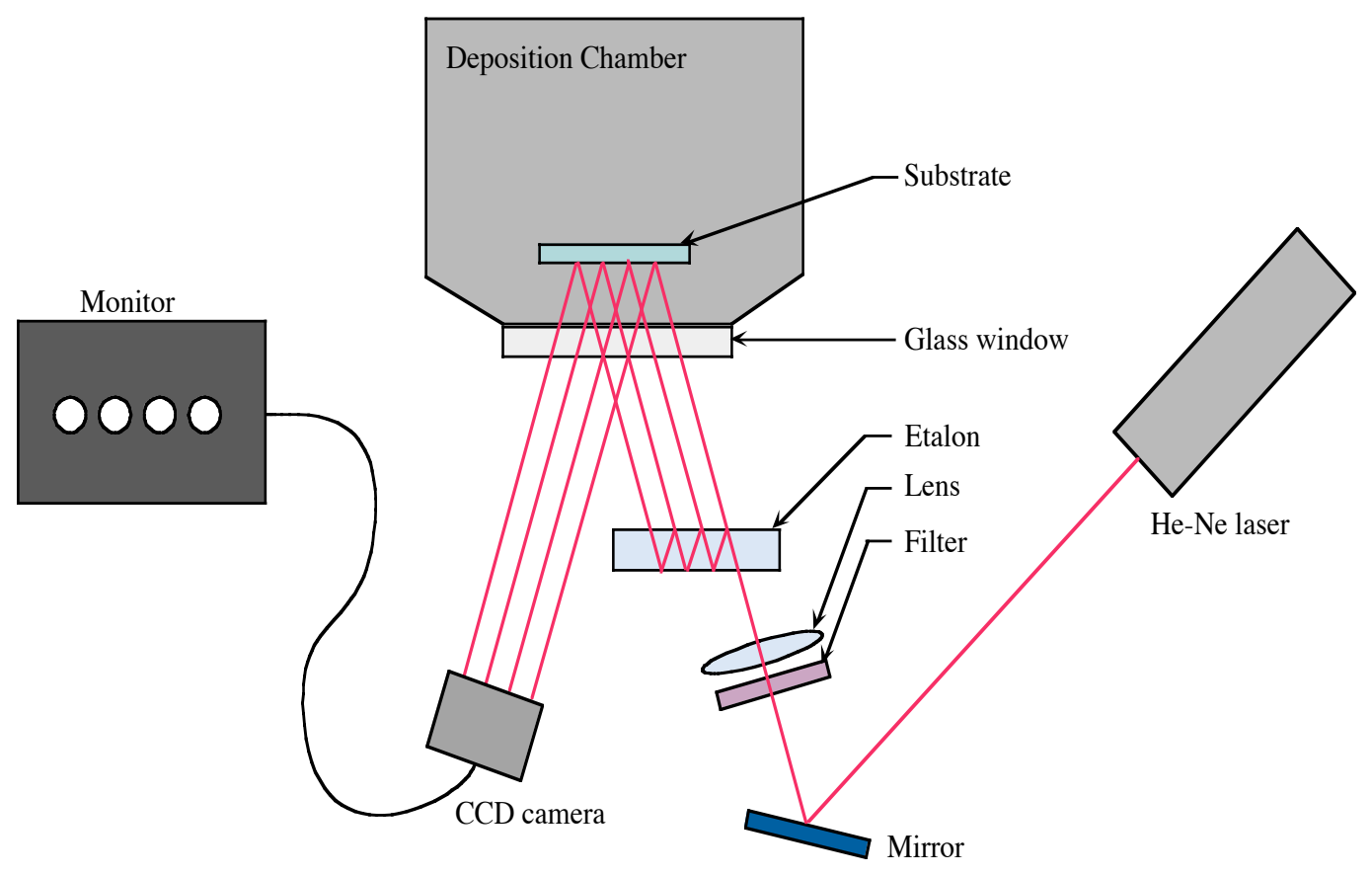

\section{Resolution of Wafer Curvature Technique}

As shown in the figures above, the curvature can be found by measuring the deflection of a reflected laser beam as the incident beam is scanned over the substrate:

$$
|\kappa|=\frac{d}{d y}\left(\frac{L_{s}}{2 L_{b}}\right)=\frac{\Delta\left(\frac{L_{s}}{2 L_{b}}\right)}{\Delta y} .
$$

From this the minimum curvature that can be detected is

$$
\kappa_{\min }=\frac{\frac{1}{2 L_{b}}\left(\Delta L_{s}\right)_{\min }}{(\Delta y)_{\max }}
$$

where $\left(\Delta L_{s}\right)_{\min }$ is the smallest laser motion that can be detected on the position sensitive detector and $(\Delta y)_{\max }$ is the largest positional displacement. For position sensitive detectors we have the following resolution: 


$$
\left(\Delta L_{s}\right)_{\min } \approx 5 \mu \mathrm{m}=5 \times 10^{-6} \mathrm{~m} .
$$

For typical laser scanning geometries we have

$$
L_{b}=1 \mathrm{~m} \quad \text { and } \quad(\Delta y)_{\max } \approx 10 \mathrm{~cm}=0.1 \mathrm{~m} .
$$

This leads to

$$
\begin{aligned}
& \kappa_{\min }=2.5 \times 10^{-5}\left(\mathrm{~m}^{-1}\right) \\
& R_{\text {max }}=\frac{1}{\kappa_{\min }}=4 \times 10^{4}=40 \mathrm{~km} !
\end{aligned}
$$

What stress resolution does this give for a typical $1 \mu \mathrm{m} \mathrm{Al} \mathrm{film} \mathrm{on} 0.5 \mathrm{~mm} \mathrm{Si}$ substrate? This can be calculated as follows:

$$
\left(\sigma_{f}\right)_{\min }=\left(\frac{E}{1-v}\right)_{s} \frac{t_{s}^{2}}{6 t_{f} R_{\max }}
$$

for the following typical parameters:

$$
t_{s}=500 \times 10^{-6} \mathrm{~m} \quad t_{f}=1 \times 10^{-6} \mathrm{~m}\left(\frac{E}{1-v}\right)_{s}=200 \mathrm{GPa}
$$

we have

$$
\left(\sigma_{f}\right)_{\min } \approx 0.2 M P a .
$$

The corresponding strain resolution is

$$
\left(\varepsilon_{f}\right)_{\min } \approx 1 \times 10^{-6} \text {. }
$$

It might be noted in closing that the etalon-beam splitting system described above typically does not have as much resolution as the scanning methods discussed earlier because of the limited size of most etalons and CCD cameras.

Thus $(\Delta y)_{\text {max }}$ in the above estimates is usually much less than $10 \mathrm{~cm}$, and this results in less curvature resolution. When better stress resolution is needed, reducing the substrate thickness is usually the best course of action. 
Application of the substrate curvature technique for the study of deformation of thin films on substrates.

A typical plot of stress versus temperature for a 1 micron Al - $1 \%$ Si film on a silicon substrate. The open diamonds are heating data and the filled diamonds are cooling data. (J.F. Turlo, Ph.D Dissertation)

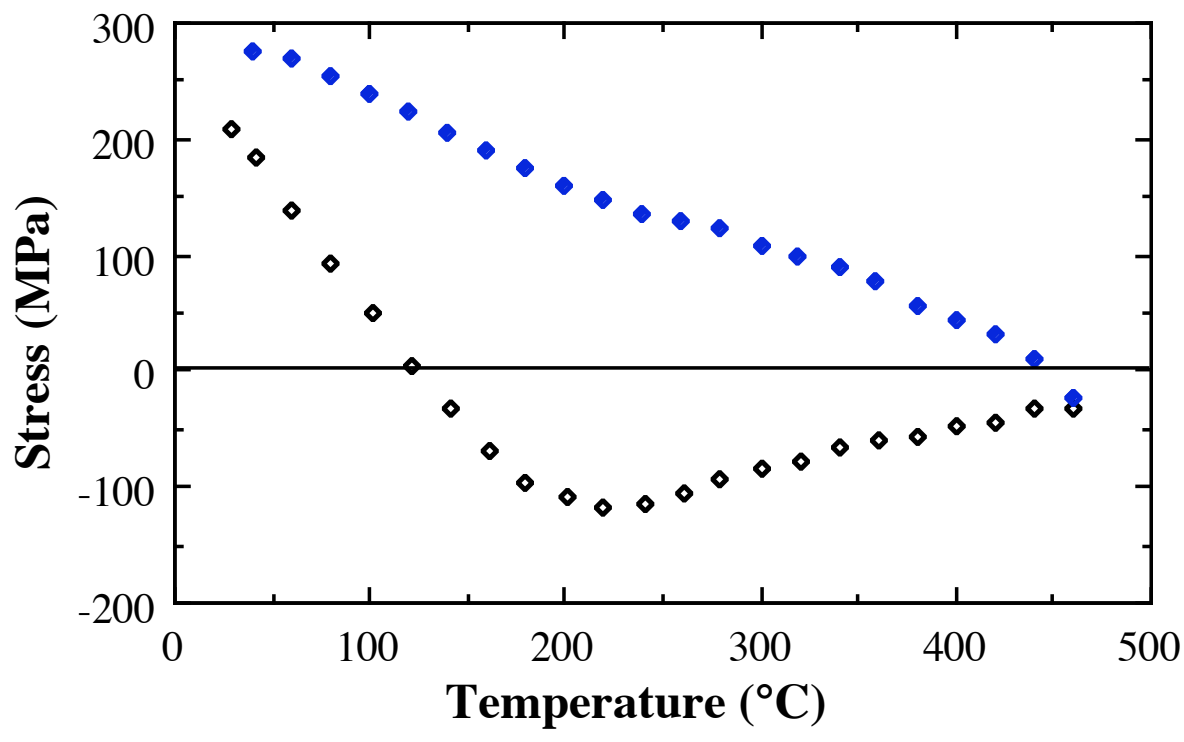


Stress relaxation data for a 1 micron aluminum film on a silicon substrate. (J.F. Turlo, Ph.D Dissertation)

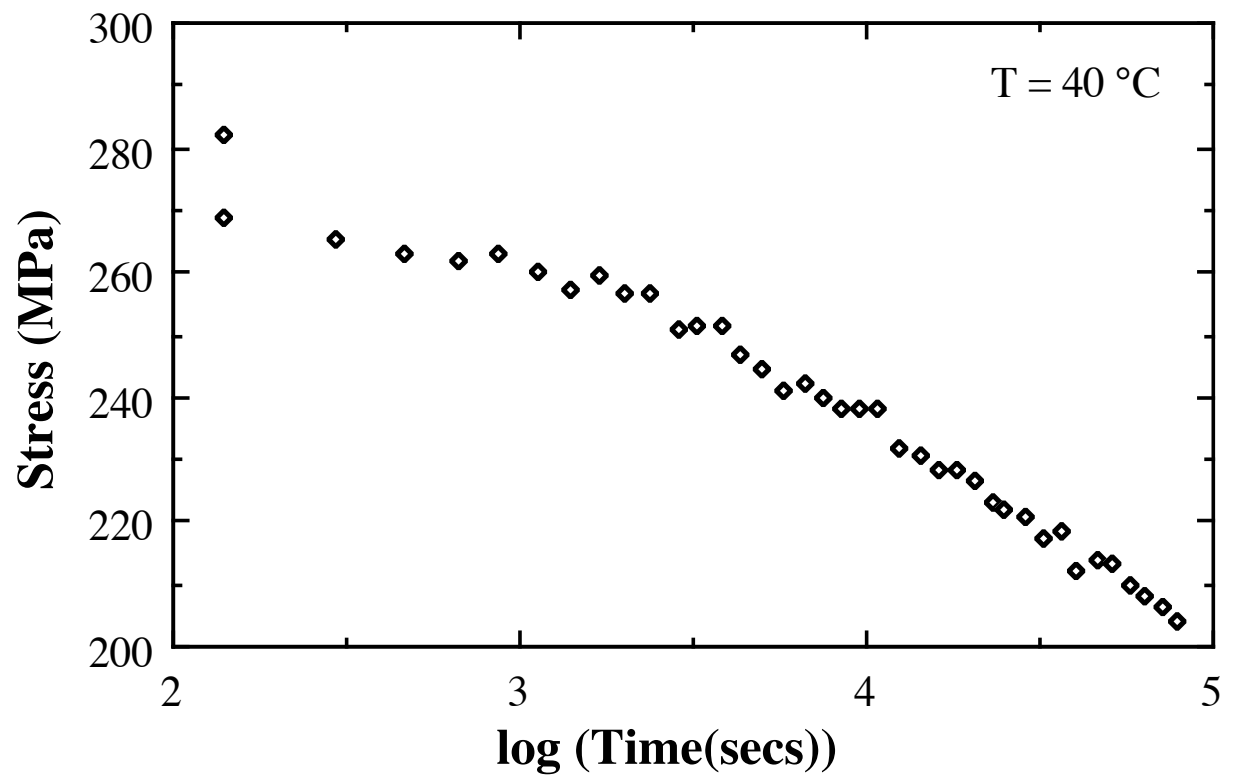

\section{Strain Relaxation and Buckling Effects}

Consider film deposition on a thin substrate clamped to a rigid support.

Films deposited on a thin substrate clamped to a rigid support

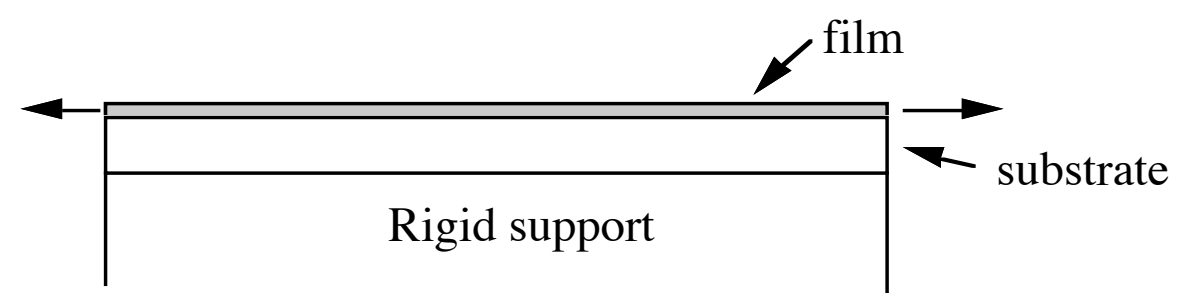

Because of misfit between the film and substrate, a stress may develop within the film (and in substrate and rigid support too). If the support is sufficiently massive, the structure will not bend significantly. However, if the thin substrate is removed from the support, significant bending can occur. 


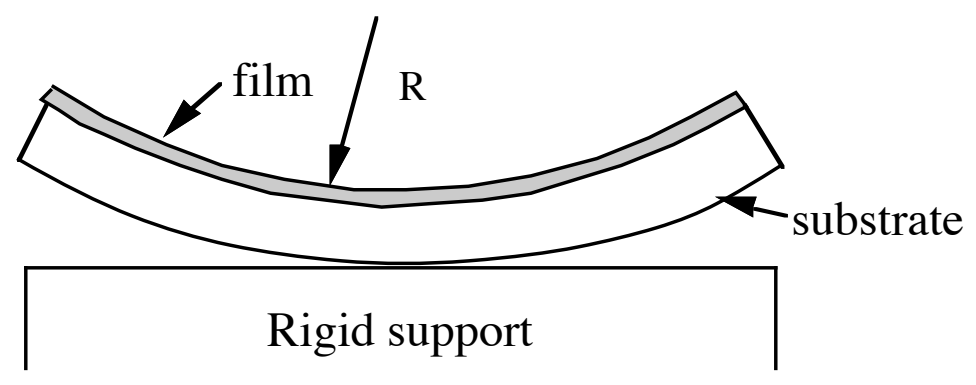

Measurement of $R$, and thicknesses and elastic properties of substrate gives $\sigma_{f}$.

If $t_{f} \approx t_{s}$, then the "thin film" approximation made earlier does not hold - a more complete analysis is required. In particular, the neutral axis of the bimaterial beam needs to be determined.

This technique has been used in the past to measure the stresses in thin oxides during oxidation of metals. For example - See : R.E. Pawel and J.J. Campbell, " Stress Measurements during Oxidation of Ta and Nb", Acta Metall. 14, 1827 (1966).

$\mathrm{Nb}$ specimens oxidized on one side at $425^{\circ} \mathrm{C}$ for various times. Specimens were protected on underside by vapor deposited Al layer

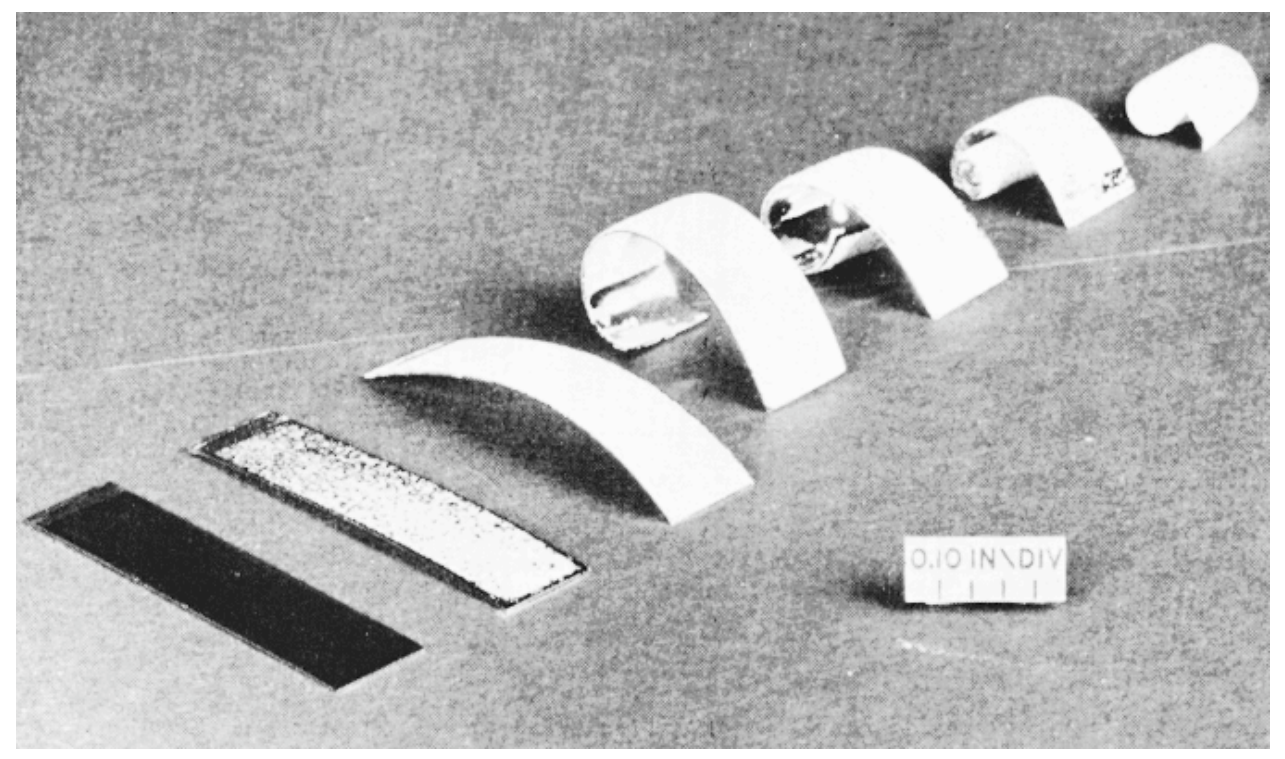

The technique has been used more recently in the study of stresses in metal multilayers. Shown below is an SEM photograph of $\mathrm{SiO}_{2}$ microbeams extending 
over an etch pit in the underlying Si Substrate. The curvature of the beams results from the compressive stresses in the $\mathrm{Au} / \mathrm{Ni}$ metal multilayer film that was sputter deposited on top of the beams after they had been fabricated. The stress can be calculated from the measured radius of curvature.

\section{Au-Ni/SiO2 Bilayer Beams (Shefford Baker, Ph.D. Dissertation)}

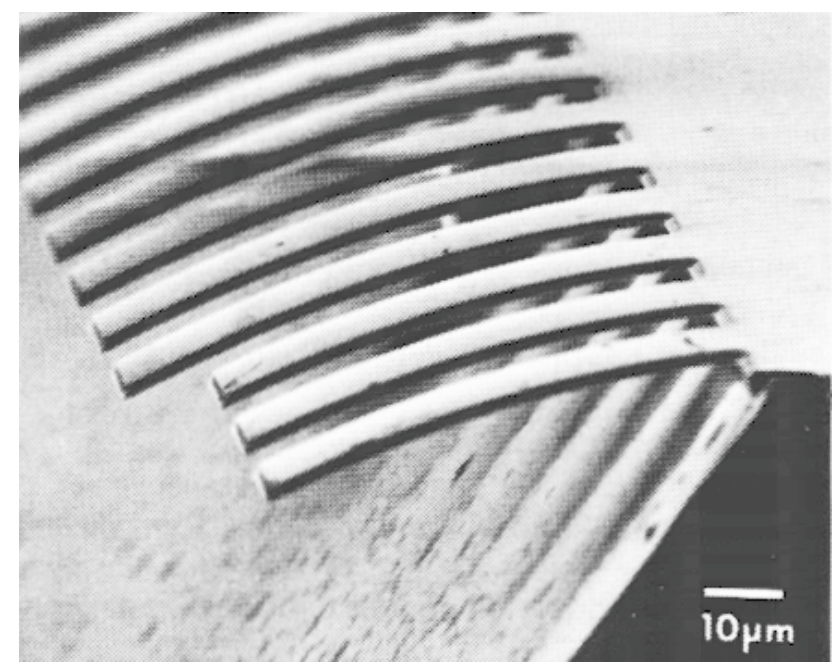

Comment on strain relaxation technique

In the oxidation experiments described above, the bending forces are very large and the thin substrate bends to a very small radius of curvature. Special shapes develop when the radius of curvature of the substrate is of the same order as the lateral dimension of the substrate. One consequence is that the substrate bends into a cylindrical shape, rather than a spherical shape, even though the stresses in the film may have been perfectly equal-biaxial before the thin substrate was released from the rigid support. This occurs whenever the stresses are extremely high or, equivalently, when the substrate is thin or very compliant. It is common to observe such cylindrical shapes in practical thin film delamination problems.

When a thin substrate bends into the cylindrical shape only one component of the strain in the film is relaxed by bending. 


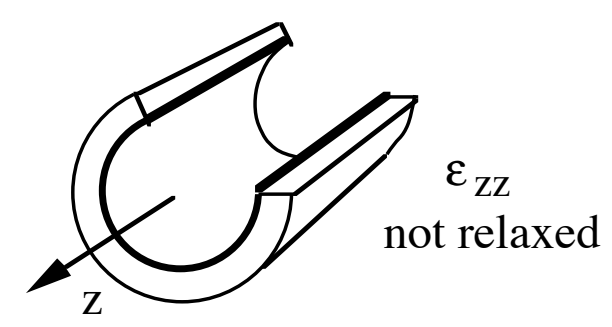

Why does this occur? The reasons are associated with the large strains or displacements associated with the bending. Equivalently, they occur when the radius of curvature of the substrate is of the same order as the lateral dimension of the substrate. Consider the relaxed shape when only a small bending force is present, corresponding to a very large radius of curvature.

\section{Substrate with slight bending}

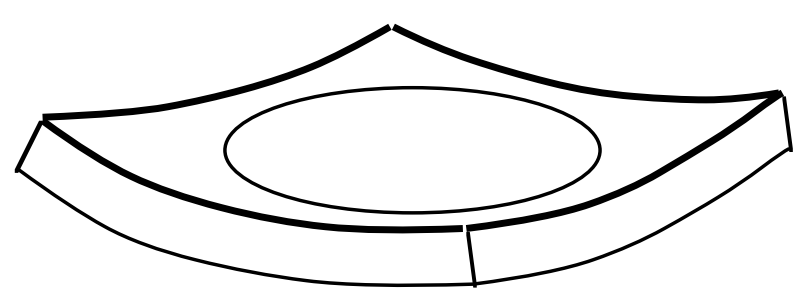

Now imagine that the stress in the film gets bigger and bigger, and the radius of curvature gets smaller and smaller. But if the bending displacements are large or if the lateral dimensions of the substrate are very large, the spherical bending of the substrate cannot be accommodated. The outer edges of the substrate are subjected to hoop compressive stresses, $\sigma_{\theta \theta}$. At a critical bending moment or for a critical sized substrate the substrate will snap into a nearly cylindrical shape. Fahnline at Penn State (C.B. Masters, N.J. Salamon and D.E. Fahnline, MRS Proceedings, Vol. 188, 21-26 (1990)) has studied this effect. According to his large strain analysis, the equilibrium curvature of the substrate varies with the size of the substrate in the following way. It also varies with film stress as shown below. 


\section{Large strain bending analysis}

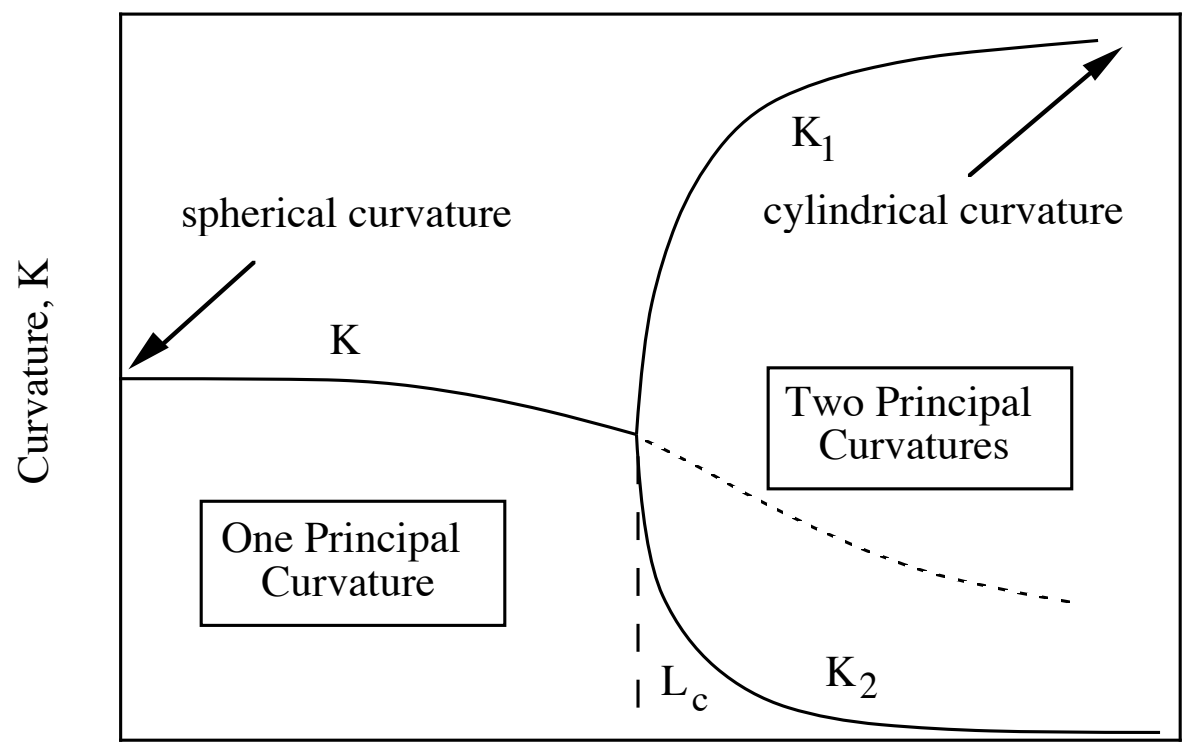

Lateral Dimension of Substrate, L

\section{Large strain bending analysis}

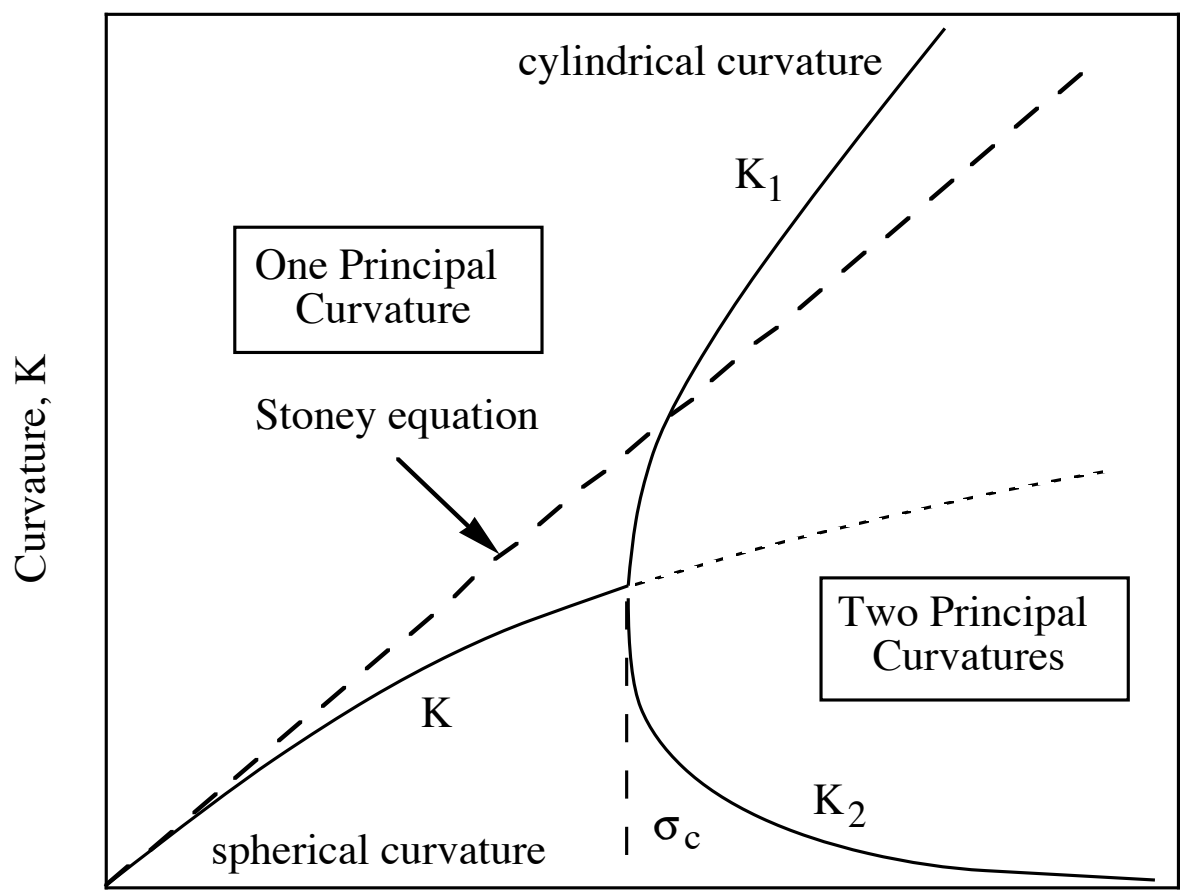

Initial Film Stress 


\section{Buckling Technique for Measuring Compressive Stresses in Films}

Consider deposited film with biaxial compressive stress $\sigma_{c}$. (Typical of $\mathrm{SiO}_{2}$, $\mathrm{Si}_{3} \mathrm{~N}_{4}$ and polycrystalline $\mathrm{Si}$ )

Film under biaxial compressive stress

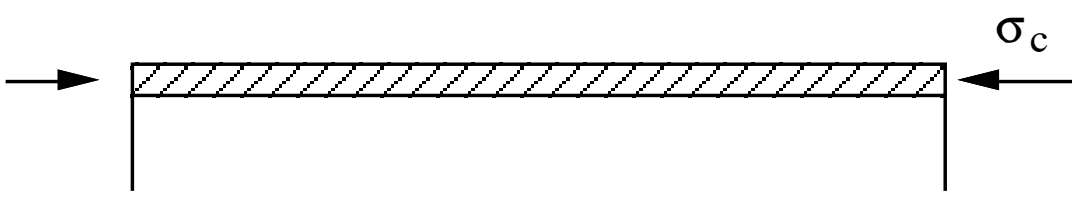

H. Guckel, T. Randazzo and D.W. Burns, J. Appl. Phys. 57 (5) 1671 (1985) - used I.C. fabrication techniques to make doubly supported beams to measure the critical length for buckle.

Buckling of doubly supported beams under compression
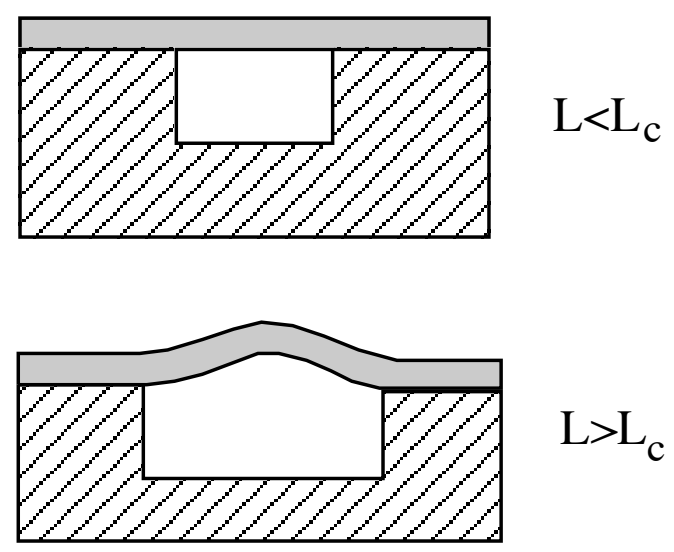

The phenomenon of buckling can be understood as follows. If the work done by axial load $p \Delta L$ is greater than the increase in strain energy associated with bending, then system is unstable with respect to buckling. 


\section{Mechanics of buckling}

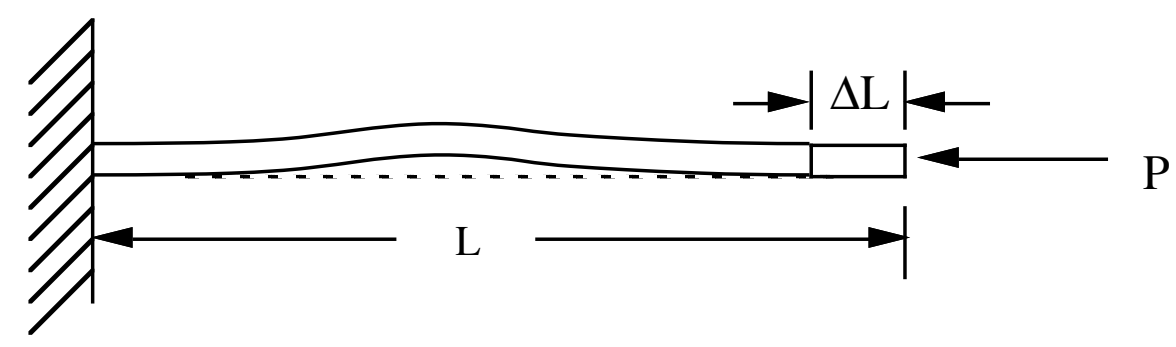

\section{Buckling of a thin substrate}

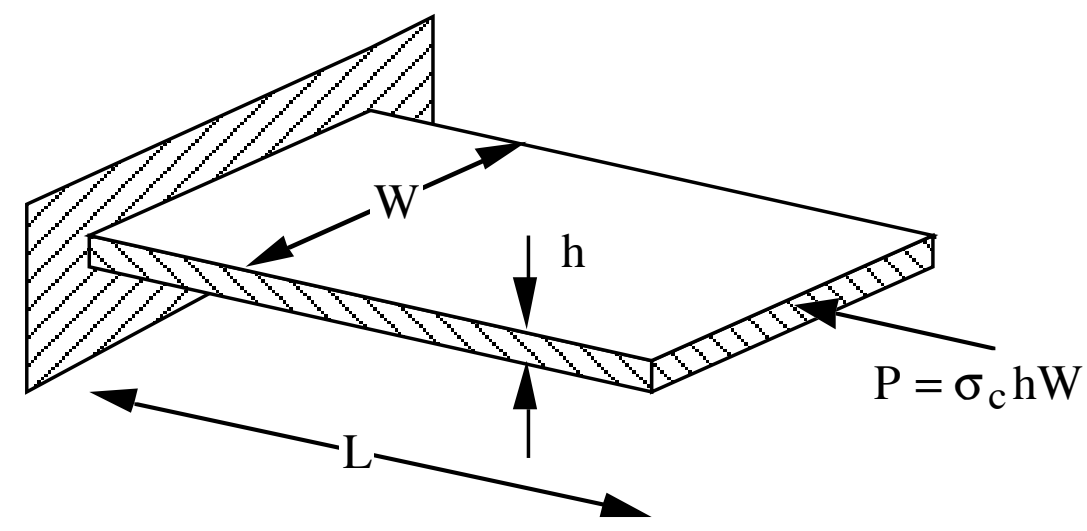

Considerations for buckling:

$$
\begin{array}{ll}
\text { Both ends free to rotate : } & \sigma_{c}=\frac{\pi}{12} \frac{h^{2}}{L_{c}^{2}} E_{f} \\
\text { Both ends constrained not to rotate : } & \sigma_{c}=\frac{\pi^{2}}{3} \frac{h^{2}}{L_{c}^{2}} E_{f}
\end{array}
$$

Guckel measured $L_{c}$ and calculate the stress $\sigma_{c}$ or the strain $\varepsilon_{c}$ (using $\varepsilon_{c}=\frac{\sigma_{c}}{E}$ for slender part of beam). The results below are for

$1.56 \mu \mathrm{m}$ polycrystalline Si on $700 \AA$ A of a masking oxide. 
Compressive strain relaxation in polysilicon

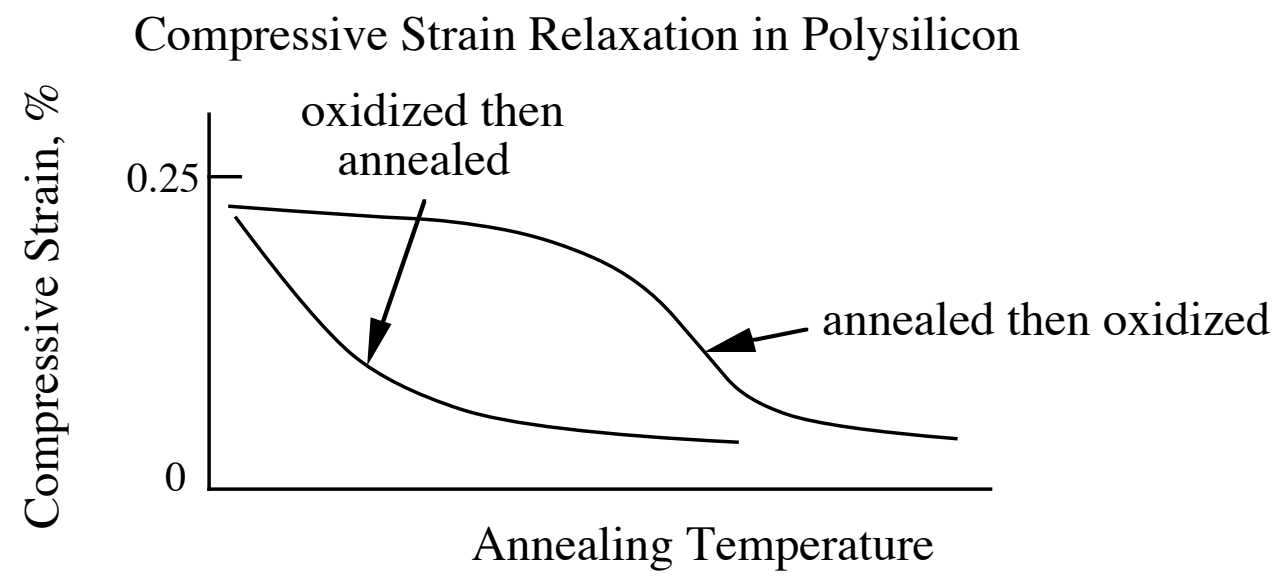

The possible effect on the $700 \AA \AA$ masking oxide appears not to have been considered by Guckel et al. 


\section{Physical Origins Of Stresses In Thin Films}

\section{Types of Films Growth}

We begin our study of the origins of stresses in thin films by considering the modes of film growth that have been observed. At the most basic level, the types of morphologies that develop in the early stages of film growth depend on the relative energies of the various interfaces involved. For a film on a substrate the relevant energies are:

$\gamma_{f}$, the energy of the surface of the film (per unit area)

$\gamma_{s}$, the energy of the surface of the substrate (per unit area)

$\gamma_{i}$, the energy of the interface between the film and the substrate (per unit area)

Consider a crystallite of the film in the shape of a hemispherical cap attached to the substrate. From basic arguments of interfacial tension, the three interfaces would be at equilibrium with respect to the cap angle $\theta$ when

$$
\gamma_{f} \cos \theta+\gamma_{i}=\gamma_{s}
$$

Crystallite on a substrate

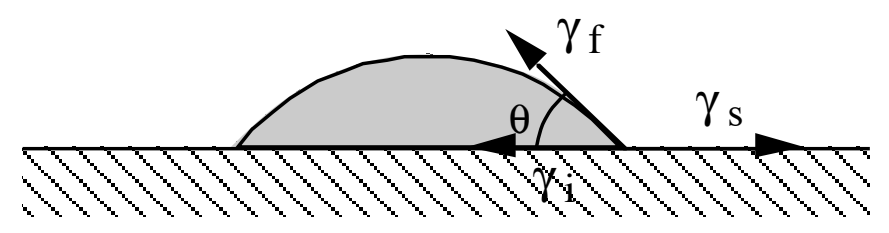

It is apparent that the crystallite will fully wet the substrate when the angle $\theta$ goes to zero. This leads to

$$
\cos \theta=1 \quad \text { and } \quad \gamma_{i}=\gamma_{s}-\gamma_{f}
$$

Similarly, a completely non-wetting condition (the film would ball up) occurs when $\theta=\pi$. This leads to 


$$
\cos \theta=-1 \text { and } \gamma_{i}=\gamma_{s}+\gamma_{f}
$$

From these considerations we may state that complete wetting is expected when

$$
\gamma_{i} \leq \gamma_{s}-\gamma_{f}
$$

and that a complete non-wetting condition will be found when

$$
\gamma_{i} \geq \gamma_{s}+\gamma_{f}
$$

Clearly, a low interface energy favors wetting and a high interface energy favors non-wetting. The observed modes of film growth can be rationalized in terms of these energies.

\section{Volmer-Weber Growth (VW) (Island Growth)}

On the basis of the discussion above, island growth is expected when neither complete wetting nor complete non-wetting occurs. This is found when $\gamma_{s}-\gamma_{f}<\gamma_{i}<\gamma_{s}+\gamma_{f}$. This corresponds to the classical nucleation and growth of isolated crystallites on substrates, commonly called Volmer-Weber type growth.

\section{Volmer-Weber Growth}

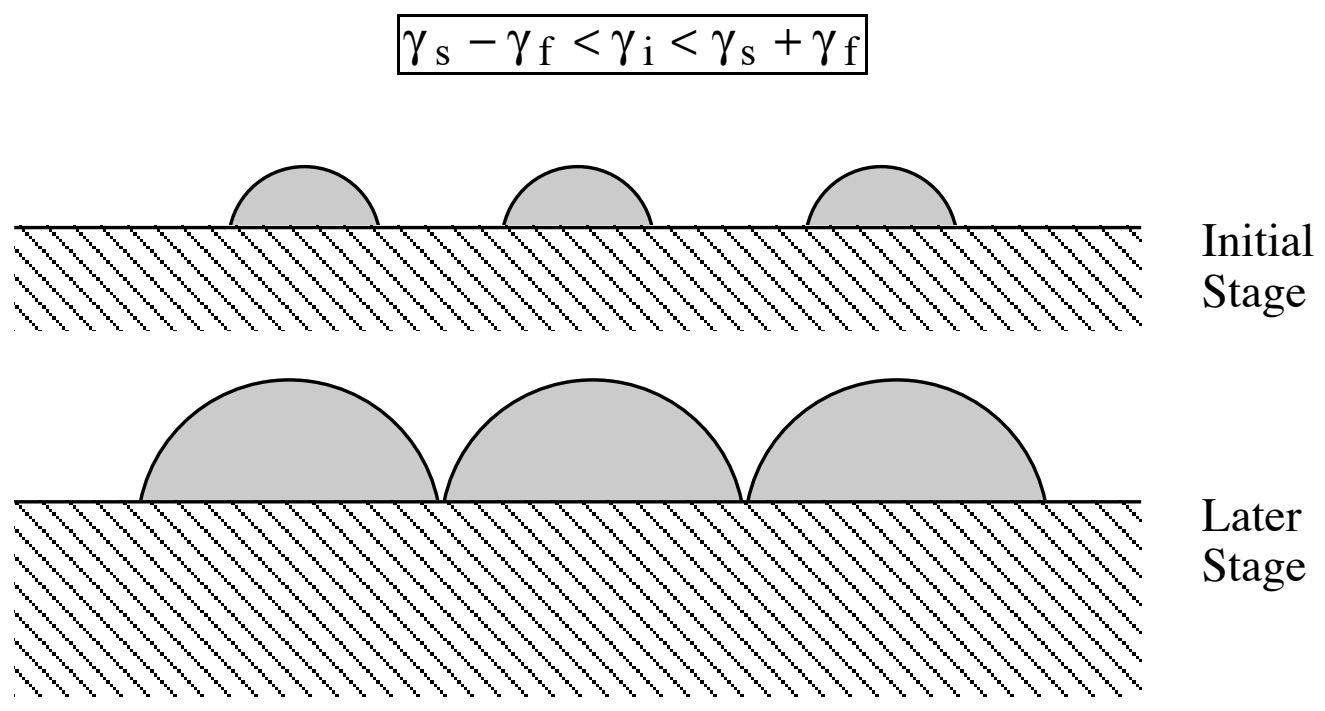




\section{Frank-VanderMerwe Growth (FV) (Layer Growth)}

When $\gamma_{i} \leq \gamma_{s}-\gamma_{f}$, complete wetting is expected. The film is expected to grow in an atomic layer by atomic layer manner. This is called Frank-VanderMerwe growth.

\section{Frank-VanderMerwe Growth}
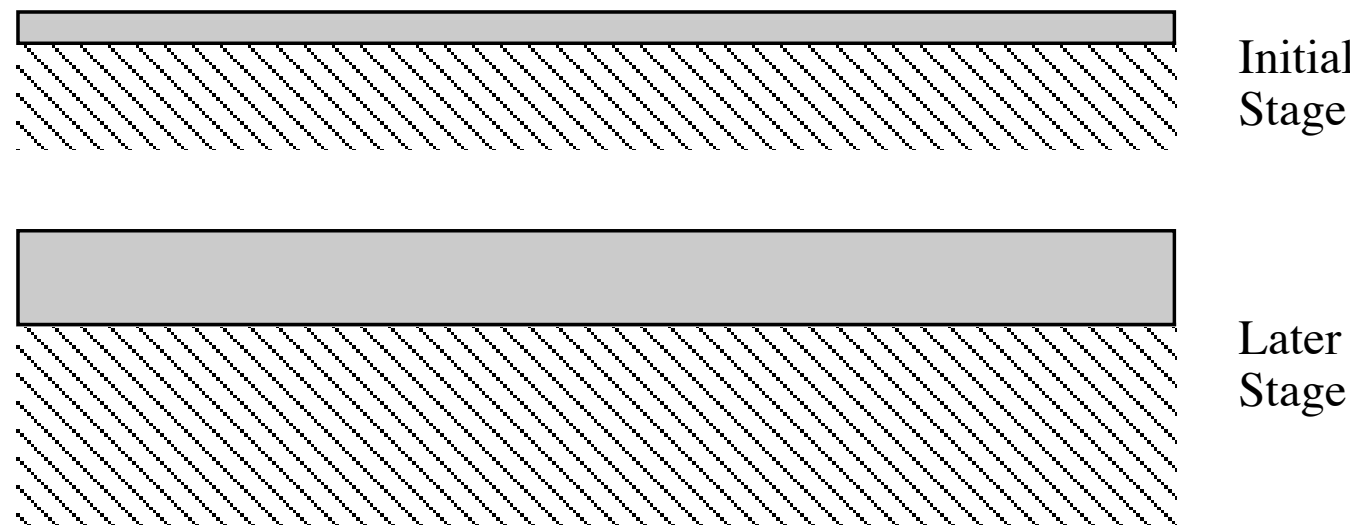

\section{$\underline{\text { Stranski-Kranstanov Growth (SK) (Layer-Island Growth) }}$}

Another mode of growth, in between the VW and FV forms of growth, is the Stranski-Kranstanov mode of growth. Here layer growth in the initial stages is followed by island growth in the later stages. The mechanism of this form of growth will be discussed later.

\section{Stranski-Kranstanov Growth}

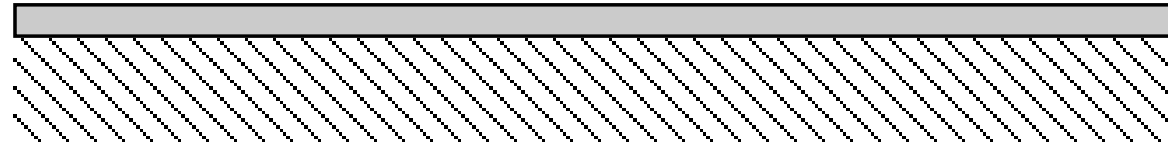

Initial

Stage

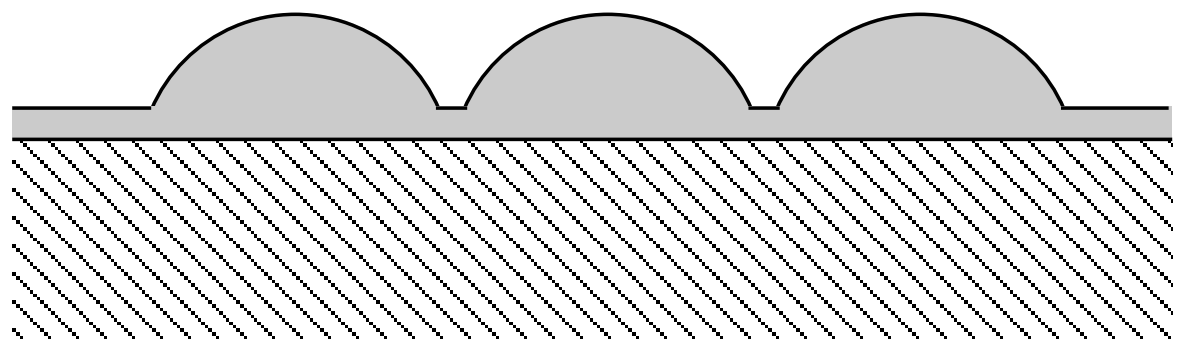

Later

Stage 


\section{Epitaxial stresses :}

Very thin crystalline films with the same crystal structure as the substrate may form perfectly coherent boundaries with the substrate. For this to occur, the film must be elastically strained for the two lattices to match up at the interface. Consider how these elastic strains develop in a growing film. Assume that the film growth occurs by the Volmer-Weber mode of growth and that the equilibrium lattice parameter of the film is smaller than that of the substrate: $a_{f}<a_{s}$. For this case the atomic positions in two crystallites about to coalesce would be as shown in the following diagram.

Crystallite coalescence $a_{f}<a_{s}$

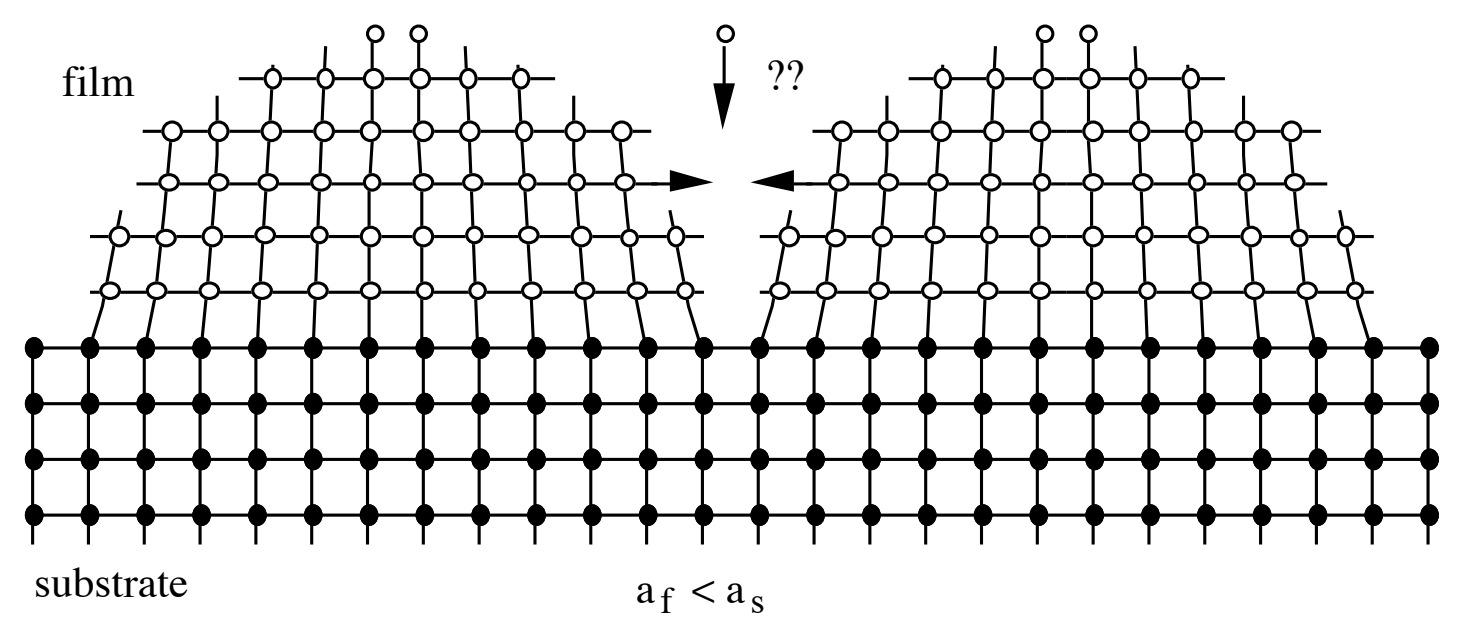

If the arriving atoms continue to attach themselves to the growing crystallites the two crystallites will eventually be pulled together and the film will develop a homogeneous elastic strain. Alternatively, a new column of atoms may be inserted in between the two crystallites. Such an extra column of atoms (an extra plane in 3D) will allow the two crystallites to have lattice parameters much closer to the equilibrium values. The incorporation of such defects (dislocations) relaxes the strain in the film.

The case of $a_{f}>a_{s}$ is shown in the diagram below. Here we see that an arriving atom must squeeze in between the two crystallites for the film to be fully strained. Such arriving atoms might not be able to reach the substrate in between the two crystallites. This corresponds to the incorporation of a dislocation in the film. In this case a column of atoms (a plane in 3D) is omitted from the film and the strain is correspondingly relaxed. 
Crystallite coalescence $a_{f}>a_{s}$

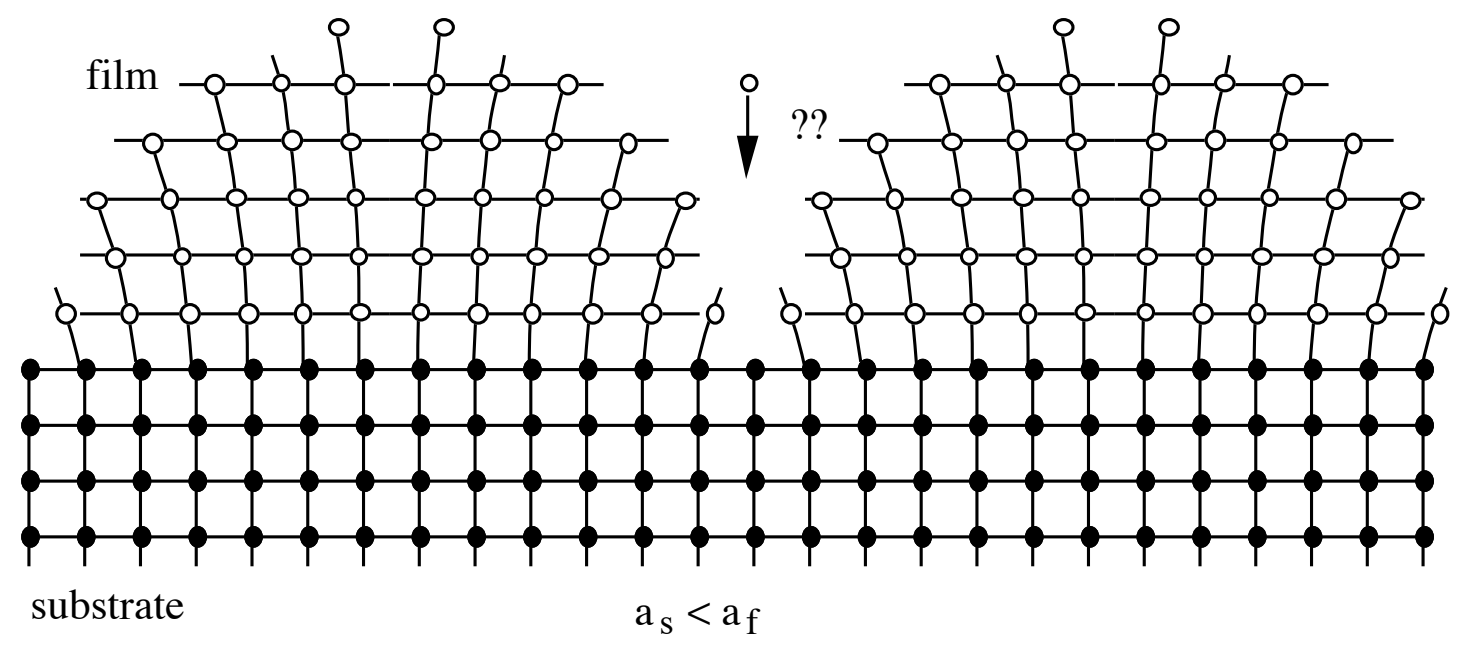

We conclude from this discussion that defect-free films can be produced only if $a_{f} \approx a_{s}$. Otherwise dislocations and stacking faults and possibly even new crystallites with different orientations might be formed in the growing film.

\section{Continuous Heteroepitaxial Films}

We consider now thin heteroepitaxial films without defects. Such films are now grown regularly by $\mathrm{MBE}$ and CVD techniques, provided that the substrate is dislocation-free. For $a_{f}<a_{s}$, the following analyses apply.

\section{Heteroepitaxial film and substrate}

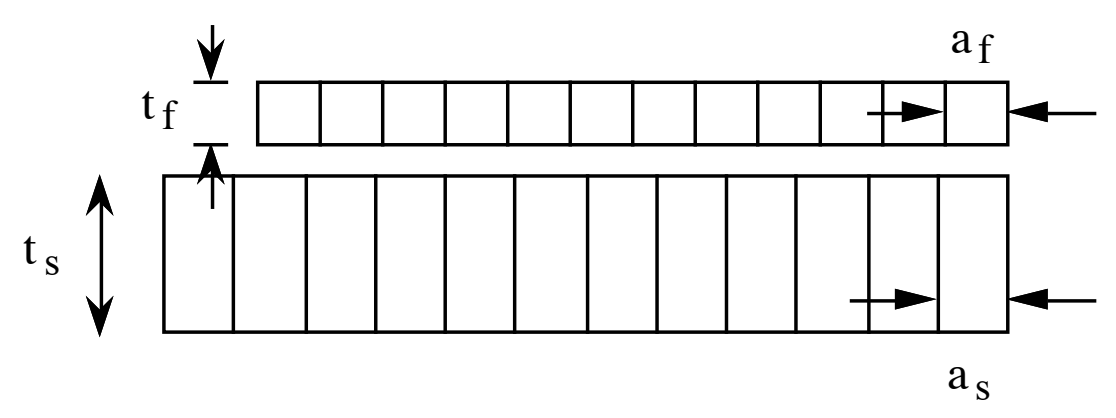

For $t_{f}<<t_{s}$ all of the strain is in the film. 


\section{Elastically strained film}

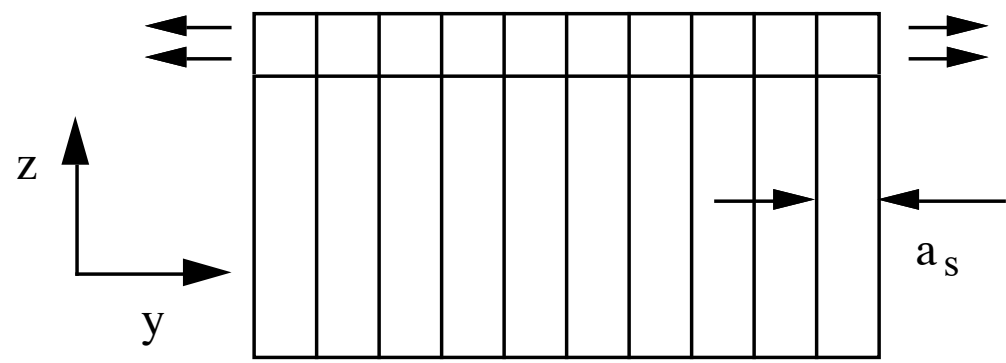

The biaxial elastic strain in the film is

$$
\varepsilon_{f}=\frac{a_{s}-a_{f}}{a_{f}}=\frac{a_{\text {final }}-a_{\text {initial }}}{a_{\text {initial }}}=\frac{\Delta a}{a} \approx \frac{a_{s}-a_{f}}{a_{s}}
$$

Note $\quad$ Even if the (001) does not coincide with the plane of the film, the lattice parameter, $\underline{\mathrm{a}}$, (the cube dimension) can be used as a measure of plane spacing because all planar spacings are proportional to $\underline{a}$.

The corresponding stresses in the film depend on the biaxial elastic modulus in the plane of the crystalline film. These relations are:

(001) films on (001) substrates

$$
\begin{aligned}
& \sigma_{y y}=\left[c_{11}+c_{12}-\frac{2 c_{12}^{2}}{c_{11}}\right] \varepsilon_{y y} \\
& \varepsilon_{z z}=-\frac{2 c_{12}}{c_{11}} \varepsilon_{y y}
\end{aligned}
$$

where

$$
\varepsilon_{y y}=\frac{a_{s}-a_{f}}{a_{f}} .
$$


For (111) films on (111) substrates

$$
\begin{aligned}
\sigma_{y y} & =\left[\frac{6 c_{44}\left(c_{11}+2 c_{12}\right)}{c_{11}+2 c_{12}+4 c_{44}}\right] \varepsilon_{y y} \\
\varepsilon_{z z} & =-\left[\frac{2 c_{11}+4 c_{12}-4 c_{44}}{c_{11}+2 c_{12}+4 c_{44}}\right] \varepsilon_{y y}
\end{aligned}
$$

Relations for (011) films on (011) substrates have been given earlier. Misfit dislocations (to be discussed later) form if the film is sufficiently thick and the misfit strain is sufficiently large.

\section{Tetragonal strains in heteroepitaxial Strains}

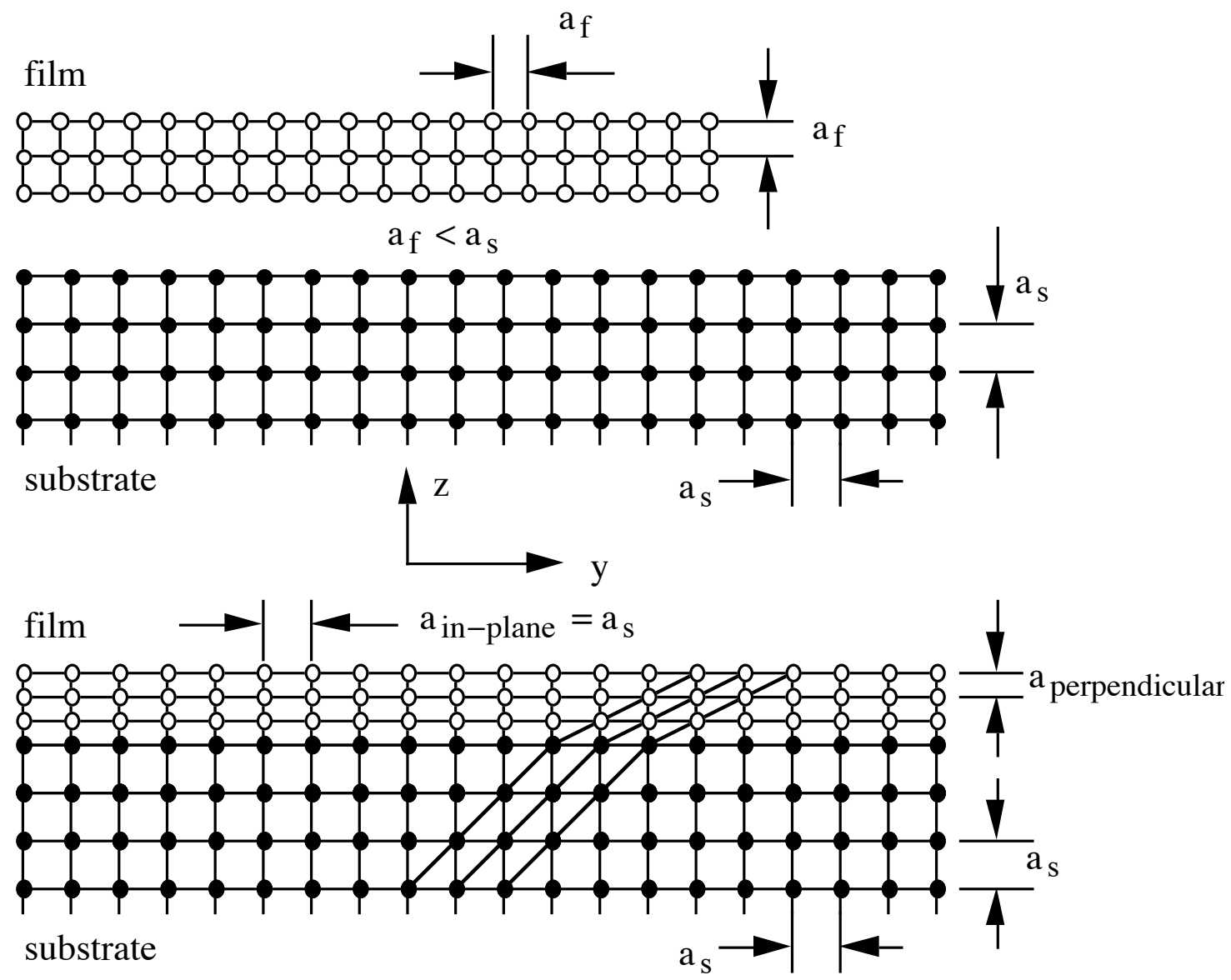


The strains in the epitaxial film can be related to the lattice parameters of the film as follows:

$$
\varepsilon_{y y}=\frac{a_{\text {in-plane }}-a_{f}}{a_{f}} \quad \text { and } \quad \varepsilon_{z z}=\frac{a_{\text {perpendicular }}-a_{f}}{a_{f}}
$$

Using

$$
\varepsilon_{z z}=-\frac{2 c_{12}}{c_{11}} \varepsilon_{y y}
$$

for the case of (001) films, the plane spacings become

$$
\begin{aligned}
& \frac{a_{\text {perpendicular }}-a_{f}}{a_{f}}=-\frac{2 c_{12}}{c_{11}}\left[\frac{a_{\text {in-plane }}-a_{f}}{a_{f}}\right] \\
& a_{\text {perpendicular }}-a_{f}=-\frac{2 c_{12}}{c_{11}}\left[a_{\text {in-plane }}-a_{f}\right]
\end{aligned}
$$

or

$$
\frac{\Delta a_{\text {perpendicular }}}{\Delta a_{\text {in-plane }}}=-\frac{2 c_{12}}{c_{11}}
$$

This relation expresses the degree of tetragonality in the strained film. Ion channeling experiments can be used to determine the angle of certain atomic channels on the strained lattice. From such measurements the strain in the film can be determined.

If we define $\theta$ as the angle between the direction of channeling and the plane of the film, for the simple (001) film described above, then we may write

$$
\tan \theta=a_{\text {perpendicular }} / a_{\text {parallel }} .
$$

\section{Using}

$$
\varepsilon_{y y}=\frac{a_{\text {in-plane }}-a_{f}}{a_{f}} \text {, and } \varepsilon_{z z}=\frac{a_{\text {perpendicular }}-a_{f}}{a_{f}}
$$


we find

$$
\begin{aligned}
& a_{\text {perpendicular }}=a_{f}\left(1+\varepsilon_{z z}\right) \\
& a_{\text {in-plane }}=a_{f}\left(1+\varepsilon_{y y}\right)
\end{aligned} .
$$

Then the channeling angle can be expressed as

$$
\tan \theta=\frac{1+\varepsilon_{z z}}{1+\varepsilon_{y y}} .
$$

Again using

$$
\varepsilon_{z z}=-\frac{2 c_{12}}{c_{11}} \varepsilon_{y y},
$$

we have

$$
\begin{gathered}
\tan \theta=\frac{1-\frac{2 c_{12}}{c_{11}} \varepsilon_{y y}}{1+\varepsilon_{y y}} \\
\left(1+\varepsilon_{y y}\right) \tan \theta=1-\frac{2 c_{12}}{c_{11}} \varepsilon_{y y}
\end{gathered}
$$

Solving for the in-plane strain we have

$$
\varepsilon_{y y}=\frac{1-\tan \theta}{\tan \theta+\frac{2 c_{12}}{c_{11}}} \text {. }
$$

With this relation the in-plane strain can be found from a measurement of the channeling angle and the elastic constants. Note that the strain is zero when $\tan \theta=1$, as expected.

\section{Capping Layers}

We have shown that elastic strains and stresses exist in heteroepitaxial thin films on substrates because the film is required to adopt the in-plane lattice parameter of the substrate. If a second film of the substrate material, called a capping layer, is grown on top of the strained film, it is unstrained because it is perfectly 
commensurate with the film with its own equilibrium lattice parameter. In the case shown below, only the Si-Ge film is under strain. The substrate and the capping layers are essentially unstrained. One consequence of such structures is that dislocations would be expected to propagate only in the strained layer and not in the substrate or capping layer.

Unstrained Si capping layer
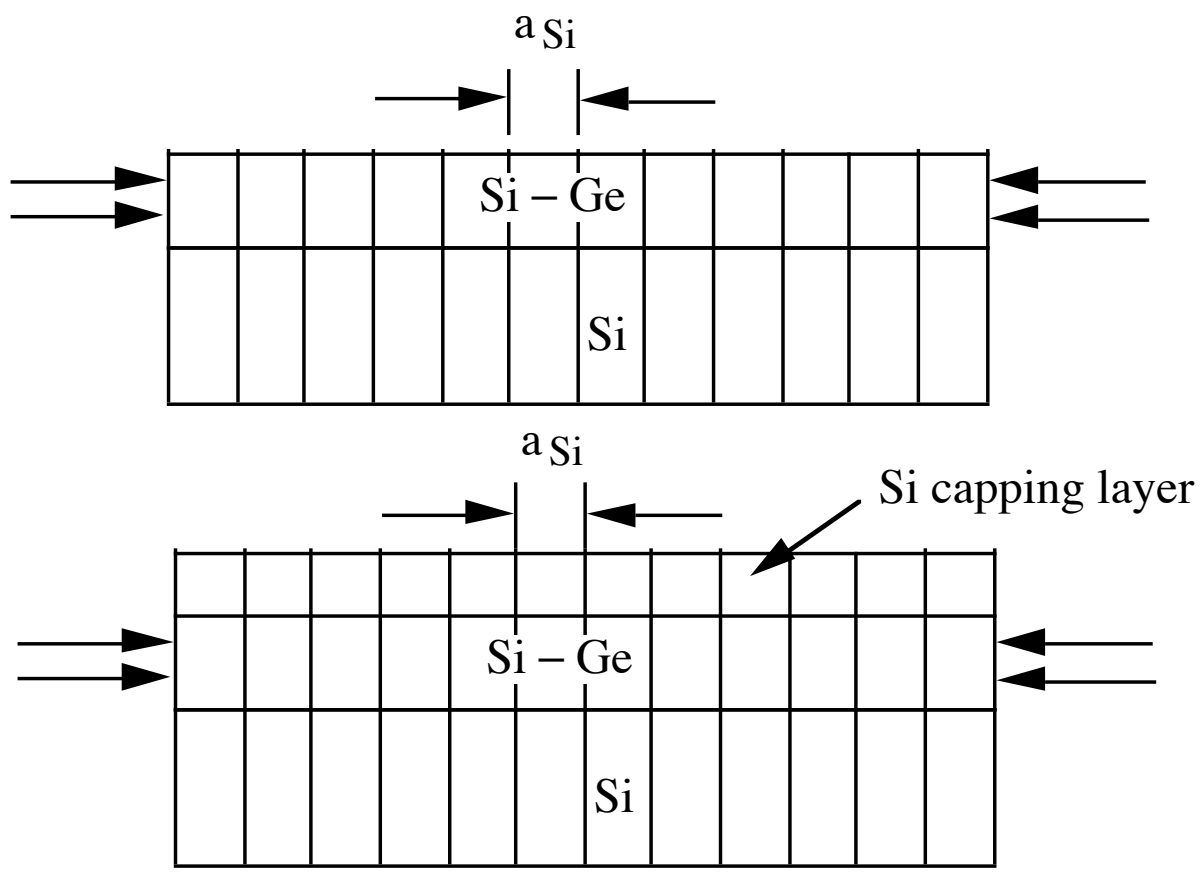

\section{Free-standing multilayered epitaxial films}

We have to assumed that $t_{f}<<t_{s}$. This is good for thin films on thick substrates. It is inappropriate for multilayers composed of alternating single crystal films of comparable thickness, free of the substrate on which they might have been grown. In such cases, both layers (or films) deform significantly when the two crystal lattices are joined at their interfaces. 
Consider a multilayer structure:

Multilayered structure (in hypothetical separated state)

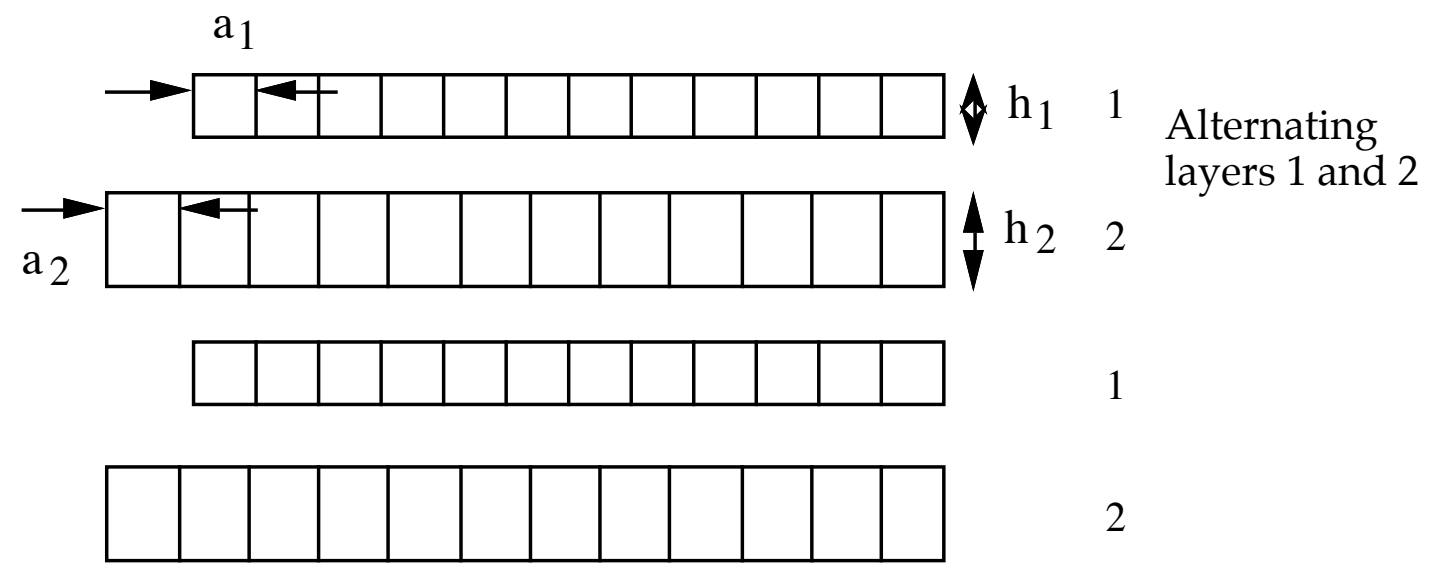

When a coherent multilayer structure is formed

Coherent multilayer
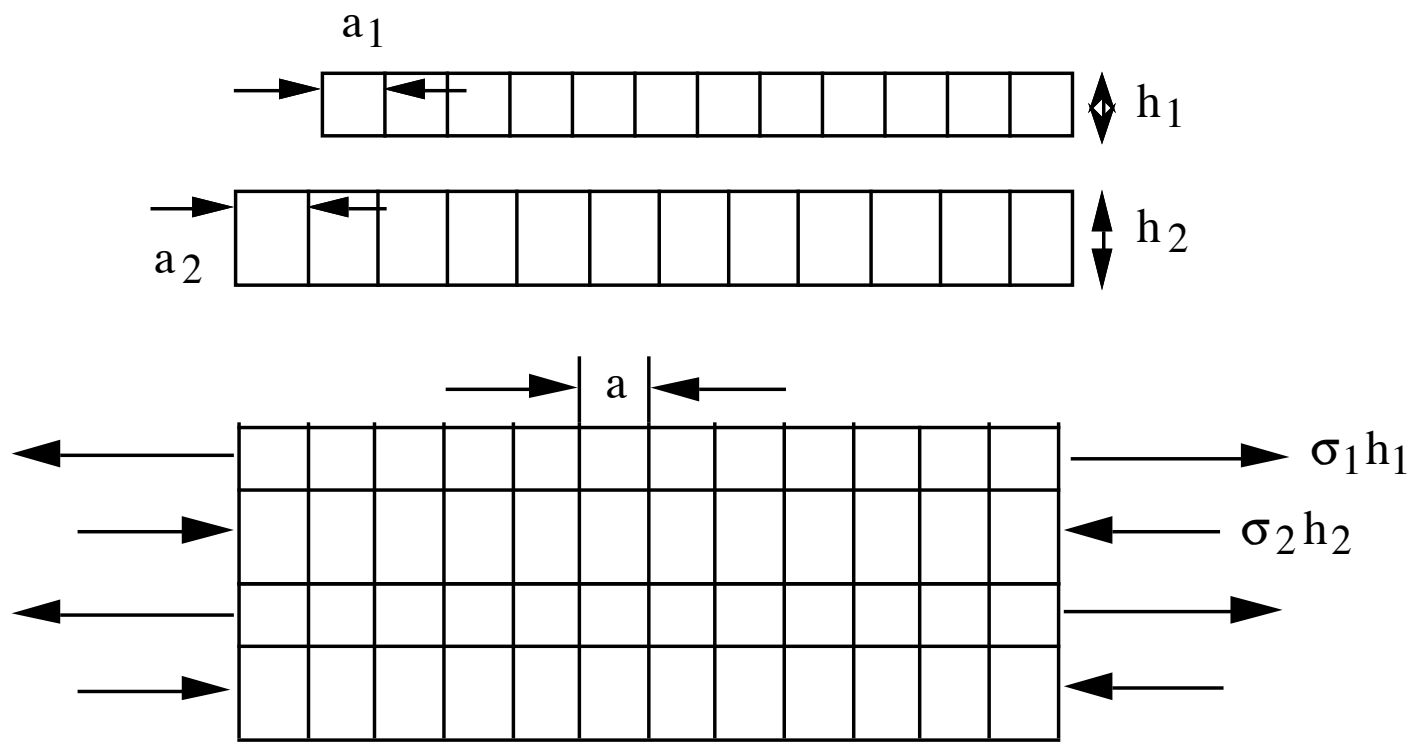

Film 1 is biaxially stretched, and Film 2 biaxially compressed.

When the multilayer is formed, the "in-plane" lattice parameters are the same in the two films. Thus the biaxial strains in the two layers are 


$$
\varepsilon_{1}=\frac{a-a_{1}}{a_{1}} \text { and } \varepsilon_{2}=\frac{a-a_{2}}{a_{2}}
$$

The corresponding biaxial stresses are

$$
\sigma_{1}=B_{1} \varepsilon_{1} \text { and } \quad \sigma_{2}=B_{2} \varepsilon_{2}
$$

where $B_{1}$ and $B_{2}$ are the biaxial moduli of the two layers (anisotropic).

If no forces are applied to the edges of the multilayer, then

$$
\sigma_{1} h_{1}+\sigma_{2} h_{2}=0
$$

so,

$$
\begin{aligned}
& B_{1} \varepsilon_{1} h_{1}+B_{2} \varepsilon_{2} h_{2}=0 \\
& B_{1} h_{1}\left(\frac{a-a_{1}}{a_{1}}\right)+B_{2} h_{2}\left(\frac{a-a_{2}}{a_{2}}\right)=0
\end{aligned}
$$

so we can solve for the "in-plane" lattice parameter in the multilayer.

$$
B_{1} h_{1}\left(\frac{a}{a_{1}}-1\right)+B_{2} h_{2}\left(\frac{a}{a_{2}}-1\right)=0
$$

Solving for the resulting in-plane lattice parameter, $a$, we get

$$
a=\frac{a_{1} a_{2}\left(1+\frac{B_{2} h_{2}}{B_{1} h_{1}}\right)}{a_{2}+\left(\frac{B_{2} h_{2}}{B_{1} h_{1}}\right) a_{1}}
$$

If $\quad \frac{B_{2} h_{2}}{B_{1} h_{1}} \Rightarrow \infty \quad a \Rightarrow a_{2}$ (film 1 adopts lattice parameter of 2)

If $\quad \frac{B_{2} h_{2}}{B_{1} h_{1}} \Rightarrow 0 \quad a=a_{1} \quad($ film 2 adopts lattice parameter of 1$)$ 
Once the lattice parameter of the multilayer is found, one can calculate the biaxial strains and stresses in each layer. Then, other lattice dimensions can be calculated.

\section{Distortions in the layers}

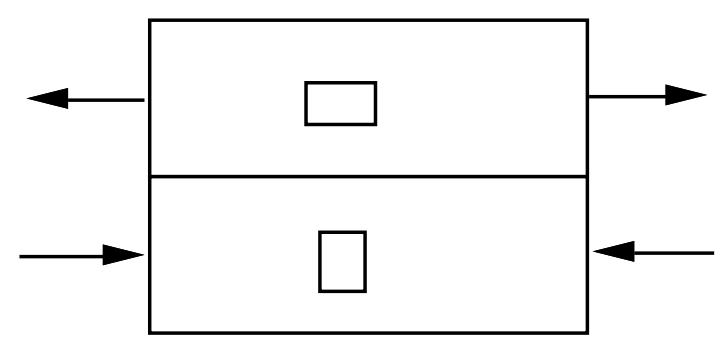

\section{Buffer Layers}

It is frequently desirable to grow unstrained single crystal films onto substrates. Epitaxy is needed to obtain the desired film structure and orientation but the epitaxial strains need to be avoided. The diagram below shows two ways in which the strain in the film can be relaxed. As shown in the diagram, the introduction of misfit dislocations into the film, perhaps in a "buffer layer" of a different material effectively isolates the film from the substrate, allowing the film to have its equilibrium lattice parameter. Another method might involve the use of small islands in which the lateral dimension of the island is comparable to the thickness of the film. In this case relaxation at the edges of the film allows the top of the island to adopt the stress-free lattice parameter. 
Relaxation of strain by buffer layer and island effect

Fully Strained

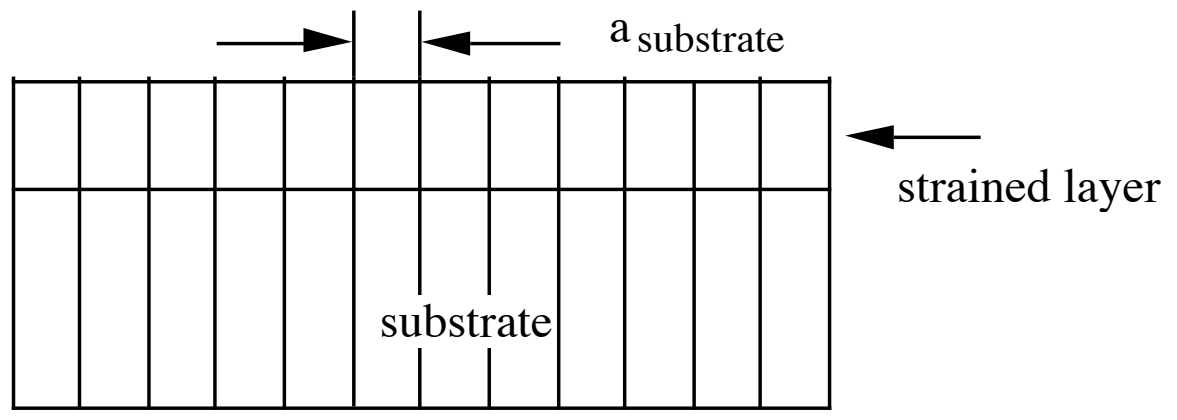

Buffer Layer
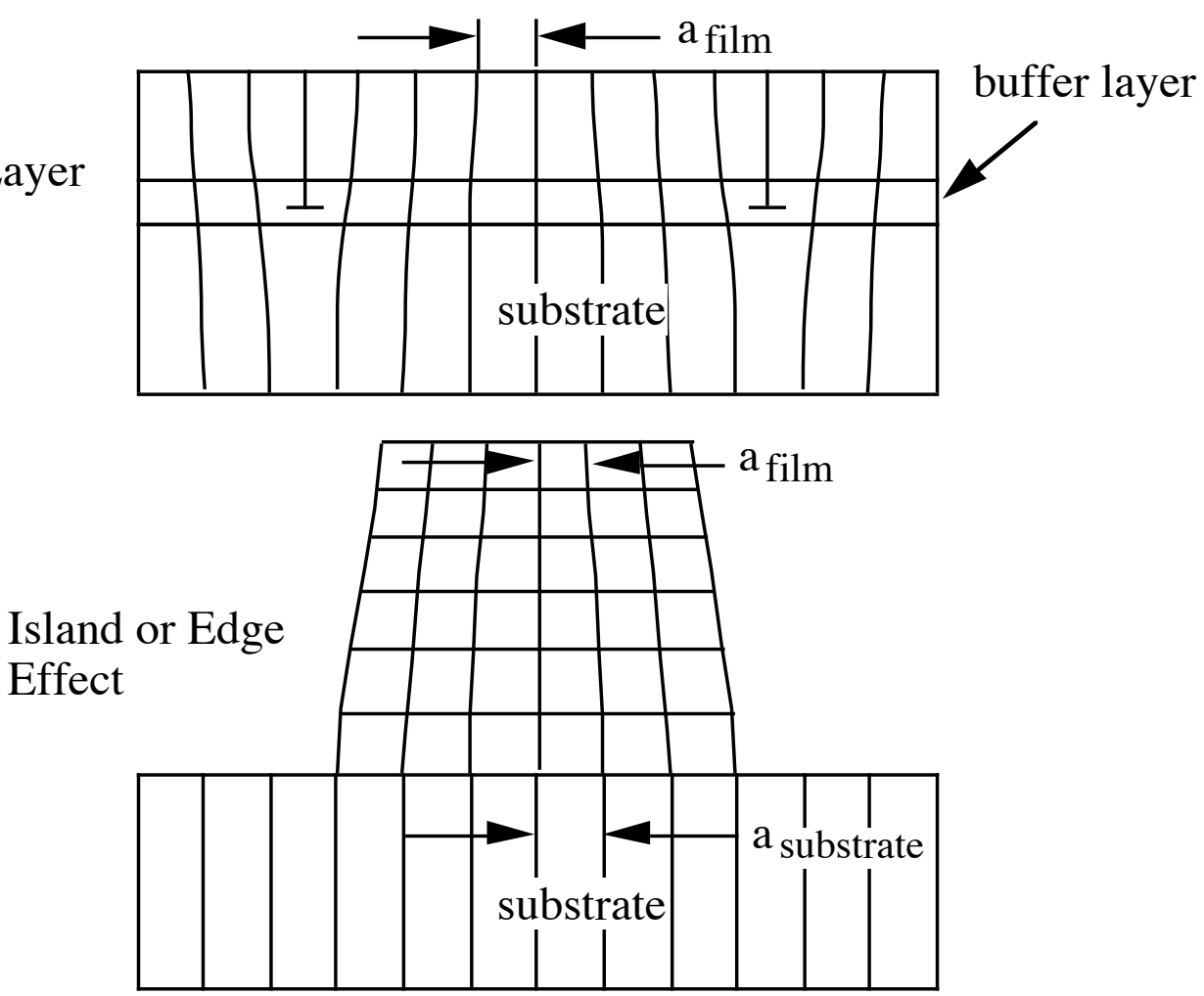

\section{Compliant Substrates}

An alternate method of strain accommodation involves the use of a very thin substrate that strains to accommodate the lattice parameter of the film during growth. This would be called a "compliant substrate." The figure shows that if a very thin single crystal substrate is bonded to a supporting substrate through a "fluid-like" layer of material that allows for free interfacial sliding at the epitaxial growth temperature, then most of the elastic strain and stress is forced to reside in the compliant substrate. Through this strategy it might be possible to grow very thick epitaxial films without ever creating dislocations in the film of interest. Having a freely sliding interface between the compliant substrate and the 
supporting substrate is critical for this to work. Some progress has apparently been made recently using glass interlayers that are able to slide easily at the epitaxial growth temperatures.

\section{$\underline{\text { Relaxation of strain by buffer layer and island effect }}$}

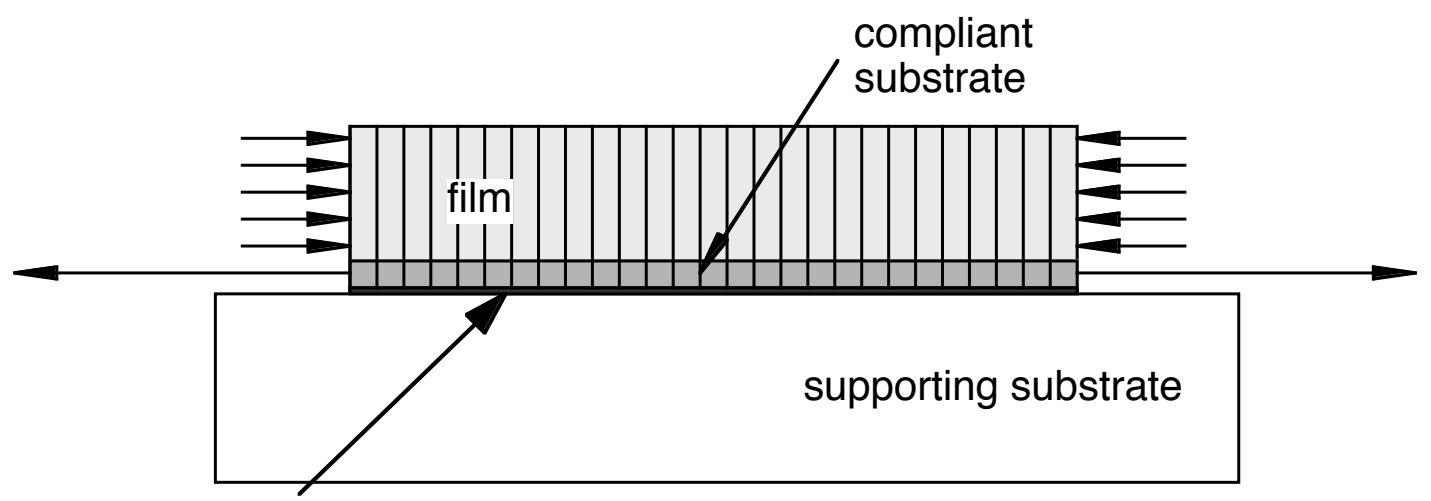

"fluid-like" layer between compliant

substrate and supporting substrate

For free sliding at the interface between the compliant substrate and the supporting substrate, the bilayer structure is subject to the condition of zero edge force. Thus we may write

$$
\varepsilon_{m i s f i t}+\varepsilon_{f}=\varepsilon_{s}
$$

and

$$
B \varepsilon_{f} t_{f}+B \varepsilon_{s} t_{s}=0
$$

where $B$ is the biaxial modulus. These two relations lead to

$$
\varepsilon_{f}=-\varepsilon_{\text {misfit }}\left(\frac{t_{s}}{t_{s}+t_{f}}\right), \varepsilon_{s}=\varepsilon_{\text {misfit }}\left(\frac{t_{f}}{t_{s}+t_{f}}\right) \text {. }
$$

For a film like Si-Ge on a Si substrate, the misfit strain is positive, so that the elastic strain in the film is negative (compressive) and the elastic strain in the substrate positive (tensile). For a very thin, compliant substrate, most of the strain resides in the substrate. 


\section{Equilibrium Critical Thickness (thin film on thick substrate)}

We wish to analyze the thermodynamic stability of strained epitaxial films with respect to the formation of misfit dislocations. As shown in the following diagram, a film with a misfit strain relative to the substrate can be made perfectly commensurate with the substrate if it is homogeneously strained or it may be matched to the substrate through the formation of misfit dislocations.

\section{Misfit accommodation processes}
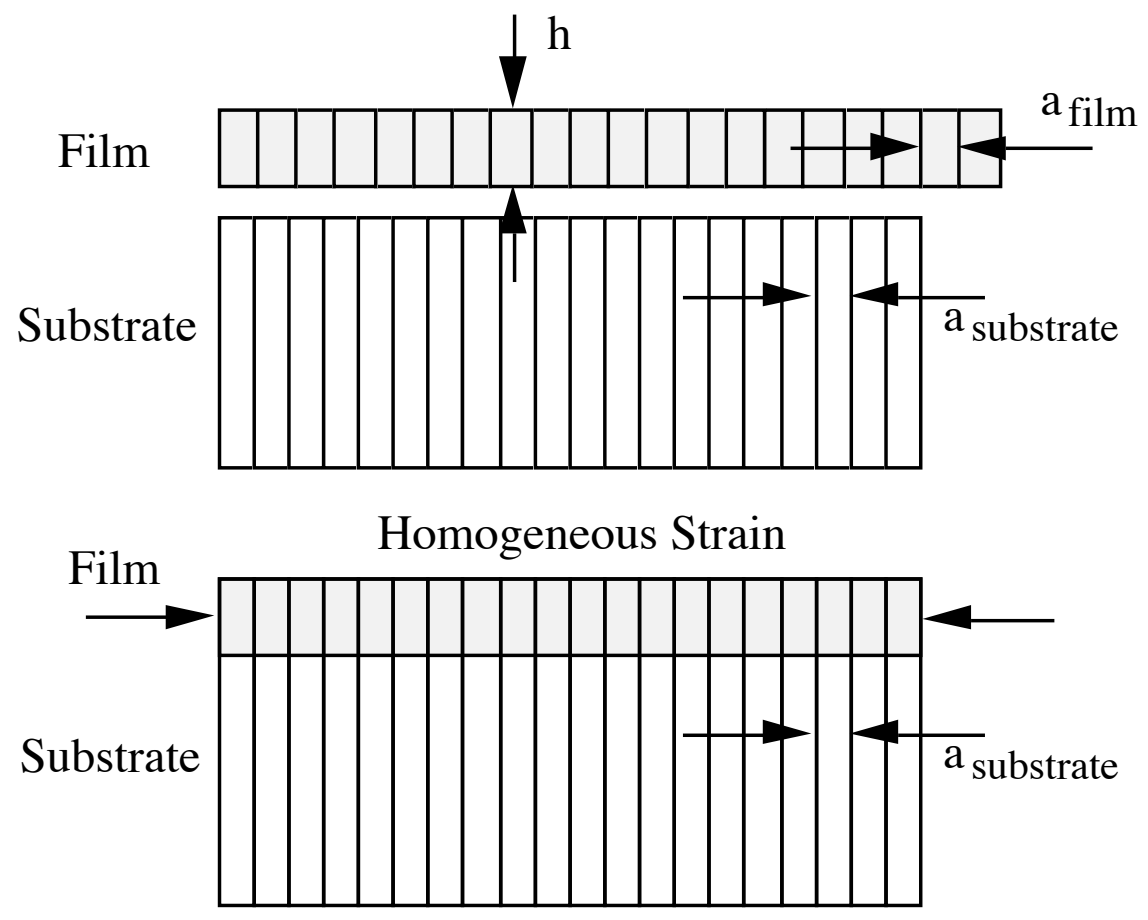

Film with Misfit Dislocations

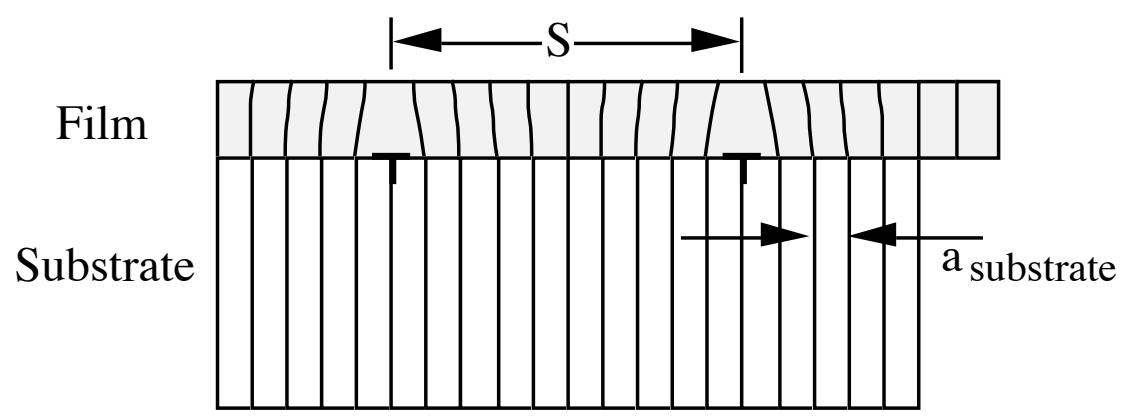

For a film below the critical thickness, $h<h_{c}$, the elastic strain energy (per unit area of film) is 


$$
\begin{aligned}
& E_{\text {hom ogeneous }}=\left[\frac{1}{2} \sigma_{1} \varepsilon_{1}+\frac{1}{2} \sigma_{2} \varepsilon_{2}\right] h \\
& E_{\text {hom ogeneous }}=\sigma_{1} \varepsilon_{1} h=B h \varepsilon_{1}^{2}
\end{aligned}
$$

where the in-plane biaxial stress is $\sigma_{1}$ and the corresponding in-plane biaxial strain is $\varepsilon_{1}$.

For a film above the critical thickness $h>h_{c}$ the total energy (per unit area) can be expressed approximately as the sum of two terms: the strain energy of the partially relaxed film and the energy associated with the misfit dislocations:

$$
E=E_{\text {strain }}+E_{\text {dislocation }}
$$

For the partially relaxed film, the remaining homogeneous strain in the film is

$$
\varepsilon=\varepsilon_{1}-\frac{b}{S}
$$

Thus the corresponding strain energy is

$$
E_{\text {strain }}=B h\left(\varepsilon_{1}-\frac{b}{S}\right)^{2}
$$

The energy (per unit length) of each misfit dislocation is $\frac{\mu b^{2}}{4 \pi(1-v)} \ln \left(\frac{\beta h}{b}\right)$, where the terms have their usual meanings in dislocation theory. The logarithmic term involves the cut-off radii for the elastic field of the dislocation. In particular, the outer cut-off radius scales with the thickness of the film because the surface of the film is traction free. Since the misfit line length per unit area is $2 / S$ (for a square array of misfit dislocations), it follows that the dislocation energy per unit area is

$$
E_{\text {dislocation }}=\frac{\mu b^{2}}{4 \pi(1-v)} \frac{2}{S} \ln \left(\frac{\beta h}{b}\right)
$$

Adding these two terms together gives 


$$
E=B h\left(\varepsilon-\frac{b}{S}\right)^{2}+\frac{\mu b^{2}}{4 \pi(1-v)} \frac{2}{S} \ln \left(\frac{\beta h}{b}\right)
$$

for the total energy per unit area of film. Here the strain $\varepsilon$ is the biaxial strain in the film. We are interested in the way the energy of the system varies as misfit dislocations are introduced. The linear density of misfit dislocations, $1 / S$, can be taken as a measure of the density of misfit dislocations. We need to determine how the energy varies as the misfit density is increased from zero. For this we need

$$
\left(\frac{\partial E}{\partial(1 / S)}\right)=-2 b B h\left(\varepsilon-\frac{b}{S}\right)+\frac{\mu b^{2}}{2 \pi(1-v)} \ln \left(\frac{\beta h}{b}\right)
$$

We note that the positive term dominates when $h$ is small while the negative term dominates when $h$ is large. The critical film thickness is that thickness for which the introduction of the first dislocation causes no variation in the energy of the film:

$$
\left(\frac{\partial E}{\partial(1 / S)}\right)_{b / S=0}=0
$$

Applying this condition

$$
0=-2 b B h \varepsilon+\frac{\mu b^{2}}{2 \pi(1-v)} \ln \left(\frac{\beta h}{b}\right)
$$

we can solve for the critical film thickness:

$$
\frac{h_{c}}{\ln \left(\beta h_{c} / b\right)}=\frac{\mu b}{4 \pi(1-v) B \varepsilon}=\frac{\mu b}{4 \pi(1-v) \sigma} \text {. }
$$

The critical thickness for a film with a misfit strain of $1 \%$ is about $10 \mathrm{~nm}$. This analysis may be understood graphically by considering the way in which the total energy varies with increasing misfit dislocation density. 


\section{Illustration of energy variations with misfit dislocation formation}

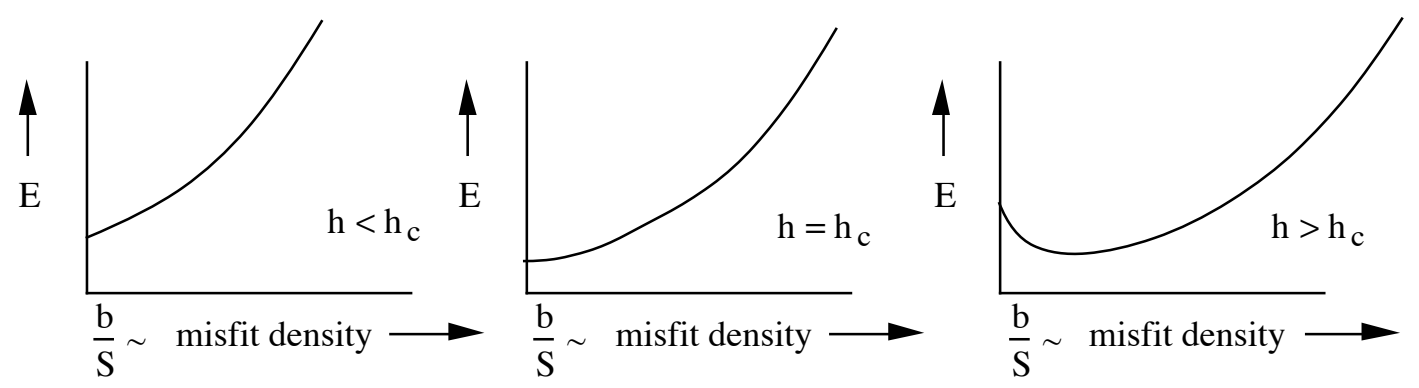




\title{
Heteroepitaxial Interfaces
}

In the discussions above we have considered only simple cubic lattices. We now consider the different crystal structures. A common heteroepitaxial relationship for semiconductors is illustrated by the case of Si-Ge alloy films on Si substrates. This is shown in the figure below. It is a case in which the film and substrate have the same crystal structure and same orientation.

\section{Si-Ge/Si orientation relationship}

\section{Si/Ge Orientation Relationship}

\author{
Ideal Matching: $\mathrm{a}_{\mathrm{Si}}=\mathrm{a}_{\mathrm{SiGe}}$
}

$$
\text { Mismatch Parameter: } \quad \delta_{\mathrm{SiGe}}=\frac{\mathrm{a}_{\mathrm{SiGe}}-\mathrm{a}_{\mathrm{Si}}}{\mathrm{a}_{\mathrm{Si}}}
$$

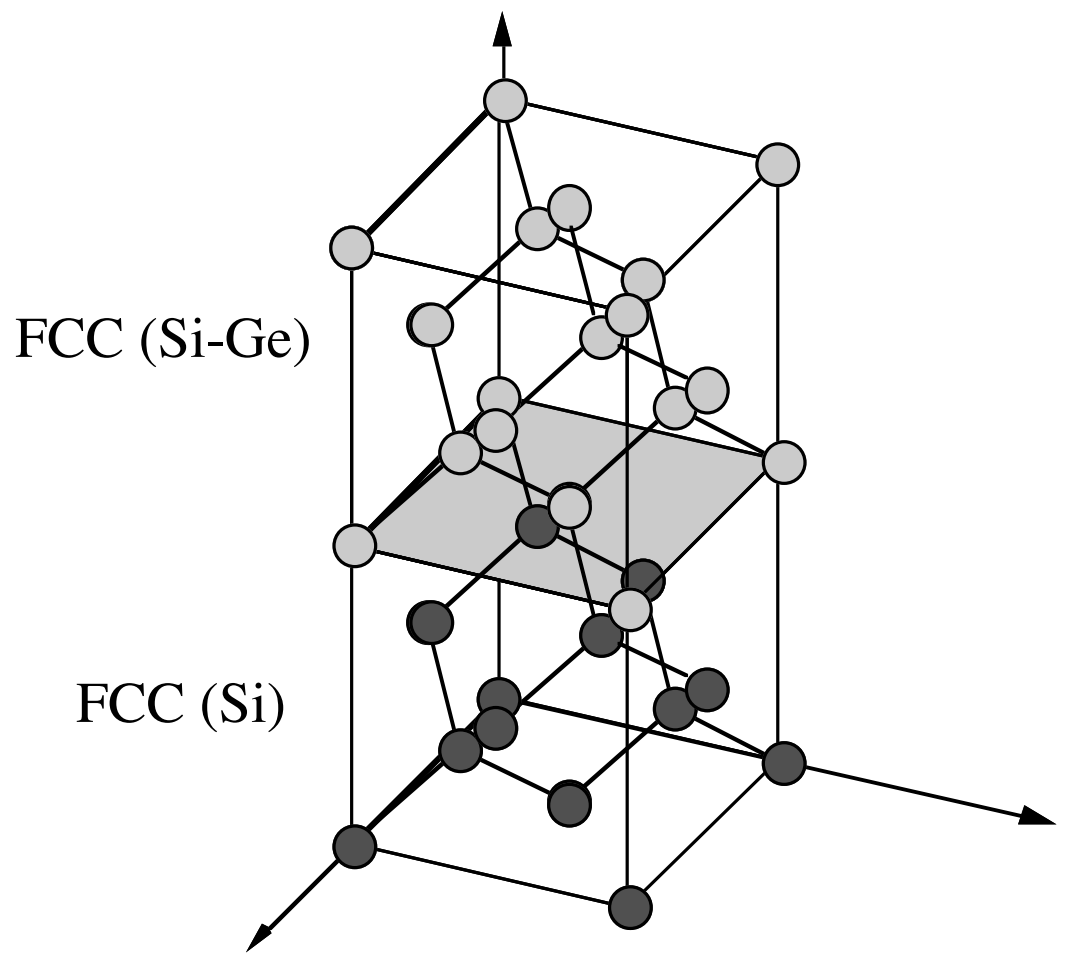


This orientation relationship is shown below for the GaAs/CdTe interface A careful examination of the positions of the atomic columns will show that the bright regions are edge misfit dislocations at the interface between the two crystals. The difference in lattice parameters is evident in the micrograph.

Misfit dislocations at the interface between GaAs and CdTe

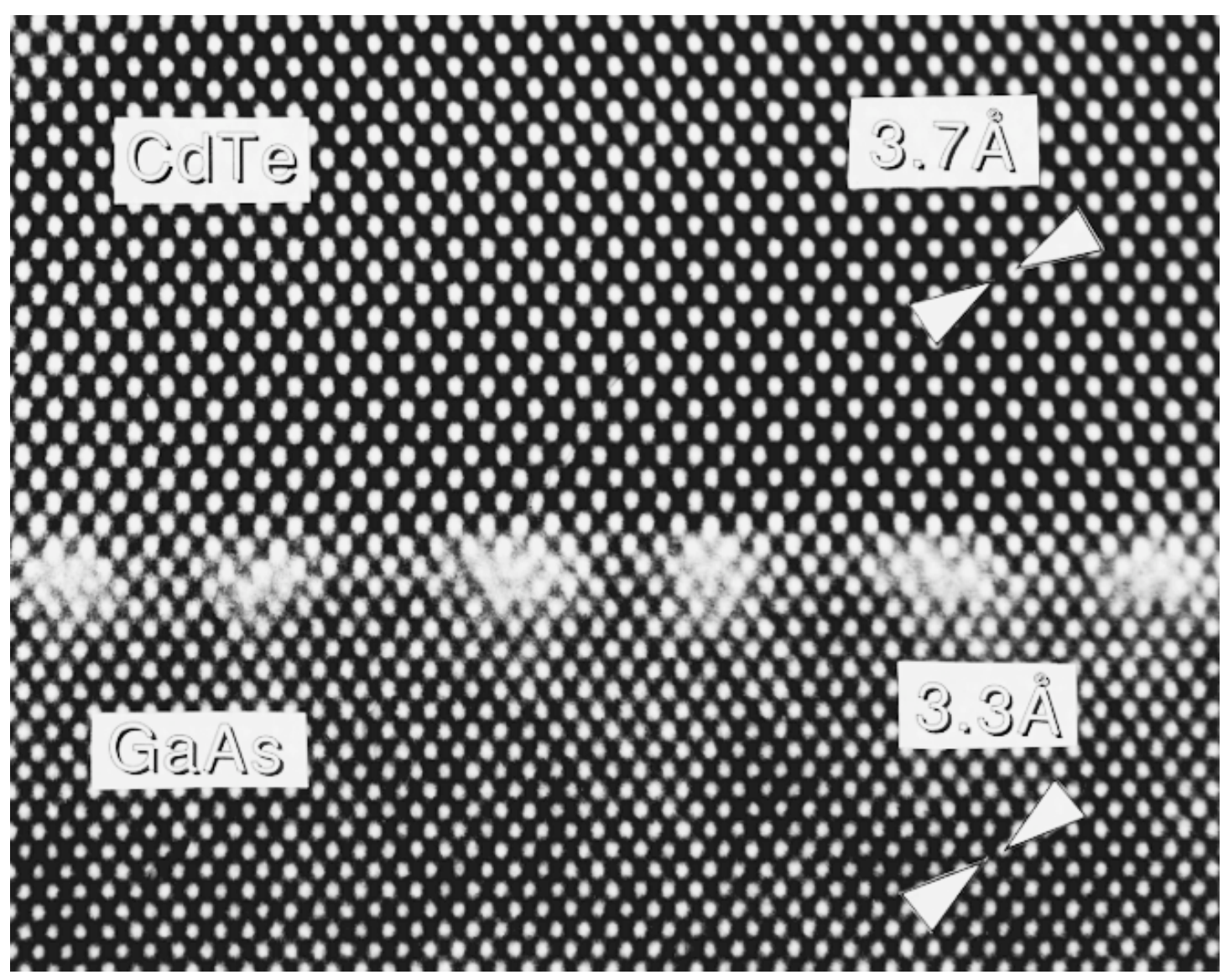


In some cases the epitaxy may create such a large strain in the film that is effectively adopts a different crystal structure. Very thin layers of $\mathrm{Cu}$ (usually FCC) grown epitaxially onto BCC Cr cause the $\mathrm{Cu}$ layers to adopt the BCC structure. This is shown below.

Pseudomorphic $\mathrm{Cu} / \mathrm{Cr}$ orientation relationship

\section{Pseudomorphic $\mathrm{Cu} / \mathrm{Cr}$ Orientation Relationship}

$$
\text { Ideal Matching: } \quad \mathrm{a}_{\mathrm{FCC}} \sqrt{2}=\mathrm{a}_{\mathrm{BCC}} \sqrt{3}
$$

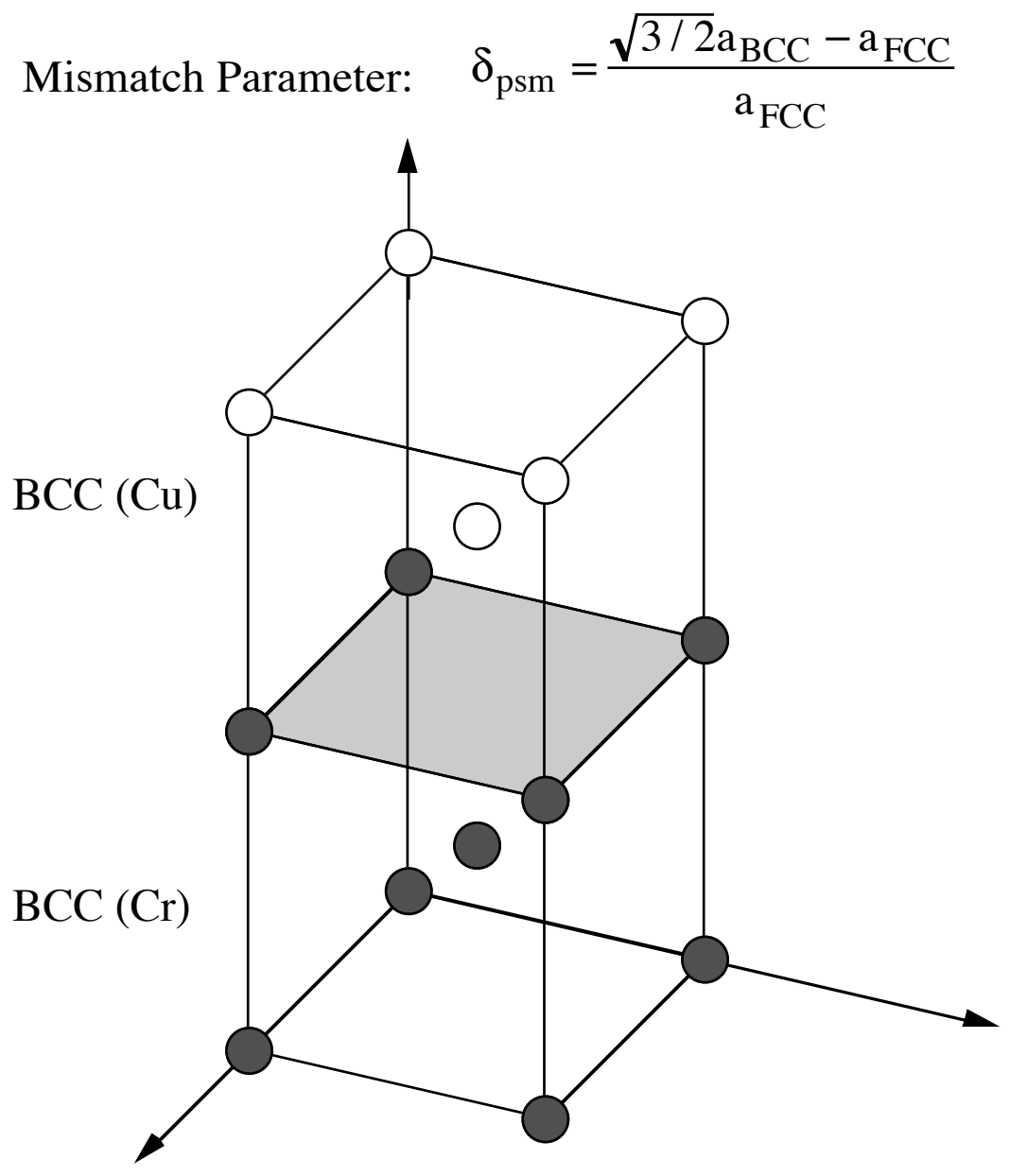


One may think of the FCC Cu as being strained in biaxial tension so that it is almost perfectly BCC. Consider the tetragonal element of the FCC structure shown in the diagram below. If the structure is subjected to very large in-plane tensile strains, the in-plane lattice parameter increases while the out-of-plane lattice parameter decreases. The result is a heavily strained FCC structure that is equivalent to a BCC structure.

\section{The Bain Strain coverting FCC to BCC}
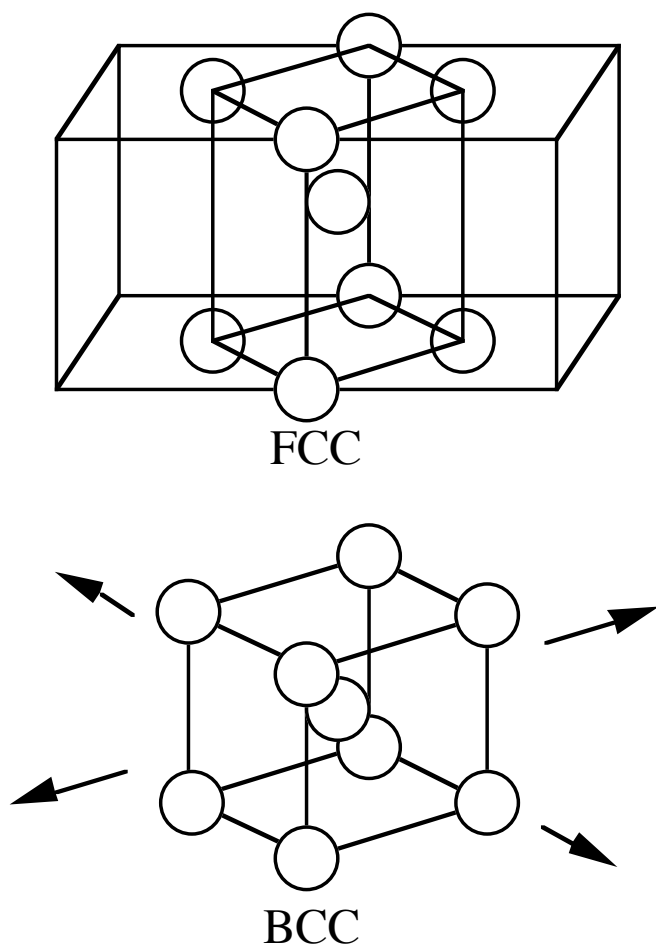

The pseudomorphic $\mathrm{Cu} / \mathrm{Cr}$ orientation relationship is expected to be most favorable when the body diagonal distance in the $\mathrm{BCC}$ structure is about equal to the face diagonal distance in the FCC structure. 
In some cases metals with different crystal structures will adopt an orientation relationship that maximizes the degree of atomic fit at the interface. The Nishiyama-Wasserman relationship shown below is common for some FCC/BCC pairs.

Nishiyama-Wasserman relationship

\section{Nishiyama-Wasserman FCC/BCC Orientation Relationship}

Ideal Matching: $\quad \mathrm{a}_{\mathrm{FCC}} \sqrt{6}=\mathrm{a}_{\mathrm{BCC}} 2 \sqrt{2}$

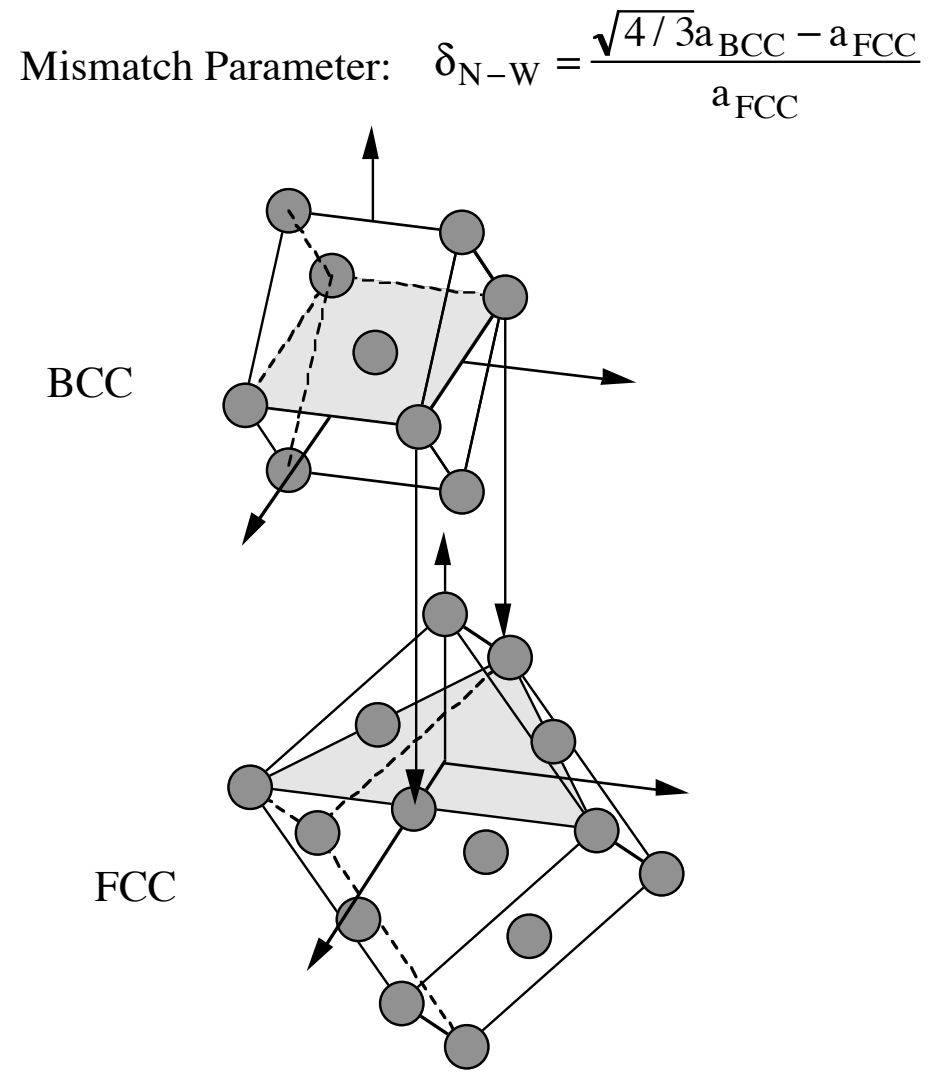


In still other cases the lattice parameters of the two metals are such that a good atomic fit can be obtained at the interface in all in-plane directions. An example is the rotated (001) FCC/BCC heteroepitaxial interface shown below. The Ag/ Cr interface displays this orientation relationship perfectly. The degree of mismatch is less than $0.2 \%$.

\section{Rotated (001) FCC/BCC heteroepitaxial interface}

\section{Rotated (001) FCC/BCC Heteroepitaxial Interface}

$$
\begin{array}{ll}
\text { Ideal Matching: } & \mathrm{a}_{\mathrm{FCC}}=\mathrm{a}_{\mathrm{BCC}} \sqrt{2} \\
\text { Mismatch Parameter: } & \delta_{(001)}=\frac{\sqrt{2} \mathrm{a}_{\mathrm{BCC}}-\mathrm{a}_{\mathrm{FCC}}}{\mathrm{a}_{\mathrm{FCC}}}
\end{array}
$$

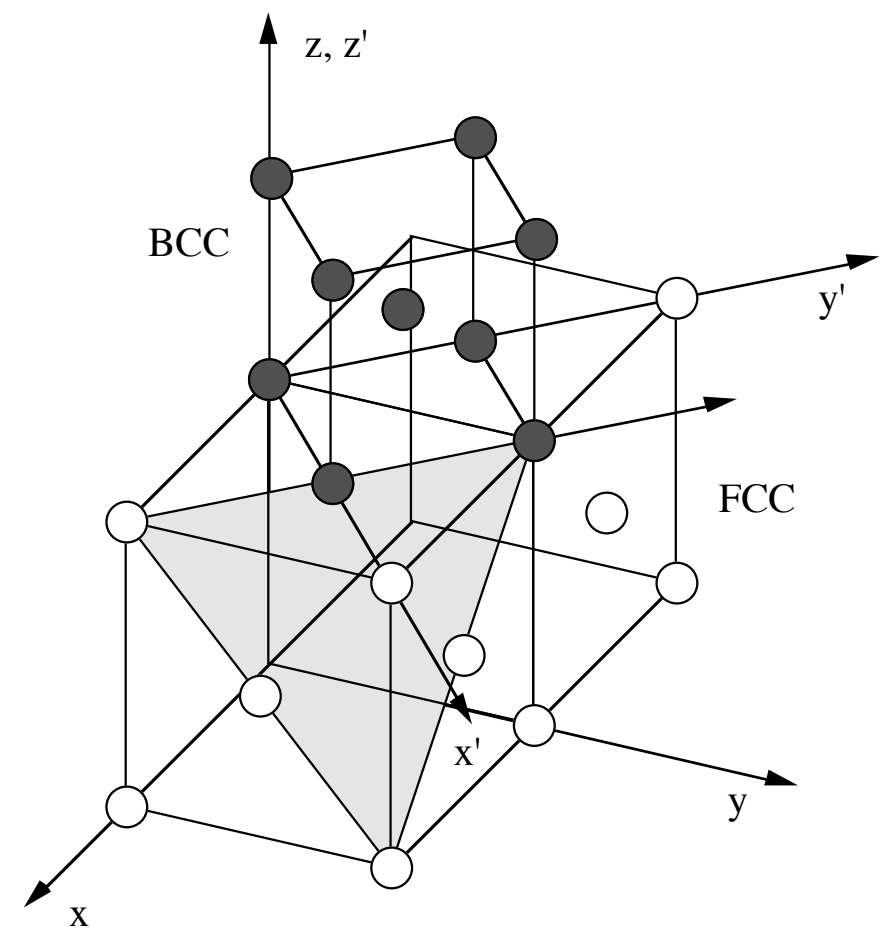


$\underline{\text { Some Misfit Parameters for FCC/BCC Pairs }}$

Table 1. Lattice mismatch $\delta N-W$ in percent from the ideal Nishiyama-Wasserman lattice parameter ratio.

\begin{tabular}{cc|c|c|} 
& & \multicolumn{1}{c}{$\mathrm{Nb}$} & $\mathrm{Mo}$ \\
$\mathrm{Cu}$ & $\mathrm{a}(\mathrm{nm})$ & 0.3301 & 0.3147 \\
\cline { 2 - 4 } $\mathrm{Ni}$ & 0.3615 & 5.422 & 0.5 \\
\hline & 0.3524 & 8.159 & 3.11 \\
\hline
\end{tabular}

Table 2. Lattice mismatch $\delta(100)$ in percent from the ideal rotated (100) lattice parameter ratio.

\begin{tabular}{ll|l|l|} 
& \multicolumn{1}{c}{$\mathrm{Cr}$} & $\mathrm{Fe}$ \\
& $\mathrm{a}(\mathrm{nm})$ & 0.2885 & 0.2866 \\
\hline $\mathrm{Ag}$ & 0.4086 & -0.15 & -0.78 \\
$\mathrm{Au}$ & 0.4078 & 0.024 & -0.6 \\
\hline
\end{tabular}

Table 3. Lattice mismatch $\delta_{p s m}$ in percent from the ideal pseudomorphic lattice parameter ratio.

\begin{tabular}{cc|c|c|} 
& & \multicolumn{1}{c}{$\mathrm{Cr}$} & $\mathrm{Fe}$ \\
& $\mathrm{a}(\mathrm{nm})$ & 0.2885 & 0.2866 \\
\cline { 2 - 4 } $\mathrm{Cu}$ & 0.3615 & -2.26 & -2.9 \\
$\mathrm{Ni}$ & 0.3524 & 0.27 & 0.39 \\
\cline { 2 - 4 } & & &
\end{tabular}


Room Temperature Elastic Constants, Lattice Parameters and Linear Expansion Coefficients for Some Cubic Crystals

\begin{tabular}{|c|c|c|c|c|c|}
\hline & $\mathrm{c}_{11}$ & $\mathrm{c}_{12}$ & $\mathrm{c}_{44}$ & $\mathrm{a}_{\mathrm{o}}$ & $\alpha$ \\
\hline & $(\mathrm{GPa})$ & $(\mathrm{GPa})$ & $(\mathrm{GPa})$ & $(\mathrm{nm})$ & $\left(10^{-6} \mathrm{~K}^{-1}\right)$ \\
\hline $\mathrm{InSb}$ & 66 & 38 & 30 & 0.6483 & 5.423 \\
\hline $\mathrm{InAs}$ & 83 & 45 & 39 & 0.6058 & 4.957 \\
\hline $\mathrm{GaSb}$ & 88 & 40 & 43 & 0.6095 & 5.383 \\
\hline $\mathrm{GaAs}$ & 118 & 53 & 59 & 0.5653 & 5.465 \\
\hline $\mathrm{AlSb}$ & 89 & 44 & 41 & 0.6135 & 4.500 \\
\hline $\mathrm{Si}$ & 166 & 64 & 80 & 0.5431 & 2.616 \\
\hline $\mathrm{Ge}$ & 129 & 48 & 67 & 0.5640 & 5.607 \\
\hline $\mathrm{Cu}$ & 168 & 121 & 75 & 0.3520 & 16.5 \\
\hline $\mathrm{Au}$ & 186 & 157 & 42 & 0.4078 & 14.0 \\
\hline $\mathrm{Pt}$ & 346.7 & 250.7 & 76.5 & 0.3924 & 9.0 \\
\hline $\mathrm{Ni}$ & 246 & 147 & 124 & 0.3620 & 13.4 \\
\hline $\mathrm{V}$ & 228 & 119 & 43 & 0.30282 & 8.4 \\
\hline $\mathrm{Mo}$ & 440 & 172 & 122 & 0.31468 & 4.8 \\
\hline $\mathrm{Nb}$ & 440 & 172 & 122 & 0.333007 & 7.3 \\
\hline $\mathrm{Ta}$ & 261 & 157 & 82 & 0.33026 & 6.3 \\
\hline $\mathrm{C}$ & 1076 & 125 & 577 & 0.3567 & 1.00 \\
\hline $\mathrm{GaP}$ & 144 & 65 & 71 & 0.5450 & 3.96 \\
\hline $\mathrm{InP}$ & 1025 & 58 & 46 & 0.5868 & 4.040 \\
\hline $\mathrm{ZnS}$ & 98 & 63 & 45 & 5.419 & 6.36 \\
\hline $\mathrm{ZnTe}$ & 72 & 41 & 31 & 6.101 & 7.500 \\
\hline $\mathrm{HdTe}$ & 61 & 43 & 20 & 6.480 & 4.750 \\
\hline & 59 & 41 & 23 & 6.462 & 4.683 \\
\hline
\end{tabular}

Some of these thermal expansion coefficients were taken from: R.S. Krisnan, R. Srinivasan and S. Devanarayanan, "Thermal Expansion of Crystals," Pergamon Press (1979). The other parameters were found by Chris Litteken. 


\section{"Intrinsic" or Growth Stresses}

It is commonly observed that stresses develop in polycrystalline and amorphous films during isothermal deposition or growth. Since these stresses cannot be attributed to thermal mismatch strains or epitaxial effects they are called "intrinsic". The term "intrinsic" is a poor choice since it implies that a freestanding film can have stresses in it as a consequence of its growth structure. As we have seen, stresses in films arise from misfit with the substrate on which they are deposited. Thus, a better term for "intrinsic" stresses is "growth" stresses. However, the term "intrinsic" is so heavily entrenched in the literature that it will probably not be changed. We consider now various factors that contribute to stresses in films on substrates during growth.

\section{Interfacial Tension Effects}

For extremely thin films, effects associated with the free surfaces and interfaces cause substrate bending to occur and stresses to develop in the film.

\section{$\underline{\text { Surface Energy }}$}

Define $\gamma_{s}$ as the reversible work needed to form (or create) a surface of unit area:

\section{Formation of two surfaces of unit area}

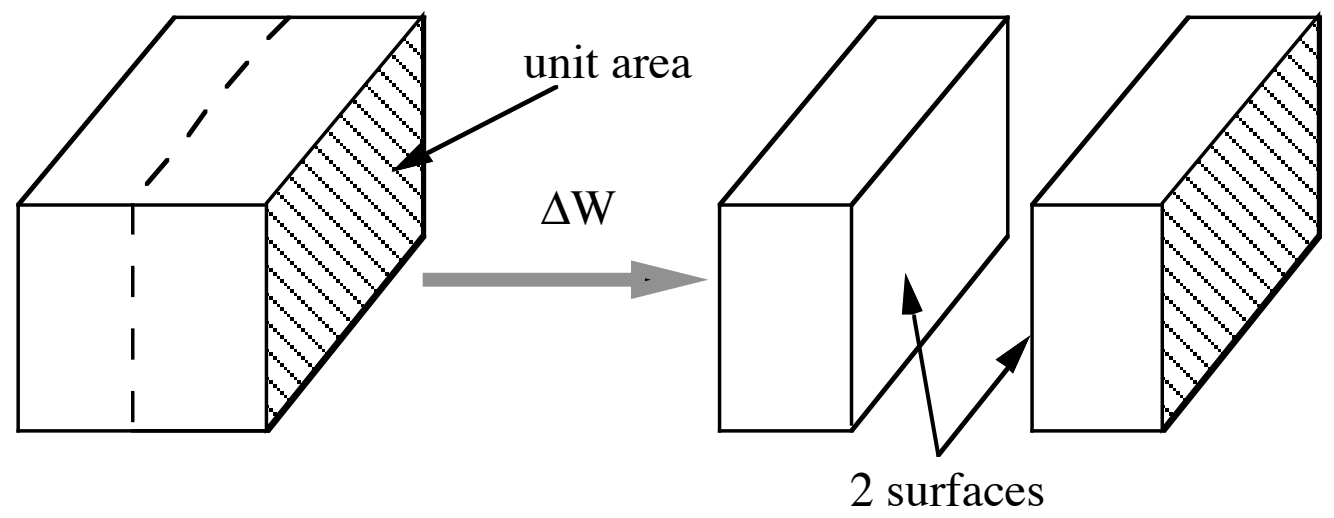

The surface energy is defined as:

$$
\gamma_{s} \equiv \frac{\Delta W}{2} ;
$$

it involves the creation of a surface. 


\section{$\underline{\text { Surface Stress }}$}

Surface stress involves the reversible extension or stretching of a surface.

\section{Stretching of surfaces}

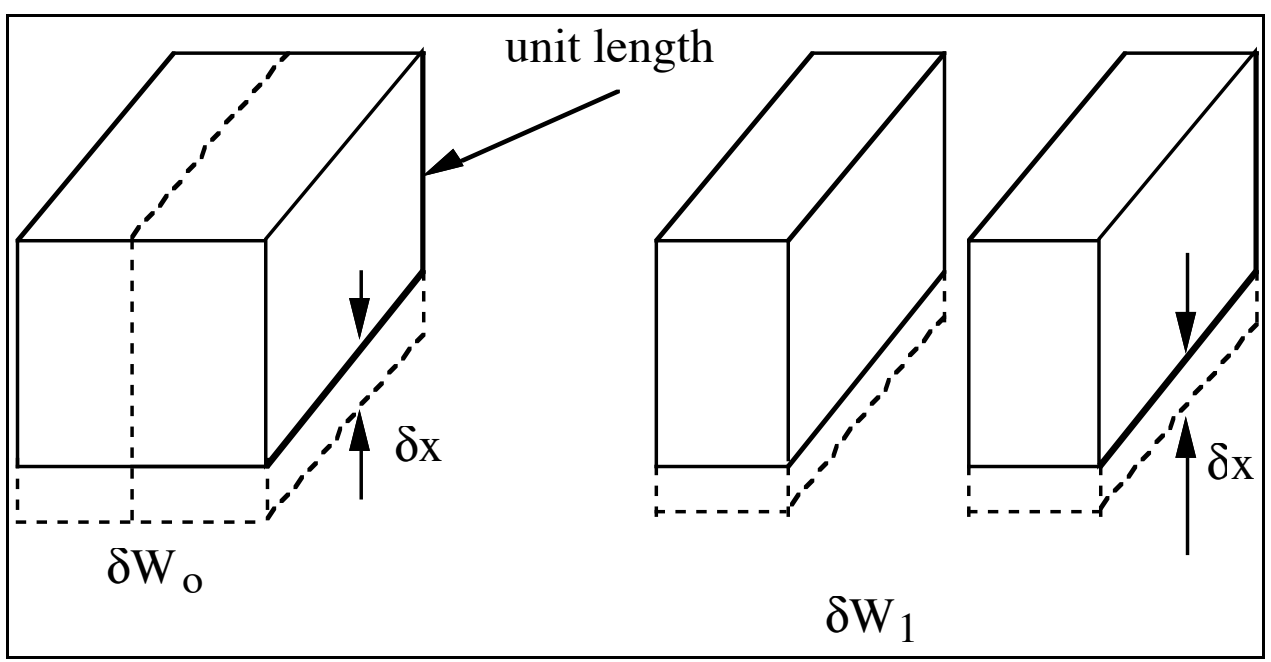

The surface stress is defined as

$$
f_{s} \equiv \frac{\delta W_{1}-\delta W_{o}}{2 \delta x}
$$

it involves the stretching of a surface. 


\section{$\underline{\text { Relation between Surface Energy and Surface Stress }}$}

Consider reversible deformation for two paths: creation of surfaces then stretching of surfaces

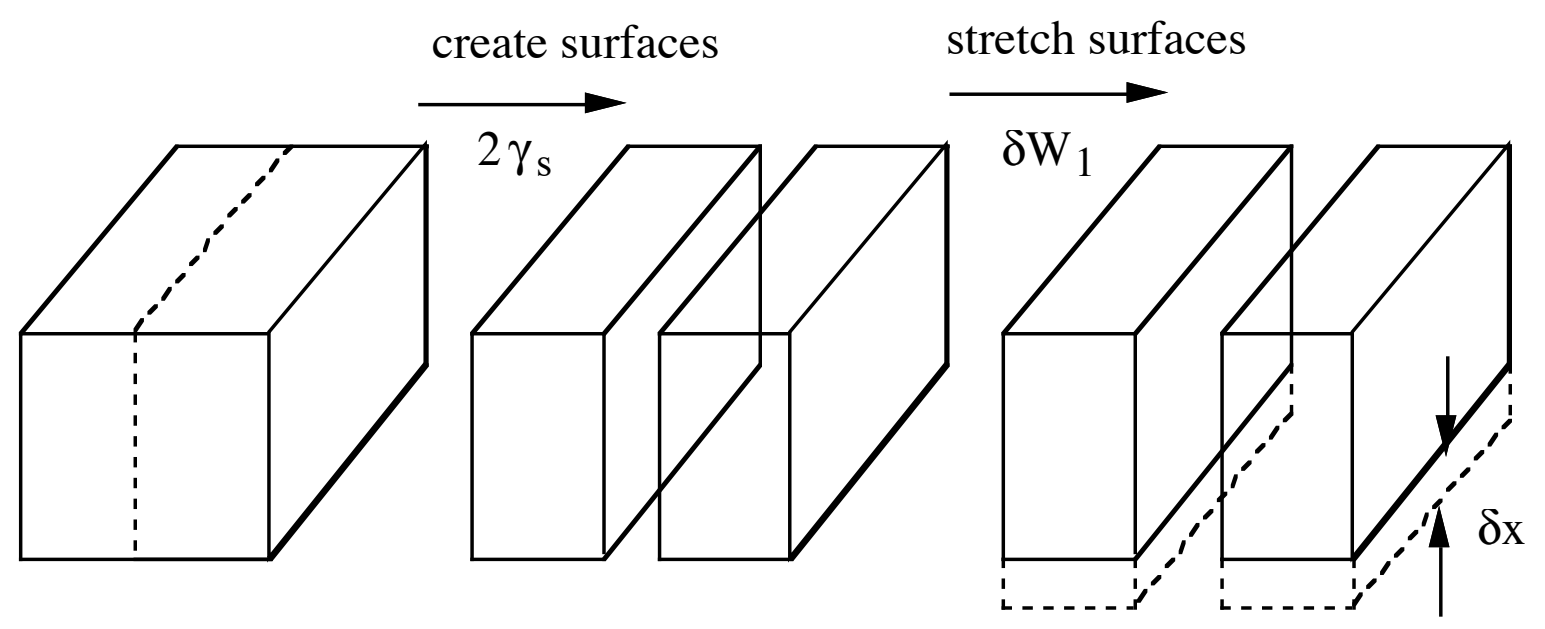

The total change of free energy for this path is: $2 \gamma_{s}+\delta W_{1}$.

Stretching of solid then creation of surfaces

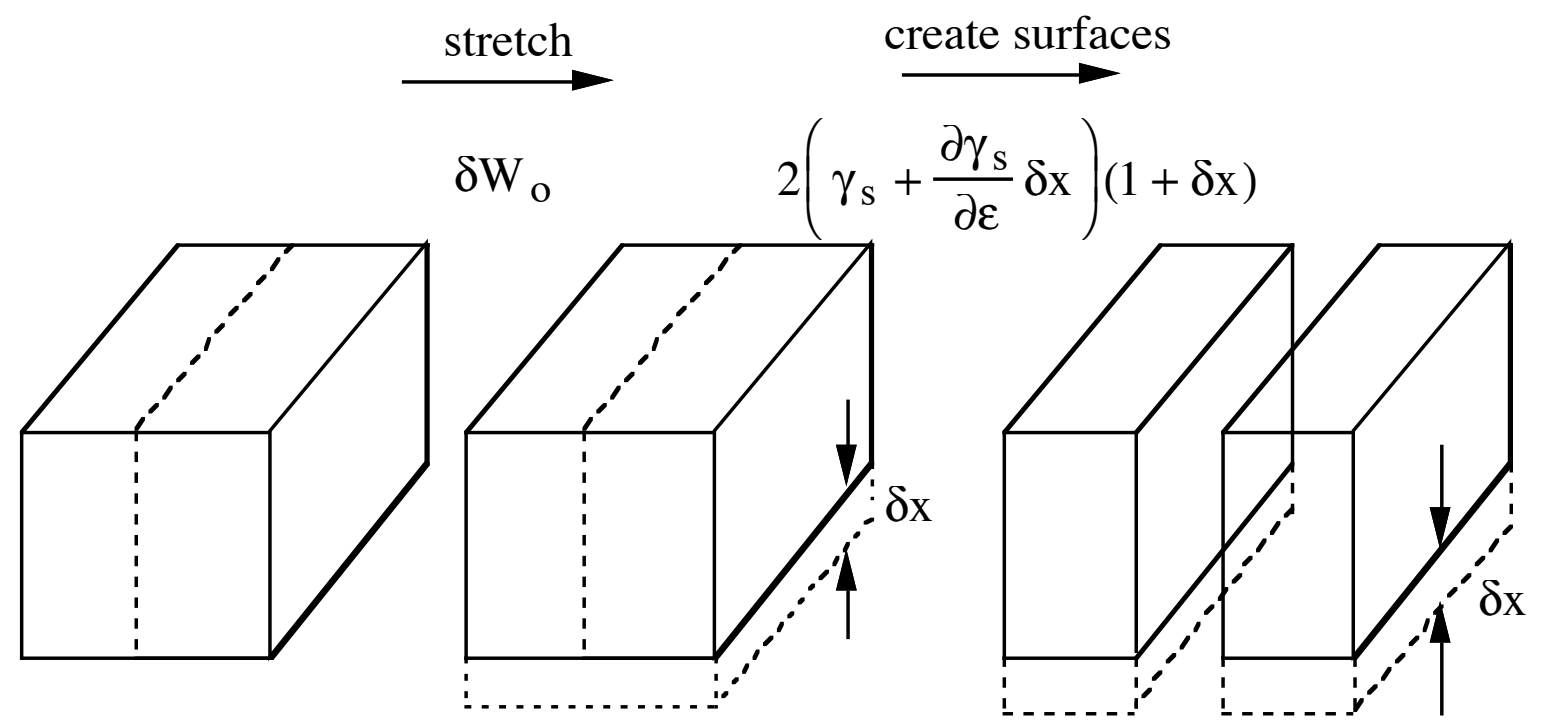

The total change of free energy for this path is:

$$
\delta W_{o}+2\left(\gamma_{s}+\frac{\partial \gamma_{s}}{\partial \varepsilon} \delta x\right)(1+\delta x)
$$


The reversible work for two paths is the same, so

$$
\begin{aligned}
& 2 \gamma_{s}+\delta W_{1}=\delta W_{o}+2\left(\gamma_{s}+\frac{\partial \gamma_{s}}{\partial \varepsilon} \delta x\right)(1+\delta x) \\
& \delta W_{1}-\delta W_{o}=2 \gamma_{s} \delta x+2 \frac{\partial \gamma_{s}}{\partial \varepsilon} \delta x+2 \frac{\partial \gamma_{s}}{\partial \varepsilon}(\delta x)^{2}
\end{aligned}
$$

Omitting the higher order terms and using the definition of surface stress:

$$
\begin{aligned}
& f_{s} 2 \delta x=2 \gamma_{s} \delta x+2 \frac{\partial \gamma_{s}}{\partial \varepsilon} \delta x \\
& f_{s}=\gamma_{s}+\frac{\partial \gamma_{s}}{\partial \varepsilon}
\end{aligned}
$$

This is the surface stress. This relation is called the Shuttleworth relation, after the person who first derived it in 1950.

\section{Interface Stress and the Coordinate System}

Some may be confused by the form of the standard equation for the interface stress. The second term indicates a variation of an energy with respect to elastic strain, but the first term seems to suggest no such variation. Since the interface stress must represent an energy variation with respect to elastic strain, in order to exert stresses on the rest of the body, it might be helpful to clarify the origin of the first term in this expression. This has been discussed by Nix and Gao (Scripta Materialia, 39, 1653 (1998)).

We can show that if Lagrangian coordinates, imbedded in the elastically deforming material, are used in the analysis, then the interface stress appears explicitly as a variation of interfacial free energy with elastic strain, as expected intuitively. In this description the reference area does not change with strain. It is like using engineering strains, where everything is referenced to the original dimensions of the sample. But if a fixed, Eulerian frame of reference is used, such that the interface area changes with strain, then a second term involving the interfacial free energy itself appears in the resulting expression, as shown above. This is like using the true strain, in which the reference dimensions of the sample change in the course of deformation. 


\section{Lagrangian and Eulerian frames of reference}

Consider a simple one dimensional system to be described using both the imbedded, Lagrangian coordinates and the fixed, Eulerian coordinates. We imagine that the body is stretched in both cases.
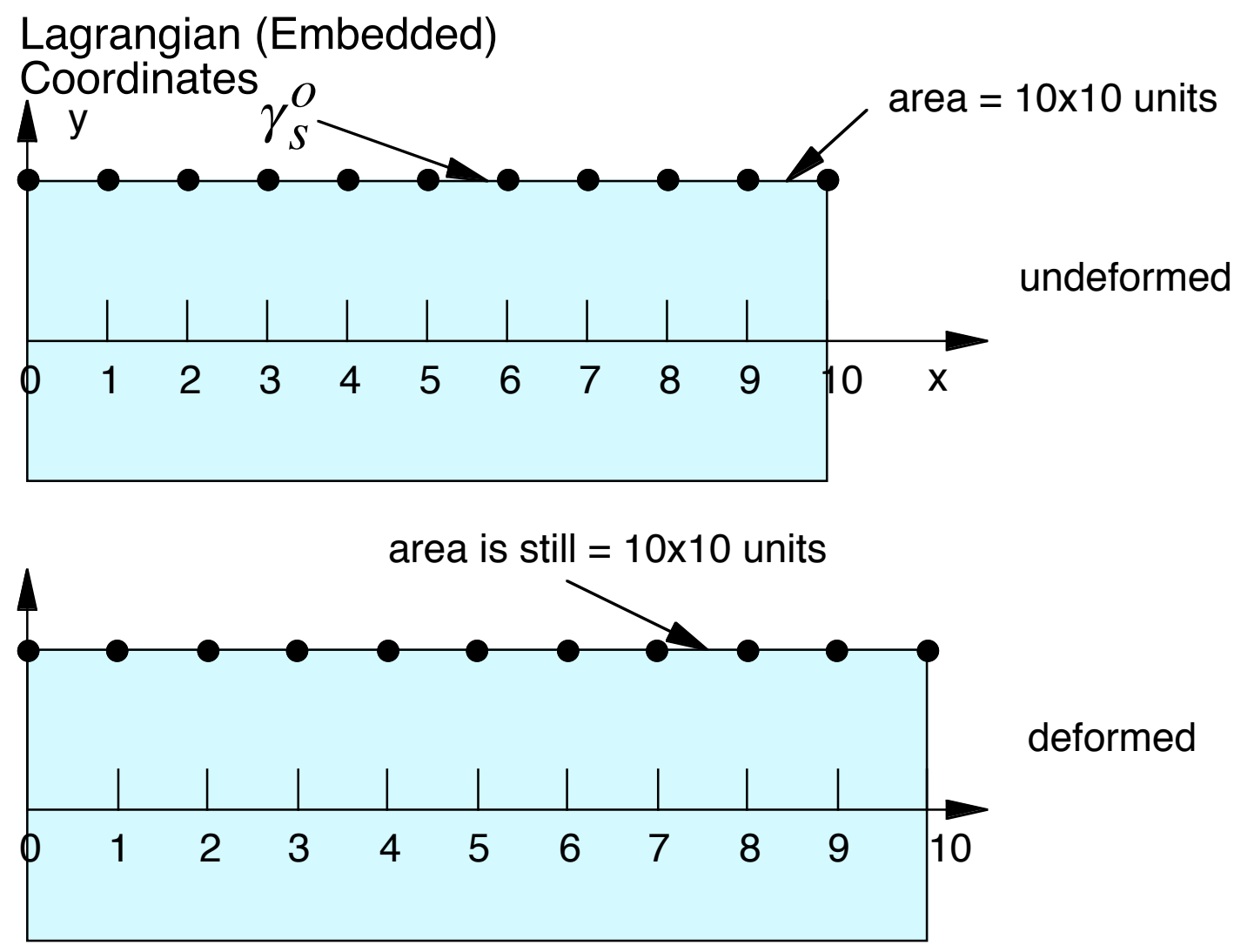

Reference area does not change with deformation

$f_{S}=\frac{\partial \gamma_{S}^{O}}{\partial \varepsilon^{L}} \quad \varepsilon^{L} \begin{aligned} & \text { is the Lagrangian strain, } \\ & \text { based on initial, embedded } \\ & \text { coordinates }\end{aligned}$

$\gamma_{S}^{O}$ is the surface energy per unit undeformed area 


\section{Eulerian (Fixed) Coordinates}

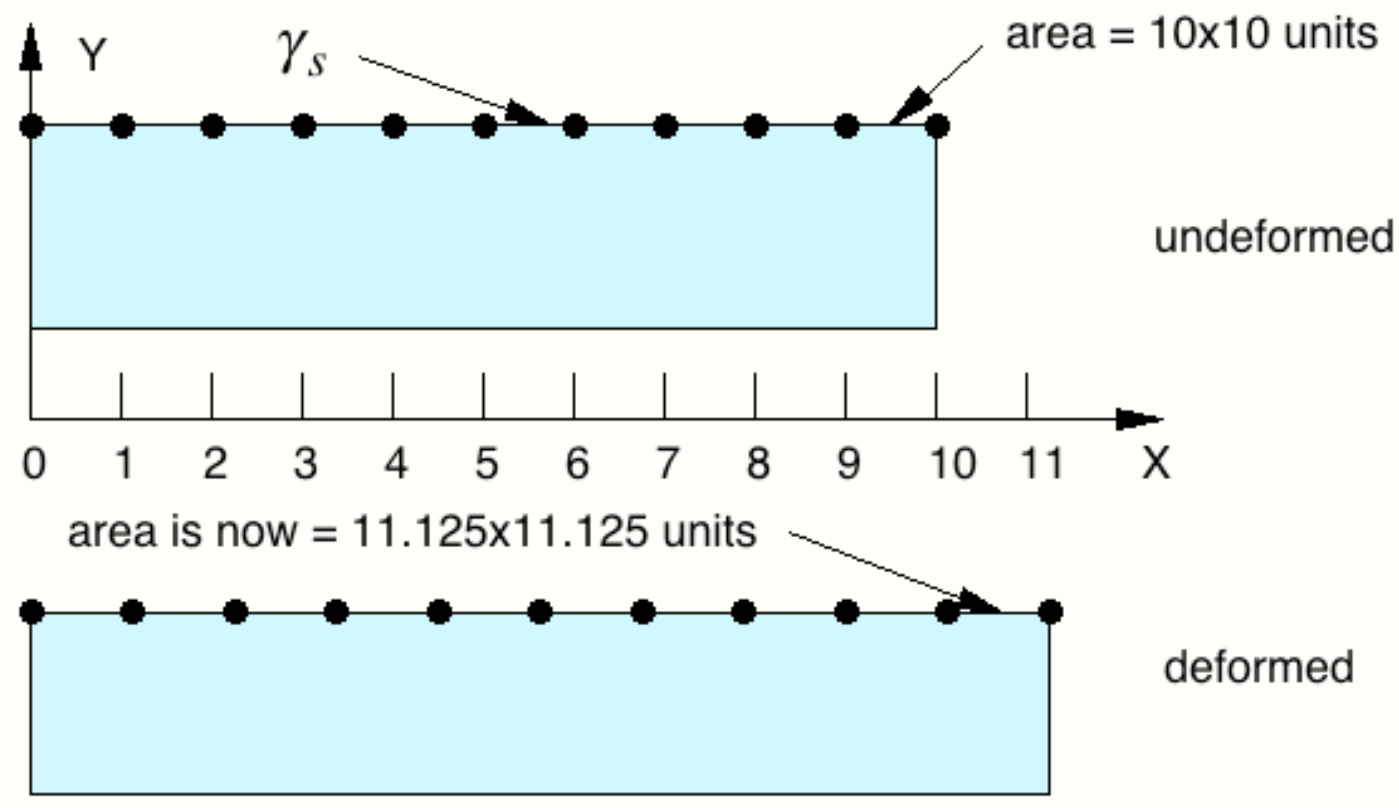

Reference area does change with deformation

$$
f_{s}=\gamma_{s}+\frac{\partial \gamma_{s}}{\partial \varepsilon^{E}} \quad \varepsilon^{E} \begin{aligned}
& \text { is the Eulerian strain, } \\
& \text { based on current, fixed } \\
& \text { coordinates }
\end{aligned}
$$

$\gamma_{s}$ is the surface energy per unit deformed area

In the case of the Lagrangian coordinate system the reference area does not change with deformation. Essentially the initial coordinate system is used, such that the area (in that coordinate system) does not change with strain. Only the energy per unit undeformed area changes with strain. But when the Eulerian (fixed) coordinate system is used, both the reference area and the surface energy per unit deformed area change with strain.

With these distinctions it is possible to show that, in general, the interface stress may be expressed either as

$$
\sigma_{i j}^{L}=\frac{\partial \gamma_{o}}{\partial \varepsilon_{i j}^{L}}
$$


where $\sigma_{i j}^{L}$ and $\varepsilon_{i j}^{L}$ are the stress and strain defined using the Lagrangian, embedded coordinate system and $\gamma_{o}$ is the energy per unit reference area,

or as

$$
\sigma_{i j}^{E}=\delta_{i j} \gamma+\frac{\partial \gamma}{\partial \varepsilon_{i j}^{E}},
$$

where $\sigma_{i j}^{E}$ and $\varepsilon_{i j}^{E}$ are the stress and strain defined using the fixed coordinate system and $\gamma$ is the energy per unit deformed area and where $\delta_{i j}$ is the Kronecker delta defined as

$$
\delta_{i j}=\left(\begin{array}{ll}
1 & 0 \\
0 & 1
\end{array}\right) .
$$

This is the most common form for the interface stress, expressed using the fixed, Eulerian, frame of reference.

Either approach may be used to account for energy changes associated with the stretching of interfaces, but care must be taken in the definitions and in the calculations. We note that the first term on the right hand side of this equation arises from the Eulerian coordinates used to define the interface stress and strain.

\section{Effects of surface stresses on elastic strains in solids}

Consider a small spherical particle of a crystalline material under an internal pressure $p$ and with a surface stress $f_{s}$ :

$$
f_{s}=\gamma_{s}+\frac{\partial \gamma_{s}}{\partial \varepsilon}
$$




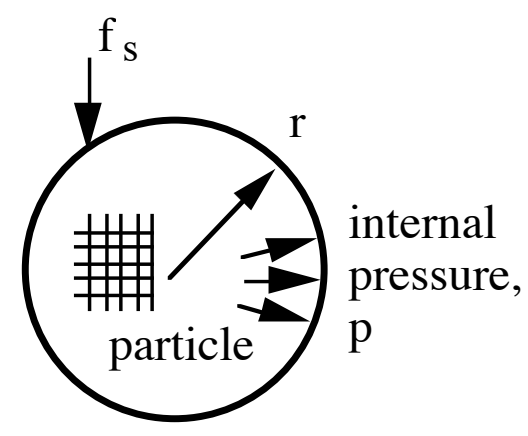

We consider a virtual size variation, $d r$. The corresponding energy variation would be

$$
\delta E=f_{s} \delta A-p \delta V
$$

The surface area $A$ and volume $V$ are:

$$
A=4 \pi r^{2} \quad \text { and } \quad V=\frac{4}{3} \pi r^{3}
$$

which lead to

$$
\delta A=8 \pi r \delta r \quad \text { and } \quad \delta V=4 \pi r^{2} \delta r
$$

Using these relations and the condition that the energy variation must be zero at equilibrium, we have

$$
0=f_{s} 8 \pi r \delta r-p 4 \pi r^{2} \delta r
$$

Solving for the pressure we have

$$
p=\frac{2 f_{s}}{r}
$$

which is called the LaPlace-Young equation. This relation shown that the surface stress creates an internal pressure in a small particle. The pressure can be enormous if the particle is very small. Vermaak et al. (J.S. Vermaak, C.W. Mays, and D. Kuhlmann-Wilsdorf, Surface Science 12 pp. 128-133 and pp 134-140 (1968)) have shown that this effect causes the lattice parameter in very small 
particles of gold to be smaller that the stress-free lattice parameter. This is direct evidence that surface stress produces real elastic strains in a material.

Vermaak et al. measured lattice parameters in small gold crystallites.

\section{Small particle under capillarity forces}

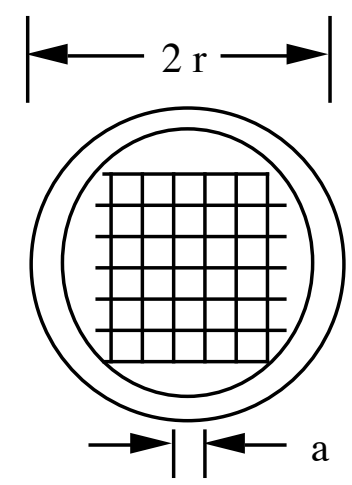

Starting with the pressure,

$$
p=\frac{2 f_{s}}{r}
$$

we also express the pressure in terms of the volumetric strains in the particle:

$$
p=-B \frac{\Delta V}{V}=-3 B \frac{\Delta a}{a}
$$

where $B$ is the bulk modulus of the particle. Equating these expressions for the pressure we get

$$
p=-\frac{3}{K} \frac{\Delta a}{a}=\frac{2 f_{s}}{r}
$$

where $\mathrm{K}$ is the compressibility of the particle. Thus

$$
\frac{\Delta a}{a}=-\left(\frac{2 K}{3}\right) \frac{f_{s}}{r}
$$

This relation suggests that the lattice parameter would vary linearly with the reciprocal of the particle radius. This is exactly what Vermaak et al. found. 
Schematic of Vermaak et al. data

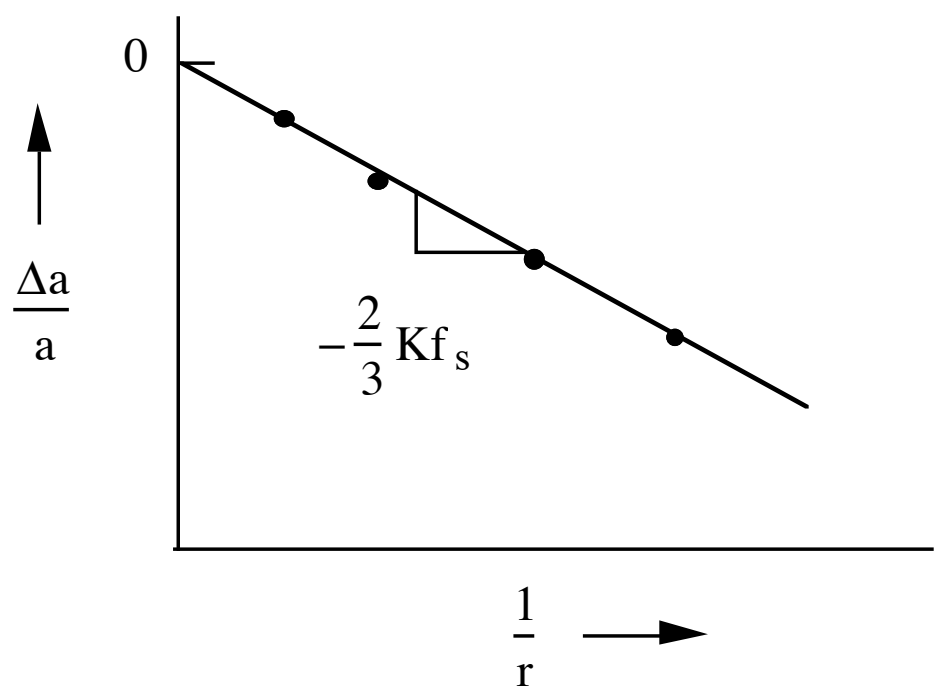

From their measurements they found for gold:

$$
f_{s}=1.175 \mathrm{~N} / \mathrm{m} .
$$

\section{$\underline{\text { Surface Stress as a Source of Intrinsic Stress }}$}

Consider a free-standing multilayer with interfaces having an interfacial stress $\mathrm{f}$. The surface stress induces an in-plane biaxial compression in the multilayer as shown by the following diagram

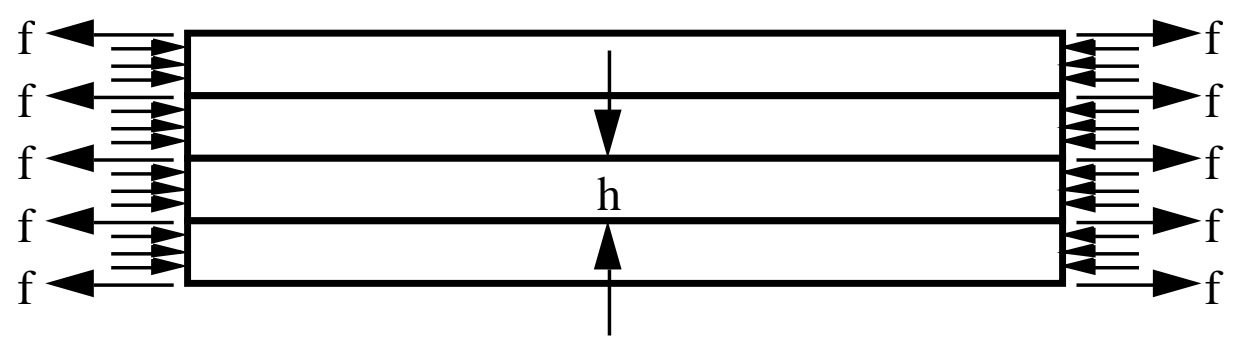

The biaxial stress induced in the multilayer is

$$
\sigma=\frac{f}{h}
$$

Using $h=100 \AA=10^{-2} \mu m=10^{-8} \mathrm{~m}$ and $\quad f=1 \mathrm{~J} / \mathrm{m}^{2}$ 
we have

$$
\sigma=-10^{8} \mathrm{~N} / \mathrm{m}^{2}=-100 \mathrm{MPa}
$$

a significant stress!

Alternatively if $h=1 \mu \mathrm{m}=10^{-6} \mathrm{~m}$, we have

$$
\sigma=-10^{6} N / m^{2}=-1 M P a
$$

a relatively small stress

Now consider a multilayered film deposited onto a solid substrate. Each of the interfaces in the sample will have an interfacial stress and each will exert forces on the entire composite. 
Multilayered film on substrate (capillarity effect)
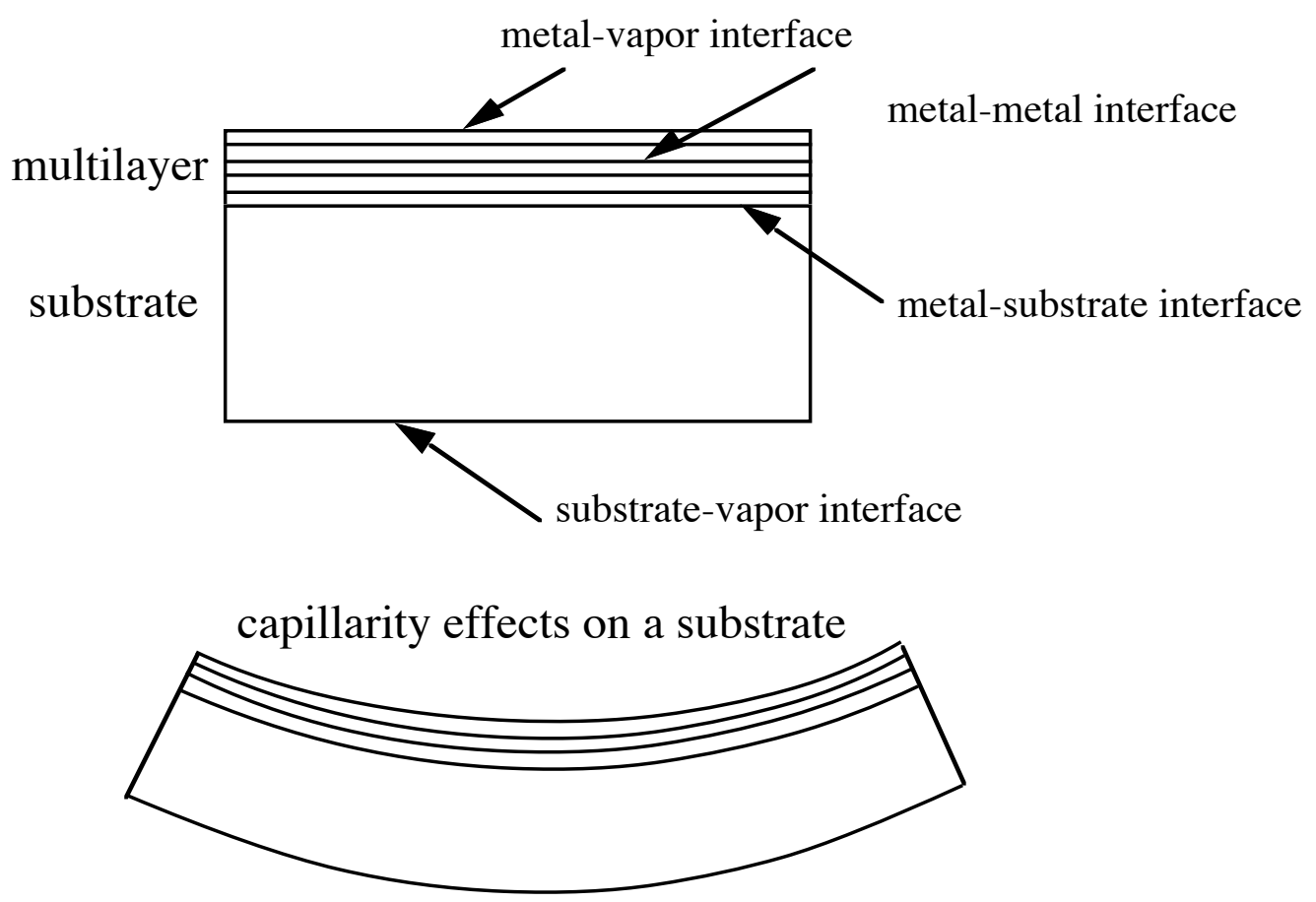

The interfaces shown would have interfacial stresses: $f_{m-v}, f_{m-m}, f_{m-s}$ and $f_{s-v}$ respectively. Because many more interfaces are located on top of the substrate the interfacial stresses would cause the substrate to bend in the way shown. Such a curvature would be created if the films were in biaxial tension, but in this case the films are actually in biaxial compression as all of the deformation is caused by the interfacial stresses.

\section{Observation of Stresses in Thin Films During Vapor Deposition of Metals - intrinsic stress mechanisms}

R. Abermann and R. Koch, Thin Solid Films, 62195 (1979)

R. Abermann and R. Koch, Thin Solid Films, $\underline{66} 217$ (1980)

R. Abermann, Proc. MRS Symposium, 239, 25 (1992).

T. Ueda, G.F. Simenson, W.D. Nix and B.M. Clemens, MRS Symposium, 382, 279 (1995)

V. Ramaswamy, W.D. Nix, B.M. Clemens, Scripta Materialia; vol.50, no.6, p.711-15 (2004). 
Beam deflection and wafer curvature techniques have been used to monitor film stress in $\mathrm{Ag}, \mathrm{Cu}, \mathrm{Au}$ and other metals and multilayers as a function of deposition time (or thickness), in the very early stages of growth.

\section{Beam deflection technique for in-situ stress measurement}

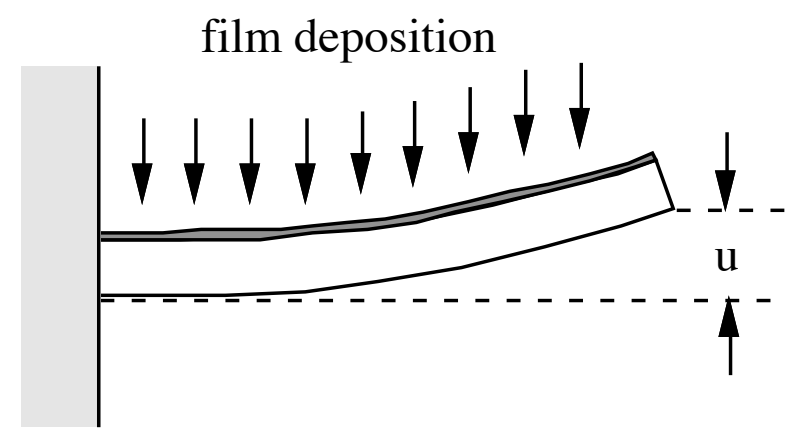

We have seen that this deflection is directly proportional to the product of the stress in the film and the film thickness:

$$
\sigma_{f} t_{f}=\left(\frac{E}{1-v}\right)_{s} \frac{t_{s}^{2}}{3 L^{2}} u
$$

where $\mathrm{u}$ is the measured deflection at the end of the beam. Thus we measure the product of the film stress and film thickness - this is force per unit width $F / W$ $(\mathrm{N} / \mathrm{m})$. More precisely, the stress in question is the average stress through the thickness of the film. If the biaxial stress in the film varies significantly with position through the thickness then this expression should be written as

$$
\bar{\sigma}_{f} t_{f}=\left(\frac{E}{1-v}\right)_{s} \frac{t_{s}^{2}}{3 L^{2}} u=\frac{F}{W}
$$

where $\bar{\sigma}_{f}$ is the average biaxial stress in the film and corresponding the force per unit width, $F / W$ is expressed. This may be expressed as

$$
\int_{0}^{t_{f}} \sigma_{f}(z) d z=\left(\frac{E}{1-v}\right)_{s} \frac{t_{s}^{2}}{3 L^{2}} u=\frac{F}{W}
$$

Here $\sigma_{f}(z)$ is the current stress distribution in the film and $u$ is the current displacement. If the stresses are "frozen in" and invariant with time after they have been established, then each increment in the thickness of the growing film 
will produce a corresponding increment in displacement which must be associated with the stress in the added layer; thus

$$
\delta \int_{0}^{t_{f}} \sigma_{f}(z) d z=\left(\frac{E}{1-v}\right)_{s} \frac{t_{s}^{2}}{3 L^{2}} \delta u=\delta\left(\frac{F}{W}\right)
$$

or

$$
\begin{gathered}
\sigma_{f}\left(t_{f}\right) \delta t_{f}=\left(\frac{E}{1-v}\right)_{s} \frac{t_{s}^{2}}{3 L^{2}} \delta u=\delta\left(\frac{F}{W}\right) \\
\sigma_{f}\left(t_{f}\right)=\left(\frac{E}{1-v}\right)_{s} \frac{t_{s}^{2}}{3 L^{2}}\left(\frac{\delta u}{\delta t_{f}}\right)=\frac{\delta\left(\frac{F}{W}\right)}{\delta t_{f}} .
\end{gathered}
$$

So the variation of the force per unit width with respect to an increment in thickness at any point would be the biaxial stress in the last layer to be added, provided that all of the assumptions about frozen in stresses are valid. Some hypothetical force (per unit width) vs. deflection curves are shown below:

If the stress (tension) in the film is constant through the thickness of the film and invariant with time, then the following force - thickness curve would be obtained. 
Force-thickness curve for constant stress
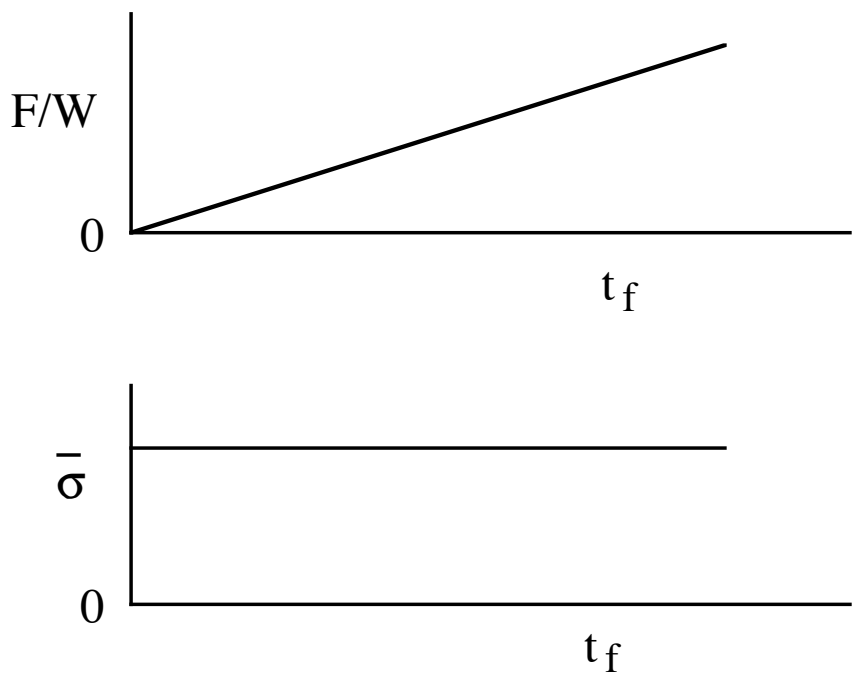

The average stress in the film is given by the slope of this line.

If the stress in the film varies with depth but is invariant with time then the force - thickness curve would be as follows:

\section{Force-thickness curve for position dependent stress}

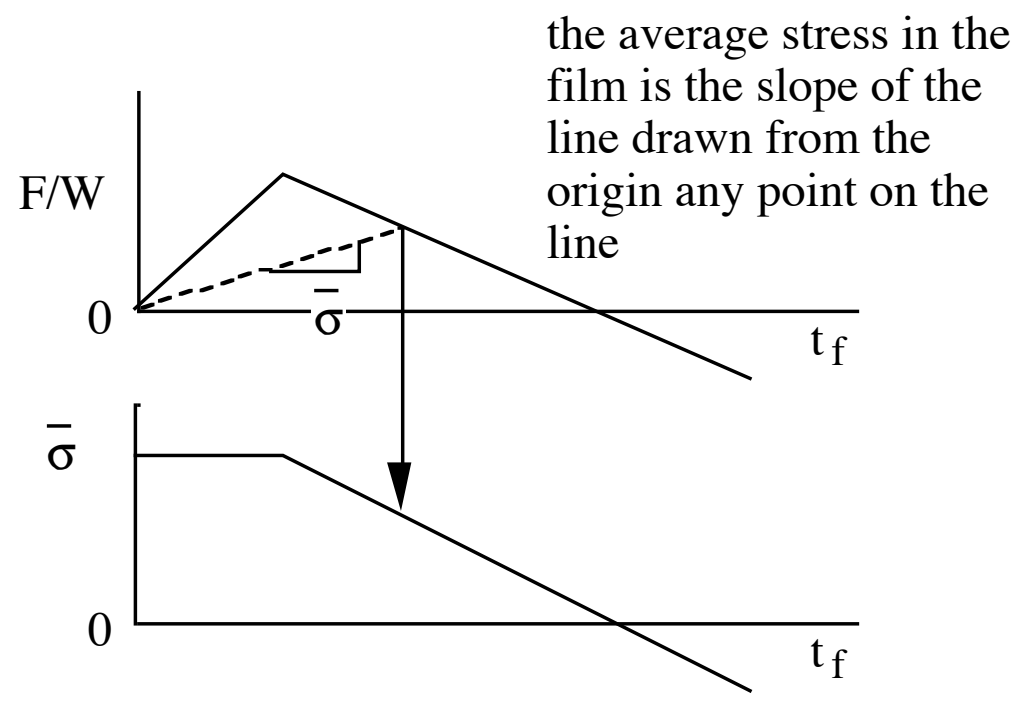

The slope of line drawn from origin to the curve gives the average stress in the film at that thickness. The slope of the curve at any point gives the stress in the last layer to be added at that point. 
As a third example consider a film in which the stress changes sign during growth. The corresponding force-thickness curve would appear as follows:

\section{Force-thickness curve for position dependent stress}

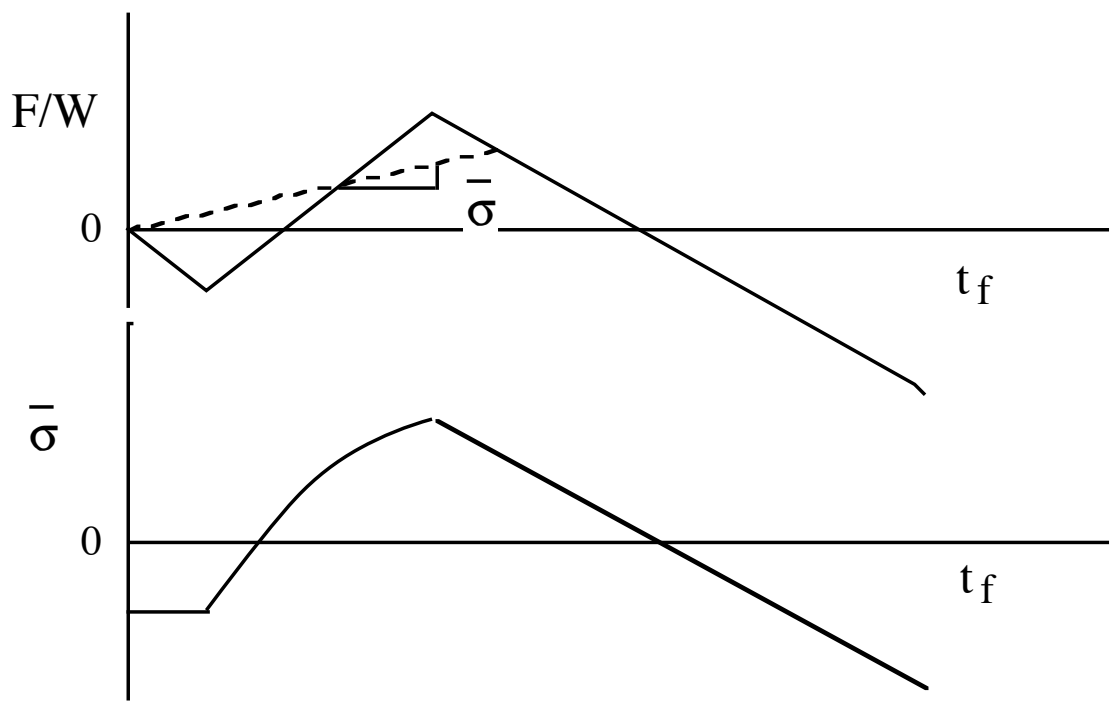

We now consider now results found by Abermann and Koch. They found that the force (per unit width) varies about linearly for refractory materials with low adatom mobility, suggesting that the stress developed in these materials is essentially constant and independent of position through the thickness.

\section{Typical results for materials with low adatom mobility}

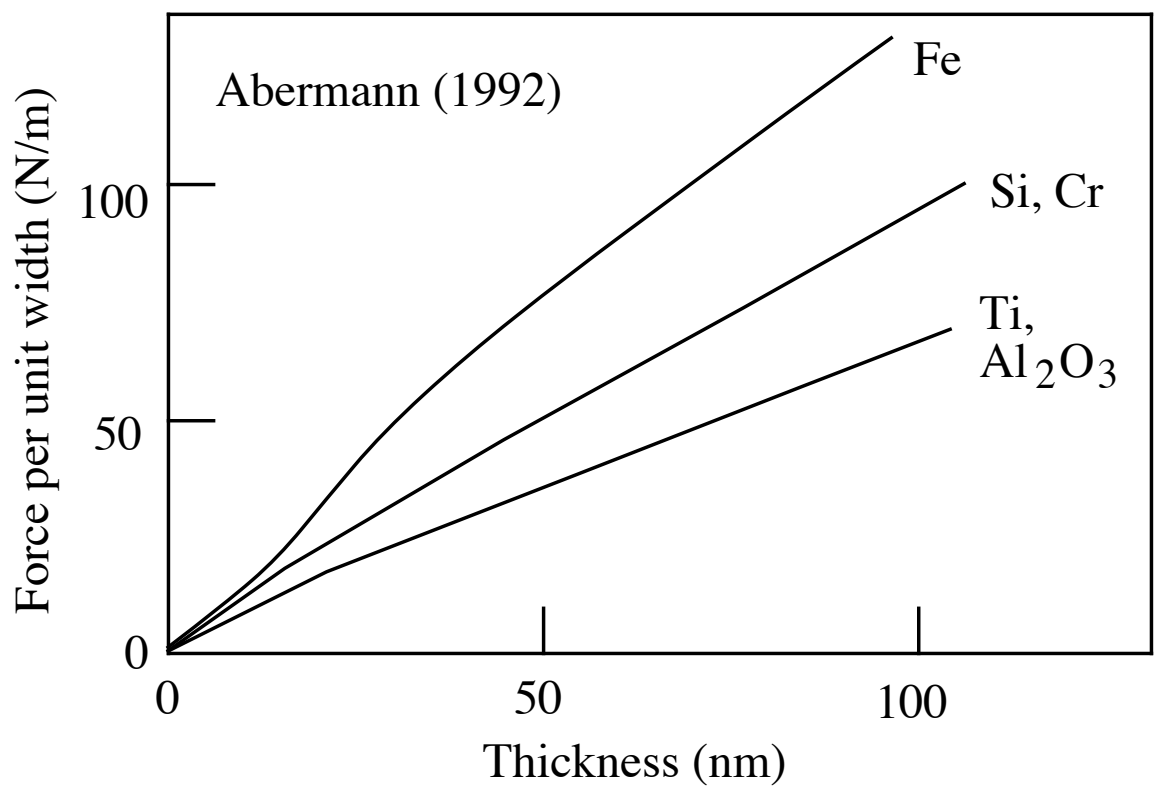


Abermann has shown that the stress generation in metal films is very much less if the temperature is raised. This is shown for the evaporation of $\mathrm{Cr}$ as a function of temperature.

Stress generation in $\mathrm{Cr}$ as a function of temperature

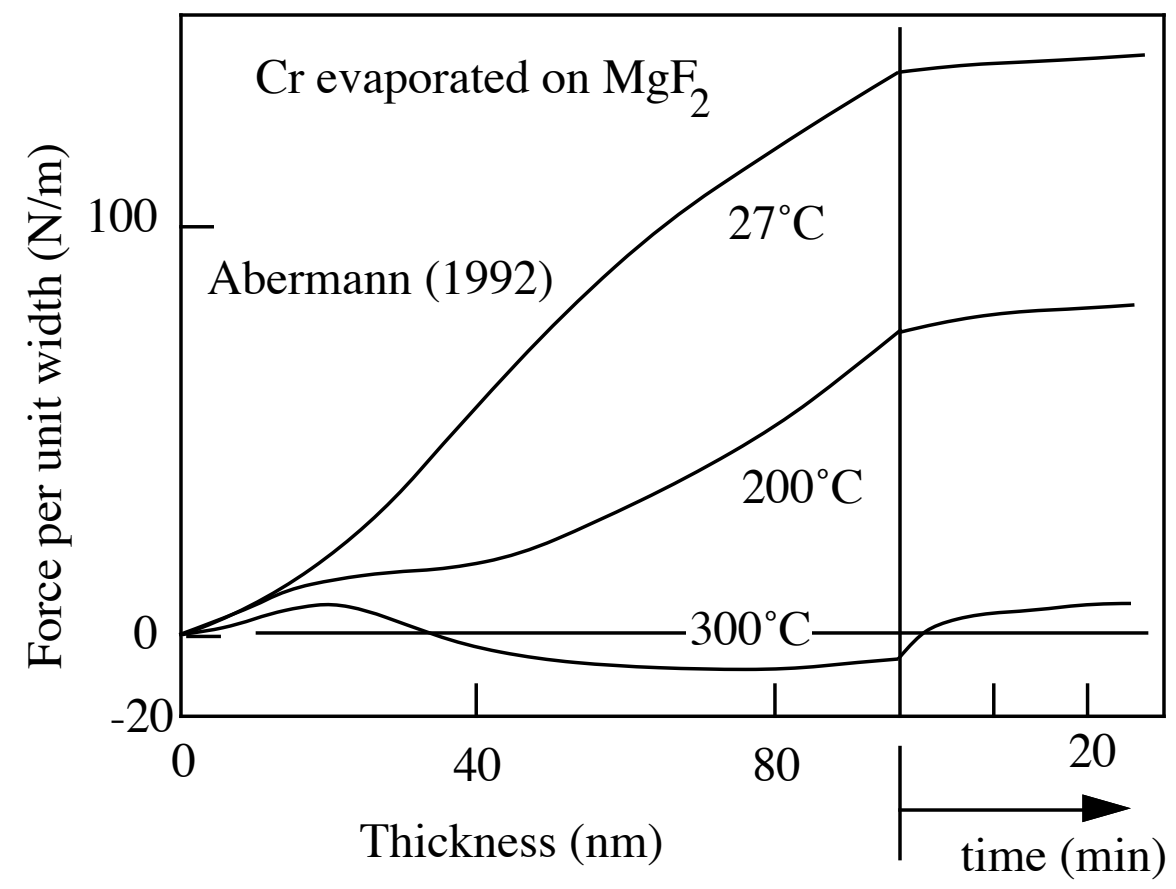

This suggests that high adatom mobility leads to much smaller stresses in evaporated films. This is also suggested by the results for $\mathrm{Cu}$ and $\mathrm{Au}$ shown below: 
Abermann results for $\mathrm{Cu}$ and $\mathrm{Au}$

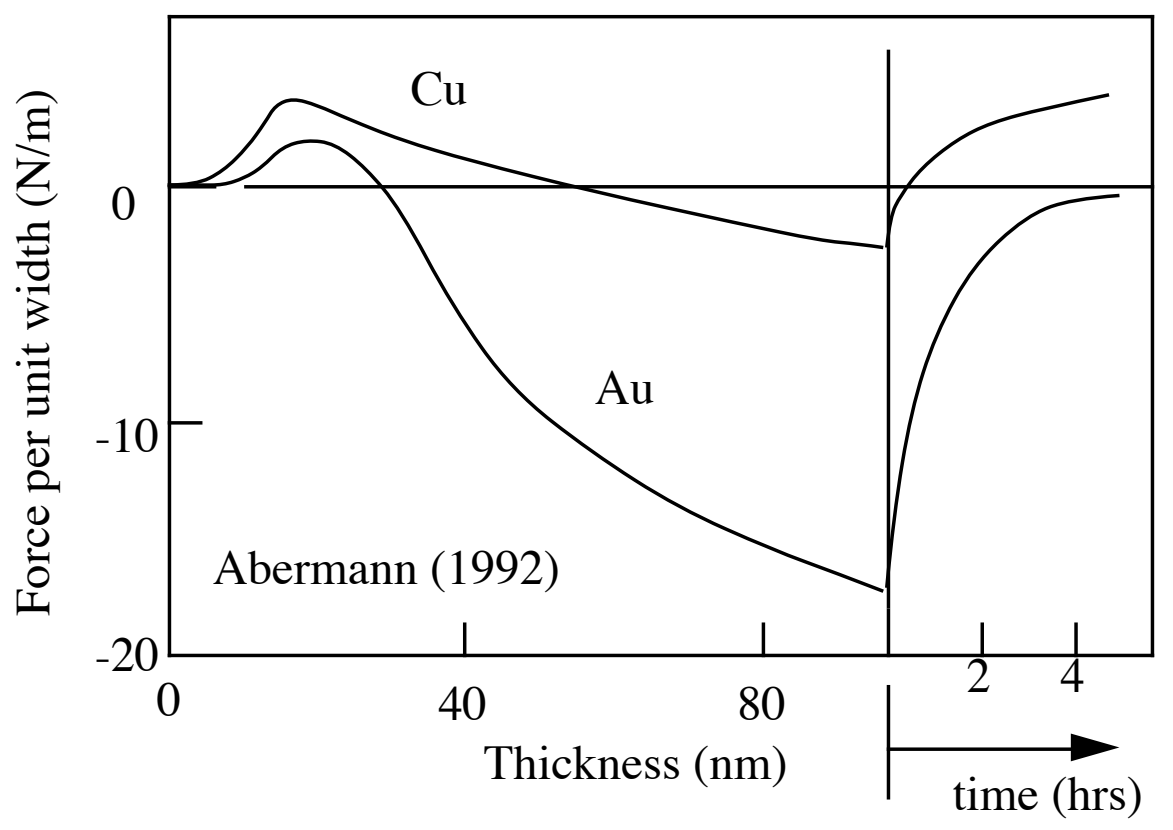

Schematic representation of Abermann's results for metals with high adatom mobility

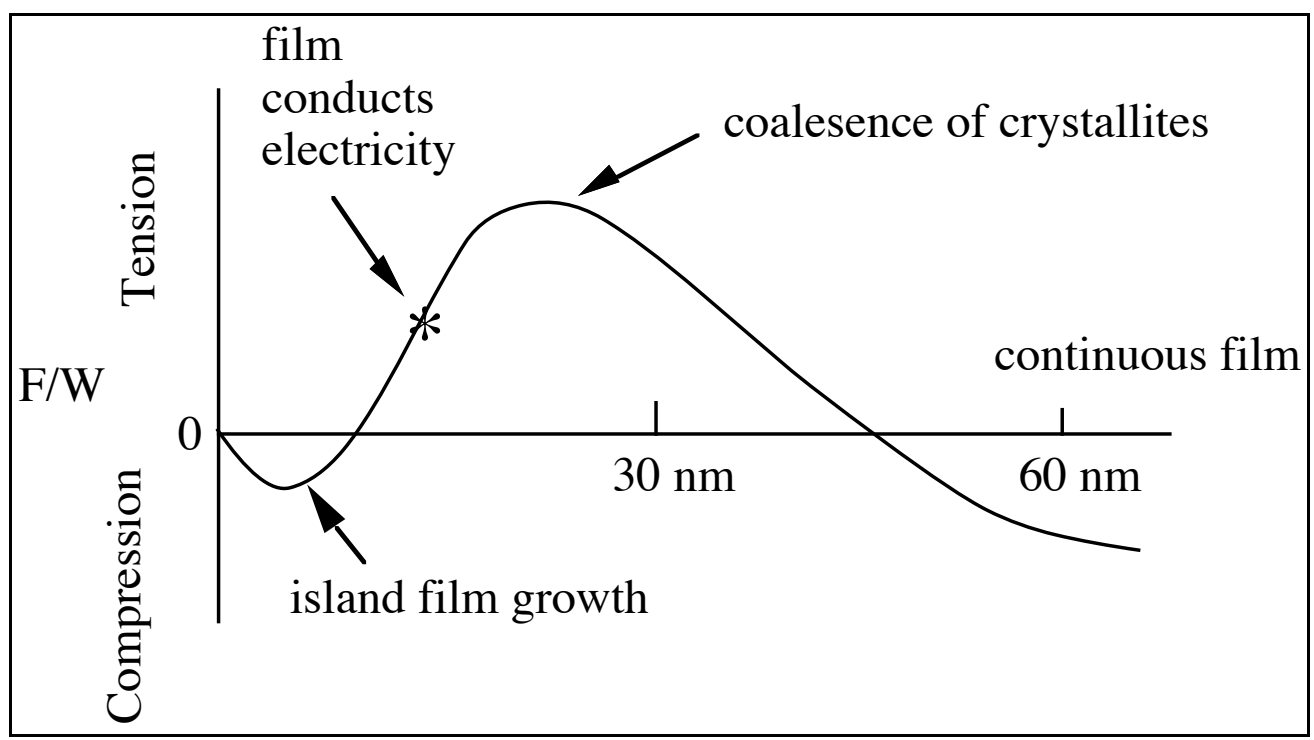

It is widely believed that the tensile hump in the curve is associated with the coalescence of crystallites. As noted in the figure, the metal films will conduct electricity only after a significant tension has developed. This strongly suggests that the tensile hump is associated with crystallite coalescence. 
Recent studies by Ramaswamy have revealed very similar stress-thickness properties.

\section{Stress Evolution for a sputtered Pt film on a thin glass substrate}

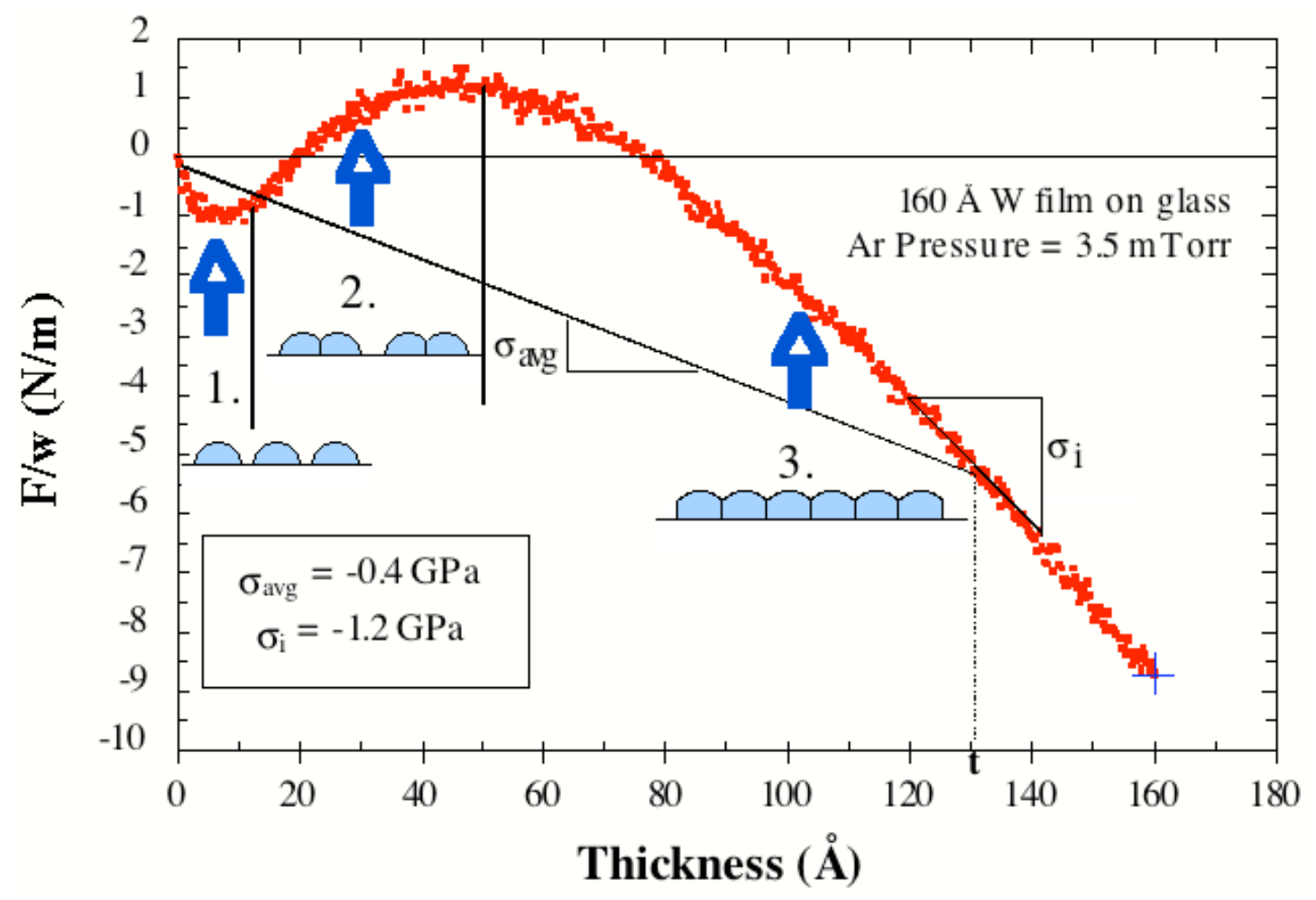

Below we discuss how crystallite coalescence can be expected to create tensile stresses.

In the experiments of Abermann and Koch the compressive stresses in the growing film and the time dependence of stress after $100 \mathrm{~nm}$ film growth may be associated with adsorption or evolution of gaseous species such as oxygen or water vapor into or from the film. This is still controversial. Very early in the growth the discontinuous film appears to be in compression. A mechanism for this is described below.

In spite of confusion about the exact meaning of the experiments, it is clear that both tensile and compressive stresses are produced in growing films. We study the possible mechanisms. 


\section{Compressive Stresses in Discontinuous Films Consisting of Small Crystallites}

We refer here to the work of R. Abermann et al., Thin Solid Films $\underline{52}, 215$ (1978). For very small crystallites, the surface stress compresses the lattice as suggested by the following figure.

Compression of a small crystallite by the surface stress

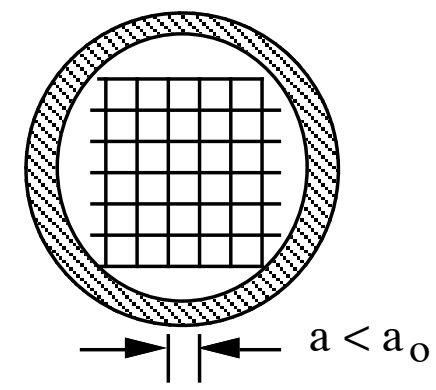

During growth of isolated crystallites, lattice is allowed to expand back to the equilibrium lattice parameter.

Lager crystallite with a near equilibrium lattice parameter

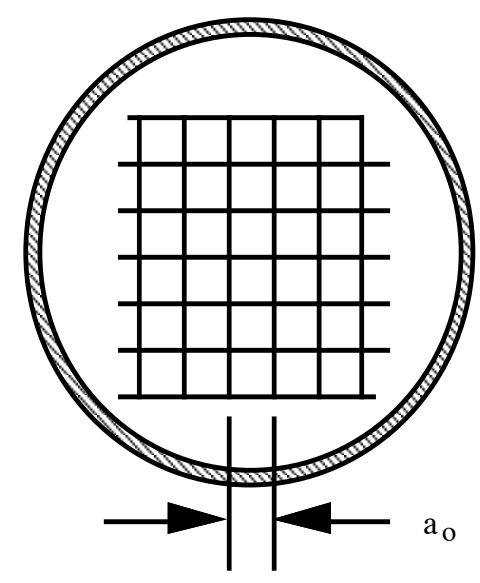

When these crystallites are bonded to substrates, the lattice relaxation associated with growth to a larger radius of curvature is expected to lead to compressive stresses in the film. This causes the substrate to bend in the way shown below. 


\section{Small crystallites on a substrate}

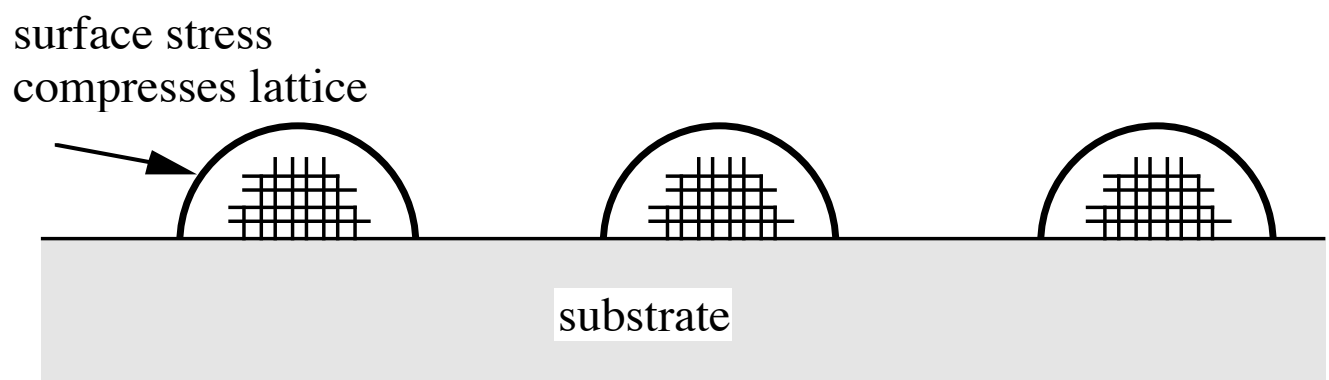

After some growth the strain in the crystallites is relieved and this induces forces in the substrate causing it to bend.

Relaxation of crystallites and forces in the substrate

relaxation of the lattice for larger particles induces forces in the substrate

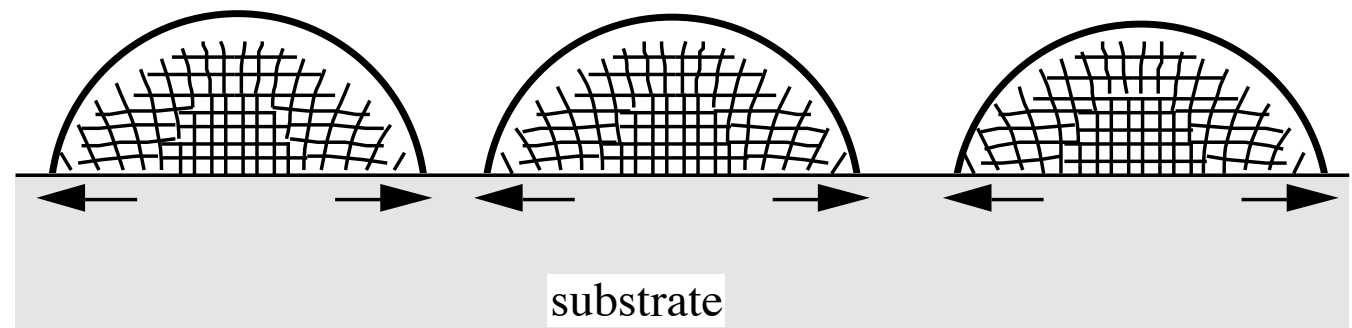

The effect on substrate is to induce a curvature.

Curvature of the substrate

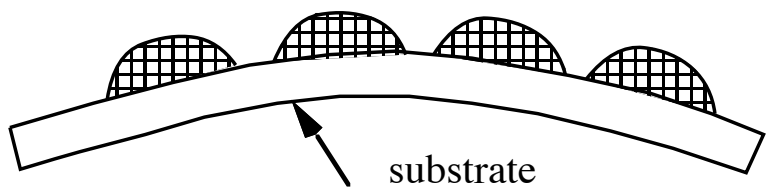

Attachment to the substrate restrains lattice expansion and this leads to compressive stresses in the film. 


\section{Another Example of Capillarity Effects}

We start this discussing by presenting evidence for effect of water on stress in a phosphosilicate glass The glass was saturated in water vapor at $90^{\circ} \mathrm{C}$ for $12 \mathrm{hrs}$ prior to testing. Then the change in stress was measured (by the substrate curvature technique) while the sample was heated and cooled in a dry environment. On heating, water is driven from the film and the stress becomes more tensile. The glass shrinks as it loses water and the constraint of the substrate leads to tensile stresses. Holding at high temperature allows more water to be driven from the film. If water is introduced at room temperature after the sample is cooled, the stress becomes more compressive as the water diffuses back into the glass film. 
Stress changes associated with water in a phosphosilicate glass

\section{Stress changes in a phosphosilicate glass}

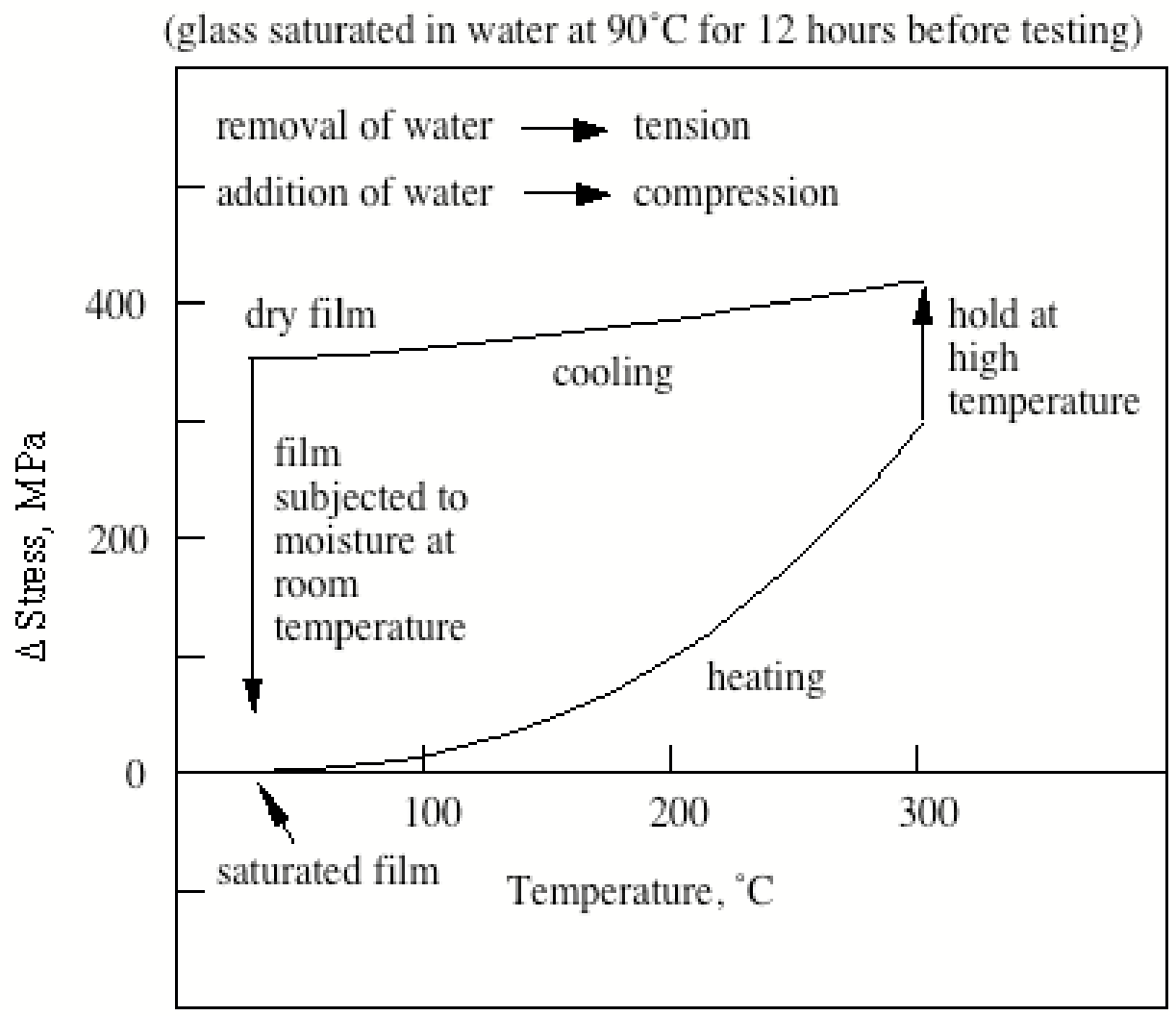

These effects have been used to measure the diffusion of water into phosphosilicate glasses by von Preissig. Shown below are the stress changes associated with isothermal annealing an initially dry glass film in a moist environment. The line through the data corresponds to the solution to the diffusion equation for this problem. 


\section{Diffusion of water in a phosphosilicate glass}

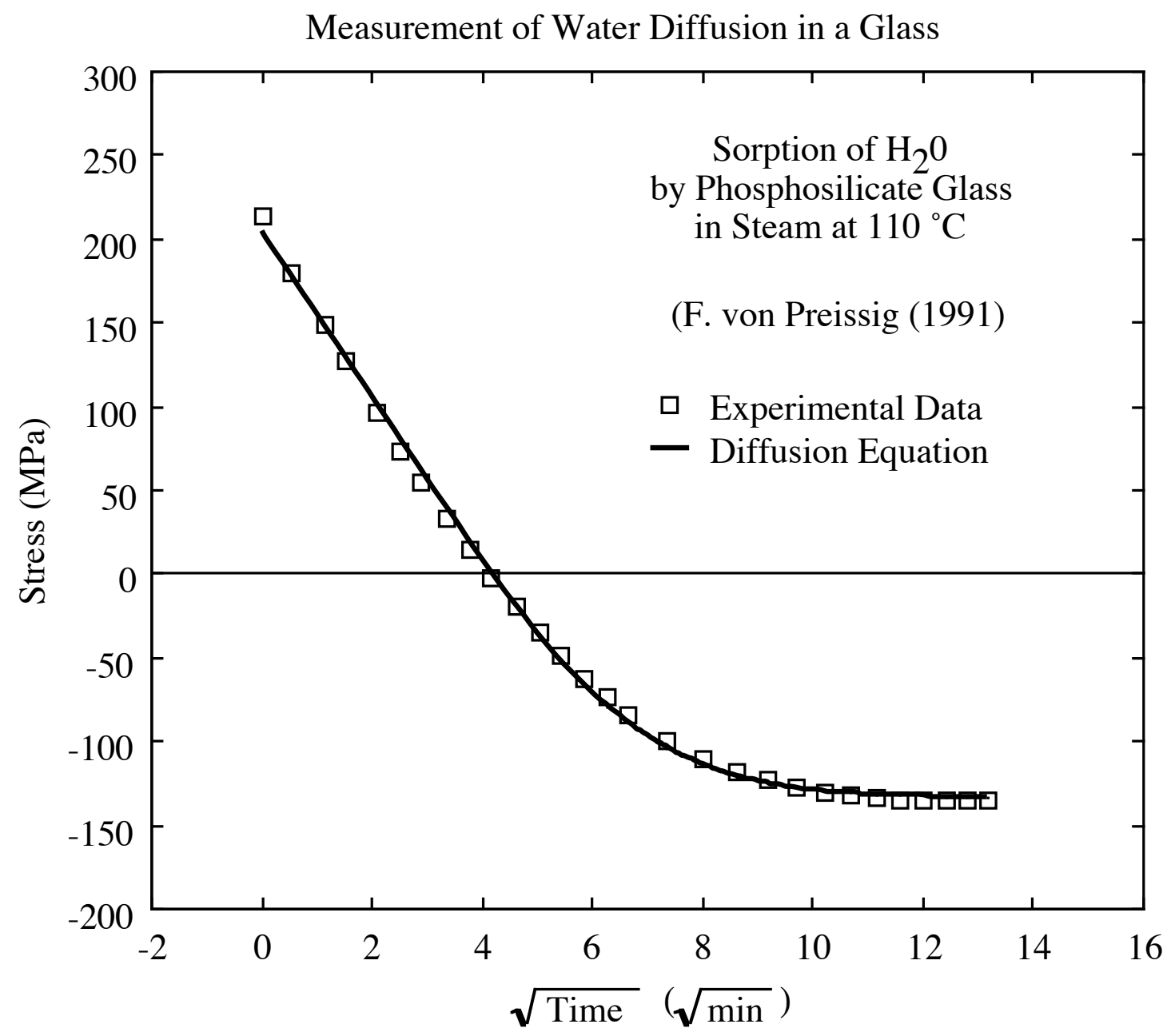

We may understand the stresses in glasses by considering that $\mathrm{H}_{2} \mathrm{O}$ affects the energies (surface stesses) of the internal surfaces. We may think of the glass as being porous, containing internal cavities with free surfaces. When water diffuses into the glass it adsorbs onto the internal surfaces and changes the surface stress. The driving force for adsorption is the reduction of the surface energy (and stress). This causes mechanical forces to be exerted on the glass which would cause it to swell if it were not attached to a substrate. This a mechanical model of the swelling associated with the adsorption of water and the associated stresses. 


\section{Mechanical picture of a porous glass}

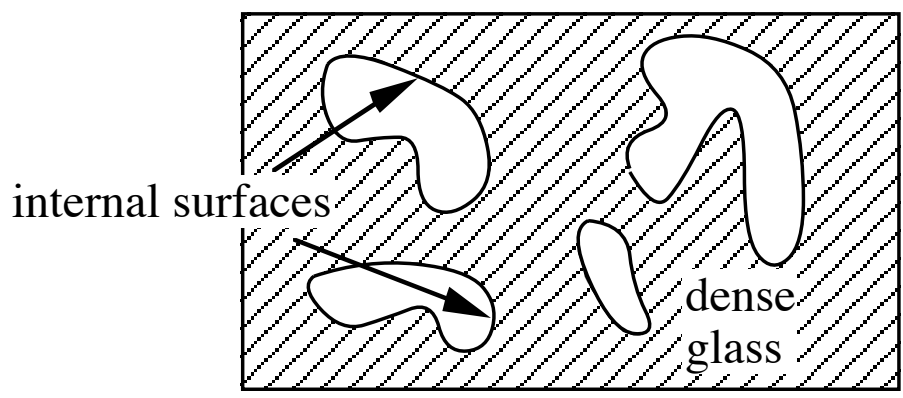

It is known that $\underline{\mathrm{H}}_{2} \mathrm{O}$ adsorbs onto surfaces and decreases surface energy and surface stress. This reduction of surface stress allows the solid to expand and this in turn leads to the development of compressive stresses in the glass if it constrained by being attached to a substrate.

We model this by treating the glass as a collection of small spheres with internal spherical cavities.

\section{Mechanical model of a porous glass}

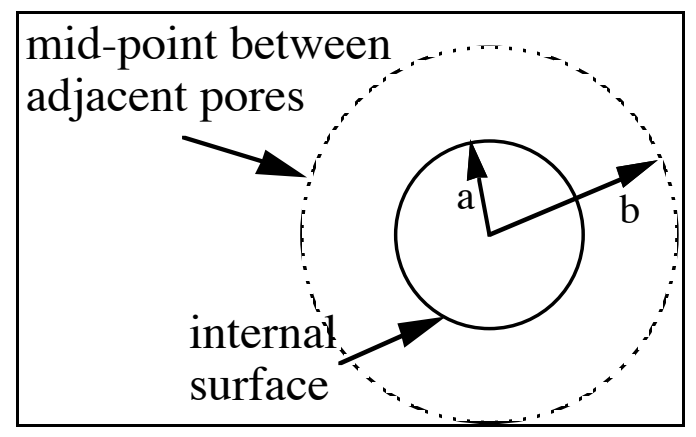

The volume fraction of voids or cavities is

$$
V_{f}=\left(\frac{a}{b}\right)^{3}
$$

where $a$ is the void size and $b$ represents the dimensional scale of the porosity. 


\section{$\underline{\text { Calculate Dilatation Associated with } \mathrm{H}_{2}} \underline{\mathrm{O} \text { Adsorption on Internal Surfaces }}$}

The surface stress for the internal surface is equivalent to tension stresses or tractions applied to that surface. The outer surface should be considered traction free (no surface stress effect) because the outer surface in the model is not a real surface in the material - it is merely the mid-point between adjacent internal cavities.

From the solution for the misfitting sphere (discussed in MSE 206) we can describe the shrinkage of sphere caused by the surface stress acting in the surface of the internal cavity.

\section{Shrinkage of an internal cavity due to surface stresses}

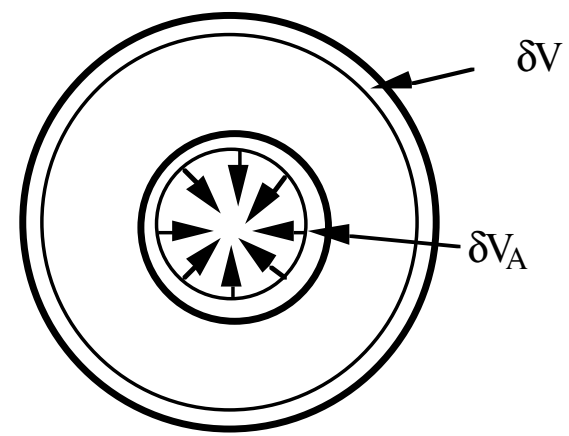

We set the isotropic elastic properties of the glass as follows: $\mu=$ shear modulus, $\mathrm{B}=$ bulk modulus and $\lambda=$ Lame coefficient.

The general solution for displacement $\mathrm{u}_{\mathrm{r}}$ for a spherical problem is

$$
u_{r}=-\delta v_{A} \frac{1}{4 \pi r^{2}}\left[1+\frac{4 \mu}{3 B}\left(\frac{r}{b}\right)^{3}\right]
$$

From this we can calculate the stress $\sigma_{r r}$ as

$$
\sigma_{r r}=(2 \mu+\lambda) \varepsilon_{r r}+\lambda \varepsilon_{\theta \theta}+\lambda \varepsilon_{\varphi \varphi}
$$

where the strains can be calculated from the known displacements. This procedure leads to 


$$
\sigma_{r r}(r=a)=\frac{\mu \delta v_{A}}{\pi a^{3}}\left[1-\left(\frac{a}{b}\right)^{3}\right]
$$

This development also requires

$$
\sigma_{r r}(r=b)=0
$$

as it should. The surface stress for an internal cavity is mechanically equivalent to tractions acting on the surface as shown in the following figure.

\section{Surface stress equivalent to negative pressure}

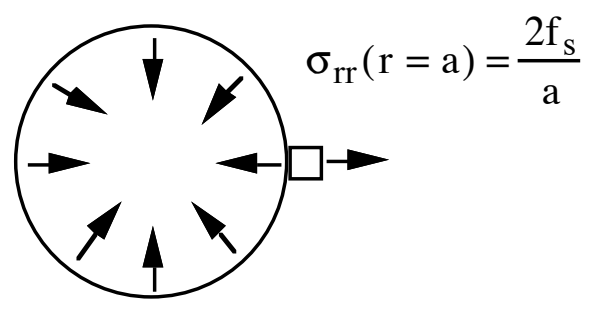

Using classical reversible work argument we can show

$$
\sigma_{r r}(r=a)=\frac{2 f_{s}}{a}
$$

where $f_{s}$ is equivalent to negative pressure inside the cavity.

Now we can calculate $\delta V_{A}$ by equating the two expressions we have for $\sigma_{r r}(r=a)$ :

$$
\delta v_{A}=\frac{2 \pi a^{2} f_{s}}{\left[1-\left(\frac{a}{b}\right)^{3}\right] \mu}
$$

and the corresponding contraction of the sphere is (see MSE206)

$$
\delta V=\delta v_{A}\left[1+\frac{4 \mu}{3 B}\right]=\frac{2 \pi a^{2} f_{s}}{\left[1-V_{f}\right] \mu}\left[1+\frac{4 \mu}{3 B}\right]
$$


Therefore, the corresponding volumetric strain is

$$
\begin{aligned}
& e=-\frac{\delta V}{V}=-\frac{\delta V}{\frac{4}{3} \pi b^{3}}=-\frac{3}{2} \frac{a^{2} f_{s}}{\left[1-V_{f}\right] b^{3} \mu}\left[1+\frac{4 \mu}{3 B}\right] \\
& e=-\frac{3}{2} \frac{a^{2} f_{s}}{\left[b^{3}-a^{3}\right] \mu}\left[1+\frac{4 \mu}{3 B}\right]
\end{aligned}
$$

This measure of strain is relative to hypothetical reference $f_{S}=0$. This gives the volumetric strain associated with the presence of internal surfaces with surface stress $f_{s}$. In the figure below we show how this strain might vary as water is adsorbed onto the internal surfaces.

\section{Variation of dilatation with surface stress (effects of water adsorption)}

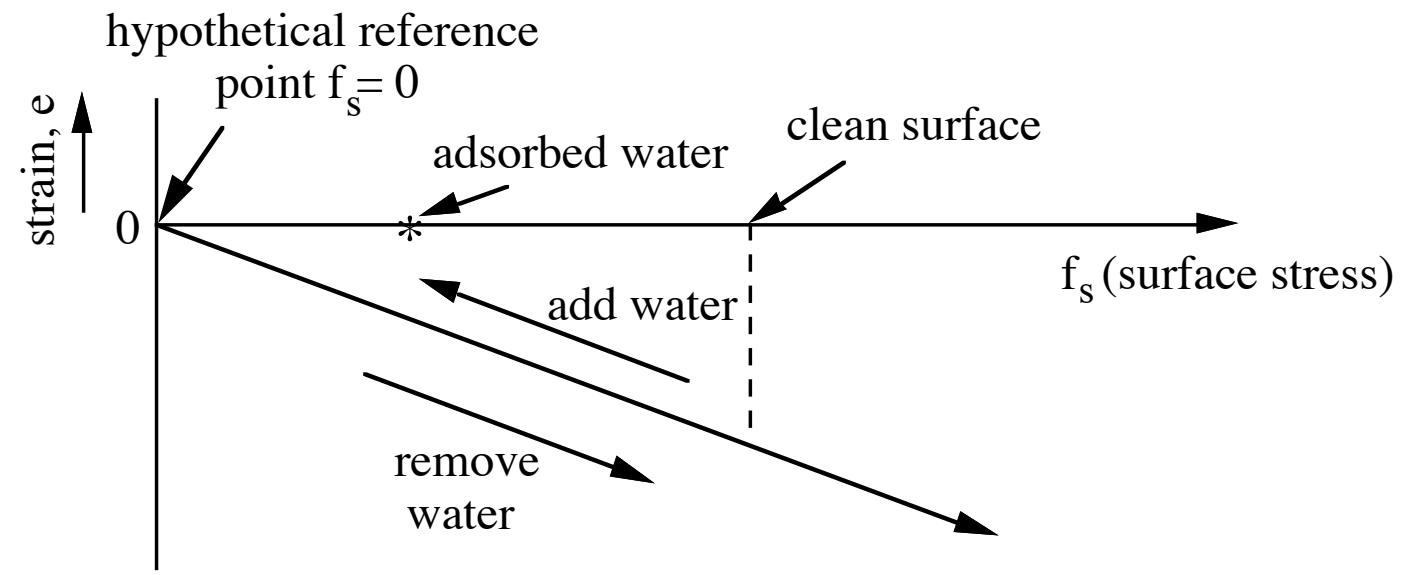

When gaseous species are adsorbed onto internal surfaces, the driving force for absorption is the reduction of the surface stress, $\mathrm{f}_{\mathrm{s}}$. Thus $\mathrm{f}_{\mathrm{s}}$ decreases and the glass expands if not constrained by the substrate. This explains how driving $\mathrm{H}_{2} \mathrm{O}$ out of a glass film causes contraction while saturating the glass with $\mathrm{H}_{2} \mathrm{O}$ causes volumetric expansion.

The change in dilatation associated with change of surface stress $\Delta \mathrm{f}_{\mathrm{S}}$ is

$$
\Delta e=-\frac{3}{2} \frac{a^{2}}{\left[b^{3}-a^{3}\right] \mu}\left[1+\frac{4 \mu}{3 B}\right] \Delta f_{s}
$$


When the film is attached to a substrate, this leads directly to stresses in the film.

\section{Swelling of a film detached from a substrate}

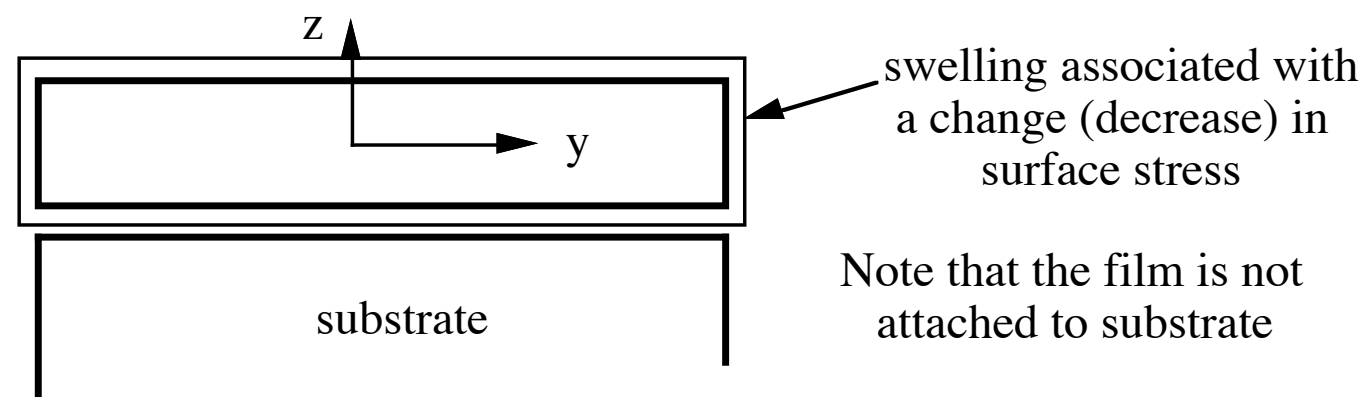

The figure illustrates welling associated with a change (decrease) in surface stress - if not attached to substrate.

The biaxial strain needed to fit the film back to the substrate is:

$$
\Delta \varepsilon=\Delta \varepsilon_{y y}^{e l}=\Delta \varepsilon_{x x}^{e l}=-\frac{\Delta e}{3}
$$

so the biaxial strain is

$$
\Delta \varepsilon=\frac{1}{2} \frac{a^{2}}{\left[b^{3}-a^{3}\right] \mu}\left[1+\frac{4 \mu}{3 B}\right] \Delta f_{s}
$$

and the corresponding increment in film stress is

$$
\Delta \sigma=\left(\frac{E}{1-v}\right)_{\text {film }} \frac{1}{2} \frac{a^{2}}{\left[b^{3}-a^{3}\right] \mu}\left[1+\frac{4 \mu}{3 B}\right] \Delta f_{s}
$$

The expression $(E / 1-v)_{f i l m}$ applies to the porous glassy film, whereas the elastic properties in the analysis apply to the fully dense parts of the glass. To be consistent, we should use a rule of mixtures to convert $(E / 1-v)_{f i l m}$ to $(E / 1-v)$ for fully dense material.

$$
\left(\frac{E}{1-v}\right)_{f i l m}=\left(1-V_{f}\right) \frac{E}{1-v}+V_{f}(0) \quad \text { Voids have no stiffness }\left(V_{f}(0)\right)
$$




$$
\left(\frac{E}{1-v}\right)_{f i l m}=\left(\frac{b^{3}-a^{3}}{b^{3}}\right) \frac{E}{1-v}
$$

Using this, together with $E=2 \mu(1+v)$ gives

$$
\begin{aligned}
& \Delta \sigma=\frac{1}{2}\left(\frac{a^{2}}{b^{3}}\right) \frac{E}{1-v} \frac{1}{\mu}\left[1+\frac{4 \mu}{3 B}\right] \Delta f_{s} \\
& \Delta \sigma=\left(\frac{a^{2}}{b^{3}}\right)\left(\frac{1+v}{1-v}\right)\left[1+\frac{4 \mu}{3 B}\right] \Delta f_{s}
\end{aligned}
$$

and using $B=\frac{2 \mu(1+v)}{3(1-2 v)}$,

this leads to $\Delta \sigma=3\left(\frac{a^{2}}{b^{3}}\right) \Delta f_{s}$.

Suppose $\Delta f_{S} \sim 0.5 \mathrm{~J} / \mathrm{m}^{2}, a=10 \AA=10^{-9} \mathrm{~m}, b=20 \AA=2 \times 10^{-9} \mathrm{~m}$, then

$\Delta \sigma \approx 0.3 \mathrm{GPa}=300 \mathrm{MPa}$. This is within the correct order of magnitude. 


\section{Mechanisms That Lead to Tension}

Here we begin to discuss mechanisms that can produce tensile intrinsic stresses.

\section{Grain Boundary Relaxation}

The most widely quoted model of intrinsic stress development is Hoffman's grain boundary relaxation model.

References:

R.W. Hoffman, Phys. Thin Films 3211 (1966)

H.K. Pulker , Thin Solid Films 89191 (1982)

R.W. Hoffman, Thin Solid Films 34185 (1976)

W.D. Nix and B.M. Clemens, J. Materials Research, 14, 3467 (1999).

S.C. Seel, C.V. Thompson, S.J. Hearne and J.A. Floro, JAP, 88, 7079 (2000)

L.B. Freund and E. Chason, Journal of Applied Physics, 89, 4866 (2001)

We focus on the cohesion between crystallites as they grow together (ignoring surface stress effects). Consider first isolated crystallites as shown in the following figure.

Isolated crystallites on a substrate

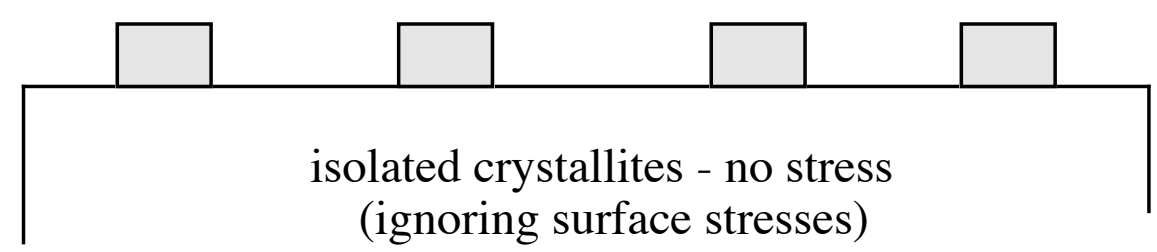

Now consider what happens when the crystallites begin to grow together. If the surfaces of the crystallites are very close to each other, cohesive forces acting between the crystallites can pull the surfaces together to make a grain boundary.

On-set of crystallite coalescence

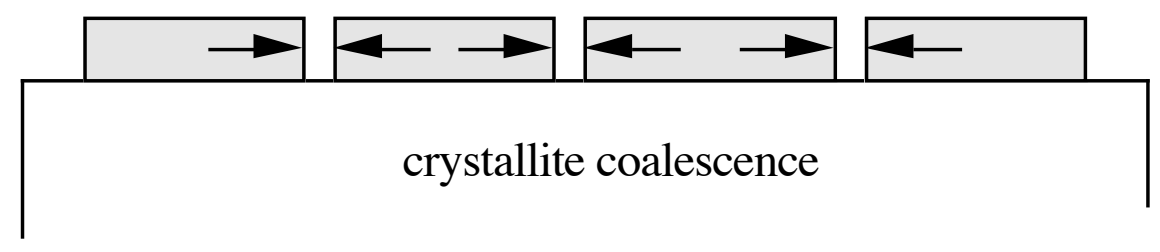


If the crystallites are not free to slide on the interface or if the cohesive forces act simultaneously on either side of the crystallite, the joining of the surfaces will occur at the expense of tensile elastic strain in the film.

Hoffman has claimed that when the surfaces of the crystallites get within atomic distances, they can move together spontaneously to form a grain boundary. This process leads to tensile strain in the film.

\section{Hoffman Analysis}

Consider hexagonal crystallites of "diameter" $L$ growing together. When the crystallite surfaces grow to within a distance $\Delta$, the surfaces may be joined to form grain boundaries. In the following figure $\Delta<<L$.

\section{Model for crystallite coalescence}
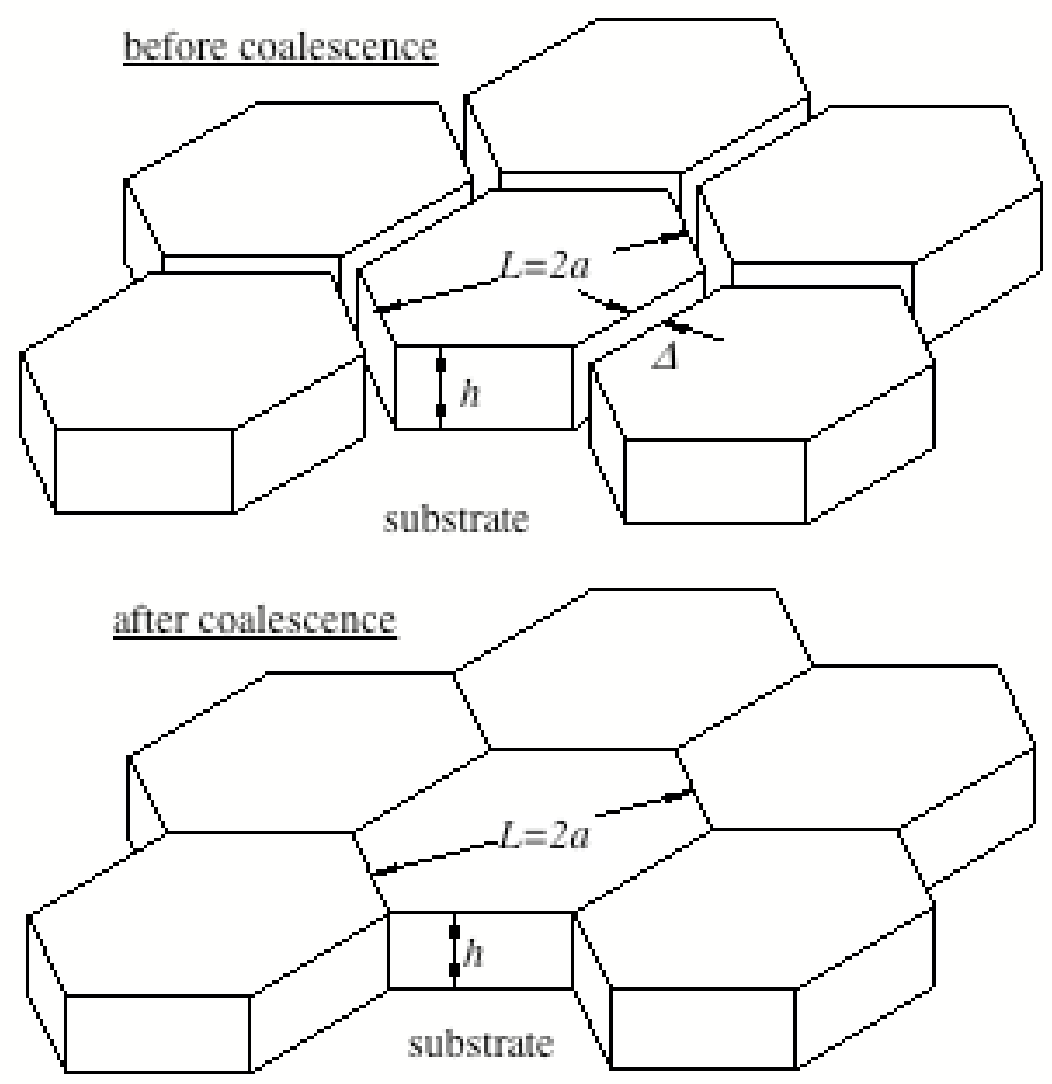

Although Hoffman's original suggestion started with the idea that crystallites snap together because the surface free energies are greater than the free energy of the grain boundary created by the coalescence, he did not use that idea to estimate the stress so generated. Instead he argued that crystallites would 
remain separated until the space between them becomes smaller than a critical dimension, which he based on atomic size considerations. He assumed that the atoms on the surface of a crystallite interact with atoms arriving from the vapor through an asymmetric potential well. Because more atoms would be expected to arrive in the attractive part of the potential well and fewer in the repulsive part of the potential well, an attractive interaction would dominate and tensile stresses would be naturally created. Thus, Hoffman estimated the gap, $\Delta$, between adjacent crystals at the point of coalescence using atomic size considerations and computed the tensile stresses as

$$
\sigma=\frac{E}{1-v} \frac{\Delta}{L},
$$

where $E / 1-v$ is the biaxial modulus of the film and $L$ is the grain size.

Here we describe an alternative picture of the crystallite coalescence process, one in which the free energies of the crystallite surfaces and grain boundaries play a direct role in determining the tensile stresses in the film. With this reinterpretation of Hoffman's model we obtain a result in which the stress can be estimated from the interfacial free energies, elastic properties and grain size alone. It is not necessary to use atomic size arguments to estimate the size of the gap at the point of crystallite coalescence.

\section{Global Energetic Analysis}

Consider a perfectly regular array of hexagonal crystallites growing on a substrate, as shown above. The crystallites are of height h and size $L=2 a$ and, before coalescence, are separated by a gap of size $\Delta$. Before coalescence the free energy per unit film area of such an array may be expressed as

$$
E_{1}=E_{0}+\frac{4 h \gamma_{s-v}}{L},
$$

where $E_{0}$ is the free energy per unit film area associated with both the top surface of the film and the film/substrate interface and the second term represents the free energy of the side faces of the crystallites, per unit film area. We use $\gamma_{s-v}$ to represent the surface free energy of the crystallites. If the spaces between the crystallites are closed by elastic displacements of the side surfaces, grain boundaries are formed in the film and each of the crystals is subjected to a biaxial strain, $\varepsilon_{\text {elastic }}=\Delta / L$. After coalescence the free energy per unit film area becomes 


$$
E_{2}=E_{0}+\frac{2 h \gamma_{g b}}{L}+\frac{E}{1-v} h\left(\frac{\Delta}{L}\right)^{2}
$$

where the second term is the grain boundary free energy and the third term is the strain energy per unit film area. The crystallites are assumed to be elastically isotropic with Young's modulus E and Poisson's ratio, v. Here we ignore the constraints imposed by the substrate in calculating the strain energy. In effect we assume that the crystallites are free to slide along the film/substrate interface when the gap is closed by the elastic displacements. This approximation may not be too severe if $h \geq L$ but is certainly not valid when $h<<L$ unless the crystallites are actually free to slide on the substrate.

We may compute the maximum gap size, $\Delta_{\max }$, that could be closed spontaneously by crystallite coalescence by noting that such a process must not increase the free energy of the system. This is found by setting $E_{2}-E_{1}=0$. The result is

$\Delta_{\max }=\left(2 L\left(2 \gamma_{s-v}-\gamma_{g b}\right) \frac{1-v}{E}\right)^{1 / 2}$,

which leads to the following upper bound estimate of the stress in the film:

$\sigma_{\max }=\frac{E}{1-v} \frac{\Delta_{\max }}{L}=\left(\frac{2\left(2 \gamma_{s-v}-\gamma_{g b}\right)}{L} \frac{E}{1-v}\right)^{1 / 2}$

In the following we suppose that the biaxial modulus may be estimated as $E / 1-v=100 \mathrm{GPa}$ and that the interfacial energies are $\gamma_{s-v}=1 \mathrm{~J} / \mathrm{m}^{2}$ and $\gamma_{g b}=0.5 \mathrm{~J} / \mathrm{m}^{2}$, respectively. With these quantities and for a grain size of $L=10 \mathrm{~nm}$, the upper bound estimates are

$$
\Delta_{\max }^{a=5 n m}=0.55 \mathrm{~nm} \text { and } \sigma_{\max }^{a=5 \mathrm{~nm}}=5.5 \mathrm{GPa} .
$$

This simple model accounts for the fact that the maximum intrinsic tensile stress develops at the point of crystalline coalescence. We note immediately that for very small grains, crystallite coalescence can lead to very high tensile stresses in the film. We also note that this relation takes the form of a Griffith equation, with the grain size taking the role of the crack length in the expression. This similarity suggests the crack-like analysis used in the following section. Also, this relation gives an upper bound estimate. The global energetic analysis does not provide any guidance on how much smaller the gap is when the crystallites 
snap together. For this we need to look more closely at the process by which the crystals might grow together during crystallite coalescence.

\section{Nix/Clemens Extension of the Hoffman model}

We consider now a continuum model of crystallite coalescence. Assume that isolated crystallites are forming on a substrate by nucleation and growth from the vapor. We will assume here that atomic diffusion does not occur in either the bulk of the crystallites or the grain boundaries that form between them. We also assume that the crystallites are perfectly stress-free when they are isolated. As the crystallites grow, their surfaces come into close proximity and eventually make contact at the point of coalescence.

\section{Isolated crystallites}

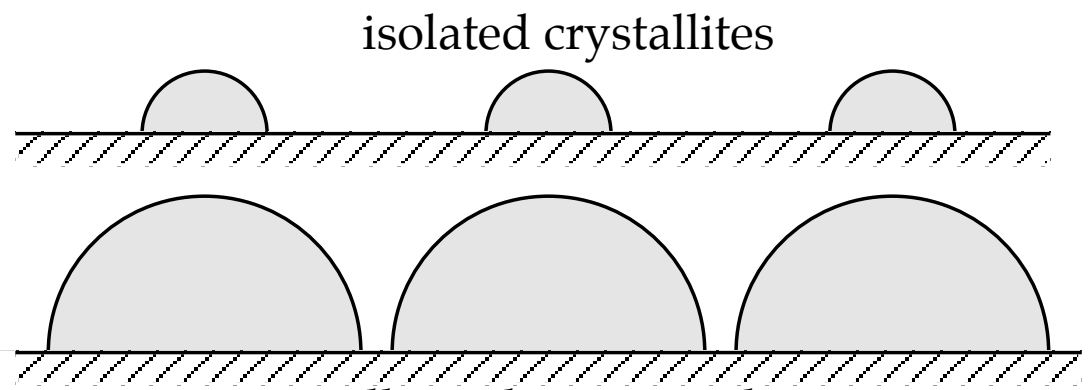

crystallites about to coalesce

We wish to consider what happens when the crystallites begin to touch each other. Because the grain boundary energy is smaller than the energies of the two surfaces, there is a driving force for the crystallites to snap together as soon as they make contact. If the crystallites are already anchored to the substrate or if contacts are made simultaneously on either side the a given crystal, then the crystallite will be spontaneously strained.

\section{Elastic straining at the point of crystallite coalescence}

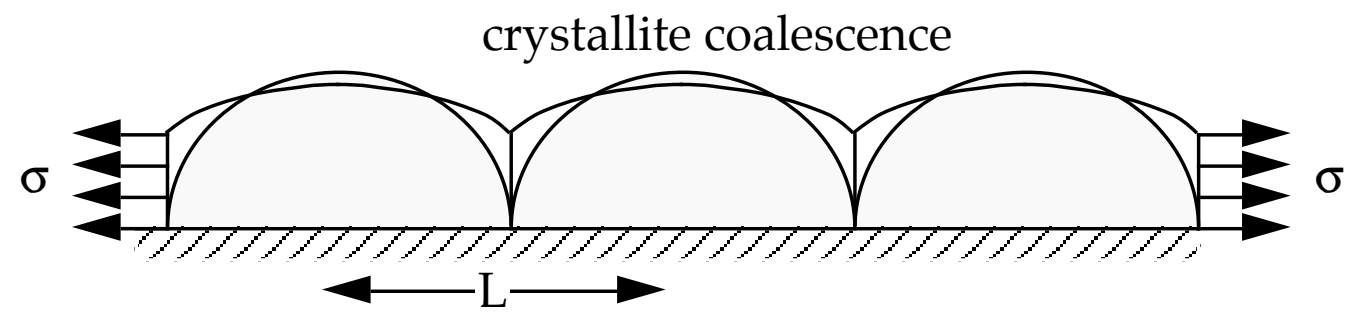


We can estimate the elastic strains and stresses at the point of coalescence using the following figure. When the circular crystals make touching contact the two surfaces close up, causing a grain boundary to be formed. This occurs by elastically stretching of the crystallites and this leads to stress in the film.

\section{Approximate analysis of crystallite coalescence}

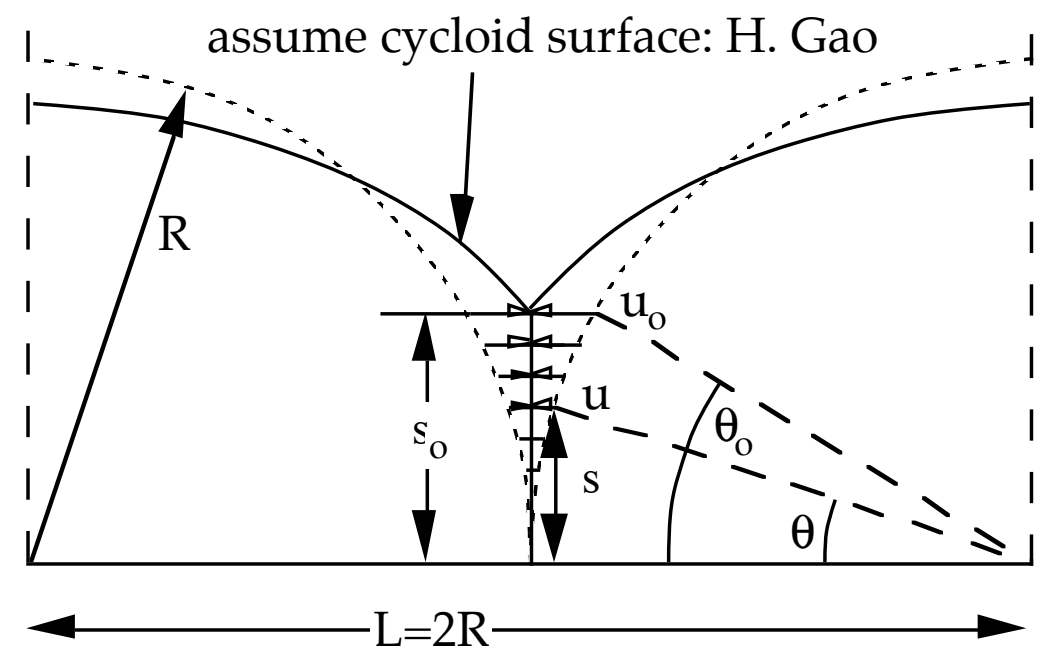

When the surfaces come together to form the grain boundary, a sharp cusp is formed at the intersection of the grain boundary and the free surfaces. If we assume that the shape of the surface is in the form of a cycloid (the path taken by a point on a circle as the circle rolls along) then the cusp is equivalent to a sharp crack (C-H. Chiu and H. Gao, Int. J. Solids and Structures, 30, 2983-3012 (1993)). This fact allows us to determine the extent to which the crystals snap together and, in turn, this allows us to determine the stress in the film.

At a given distance, $\mathrm{s}$, from the substrate each surface must be displaced by a distance $u$ in order to form the grain boundary. The strain in the film at that point would then be

$$
\varepsilon=\frac{u}{R} .
$$

Using the geometry in the figure above we have

$$
\varepsilon=\frac{u}{R}=1-\cos \theta \approx \frac{1}{2} \theta^{2} .
$$

The average strain in the continuous part of the film is 


$$
\bar{\varepsilon}=\frac{1}{s_{O}} \int_{0}^{s_{o}} \varepsilon(\theta) d s=\frac{1}{6} \theta_{o}^{2}=\frac{1}{6}\left(\frac{s_{O}}{R}\right)^{2}
$$

and the corresponding stress is

$$
\bar{\sigma}=\frac{E}{1-v^{2}} \bar{\varepsilon}=\frac{E}{6\left(1-v^{2}\right)}\left(\frac{s_{O}}{R}\right)^{2}
$$

Closure of the surfaces to form the grain boundary stops when the crack extension force at the cycloid cusp is equal to the critical crack extension force for the Griffith equation:

$$
G_{c}=2 \gamma_{s-v}-\gamma_{g b}
$$

For a cycloid surface of a strained film Chiu and Gao (Int. J. Solids and Structures, 30, 2983-3012 (1993)) have shown that the stress intensity factor for the crack-like singularity is

$$
K=\frac{1}{1+v} \bar{\sigma} \sqrt{\frac{L}{2}}=\frac{1}{1+v} \bar{\sigma} \sqrt{R},
$$

so the crack extension force is

$$
G=\left(1-v^{2}\right) \frac{K^{2}}{E}=\left(\frac{1-v}{1+v}\right) \frac{\bar{\sigma}^{2} R}{E}
$$

Solving for the stress using the condition $G=G_{C}$ gives

$$
\bar{\sigma}=\left(\frac{(1+v)}{(1-v)} E \frac{\left(2 \gamma_{s-v}-\gamma_{g b}\right)}{R}\right)^{1 / 2}
$$

Equating this relation to the expression above allows us to determine $s_{0}$, the length of the "zipped up" grain boundary segment: 


$$
\bar{\sigma}=\frac{E}{6\left(1-v^{2}\right)}\left(\frac{s_{O}}{R}\right)^{2}=\left[\frac{(1+v)}{(1-v)} E \frac{\left(2 \gamma_{s-v}-\gamma_{g b}\right)}{R}\right]^{1 / 2} .
$$

The result is

$$
\frac{s_{o}}{R}=\left[\frac{36\left(1-v^{2}\right)(1+v)^{2}\left(2 \gamma_{s}-\gamma_{g b}\right)}{E R}\right]^{\frac{1}{4}} .
$$

Using

$$
\begin{aligned}
& 2 \gamma_{s}-\gamma_{g b}=1.5 \mathrm{~J} / \mathrm{m}^{2} \\
& E /\left(1-v^{2}\right)=100 \mathrm{GPa} \\
& v=0.33
\end{aligned}
$$

we find the results given in the table below.

Grain boundary segment lengths and average film stress for different grain sizes

\begin{tabular}{|c|c|c|}
\hline Grain Size & $\begin{array}{l}\text { Grain Boundary Segment } \\
\text { Length }\end{array}$ & Average Film Stress \\
\hline$L=2 a(n m)$ & $s_{o} / R$ & $\bar{\sigma}(G P a)$ \\
\hline 2 & 0.831 & 11.5 \\
4 & 0.698 & 8.3 \\
10 & 0.556 & 5.1 \\
20 & 0.467 & 3.6 \\
100 & 0.312 & 0.5 \\
\hline
\end{tabular}

As expected physically, after the zipping process is complete, the length of the grain boundary segments relative to the height of the crystallites depends strongly on the crystallite size. For extremely small crystallites, the interfacial forces are sufficiently strong to almost completely close the gap between the crystals at the point of touching. For larger grains, only a small portion of the crystallites are joined together to form a continuous film. The stresses in the table are all overestimates. All of the crystallites are imagined to touch at the same time, with the consequence that no shear stresses can be developed on the crystallite/substrate interface and no sliding of the crystallites can occur. A more realistic picture would allow different crystallites to coalesce at different times and some crystallite sliding to occur on the substrate; this would lessen the stress in the film. Subsequent work has shown that the above analysis is conceptually correct but that it overestimates the stress due to crystallite coalescence. 
$\underline{\text { Stresses in a growing film }}$

We consider now subsequent growth of the film after the crystallites have coalesced to form a continuous film. As suggested originally by Doljack and Hoffman, once the crystallites are elastically strained by the "grain boundary relaxation" process, subsequent epitaxial growth on the strained crystallites can lead to a uniformly strained film. Abermann has shown that thin films of metals with low ad-atom mobility are indeed uniformly strained in tension, suggesting the kind of epitaxial growth envisioned here. However, for metals with high adatom mobility, typically FCC metals with lower melting temperatures, the tensile stresses generated at the point of crystallite coalescence are not sustained; the stresses are usually near zero or slightly compressive at large film thicknesses. Relaxation processes associated with high ad-atom mobility are assumed to be responsible for the decline in the tensile stress. Consistent with these predictions, Abermann showed that when Fe films are evaporated and condensed onto $\mathrm{MgF}_{2}$ substrates at room temperature they continue to grow in a strained state as the films thicken, whereas at deposition temperatures of $200^{\circ} \mathrm{C}$ the tensile stresses are found only in the early stages of deposition. His results are shown in the figure below, where the average stress in the film is plotted against film thickness for three different deposition temperatures. At room temperature, iron behaves like a metal with low ad-atom mobility; the average stress in the film develops in the early stages of growth and remains approximately constant during subsequent growth. At $200^{\circ} \mathrm{C}$ the behavior is like that for a metal with high ad-atom mobility, with the average stress falling back to zero after the point of crystallite coalescence and becoming negative (compressive) with continued growth. 
Evolution of the average stress in vapor deposited Fe films with increasing film thickness for different substrate temperatures (data taken from Abermann (1990)).

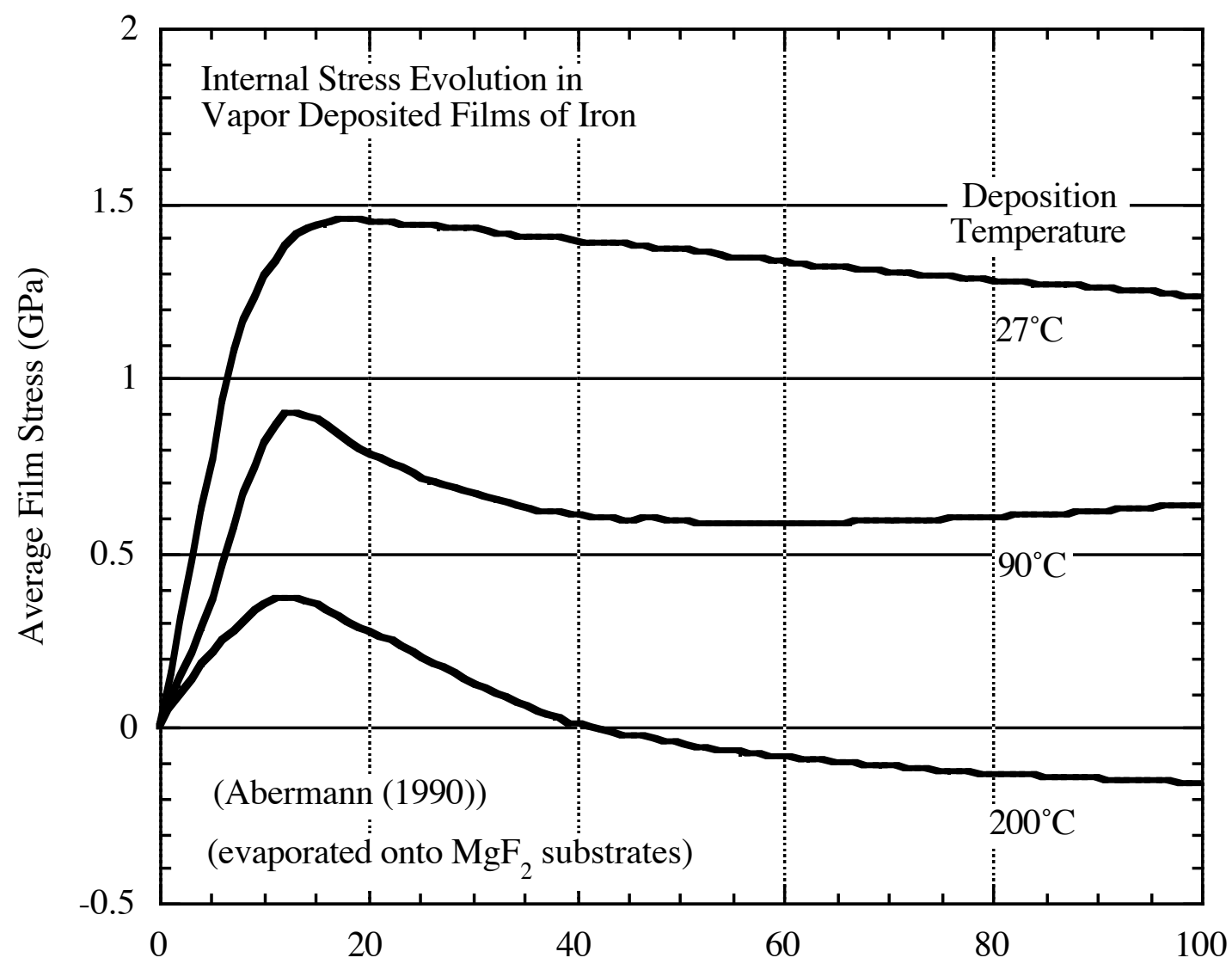

Film Thickness (nm)

Following Doljack and Hoffman, we imagine that for metals with low ad-atom mobility the rate of diffusion on the surface of the growing crystals is low compared to their rate of arrival from the vapor, with the consequence that the crystals grow epitaxially in a strained state. This behavior is illustrated schematically in the figure below. Here the arriving atoms are assumed to attach to the strained crystal at their point of arrival, thus allowing the crystal to grow in a strained state. If the mobility of the atoms on the growing surface is large compared to their rate of arrival, then the stress created in the film by the coalescence of the crystallites can be relaxed during subsequent film growth. One mechanism of strain relaxation involves the preferential attachment of atoms at the grain boundaries. The diagram shows how atoms could diffuse to the grain boundaries and relax the stress by attaching preferentially there. 
Growth of a polycrystalline film after the point of crystallite coalescence
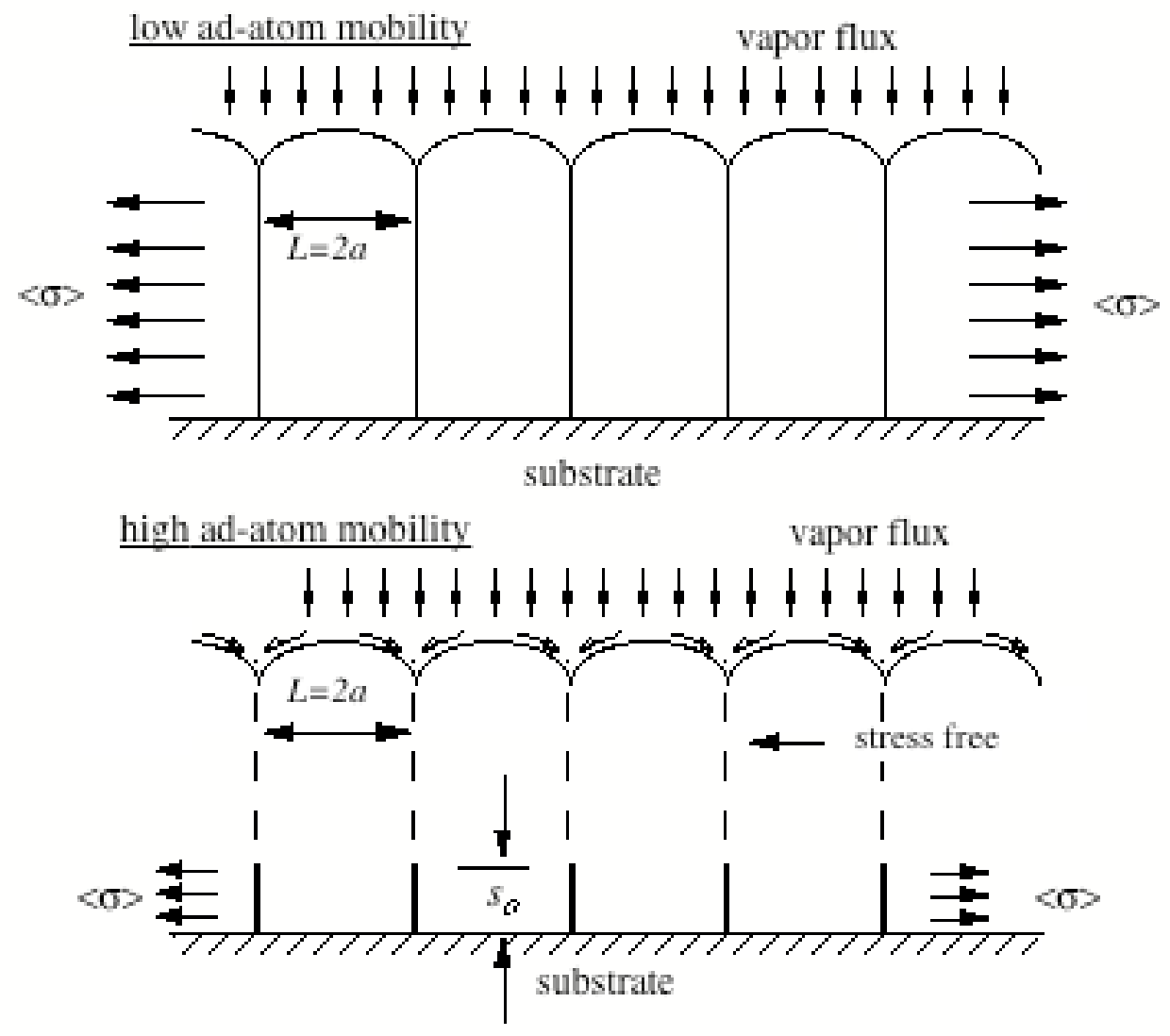

\section{General considerations of intrinsic stresses through densification}

We consider now a more general description of the kinetic processes leading to biaxial tensile stresses in deposited films. The basic idea is that the film is deposited in a non-equilibrium, non-dense state. Densification after the film material is attached to the substrate leads to tension stress in the film. Consider atoms arriving from the vapor and depositing on a growing film. Two rates are of importance and will be compared:

1. Rate of arrival of depositing atoms (growth rate). More precisely this is the rate of arrival of atoms from the vapor, expressed as the reciprocal of the time between atomic arrivals at a particular site on the surface, $R_{\text {arrival }}=1 / t_{\text {arrival }}$. 
2. Rate of atomic rearrangement on the surface of the growing film by surface diffusion ( $\left.R_{\text {surface rearrangement }}\right)$. This can be expresed as the atomic jump frequency on the surface, $R_{\text {surface rearrangement }}=1 / t_{j u m p}$.

If the rate of surface rearrangement is much greater than rate of arrival,

$$
R_{\text {surface rearrangement }}>>R_{\text {arrival }}
$$

then an equilibrium structure is produced and there is no potential for film stress (discounting epitaxy and thermal stresses in this discussion).

\section{Equilibrium film growth}

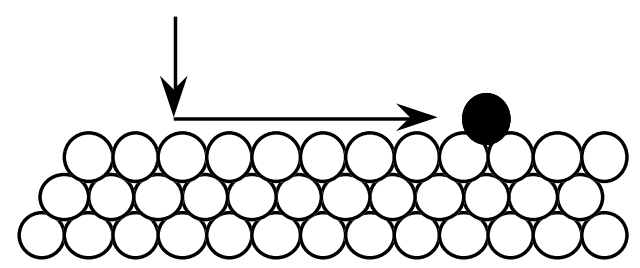

If the rate of surface rearrangement is much smaller than rate of arrival of atoms,

$$
R_{\text {surface rearrangement }}<<R_{\text {arrival }}
$$

then a non-equilibrium structure is produced and film stresses are possible.

Non-equilibrium film growth

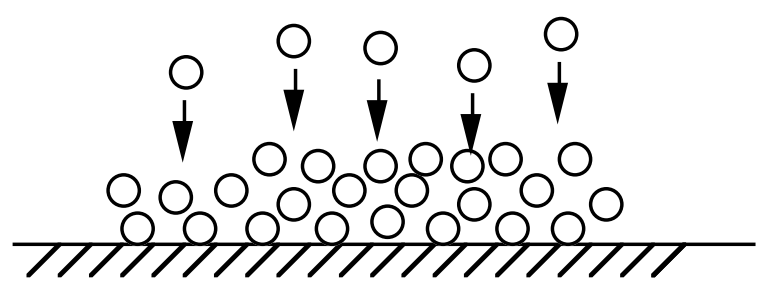

The stresses that may be present in a non-equilibrium film depend on the tendancy of the film to shrink once it is attached to the substrate. Thus the stresses that develop are associated with the internal atomic rearrangement or densification of the film $\left(R_{\text {densification }}\right)$. If the non-equilibrium structure is "frozen-in" (i.e., no atomic rearrangement), then no stresses are developed in the film. If the non-equilibrium structure relaxes to a denser state, then "intrinsic" tensile stresses are developed in the film. In addition, it is necessary to consider any relaxation processes that may occur in the film by plastic flow processes 
(slip, shear, etc $\left.\left(R_{\text {relaxation }}\right)\right)$. Thus the evolution of stresses in a growing film depends also on the competition between these two rates: If $R_{\text {densification }} \gg R_{\text {relaxation }}$ then stresses are developed in the growing film. But if $R_{\text {densification }}<<R_{\text {relaxation }}$ then stress relaxation dominates and the intrinsic stresses are not maintained.

\section{Schematic representation of intrinsic stress development in a metal film}

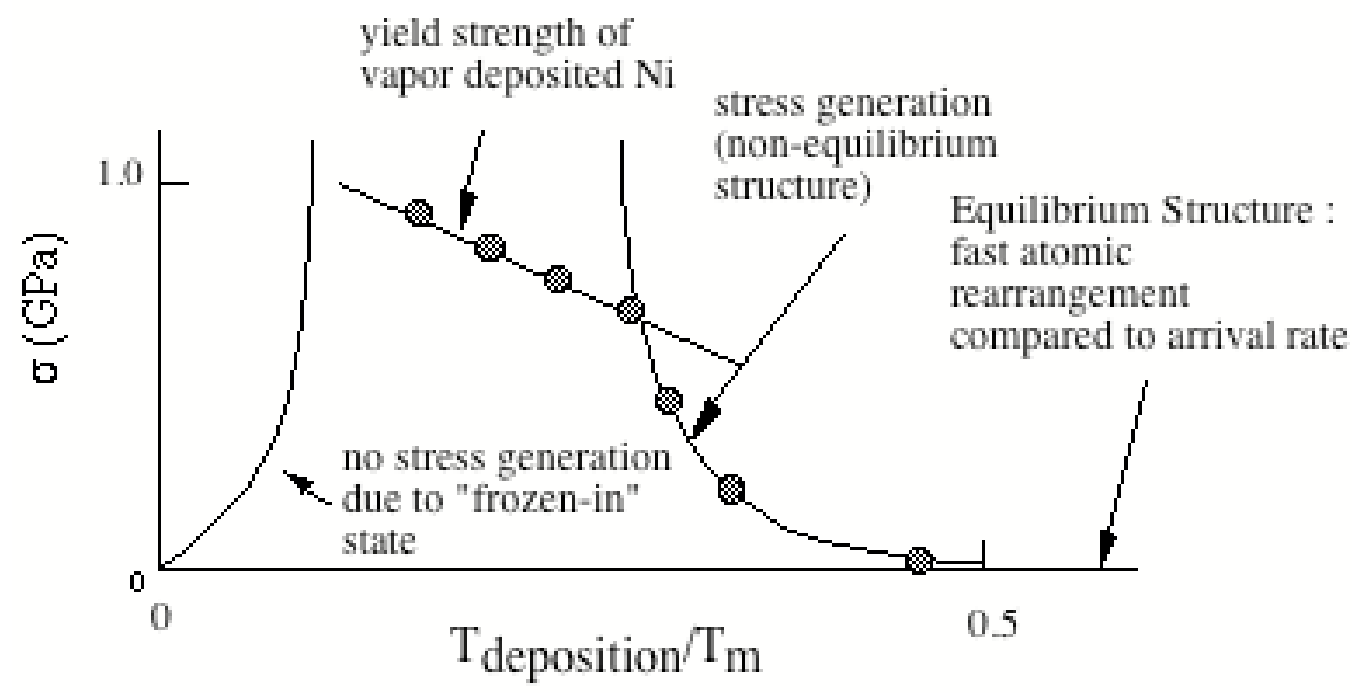

We now consider the internal atomic rearrangement processes that lead to "intrinsic" tensile stresses in films.

\section{Grain Growth}

Chaudhari was the first to recognize that grain growth in a film after it has been deposited onto a substrate can be a source of significant residual stress. [P. Chaudhari, J. Vac. Sci. Tech. 9520 (1972)]. He pointed out that grain boundaries are less dense than the crystal lattice so that grain growth (elimination of some grain boundaries) leads to densification of the film and, thus, to tensile stresses in the film.

Consider an as-deposited film with an initial grain size $L_{0}$. 


\section{Polycrystalline film on a substrate}

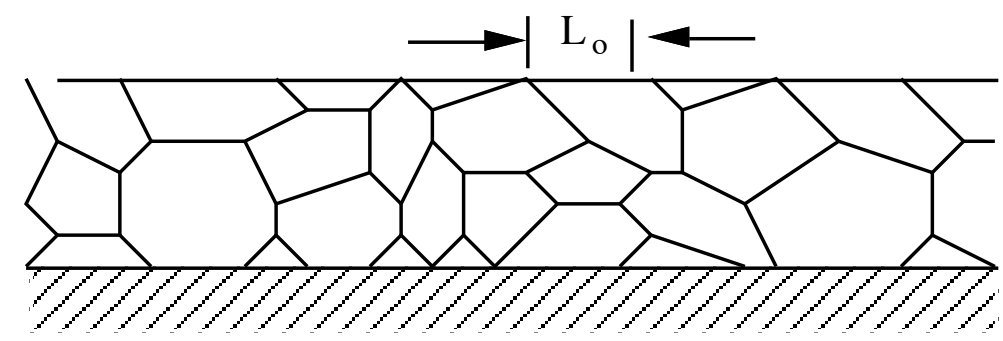

Consider grain growth to grain size $\mathrm{L}>\mathrm{L}_{\mathrm{o}}$. Let $\Delta$ a be the excess volume per unit area of grain boundary. This is equivalent to a separation distance between two ideal crystals caused by the imperfect atomic packing in the boundary.

\section{Excess volume (per unit area) in a grain boundary}
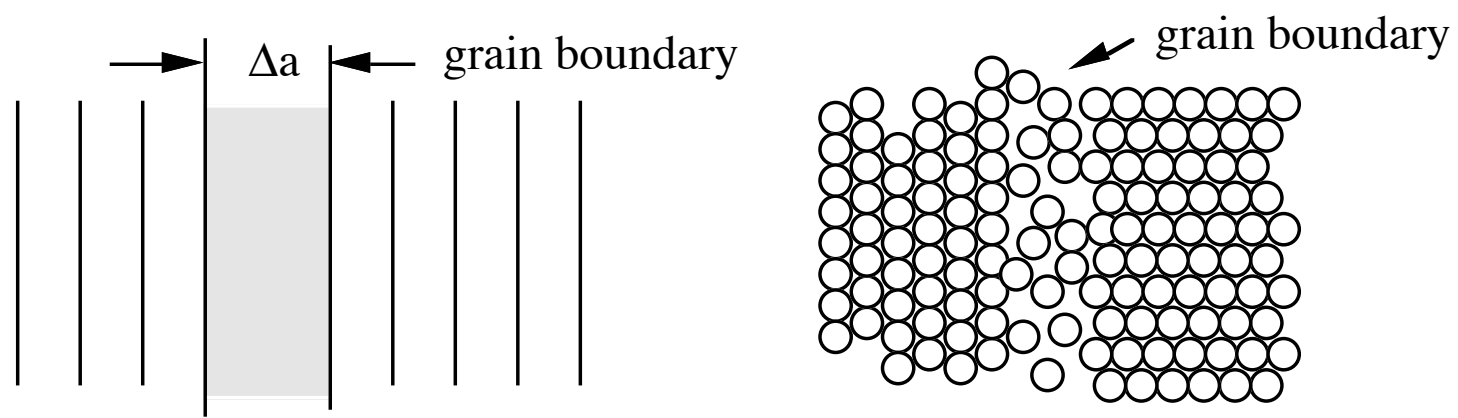

The excess volume per unit area, $\Delta a$ is expected to be of order of the atomic dimension and the grain boundary is equivalent to a gap $\Delta$ a between the adjacent crystals. Consider a reference crystal volume $V_{\text {ref }}$ of the film (Here, $V_{\text {ref }}$ is the crystal volume not including excess volume associated with the grain boundaries.) with grain diameter $L$.

Using the model of spherical grains, the grain boundary area per unit volume is

$$
\frac{A}{V}=\frac{1}{2} \frac{4 \pi R^{2}}{\frac{4}{3} \pi R^{3}}=\frac{3}{2 R}=\frac{3}{L}
$$

where the factor $1 / 2$ arises from the sharing of the grain boundary with the adjoining crystal. 
Excess volume in the reference volume $V_{r e f}$ is

$$
V_{g b}^{x s}=V_{\text {ref }} \frac{3}{L} \Delta a .
$$

The total volume of the polycrystalline aggregate is then

$$
V_{T}=V_{r e f}+V_{g b}^{x s}=V_{r e f}\left(1+\frac{3 \Delta a}{L}\right) .
$$

Thus the polycrystalline solid is dilated relative to single crystal reference by

$$
e_{*}^{T}=\frac{V_{T}-V_{\text {ref }}}{V_{\text {ref }}}=\frac{V_{T}}{V_{\text {ref }}}-1=\frac{3 \Delta a}{L} .
$$

This is the volumetric transformation strain associated with the creation of the grain boundaries.

Suppose the film is deposited in stress free state with grain size $L_{0}$. Then after grain growth to grain size $L$, the transformation strain relative to the asdeposited state is

$$
e^{T}=3 \Delta a\left(\frac{1}{L}-\frac{1}{L_{o}}\right) .
$$

These relations can be illustrated schematically as follows: 


\section{Dilatation associated with grain growth}
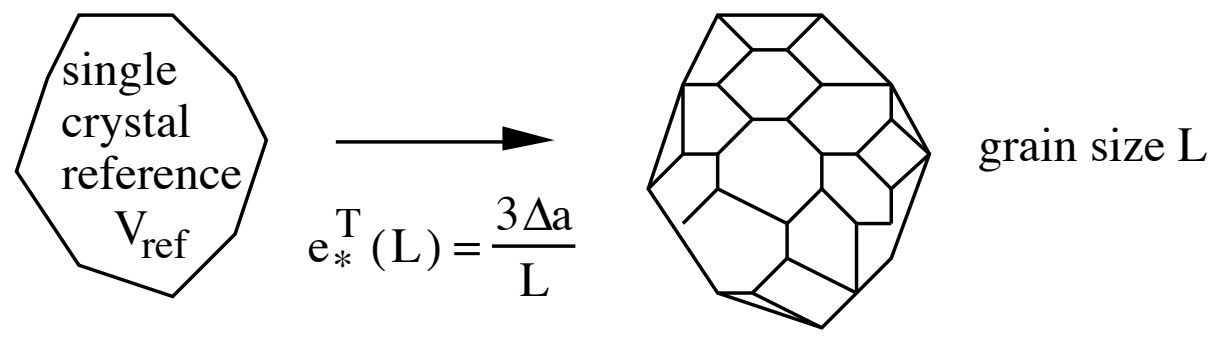

$$
\uparrow \mathrm{e}^{\mathrm{T}}=3 \Delta \mathrm{a}\left(\frac{1}{\mathrm{~L}}-\frac{1}{\mathrm{~L}_{\mathrm{o}}}\right)
$$
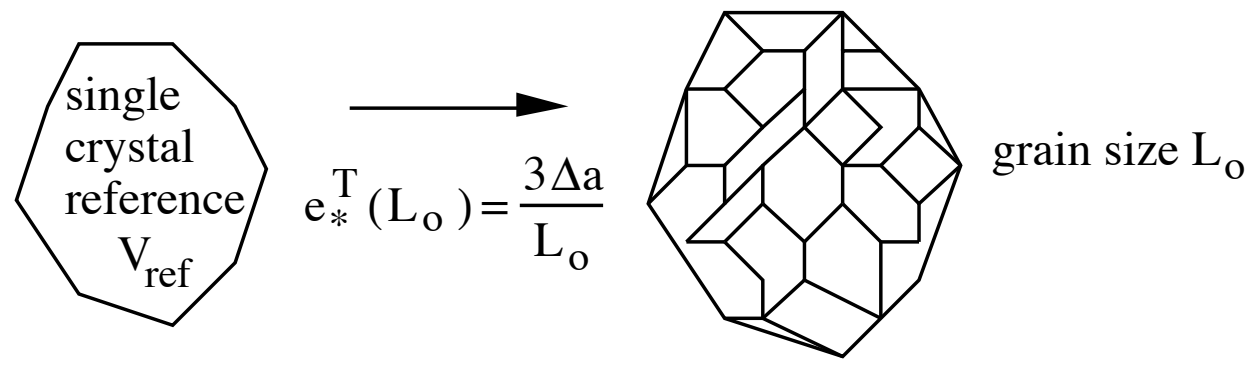

The transformation strain here is the volumetric strain associated with grain growth assuming that the film is not attached to the substrate. As discussed before, re-attaching the film to the substrate requires that elastic strains be imposed on the film. Schematically:

\section{Stresses associated with grain growth}

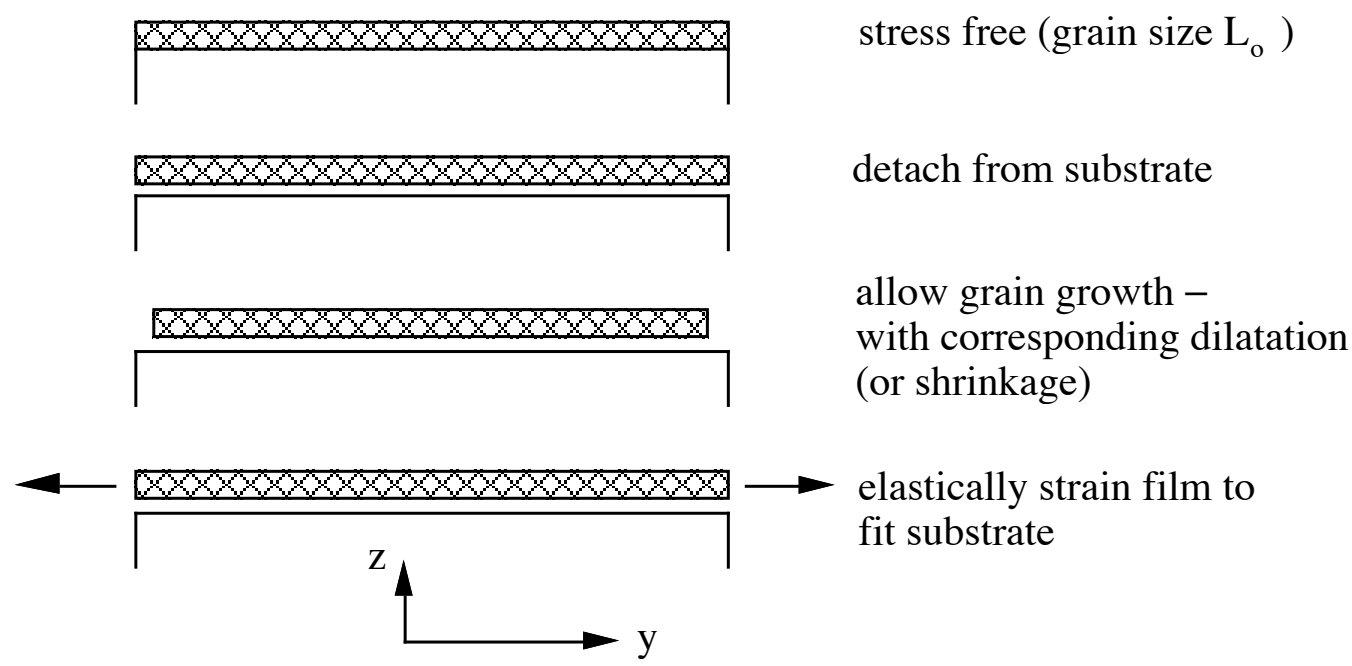


As illustrated in the figure

$$
\varepsilon_{x x}^{e l}=\varepsilon_{y y}^{e l}=-\frac{e^{T}}{3}=-\Delta a\left(\frac{1}{L}-\frac{1}{L_{o}}\right),
$$

with corresponding biaxial stresses

$$
\sigma_{x x}=\sigma_{y y}=-\left(\frac{E}{1-v}\right)_{f i l m} \Delta a\left(\frac{1}{L}-\frac{1}{L_{o}}\right)
$$

Thus, the intrinsic tensile stress caused by grain growth is

$$
\sigma=\left(\frac{E}{1-v}\right)_{f i l m} \Delta a\left(\frac{1}{L_{o}}-\frac{1}{L}\right)
$$

For

$$
\begin{aligned}
& 1 / L->0 \text { (infinite grain growth), } \\
& L_{o}=100 \AA=10 \mathrm{~nm}, \\
& \Delta a=1 \AA=0.1 \mathrm{~nm}, \\
& (E / 1-v)_{f i l m}=100 \mathrm{GPa},
\end{aligned}
$$

we find

$$
\sigma=1 G P a,
$$

a very large stress.

\section{Energetic and Kinetic Analysis}

During grain growth, the elastic strain energy increases as the stresses and strains in the film develop. The strain energy density is

$$
W_{e l}=\frac{1}{2} \sigma_{x x} \varepsilon_{x x}^{e l}+\frac{1}{2} \sigma_{y y} \varepsilon_{y y}^{e l}
$$

which, with the equations above becomes 


$$
W_{e l}=\left(\frac{E}{1-v}\right)_{\text {film }}(\Delta a)^{2}\left(\frac{1}{L_{o}}-\frac{1}{L}\right)^{2} .
$$

So $W_{e l}$ increases with grain growth - This can stop grain growth!

Now consider grain growth in a thin film with associated strain energy. The energy per unit volume in the film is

$$
E=E_{g b}+W_{e l}
$$

Using the grain boundary area per unit volume calculated above we can write

$$
E=\frac{3 \gamma_{g b}}{L}+\left(\frac{E}{1-v}\right)_{\text {film }}(\Delta a)^{2}\left(\frac{1}{L_{o}}-\frac{1}{L}\right)^{2},
$$

which can be written in more compact form as

$$
E=\frac{3 \gamma_{g b}}{L}+A\left(\frac{1}{L_{o}}-\frac{1}{L}\right)^{2},
$$

where $A$ is a constant, $L$ is the current grain size and $L_{o}$ is the initial grain size. To study the effects of grain growth on the energy of the film it is convenient to find the variation of the energy with $1 / L$ :

$$
\frac{d E}{d\left(\frac{1}{L}\right)}=3 \gamma_{g b}-2 A\left(\frac{1}{L_{o}}-\frac{1}{L}\right)
$$

If the initial grain size is below a critical value, $L_{0}<L_{\text {critical }}$, then the energy variations associated with grain growth are as shown in the following diagram. 


\section{Energetics of grain growth, $L_{0} \leq L_{\text {critical }}$}

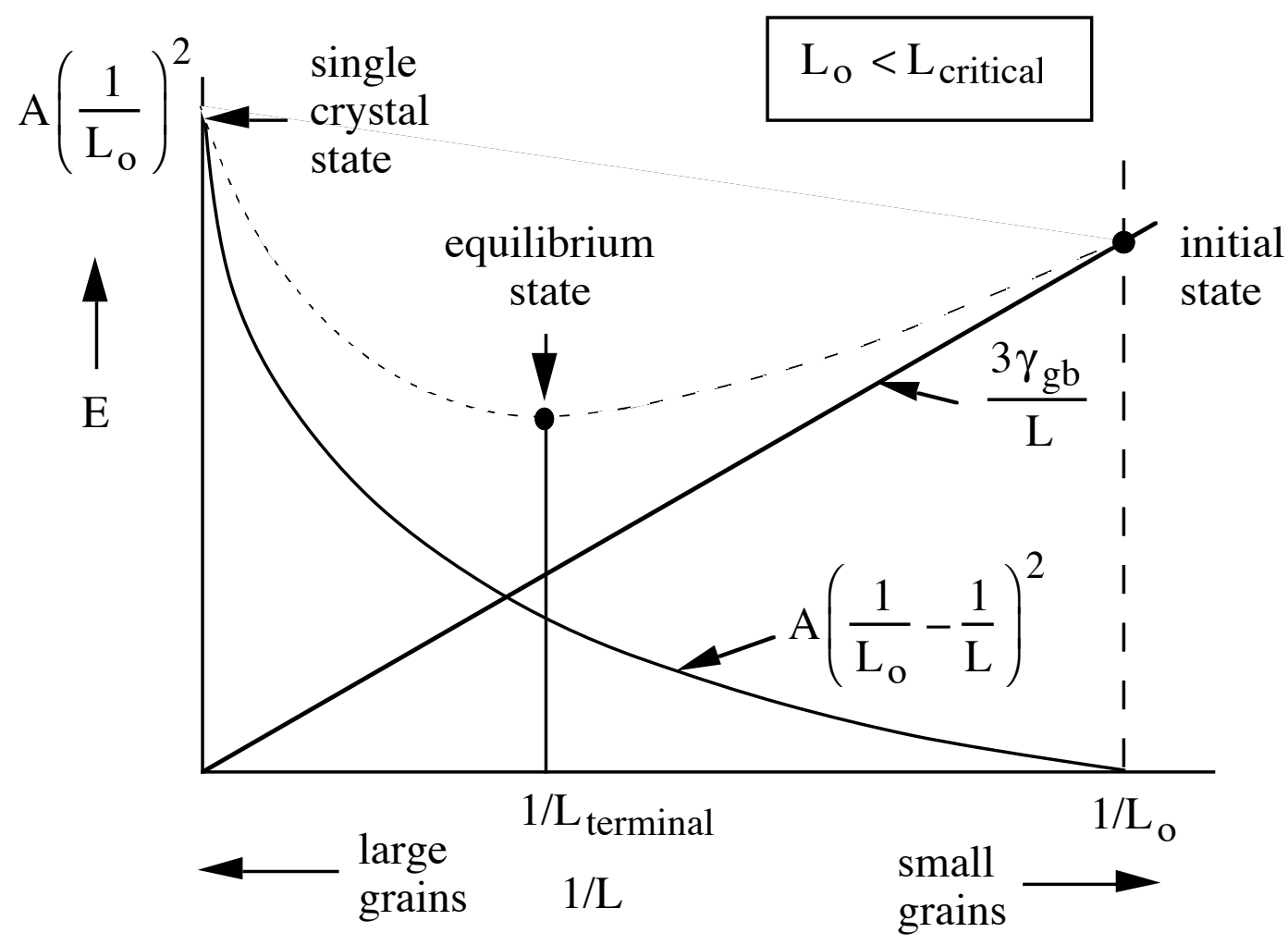

If the initial grain size is greater than the critical value, $L_{0}>L_{\text {critical }}$, then the grains will grow in an unbounded manner until a single crystal state is reached. In this case the strain energy that develops in the film is not great enough to stop the grain growth. 


\section{Energetics of grain growth, $L_{0}>L_{c r i t i c a l}$}

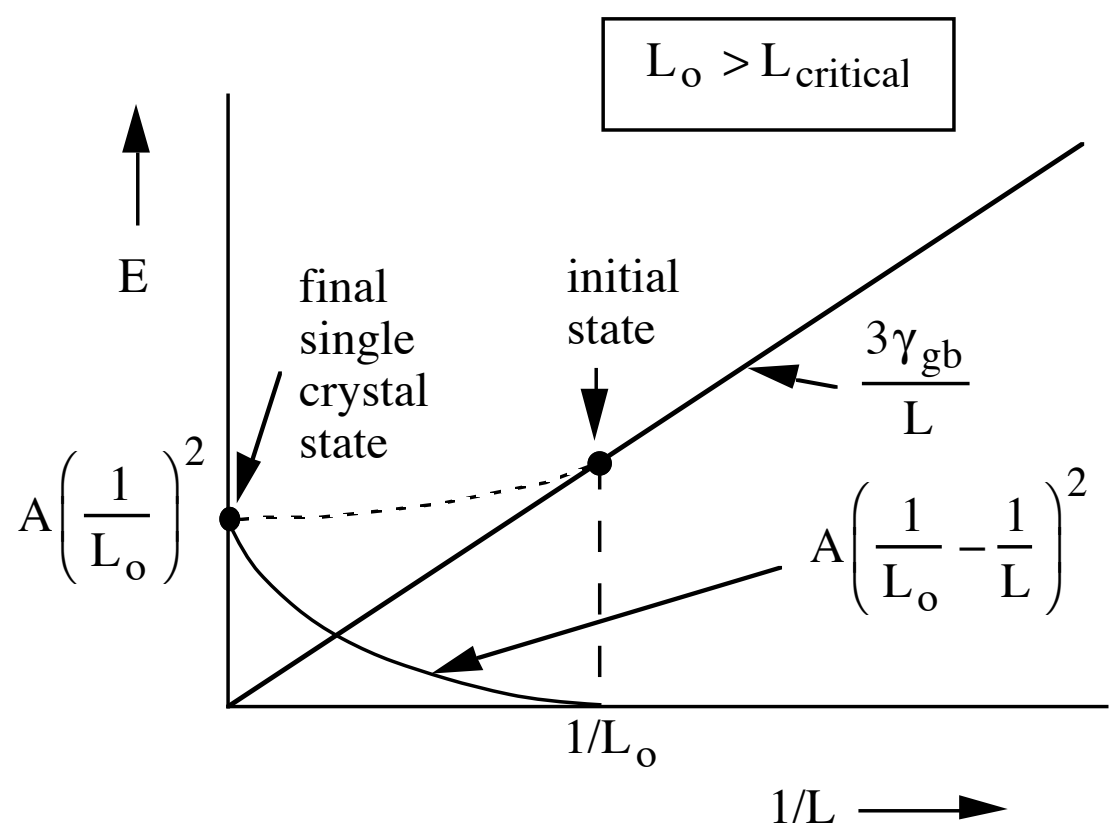

For $L_{0}<L_{\text {critical }}$ the terminal grain size can be found by

$$
\frac{d E}{d\left(\frac{1}{L}\right)}=0=3 \gamma_{g b}-2 A\left(\frac{1}{L_{o}}-\frac{1}{L_{\text {ter } \min a l}}\right)
$$

which leads to

$$
\frac{1}{L_{o}}-\frac{1}{L_{\text {ter } \min a l}}=\frac{3 \gamma_{g b}}{2 A}=\frac{3 \gamma_{g b}}{2\left(\frac{E}{1-v}\right)_{f i l m}(\Delta a)^{2}} .
$$

We find $L_{\text {critical }}$ by solving for the value of $L_{0}$ for which $1 / L_{\text {terminal }}=0$. This gives

$$
\frac{1}{L_{\text {critical }}}=\left(\frac{1-v}{E}\right)_{\text {film }} \frac{3 \gamma_{g b}}{2(\Delta a)^{2}} \text {. }
$$

Using 


$$
\begin{aligned}
& \gamma_{g b}=0.3 \mathrm{~J} / \mathrm{m}^{2} \\
& \Delta a=0.1 \mathrm{~nm} \\
& \left(\frac{E}{1-v}\right)_{\text {film }}=100 \mathrm{GPa}
\end{aligned}
$$

we have

$$
L_{\text {critical }}=2 n m=20 \AA .
$$

$\underline{\text { Intrinsic stress due to grain growth }}$

The largest intrinsic stress is developed when the initial grain size is smaller than the critical grain size and the grains grow to the terminal value:

$$
\sigma_{\text {ter } \min a l}=\left(\frac{E}{1-v}\right)_{f i l m} \Delta a\left(\frac{1}{L_{o}}-\frac{1}{L_{t e r \min a l}}\right)
$$

Using

$$
\frac{1}{L_{o}}-\frac{1}{L_{\text {ter } \min a l}}=\frac{3 \gamma_{g b}}{2\left(\frac{E}{1-v}\right)_{f i l m}(\Delta a)^{2}}
$$

we have

$$
\sigma_{\text {ter } \min a l}=\left(\frac{3 \gamma_{g b}}{2(\Delta a)}\right)
$$

Again using

$$
\begin{aligned}
& \gamma_{g b}=0.3 \mathrm{~J} / \mathrm{m}^{2} \\
& \Delta a=0.1 \mathrm{~nm}
\end{aligned}
$$

we have

$$
\sigma_{\text {ter } \min a l}=5 G P a \text {. }
$$


For $L o=100 \AA=10 \mathrm{~nm}$, the terminal grain size is infinite (single crystal) and the corresponding terminal stress is

$$
\sigma_{\text {ter } \min a l}=\left(\frac{E}{1-v}\right)_{\text {film }} \Delta a\left(\frac{1}{10 n m}-0\right)=1 G P a
$$

where again we have used

$$
\begin{aligned}
& \left(\frac{E}{1-v}\right)_{f i l m}=100 \mathrm{GPa} \\
& \Delta a=0.1 \mathrm{~nm}
\end{aligned}
$$

\section{Kinetics of grain growth}

\section{Grain growth - driving force}
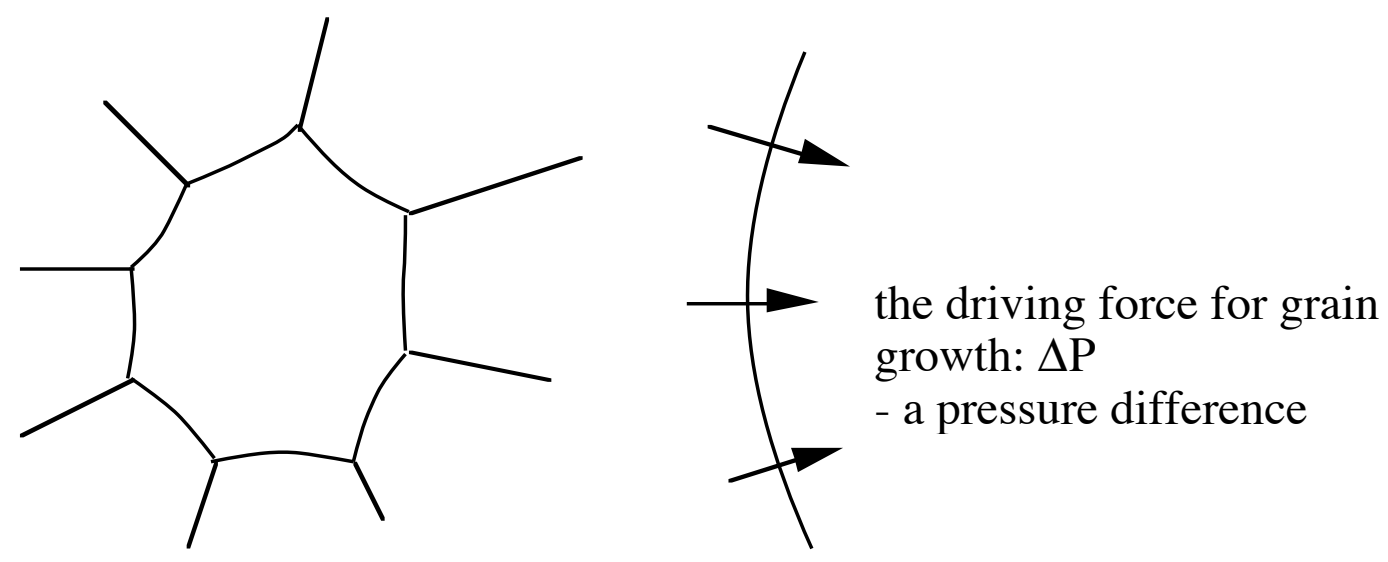

grain growth 
The kinetics of grain growth can be described by

$$
\text { velocity }=\frac{d R}{d t}=\frac{D^{*} \Omega}{k T \delta} \bullet \Delta P=\frac{1}{2} \frac{d L}{d t},
$$

where:

$$
\begin{aligned}
& \Delta P=\text { pressure difference (driving grain growth) } \\
& \Omega=\text { atomic volume } \\
& \delta=\text { atomic jump distance in boundary } \\
& D^{*}=\text { grain boundary diffusivity }
\end{aligned}
$$

which is expressed as

$$
D^{*}=\delta^{2} v \exp \left(-\frac{\Delta H}{k T}\right)
$$

and where

$$
v=\text { vibration frequency. }
$$

This relation can be understood by noting that one term in the expression is an atomic force

$$
F=\frac{\Omega}{\delta} \bullet \Delta P
$$

which through the Einstein relation yields a velocity

$$
\text { velocity }=\frac{D^{*}}{k T} \bullet F \text {. }
$$

This relation can also be justified microscopically by noting that the mechanism of grain boundary migration involves atoms jumping across the boundary. The diagram shows this to be the elementary process of grain boundary migration. 


\section{Grain boundary migration}

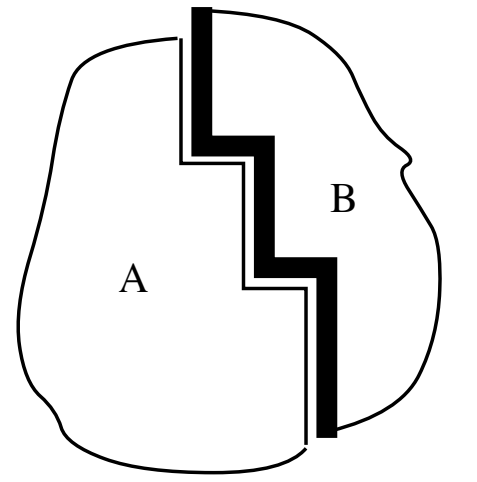

Reference atoms in crystal B

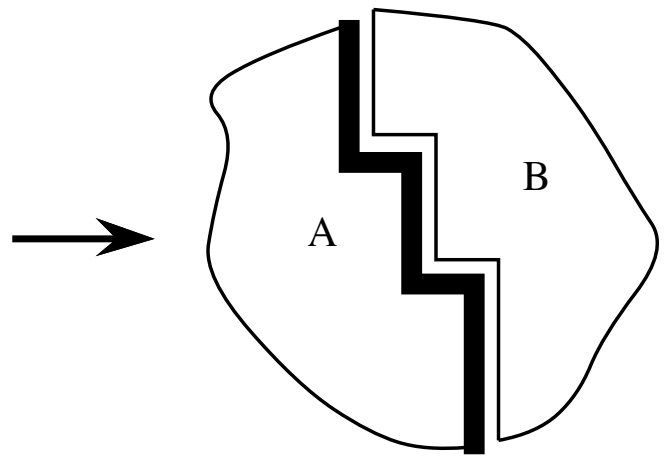

Reference atoms in crystal A

\section{Driving force for grain growth}

First consider a simple case of no strain energy (standard grain boundary). As discussed above, the grain boundary energy per unit volume is

$$
E_{g b}=\frac{3 \gamma_{g b}}{L},
$$

where $L$ is the grain diameter. For an infinitesimal amount of grain growth, $d L$,

$$
d E_{g b}=-\frac{3 \gamma_{g b}}{L^{2}} d L .
$$

This energy variation is the same as that produced by the migration of the boundaries in response to a driving pressure difference, $\Delta \mathrm{P}$ :

$$
d E_{g b}=-\Delta P \bullet \frac{3}{L} \bullet d R=-\Delta P \bullet \frac{3}{L} \bullet \frac{d L}{2},
$$

where the term $3 / L$ is the boundary area per unit volume and the term $d R=d L / 2$ represents an incremental movement of the boundary. 


\section{Equivalent work effect for grain growth}

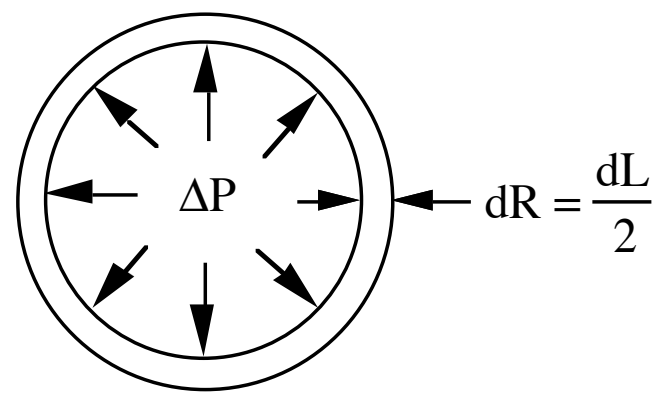

Equating the two work effects gives,

$$
\Delta P=\frac{2 \gamma_{g b}}{L} .
$$

This is the classical result for the driving force for grain growth.

Now consider grain growth in a thin film with associated strain energy. The energy per unit volume is

$$
\begin{aligned}
& E=E_{g b}+W_{e l}, \\
& E=\frac{3 \gamma_{g b}}{L}+(\Delta a)^{2}\left(\frac{1}{L_{o}}-\frac{1}{L}\right)^{2}\left(\frac{E}{1-v}\right)_{\text {film }} .
\end{aligned}
$$

Here an infinitesimal amount of grain growth produces the energy variation

$$
d E=-\frac{3 \gamma_{g b}}{L^{2}} d L+2(\Delta a)^{2}\left(\frac{1}{L_{o}}-\frac{1}{L}\right)\left(\frac{E}{1-v}\right)_{f i l m} \frac{d L}{L^{2}} .
$$

Again this energy variation can be represented by

$$
d E=-\Delta P \bullet \frac{3}{L} \bullet \frac{d L}{2} .
$$

Equating the two, we obtain 


$$
\Delta P=\frac{2 \gamma_{g b}}{L}-\frac{4(\Delta a)^{2}}{3 L}\left(\frac{1}{L_{o}}-\frac{1}{L}\right)\left(\frac{E}{1-v}\right)_{f i l m} .
$$

Using this in the equation for grain growth, we have

$$
\begin{aligned}
& \frac{d L}{d t}=2 \bullet \frac{D^{*} \Omega}{k T \delta} \bullet \Delta P \\
& \frac{d L}{d t}=2 \bullet \frac{D^{*} \Omega}{k T \delta} \bullet \frac{2 \gamma_{g b}}{L}\left[1-\frac{2(\Delta a)^{2}}{3 \gamma_{g b}}\left(\frac{1}{L_{o}}-\frac{1}{L}\right)\left(\frac{E}{1-v}\right)_{f i l m}\right] .
\end{aligned}
$$

The development of intrinsic stress is found by differentiation of the expression for intrinsic stress

$$
\frac{d \sigma}{d t}=\left(\frac{E}{1-v}\right)_{\text {film }} \Delta a \frac{1}{L^{2}} \frac{d L}{d t},
$$

and using the grain growth law

$$
\frac{d \sigma}{d t}=\left(\frac{E}{1-v}\right)_{\text {film }} \Delta a \frac{D^{*} \Omega}{k T \delta} \cdot \frac{4 \gamma_{g b}}{L^{3}}\left[1-\frac{2(\Delta a)^{2}}{3 \gamma_{g b}}\left(\frac{1}{L_{o}}-\frac{1}{L}\right)\left(\frac{E}{1-v}\right)_{\text {film }}\right] .
$$

We note that grain growth stops when

$$
1=\frac{2(\Delta a)^{2}}{3 \gamma_{g b}}\left(\frac{1}{L_{o}}-\frac{1}{L}\right)\left(\frac{E}{1-v}\right)_{f i l m},
$$

or

$$
\frac{1}{L_{o}}-\frac{1}{L_{\text {ter } \min a l}}=\frac{3 \gamma_{g b}}{2\left(\frac{E}{1-v}\right)_{f i l m}(\Delta a)^{2}} .
$$

as discussed above. This condition is satisfied only if the initial grain size is sufficiently small to satisfy this relation. The expression above for the rate of change of the stress due to grain growth can be integrated numerically to obtain the evolution of stress in the film during a heating cycle in which the grain size changes. 


\section{Excess Vacancy Annihilation}

When crystalline films are formed by deposition onto cold substrates, the conditions are far from equilibrium and it is reasonable to expect a nonequilibrium, excess vacancy concentration to be established in the film. As these vacancies annihilate, the associated volume changes cause a stress to develop within the film.

Deposited film with excess vacancies

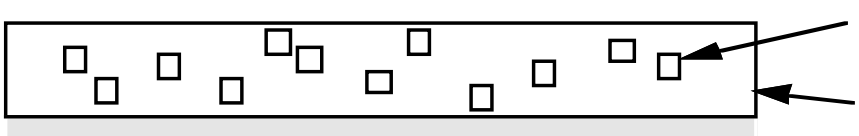

excess vacancies

as-deposited film

(non-equilibrium, but

stress free state)

The sign and magnitude of the stress associated with excess vacancy annihilation depends both on vacancy volume and site of annihilation.

Consider: $\Omega_{v}=\Omega$ (atomic volume), a perfectly rigid lattice.

Vacancy annihilation at a surface

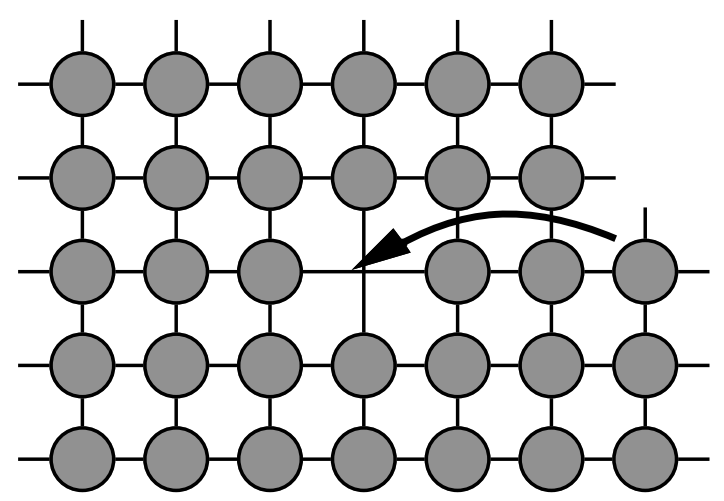


There are various possible annihilation sites:

1. Free surface

2. Film/substrate interface

3. Voids

4. Grain boundaries

1. Annihilation at free surface :

Vacancy annihilation at a free surface

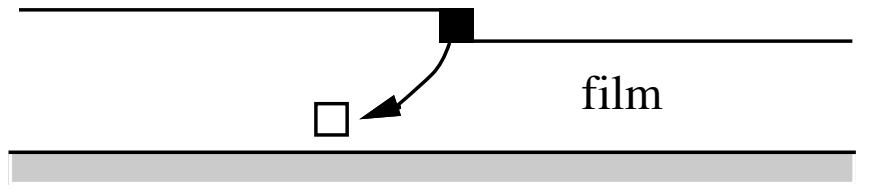

Here there is a volume change - but no stress is developed - the planar dimensions at the film are not changed with respect to those of the substrate.

2. Annihilation at voids:

Vacancy annihilation at an internal void

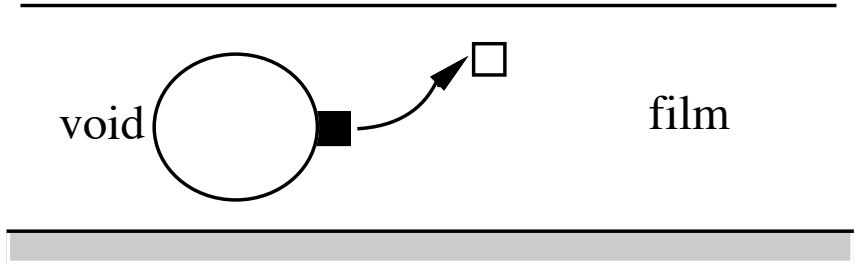

No volume change occurs and no stress is developed. 
3. Annihilation at substrate/film interface (or at grain boundaries parallel to free surface at film ):

Vacancy annihilation at parallel grain boundaries

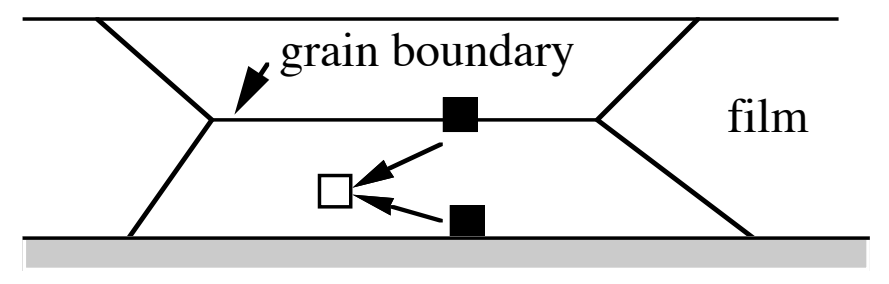

This is approximately the same as annihilation at free surface. No stress is expected to develop.

4. Annihilation at grain boundaries perpendicular to the film:

Annihilation at grain boundaries perpendicular to the film

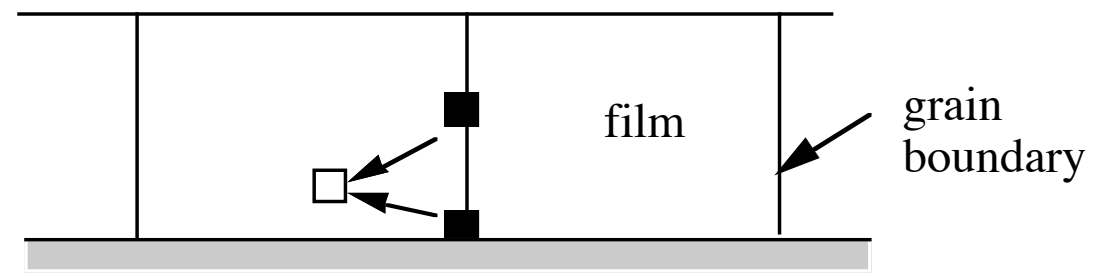

In this case a biaxial tension stress develops - removing atoms from the grain boundary creates "gaps" which must be closed by elastic stretching of the grains.

5. Annihilation at edge dislocations:

Annihilation at edge dislocations

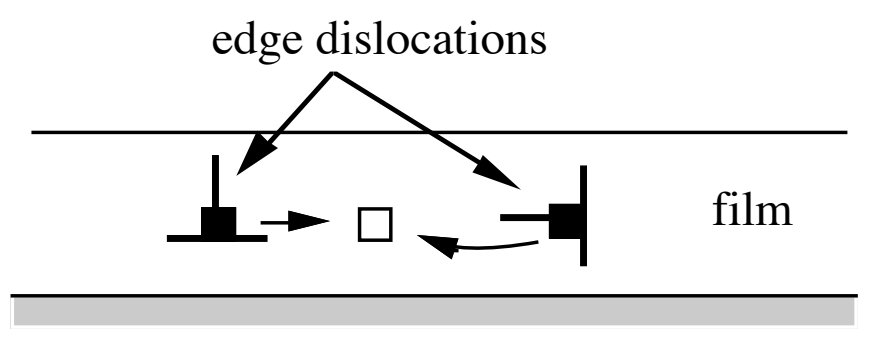


This is analogous to grain boundaries parallel and perpendicular to the film. Tension stress develops for a Burgers vector, $\vec{b}$, parallel to the film. No stress develops for the Burgers vector, $\vec{b}$, perpendicular to the film.

\section{Consider: $\Omega_{v}<\Omega$ (atomic volume). Relaxation Effects}

Before treating vacancy annihilation in detail, consider also the case in which the vacancy volume is less than the atomic volume: $\Omega_{v}<\Omega$ (atomic volume). This is expected for lattices that have finite rigidity because the vacancy tends to contract the lattice around it. Each time a vacancy annihilates (i.e. when the vacancy is replaced by a lattice atom) a volume expansion $\Omega-\Omega_{v}$ occurs, regardless of where the vacancy annihilates. So, in addition to the effects discussed above (which are annihilation site specific), there is a volume expansion $\left(\Omega-\Omega_{v}\right)$ associated with each annihilation event. The relaxational dilatation associated with the annihilation of $\Delta c$ vacancies per unit volume is

$$
\Delta e^{T}=\Delta c \bullet\left(\Omega-\Omega_{v}\right)
$$

This dilatational strain leads to a misfit strain which in turn leads to stresses in the film.

Misfit associated with vacancy annihilation

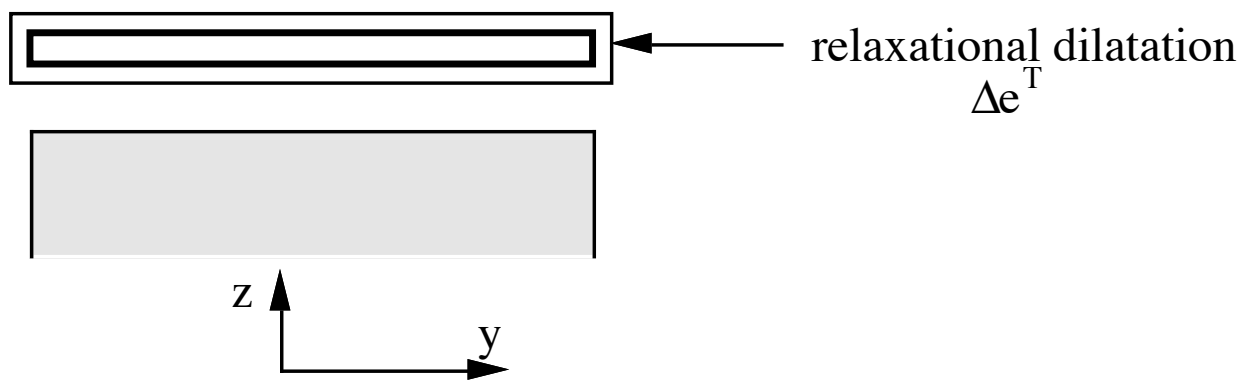

The corresponding elastic strains in the film are

$$
\varepsilon_{x x}^{e l}=\varepsilon_{y y}^{e l}=-\frac{e^{T}}{3}
$$

These must be imposed on the film so that it will fit the substrate. The corresponding biaxial stress in the film is 


$$
\sigma=-\left(\frac{E}{1-v}\right)_{\text {film }} \bullet \frac{e^{T}}{3}=-\left(\frac{E}{1-v}\right)_{\text {film }} \bullet \frac{\Delta c \bullet\left(\Omega-\Omega_{v}\right)}{3}
$$

Here $\Delta c$ represents all of the annihilating vacancies per unit volume, regardless of where they annihilate. This compressive stress should be added to any stresses that develop due to vacancy annihilation at grain boundaries perpendicular to the film.

\section{Annihilation of Vacancies at Grain Boundaries Perpendicular to Film $\left(\Omega_{v}=\Omega\right)$}

Consider a polycrystalline film with a grown-in vacancy concentration $c_{i}>c_{0}$, where $c_{o}$ is the (stress free) equilibrium vacancy concentration at the deposition temperature.

\section{$\underline{\text { One-dimensional model }}$}

Model for stress generation due to vacancy annihilation at perpendicular grain boundaries

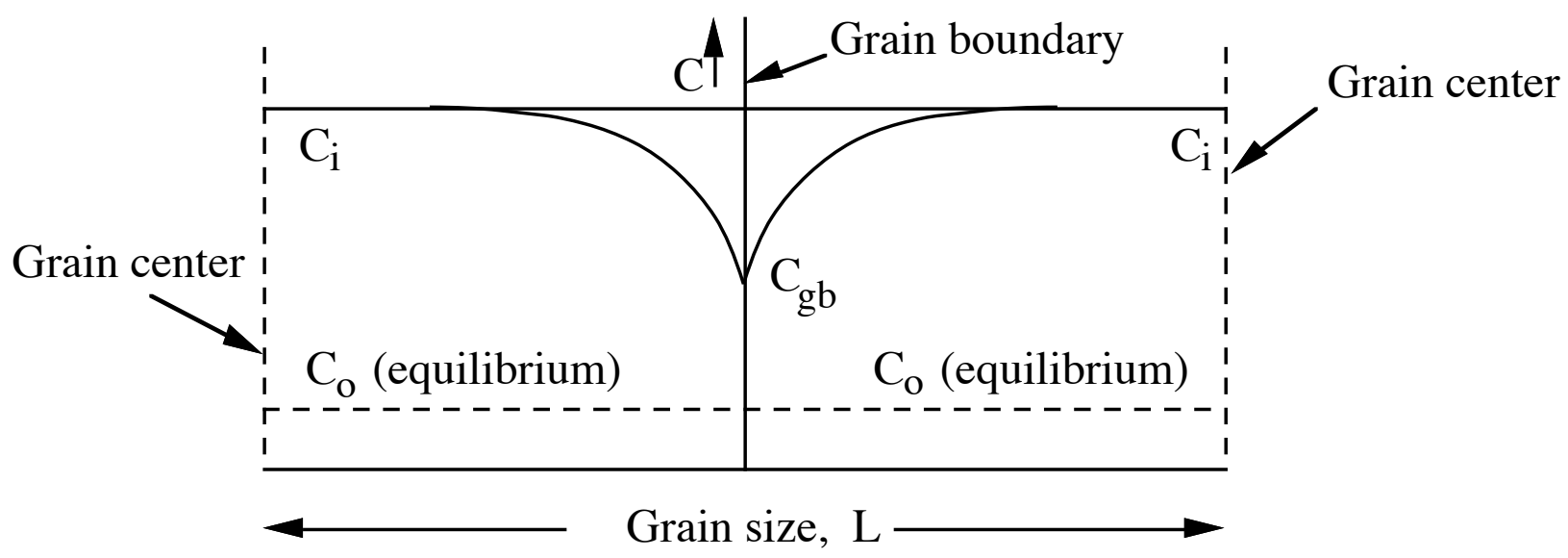

Some of the vacancies near the grain boundary diffuse to the boundary and annihilate there. This reduces the vacancy concentration toward the equilibrium value. But, annihilating vacancies at the grain boundaries causes gaps to form between the grains unless the adjoining grains are free to move toward each other to eliminate the gap. 
Gap formation due to vacancy annihilation

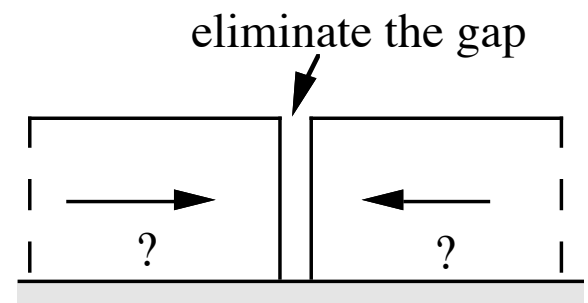

But the grains are attached to the substrate and are not free to move. The only way to keep the gap closed is by elastically straining the adjoining crystals. In the present simple analysis, we assume that the grains are rigidly attached to the substrate only at the centers of the grains. Then,

\section{Elastic displacements to maintain grain-grain contact}

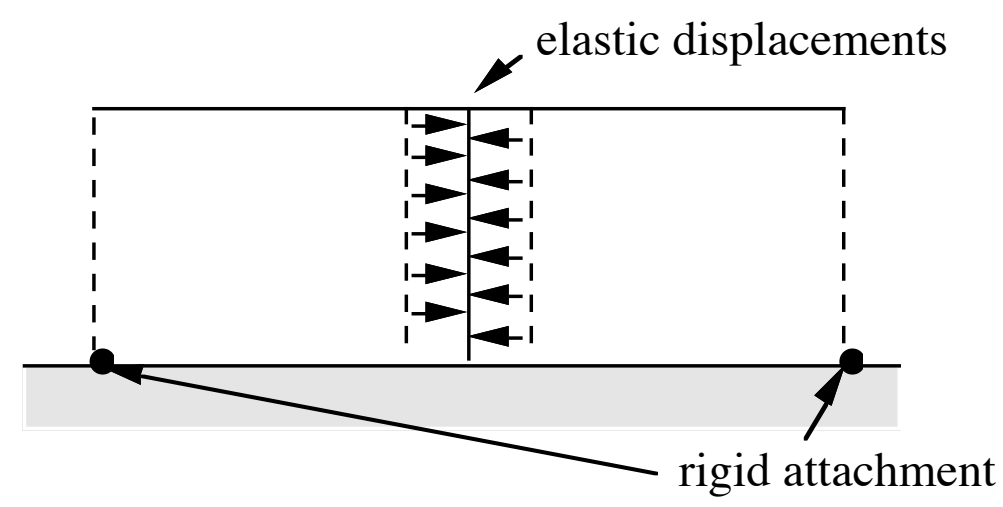

If the grains were everywhere bonded to the substrate, a more complex stress state would develop.

Grains attached to substrate

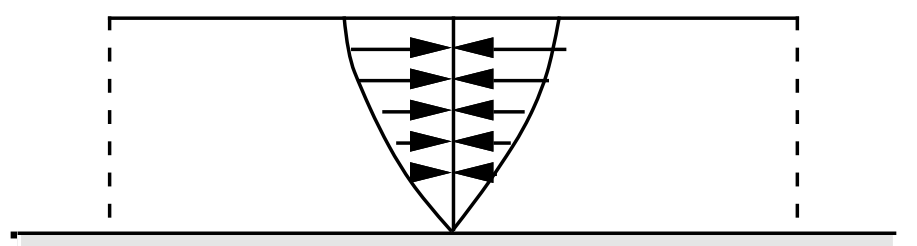

rigid attachment of film to substrate 
Consider the simplest case - the development of tension stress in the film causes the equilibrium vacancy concentration in the grain boundary $c_{g b}$ to increase, and we have

$$
c_{g b}=c_{o} \exp \left(\frac{\sigma \Omega}{k T}\right) \approx c_{o}\left[1+\frac{\sigma \Omega}{k T}\right],
$$

where

$$
\begin{aligned}
& \sigma=\text { biaxial stress in film } \\
& \Omega=\text { atomic volume } \\
& k=\text { Boltzmann's constant } \\
& T=\text { absolute temperature }
\end{aligned}
$$

The vacancy flux to the grain boundary results is the following vacancy concentration profile:

\section{Vacancy diffusion profile}

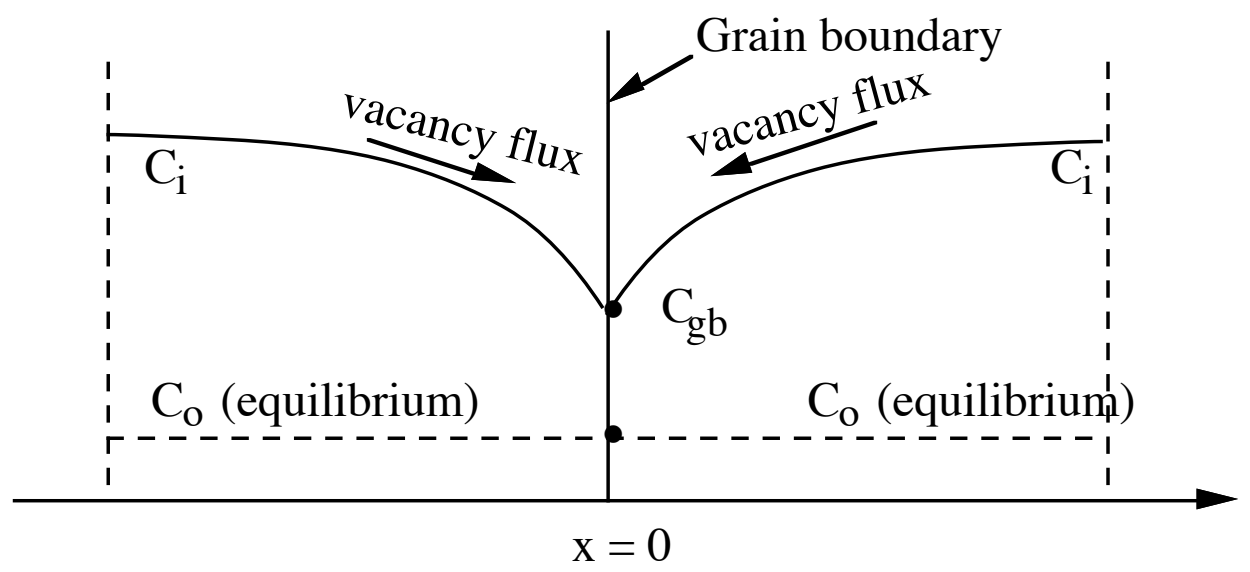

The kinetics of stress generation are controlled by the kinetics of vacancy diffusion to the grain boundary:

Considering vacancy flow in the $x>0$ domain, the rate of "gap" generation is given by

$$
\dot{u}=-2 J_{v}(x=0) \Omega \text {. }
$$


This relation is understood by noting that volume rate / unit area $=$ displacement rate. Also, $J_{v}$ is expressed as vacancies/ unit area-sec. The factor 2 come from diffusion from both sides of the boundary and the negative sign is related to the negative vacancy flux in the $x>0$ domain.

If the gap is kept closed by elastic straining of the crystals:

$$
\frac{d \sigma}{d t}=\frac{E}{1-v} \bullet \frac{\dot{u}}{L}
$$

Using Fick's first Law

$$
J_{v}(x=0)=-D_{v}\left(\frac{\partial c}{\partial x}\right)_{x=0}
$$

so

$$
\frac{d \sigma}{d t}=\left(\frac{E}{1-v}\right) \bullet\left(\frac{-2 \Omega}{L}\right)\left(-D_{v}\left(\frac{\partial c}{\partial x}\right)_{x=0}\right)=\left(\frac{E}{1-v}\right) \bullet\left(\frac{2 \Omega D_{v}}{L}\right) \bullet\left(\frac{\partial c}{\partial x}\right)_{x=0}
$$

But the stress is directly related to the grain boundary vacancy concentration through

$$
\sigma=\frac{k T}{\Omega}\left[\frac{c_{g b}}{c_{o}}-1\right]
$$

so

$$
\frac{d \sigma}{d t}=\frac{k T}{\Omega} \cdot \frac{1}{c_{o}} \cdot\left(\frac{d c_{g b}}{d t}\right)
$$

Using this we have

$$
\frac{d c_{g b}}{d t}=\frac{2 E \Omega D_{L}}{(1-v) k T \cdot L}\left(\frac{\partial c}{\partial x}\right)_{x=0}
$$

for the condition at the grain boundary. (Here we have used $D_{L}=\Omega \bullet c_{o} \bullet D_{v}$ the lattice diffusion coefficient.) 
Thus the relevant initial-boundary value problem is

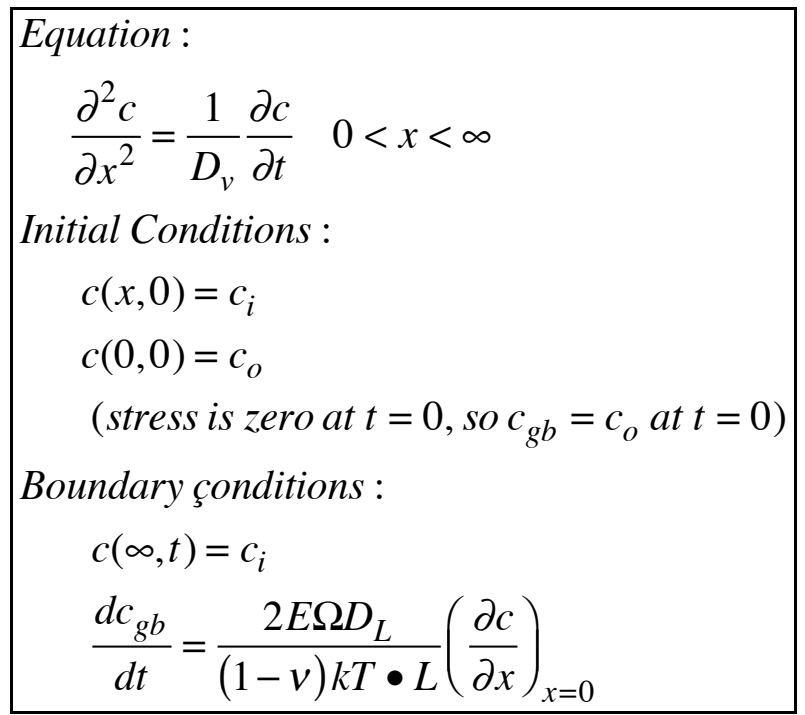

A few years ago D.M. Barnett produced a solution to this problem. The result is

$$
\frac{\sigma \Omega}{k T}=\left[\frac{c_{i}}{c_{o}}-1\right] \cdot\left[1-\exp \left(z^{2}\right) \operatorname{erfc}(z)\right],
$$

with

$$
z=\frac{2}{1-v} \bullet \frac{E \Omega}{L k T} \bullet \frac{D_{L}}{D_{v}}\left(D_{v} t\right)^{1 / 2} .
$$

For small $z$, short times, the stress varies as

$$
\sigma=\frac{4 E}{L(1-v)}\left[\frac{c_{i}}{c_{o}}-1\right] \frac{D_{L}}{D_{v}}\left(\frac{D_{v} t}{\pi}\right)^{1 / 2} .
$$

Schematically, this is a simple parabolic stress evolution law: 


\section{Stress evolution due to vacancy annihilation}

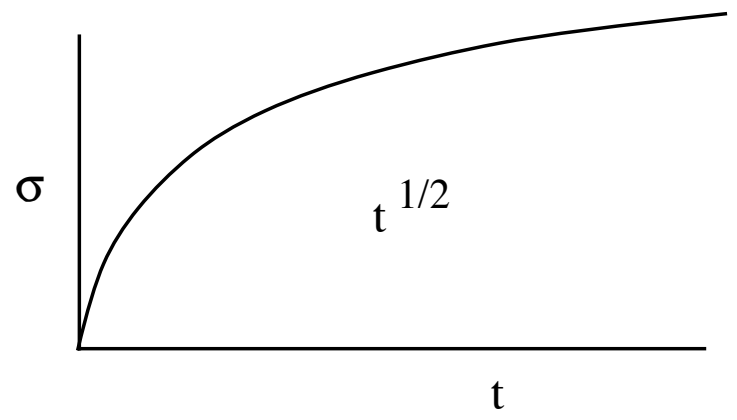

Writing $c_{i}-c_{o}=\Delta c$ and using $D_{L}=D_{v} \Omega c_{o}$ we have

$$
\sigma=\frac{4 E \Omega}{L(1-v)} \bullet \Delta c \bullet\left(\frac{D_{v} t}{\pi}\right)^{1 / 2} .
$$

The magnitude of stress depends on $\Delta c$; the kinetics depend on $D_{v}$.

\section{Shrinkage of Grain Boundary Cavities}

We suppose a thin polycrystalline film is formed with micro-porosity in the grain boundaries. The shrinkage of such cavities by diffusional processes will lead to stresses in the film. To model this we consider a cylinder of radius $R$ with a single grain containing a single hemispherical capped void.

\section{Model for grain boundary porosity}

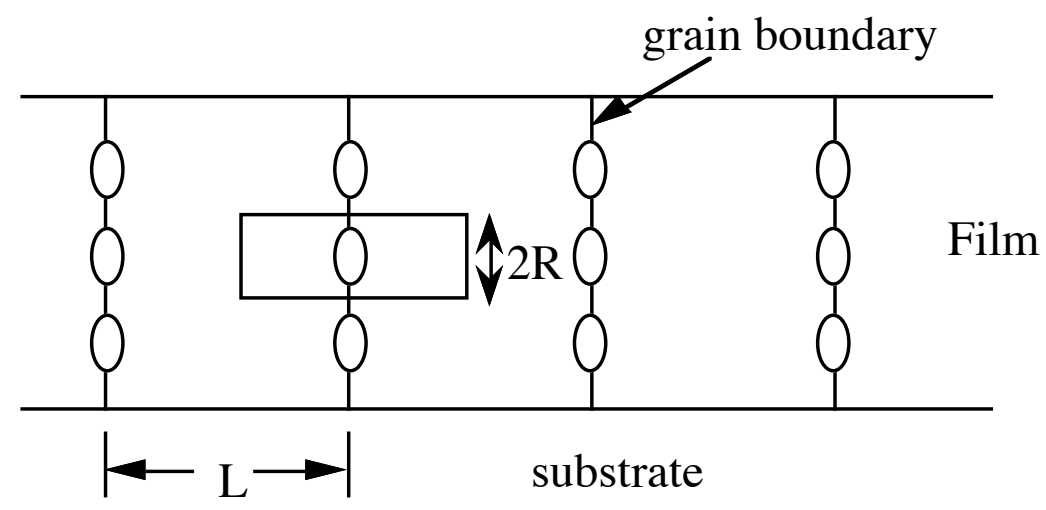

As atoms in the grain boundary diffuse into the voids, a biaxial tension stress develops in the film. Because the grain centers are attached to the substrate, the ends of the cylinder in question can be fixed. Let the grain boundary voids have a radius $a$ in the plane of the grain boundary, as shown in the next figure. 
Single grain boundary void

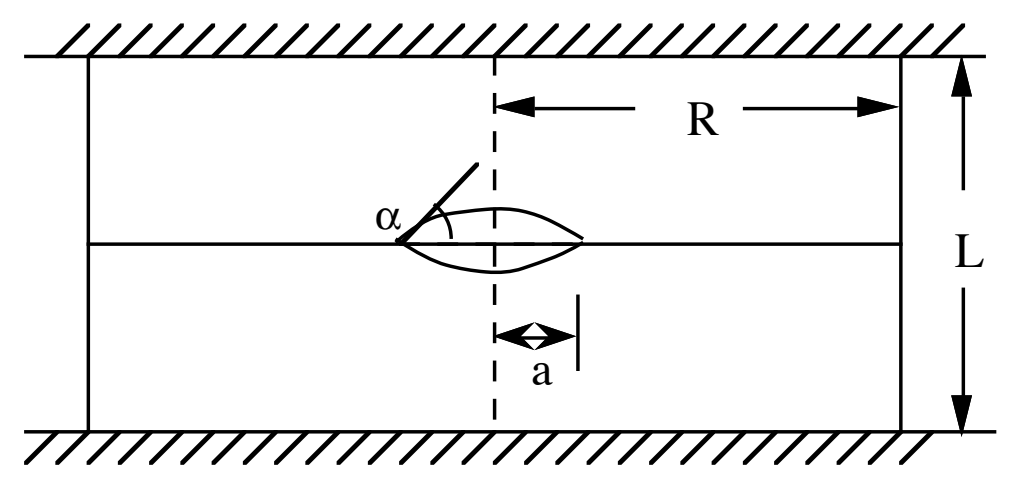

\section{Analysis of Stress Associated with Void Closure}

The void volume fraction is

$$
f=\frac{\beta a^{3}}{\pi R^{2} L}=\frac{V_{\text {void }}}{V_{\text {cylinder }}}
$$

where $\beta$ is a shape factor given by

$$
\beta=\frac{2 \pi}{3 \sin ^{3} \alpha} \bullet\left(2-3 \cos \alpha+\cos ^{3} \alpha\right) .
$$

This was given by Raj and Ashby (1975).

First assume that the length of the cylinder is unconstrained and that the length changes when the void grows by $d a$ 
Incremental viod growth

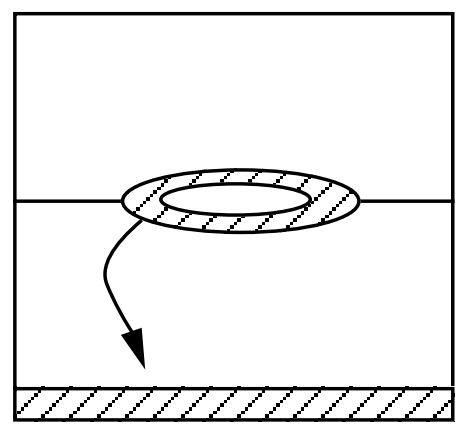

The incremental volume growth is

$$
d V=d\left(\beta a^{3}\right)=3 \beta a^{2} d a .
$$

We need to determine the change in length of the cylinder when the void grows by a given amount. Physically, atoms are removed from the surface of the void and deposited onto the adjoining grain boundary, around the cavity. But when the atoms removed from the cavity surface are deposited on the grain boundary they cause an additional amount of cavity growth, as the surfaces of the cavity are displaced away from each other. This is called a "jacking" term, as if the insertion of matter on the grain boundary were lifting one grain relative to the other, like jacking up a car. But for a fixed cavity volume change one may compute the length change in a much simpler way, by imagining that the material removed from the cavity surface is deposited onto the end of the cylinder. This is the same as deposition on the adjoining grain boundary. The corresponding length change is then

$$
d u=\frac{d V}{\pi R^{2}}=\frac{3 \beta a^{2} d a}{\pi R^{2}} .
$$

If the length of the cylinder is constrained not to change, then a compressive stress would be associated with an increment of cavity growth (or a tension stress associated with cavity shrinkage),

$$
d \sigma=-\left(\frac{E}{1-v}\right)_{f i l m} \bullet \frac{d u}{L} .
$$

where 


$$
E_{f i l m}=(1-f) \cdot E=\left(1-\frac{\beta a^{3}}{\pi R^{2} L}\right) \bullet E
$$

where we account for the effect of the cavity on the elastic modulus of the film, and

$$
d u=\frac{3 \beta a^{2} d a}{\pi R^{2}}
$$

Thus

$$
d \sigma=-\left(\frac{E}{1-v}\right) \cdot \frac{1}{L} \bullet\left(1-\frac{\beta a^{3}}{\pi R^{2} L}\right) \cdot \frac{3 \beta a^{2}}{\pi R^{2}} \bullet d a
$$

Thus the kinetics of intrinsic stress development depend on the kinetics of cavity shrinkage, which in turn depends on grain boundary diffusion of atoms to the cavity. This is a classical problem in the field of creep fracture that has been extensively studied. Speight \& Beere have given the most exact solution for stress driven cavity growth:

$$
\frac{d V}{d t}=\frac{2 \pi \bullet \delta D_{g b} \bullet \Omega}{k T} \bullet \frac{\left(\sigma-\frac{2 \gamma_{s}}{a}\right)}{\left\{\ln \left(\frac{R}{a}\right)-\frac{a^{4}}{4 R^{4}}+\frac{a^{2}}{R^{2}}-\frac{3}{4}\right\}}
$$

where:

$$
\begin{aligned}
& \sigma=\text { biaxial stress } \\
& \delta D_{g b}=\text { grain boundary width } \bullet \text { diffusivity } \\
& 2 \gamma_{s} / a=\text { capillarity stress for spontaneous cavity shrinkage. }
\end{aligned}
$$

Void shrinkage by grain boundary diffusion

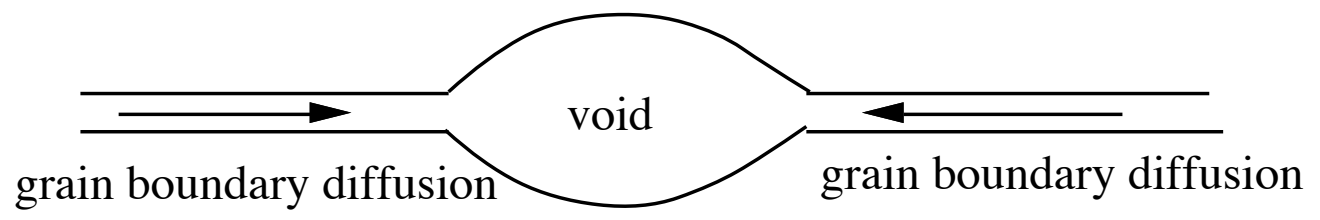


Initially, $\sigma=0$ and the void starts to shrink. If the void is sufficiently small it will continue to shrink even when the tension stress develops in the film. For larger cavities, cavity shrinkage is eventually stopped by the stress that develops. The Speight-Beere expression for volumetric growth can be expressed in terms of the change in void size.

The incremental volume change is

$d V=3 \beta a^{2} d a$

and this can be directly related to the stress increment

$$
d \sigma \sim-d a .
$$

This leads to:

$$
\frac{d \sigma}{d t}=-\frac{E}{1-v} \cdot \frac{1}{L} \bullet\left(1-\frac{\beta a^{3}}{\pi R^{2} L}\right) \cdot \frac{2 \pi \bullet \delta D_{g b} \bullet \Omega}{\pi R^{2} \bullet k T} \cdot \frac{\left(\sigma-\frac{2 \gamma_{s}}{a}\right)}{\left\{\ln \left(\frac{R}{a}\right)-\frac{a^{4}}{4 R^{4}}+\frac{a^{2}}{R^{2}}-\frac{3}{4}\right\}} .
$$

The corresponding expression for the change of void size is:

$$
\frac{d a}{d t}=\frac{2 \pi \bullet \delta D_{g b} \bullet \Omega}{3 k T \beta a^{2}} \cdot \frac{\left(\sigma-\frac{2 \gamma_{s}}{a}\right)}{\left\{\ln \left(\frac{R}{a}\right)-\frac{a^{4}}{4 R^{4}}+\frac{a^{2}}{R^{2}}-\frac{3}{4}\right\}} .
$$

These two equations must be solved simultaneously to calculate the development of intrinsic stresses by void shrinkage.

The cavity shrinkage equations can be expressed approximately as:

$$
\begin{aligned}
& \frac{d a}{d t}=A \bullet \frac{1}{a^{2}} \bullet\left(\sigma-\frac{2 \gamma_{s}}{a}\right) \\
& \frac{d \sigma}{d t}=-B \bullet\left(\sigma-\frac{2 \gamma_{s}}{a}\right)
\end{aligned}
$$


The solution of these equations (for a fixed volume fractions of voids and fixed grain size) should be as shown in the following figure.

Intrinsic stress development by shrinkage of grain boundary voids

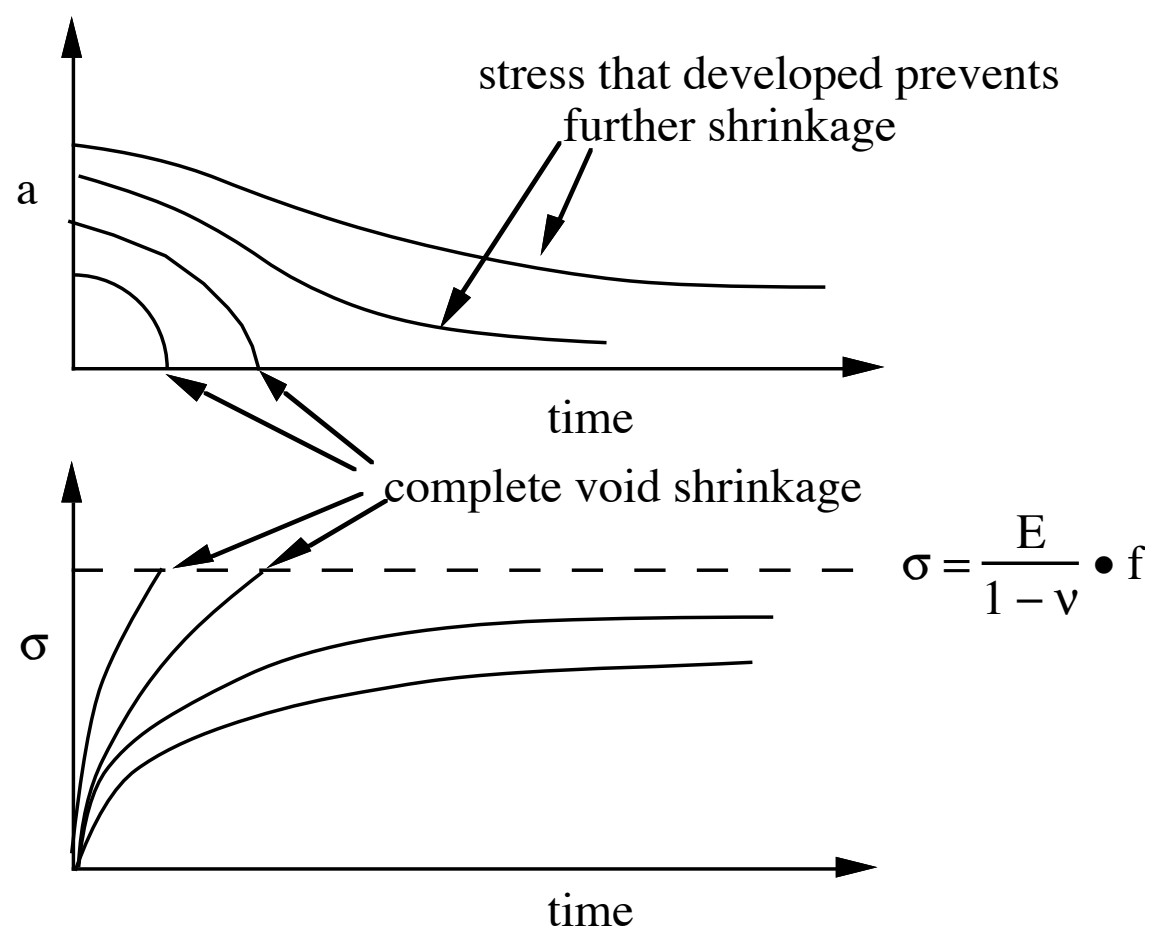

\section{Phase Transformations}

A major source of stress in alloy thin films involves the volume changes associated with phase changes. Films are often deposited in an amorphous or microcrystalline state. On heating, the film can crystallize or the more stable phase can form. A stress develops if the more stable phases that form have a different volume (compared to the initial state). Below are shown some examples of these effects. 
Sputter Deposited Al + 1\%Si + 2.5\%Ti Films - Densification on First Heating ( $\mathrm{TiAl}_{3}$ precipitation)

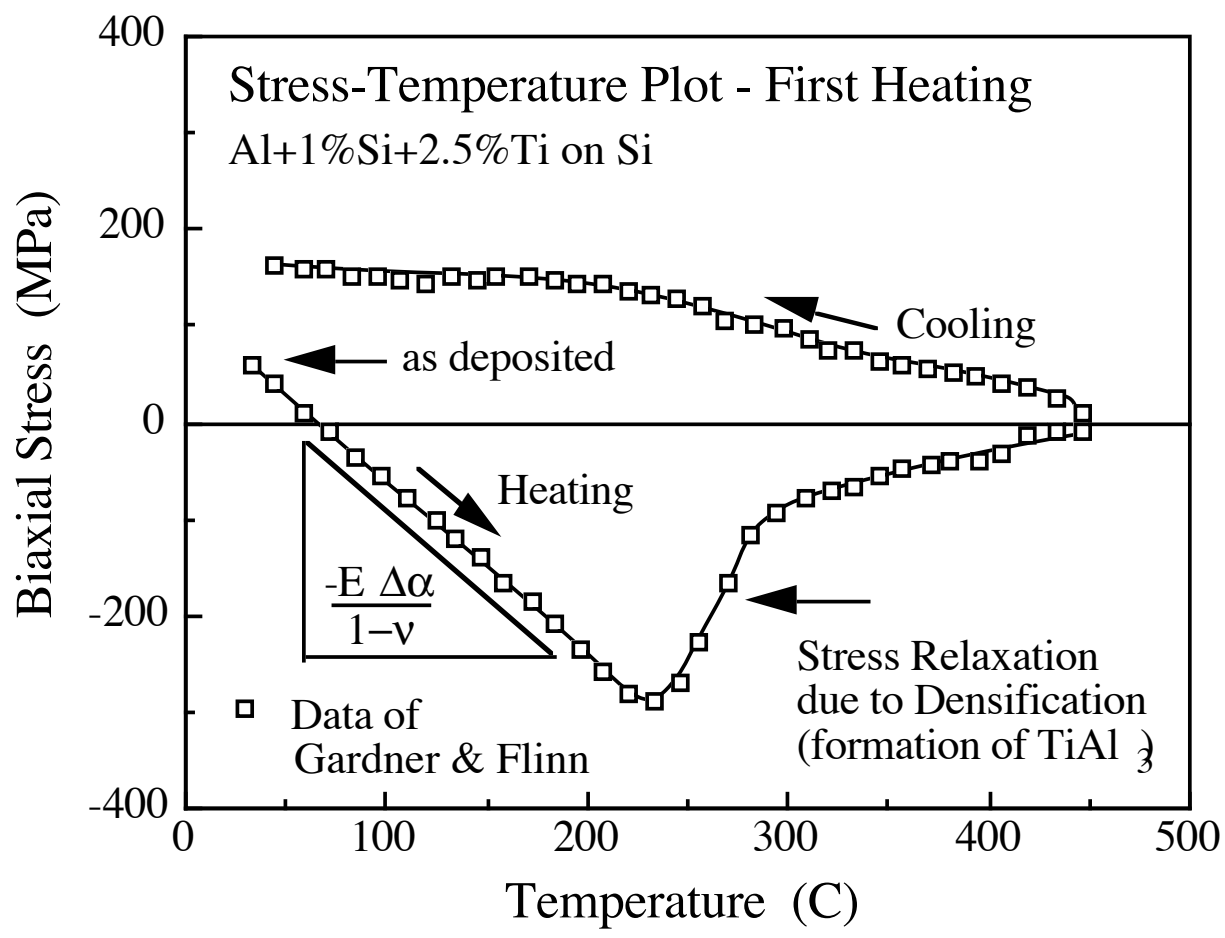

Paul Townsend has studied these densification processes in a number of CVD films. 
Densification of CVD WSi ${ }_{2}$ Films on Heating

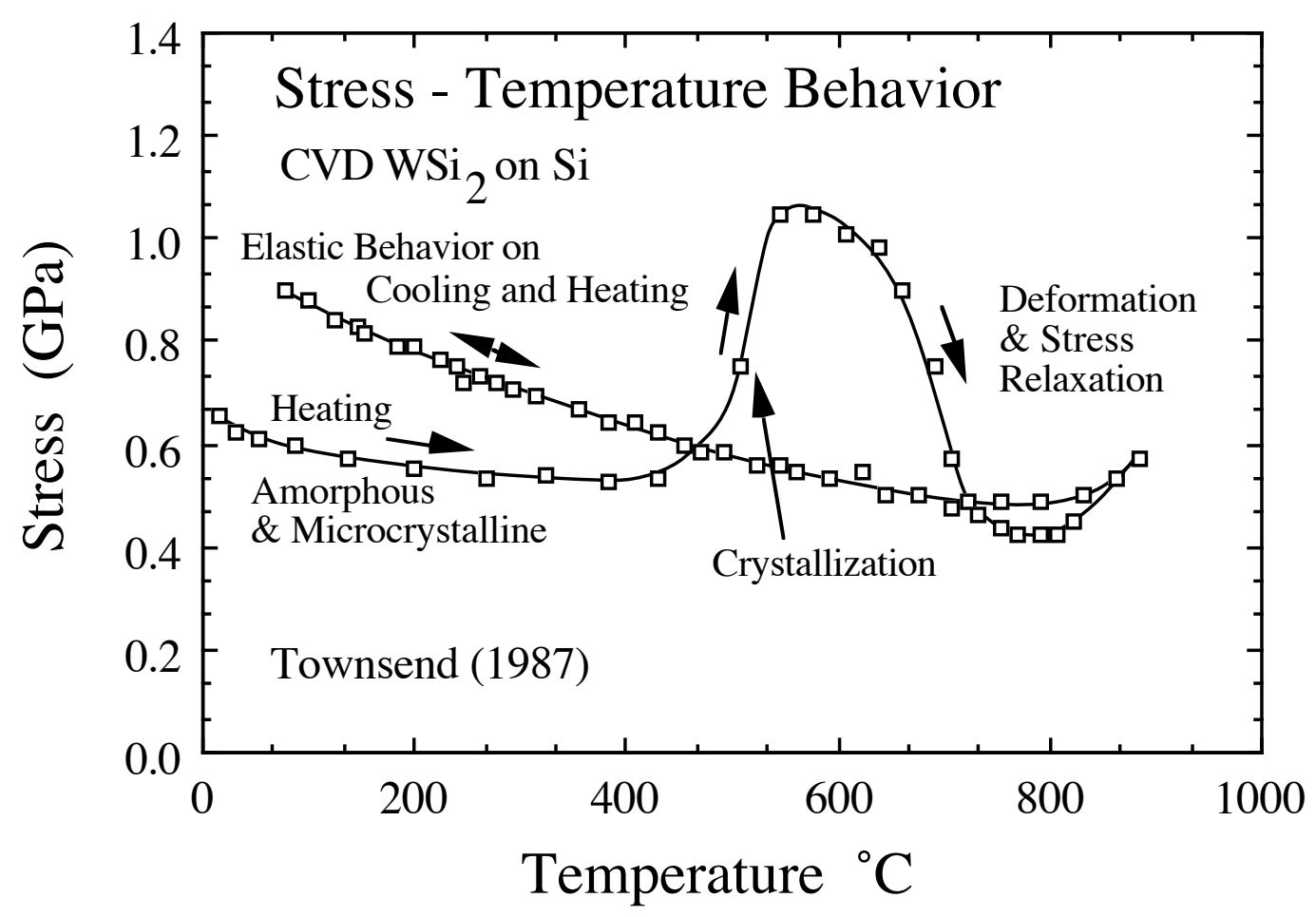

Similar behavior has been observed for $\mathrm{TaSi}_{2}$ films 
Evolution of Stress with Temperature in a TaSi 2 Film During First and Second Annealing Cycles to $920^{\circ} \mathrm{C}$.

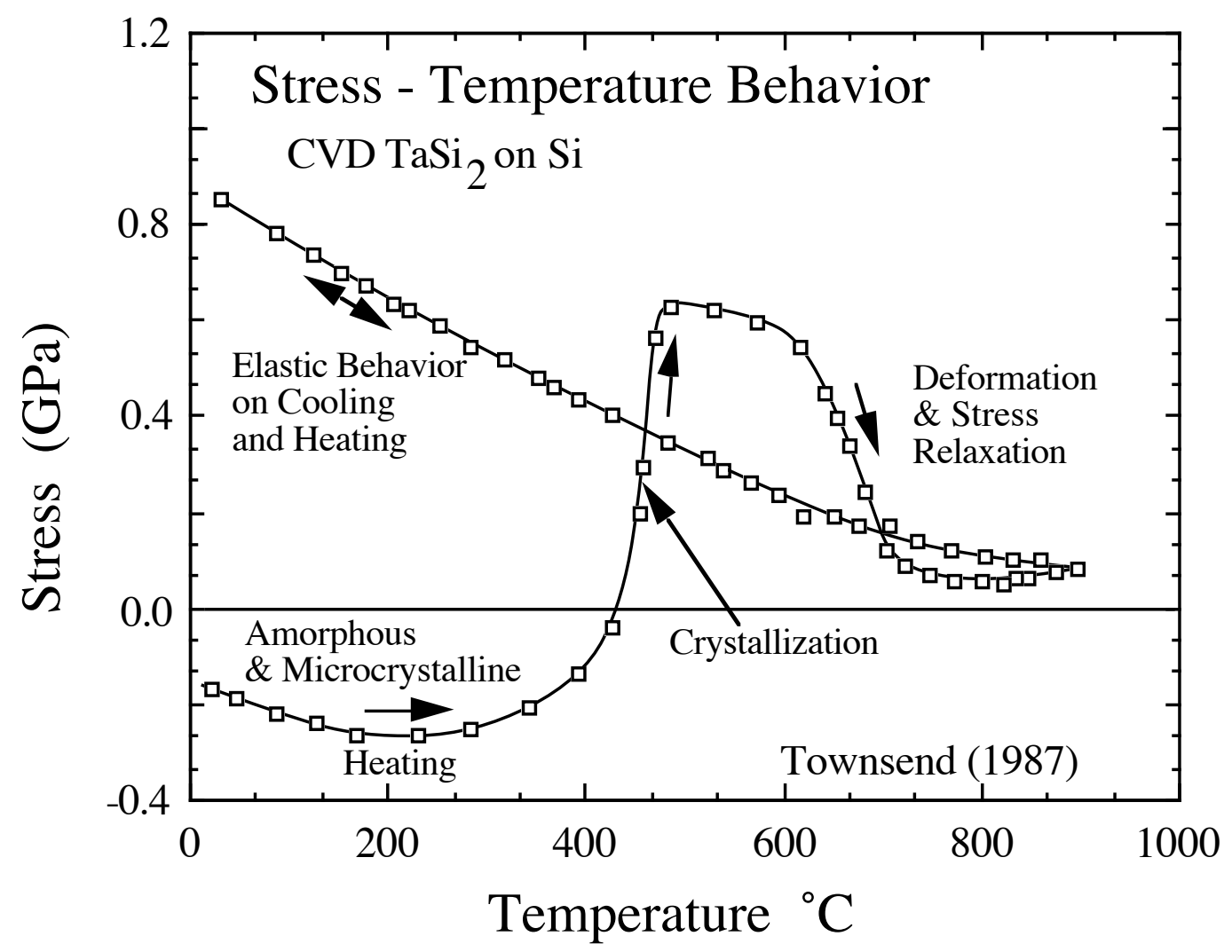


Evolution of Stress with Temperature in a $\mathrm{TaSi}_{2}$ Film During First and Second Annealing Cycles to $500^{\circ} \mathrm{C}$.

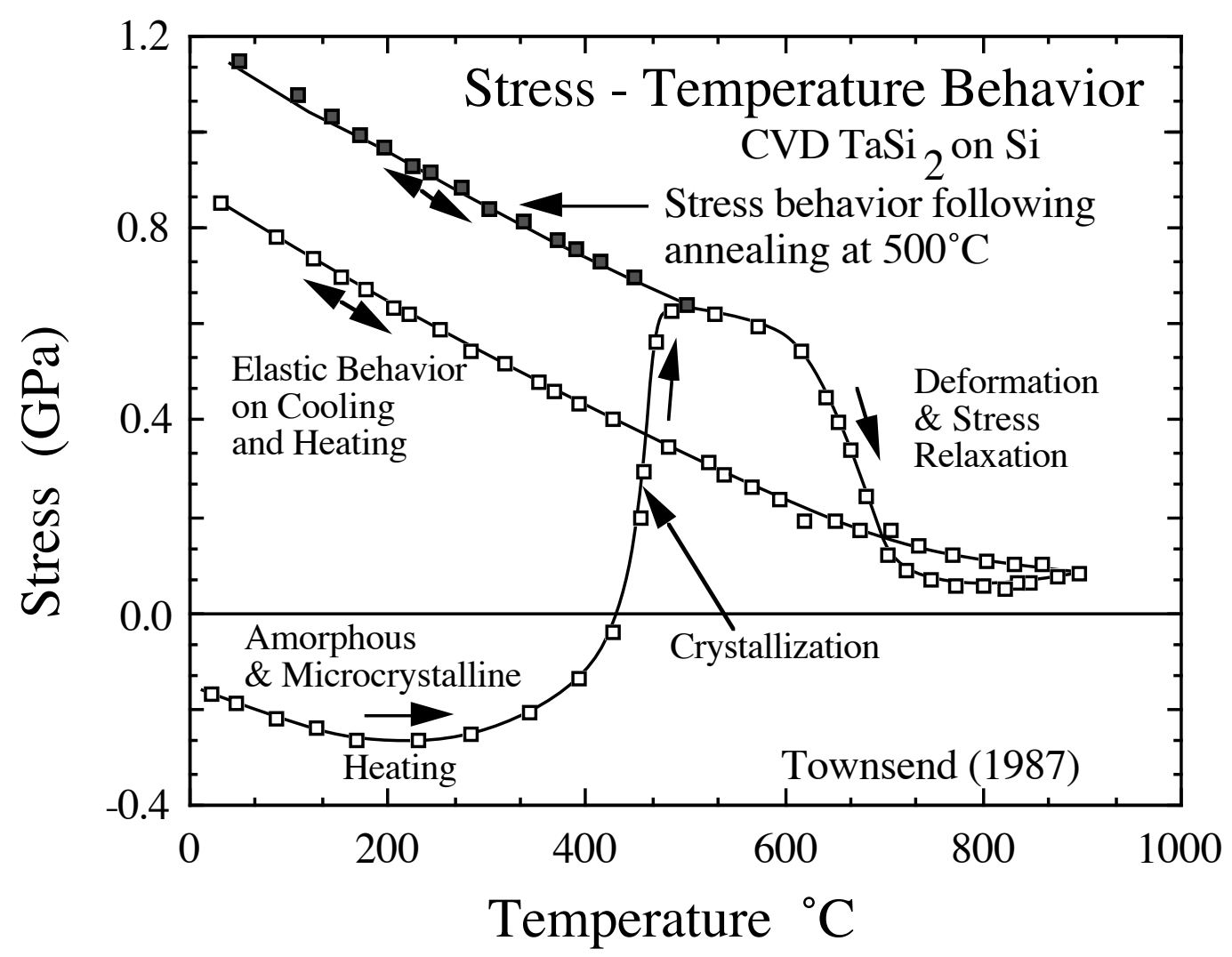

Below we show evidence for the formation of crystalline $\mathrm{TaSi}_{2}$ during annealing of CVD films. This provides strong evidence that the stress behavior is associated with the densification associated with formation of the intermetallic compound. 
XRD Spectra Of $\mathrm{TaSi}_{2}{ }_{2}$ Film Deposited In Homogeneous Condition and Annealed at Several Temperature

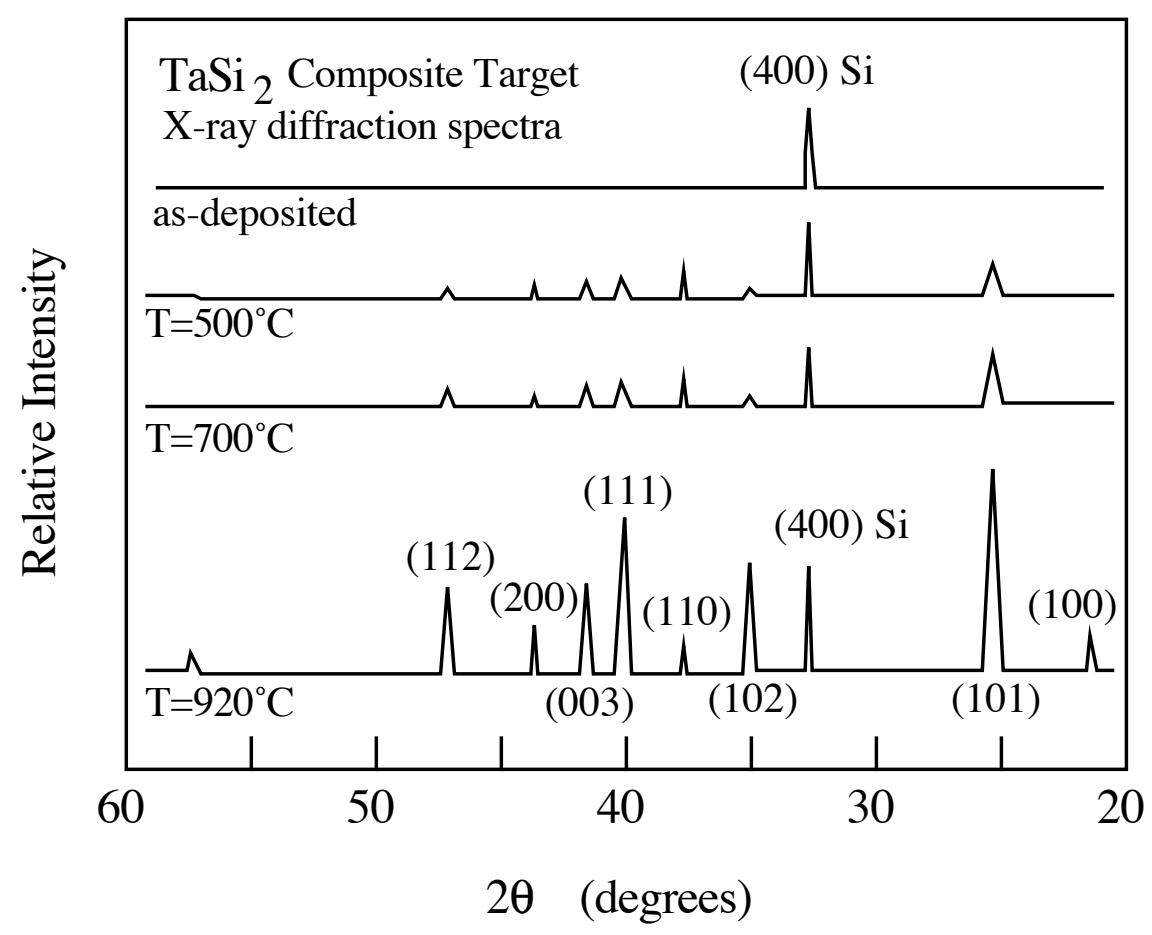


Electron diffraction patterns for a $\mathrm{TaSi}_{2}$ film heated to various temperatures

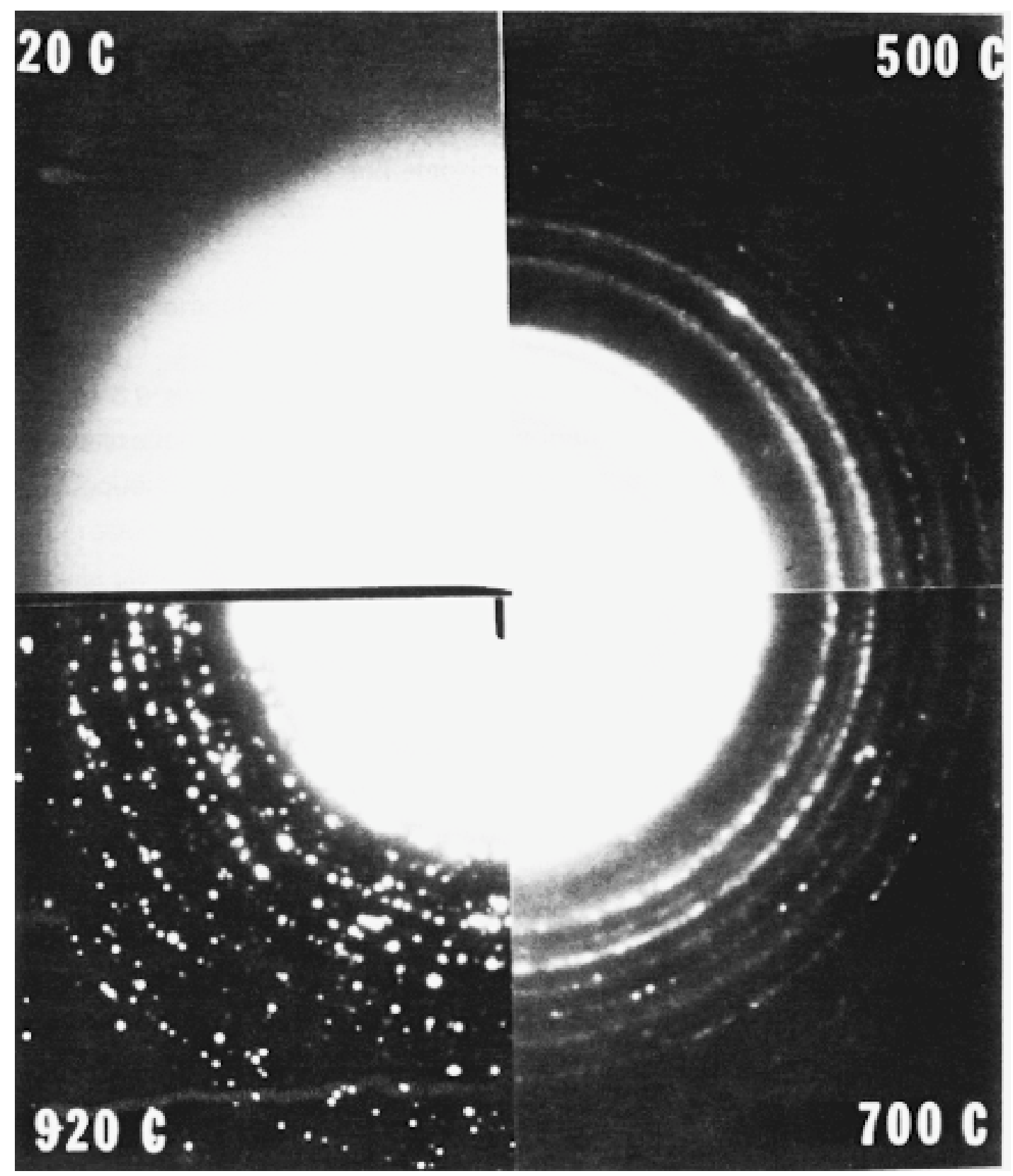




\section{Stresses in Sputtered Films-}

Most of mechanisms discussed above lead to tension stresses in deposited films. For sputtered films very large compressive stresses can be generated under certain conditions. Here we discuss the factors controlling the stresses in sputtered films. An excellent review of this subject is available in the classic paper by Thornton and Hoffman:

J.A. Thornton and D.W. Hoffman. Stress-Related Effects in thin Films., Thin Solid Films 171 (1989), 5-31. (Reprinted from: Proc. 22 Ann. Tech. Conf. Soc. of Vac. Coaters, 1979).

Other good references include:

J.A. Thornton, J. Tabock and D.W. Hoffman, Thin Solid Films $\underline{64} 111$ (1979)

D.W. Hoffman and J.A. Thornton, J. Vac. Sci. Tech. 17380 (1979)

The sputtering process can be illustrated as shown in the following diagram.

\section{Schematic Diagram of Sputtering Process}

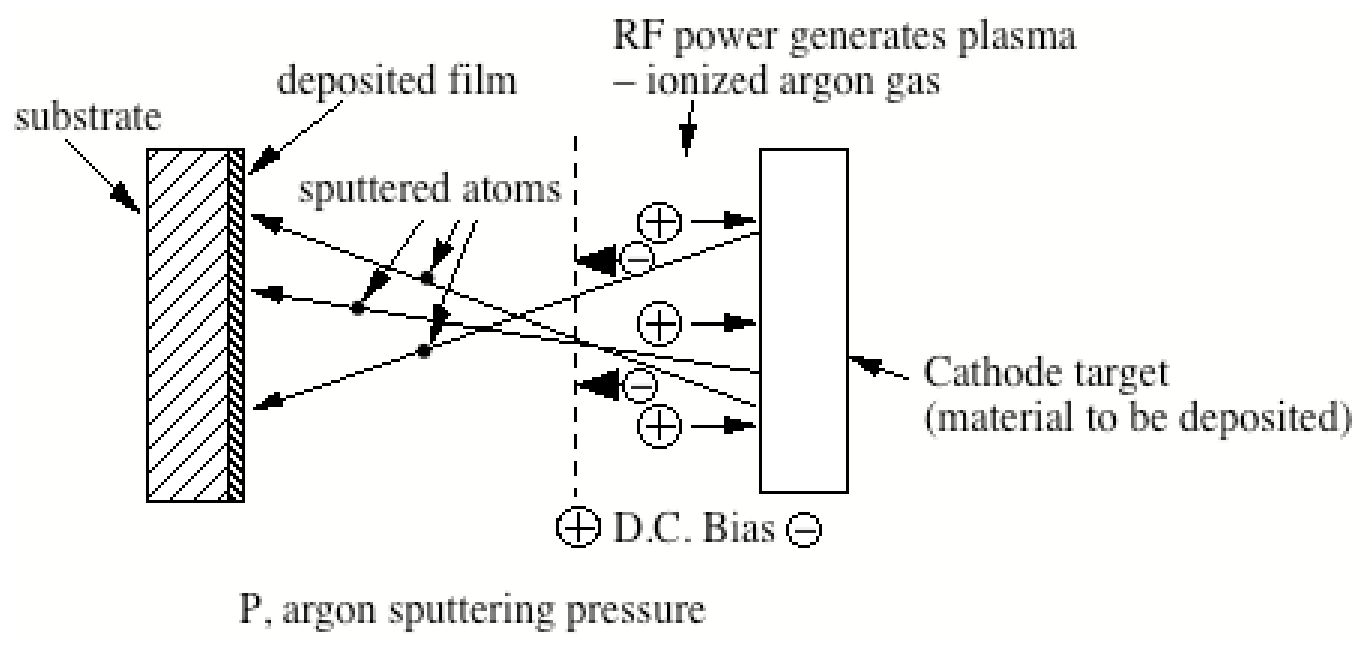

Students interested in the physics of the sputtering process should consult the book by M. Ohring, ("The materials science of thin films,", Academic Press, 1991). Here we give a very qualitative description of the sputtering process. Radio Frequency (RF) sputtering involves applying an RF voltage to a target material in a chamber filled with a working gas such as argon. The voltage on the target is applied relative to the chamber in which the sputtering is being done. A plasma, consisting of electron-ion pairs is created in the chamber as the 
RF power is applied. Because the electrons in the plasma are more mobile than the ions, more electrons will tend to arrive at the target when the RF voltage is positive than will ions arrive when the RF voltage on the target is negative. Such an imbalance in charge transfer cannot be sustained because no dc voltage is applied and no dc current is flowing from or to the target material. As a consequence, the target will naturally develop a negative dc voltage during RF sputtering so that the flow of electrons and ions to the target are balanced. This dc negative bias will cause positively charged gas ions to be accelerated to the target with high energy. As the arriving gas ions collide with the atoms on the surface of the target, the energy of the collision causes the surface atoms to be sputtered from the surface. The sputtered atoms then travel in a "line-of-sight" to the substrate, where they deposit onto the growing film.

As shown in some of the diagrams below, the stresses in sputtered films can be either compressive or tensile, depending on argon sputtering pressure (as well as power, geometry, relative atomic mass of target material and working gas). The transition from compressive to tensile stress can be understood by

1. The natural tendency of the film to develop tensile stresss (through the various mechanisms discussed before),

2. The development of compressive stresses in the film by "atomic shot peening" associated with the collision of neutral inert gas atoms (created in the sputtering process, as described below) with the growing film. 
Atomic Peening leads to compressive stresses

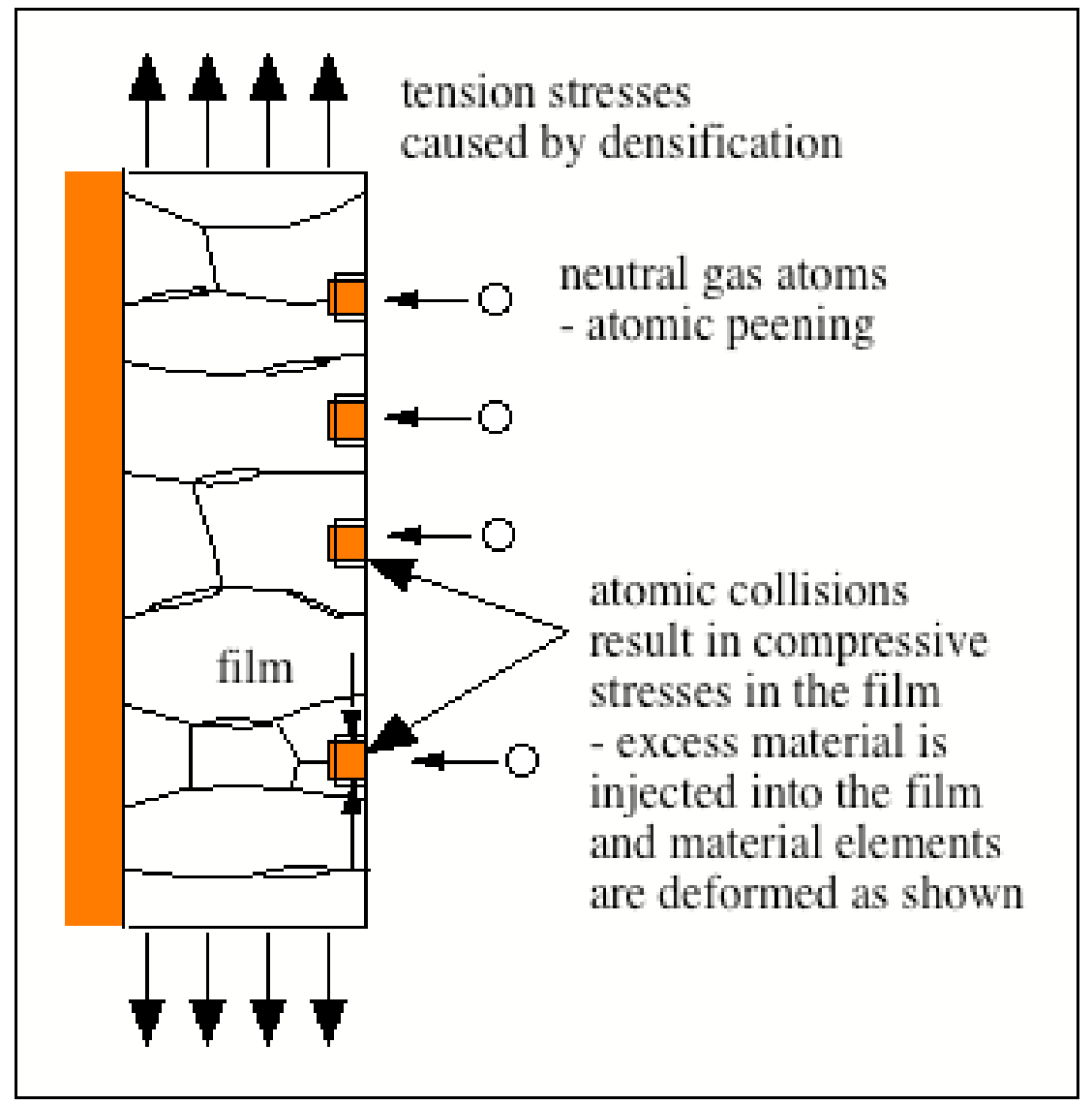

The production of compressive stresses can be understood qualitatively by considering both the implantation of atoms in the film during deposition and the micro-plastic deformation associated with the atomic collision process. Energetic argon atoms become implanted into the film and this results in compressive stresses. The excess atoms dilate the material near the surface and this leads to compressive stresses through the constraint of the underlying film. Also, atomic collisions with the growing surface result in plastic deformation events. Each surface element can be considered to deform as shown in the figure. This deformation would occur by the incremental movements of dislocations in the film or other shear processes. Again the constraint of the underlying film leads to biaxial compression in the film.

\section{Effect of Sputtering Gas Pressure on Stress in Film:}

Low sputtering gas (Argon) pressure results in compressive stresses in the film while high background gas pressures result in tension. Also, transition pressures 
from compression to tension are higher for target materials with greater atomic mass. These results are shown in the following figure and are explained below.

Force per unit width produced by internal stress in coatings deposited at various pressures using cylindrical post magnetrons

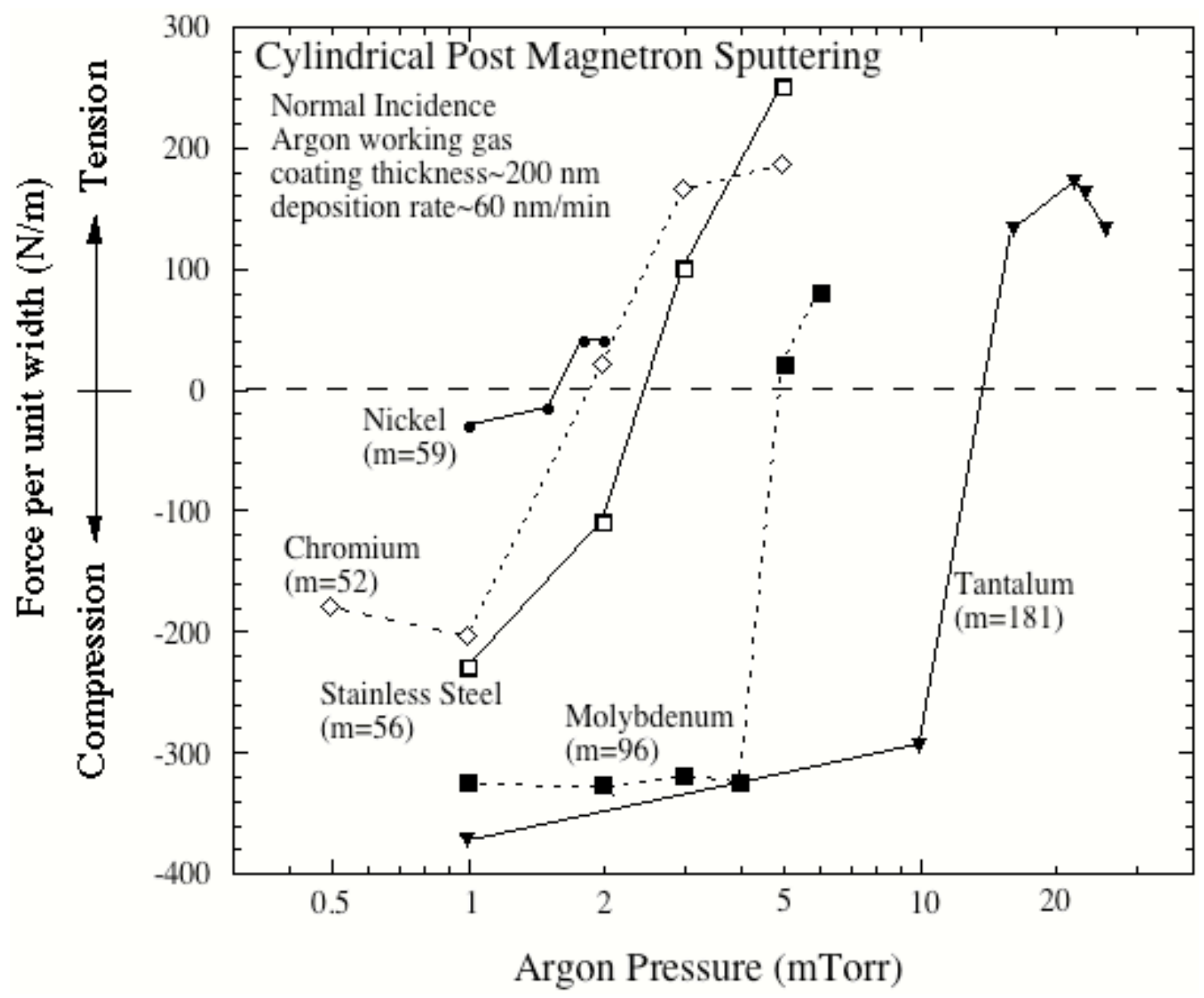

The transition pressure (from compression to tension) and the amount of entrapped argon in films both depend on atomic mass of target material. This is shown in the following figure. 
Dependence of the critical pressure for the transition from compressive stresses to tensile stresses on the atomic mass of the sputtering target

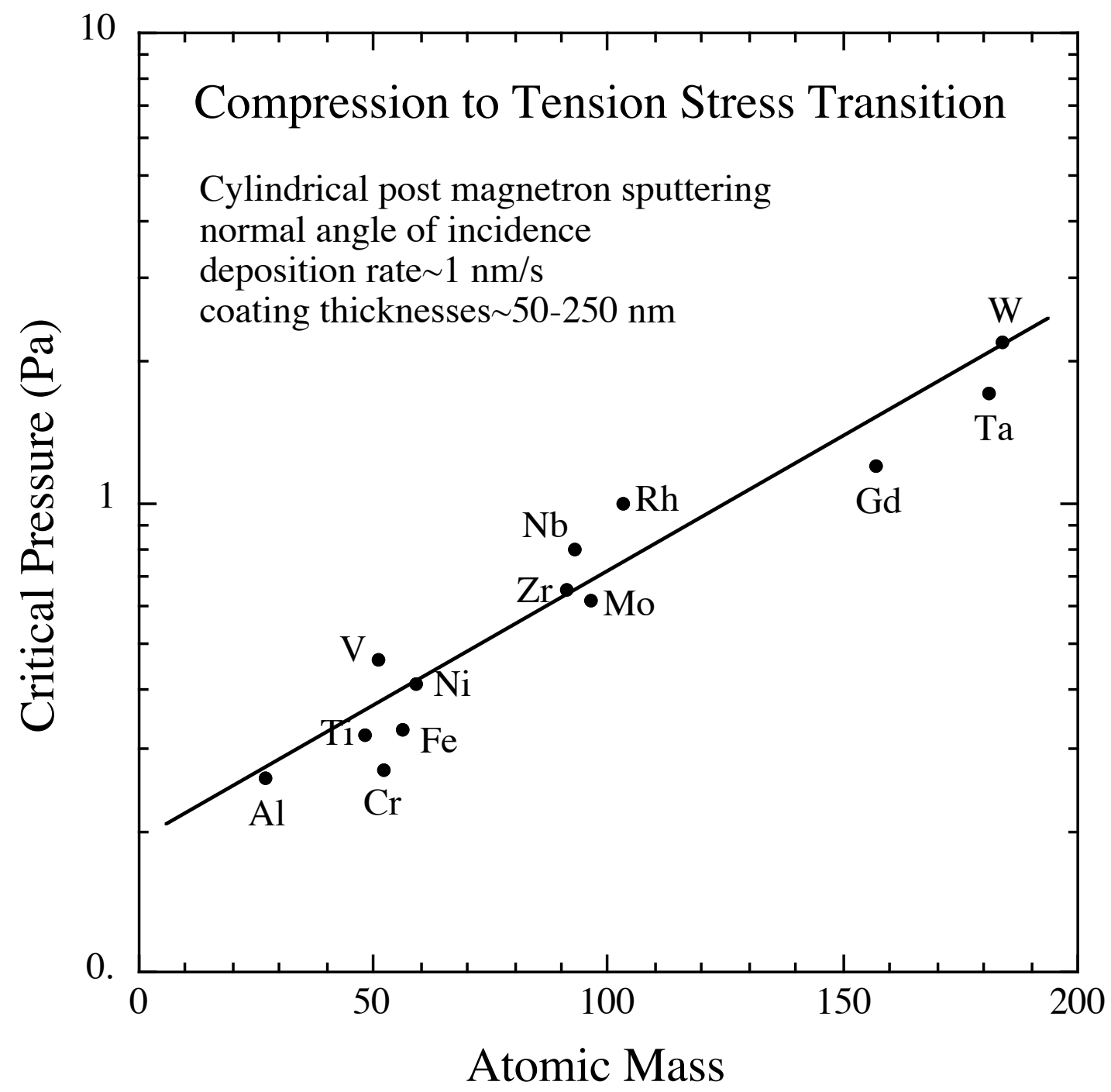

These effects are related to the kinetic energies of the neutralized gas (argon) atoms that are reflected from the sputtering target. When the argon ions collide with the sputtering target they become neutralized and they also experience an elastic collision with the target atoms on the surface. The following analysis shows that the kinetic energies of the reflected neutral gas atoms depend strongly on the atomic mass of the target atoms. Consider the elastic collision shown in the figure. 
Elastic collision of an Argon ion of atomic mass $m_{1}$ with a target material of atomic mass $\mathrm{m}_{2}$

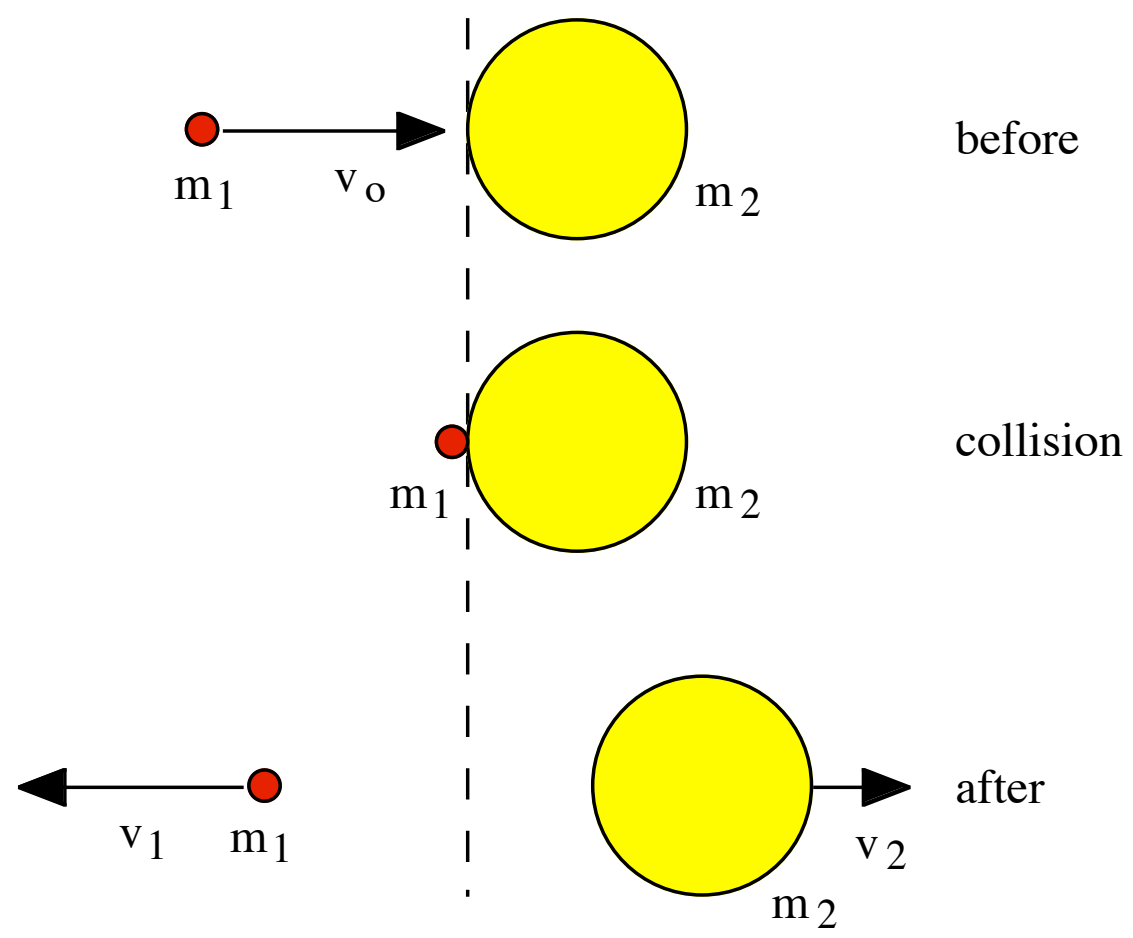

For such elastic collisions the momentum and the kinetic energy must be conserved. For energy conservation we have

$$
E_{o}=\frac{1}{2} m_{1} v_{o}^{2}=\frac{1}{2} m_{1} v_{1}^{2}+\frac{1}{2} m_{2} v_{2}^{2},
$$

and for conservation of momentum we have

$$
m_{1} v_{o}=m_{1}\left(-v_{1}\right)+m_{2} v_{2} .
$$

Solving these equations we have

$$
\begin{aligned}
& m_{1}\left(v_{o}^{2}-v_{1}^{2}\right)=m_{2} v_{2}^{2} \\
& m_{1}\left(v_{o}+v_{1}\right)=m_{2} v_{2}
\end{aligned}
$$


Solving simultaneously we have

$$
\begin{aligned}
& v_{1}=\left(\frac{m_{2}-m_{1}}{m_{2}+m_{1}}\right) v_{o} \\
& v_{2}=\left(\frac{2 m_{1}}{m_{2}+m_{1}}\right) v_{o}
\end{aligned}
$$

for the resulting velocities. The kinetic energy of the neutralized gas atom is then

$$
E_{1}=\frac{1}{2} m_{1} v_{1}^{2}=\frac{1}{2} m_{1} v_{o}^{2} \bullet\left(\frac{m_{2}-m_{1}}{m_{2}+m_{1}}\right)^{2}=E_{o}\left(\frac{m_{2}-m_{1}}{m_{2}+m_{1}}\right)^{2}
$$

Thus we have

$$
\begin{aligned}
& E_{1}=0 \quad \text { when } m_{2}=m_{1} \\
& \text { and } \\
& E_{1}>0 \quad \text { when } m_{2}>m_{1}
\end{aligned}
$$

This little analysis shows that the kinetic energy of the reflected neutral gas atoms increases with the atomic mass of the target atoms. This explains why the resulting stresses are more compressive for target materials with greater atomic mass.

For a long time it was commonly thought that stresses were caused by the incorporation of argon in the films. However, Thornton, et al. have shown that it is the "peening" action of the argon atoms that produces the compressive stress. Thornton and his colleagues conducted a number of experiments to prove that it is the neutral gas atoms that create both the entrapped gas atoms and the compressive stress.

\section{Thornton's Special Experiment}

Neutralized gas atoms scatter from the target at a very low angle, as shown in the diagram below. This is equivalent to what would happen if a small steel ball were shot at a surface composed of an array of much larger steel balls. Because collisions at a glancing angle are much more likely because of the small crosssection of head-on collisions, most reflections would occur at a small angle to the surface. 
Influence of sputtering apparatus geometry on flux of energetic reflected argon gas atoms which reach the substrate
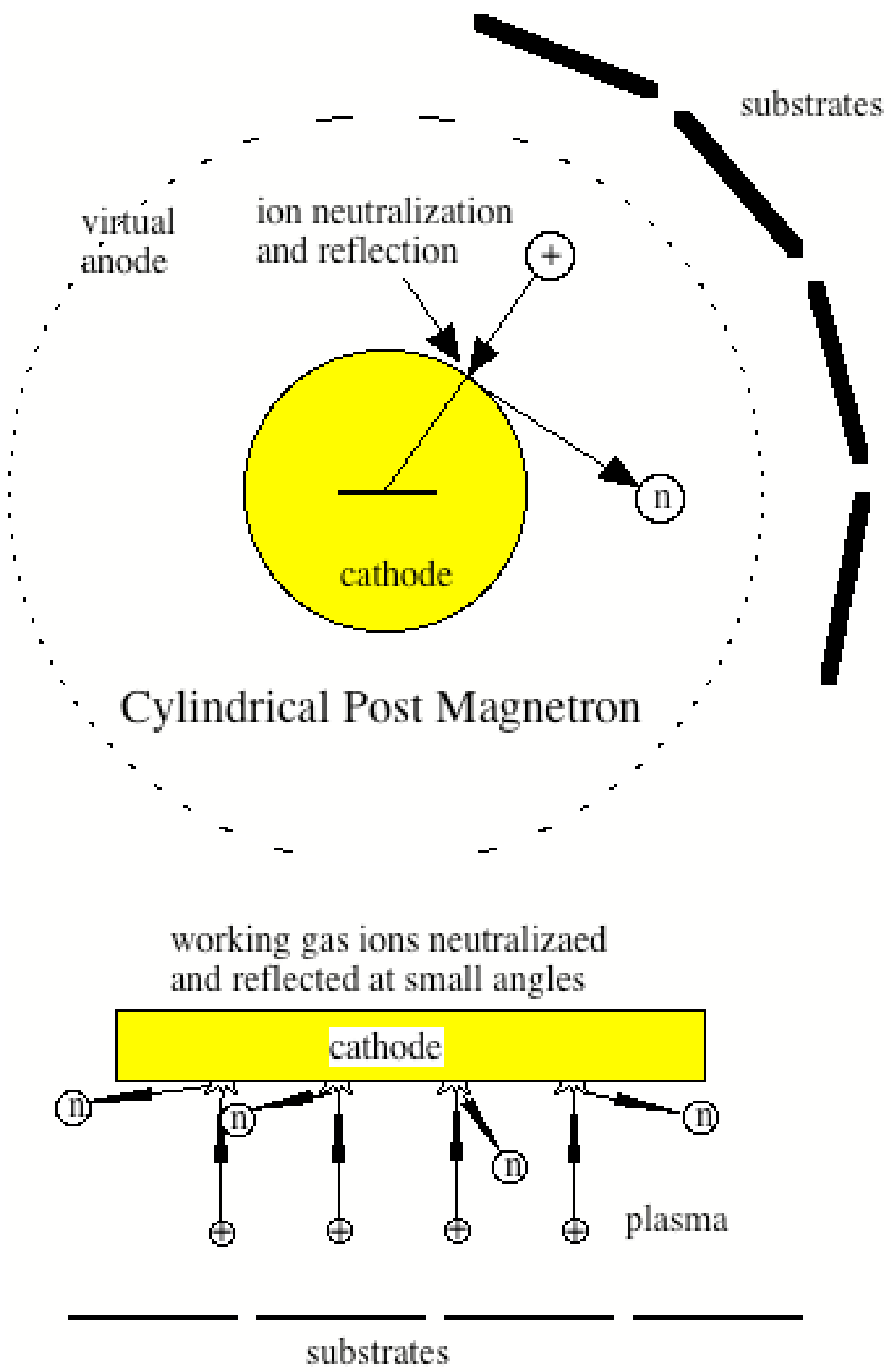

Some argon ions become neutralized and are scattered at low angles from the surface of the cathode. Using a cylindrical post sputtering geometry and a shield over the substrate with a small slit, Thornton et al. showed that the highest argon 
concentrations in the film and the highest compressive stresses are found at the edges of the film where the highest flux of neutral argon atoms is expected.

The slit camera configuration was used to resolve the influence of the argon that is reflected from the cathode at small angles on the entrapped argon content of the coatings

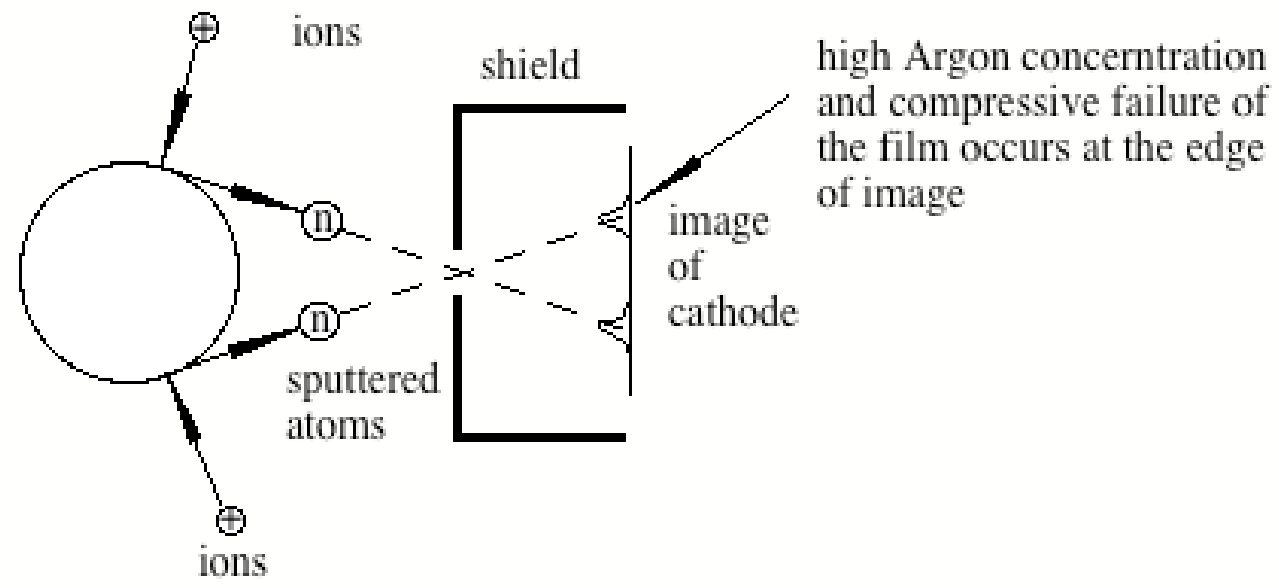

The argon concentration is highest at the edges of the image, where the compressive failures are also observed. 
Microprobe scans of the Mo image and the entrapped argon content across the width of the image formed using the slit camera configuration shown in the previous figure. The deposition pressure was $0.13 \mathrm{~Pa}$

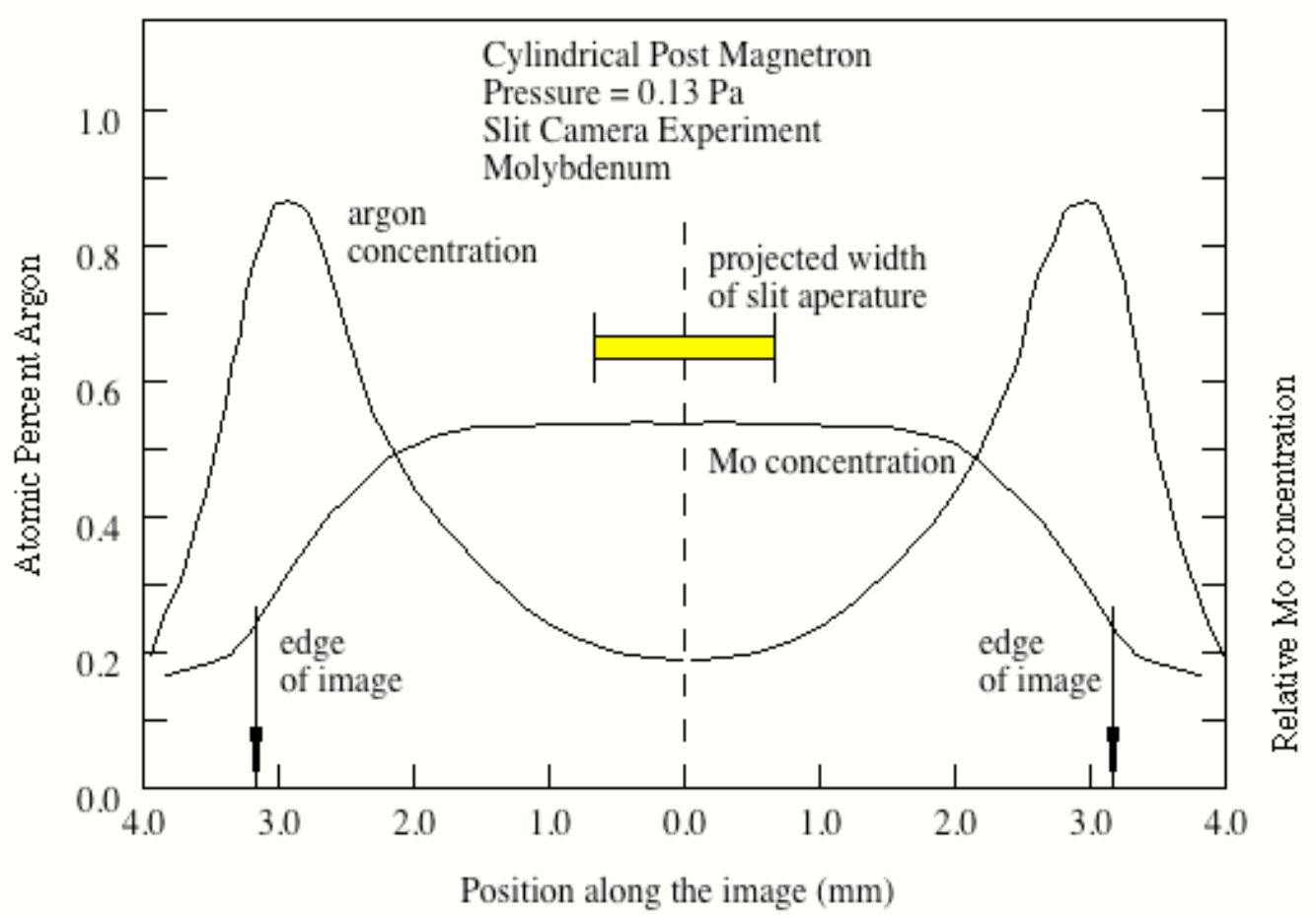

\section{Hollow Cathode Experiments}

In the figure below a hollow cathode geometry is used to control the stress in a sputter deposited 304 stainless steel film. The geometry of the cathode is such that only a few neutralized argon atoms are able to reach substrate - so large compressive stresses are not produced. For the case of an unshielded substrate, tension is created in the film due mainly to the oblique incidence of sputtered atoms (the porosity is greater for oblique deposition because of shadowing effects). For the shielded substrate, no oblique deposition occurs. This results in weak compressive stresses caused by the small flux of neutralized gas atoms reflecting from the cathode. The stresses at a given argon pressure are much smaller than for films created by cylindrical post magnetron sputtering. 


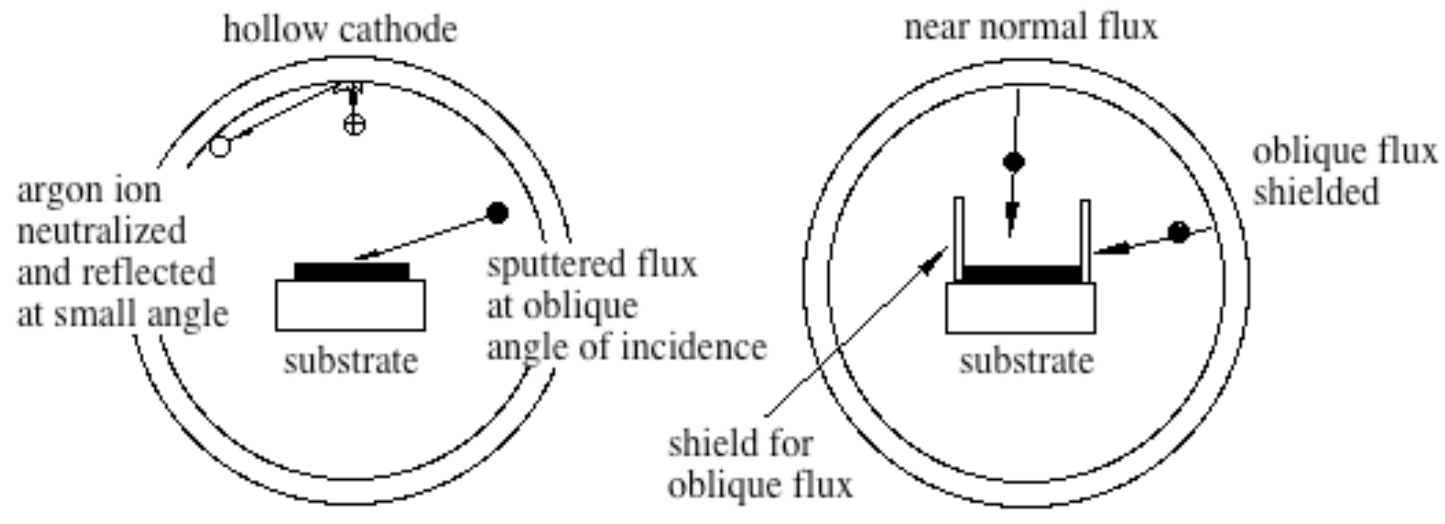

The internal stress vs, the argon pressure for stainless steel (304) coatings deposited onto shielded and unshielded substrates mounted on the axis of a cylindrical hollow-cathode magnetron $0.1 \mathrm{~m}$ in diameter and $0.3 \mathrm{~m}$ long

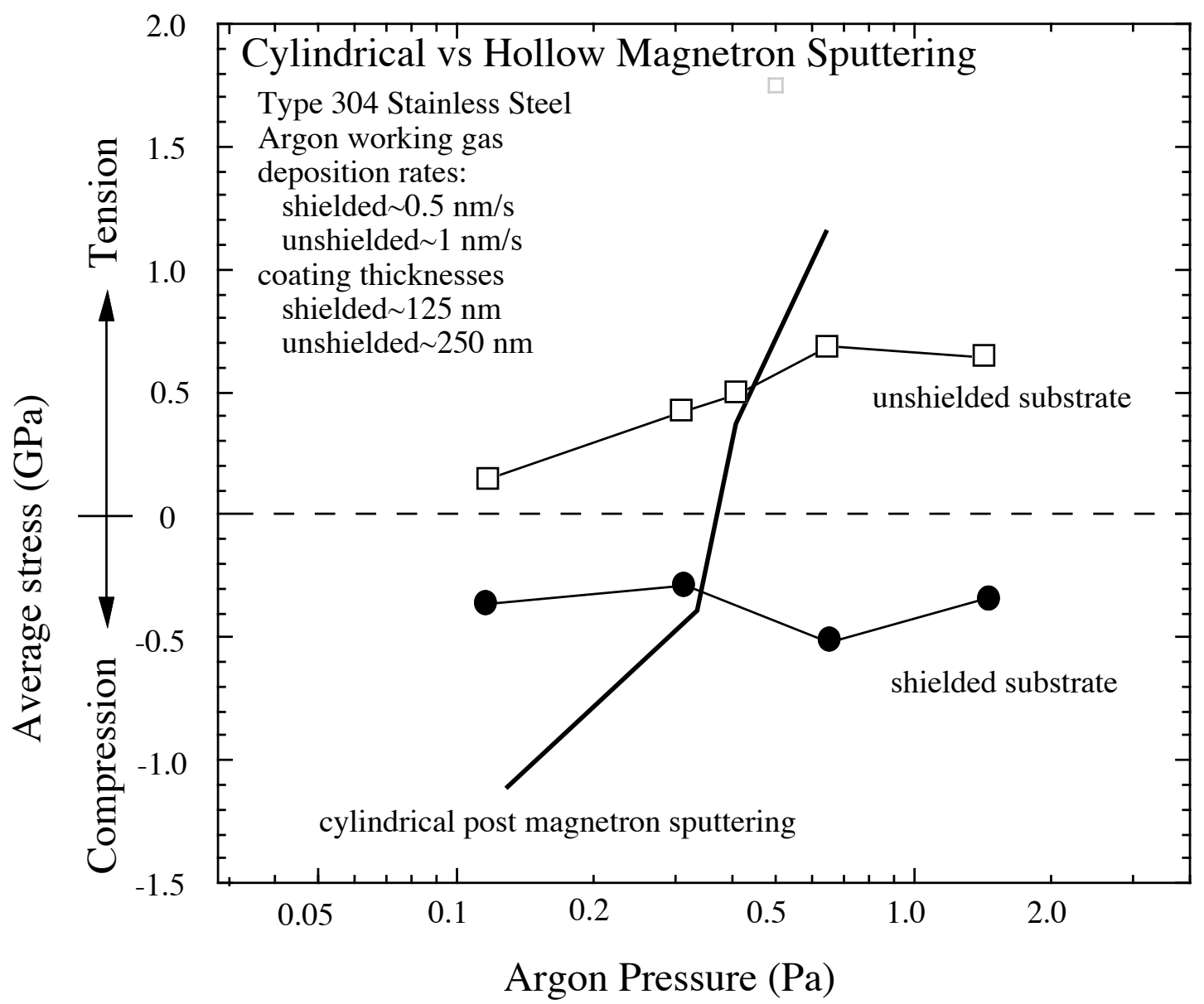


Therefore, the conclusions are:

1. Low argon pressure - The neutral argon atoms reflected from the target suffer few collisions with other gas atoms on their way to the growing film, with the consequence that many energetic argon atoms arrive at the growing film and produce high compressive stresses by "atomic peening".

2 High argon pressure - Many collisions in the gas prevent energetic argon atoms from arriving at growing film - so natural processes leading to tension prevail.

3. For targets with large atomic mass, the reflected argon atoms are more energetic - so bigger compressive stresses are observed and and higher argon pressures are needed to produce tension.

Entrapped argon gas also depends on the mass of the target. 
Entrapped Argon Content of Sputtered Coatings as a Function Of Atomic Mass of Coating Material .

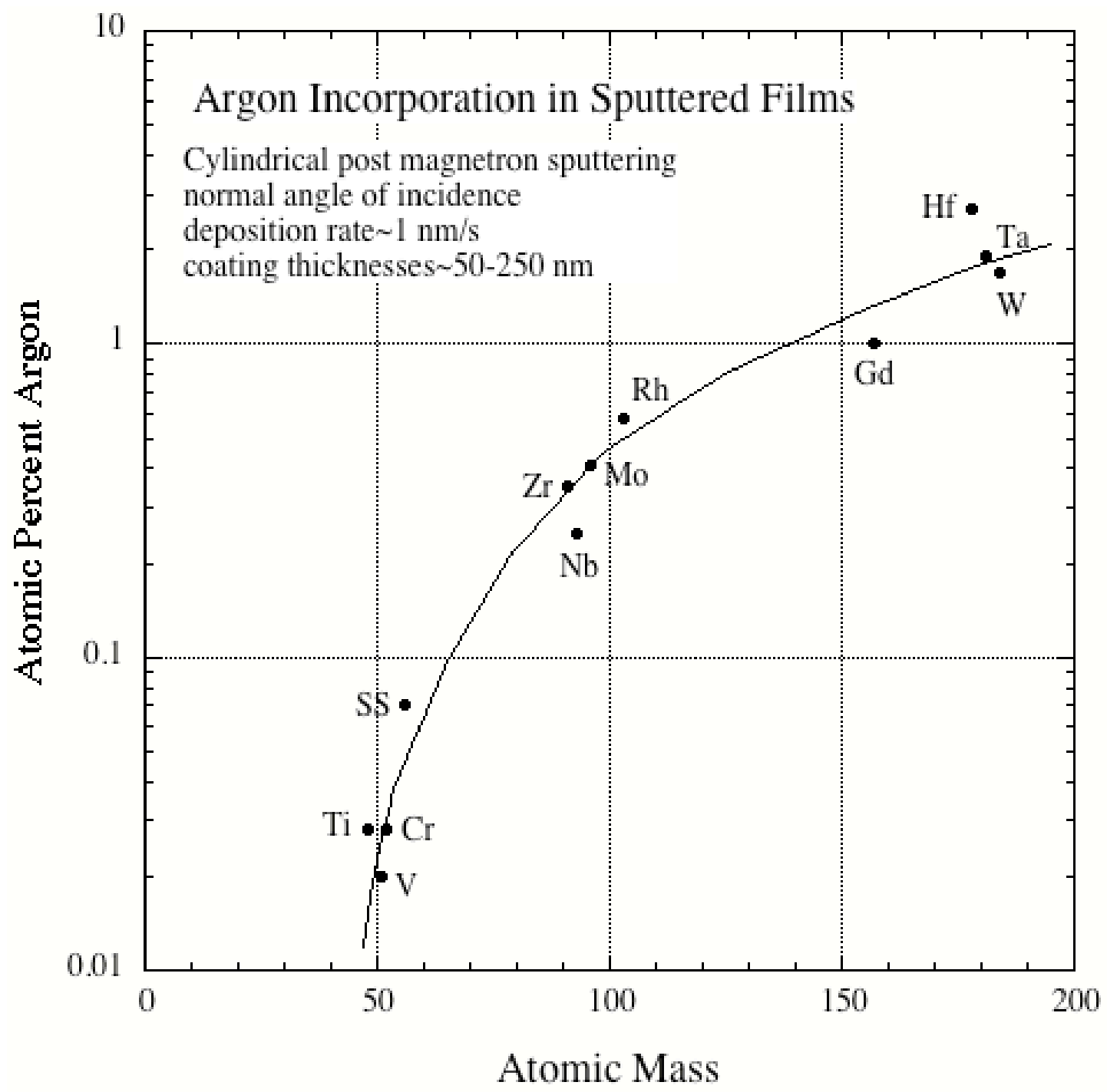




\section{Techniques for the Study of Mechanical Properties of Thin Films}

\section{The Bulge Test}

Some of the following notes on bulge testing were kindly prepared by Robert Hohlfelder when he was a student at Stanford.

The bulge test is a way of measuring "in-plane" mechanical properties of thin films, including:

- $\quad$ Biaxial Elastic Modulus

- $\quad$ Residual Stress

- $\quad$ Yield Stress

Pressure is applied to one side of a free-standing thin-film "window", causing it to "bulge" upward.

\section{The Bulge Test}

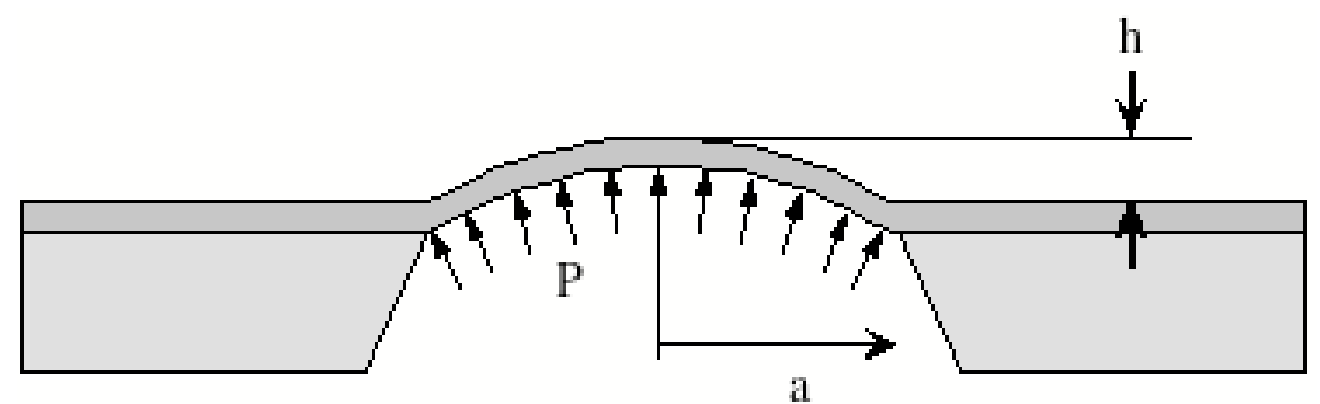

The center deflection $(h)$ and the applied pressure $(p)$ are directly related to the stress and strain in the window. Thus, information about mechanical properties can be extracted from measurements of pressure and deflection. 


\section{View of a silicon nitride window from the back of the wafer}

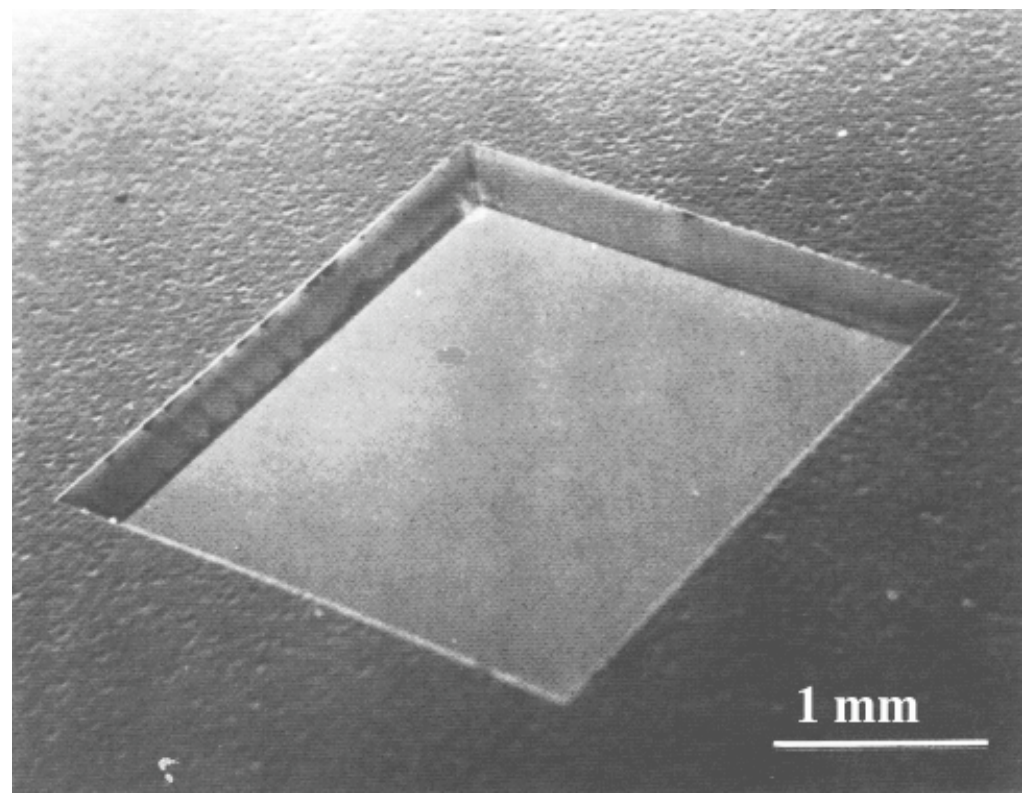

\section{Bulge-Test Apparatus}

We use interferometry to measure the deflection of the bulge as a function of applied pressure.

Deflection of the window can be measured using laser interferometry (this requires a reflective film) or through other methods:

- Optical (focus microscope onto the film)

- $\quad$ Volumetric

Pressure is easy to measure accurately. 


\section{Schematic of the Bulge Test Apparatus at Stanford}

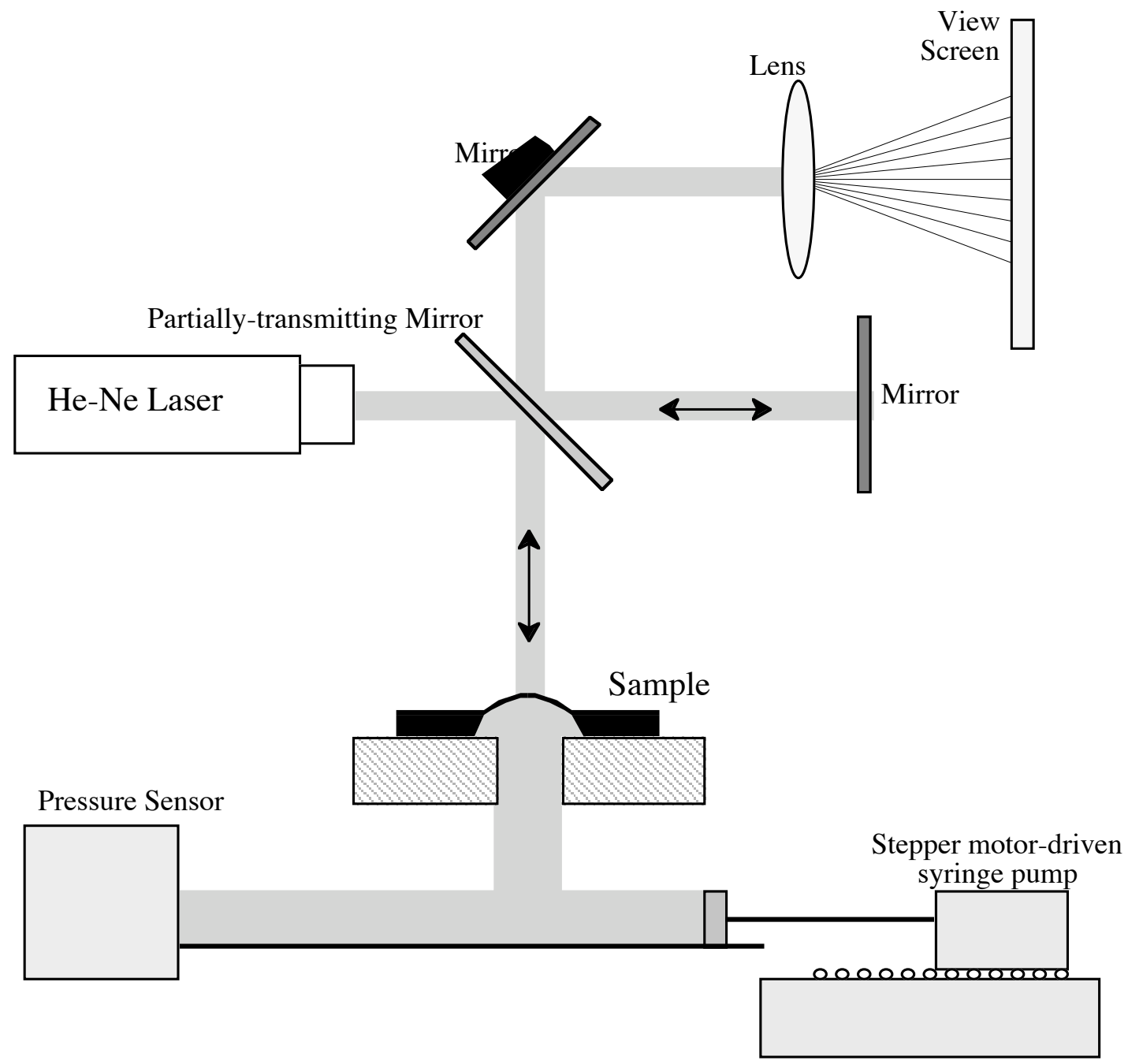




\section{The "Spherical Cap" model of the Bulge Test}

A pressurized thin-film window can be modeled as a section of a thin-walled spherical pressure vessel.

\section{Thin-walled spherical pressure vessel}

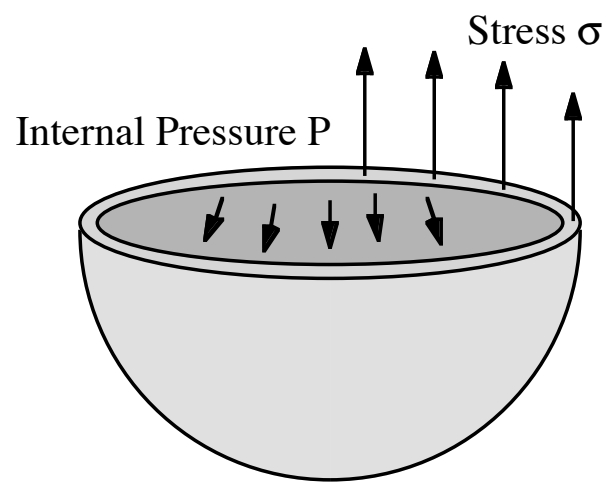

For wall thickness $\mathrm{t}$ and radius $\mathrm{R}$, the stress in the wall is obtained by balancing the forces due to stress and pressure:

$$
\begin{aligned}
& p \cdot \pi R^{2}=\sigma \cdot 2 \pi R t \\
& \sigma=\frac{p R}{2 t}
\end{aligned}
$$

Note: Stress is biaxial $\left(\sigma_{x x}=\sigma_{y y}\right)$. This gives us a relationship between the pressure, radius of curvature, and stress in a pressurized membrane. We can model a circular thin film window by "slicing off" a piece of the pressure vessel.

Geometry of a bulged film

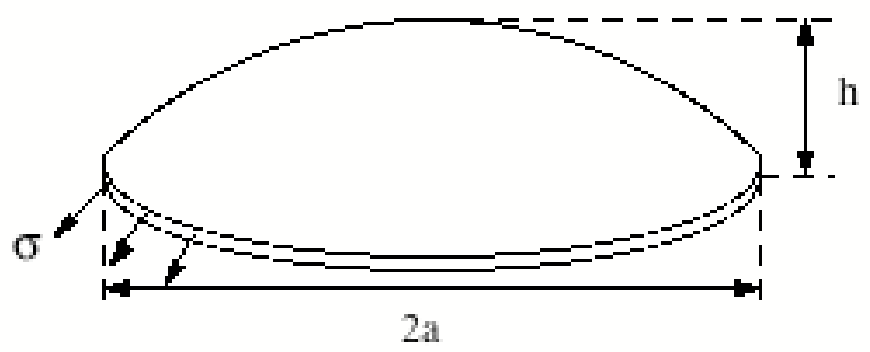


Consider the limit of relatively small deflections $(h<<a)$ :

Geometry for bulge analysis

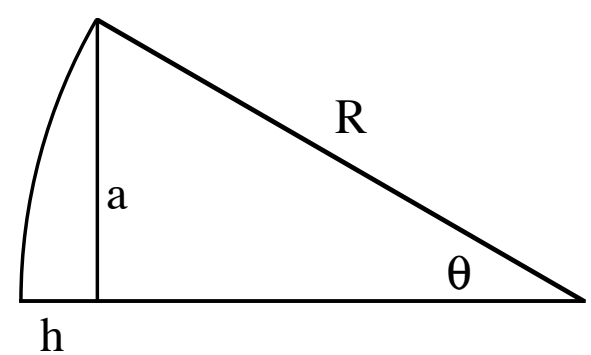

The relation between the height of the bulge and the radius of curvature is then:

$$
\begin{aligned}
& R^{2}=(R-h)^{2}+a^{2} \quad(\text { Pythagorean Theorem }) \\
& R^{2}=R^{2}-2 R h-h^{2}+a^{2} \approx R^{2}-2 R h+a^{2} \\
& a^{2}=2 R h \\
& R=\frac{a^{2}}{2 h}
\end{aligned}
$$

We can then make a substitution:

$$
\sigma=\frac{p R}{2 t} \Rightarrow \sigma=\frac{p a^{2}}{4 t h}
$$

There is also a relationship between the center deflection $(h)$ and the film strain $\varepsilon$. This is obtained purely from geometry:

$$
\varepsilon=\frac{R \theta-a}{a}=\frac{\theta-(a / R)}{(a / R)}
$$

Note that:

$$
\theta=\arcsin (a / R)=(a / R)+\frac{(a / R)^{3}}{6}+\ldots
$$

This leads to: 


$$
\begin{aligned}
& \varepsilon=\frac{(a / R)+\frac{(a / R)^{3}}{6}-(a / R)}{(a / R)}=\frac{a^{2}}{6 R^{2}} \\
& \varepsilon=\frac{a^{2}}{6 R^{2}}=\frac{2 h^{2}}{3 a^{2}}
\end{aligned}
$$

For biaxial elastic strain

$$
\sigma=B \varepsilon
$$

If we substitute for $\sigma$ and $\varepsilon$ and rearrange, we obtain:

$$
p=\frac{8 B t}{3 a^{4}} h^{3}
$$

which is the relationship between pressure and deflection when there is no residual stress present. Residual stress in the film can be accounted for by using:

$$
\sigma=B \frac{2 h^{2}}{3 a^{2}}+\sigma_{0}
$$

or

$$
\sigma=B \frac{2 h^{2}}{3 a^{2}}+\sigma_{0}=\frac{p a^{2}}{4 t h}
$$

In which case the bulge equation becomes:

$$
p=\frac{8 B t}{3 a^{4}} h^{3}+\frac{4 \sigma_{0} t h}{a^{2}}
$$

This is the "bulge equation."

We note that the equation for pressure contains two terms. The first term is proportional to $h^{3}$ and the biaxial modulus and the second term is proportional to $h$ and to the residual stress. At small deflections, $\left(\sigma_{o} h>B h^{3}\right)$ the test behavior is dominated by the residual stress in the film, and the pressure varies linearly with 
height. At larger deflections $\left(B h^{3}>\sigma_{o} h\right)$, the behavior is dominated by the modulus of the film, and the pressure increases with the cube of height. This behavior is illustrated below for an $A l+S_{i N}$ composite thin film. The bulge behavior for a bare $\operatorname{SiN}_{x}$ membrane is also shown for comparison. In the next graph the bulge behavior for a $A g-P d$ multilayered film on a $S i N_{x}$ membrane is shown.

\section{Bulge Test Data for a $\mathrm{Al}+\mathrm{SiN}_{\underline{x}} \underline{\text { membrane }}$}

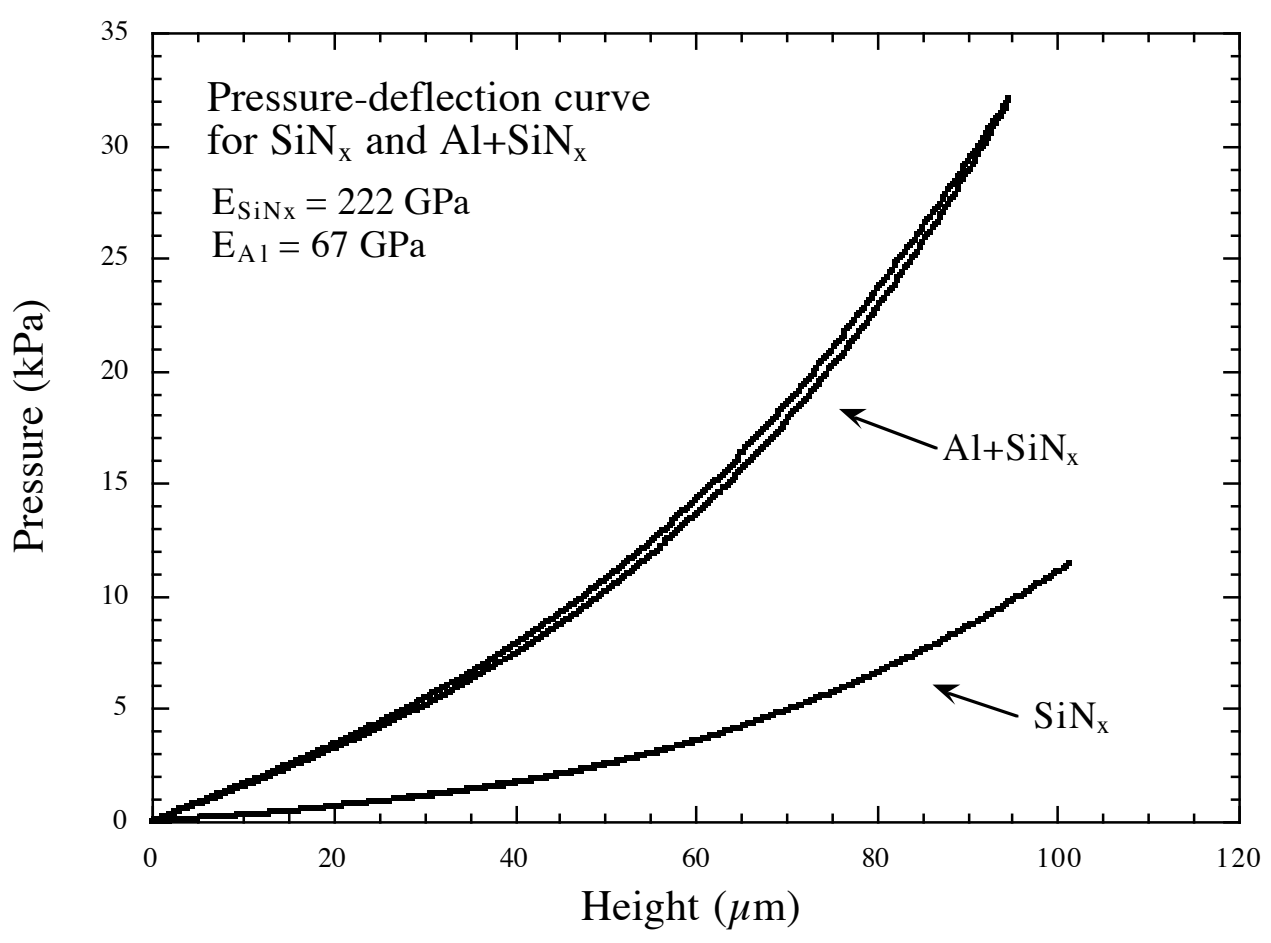




\section{Bulge test data for a multilayered $\mathrm{Ag}-\mathrm{Pd}$ thin film on a $\mathrm{SiN}_{\mathrm{x}}$ membrane}

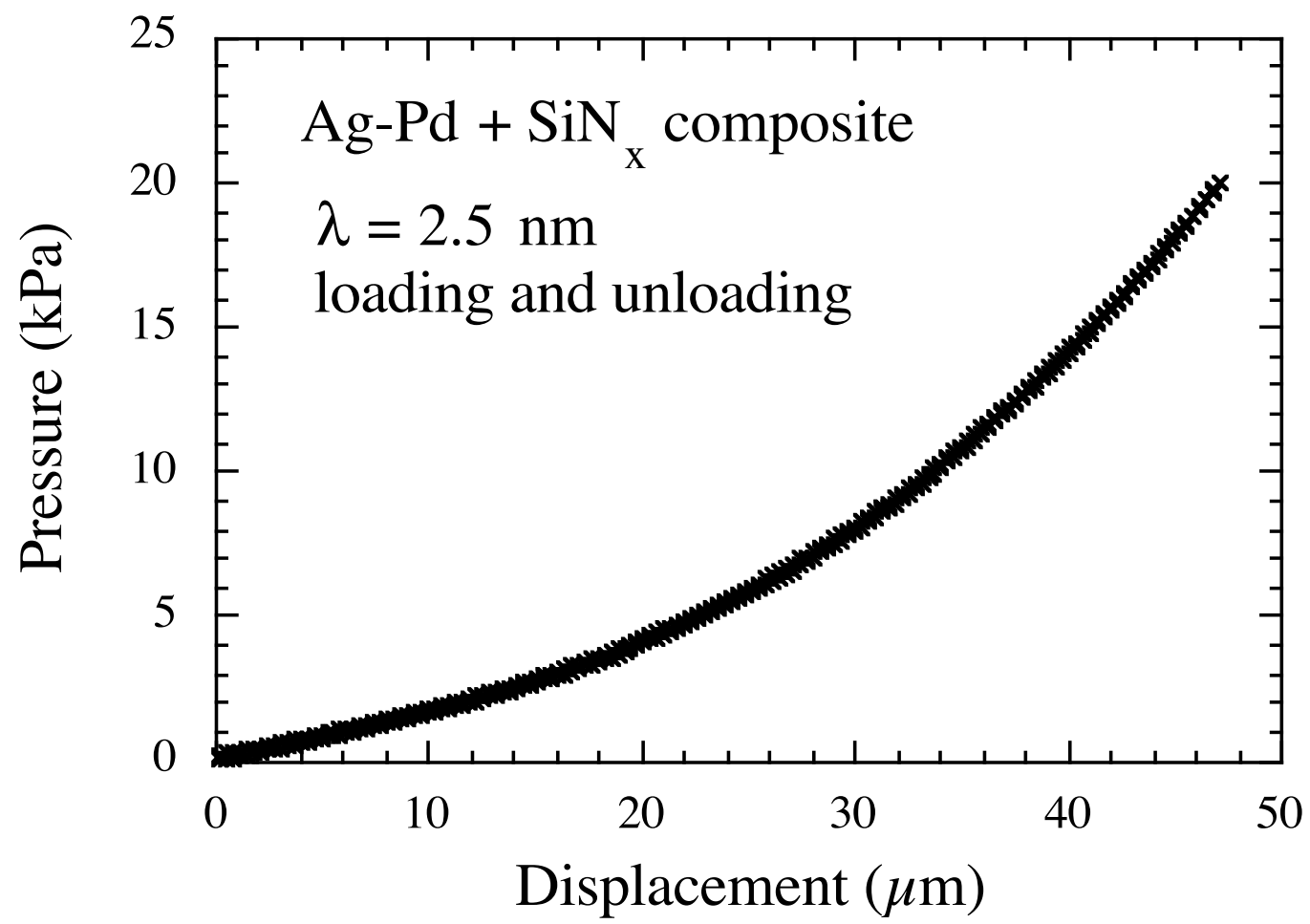

The biaxial modulus and residual stress can easily be determined from bulge test data. Divide the bulge equation by $h$ :

$$
\frac{p}{h}=\frac{8 B t}{3 a^{4}} h^{2}+\frac{4 t \sigma_{0}}{a^{2}}
$$

This means that a plot of $p / h v s . h^{2}$ will yield a straight line. As long as we know the film thickness $(t)$ and the window radius $(a)(a$, must be known very accurately because it appears in the bulge equation to the fourth power!), we can obtain the residual stress and biaxial modulus from the slope and intercept of that line. This relation is shown below for a $A g-P d$ multilayered film on a $S i N_{x}$ membrane. 


\section{Bulge test data for $\mathrm{Ag}-\mathrm{Pd} / \mathrm{SiN}_{\mathrm{x}}$}

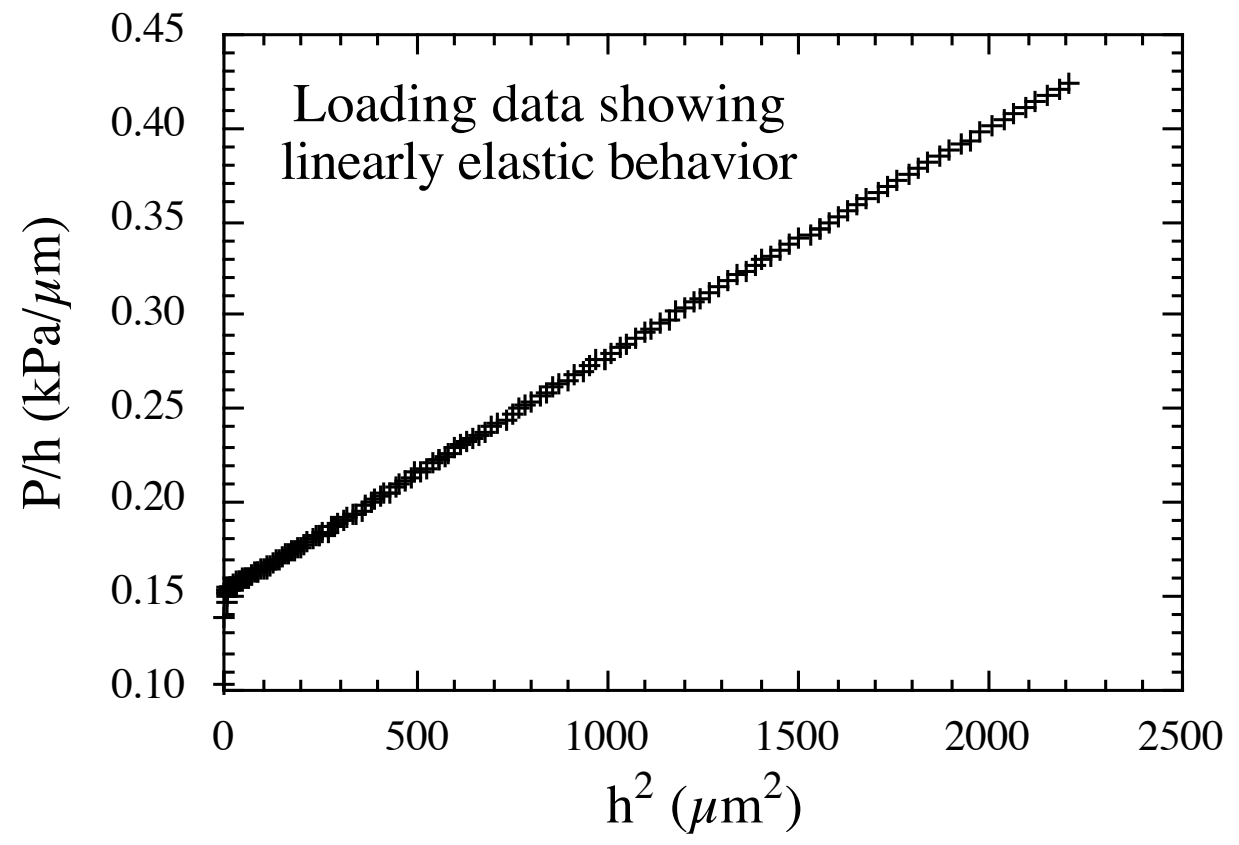

\section{Limitations of the Spherical Cap Model}

In a spherical pressure vessel, stress is biaxial $\left(\sigma_{x x}=\sigma_{y y}, \varepsilon_{x x}=\varepsilon_{y y}\right)$. The relation between stress \& strain is:

$$
\varepsilon_{x x}=\sigma_{x x} \cdot \frac{1-v}{E}
$$

In a pressurized circular window, stress is perfectly biaxial only at the center. Near the edges, the film is bonded to the substrate and plane-strain conditions prevail $\left(\varepsilon_{\theta \theta}=0\right)$. 


\section{Section of a window near the outer edge}

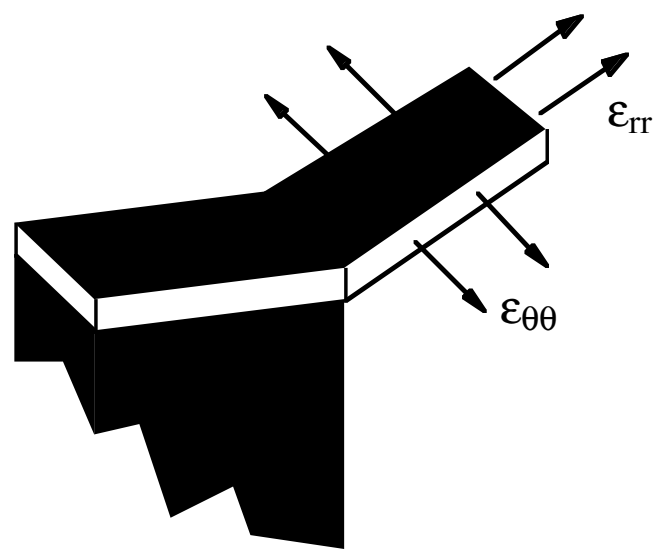

The stress-strain relation for plane strain is:

$$
\varepsilon_{r r}=\frac{1-v^{2}}{E} \sigma_{r r} \quad \varepsilon_{\theta \theta}=0
$$

For a given stress $\sigma_{r r}$, strain $\left(\varepsilon_{r r}\right)$ in the radial direction will be higher near the edge of a window than would be predicted by the idealized spherical cap model. In other words, actual windows are slightly more compliant than predicted by the spherical cap model. Use of the spherical cap model to fit experimental data results in values for modulus which are a few percent too low. Martha Small conducted finiteelement simulations of bulge tests and obtained an empirical correction factor:

$$
B_{\text {actual }}=\frac{B_{\text {calculated }}}{1-0.241 v}
$$


Correction factors for edge constraint

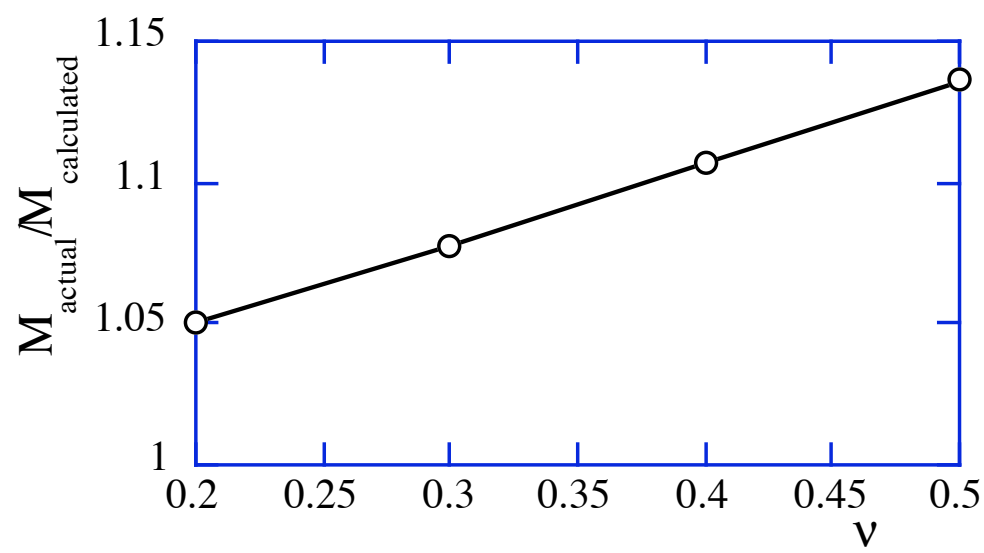

\section{Stresses in a circular window}

The finite element technique has been used to find the stress distribution in a circular membrane subjected to bulging.

\section{Stresses in a circular membrane}

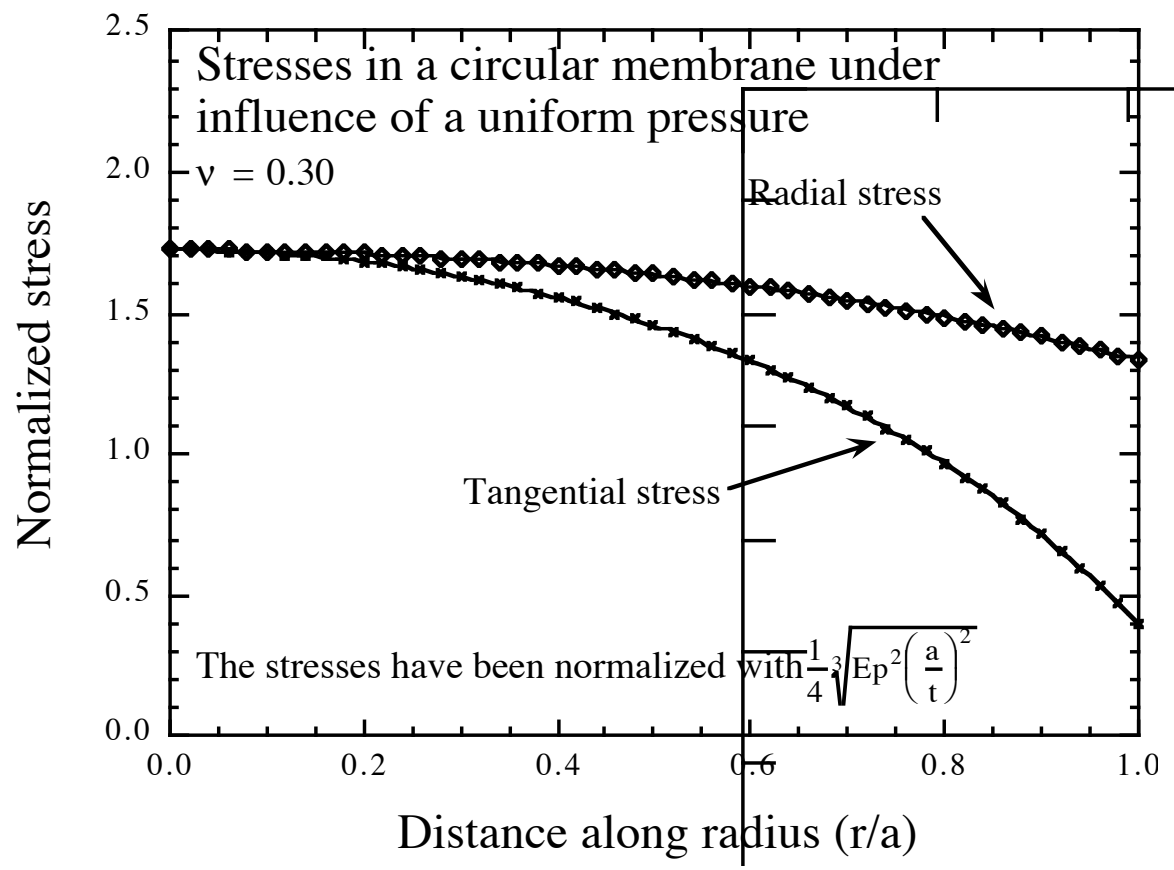




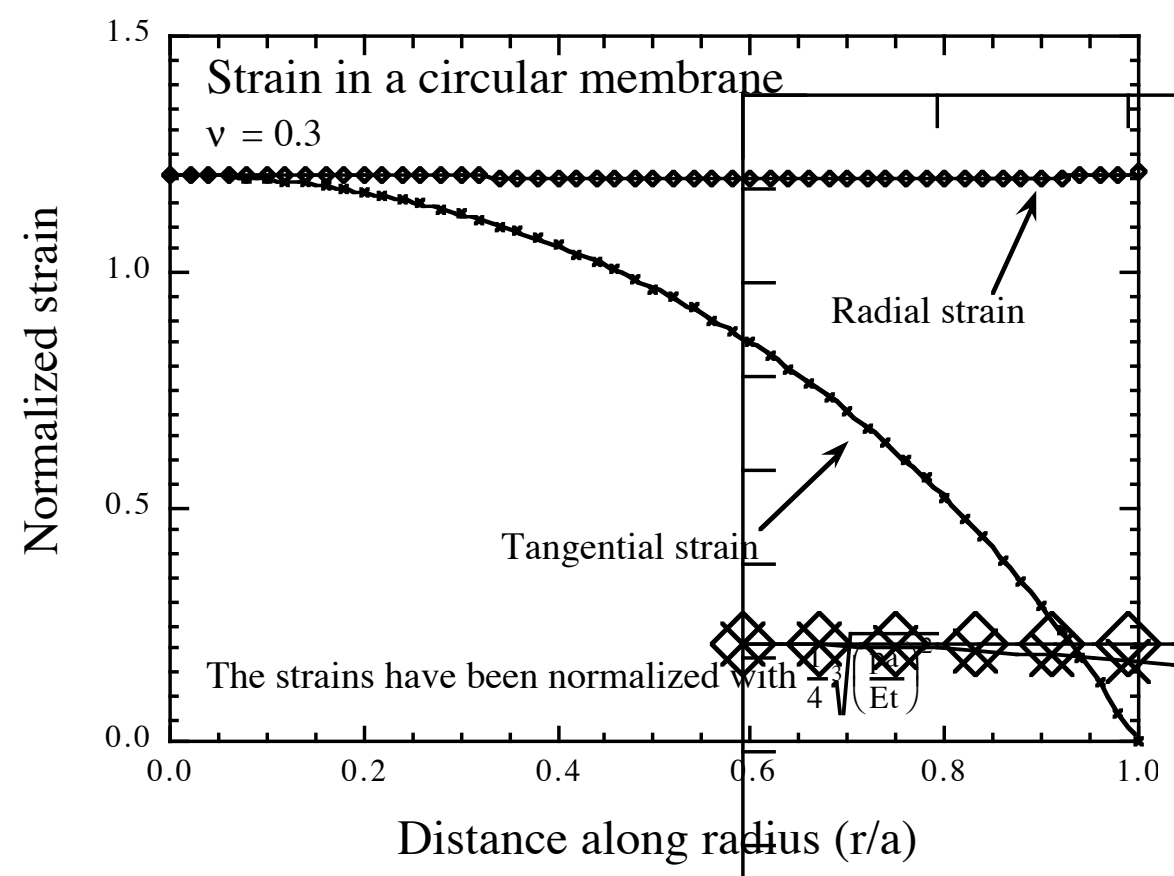

\section{Sample Fabrication}

Bulge test samples must be very flat, wrinkle-free and have precise dimensions. Micomachining is the best way to create such test structures. 
Example of a process for making free-standing windows:

1) Start w/ (100) Si substrate

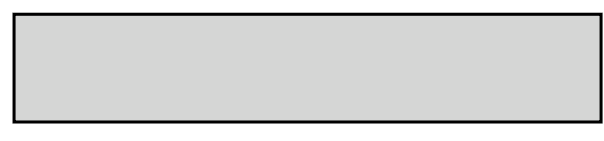

3) Spin on \& pattern photoresist
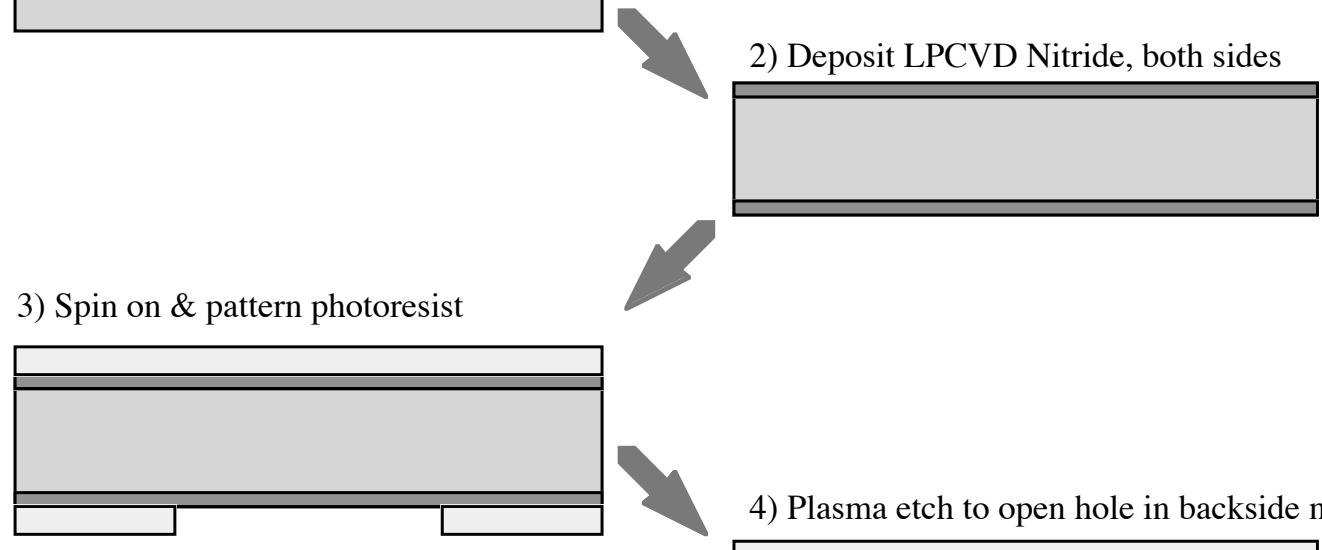

4) Plasma etch to open hole in backside nitride

5) $\mathrm{KOH}+$ Methanol etch to open hole through silicon
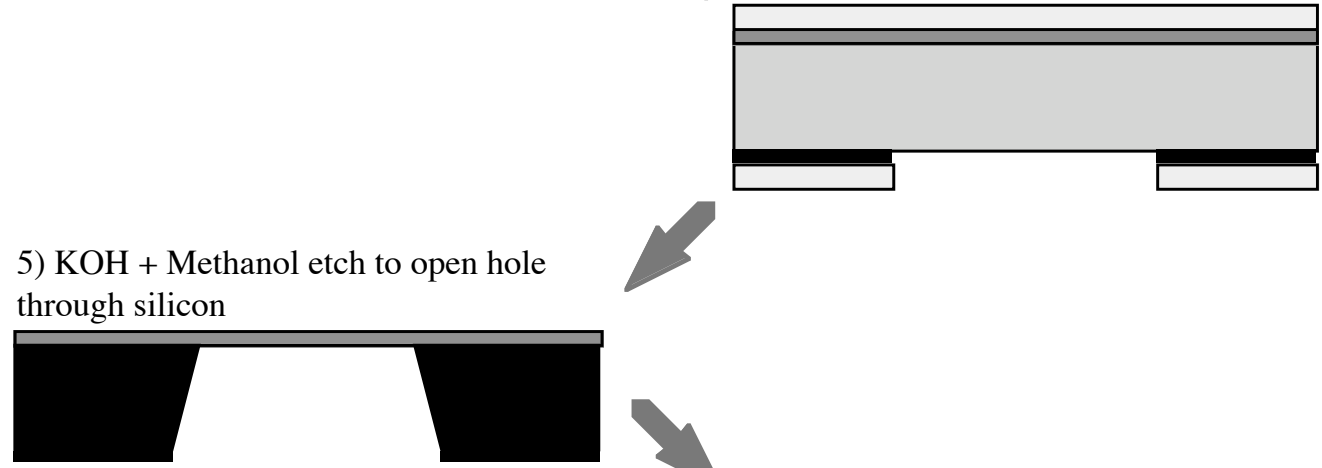

6) Deposit other films on nitride, if desired

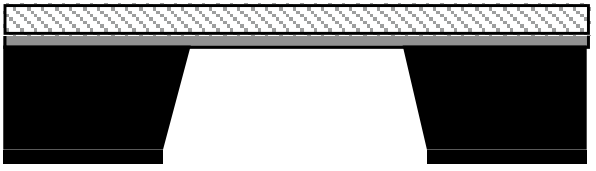

The $\mathrm{KOH} /$ Methanol etch is highly anisotropic. The (100) planes are etched much faster than (111) planes. The sides of the hole through the Si substrate are defined by (111) planes. The (111) planes intersect the surface along $<110>$ directions. For this reason, it is easiest to make square or rectangular windows, with sides along the $<110>$ directions. 


\section{Square Windows}

\section{Energy minimization methods:}

It is difficult to solve analytically the problem of the deflection of a square thin-film window. In a circular window, the deflection at any point is a function of one coordinate, its distance from the center. In square windows, deflection varies with $\underline{\text { two }}$ coordinates $(x \mathcal{E} y)$. Approximate solutions can be found using an energyminimization method. The full solution is quite involved, but it's useful to outline the procedure.

Procedure:

1) Assume that the displacement functions take a certain form. The functions contain unknown coefficients $\left(A, w_{0}, w_{1}\right)$ which we need to determined. These functions don't just pop out of thin air; they're equivalent to the first two terms of a Taylor series expansion. The quantities $(u, v, w)$ are displacements in the $(x, y, z)$ directions:

$$
\begin{aligned}
& u=\frac{A}{a^{4}} x\left(a^{2}-x^{2}\right)\left(a^{2}-y^{2}\right) \\
& v=\frac{A}{a^{4}} y\left(a^{2}-x^{2}\right)\left(a^{2}-y^{2}\right) \\
& w=\frac{w_{0}}{a^{4}}\left(1+\frac{w_{1}}{a^{2}}\left(x^{2}+y^{2}\right)\right)\left(a^{2}-x^{2}\right)\left(a^{2}-y^{2}\right)
\end{aligned}
$$

2) Strains are determined by the displacement functions:

$$
\begin{aligned}
\varepsilon_{x x} & =\frac{\partial u}{\partial x}+\frac{1}{2}\left(\frac{\partial w}{\partial x}\right)^{2} \\
\varepsilon_{y y} & =\frac{\partial v}{\partial y}+\frac{1}{2}\left(\frac{\partial w}{\partial y}\right)^{2} \\
\varepsilon_{x y} & =\frac{\partial u}{\partial y}+\frac{\partial v}{\partial x}+\frac{\partial w}{\partial x} \frac{\partial w}{\partial y}
\end{aligned}
$$

3) The total potential energy of the membrane is equal to the total strain energy minus the work done by the applied pressure:

$$
\begin{aligned}
V= & \frac{E t}{2\left(1-v^{2}\right)} \iint\left(\varepsilon_{x x}^{2}+\varepsilon_{y y}^{2}+2 v \varepsilon_{x} \varepsilon_{y}+\frac{1}{2}\left(1-v^{2}\right) \varepsilon_{x y}\right) d x d y \\
& -\iint p w d x d y
\end{aligned}
$$


4) To solve for the unknown coefficients, minimize the total potential energy with respect to each of the coefficients:

$$
\frac{\partial V}{\partial A}=0 \quad, \quad \frac{\partial V}{\partial w_{0}}=0 \quad, \quad \frac{\partial V}{\partial w_{1}}=0
$$

5) This gives three equations \& three unknowns... solve for $\left(A, w_{0}, w_{1}\right)$. (Easier said than done!)

What's interesting about this analysis is that the final result is quite similar to that obtained for the idealized spherical cap model:

$$
\begin{aligned}
& p=g(v) \frac{B t}{a^{4}} h^{3}+3.393 \frac{\sigma_{0} t h}{a^{2}} \\
& g(v)=\frac{1}{(0.792+0.085 v)^{3}}
\end{aligned}
$$

The functional form of this expression is exactly the same as that for a "spherical cap". The coefficient $(8 / 3)$ has been replaced by $g(v)$, and the coefficient $(4)$ has been replaced by (3.393).

\section{Bulge parameter for a circular membrane}

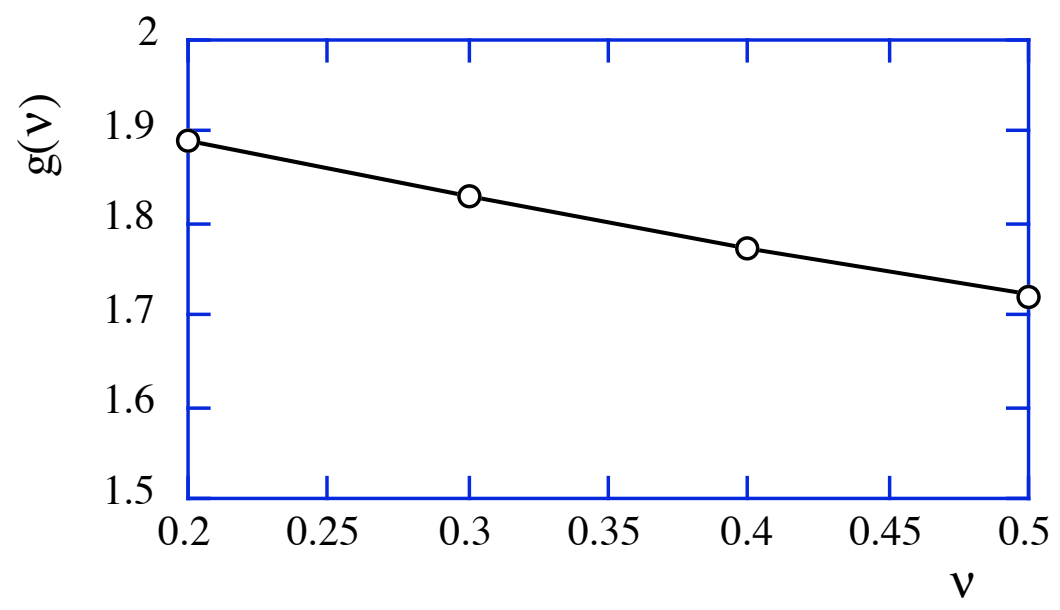




\section{$\underline{\text { Stresses in a square membrane }}$}

The analysis technique described here has been used to determine the stresses in square and rectangular membranes. The following results are for square membranes.

$\sigma_{x x}$ (Normalized) from Joost Vlassak's dissertation

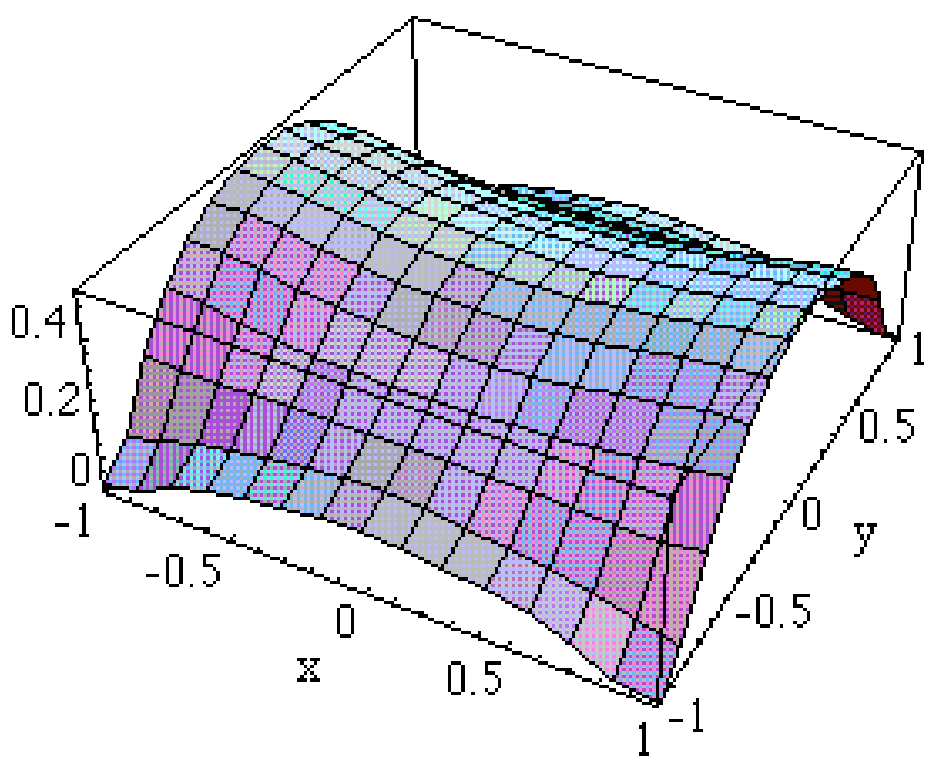

\section{Maximum Principal Stress (Normalized) from Joost Vlassak's dissertation}

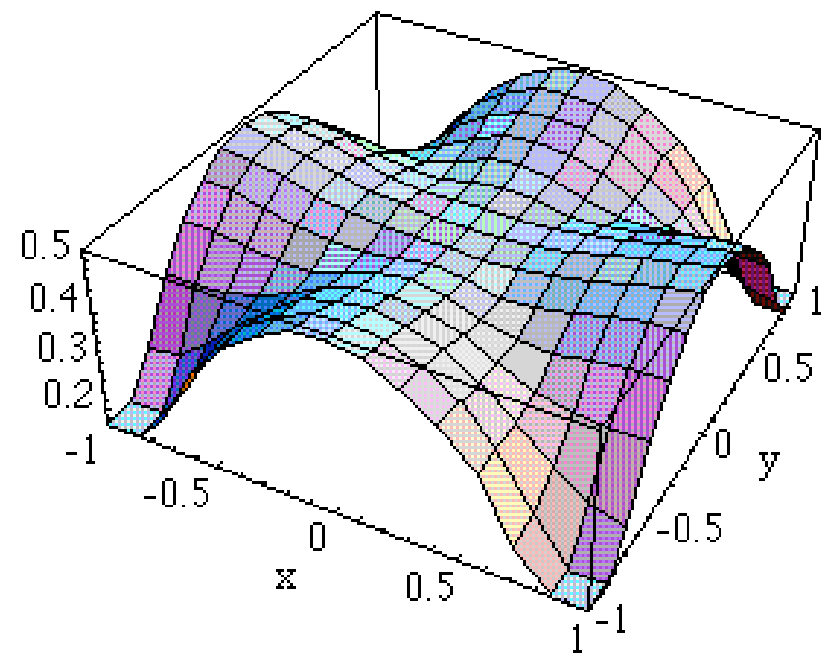


Yielding is expected to occur first at the centers of the edges. Plastic deformation of metal films is observed occur first at the centers of the edges of the membrane.

\section{Rectangular Membranes}

We've considered the problem of square membranes. What about rectangular membranes, in which one side might be significantly longer than the other? (aspect ratio $=b / a) \quad$ What will the strains in the membrane look like?

\section{Deflection of a rectangular membrane}

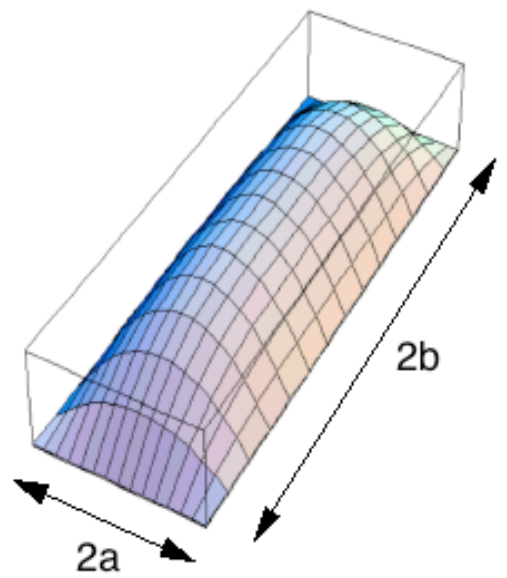

Look at cross sections of the window along the axes:

Deflections and strains in different directions

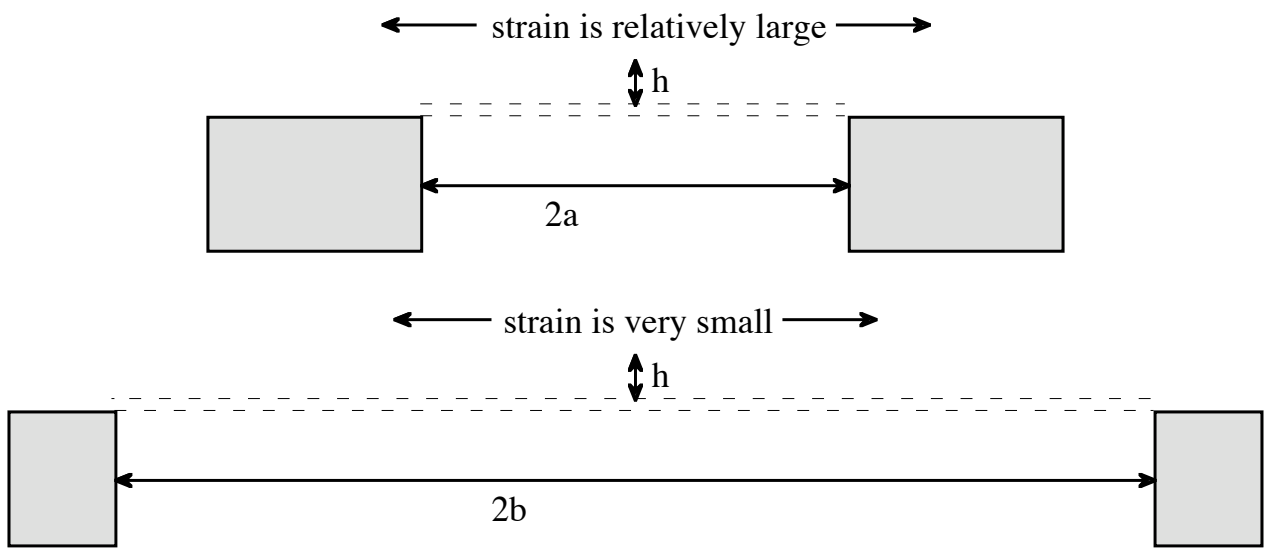


We might guess that if the aspect ratio is high enough, the deflection of the window will produce no strain in the direction of the long axis. Furthermore, away from the ends of the rectangle, the film should assume a uniform radius of curvature.

\section{$\underline{\text { Plane Strain }}$}

Plane strain conditions prevail when the strain in one direction is exactly zero. We can write out the stress-strain relationships for plane strain:

\section{Plane strain conditions}

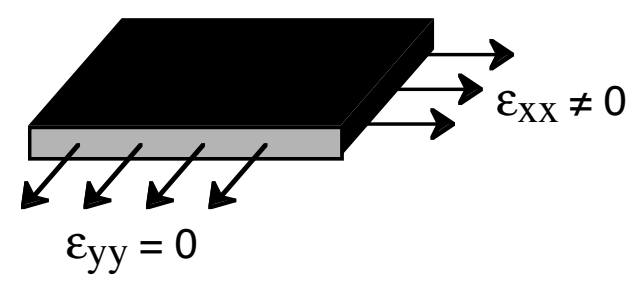

The mechanics of plane strain is described by the following equations:

$$
\begin{aligned}
& \varepsilon_{x x}=\frac{1}{E}\left(\sigma_{x x}-v \sigma_{y y}\right), \\
& \varepsilon_{x x}=\frac{1}{E}\left(\sigma_{x x}-v \sigma_{y y}\right), \\
\varepsilon_{y y}= & \frac{1}{E}\left(\sigma_{y y}-v \sigma_{x x}\right)=0 \\
\therefore \sigma_{y y}= & v \sigma_{x x}, \\
\varepsilon_{x x}= & \sigma_{x x} \frac{\left(1-v^{2}\right)}{E}
\end{aligned}
$$

$\underline{\text { Stress in a thin-walled cylindrical pressure vessel }}$

Previously we looked at the stress in a thin-walled spherical pressure vessel and found that stress is equi-biaxial. We were able to model deflection of a circular thinfilm window by treating it as a section of a spherical pressure vessel. This worked fairly well, even though the strain in a circular window is not everywhere equibiaxial. In long rectangular windows, strain closely approximates plane-strain conditions. We can model a rectangular window by treating it as a section of a thinwalled cylindrical pressure vessel. 
Let's find the stress in a cylindrical thin-walled pressure vessel under plane-strain conditions $\left(\varepsilon_{y y}=0\right)$.

\section{Thin-walled pressure vessel}

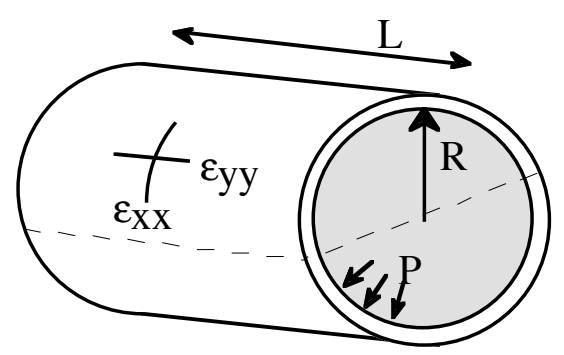

Imagine slicing the cylinder in half along the dotted line. The forces on each half of the cylinder must add to zero.

$$
\begin{aligned}
& 2 R \cdot L \cdot p=2 t \cdot L \cdot \sigma_{x x} \\
& \sigma_{x x}=\frac{R p}{t}
\end{aligned}
$$

where $t$ is the wall thickness.

\section{Bulge equation for plane strain conditions}

Now we can derive a bulge equation to describe long rectangular windows in which plane-strain conditions hold. 


\section{$\underline{\text { Plane strain bulge }}$}

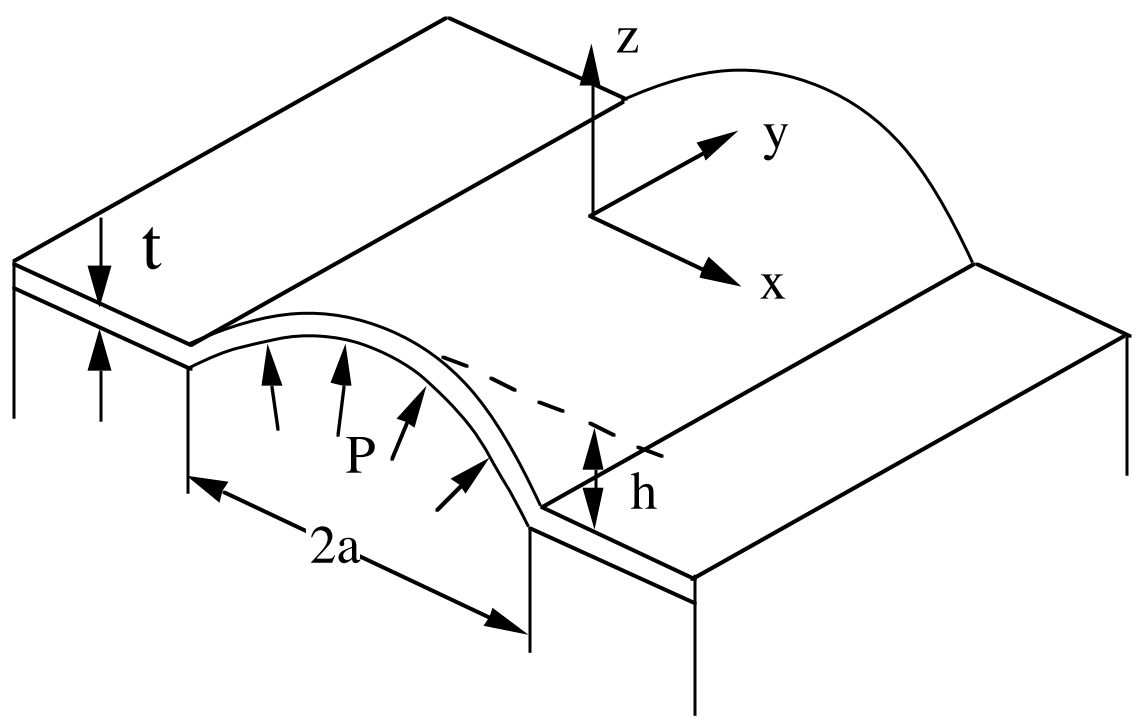

The window is in plane strain, so that:

$$
\sigma_{x x}=\varepsilon_{x x} \frac{E}{1-v^{2}}
$$

For a cylindrical pressure vessel:

$$
\sigma_{x x}=\frac{R p}{t}
$$

In the notes above we showed through geometric arguments that for small deflections:

$$
\varepsilon_{x x}=\frac{2 h^{2}}{3 a^{2}} \quad, \quad R=\frac{a^{2}}{2 h} .
$$

Using these expressions with the stress, we can solve for pressure:

$$
p=\frac{4}{3} \frac{h^{3}}{a^{4}} \frac{E t}{1-v^{2}}
$$

In order to include the effects of residual stress in our model, add $\sigma_{O}$ to the stress resulting from strain change: 


$$
\sigma_{x x}=\sigma_{o}+\frac{E}{1-v^{2}} \cdot \frac{2 h^{2}}{3 a^{2}}
$$

Inserting this into the relation $\sigma_{x x}=R p / t$, we find a bulge equation which is valid for long rectangular windows:

$$
p=\frac{2 h t \sigma_{o}}{a^{2}}+\frac{4}{3} \frac{h^{3}}{a^{4}} \frac{E t}{\left(1-v^{2}\right)}
$$

By now, it should not come as a surprise that this equation is very similar in form to the one that was derived for a pressurized spherical cap. The differences are:

- Different numerical coefficients preceding the two terms

- $\quad E /\left(1-v^{2}\right)$ appears instead of $E /(1-v)$

The quantity $E /\left(1-v^{2}\right)$ can be determined from bulge experiments on rectangular windows, and $E /(1-v)$ can be determined from experiments on round or square windows. If we perform both kinds of experiments, then in theory we can solve for $E \mathcal{E} v$. In practice, it doesn't take much experimental error to throw off the measurements of $v$.

\section{Sources of Error in the Bulge Test}

There are some "usual suspects" which can cause problems with the bulge test.

Mounting the film

One of the biggest motivations for making windows in situ by etching away the substrate is to ensure that the film is well-supported and not wrinkled. Any wrinkling of the film can result in significant errors. 


\section{$\underline{\text { Non-flat Films }}$}

Performing a bulge test on a film which is not initially flat can result in fairly large errors. Using laser interferometry to measure deflection, you can ensure that the film is flat to within the resolution of the interferometer. If the film isn't flat, the bulge equation needs to be modified by an "initial height" $\left(h_{i}\right)$ :

$$
P=c_{1} \frac{\sigma_{0} t}{a^{2}}\left(h-h_{i}\right)+c_{2} \frac{E t}{(1-v) a^{4}}\left(h-h_{i}\right)^{3}
$$

\section{$\underline{\text { Residual Stress }}$}

A film window which has a compressive residual stress will not remain flat once the substrate is etched away. Films are too thin to support much compressive stress without buckling.

\section{Buckling of a compressive film}

Compressive Stress

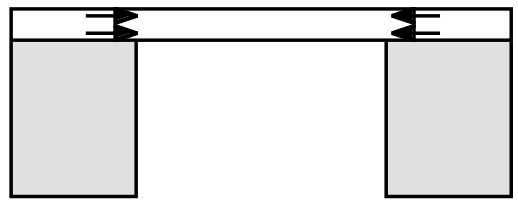

Buckling

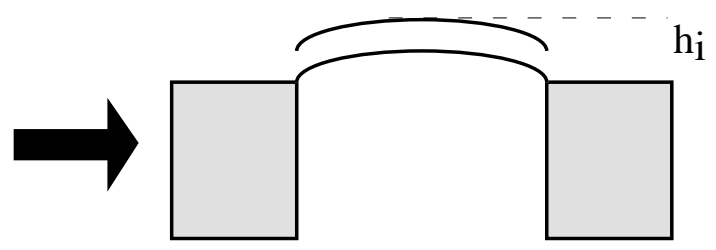

At the start of a bulge test, a film with residual compressive stress may be buckled and will have an "initial height" $\left(h_{i}\right)$ which must be accounted for in the data fit. Even after pressure is applied, there will still be buckling near the edges, where the film is constrained by the substrate. It will consequently be very difficult to get good data.

Failure to account for the effects of residual stress have led to reports of a "supermodulus effect".

Supermodulus Effect- A reported enhancement ( 400\%) in the modulus of multilayer films (Ag-Pd, Au-Ni) which appeared at low compositional wavelengths. This effect was observed in bulge test data but could not be corroborated by other testing techniques. In actuality, the supermodulus effect was an artifact resulting from a failure to account for the effects of residual stress on the bulge test.

How can these problems be avoided? One approach involves the deposition of a compressive film on top of a window which is under a tensile stress. If you choose 
stresses \& thicknesses correctly, the composite structure will have net tensile stress and will not buckle. For example, to refute the supermodulus effect Martha Small looked at Ag-Pd films deposited on tensile silicon nitride membranes. Her results are shown in the following figure.

Biaxial modulus of $\mathrm{Ag}-\mathrm{Pd}$ multilayered films (no supermodulus effect)

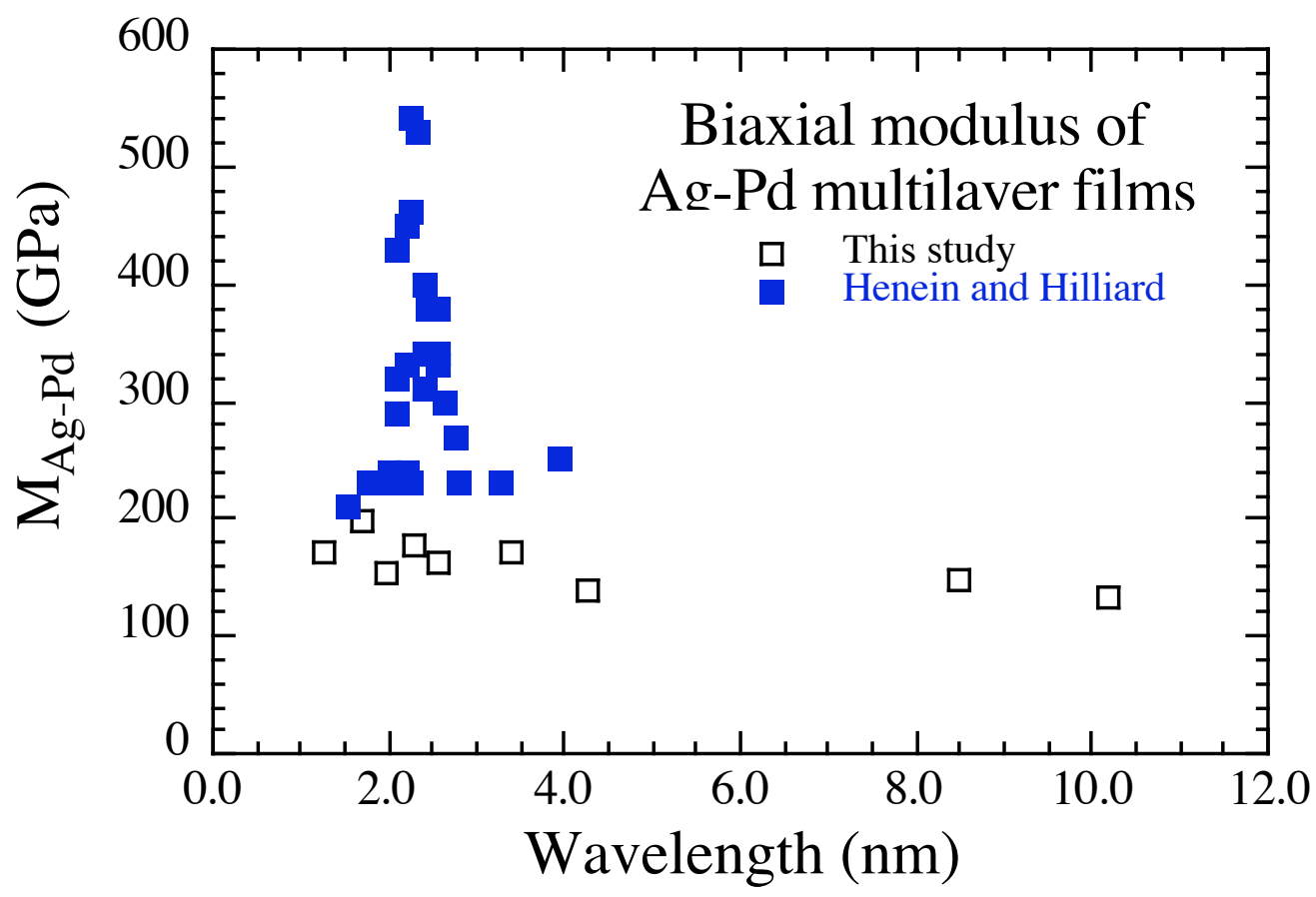

\section{Plastic Flow and Rectangular Windows}

Plastic flow in a bulge test is indicated by loading/ unloading hysteresis in the pressure-height relation. This is shown in the following graph for the bulge response of an evaporated gold film in the form of a square window. Notice that on unloading an elastic bulge relation is observed. On reloading, the elastic displacements are recovered and the elastic-plastic bulge relation is again followed. This is analogous to the elastic-plastic response in an ordinary stress-strain curve. 


\section{Bulge relation for a square Au membrane}

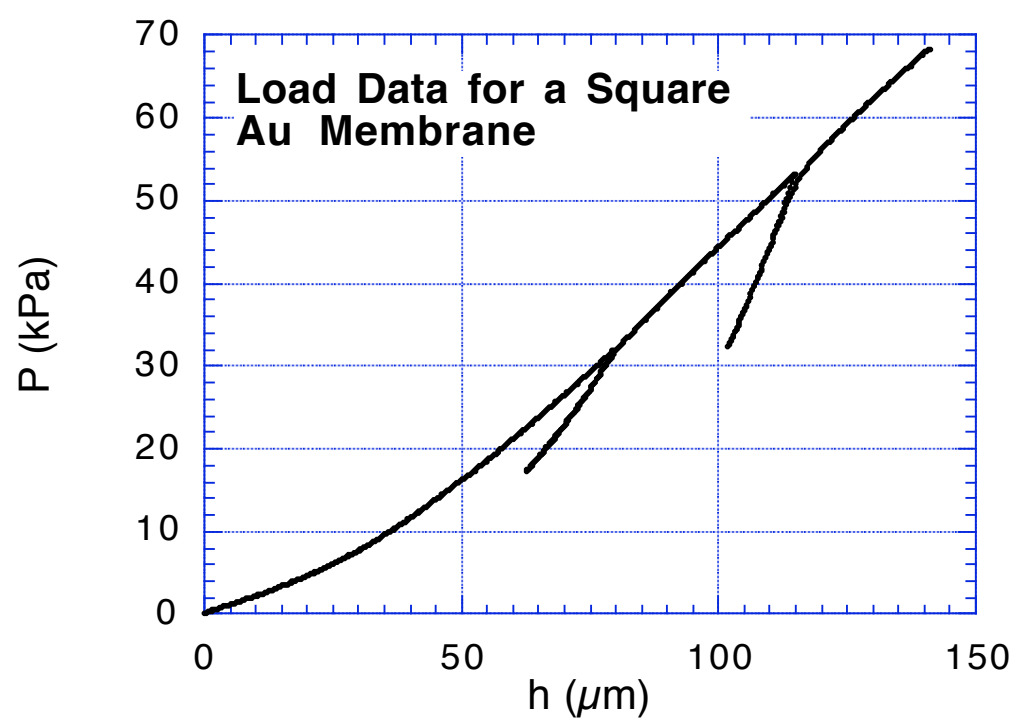

To measure the yield stress of a material, we need to be able to detect the onset of yielding. Typically the yield stress is defined by the stress at which a certain amount of permanent strain (usually $0.2 \%$ ) takes place. In circular and square windows, stress \& strain vary throughout the window. Areas of the window where stress is high will start to yield before other areas where stress is lower. Not only does this make it difficult to tell exactly when yielding begins, it is also very hard to determine local values of strain from the total deflection of the window $(h)$.

In rectangular windows, the strain state is quite uniform. To a good approximation, plane-strain conditions hold everywhere. Yielding will initiate uniformly, and for this reason such windows can be used to study plastic flow as well as elastic deformation. Remember: $\sigma_{x x}$ and $\varepsilon_{x x}$ can be determined directly from measurements of $p$ and $h$.

Next we show some experimental data for yielding of thin metal films. 
Representative data from tests of rectangular membranes: Evaporated Au film, 1.1 $\mu \mathrm{m}$ thick, $\{111\}$ texture, pressure vs. deflection

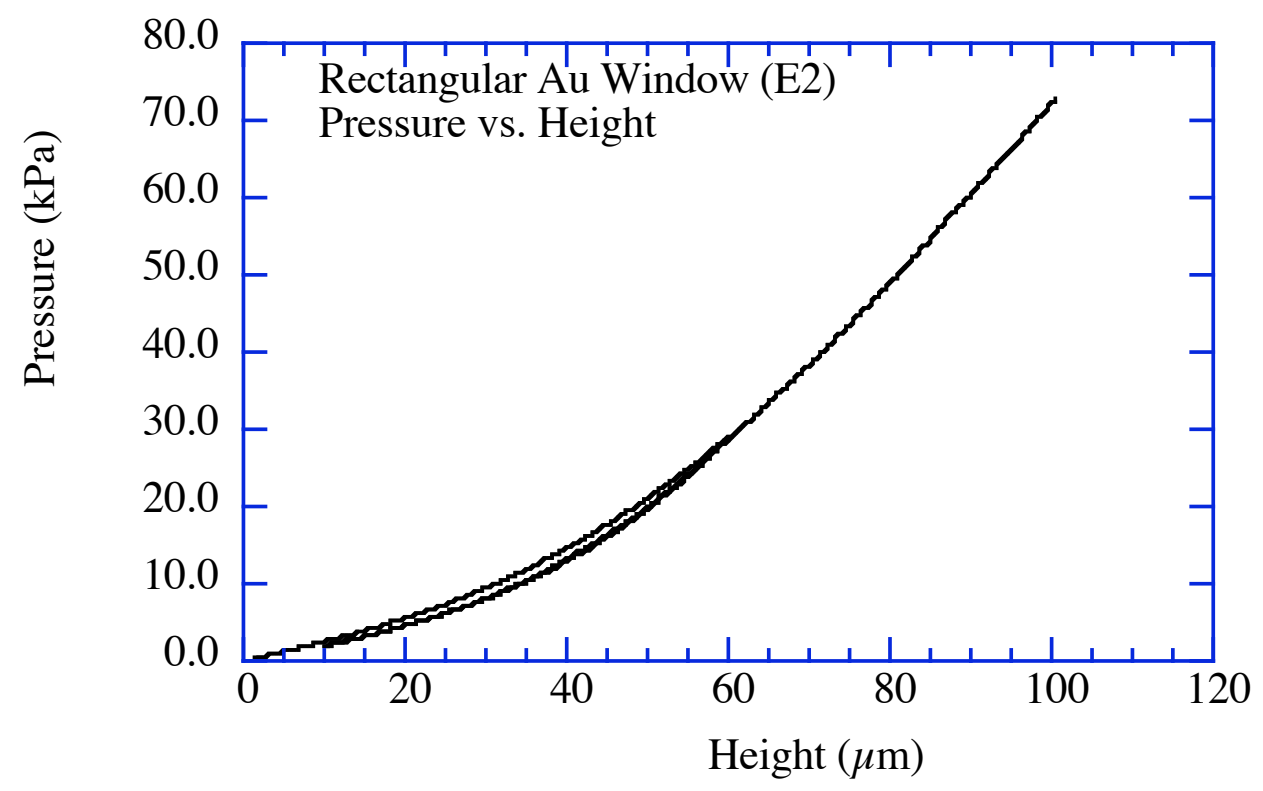

Representative data from tests of rectangular membranes: Evaporated Au film, 1.1 $\mu \mathrm{m}$ thick, $\{111\}$ texture, stress vs. strain

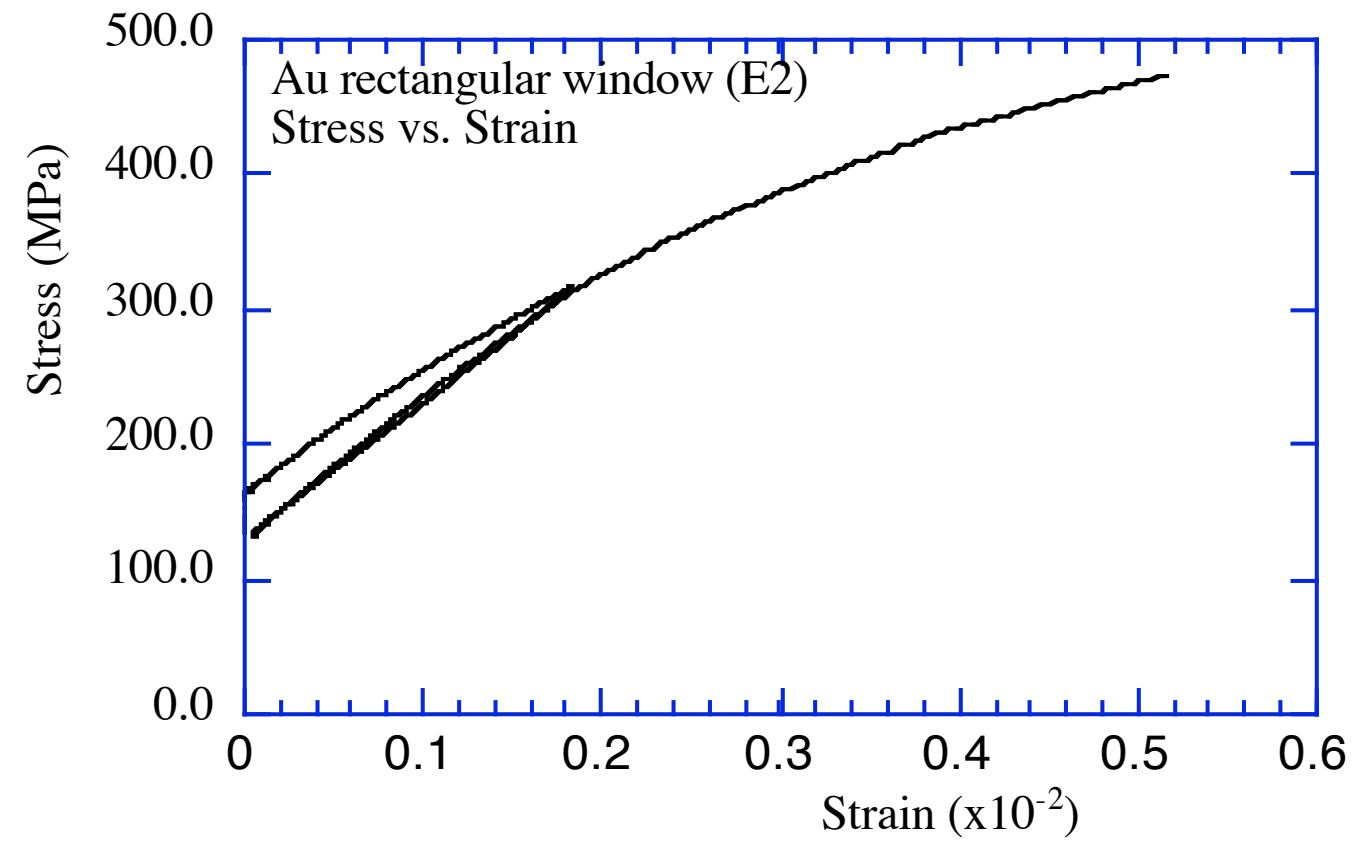


Representative data from tests of rectangular membranes: Evaporated Au film, 1.1 $\mu \mathrm{m}$ thick, $\{111\}$ texture, pressure vs. deflection

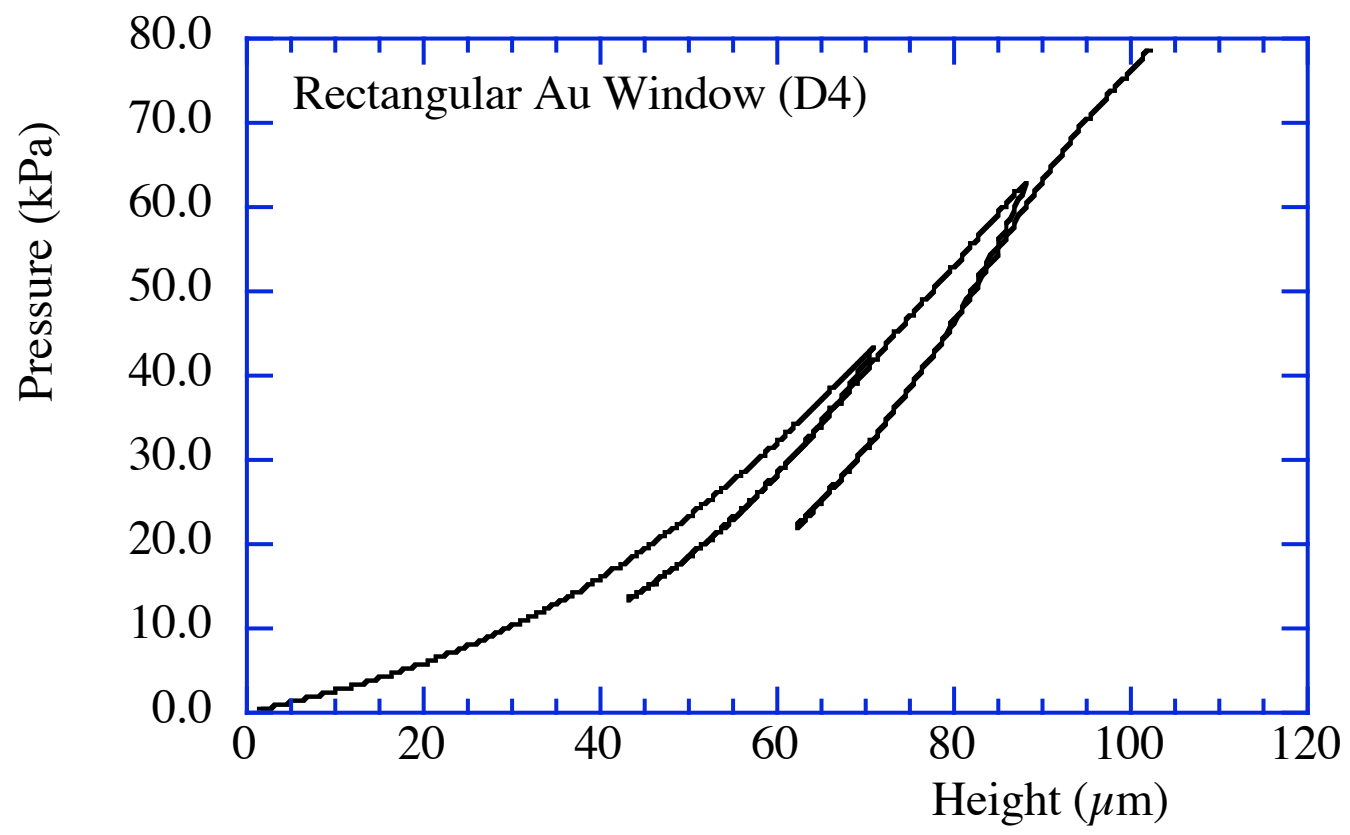

Representative data from tests of rectangular membranes: Evaporated Au film, 1.1 $\mu \mathrm{m}$ thick, $\{111\}$ texture, stress vs. strain

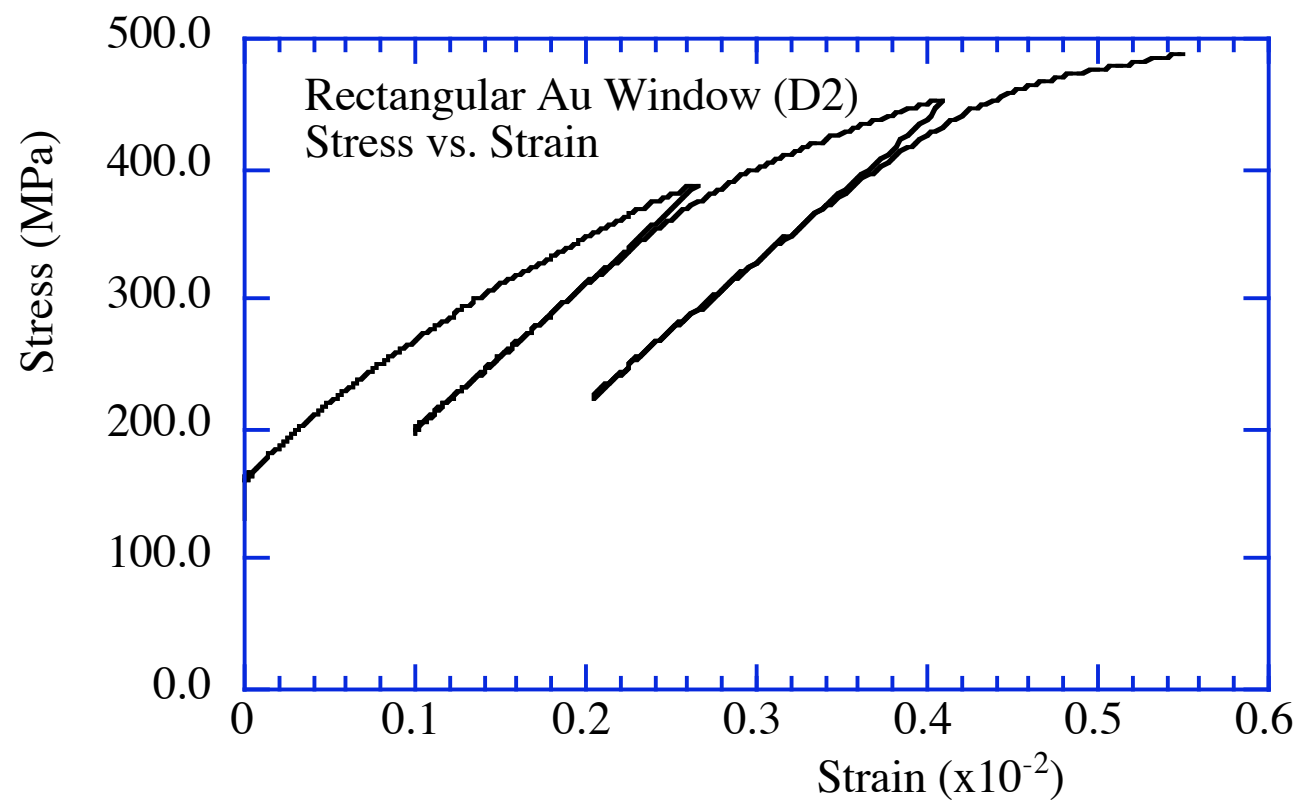


Mechanical properties of $\mathrm{Au}$ and $\mathrm{Al}$ thin films
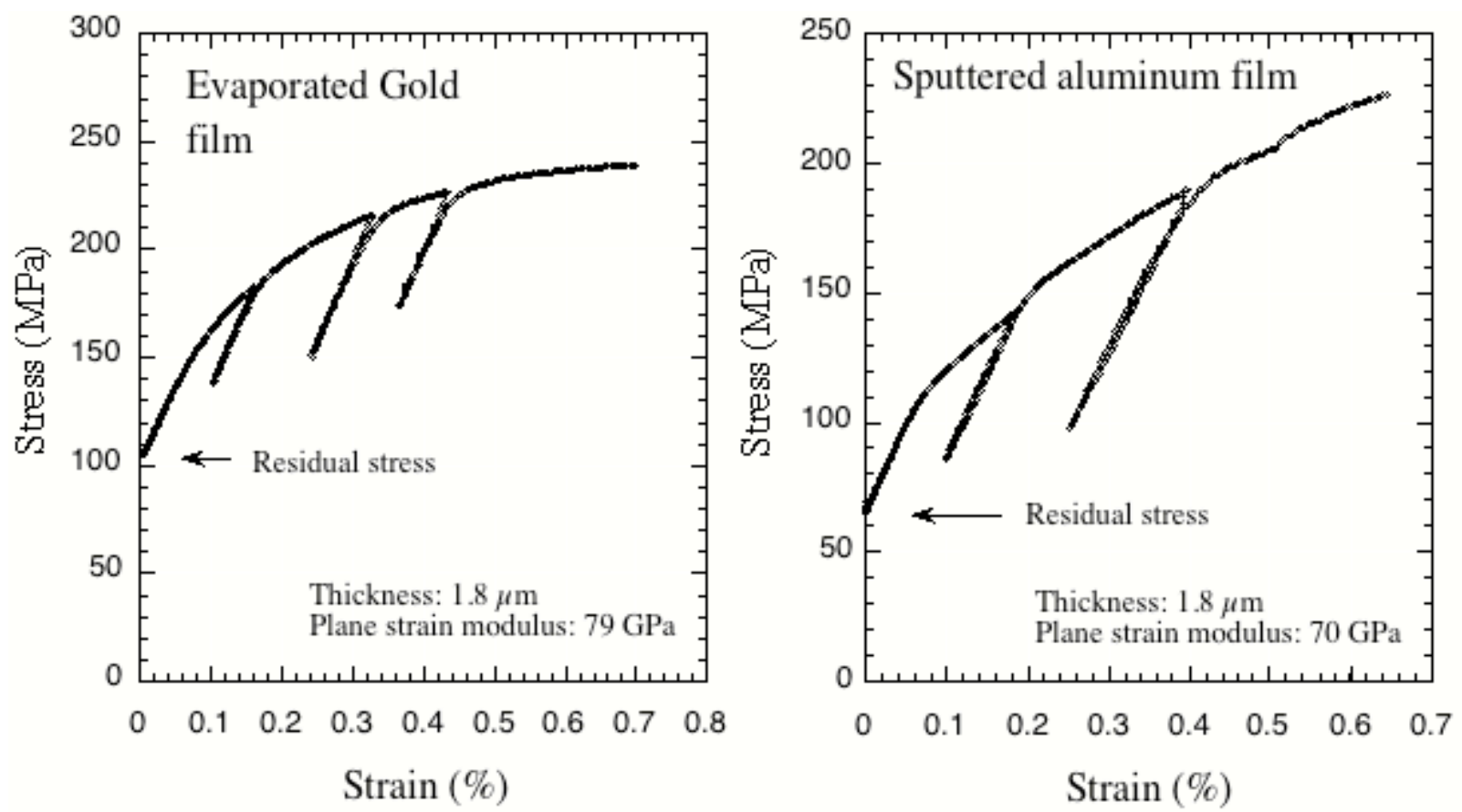

\section{Equivalent Stress- Equivalent Plastic Strain Curve (Rectangular Membranes)}

During bulge testing under plane strain conditions, the strain increment in the bulge direction can be measured from the increment in the bulge height

$$
\delta \varepsilon_{x x}=\delta\left(\frac{2 h^{2}}{3 a^{2}}\right)=\frac{4 h}{3 a^{2}} \delta h .
$$

Also, at each point in the bulge test the stress in the bulge direction is known

$$
\sigma_{x x}=\frac{p a^{2}}{2 h t} .
$$

In the following, we show that because the deformation occurs under plane strain conditions, it is possible to determine the equivalent stress - equivalent plastic strain curve from the bulge data.

For plane strain deformation we may write the total strain increments as 


$$
\begin{aligned}
& \delta \varepsilon_{x x}=\delta \varepsilon_{x x}^{e}+\delta \varepsilon_{x x}^{p} \\
& \delta \varepsilon_{y y}=\delta \varepsilon_{y y}^{e}+\delta \varepsilon_{y y}^{p}=0
\end{aligned}
$$

We may use Hooke's law to determine the elastic strain increments

$$
\begin{aligned}
& \delta \varepsilon_{x x}^{e}=\frac{1}{E}\left(\delta \sigma_{x x}-v \delta \sigma_{y y}\right) \\
& \delta \varepsilon_{y y}^{e}=\frac{1}{E}\left(\delta \sigma_{y y}-v \delta \sigma_{x x}\right)
\end{aligned}
$$

At this point in the analysis the transverse stress increment, $\delta \sigma_{y y}$, is not known. This will be determined below. We may find the plastic strain increments using the Levy-Mises flow law, which may be stated as

$$
\begin{aligned}
& \delta \varepsilon_{x x}^{p}=\frac{\sigma_{x x}-\frac{1}{2} \sigma_{y y}}{\bar{\sigma}} \delta \bar{\varepsilon}_{p} \\
& \delta \varepsilon_{y y}^{p}=\frac{\sigma_{y y}-\frac{1}{2} \sigma_{x x}}{\bar{\sigma}} \delta \bar{\varepsilon}_{p}
\end{aligned}
$$

where $\delta \bar{\varepsilon}_{p}$ is the equivalent plastic strain increment and $\bar{\sigma}$ is the equivalent stress, defined as

$$
\bar{\sigma}=\sqrt{\frac{1}{2}\left(\sigma_{x x}^{2}+\sigma_{y y}^{2}+\left(\sigma_{x x}-\sigma_{y y}\right)^{2}\right)} .
$$

Solving these equations simultaneously, together with the elastic (Hooke's law) and plastic (Levy-Mises) constitutive laws gives the increments $\delta \bar{\varepsilon}_{p}$ and $\delta \sigma_{y y}$

$$
\begin{gathered}
\delta \bar{\varepsilon}_{p}=\frac{2 \bar{\sigma}\left(\delta \varepsilon_{x x}-\frac{1-v^{2}}{E} \delta \sigma_{x x}\right)}{\left((2-v) \sigma_{x x}-(1-2 v) \sigma_{y y}\right)} \\
\delta \sigma_{y y}=v \delta \sigma_{x x}-\frac{E\left(2 \sigma_{y y}-\sigma_{x x}\right)\left(\delta \varepsilon_{x x}-\frac{1-v^{2}}{E} \delta \sigma_{x x}\right)}{\left((2-v) \sigma_{x x}-(1-2 v) \sigma_{y y}\right)}
\end{gathered}
$$


With these relations, the bulge data taken from a rectangular bulge experiment can be numerically transformed into an equivalent stress- equivalent strain curve. This is the continuum equivalent of a uniaxial stress-strain test.

Next we show some rectangular bulge results where the equivalent stress-equivalent plastic strain relation has been extracted from the basic bulge data.

Plane strain, stress-strain curve for an Al thin film

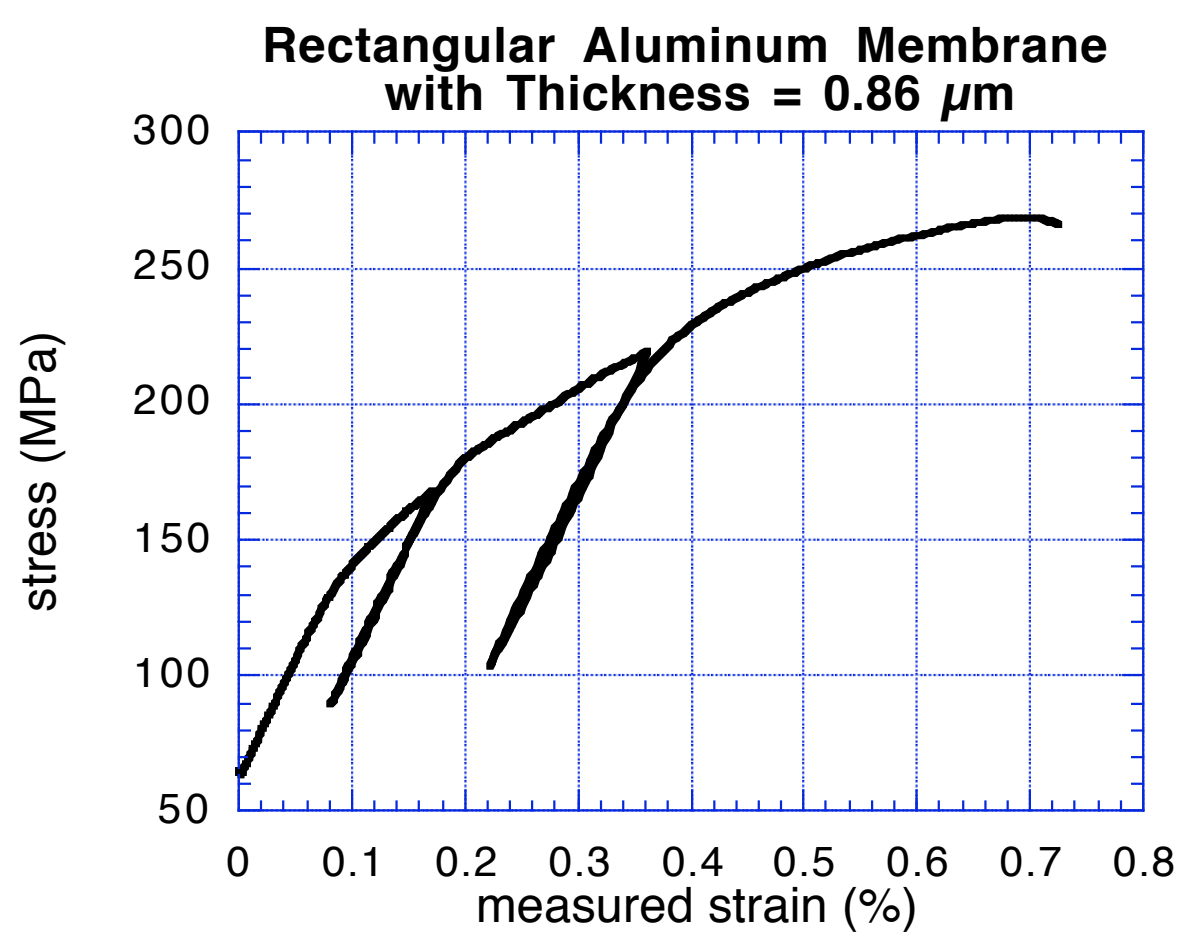


Equivalent stress-equivalent plastic strain curve for an Al thin film

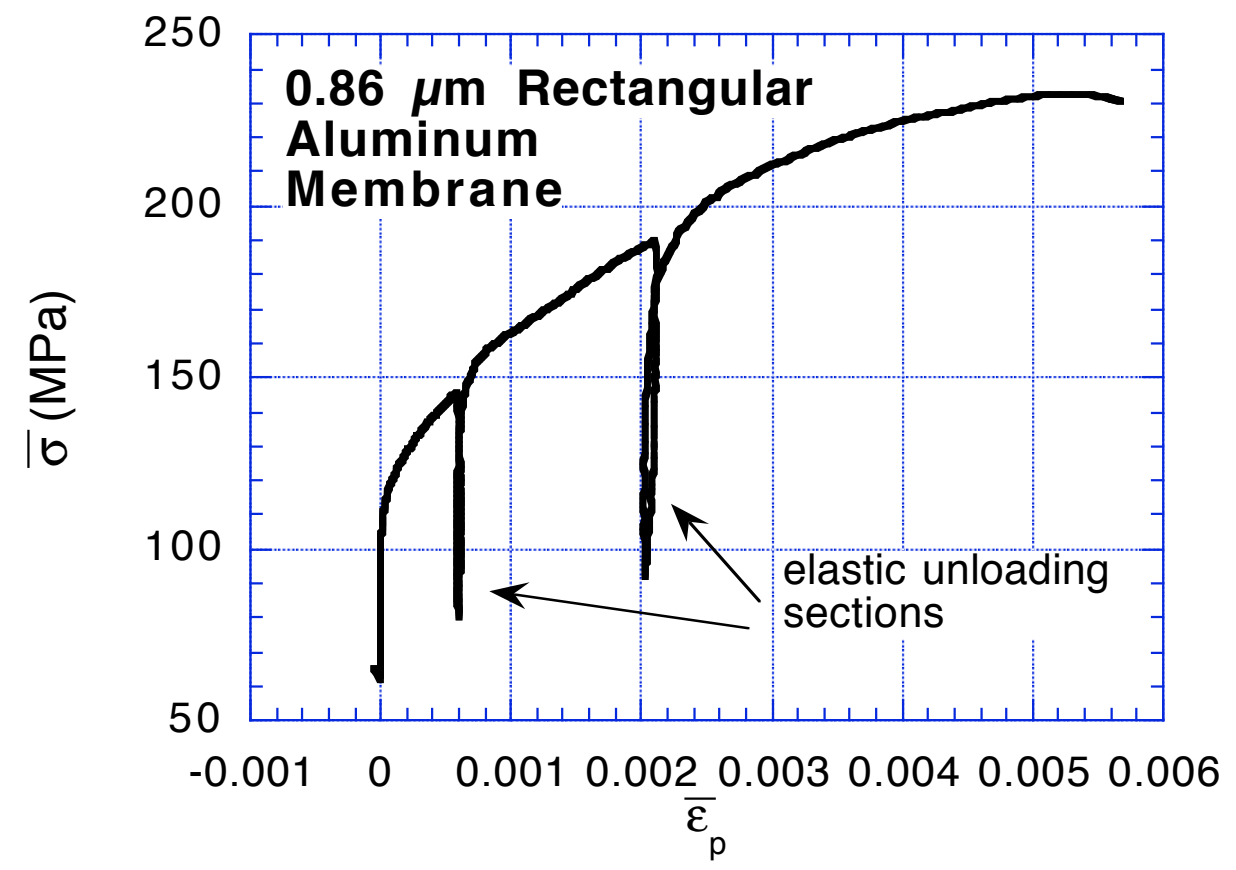

Plane strain, stress-strain curve for a Au thin film

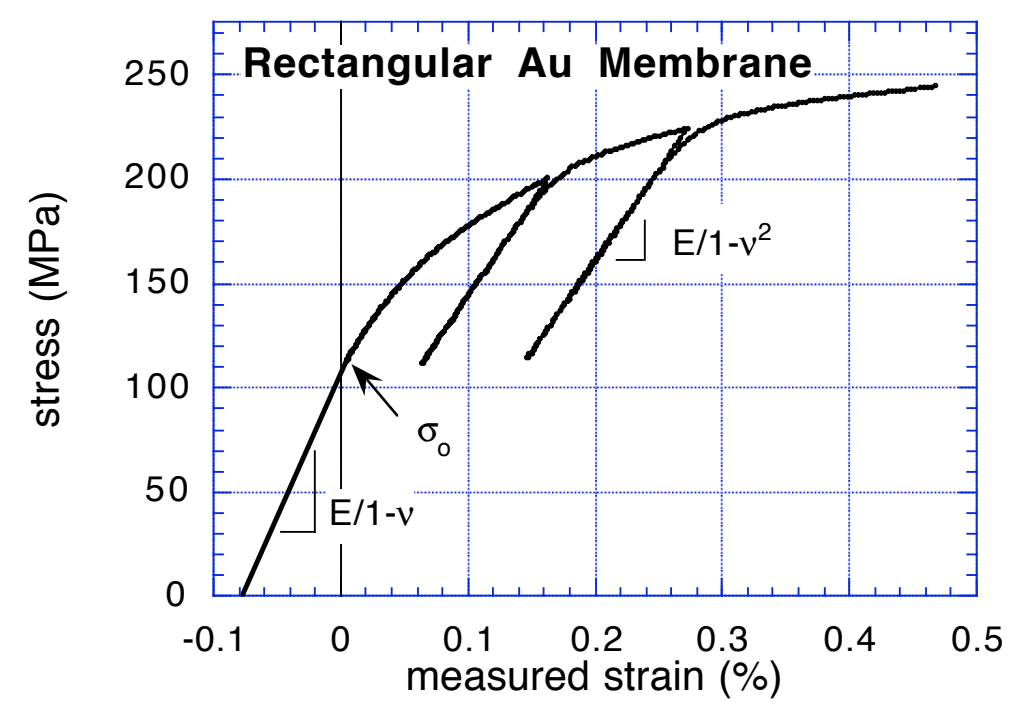


Equivalent stress-equivalent plastic strain curve for a Au thin film

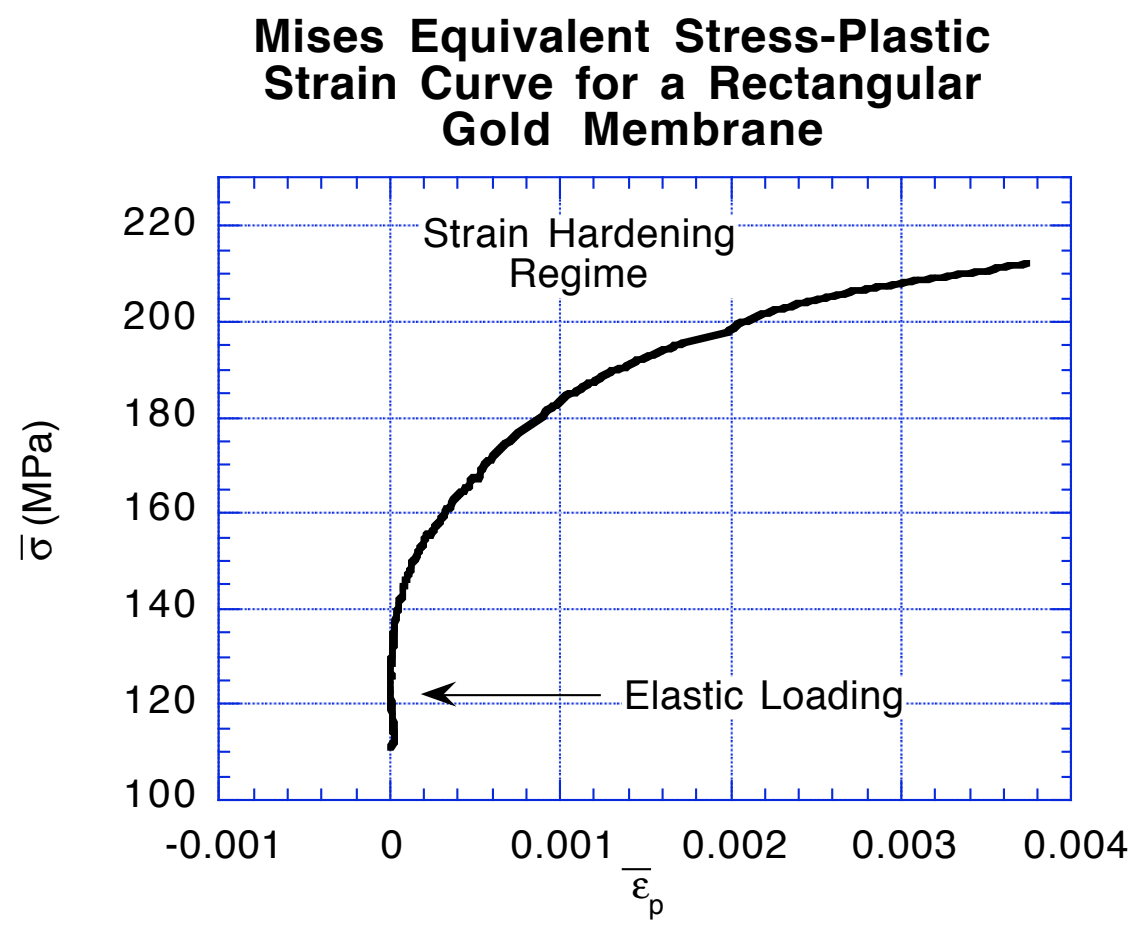

Power Law Hardening of a Au thin film

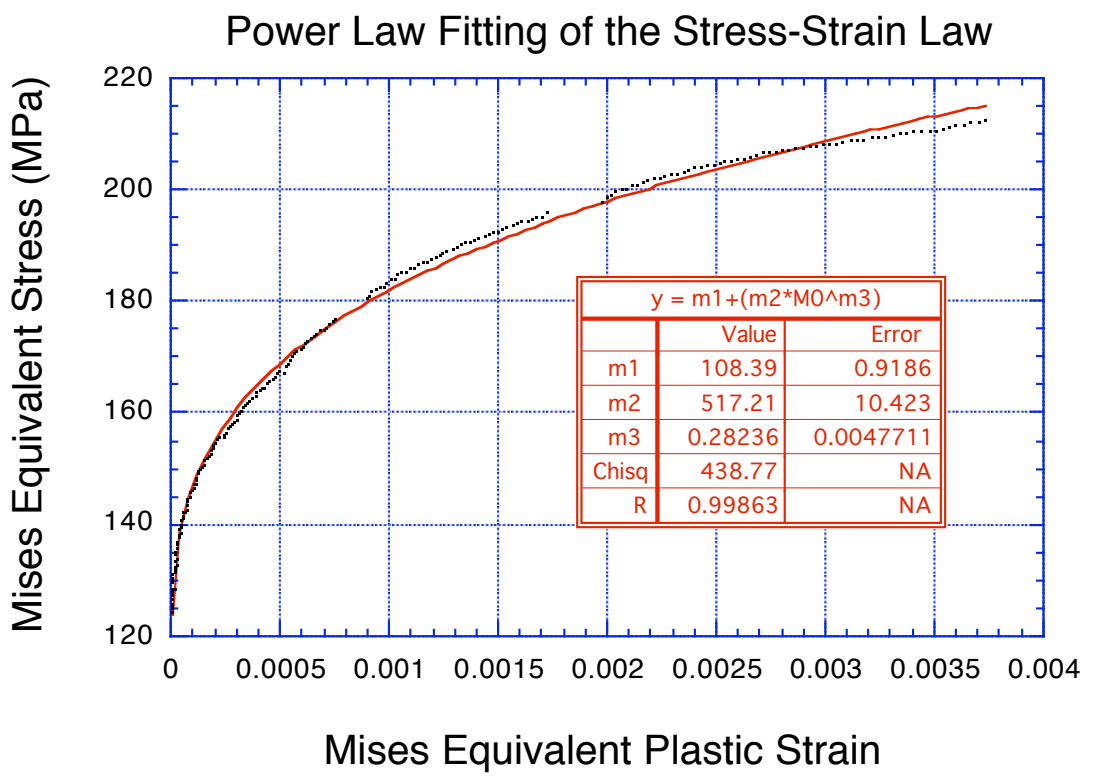




\section{Nanoindentation (sub-micron, depth-sensing indentation)}

Nanoindentation is now one of the most widely used techniques to study mechanical properties of thin films. It involves forcing a sharp diamond indenter into the surface of a thin film on a substrate, while measuring the force imposed and the corresponding displacement of the indenter. The load and displacement resolution of such instruments is sufficient to obtain useful mechanical property data for films as thin as about $100 \mathrm{~nm}$. Because the indentations made are too small to be easily observed, the size of the indentation is determined from the measured depth of indentation and the calibrated shape of the indenter. Thus nanoindentation is called a depth-sensing indentation technique. The technique provides information about the following mechanical properties:

1. Hardness

2. Elastic properties

3. Time-dependent deformation

In addition, when coupled with finite element analysis methods, the technique can provide information about the yield strength of the film in question, although the effects of the fine scale of the testing geometry must be taken into account in interpreting the measurements. Under some conditions nanoindentation can also be used to induce fracture in the film or at the filmsubstrate interface and this can lead to methods for measuring fracture toughness. Since the mid-1980's, commercial instruments have been available for nanoindentation and the technique is now widely used to study the mechanical properties of thin films.

\section{Basic Hardness Testing}

Ordinary hardness testing involves forcing a sharp indenter (usually diamond) into the surface of a solid with a fixed load or force and then measuring the projected area of the impression left in the surface. 


\section{Basic methodology for hardness testing}
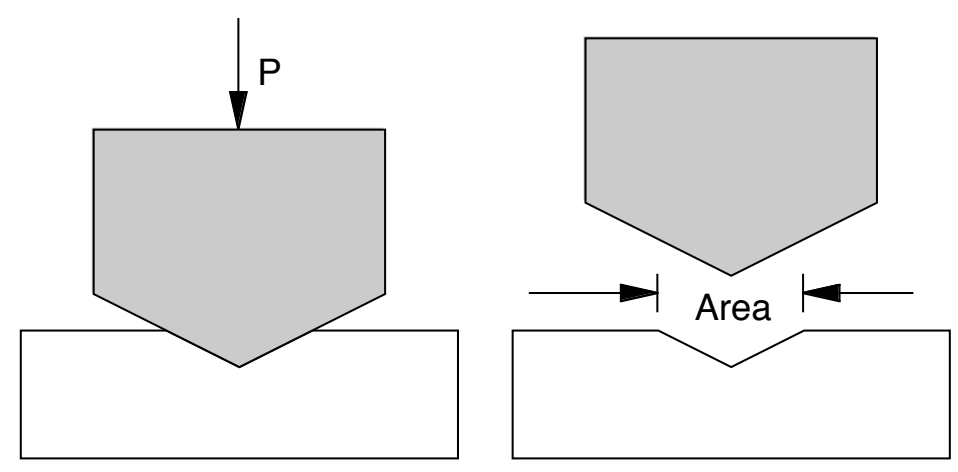

With this testing methodology, the hardness is usually defined as

$$
\text { Hardness }=\frac{\text { Load }}{\text { Area }}=\frac{P}{A}
$$

where the load $P$ is the maximum load applied and the area $A$ is the projected area of the resulting impression. For ordinary microhardness measurements Vickers and Knoop indenters are commonly used. The projected shapes or the impressions made by these indenters are shown in the figure below. Often the diagonal lengths of these impressions are measured and the corresponding areas are calculated to obtain the hardness.

\section{Vickers and Knoop Impressions}

\section{Vickers}
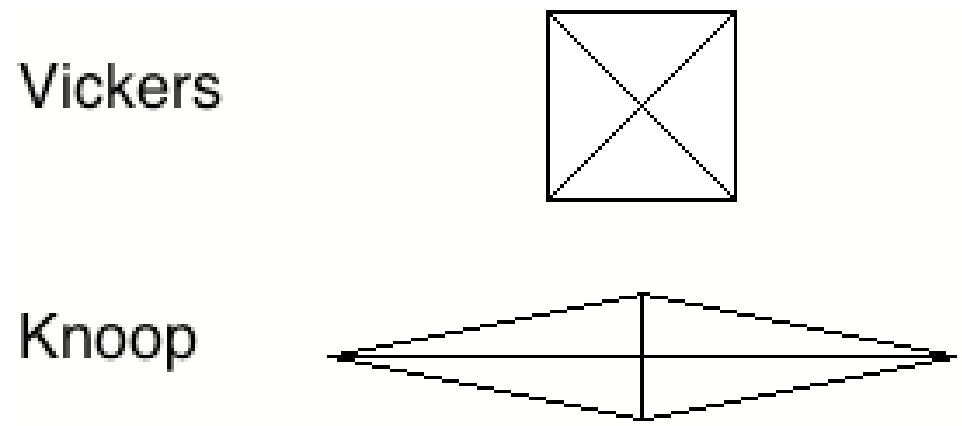

Ordinary microhardness testing is of limited use in the study of mechanical properties of thin films for several reasons. Typical loads available cause the indenters to push through the film and into the substrate, with the consequence that the film properties cannot be measured. Even if smaller loads could be applied, the indentations made would be so small that it would be difficult to observe and accurately measure the area of the resulting impression. Also, 
imposing the force and subsequently measuring the resulting impression does not provide any information about the elastic properties or the time-dependent plastic properties of the film.

\section{Depth-Sensing Indentation Testing at the Sub-Micon Scale (nanoindentation)}

The Nanondenter, which was developed by John Pethica and Warren Oliver in the early 1980's when they were at Brown-Boveri in Switzerland, has the following characteristics:

- $\quad$ Load controlled (current in a loading coil/magnet assembly is controlled)

- Indenter displacement measured (capacitance measurement)

- $\quad$ Computer controlled (positioning is controlled and the entire indentation experiment (load-displacement-time relation) is controlled.

- $\quad \mathrm{AC}$ force oscillation and corresponding oscillating displacement is available for measuring contact stiffness throughout the indentation experiment.

The principal resolution figures are:

- $\quad$ Load resolution $-0.25 \mu \mathrm{N}$

- $\quad$ Displacement resolution $-0.3 \mathrm{~nm}$

- $\quad$ Position resolution - $1 \mu \mathrm{m}$

In the early 1990's several new instruments became commercially available. The most successful of these is the Hysitron System which is an AFM type system with excellent imaging capabilities. The principal advantage of the Hysitron system is the ability to image the feature to be studied before the indentation is made and also to directly observe the impression left in the material after the indentation experiment is completed. This provides very useful qualitative information about the indentation experiment, but it does not permit sufficiently accurate measurements of the contact area to obtain reliable measurements of elastic modulus and hardness. For that it is best to use the measured stiffness and the calibrated shape of the indenter, together with various relations from the literature on contact mechanics, as described below. The scanning and imaging feature is now included with the Nanoindenter as well. 
Below we show a schematic diagram of the Nanoindenter. The load or force is applied by controlling the current in the coil mounted on top of the indenter column. The field of the coil acts against the permanent magnet and a force is exerted onto the column. The instrument is load controlled. The indenter column is supported by very delicate leaf springs, which are part of the capacitance device for measuring the displacement of the indenter column.

\section{The Nanoindenter}

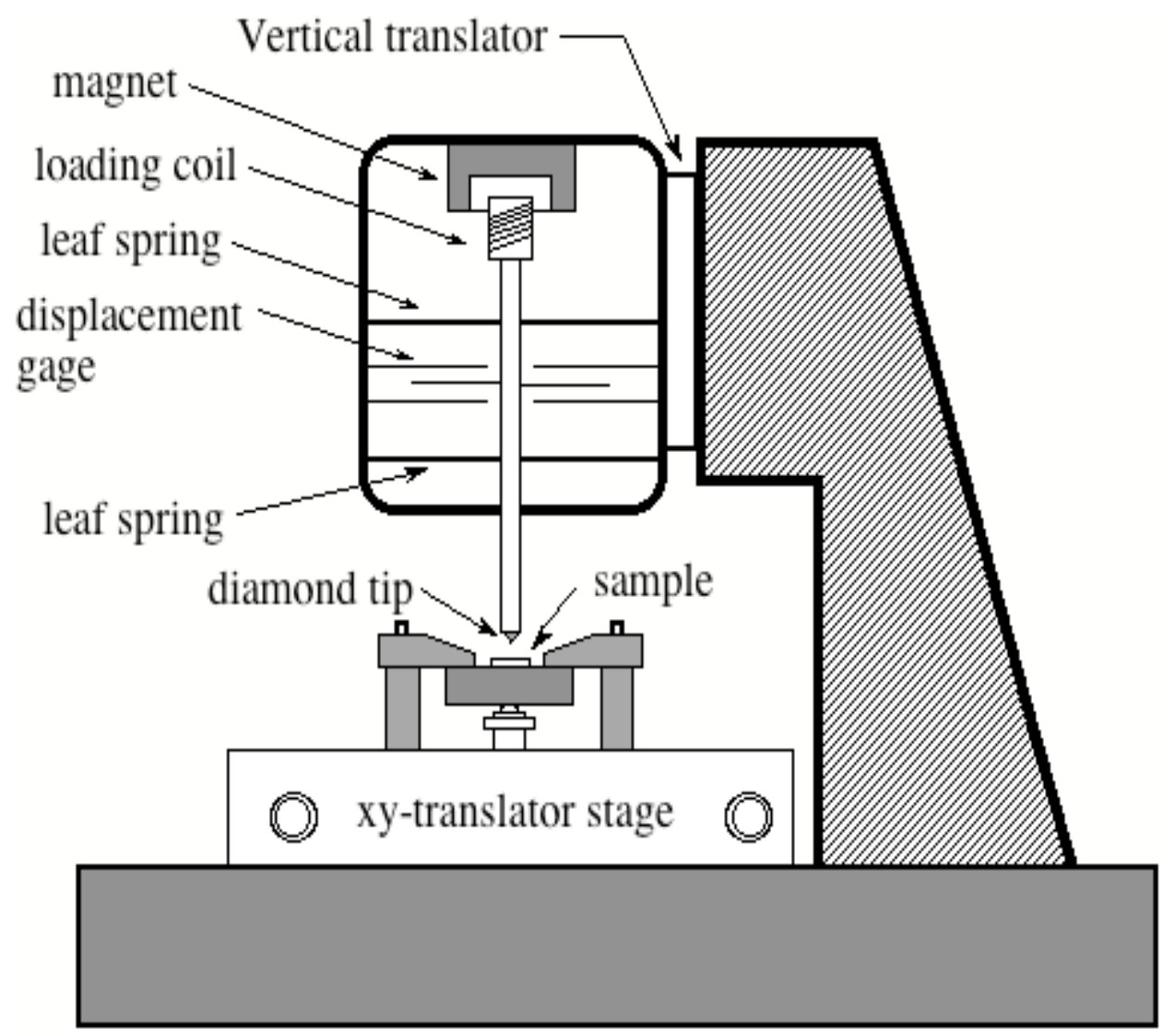

A typical load-displacement curve from a nanoindentation experiment is shown in the following figure. The indentation load is found by subtracting any forces associated with the deflection of the suspending springs and including the weight of the indenter column itself. The indentation depth is the displacement measured from the point at which the tip of the indenter first reaches the surface of the solid. This point is found by observing a dramatic increase in the stiffness of the system when the indenter makes contact with the material.

As shown in the load-displacement diagram below for the indentation of the $\{100\}$ surface of a single crystal of tungsten, nanoindentation provides information about the elastic-plastic response on loading, the time-dependent creep response when the load is held fixed, and the elastic response when the 
load is decreased. For a hard substance like fused quartz, there is very little timedependent creep.

\section{Load-depth curve for nanoindentation of single crystal tungsten}

\section{Typical indentation plot for the $\{100\}$ surface of a tungsten single crystal}

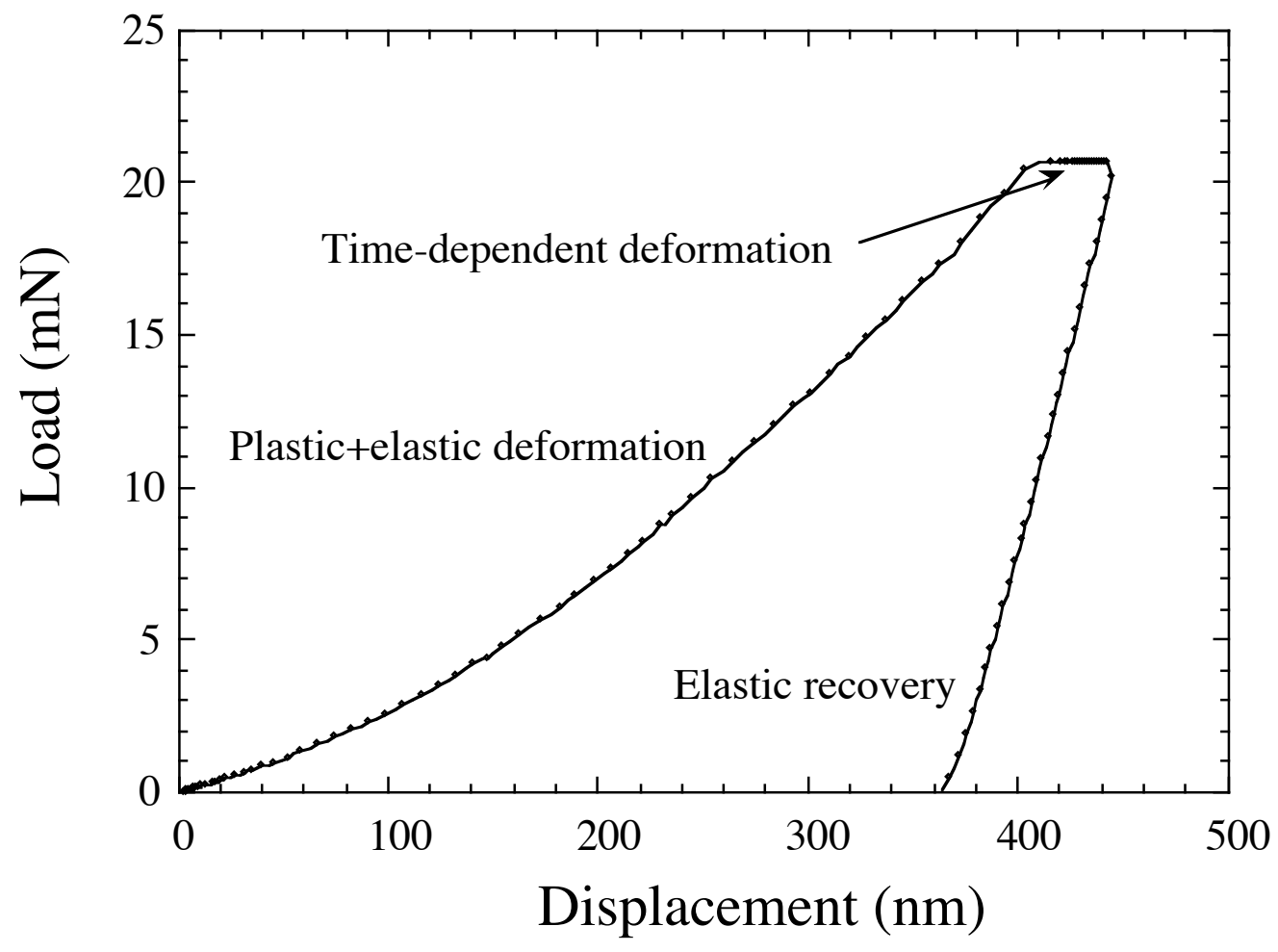




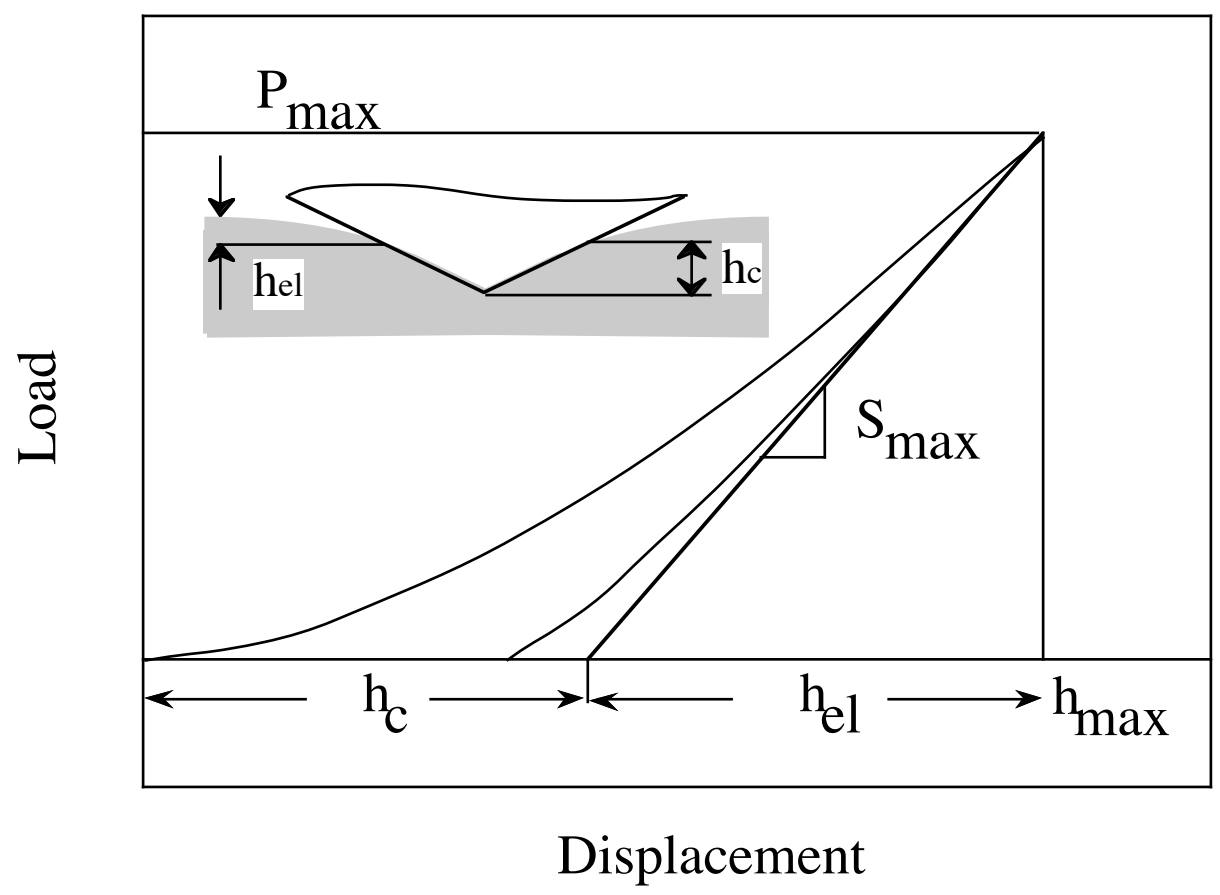

As discussed below, the projected contact area between the indenter and the material being indented is of crucial importance in nanoindentation. For example the hardness is given by

$$
\text { Hardness }=\frac{P_{\max }}{A_{\text {contact }}}
$$

where $P_{\max }$ is the maximum load imposed on the indenter and $A_{\text {contact }}$ is the projected contact area between the indenter and the material being indented. We note that this definition of hardness involves the contact area under load and not the residual projected area of the impression left by the indenter, as in the case of ordinary microhardness measurements. For very hard or compliant materials this can lead to meaningless values of hardness. Think of the indentation of rubber - here the indenter makes contact with the material and a load is applied, but no plastic flow at all occurs. In this case the "hardness" as defined above is merely the mean pressure at the point of maximum load. It is no measure of the real hardness of the material.

The elastic properties of the material being indented can also be found from the unloading portion of the load-displacement curve, provided the projected contact area is known. Procedures have been developed for determining the 
contact area from the load-displacement curve and knowledge of the shape of the indenter. These will be described below.

The elastic and plastic displacements associated with nanoindention are illustrated in the following diagram.

Elastic and plastic response during indentation
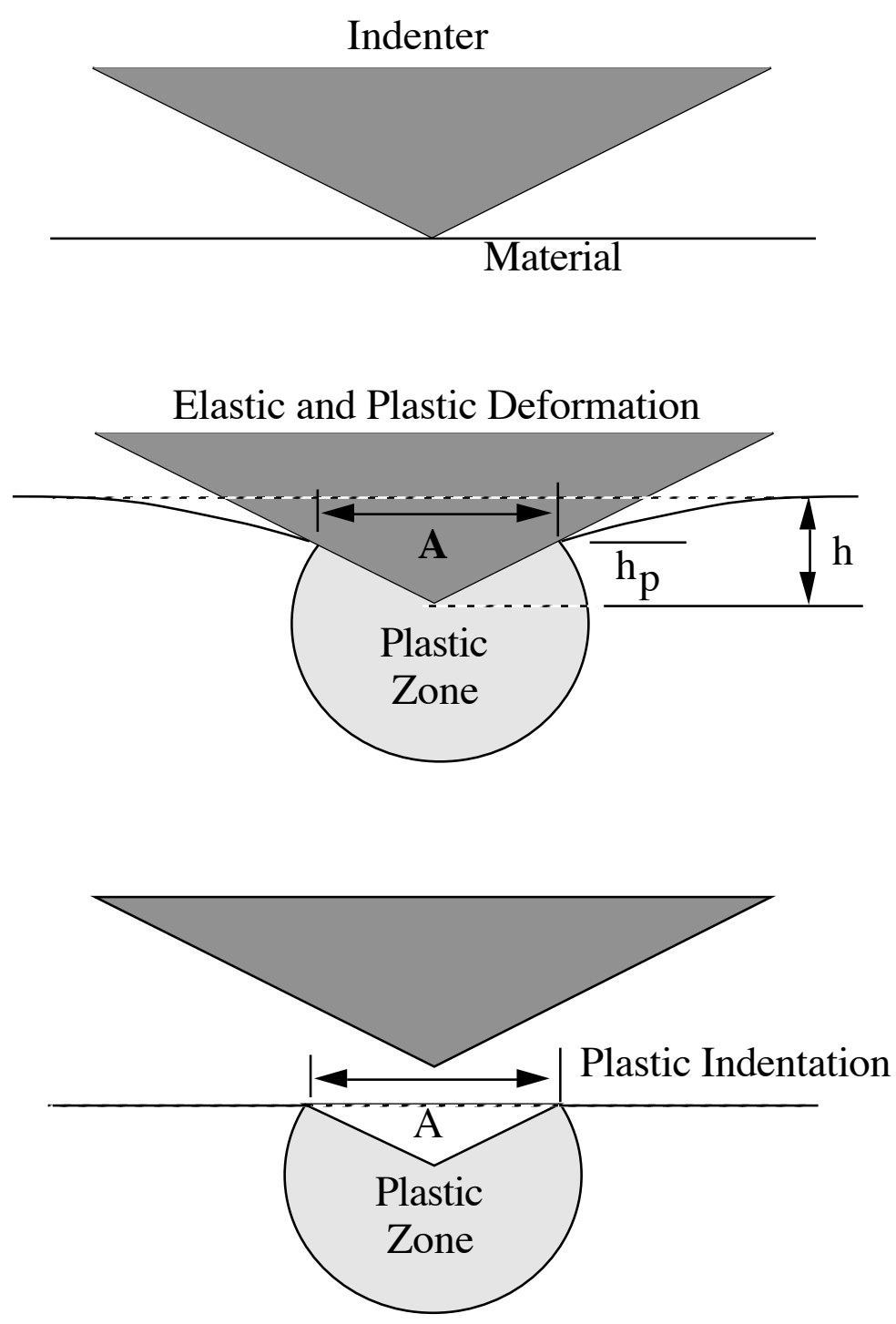

For nanoindentation we need to have a very sharp and well-defined tip. The best geometry for this is the Berkovich three-sided indenter geometry as shown in the figure below. If a point is to be created at the tip of the diamond indenter, three faces (rather than four for the Vickers and Knoop indenters) are much 
preferred because three planes will always meet at a single point, whereas the grinding of four surfaces to meet at a single point is a very difficult proposition.

\section{Impression left in the surface by a Berkovich indenter}

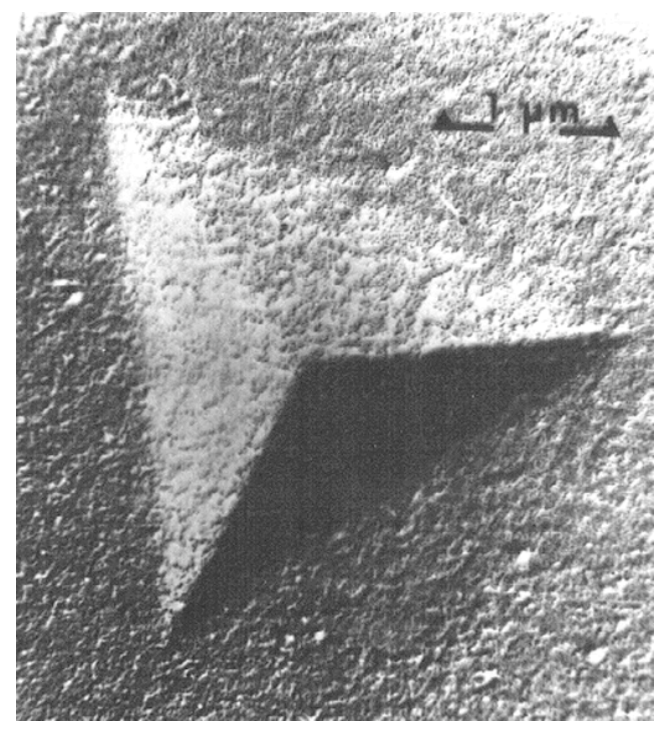

Even very sharp Berkovich indenters are not perfectly sharp. They are rounded at the tip and this tip shape must be taken into account when determining material properties at very small indentations. Usually the radius of curvature at the tip of a sharp Berkovich indenter is about $100 \mathrm{~nm}$. As shown in the indenter tip shape diagram below, the contact area, $A_{c}$, must be known as a function of the distance from the end of the indenter, called the contact depth, $h_{c}$. This is usually described as a tip shape area function of the form

$$
A_{c}=f\left(h_{c}\right) \text {, }
$$

where the function $\mathrm{f}$ is usually expressed as a polynomial. The nominal shape of the Berkovich indenter is such that the area-depth relation is the same as that for a Vickers indenter:

$$
A_{c}=24.5 h_{c}^{2} .
$$


Indenter tip shape function

Contact Depth and Contact Area

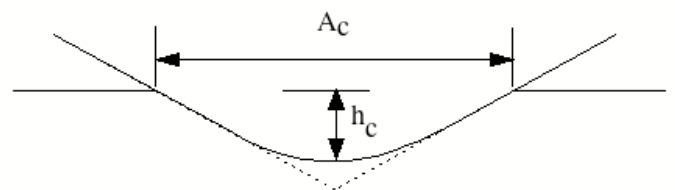

\section{Doerner-Nix Method of Analysis}

The load-depth curve for nanoindentation shows elastic recovery when the load is decreased from the maximum value. The slope of the unloading curve represents the stiffness of the system. That stiffness, in turn, is determined both by the stiffness of the contact and by the stiffness of the loading mechanism itself. When force is exerted onto the indenter column by the coil/magnet assembly, displacements occur both at the contact and elsewhere in the machine. The compliances (reciprocal of the stiffnesses) in the system may be illustrated as follows. Careful thinking about the structure of the Nanoindenter will show that this is the correct model.

\section{Compliances in the Nanoindenter}

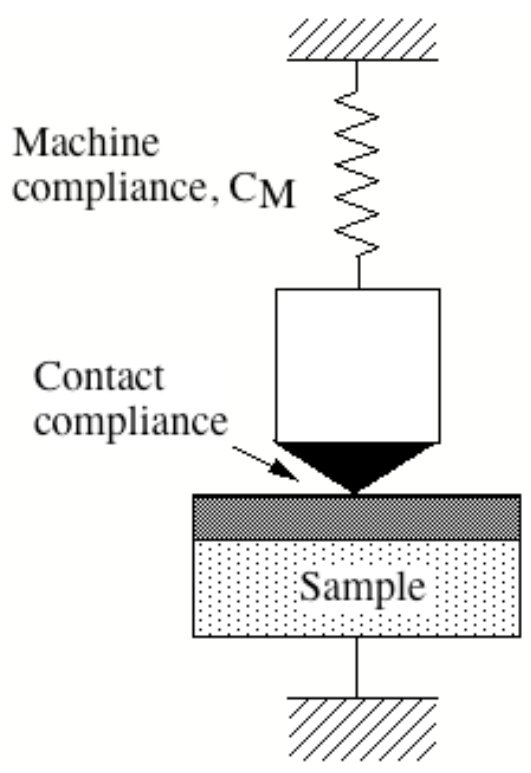

In a mechanical system such as this, the same force is imposed on both elements and the displacements add. Thus the compliances also add: 


$$
C_{\text {experimental }}=\frac{d h}{d P}=C_{\text {contact }}+C_{\text {machine }} \text {. }
$$

This relation shows that the compliance of the machine itself must be determined and substracted from the measured unloading compliance to determine the contact compliance.

\section{Doerner and Nix Constant Unloading Stiffness/Constant Contact Area}

\section{Assumption}

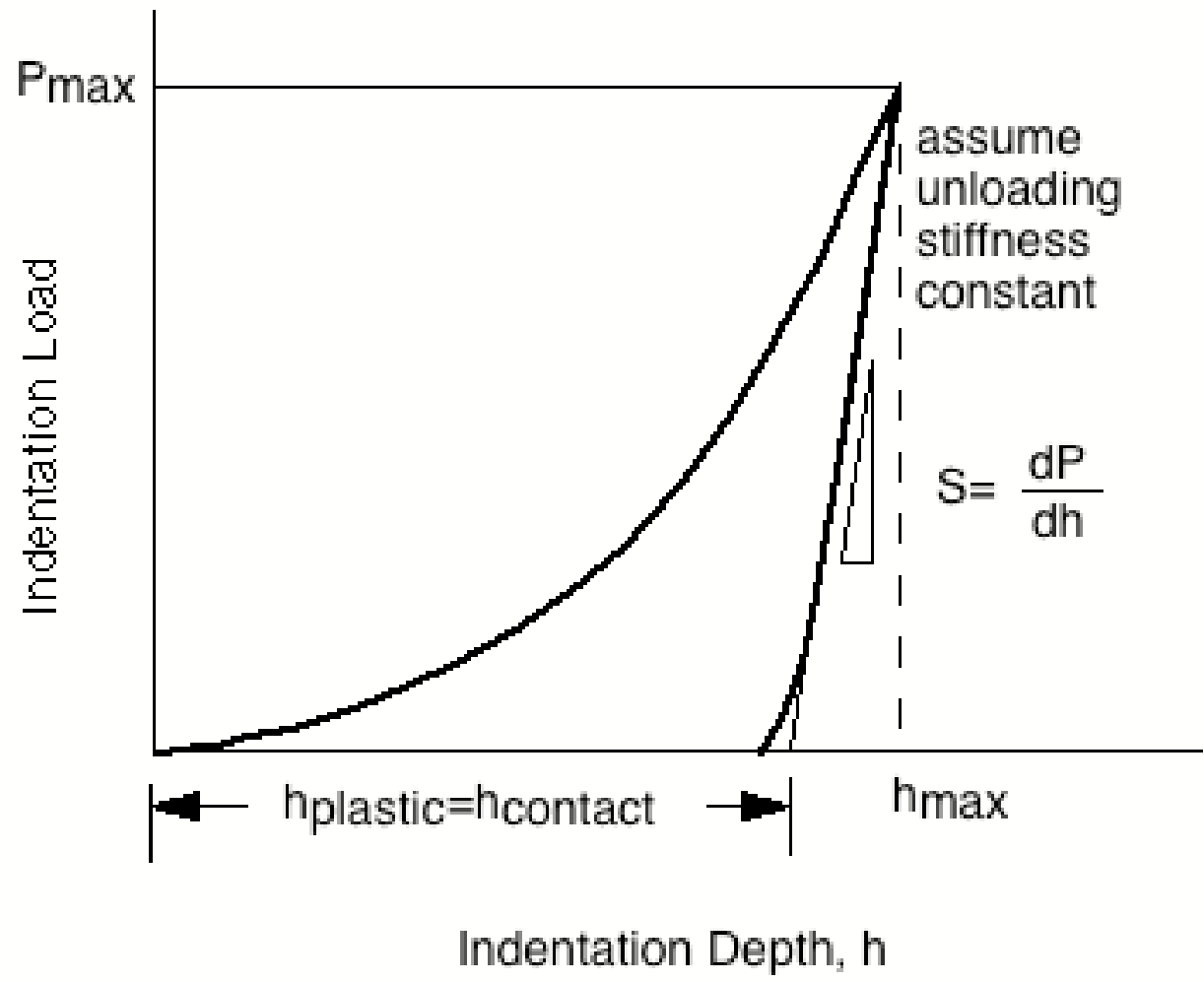

We now examine the contact compliance following the method of Doerner and Nix (1986). They assumed that the stiffness on unloading is approximately constant and treated the unloading stiffness or compliance using the flat punch model of Sneddon. We imagine that the slope of the unloading curve (after the machine compliance has been subtracted) can be treated as a contact between a circular, flat punch (the indenter) and the surface of the material. 


\section{Sneddon's Flat Punch Model}

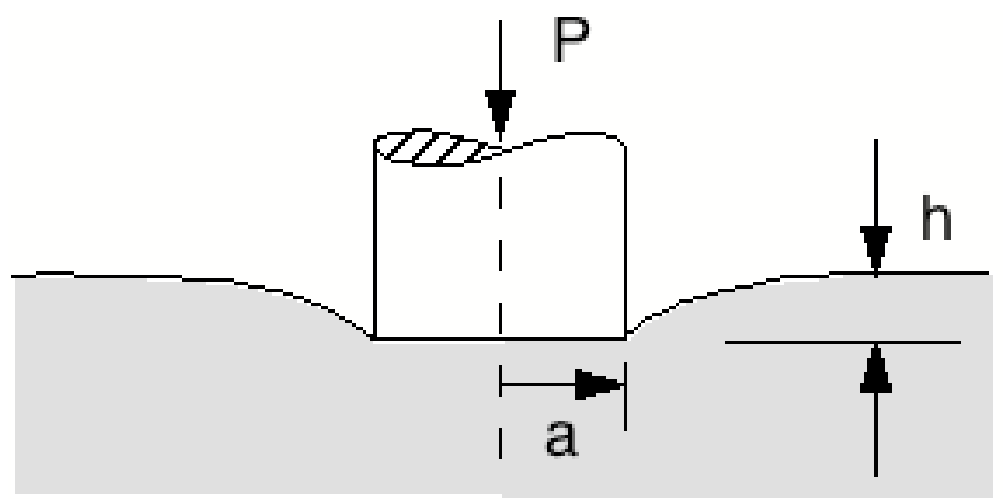

Sneddon (I, Sneddon, Int. J. Engr. Sci. vol 3, 47 (1965)) showed that for a perfectly rigid circular flat punch the indenting an isotropic elastic half space, the loaddisplacement relation is

$$
P=\frac{2 E \sqrt{A}}{\sqrt{\pi}\left(1-v^{2}\right)} h
$$

where $E /\left(1-v^{2}\right)$ is the plane strain modulus. A simple dimensional argument proves that a relationship of this form is to be expected. The corresponding contact stiffness, $S_{c}$, and contact compliance, $C_{c}$ are then described as follows:

$$
\begin{aligned}
& S_{c}=\frac{d P}{d h}=\frac{2 E \sqrt{A}}{\sqrt{\pi}\left(1-v^{2}\right)} \\
& C_{c}=\frac{1}{S_{c}}=\frac{\sqrt{\pi}\left(1-v^{2}\right)}{2 E \sqrt{A}}
\end{aligned}
$$

In addition, Sneddon provided a solution for the elastic displacement (or sink-in) of the surface outside of the contact radius:

$$
h_{e l}(r, 0)=\frac{2}{\pi}\left[\sin ^{-1}\left(\frac{a}{r}\right)\right] h \quad r>a .
$$

This sink-in is shown in the diagram. For the case in which the indenter is not perfectly rigid, the plane strain compliance in the above relations should be replaced by 


$$
\frac{\left(1-v^{2}\right)}{E}=\frac{1}{E_{r}}=\left(\frac{1-v^{2}}{E}\right)_{\text {material }}+\left(\frac{1-v^{2}}{E}\right)_{\text {indenter }}
$$

where the composite modulus, $E_{r}$, describes the properties of the materials in contact.

Elastic and plastic indentation deformation in indentation (Doerner and Nix)
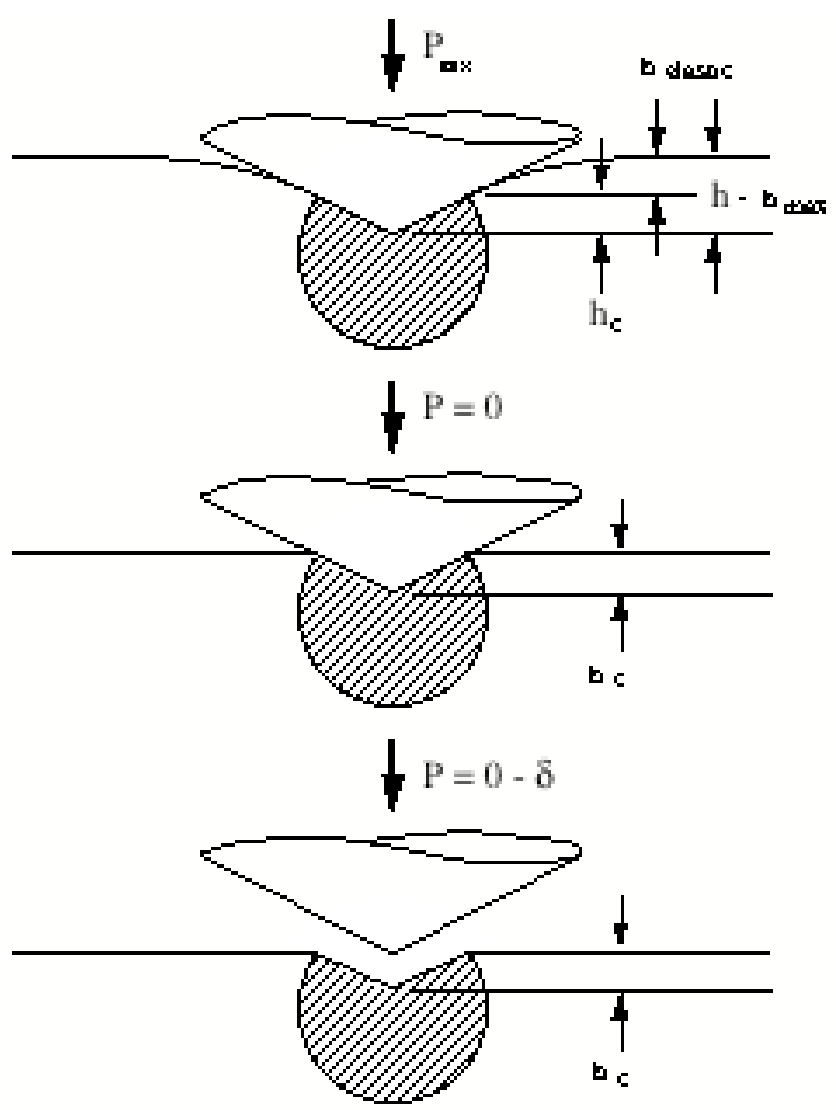

As illustrated in the diagram above, Doerner and Nix assumed that once a plastic impression had been made into the surface during nanoindentation, the contact area at the maximum load would not change significantly on unloading and the contact area could be determined from a measurement of the stiffness of the contact and the elastic properties of the materials, using Sneddon's relations. With these assumptions the measured compliance on unloading is predicted to be

$$
C_{\text {exp erimental }}=\frac{d h}{d P}=\frac{\sqrt{\pi}}{2} \frac{1}{E_{r} \sqrt{A}}+C_{\text {machine }}
$$


Alternatively, this relation can be used to find the contact area, if the elastic moduli of the indenter and the material are known. The following graphs shows that this contact relation is very well obeyed.

\section{Depth dependence of the indentation compliance for sapphire}

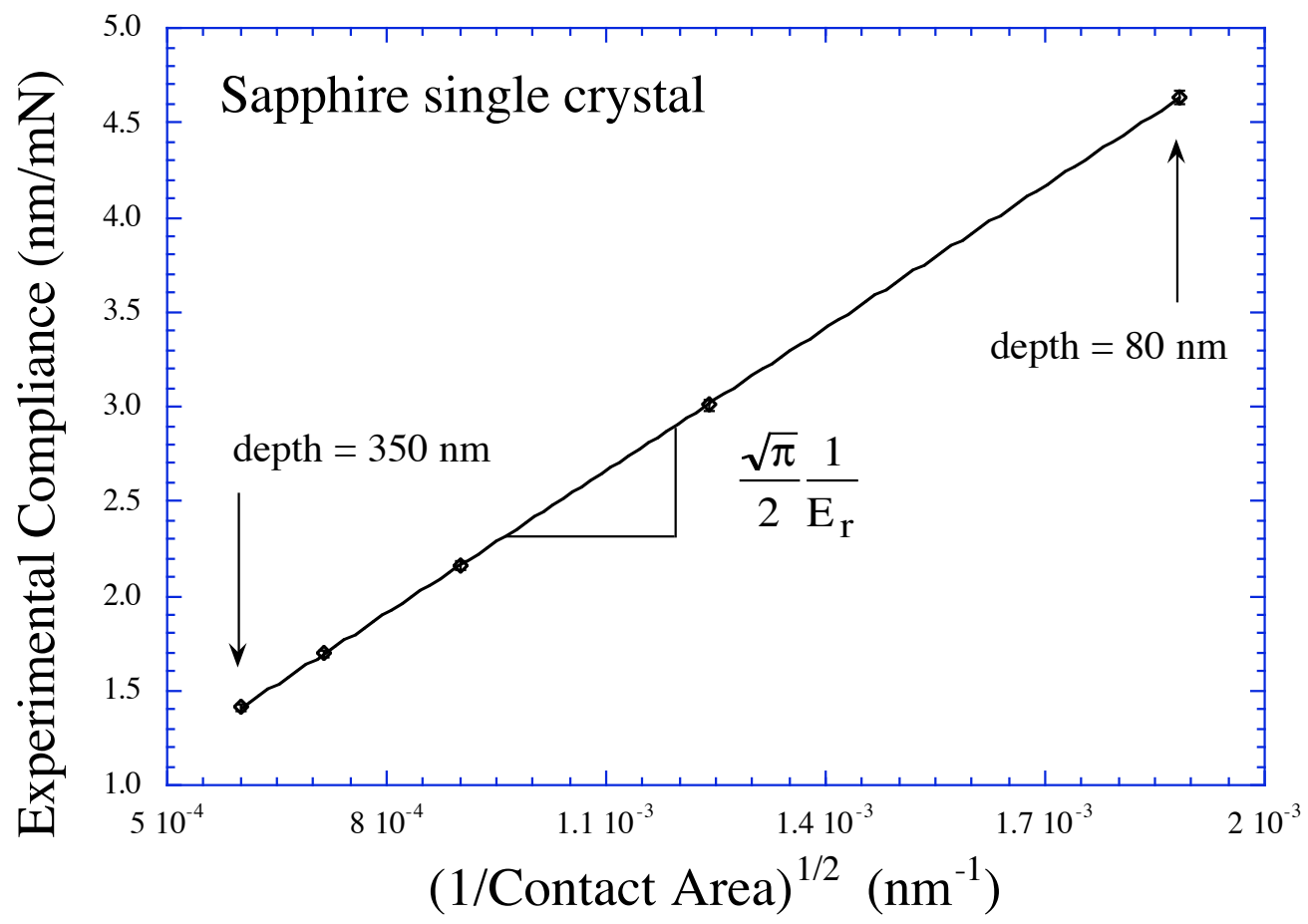

We note that the slope of the line in this figure is directly proportional to the reciprocal of the composite modulus, Er, and that the intercept at $1 / \sqrt{A_{c}} \rightarrow 0$ gives the machine compliance. For the case of a perfectly sharp indenter, the contact area, $A_{c}$, can be related to the contact depth $h_{c}$ through

$$
A_{c}=24.5 h_{c}^{2}
$$

leading to

$$
C_{\text {experimental }}-C_{\text {machine }}=\frac{1}{2} \sqrt{\frac{\pi}{24.5}} \frac{1}{E_{r}} \frac{1}{h_{c}} .
$$

for the depth dependence of the compliance. 
In the original Doerner and Nix method of analysis, the indenter tip area function was determined by making indentations of various plastic depths into a material and directly measuring the projected area of the impression by replica TEM techniques. The plastic or contact depth was determined by a simple linear fit to the unloading data, as shown in the diagram above. A master curve of observed contact area vs. plastic depth served as a calibration of the indenter shape. This method of analysis did account for the rounding of the tip of the diamond indenter and permitted accurate measurements of mechanical properties even at small depths where the shape of the diamond indenter is not ideal. Present methods of tip shape calibration involve the determination of the contact area and its dependent on contact depth from measurements of the contact stiffness in a material with known elastic properties (fused quartz).

\section{Oliver-Pharr Method of Analysis}

Warren Oliver and George Pharr pointed out that the slope of the unloading curve in nanoindentation experiments is not constant, as assumed by Doerner and Nix, and that the contact area is therefore also not constant on unloading. This is particularly obvious in the data for a hard material like fused quartz but it is also true for soft metals. Oliver and Pharr reasoned that a better contact model is one in which the contact area is permitted to change on unloading. As described below, they used Sneddon's treatment of a rigid cone indenting an elastic half space to model the contact area on unloading.

\section{Load-depth curve for nanoindentation of fused quartz}

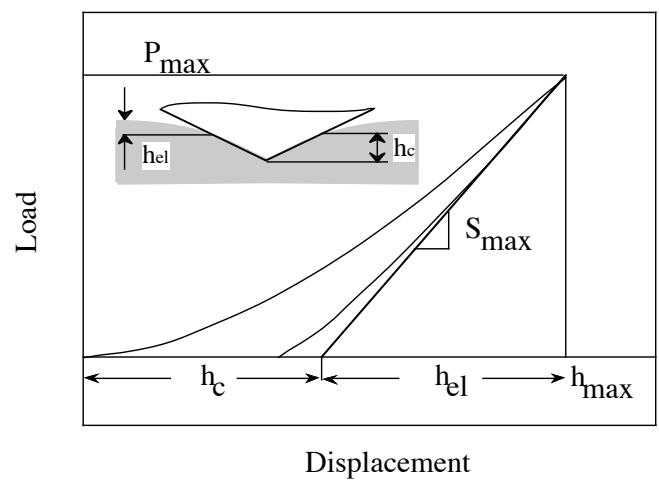

The Oliver-Pharr method of analysis can be understood best by considering the shape of the impression both under load and in the unloaded state. This is shown in the following diagram. 


\section{Oliver-Pharr analysis of unloading mechanics}
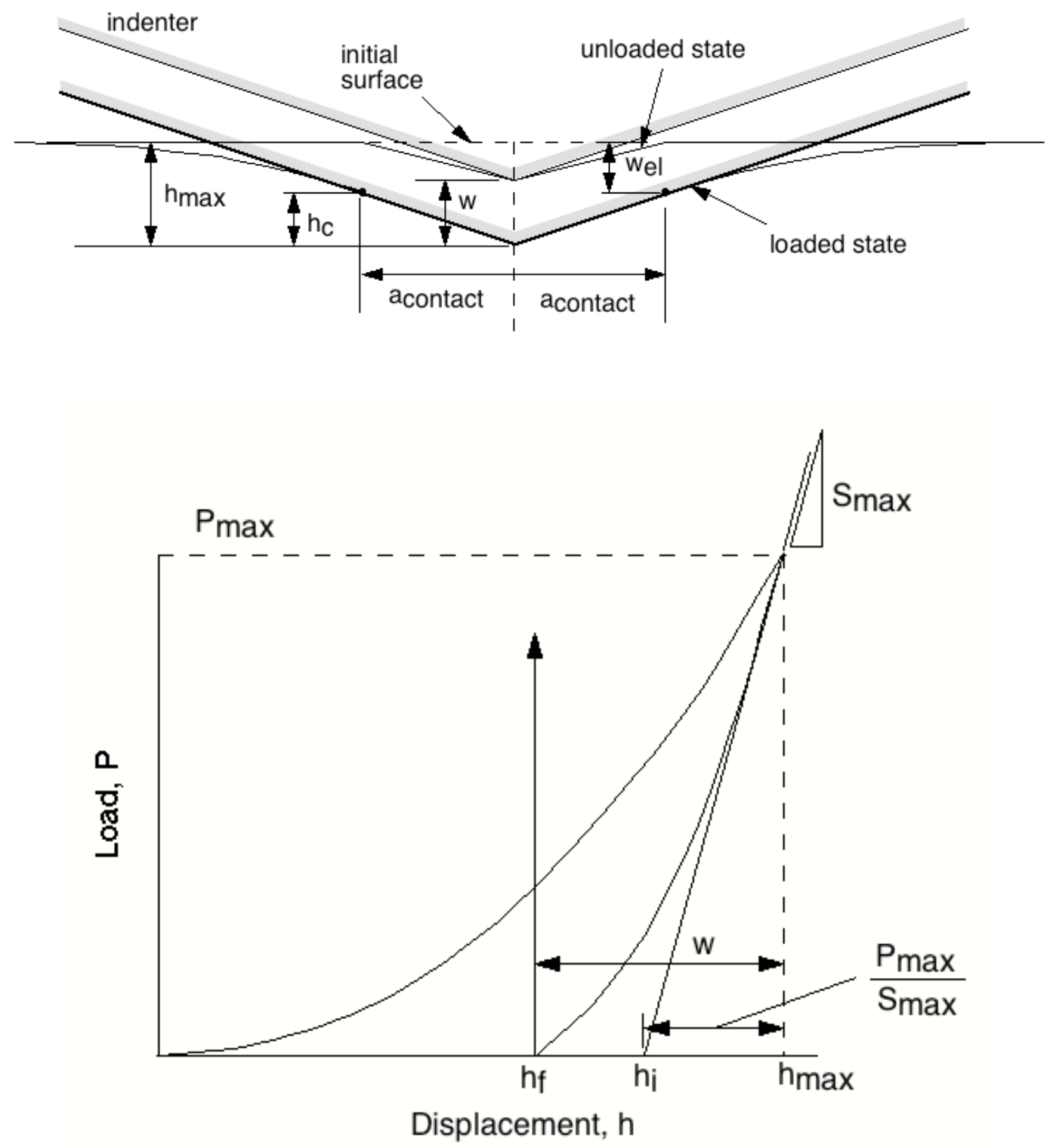

The changing contact area during unloading can be modeled by treating the contact problem as equivalent to the indentation of a flat elastic half space with a rigid cone. Sneddon has given the solution to this contact problem. 


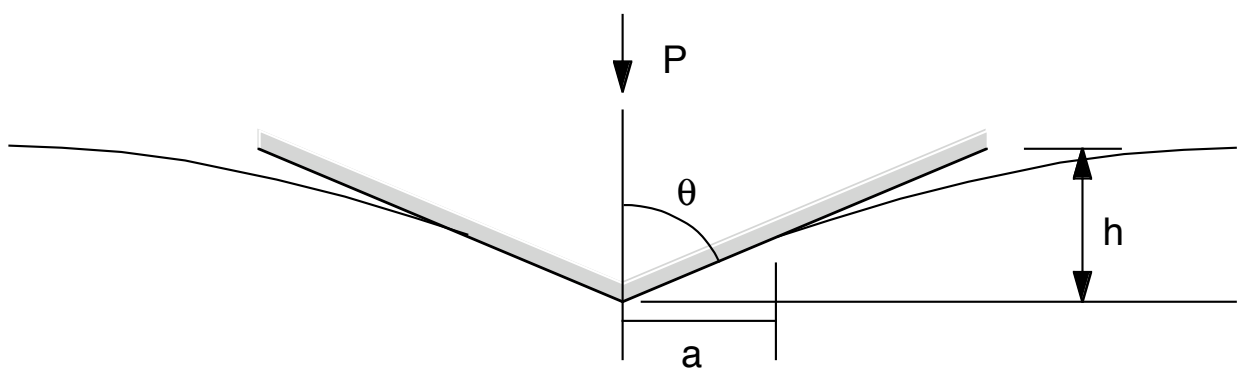

Sneddon gave the following solution for the endentation of an elastic half space by a rigid cone. The relationship between the depth of indentation, $h$, and the contact radius, a, is given by

$$
h=\frac{\pi a}{2 \tan \theta},
$$

where $\theta$ is the half angle for the cone indenter, as shown in the figure. The corresponding load at that depth is given by

$$
P=\frac{\pi a^{2} E}{2\left(1-v^{2}\right) \tan \theta} .
$$

Combining these two relations gives the load-depth relation for cone indentation:

$$
P=\frac{2 E \tan \theta}{\pi\left(1-v^{2}\right)} h^{2} .
$$

The stiffness of the contact at this point is then

$$
\frac{d P}{d h}=\frac{4 E \tan \theta}{\pi\left(1-v^{2}\right)} h .
$$

Sneddon also provided a solution for the displacement of the surface surrounding the indenter. 


$$
h_{e l}(r, 0)=\frac{2}{\pi}\left[\sin ^{-1}\left(\frac{a}{r}\right)-\frac{r}{a}+\frac{\sqrt{r^{2}-a^{2}}}{a}\right] h \text { for } r>a \text {. }
$$

Referring to the diagrams which describe the Oliver-Pharr method, we imagine the unloading curve (or, equivalently, the reloading curve if the indenter is pushed back into the impression) as being the same as the loading of an elastic half space with a rigid cone. With this picture, the unloading (reloading) curve can be described as

$$
P=\frac{2 E \tan \theta}{\pi\left(1-v^{2}\right)}\left(h-h_{f}\right)^{2}
$$

where $h_{f}$ is the final depth in the unloaded state. The stiffness at any point on the unloading curve is then

$$
\frac{d P}{d h}=S=\frac{4 E \tan \theta}{\pi\left(1-v^{2}\right)}\left(h-h_{f}\right)
$$

Again referring to the Oliver-Pharr diagrams, the contact depth $h_{c}$ is given by

$$
h_{c}=h_{\max }-w_{e l}
$$

where $h_{\max }$ can be directly measured and $w_{e l}$ can be found using the Sneddon model. Using the surface displacement formula given above and calling the surface displacement at the edge of the contact $w_{e l}$ and the total displacement for reloading $w$, we can write

$$
\begin{aligned}
& w_{e l}=\frac{2}{\pi}\left[\sin ^{-1}(1)-1\right]\left(h_{\max }-h_{f}\right) \\
& w_{e l}=\frac{2}{\pi}\left[\frac{\pi}{2}-1\right]\left(h_{\max }-h_{f}\right) \\
& w_{e l}=\left(1-\frac{2}{\pi}\right)\left(h_{\max }-h_{f}\right)
\end{aligned}
$$

But we also know from Sneddon's treatment that

$$
\frac{P_{\max }}{S_{\max }}=\frac{\left(h_{\max }-h_{f}\right)}{2},
$$


so the elastic displacement at the edge of the contact can be written as

$$
w_{e l}=\left(1-\frac{2}{\pi}\right) 2 \frac{P_{\max }}{S_{\max }} .
$$

Finally, the contact depth by the Oliver-Pharr method is given by

$$
\begin{aligned}
& h_{c}=h_{\max }-w_{e l} \\
& h_{c}=h_{\max }-2\left(1-\frac{2}{\pi}\right) \frac{P_{\max }}{S_{\max }} . \\
& h_{c}=h_{\max }-0.7268 \frac{P_{\max }}{S_{\max }}
\end{aligned}
$$

With this treatment, the contact depth can be determined directly from the loaddisplacement data. The contact area is then found using the calibrated tip area function. The Oliver and Pharr method of analysis described here is now universally adopted as the best way to analyse nanoindentation data.

As noted above, fused quartz is commonly used to calibrate the tip shape. In the figure below the measured contact compliance (the reciprocal of the stiffness) for fused quartz is shown as a function of the contact area based on the Oliver-Pharr method of finding the contact depth. The parameter $\varepsilon$ takes the value of $\varepsilon=0.75$ in this analysis instead of the value $\varepsilon=0.7268$ given for the ideal cone model

\section{Depth dependence of the unloading compliance for fused quartz}

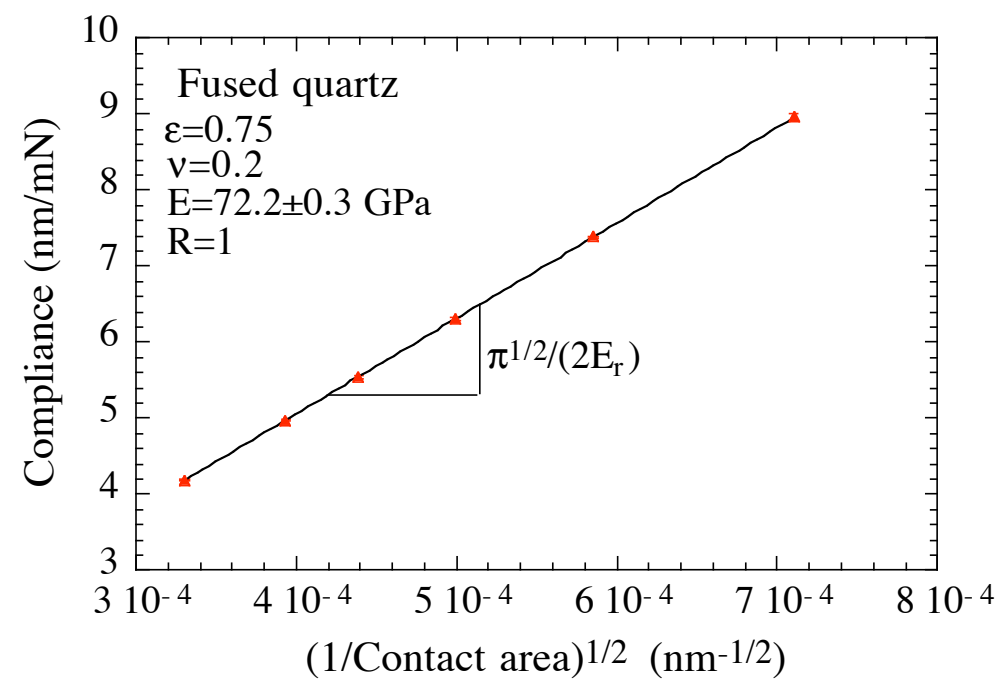


With a knowledge of the contact area, the hardness can be accurately determined.

\section{Hardness of fused quartz as a function of indentation depth}

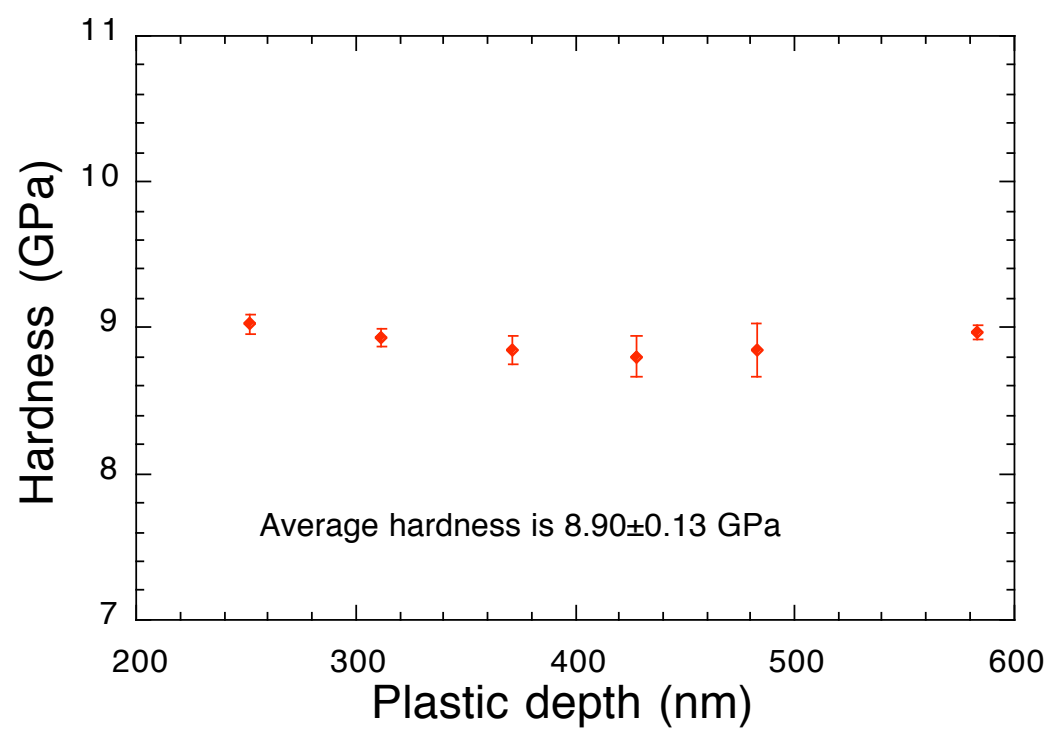

We note that the hardness of a hard material like fused quartz does not depend on the depth of indentation or the area of the contact. The hardness is an intrinsic material property.

In the following pages we give some typical results of nanoindentation experiments on various thin film materials. The methods of analysis given above have been used to obtain these results.

First we examine the indentation properties of various microelectronic thin films. In the following figures the hardnesses of $\mathrm{TiN}, \mathrm{Ti}$ and various $\mathrm{Al}-\mathrm{Cu}$ metallizations are shown as function of indentation depth. Clearly, TiN is much harder than $\mathrm{Ti}$, which is also harder than $\mathrm{Al}-\mathrm{Cu}$, as expected. The variation in hardness with depth at shallow indentations is an artifact of the contact area. These films are not ideally smooth so the contact area made after the indenter is pushed past the point of first contact is less than if the films were perfectly flat and smooth. If the contact area had been based not on the depth of indentation and the calibrated tip shape, but on the measured stiffness, then this artifact could be removed. However, the contact stiffness is not constant for a film on a substrate, so this method of analysis is not easy to use. Below we discuss the problem of contact stiffness for film/substrate structures. 


\section{Nanoindentation of various microelectronic thin films}

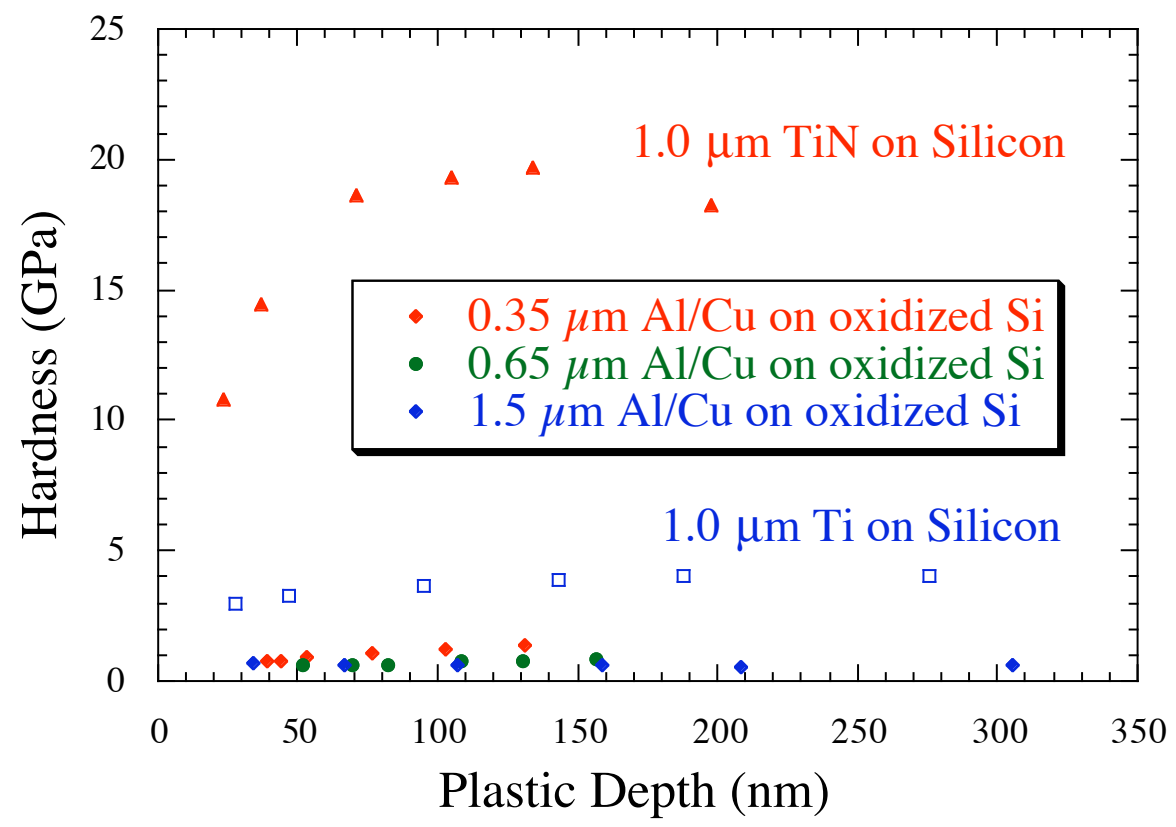

Nanoindentation of Al-Cu thin film metallizations

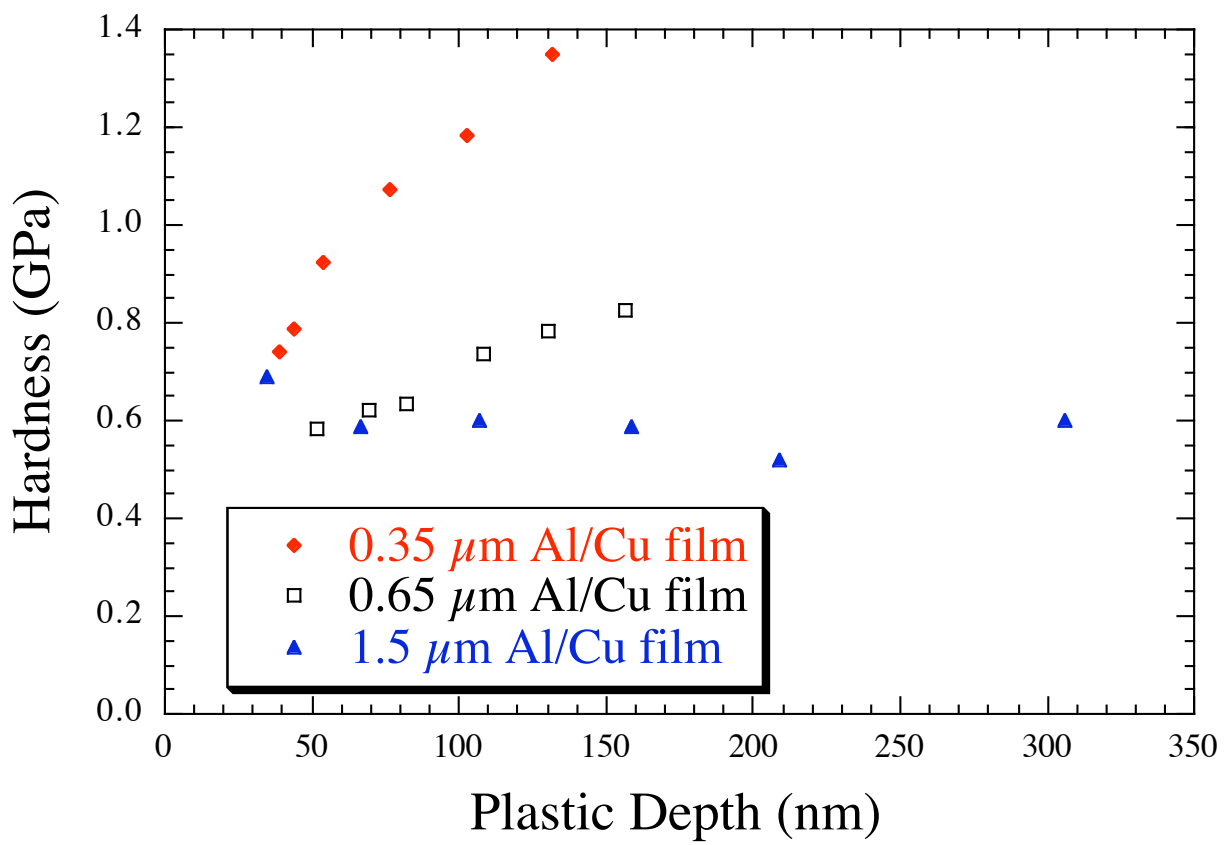

The data show that thinner Al-Cu films are stronger than thicker ones. We will see this relationship again when we study plasticity in thin metal films on substrates. 
Nanoindentation can be used to study the mechanical properties of strong metal multilayers. Shown below are data taken from the work of Jerry English, who studied the mechanical properties of epitaxial $\mathrm{Ag} / \mathrm{Cr}$ multilayers grown by sputter deposition onto $\mathrm{MgO}$ substrates. As discussed in earlier sections of these notes, when $\mathrm{Ag} / \mathrm{Cr}$ multilayers are grown onto (001) $\mathrm{MgO}$, the lattice match, after a 45 degree rotation is almost perfect. The hardening effects shown in these figures can be attributed both to the slip misorientation that is created in these structures and to the variation is elastic modulus in the multilayer. Dislocations cannot move easily from one layer to the next because of the structural barrier at the interface and the image forces that arise because of the elastic mismatch.

\section{Indentation properties of $\mathrm{Ag} / \mathrm{Cr}$ multilayers on $\mathrm{MgO}$}

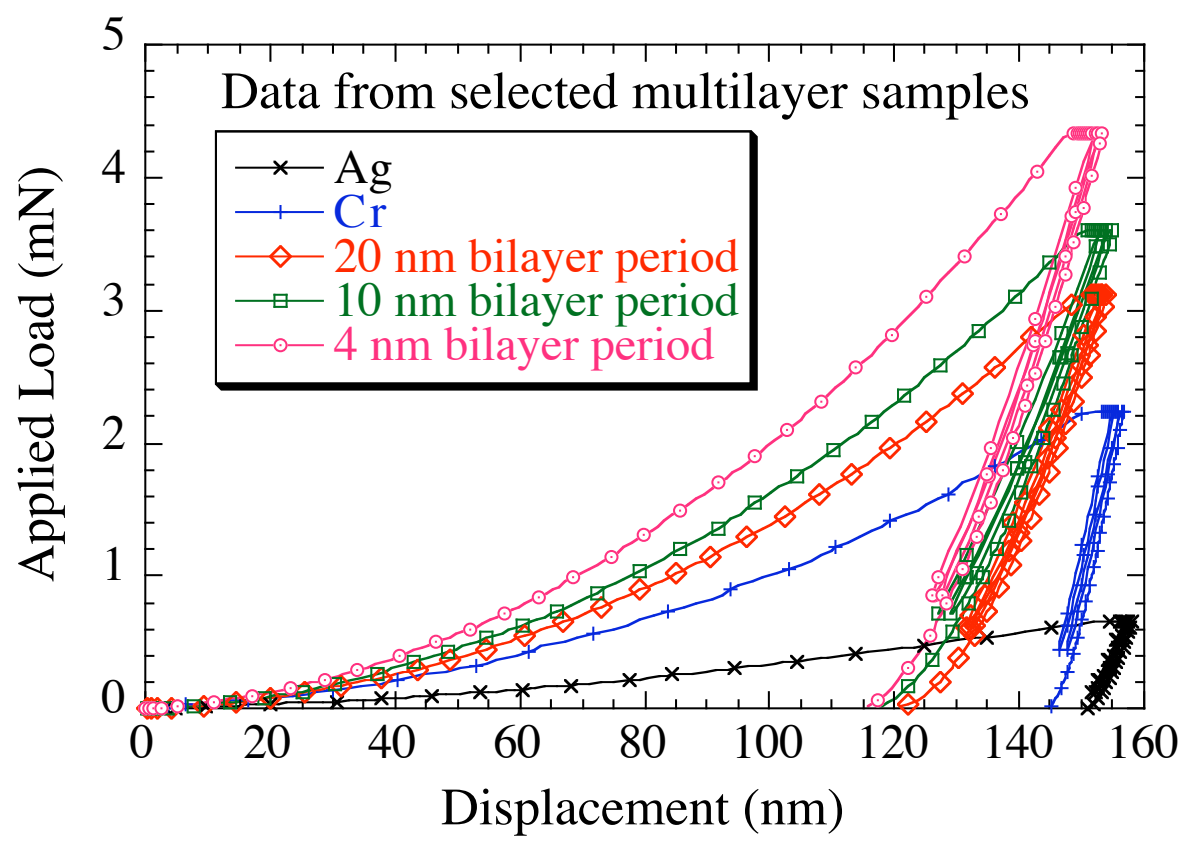

The hardness of a selected multilayer is compared with the hardness of epitaxial $\mathrm{Ag}$ and epitaxial $\mathrm{Cr}$ in the figure below. The multilayer is harder than either of the two single layers because of the fine structure of the multilayer. We also note that these hard materials do not exhibit a significant depth dependence of the hardness. This is expected because these materials are already quite hard because of their small microstructural dimensions. 
Hardness of a $\mathrm{Ag} / \mathrm{Cr}$ mulitlayer as a function of indentation depth compared to the hardness of single epitaxial layers of $\mathrm{Ag}$ and $\mathrm{Cr}$.

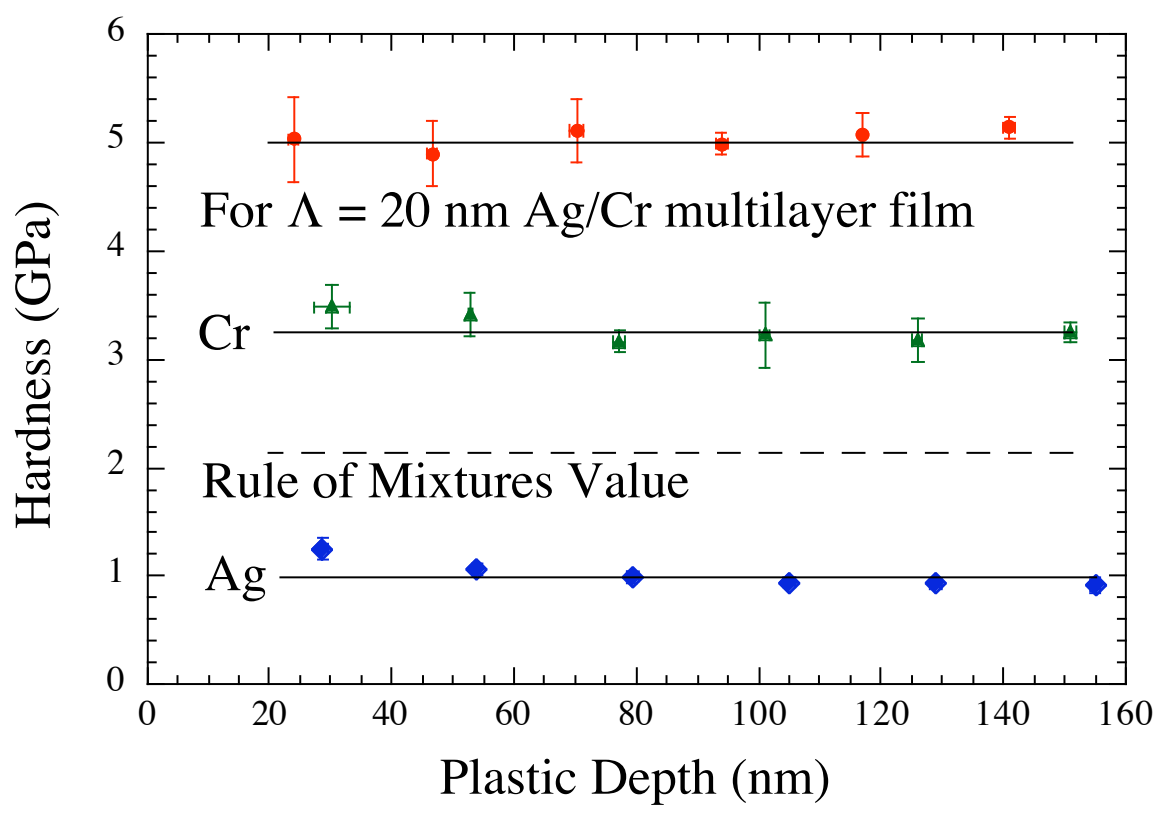

\section{Elastic Anisotropy}

It is reasonable to suppose that elastic anisotropy might be measured by nanoindentation, if single crystals of different orientatations are tested.

However, Joost Vlassak showed that such effect of elastic anisotropy are quite small, primarily because the displacement field associated with indentation experiment involves displacements in essentially all directions in the crystal. Results for the indentation of both tungsten (isotropic) and beta brass (very anisotropic) are shown in the attached diagrams. Even for highly anisotropic crystals such as beta brass, the indentation moduli are not very sensitive to the plane of indentation. 
Indentation of tungsten (isotropic) and beta brass (anisotropic) single crystals
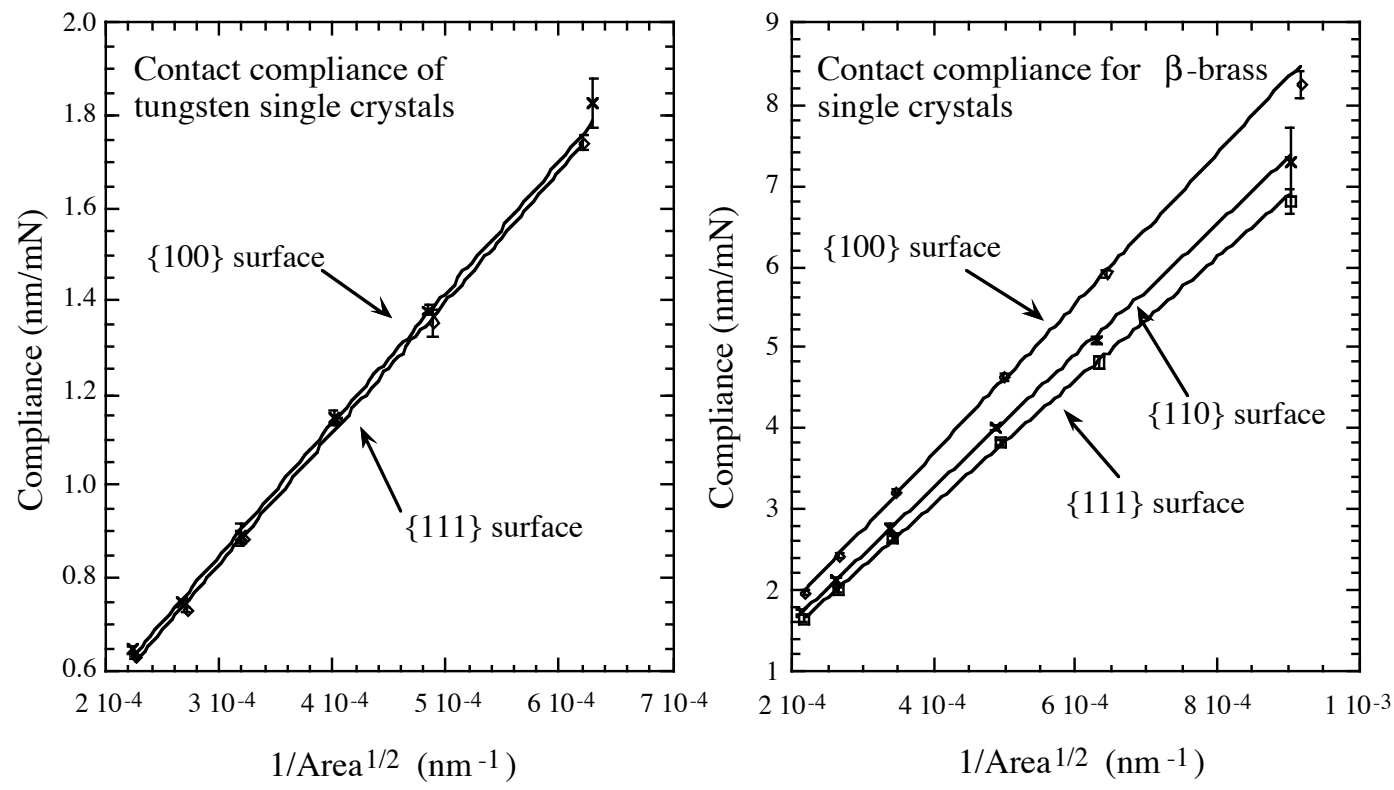

Vlassak made an analysis of the expected effect of anisotropy on indentation modulus. Vlassak calculated the indentation modulus for various crystals and compared the calculations with measured indentation moduli in different directions.

Comparison of calculated and measured indentation moduli for various crystals

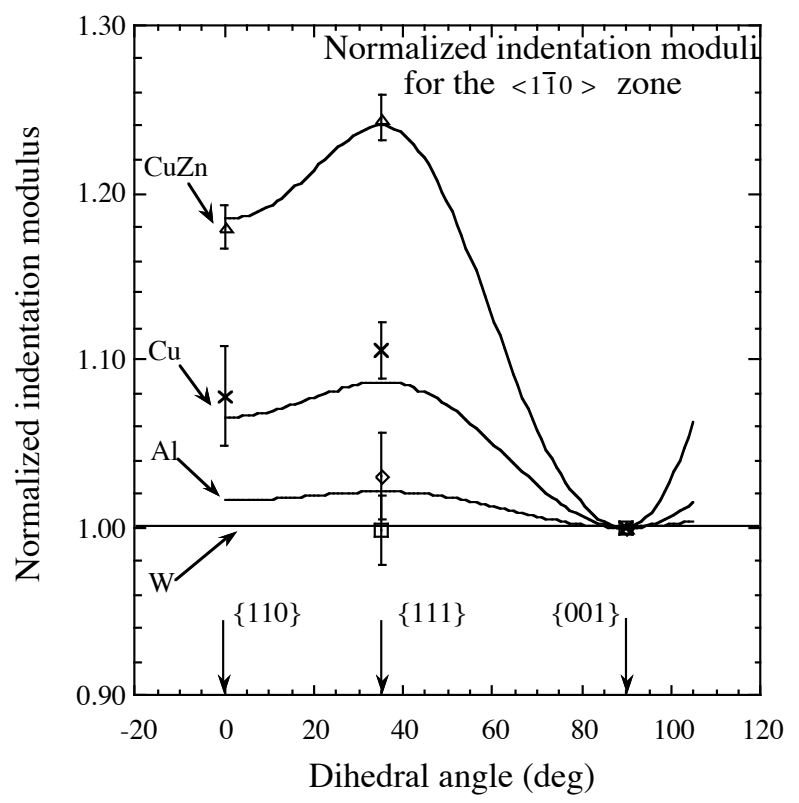




\section{Elastic Properties of Thin Films on Substrates}

The contact compliance in nanoindentation of an elastically homogeneous material can be expressed as

$$
C_{\text {contact }}=\frac{\beta}{E_{r}} \frac{1}{h_{c}},
$$

where

$$
\beta=\frac{1}{2} \sqrt{\frac{\pi}{24.5}} .
$$

Multiplying both sides of the equation by the film thickness, $t_{f}$, gives

$$
C_{\text {contact }} \bullet t_{f}=\frac{\beta}{E_{r}}\left(\frac{t_{f}}{h_{c}}\right) .
$$

As shown in the figure, if the film thickness is much greater than the indentation depth, the elastic properties of the film should be those of the film, whereas if the film thickness is small compared to the indentation depth, the elastic properties of the substrate dominate the indentation response.

Schematic indentation of a thin film on a substrate

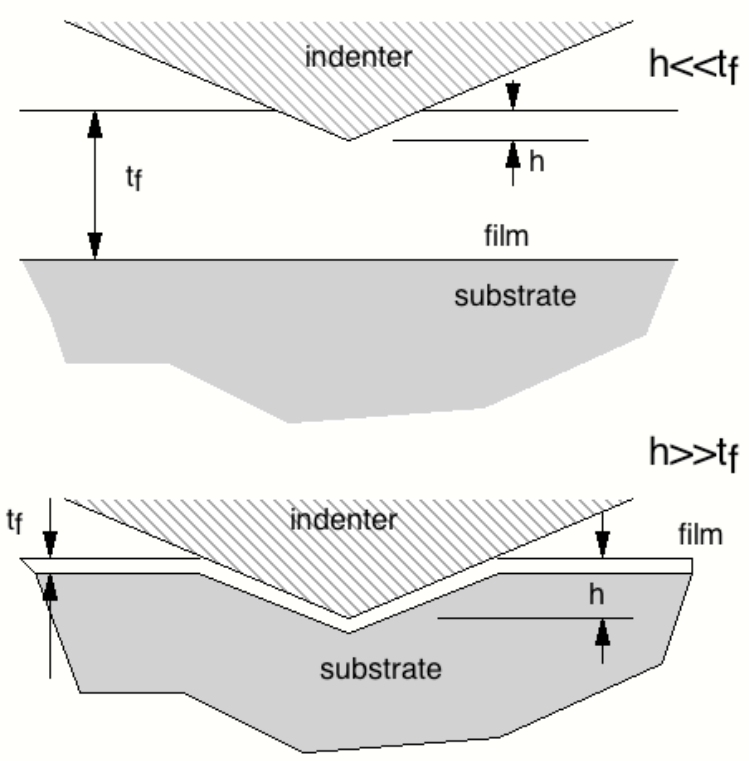


As shown for the indentation of tunsgten thin films on silicon substrates, the measured contact compliance follows the properties of the film for shallow indentations and the substrate for deep indentations.

\section{Contact compliance of W films on Si substrates}

\section{Indentation Elastic Properties of Films on Substrates}

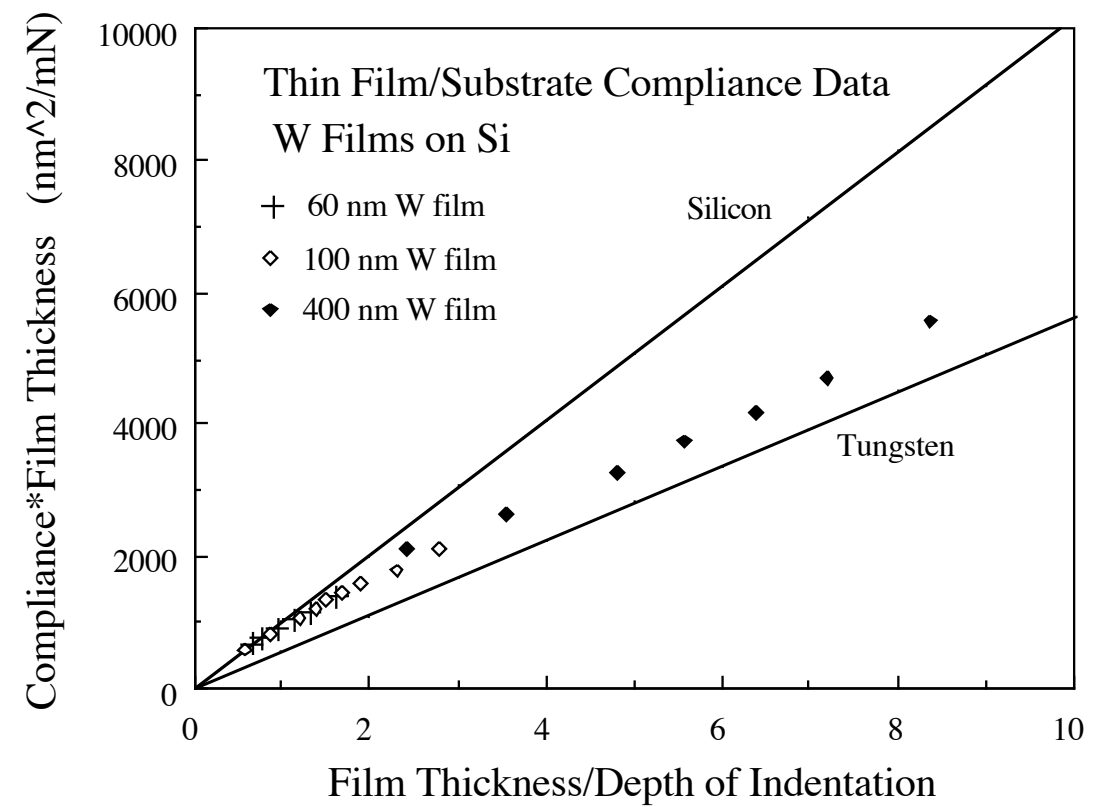

\section{Pile-up and Sink-in}

Most of the results discussed above have been based on the Oliver-Pharr method for determining the contact area. This involves determining the contact area from the measured depth of indentation and the shape of the indenter, based on calibration studies with fused quartz. This procedure will work well if the material plastically deforms like fused quartz. In the following figures the shape of the indentation in annealed and cold worked $\mathrm{Cu}$ are shown for comparison. Annealed $\mathrm{Cu}$ behaves much like fused quartz, showing a sink-in effect. Notice that the edges of the impression are not straight but instead are bowed in. This is called sink-in as the indenter sinks into the material elastically. For cold-worked $\mathrm{Cu}$ a pile-up effect is observed. Here the metal pushes up the sides of the indenter increasing the contact area with the indenter. When significant pile-up occurs, the best way to determine the contact area is to measure the stiffness of the contact and calculate the contact area using one of the Sneddon relations. 